\title{
Towards better diagnosis and monitoring of asthma and cystic fibrosis in children : the value of non- invasive inflammometry
}

Citation for published version (APA):

Robroeks, C. M. H. H. T. (2010). Towards better diagnosis and monitoring of asthma and cystic fibrosis in children : the value of non-invasive inflammometry. [Doctoral Thesis, Maastricht University]. Datawyse / Universitaire Pers Maastricht. https://doi.org/10.26481/dis.20100527cr

Document status and date:

Published: 01/01/2010

DOI:

10.26481/dis.20100527cr

Document Version:

Publisher's PDF, also known as Version of record

Please check the document version of this publication:

- A submitted manuscript is the version of the article upon submission and before peer-review. There can be important differences between the submitted version and the official published version of record.

People interested in the research are advised to contact the author for the final version of the publication, or visit the DOI to the publisher's website.

- The final author version and the galley proof are versions of the publication after peer review.

- The final published version features the final layout of the paper including the volume, issue and page numbers.

Link to publication

\footnotetext{
General rights rights.

- You may freely distribute the URL identifying the publication in the public portal. please follow below link for the End User Agreement:

www.umlib.nl/taverne-license

Take down policy

If you believe that this document breaches copyright please contact us at:

repository@maastrichtuniversity.nl

providing details and we will investigate your claim.
}

Copyright and moral rights for the publications made accessible in the public portal are retained by the authors and/or other copyright owners and it is a condition of accessing publications that users recognise and abide by the legal requirements associated with these

- Users may download and print one copy of any publication from the public portal for the purpose of private study or research.

- You may not further distribute the material or use it for any profit-making activity or commercial gain

If the publication is distributed under the terms of Article $25 \mathrm{fa}$ of the Dutch Copyright Act, indicated by the "Taverne" license above, 
Towards better diagnosis and monitoring of asthma and cystic fibrosis in children the value of non-invasive inflammometry

Charlotte MHHT Robroeks 
Financial support for the publication of this thesis was supported by - Abbott B.V.

- Astma Fonds Longstichting

- AstraZeneca B.V.

- Bayer Health Care B.V.

- Hero Nederland B.V., Friso Kindervoeding

- Luminex B.V.

- Prof.dr. Jaap Swieringa Stichting

- Romedic B.V.

- Stichting Kindergeneeskunde Oost Zuid Limburg

- Teva Pharma Nederland B.V.

ISBN 9789052789491

(C) Copyright CMHHT Robroeks, Maastricht 2010

Printed by Datawyse / Universitaire Pers Maastricht 


\title{
Towards better diagnosis and monitoring of asthma and cystic fibrosis in children the value of non-invasive inflammometry
}

\author{
PROEFSCHRIFT \\ ter verkrijging van de graad van doctor aan de Universiteit Maastricht, \\ op gezag van de Rector Magnificus, Prof. mr. G.P.M.F. Mols, \\ volgens het besluit van het College van Decanen, \\ in het openbaar te verdedigen \\ op donderdag 27 mei 2010 om 16.00 uur \\ door
}

Charlotte Magdalena Hubertus Hubertina Theodorus Robroeks geboren op 24 juni 1978 te Boxtel

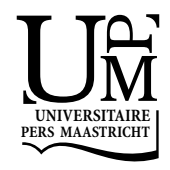


Promotor

Prof. dr. L.J.I. Zimmermann

\section{Copromotores}

Dr. E. Dompeling

Dr. Q. Jöbsis

\section{Beoordelingscommissie}

Prof. dr. C.P. van Schayck (voorzitter)

Prof. dr. C.A. Bruggeman

Prof. dr. P.J. Sterk (Universiteit van Amsterdam)

Dr. G.J. Wesseling 
Voor mijn ouders

Aan Sander 


\section{Contents}

1 General introduction 9

\section{Section 1 Methodology}

2 Feasibility of exhaled nitric oxide measurements at various flow rates in children with asthma. Pediatric Allergy and Immunology 2010;21(1):e222e228

3 Biomarker reproducibility in exhaled breath condensate collected with different condensers. European Respiratory Journal 2008;31(5):934-942

$4 \quad$ Feasibility of a new method to collect exhaled breath condensate in preschool children. Pediatric Allergy and Immunology 2010;21(1):e235-e244

\section{Section 2 Diagnosis}

$5 \quad$ Cytokines in exhaled breath condensate of children with asthma and cystic fibrosis. Annals of Allergy Asthma and Immunology 2006;96(2):349-355

6 Increased cytokines, chemokines and soluble adhesion molecules in exhaled breath condensate of asthmatic children. Clinical and Experimental Allergy 2010;40(1):77-84

7.1 Exhaled nitric oxide and biomarkers in exhaled breath condensate indicate the presence, severity and control of childhood asthma. Clinical and Experimental Allergy 2007;37(9):1303-1311

7.2 Biomarkers in exhaled breath condensate indicate presence and severity of cystic fibrosis in children. Pediatric Allergy and Immunology 2008;19(7):652659

$8 \quad$ Structural lung changes, lung function, and inflammatory markers in cystic fibrosis. Pediatric Allergy and Immunology, in press

9.1 Volatile organic compounds in exhaled breath as a diagnostic tool for asthma in children. Clinical and Experimental Allergy 2010;40(1):68-76

9.2 Metabolomics of volatile organic compounds in cystic fibrosis patients and controls. Pediatric Research, published ahead of print 


\section{Section 3 Monitoring}

10 Comparison of the anti-inflammatory effect of extra-fine HFA-beclomethasone versus fluticasone-DPI on exhaled inflammatory markers in childhood asthma. Annals of Allergy Asthma and Immunology 2008;100(6):601-607

11.1 Non-invasive inflammatory markers in exhaled breath condensate predict exacerbations of childhood asthma; results of a one year prospective study, submitted

11.2 Cystic fibrosis exacerbations are predicted by non-invasive inflammatory markers in exhaled breath condensate: a one year longitudinal study, submitted

12.1 Volatile organic compounds in exhaled breath predict exacerbations of childhood asthma in a one year prospective controlled study, submitted

12.2 Volatile organic compounds in exhaled breath predict exacerbations in cystic fibrosis: results in a one-year longitudinall study, submitted

\section{Discussion \& Summary}

13 General discussion $\quad 241$

14 Summary 265

Samenvatting 273

$\begin{array}{ll}\text { Dankwoord } & 281\end{array}$

$\begin{array}{ll}\text { Curriculum Vitae } & 285\end{array}$

List of publications $\quad 286$ 



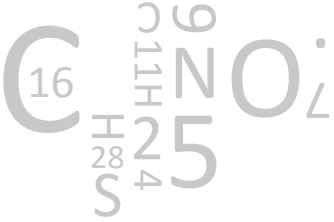

CHAPTER 1

General introduction 


\section{Contents}

1.1 Asthma in children

1.1.1 Social burden

1.1.2 Aetiology

1.1.3 Treatment

1.1.4 Problems in daily practice

1.2 Cystic fibrosis

1.2.1 Social burden

1.2.2. Aetiology

1.2.3 Treatment

1.2.4 Problems in daily practice

1.3 The additional value of non-invasive inflammometry

1.4 Methodology of non-invasive inflammometry assessments

1.4.1 Fractional exhaled nitric oxide

1.4.2 Volatile organic compounds

1.4.3 Exhaled breath condensate

1.5 Objectives of this thesis: methodology, diagnosis, monitoring 


\subsection{Asthma in children}

\subsubsection{Social burden}

Asthma is the most common occurring chronic disease in childhood with a prevalence of $5-10 \%[1,2]$. The World Health Organization (WHO) estimates that 300 million people currently suffer from asthma worldwide which makes asthma a major public health problem [1]. In the past 30 years, a marked increase in the prevalence of asthma was observed in many Western countries worldwide [3]. In the Netherlands, a 5-fold increase in the prevalence of asthma occurred during this period [4]. The reasons for this increase are poorly understood, as well as the reasons for a recent stabilisation or even a small decrease in prevalence [4]. The consequences of childhood asthma for daily life are huge and comprise respiratory complaints, diminished quality of life, disturbed physical activities, school absence or work absence for the parents, extra visits to a doctor, emergency care visits, and hospital admissions $[2,5]$. The financial burden on patients with asthma in different Western countries ranges from $\$ 300$ to $\$ 1,300$ per patient per year, mainly affecting those with the most severe disease [6].

\subsubsection{Aetiology}

Asthma is defined as a disease with intermittent symptoms of coughing, wheezing and breathlessness, based on a variable but reversible airway obstruction and a chronic airway inflammation [2]. Asthma has a multifactorial origin with both genetic and environmental causes and gene-environment interactions [7]. It is a heterogeneous disease in which classifications can be defined on basis of phenotypes. These phenotypes are based on a wide variety of parameters: 1) symptoms (age of onset, severity), 2) triggers (atopy, exercise induced, virus triggered, multiple trigger wheeze, exacerbation prone), 3) response to treatment (mainly to corticosteroids), 4) pathophysiological (eosinophilic or neutrophilic inflammation in biopsies, induced sputum samples and broncho-alveolar lavage), 5) physiological measurements (bronchial hyperresponsiveness, severity of airway obstruction), and 6) genetic polymorphisms $[8,9]$. There is a major overlap between these phenotypes [10]. At present, multivariate cluster analysis is being used to reorganize classification of asthma phenotypes in order to increase understanding of asthma aetiology [11]. An increased number of studies are being performed concerning the genetics of asthma. For example, genes discovered to be involved in the pathophysiology of asthma include toll-like receptor genes [12, 13], which may be X-chromosome linked [14] and genetics of interferon regulatory factor (IRF)-1 [15-17]. The relationship between phenotypes, genotypes and responds to treatment is not clear yet.

Asthma exacerbations during which patients experience an increase in respiratory symptoms may be provoked by: 1) viral infections, most commonly rhinovirus, respiratory syncytial virus and influenza. In 2008, it is estimated that $80 \%$ of the asthma exacerbations were associated with viral infection [18]. Mononuclear cells are activated in the airways. Consequently, airway monocytes, macrophages and dendritic cells secrete proinflammatory cytokines, adhesion molecules and chemokines excite inflammatory cell recruitment. The neutrophil response accompanied with acute respiratory infections contribute to airway obstruction and bronchial hyperresponsiveness. 2) Atopy and allergic 
rhinitis. This inflammatory process is mainly based on mast cell degranulation and an eosinophilic respons. In addition, activation of regulatory T-lymphocytes result in a dysbalance of T-helper 1 and 2 lymphocytes, which is the major immunological pathway behind atopy. 3) Non-specific stimuli like smoke, fogg, cold air, and exercise.

\subsubsection{Treatment}

Asthma cannot be cured, but it can be controlled. The goals of treatment are to prevent ongoing symptoms, asthma exacerbations, to reduce severity of asthma exacerbations, to improve quality of life and to minimize drug use. Standard care of children with asthma implies regular visits to a doctor, checks of inhalation technique and treatment compliance, education, and measurements of lung function [2]. Both general practitioners and specialists (paediatricians or paediatric pulmonologists) participate in the care of children with asthma. In addition, it is recognized that an asthma nurse or nurse practitioner contributes to the care to children with asthma [2].

National and international guidelines are available which describe treatment in a stepwise manner $[2,19]$. Non-medication treatment includes awareness of asthma triggers in order to avoid them. More knowledge of the disease improves treatment compliance. Asthma medications for long term control include: 1) Corticosteroids, 2) Mast cell stabilizers, 3) Long acting beta-agonists, 4) Theophylline, 5) Leukotriene receptor antogonists, 6) Immunoglobulin E (IgE) blockage. In addition, medications to quickly relief symptoms of asthma attack are used: 1) Inhaled short acting beta-agonists, 2) Inhaled anticholinergics, 3) Systemic corticosteroids.

\subsubsection{Problems in daily practice}

The purpose of treatment is to achieve optimal asthma control [2]. However, in spite of marked progress in several aspects of asthma management and treatment in the past 3050 years, worldwide large problems exist with the control of asthma in both adults and children. Several cross-sectional surveys demonstrated that actual levels of asthma control fall far below the goals of national and international guidelines [20, 21]. Several Asthma Insights and Reality (AIR) surveys assessed the severity, the control, and management of asthma in children and adults by means of cross-sectional surveys [20, 21]. Despite variations at a country level, all these studies indicated that the level of asthma control falls short of the goals for long-term management in international guidelines [20, 21]. This effect was also evident in children of an outpatient population studied by Hammer et al [22]. One of the most likely explanations for this finding is that current monitoring of the disease does not include parameters of airway inflammation but only symptoms and lung function $[23,24]$.

It is known that only a low correlation exists between parameters of airway inflammation and respiratory symptoms and lung function [23, 24]. So far, monitoring of asthma and the guiding of anti-inflammatory treatment is based on assessments of respiratory symptoms and lung function [2]. Probably asthma control and the exacerbation rate will greatly improve when parameters of airway inflammation are included in the titration of treatment. The question is which parameters are most useful in this respect. The standard method of airway inflammation -bronchoscopy with bronchial biopsies or bronchoalveolar lavage- 
has to be performed under general anaesthesia in children and is far too invasive for routine use. Fleming et al showed a significant decrease of the number of exacerbations and the severity of symptoms when asthma follow-up included an inflammation based approach by means of sputum samples [25]. However, sputum induction and measurements of bronchial hyperreactivity give discomfort to patients, are time-consuming, and have a variable success rate. Therefore they are not very useful in children [26, 27].

\subsection{Cystic fibrosis}

\subsubsection{Social burden}

The mean prevalence of CF disease in Europe is $0.737 / 10,000$ [28]. In the Netherlands, all patients are being treated in 7 CF centres. Data of the European lung foundations show that $34 \%$ of European CF patients are adults. As survival rates improve, there are marked age-related changes in healthcare resource use. Older patients generally require increased care and more expensive resources. Treatment costs of a newly diagnosed infant is approximately $€ 3,000$ per year. For an adult with advanced disease, costs may even rise to $€ 40,000$ per year. Admission in the hospital is nessecary in approximately $15 \%$ of CF patients each year. The mean duration of an admission is 22 days. The number of lost work days for patients with CF varies according to the degree of disability. For patients whose disease is very advanced, lung transplantation may be an option. $0.6 \%$ Of CF patients per year have an organ transplant (double lung, heart and lung or liver). Meachery et al. [29] reported outcomes of lung transplantation in CF in the United Kingdom. In $95 \%$ of the patients, a bilateral sequential lung transplantation was performed. Median age at transplantation was 26 years. $\mathrm{FEV}_{1} \%$ of predicted values improved from $21 \%$ (median pretransplantation) to $78 \%$ (1 year after transplantation). In the first month, the acute rejection rate was $41 \%$. Survival rates were, at $1,3,5$ and 10 years were $82 \%, 70 \%, 62 \%$ and $51 \%$, respectively. Bronchiolitis obliterans syndrome and sepsis were common causes of death. In addition, Bech et al [30], presented outcome data of lung transplantation over a period of 10 years in Denmark. Data were comparable with the outcomes reported by Meachery et al. Median age at transplantation was 29 years. Survival of transplanted patients at 1, 3, 5 and 8 years was $89,80,80$ and 70\%, respectively. Causes of death of transplanted patients were: respiratory failure, bronchiolitis obliterans syndrome, infection, and bronchial anastomosis dehiscence. Pulmonary function ( $\mathrm{FEV}_{1} \%$ predicted) improved from median $20 \%$ (pre-transplantation) to $71 \% 5$ years post-transplantation. Survival of non-transplanted patients on the waiting list at 1 and 2 years was 28 and $11 \%$, respectively.

\subsubsection{Aetiology}

Cystic fibrosis (CF) is an autosomal recessive disorder, caused by genetic mutations in CF transmembrane conductance regulator (CFTR), a protein responsible for the movement through the cell membranes of chloride ion. Over 600 mutations can cause CF. However, the most frequent mutation is the deltaF508 CFTR. In the Netherlands, this mutation accounts for $78 \%$ of the mutations. CFTR is present in cells that line the passage ways of the 
lungs, pancreas, colon, and genitourinary tract. Defective CFTR causes impaired or absent transport of chloride through cell-membranes, with an impaired mucociliary clearance, and viscous mucus in the airways. Mucus in CF patients is very thick and accumulates in the intestines and lungs. There is a wide variety of CF phenotypes among individuals with a similar CFTR genotype. This implies that factors independent of CFTR influence outcome in CF. To date, a definitive modifier gene for CF remains to be identified. Identifying the factors contributing to variation in pulmonary disease, the primary cause of mortality, remains a challenge for $\mathrm{CF}$.

\section{CF lung disease}

Chronic airway infection, inflammation and oxidative stress, mainly due to neutrophilic immune responses, causes a progressive decline in lung function and irreversible structural pulmonary damage [31-34]. Pulmonary disease accounts for more than $90 \%$ of deaths in CF [35]. Airway inflammation in patients with CF is persistent and already present early in life $[36,37]$. Exaggerated inflammatory responses are present, resulting in irreversible structural changes of the airways $[38,39]$. This leads to progressive airway obstruction and restriction, eventually resulting in respiratory failure. Intermittent pulmonary exacerbations and chronic infections accelerate progression of CF lung disease. Moreover, defective mucociliary action and failure of the innate defence mechanisms of the lung against inhaled bacterial organisms results in inability of the airways to clear bacteria [32, 34, 40]. The result is malnutrition, poor growth, recurrent respiratory infections, breathing difficulties, and eventually irreversible lung damage. Other problems caused by CF include sinusitis, nasal polyps, abdominal pain and discomfort, gassiness, rectal prolapse, liver disease, diabetes, inflammation of the pancreas, and gallstones. Although the genetic defect responsible for CF is well described, the exact mechanism of lung tissue damage remains unclear [41]. One of the questions is whether lung inflammation follows bacterial infections or vice versa. Accumulating evidence suggests that in CF early pulmonary inflammation may be associated with an abnormal increase of the production of pro-inflammatory cytokines in the lung, even in absence of infectious stimuli [42]. Even in stable patients, chronic airway inflammation is present, as reflected by high airway fluid concentrations of pro-inflammatory cytokines [43]. Analysis of bronchoalveolar lavage (BAL) fluid has shown a 1.000 fold increase in the number of neutrophils from the lung of patients with CF compared to controls [33].

\subsubsection{Treatment}

The main goals of treatment in CF are 1) prevention and reduction of airway infection and inflammation, 2) to optimize the nutrition status and 3) the physical condition of the patient.

To manage lung problems, physical therapy, exercise, and medications are used to reduce the mucus blockage of the airways. Drug treatment include bronchodilators, mucolytics and antibiotics. In addition, in order to optimise the gastro-intestinal condition, a highcaloric diet, low in fat and high in protein is recommended, and pancreatic enzymes combined with fat-soluble vitamines $(A, D, E, K)$ are often prescribed. 


\subsubsection{Problems in daily practice}

Although patients with CF nowadays live longer and have an improved quality of life, the disease remains progressive and CF lung disease is the major cause of mortality. Although airway inflammation is very important in the pathophysiology of CF lung disease, direct measurements of inflammation and inflammatory markers (IM) play a surprisingly small role in the diagnosis and monitoring of this disease. Methods available to obtain information about the presence of inflammation in the airways, like induced sputum or bronchoscopy with BAL and biopsies, are too invasive to apply routinely or repeatedly in children [44]. Severity and control of pulmonary CF disease is currently based mainly on lung function deteriorations, pulmonary symptoms, energy level and nutrition status [31, 32, 45]. Lung function parameters reflect presence and severity of restriction and obstruction of the overall airway status [46]. These parameters have a relative slow rate of change, and, thereby, this technique is insensitive to detect early pulmonary damage $[47,48]$. Pulmonary symptoms are the result of restriction and obstruction of the airways [31, 32]. Pathological lung changes start miniscule, and, therefore, do not always lead to pulmonary symptoms [49]. In conclusion, lung function parameters and presence of symptoms do not reflect ongoing inflammation in a direct manner and treatment cannot be adjusted based on inflammation activity but only on the consequences of CF lung disease.

\subsection{The additional value of non-invasive inflammometry}

The consequence of monitoring airway inflammation, instead of only airway obstruction or symptoms, is that exacerbations may be detected in an early stage, and the effects of treatment can be better evaluated. Early detection of exacerbations of chronic inflammatory diseases implies that early, appropriate treatment can be started, which probably can prevent the development of severe exacerbations, hospital admissions, and further deterioration of lung function. This will have a large influence on the quality of life of patients. Available techniques to measure airway inflammation, like broncho-alveolar lavage, induced sputum and lung biopsy, are to invasive to used in children regularly. In the past decade, there has been an increasing interest in non-invasive measurement of airway inflammation. By applying non-invasive inflammatory markers in exhaled breath (condensate) in order to monitor children with chronic inflammatory lung diseases like asthma and $\mathrm{CF}$, we hypothesise that there will be an improved level of disease control, a reduced number of exacerbations, less severe exacerbations and, consequently, an increase in quality of life of these children.

\subsection{Methodology of non-invasive inflammometry assessments}

Non-invasive measurements of chronic airway inflammation by means of inflammatory markers (IM) in exhaled breath and exhaled breath condensate (EBC) is completely noninvasive, has a $100 \%$ success rate in children from the age of 6 years, takes little time, and has the advantage that several parameters can be assessed simultaneously $[23,50]$. 


\subsubsection{Fractional exhaled nitric oxide}

Nitric oxide (NO) is synthesized from L-arginine by nitric oxide synthetase (NOS), which exists in two constitutive (cNOS) and one inducible (iNOS) isoform. The constitutive isoforms are localized in airway nerves and bronchial epithelium, type II alveolar epithelial cells, and in endothelial cells of pulmonary blood vessels, where they synthesize NO. In addition, induced by proinflammatory cytokines, iNOS releases large quantities of NO. iNOS reactivity has been shown in many cells: neutrophils, eosinophils, macrophages, type II alveolar epithelial cells, airway epithelial cells, mast cells, fibroblasts, endothelial cells, and airway and vascular smooth muscle cells.

Fractional exhaled nitric oxide (FeNO) can be measured with a high sensitivity and specificity by means of a chemiluminescence gas analyzer (NIOX ${ }^{\circ}$, Aerocrine, Solna, Sweden). Compared to induced sputum, FeNO is non-invasive and relatively easy to obtain in children. FeNO correlates with eosinophilia in induced sputum [51] and bronchial hyperresponsiveness in steroid-naive asthmatics [52]. Therefore, FeNO may be used as a marker to monitoring asthma control, and may be a usefull tool to tailor asthma medications in addition to conventional parameters as clinical symptoms and lung function parameters. Studies showed that FeNO is significantly influenced by atopic status [53-55], use of corticosteroids, age and height [56,57], presence of respiratory infections [58]. Because FeNO is not asthma specific, the additional value of FeNO to diagnose and monitor asthma is limited. A cochrane meta-analysis [59] based on six studies in 1053 adults and children, has showed that tailoring the dose of inhaled corticosteroids based on FeNO in comparison with clinical symptoms, did not significantly reduce frequency of asthma exacerbations, improve lung function or decrease severity of asthma symptoms. In children, a trend was found favouring the FeNO strategy in patients with one or more exacerbations. Although, higher doses of corticosteroids were prescribed to these patients.

CF lung disease disease is characterized by neutrophilic airway inflammation and chronic airway infection. Levels of FeNO are normal or decreased [60-63]. Lower FeNO levels and higher nitrite concentrations in the CF population can be explained by several hypotheses $[61,64]$. At first, the viscous mucus in CF airways may impair diffusion of gaseous NO into the airway lumen [65]. This can result in conversion of NO in nitrite, which is consistent with the higher levels of nitrite in CF than in controls. Secondly, decreased expression of iNOS II in epithelial cells may result in low FeNO levels in children with CF [53, 66-69]. Further work is needed to fully understand pulmonary NO exchange dynamics in the different stages of CF lung disease across different age groups and in larger cohorts, but also compared to other chronic lung diseases such as non-CF bronchiectasis [70].

\subsubsection{Volatile organic compounds}

Volatile mediators of airway inflammation and oxidative stress can be assessed in exhaled breath. Besides nitrogen, oxygen, carbon dioxide and water, hundreds of volatile organic compounds (VOCs) can be detected in exhaled breath [71]. The presence and the ratio of VOCs, may provide insights into different biochemical processes in healthy and diseased human lungs. Types of endogenous markers used for diagnostic purpose, described by Miekisch et al, are: saturated hydrocarbons, unsaturated hydrocarbons, oxygencontaining compounds, sulpher-containing compounds and nitrogen-containing com- 
pounds [72]. VOCs can be analysed using gas chromatograph/mass spectrometry analysis (GC/MS). In the inflammation process, VOCs are derived from peroxidation and other reactions of reactive oxygen species (ROS).

Most studies of VOCs in exhaled breath were performed in patients with lung cancer. Reported data show up to $100 \%$ sensitivity and $81 \%$ specificity for lung cancer regardless the state of disease progression [73-75].

Another method to analyse the potential of VOCs, is measurements by means of the electronic nose (eNose). This technique is based on chemical vapour sensor arrays that respond to specific chemical characteristics of individual molecules such as VOCs. By means of integrative analysis and pattern recognition, the discriminative ability of the obtained VOC profiles or 'breathprints' can be assessed [76]. Machado et al. showed that one such eNose had $71 \%$ sensitivity and $92 \%$ specificity for detecting lung cancer [76].

Breathprints of patients with asthma could be separated from VOC profiles of patients with COPD and controls with a correct classification of 93-100\% [77, 78].

Paredi et al. demonstrated elevated levels of exhaled ethane in patients with CF compared to healthy controls, which significantly correlates with $\mathrm{CO}$, a marker of inflammation and oxidative stress [79]. This indicates that oxidative stress and lipid peroxidation are increased in patients with CF. During exacerbations oxidative stress increased in patients with CF [80].

In CF, only in one study several VOCs were assessed. Authors state that VOCs may be usefull in the follow-up of CF lung disease, however, more research is needed [81].

\subsubsection{Exhaled breath condensate}

The exact origin of EBC is not known. The most accepted hypothesis of the origin of EBC is the following. During in- and exhalation, airflow turbulences are particularly present in smaller airways. By consequence, small droplets of the epithelial lining fluid, containing inflammatory markers (IM), will be transported through the airways accompanied with water vapour. By cooling exhaled breath, water vapour and the small droplets of the epithelial lining fluid will condense and may be collected. The droplets of the epithelial lining fluid consist of biomarkers of airway inflammation and oxidative stress, including nitric oxide-derived products, hydrogen peroxide, cytokines, leukotrienes, prostaglandins, and eicosanoids. Because of the non-invasive character, EBC does not influence airway function or cause inflammation. It can be easily and safely performed on children and patients with severe diseases and even allows for repeated measurements to be taken.

There are various devices to collect EBC: commercially and home-made. During tidal breathing, exhaled breath is conducted to a cooled unit in order to enhance formation of condensate. Devices are non-rebreathing; two way valves are used to separate inhaled air from exhaled breath to prevent inhalation of cold air passing through the condenser. In addition, saliva traps are used to prevent saliva contamination [82]. Commercially available devices are the ECoScreen (Viasys, Hoechberg, Germany), the RTube ${ }^{\mathrm{TM}}$ (Respiratory Research, Charlottesville, USA), and the TURBO-DECCS, (ItalChill, Parma, Italy).

There are a few drawbacks of EBC. There is uncertainty regarding the origin of EBC. Markers may originate from each part of the respiratory tract. A large variability of markers has been reported, possibly based on a dilution factor. Different methods have been used in order to correct for this dilution, e.g. based on sample volume, conductivity, exhaled ions, 
urea or protein concentrations [82, 83]. A third issue is the low concentrations of the markers. Lower detection limits of the available assays are close to the levels of markers [82].

Despite these drawbacks, markers in EBC may be valuable in diagnosing and monitoring chronic inflammatory lung diseases. Furthermore, based on markers in EBC, more knowledge of pathogenesis may be obtained.

\subsection{Objectives of this thesis: methodology, diagnosis, monitoring}

Exhaled breath analysis has become a promising new tool in monitoring airway inflammation in children with chronic airway diseases. However, there are still questions which need to be resolved. This thesis is divided in three sections:

- Section 1) methodological aspects of exhaled breath (condensate) collection and assessment of inflammatory markers;

- Section 2) diagnostic properties of these non-invasive techniques;

- Section 3) the monitoring value of these techniques in asthma and CF.

Outline of this thesis:

Section 1 consists of three chapters, in which methodological aspects of exhaled NO, EBC collection devices and feasibility of EBC collection in preschool children, are discussed:

In chapter $\mathbf{2}$ we study the feasibility, linearity and long-term repeatability of NO measurements at four different exhalation flow rates in children with asthma. To discriminate between alveolar and bronchial NO, measurements need to be assessed at various flow rates. In addition, levels of FeNO, alveolar NO and bronchial NO in asthma are given.

Different EBC collection devices are used in research, however, it is not known to what extent condenser coatings influence presence and reproducibility of inflammatory markers in EBC. Chapter 3 focusses on the influence of condenser coatings on biomarker reproducibility in order to find the most optimal system.

Partly based on the results of chapter 3, we developed a new highly efficiënt condenser which enables EBC collection in all ages (patent EP07102586: Apparatus for and method of condensing exhaled breath). In chapter 4 the feasibility of EBC collection in preschool children with and without wheezing by means of this new device is presented.

Section 2 embodies 7 chapters concerning diagnostic properties of these non-invasive techniques:

Cytokine responses are important controlling, and thereby, signalling elements of inflammatory and immune reactions. Asthma is considered as a T-helper (Th)-2 mediated disease. In contrast, in CF the Th-1 cells predominate. In chapter 5, the presence, concentration, and short-term variability of Th-1 and Th- 2 mediated cytokines in EBC of children with asthma, or CF and healthy controls was studied. In addition, we study the discriminating ability of cytokines in EBC between children with asthma or CF, and healthy children.

The utility of the cytokines has been hampered by low detection rates and low concentrations in EBC. This was coherent with the results of chapter 5 . Therefore, in chapter 6, we 
assessed cytokines, chemokines and soluble adhesion molecules by means of flow cytometry (xMAP technology) in children with asthma.

Various reports have been published concerning levels of FeNO and various markers in EBC. However, the ability to indicate an asthma diagnosis, asthma severity and control, is largely unknown. In chapter $\mathbf{7 . 1}$ we investigate the ability of FeNO and inflammatory markers in EBC considered together, to indicate the presence, severity and control of childhood asthma.

In chapter $\mathbf{7 . 2}$ the ability of FeNO and markers in EBC considered together, to indicate CF lung disease and severity of CF in children is analyzed.

The relationship between lung function on the one hand, structural lung changes as visualized by high resolution computed tomography (HRCT) scans, and non-invasive IM on the other is yet unclear in CF patients. In chapter 8 data on non-invasive IM in EBC, HRCT imaging, and static and dynamic lung function indices were analyzed in CF patients.

Another promising non-invasive method to measure airway inflammation is assessment of volatile organic compounds (VOCs) in exhaled breath. In chapter 9.1 we study the discriminating ability of VOCs in children with asthma. VOCs were assessed by gas chromatography-mass spectrometry.

In chapter 9.2 we study whether VOCs could discriminate between CF and controls, and between CF patients with and without Pseudomonas colonization.

Longitudinal data are necessary to further confirm the role of these markers for the management of chronic inflammatory diseases. In section 3, the monitoring value of exhaled breath analysis in asthma and CF is discussed:

The objective of chapter $\mathbf{1 0}$ was to compare the anti-inflammatory effects of extrafine HFA-beclomethasone and fluticasone-DPI (dry powder inhaler) in asthmatic children, on bronchial and alveolar NO levels, inflammatory markers in EBC, and conventional parameters such as lung function indices, local adverse effects and symptoms.

Based on the results of chapter 7.1, FeNO and inflammatory markers in EBC considered together, may predict exacerbations of childhood asthma. Therefore, in chapter 11.1, we studied these markers in a one year prospective study in order to analyse the predictive ability of non-invasive markers to predict an asthma exacerbation in children. In addition, the time course of changes in inflammatory markers during exacerbations was investigated.

In chapter $\mathbf{1 1 . 2}$ we analysed the ability to predicted exacerbations of CF lung disease by means of FeNO and non-invasive inflammatory markers in EBC in a one year longitudinal study.

The results of chapter 10.1 and 10.2 show that analysis of VOCs in exhaled breath has clinical potential. In chapter $\mathbf{1 2 . 1}$ we hypothesize that VOCs analysis in exhaled breath can predict asthma exacerbations in children. We investigate whether VOCs in exhaled breath are able to reliably predict asthma exacerbations, in a one year prospective controlled study. In addition, we identify the chemical background of the most predicting VOCs.

In chapter 12.2, we study the ability of VOCs to predict exacerbations of CF lung disease in a one year prospective controlled study. 
Finally in chapter 13 a general discussion of the findings in this thesis is given and recommendations of future research are made.

\section{References}

1. World Health Organization, Fact sheet asthma no 3007, may 2008 (www.who.int).

2. Global Initiative for asthma (GINA). Pocket guide for asthma management and prevention in children. National Institute of Health, National Heart, Lung, and Blood Institute 2006.

3. Asher MI, Montefort S, Bjorksten B, Lai CK, Strachan DP, Weiland SK, Williams H. Worldwide time trends in the prevalence of symptoms of asthma, allergic rhinoconjunctivitis, and eczema in childhood: ISAAC Phases One and Three repeat multicountry cross-sectional surveys. Lancet 2006: 368(9537): 733-743.

4. van Schayck CP, Smit HA. The prevalence of asthma in children: a reversing trend. Eur Respir J 2005: 26(4): 647-650.

5. Johnston NW. The similarities and differences of epidemic cycles of chronic obstructive pulmonary disease and asthma exacerbations. Proc Am Thorac Soc 2007: 4(8): 591-596.

6. Braman SS. The global burden of asthma. Chest 2006: 130(1 Suppl): 4S-12S.

7. Dietert RR, Zelikoff JT. Early-life environment, developmental immunotoxicology, and the risk of pediatric allergic disease including asthma. Birth defects research 2008: 83(6): 547-560.

8. Henderson J, Granell R, Sterne J. The search for new asthma phenotypes. Arch Dis Child 2009: 94(5): 333336.

9. Simpson JL, Grissell TV, Douwes J, Scott RJ, Boyle MJ, Gibson PG. Innate immune activation in neutrophilic asthma and bronchiectasis. Thorax 2007: 62(3): 211-218.

10. Wenzel SE. Asthma: defining of the persistent adult phenotypes. Lancet 2006: 368(9537): 804-813.

11. Spycher BD, Silverman M, Brooke AM, Minder CE, Kuehni CE. Distinguishing phenotypes of childhood wheeze and cough using latent class analysis. Eur Respir J 2008: 31(5): 974-981.

12. Phipps S, Lam CE, Foster PS, Matthaei KI. The contribution of toll-like receptors to the pathogenesis of asthma. Immunology and cell biology 2007: 85(6): 463-470.

13. Iwamura C, Nakayama T. Toll-like receptors in the respiratory system: their roles in inflammation. Curr Allergy Asthma Rep 2008: 8(1): 7-13.

14. Moller-Larsen S, Nyegaard M, Haagerup A, Vestbo J, Kruse TA, Borglum AD. Association analysis identifies TLR7 and TLR8 as novel risk genes in asthma and related disorders. Thorax 2008: 63(12): 1064-1069.

15. Contoli M, Message SD, Laza-Stanca V, Edwards MR, Wark PA, Bartlett NW, Kebadze T, Mallia P, Stanciu LA, Parker HL, Slater L, Lewis-Antes A, Kon OM, Holgate ST, Davies DE, Kotenko SV, Papi A, Johnston SL. Role of deficient type III interferon-lambda production in asthma exacerbations. Nature medicine 2006: 12(9): 10231026.

16. Johnston SL. Innate immunity in the pathogenesis of virus-induced asthma exacerbations. Proc Am Thorac Soc 2007: 4(3): 267-270.

17. Stern DA, Guerra S, Halonen M, Wright AL, Martinez FD. Low IFN-gamma production in the first year of life as a predictor of wheeze during childhood. J Allergy Clin Immunol 2007: 120(4): 835-841.

18. Sykes A, Johnston SL. Etiology of asthma exacerbations. J Allergy Clin Immunol 2008: 122(4): 685-688.

19. von Mutius E. Towards prevention. Lancet 1997: 350 Suppl 2: SII14-17.

20. Gustafsson PM, Watson L, Davis KJ, Rabe KF. Poor asthma control in children: evidence from epidemiological surveys and implications for clinical practice. Int J Clin Pract 2006: 60(3): 321-334.

21. Rabe KF, Adachi M, Lai CK, Soriano JB, Vermeire PA, Weiss KB, Weiss ST. Worldwide severity and control of asthma in children and adults: the global asthma insights and reality surveys. J Allergy Clin Immunol 2004: 114(1): 40-47.

22. Hammer SC, Robroeks CM, van Rij C, Heynens J, Droog R, Jobsis Q, Hendriks HJ, Dompeling E. Actual asthma control in a paediatric outpatient clinic population: do patients perceive their actual level of control? Pediatr Allergy Immunol 2008: 19(7): 626-633. 
23. Kharitonov SA, Barnes PJ. Biomarkers of some pulmonary diseases in exhaled breath. Biomarkers 2002: 7(1): $1-32$.

24. Kips JC, Kharitonov SA, Barnes PJ. Non invasive assessment of airway inflammation in asthma. Eur Respir Mon 2003: 13: 164-179.

25. Fleming L, Wilson N, Regamey N, Bush A. The use of non-invasive markers of inflammation to guide management in children with severe asthma. Am J Respir Crit Care Med 2009: 179: A1305.

26. Lex C, Payne DN, Zacharasiewicz A, Li AM, Wilson NM, Hansel TT, Bush A. Sputum induction in children with difficult asthma: safety, feasibility, and inflammatory cell pattern. Pediatr Pulmonol 2005: 39(4): 318-324.

27. Pianosi P, Smith CP, Almudevar A, McGrath PJ. Dalhousie dyspnea scales: Pictorial scales to measure dyspnea during induced bronchoconstriction. Pediatr Pulmonol 2006: 41(12): 1182-1187.

28. Farrell PM. The prevalence of cystic fibrosis in the European Union. J Cyst Fibros 2008: 7(5): 450-453.

29. Meachery G, De Soyza A, Nicholson A, Parry G, Hasan A, Tocewicz K, Pillay T, Clark S, Lordan JL, Schueler S, Fisher AJ, Dark JH, Gould FK, Corris PA. Outcomes of lung transplantation for cystic fibrosis in a large UK cohort. Thorax 2008: 63(8): 725-731.

30. Bech B, Pressler T, Iversen M, Carlsen J, Milman N, Eliasen K, Perko M, Arendrup H. Long-term outcome of lung transplantation for cystic fibrosis-Danish results. Eur J Cardiothorac Surg 2004: 26(6): 1180-1186.

31. Gibson RL, Burns JL, Ramsey BW. Pathophysiology and management of pulmonary infections in cystic fibrosis. Am J Respir Crit Care Med 2003: 168(8): 918-951.

32. Ratjen F, Doring G. Cystic fibrosis. Lancet 2003: 361(9358): 681-689.

33. Brown RK, Wyatt H, Price JF, Kelly FJ. Pulmonary dysfunction in cystic fibrosis is associated with oxidative stress. Eur Respir J 1996: 9(2): 334-339.

34. V. Kumar AA, N Fausto. Pathologic basis of disease. 7th ed. Elsevier Saunders, Pennsylvania, 2005.

35. FitzSimmons SC. The changing epidemiology of cystic fibrosis. Curr Probl Pediatr 1994: 24(5): 171-179.

36. Khan TZ, Wagener JS, Bost T, Martinez J, Accurso FJ, Riches DW. Early pulmonary inflammation in infants with cystic fibrosis. Am J Respir Crit Care Med 1995: 151(4): 1075-1082.

37. Armstrong DS, Grimwood K, Carzino R, Carlin JB, Olinsky A, Phelan PD. Lower respiratory infection and inflammation in infants with newly diagnosed cystic fibrosis. Bmj 1995: 310(6994): 1571-1572.

38. Birrer P, McElvaney NG, Rudeberg A, Sommer CW, Liechti-Gallati S, Kraemer R, Hubbard R, Crystal RG. Protease-antiprotease imbalance in the lungs of children with cystic fibrosis. Am J Respir Crit Care Med 1994: 150(1): 207-213.

39. Konstan MW, Hilliard KA, Norvell TM, Berger M. Bronchoalveolar lavage findings in cystic fibrosis patients with stable, clinically mild lung disease suggest ongoing infection and inflammation. Am J Respir Crit Care Med 1994: 150(2): 448-454.

40. Boucher RC. New concepts of the pathogenesis of cystic fibrosis lung disease. Eur Respir J 2004: 23(1): 146158.

41. McGrath LT, Mallon P, Dowey L, Silke B, McClean E, McDonnell M, Devine A, Copeland S, Elborn S. Oxidative stress during acute respiratory exacerbations in cystic fibrosis. Thorax 1999: 54(6): 518-523.

42. Tabary O, Zahm JM, Hinnrasky J, Couetil JP, Cornillet P, Guenounou M, Gaillard D, Puchelle E, Jacquot J. Selective up-regulation of chemokine IL-8 expression in cystic fibrosis bronchial gland cells in vivo and in vitro. Am J Pathol 1998: 153(3): 921-930.

43. Grasemann H, loannidis I, Tomkiewicz RP, de Groot H, Rubin BK, Ratjen F. Nitric oxide metabolites in cystic fibrosis lung disease. Arch Dis Child 1998: 78(1): 49-53.

44. Kharitonov SA, Barnes PJ. Exhaled markers of pulmonary disease. Am J Respir Crit Care Med 2001: 163(7): 1693-1722.

45. Shwachman $\mathrm{H}$, Kulczycki LL. Long-term study of one hundred five patients with cystic fibrosis; studies made over a five- to fourteen-year period. JAMA Dis Child 1958: 96(1): 6-15.

46. Maffessanti M, Candusso M, Brizzi F, Piovesana F. Cystic fibrosis in children: HRCT findings and distribution of disease. J Thorac Imaging 1996: 11(1): 27-38.

47. Tiddens HA. Chest computed tomography scans should be considered as a routine investigation in cystic fibrosis. Paediatr Respir Rev 2006: 7(3): 202-208. 
48. de Jong PA, Lindblad A, Rubin L, Hop WC, de Jongste JC, Brink M, Tiddens HA. Progression of lung disease on computed tomography and pulmonary function tests in children and adults with cystic fibrosis. Thorax 2006: 61(1): 80-85.

49. Brennan S, Hall GL, Horak F, Moeller A, Pitrez PM, Franzmann A, Turner S, de Klerk N, Franklin P, Winfield KR, Balding E, Stick SM, Sly PD. Correlation of forced oscillation technique in preschool children with cystic fibrosis with pulmonary inflammation. Thorax 2005: 60(2): 159-163.

50. Rosias PP, Dompeling E, Dentener MA, Pennings HJ, Hendriks HJ, Van lersel MP, Jobsis Q. Childhood asthma: Exhaled markers of airway inflammation, asthma control score, and lung function tests. Pediatr Pulmonol 2004: 38(2): 107-114.

51. Jatakanon A, Lim S, Kharitonov SA, Chung KF, Barnes PJ. Correlation between exhaled nitric oxide, sputum eosinophils, and methacholine responsiveness in patients with mild asthma. Thorax 1998: 53(2): 91-95.

52. Dupont L, Rochette F, Demedts MG, Verleden GM. Exhaled nitric oxide correlates with airway hyperresponsiveness in steroid-naive patients with mild asthma. Am J Respir Crit Care Med 1998: 157(3 Pt 1): 894-898.

53. Franklin PJ, Hall GL, Moeller A, Horak F, Jr., Brennan S, Stick SM. Exhaled nitric oxide is not reduced in infants with cystic fibrosis. Eur Respir J 2006: 27(2): 350-353.

54. Franklin PJ, Taplin R, Stick SM. A community study of exhaled nitric oxide in healthy children. Am J Respir Crit Care Med 1999: 159(1): 69-73.

55. Prasad A, Langford B, Stradling JR, Ho LP. Exhaled nitric oxide as a screening tool for asthma in school children. Respir Med 2006: 100(1): 167-173.

56. Malmberg LP, Petays T, Haahtela T, Laatikainen T, Jousilahti P, Vartiainen E, Makela MJ. Exhaled nitric oxide in healthy nonatopic school-age children: determinants and height-adjusted reference values. Pediatr Pulmonol 2006: 41(7): 635-642.

57. Strunk RC, Szefler SJ, Phillips BR, Zeiger RS, Chinchilli VM, Larsen G, Hodgdon K, Morgan W, Sorkness CA, Lemanske RF, Jr. Relationship of exhaled nitric oxide to clinical and inflammatory markers of persistent asthma in children. J Allergy Clin Immunol 2003: 112(5): 883-892.

58. Sanders SP, Proud D, Permutt S, Siekierski ES, Yachechko R, Liu MC. Role of nasal nitric oxide in the resolution of experimental rhinovirus infection. J Allergy Clin Immunol 2004: 113(4): 697-702.

59. Petsky HL, Cates CJ, Li AM, Kynaston JA, Turner C, Chang AB. Tailored interventions based on exhaled nitric oxide versus clinical symptoms for asthma in children and adults. Cochrane Database Syst Rev 2008(2): CD006340.

60. Balfour-Lynn IM, Laverty A, Dinwiddie R. Reduced upper airway nitric oxide in cystic fibrosis. Arch Dis Child 1996: 75(4): 319-322.

61. de Winter-de Groot KM, van der Ent CK. Nitric oxide in cystic fibrosis. J Cyst Fibros 2005: 4 Suppl 2: 25-29.

62. Grasemann H, Michler E, Wallot M, Ratjen F. Decreased concentration of exhaled nitric oxide (NO) in patients with cystic fibrosis. Pediatr Pulmonol 1997: 24(3): 173-177.

63. Lundberg JO, Nordvall SL, Weitzberg E, Kollberg H, Alving K. Exhaled nitric oxide in paediatric asthma and cystic fibrosis. Arch Dis Child 1996: 75(4): 323-326.

64. Ojoo JC, Mulrennan SA, Kastelik JA, Morice AH, Redington AE. Exhaled breath condensate pH and exhaled nitric oxide in allergic asthma and in cystic fibrosis. Thorax 2005: 60(1): 22-26.

65. Ho LP, Innes JA, Greening AP. Nitrite levels in breath condensate of patients with cystic fibrosis is elevated in contrast to exhaled nitric oxide. Thorax 1998: 53(8): 680-684.

66. Grasemann H, Ratjen F. Cystic fibrosis lung disease: the role of nitric oxide. Pediatr Pulmonol 1999: 28(6): 442-448.

67. Meng QH, Springall DR, Bishop AE, Morgan K, Evans TJ, Habib S, Gruenert DC, Gyi KM, Hodson ME, Yacoub $\mathrm{MH}$, Polak JM. Lack of inducible nitric oxide synthase in bronchial epithelium: a possible mechanism of susceptibility to infection in cystic fibrosis. J Pathol 1998: 184(3): 323-331.

68. Kelley TJ, Drumm ML. Inducible nitric oxide synthase expression is reduced in cystic fibrosis murine and human airway epithelial cells. J Clin Invest 1998: 102(6): 1200-1207.

69. Moeller A, Horak F, Jr., Lane C, Knight D, Kicic A, Brennan S, Franklin P, Terpolilli J, Wildhaber JH, Stick SM. Inducible NO synthase expression is low in airway epithelium from young children with cystic fibrosis. Thorax 2006: 61(6): 514-520. 
70. Hofer M, Mueller L, Rechsteiner T, Benden C, Boehler A. Extended nitric oxide measurements in exhaled air of cystic fibrosis and healthy adults. Lung 2009: 187(5): 307-313.

71. Phillips M, Herrera J, Krishnan S, Zain M, Greenberg J, Cataneo RN. Variation in volatile organic compounds in the breath of normal humans. J Chromatogr B Biomed Sci Appl 1999: 729(1-2): 75-88.

72. Miekisch W, Schubert JK, Noeldge-Schomburg GF. Diagnostic potential of breath analysis-focus on volatile organic compounds. Clin Chim Acta 2004: 347(1-2): 25-39.

73. Phillips M, Cataneo RN, Cummin AR, Gagliardi AJ, Gleeson K, Greenberg J, Maxfield RA, Rom WN. Detection of lung cancer with volatile markers in the breath. Chest 2003: 123(6): 2115-2123.

74. Phillips M, Gleeson K, Hughes JM, Greenberg J, Cataneo RN, Baker L, McVay WP. Volatile organic compounds in breath as markers of lung cancer: a cross-sectional study. Lancet 1999: 353(9168): 1930-1933.

75. Poli D, Carbognani P, Corradi M, Goldoni M, Acampa O, Balbi B, Bianchi L, Rusca M, Mutti A. Exhaled volatile organic compounds in patients with non-small cell lung cancer: cross sectional and nested short-term followup study. Respir Res 2005: 6: 71.

76. Machado RF, Laskowski D, Deffenderfer O, Burch T, Zheng S, Mazzone PJ, Mekhail T, Jennings C, Stoller JK, Pyle J, Duncan J, Dweik RA, Erzurum SC. Detection of lung cancer by sensor array analyses of exhaled breath. Am J Respir Crit Care Med 2005: 171(11): 1286-1291.

77. Dragonieri S, Schot R, Mertens BJ, Le Cessie S, Gauw SA, Spanevello A, Resta O, Willard NP, Vink TJ, Rabe KF, Bel EH, Sterk PJ. An electronic nose in the discrimination of patients with asthma and controls. J Allergy Clin Immunol 2007: 120(4): 856-862.

78. Fens N, Zwinderman AH, van der Schee MP, de Nijs SB, Dijkers E, Roldaan AC, Cheung D, Bel EH, Sterk PJ. Exhaled Breath Profiling Enables Discrimination of Chronic Obstructive Pulmonary Disease and Asthma. Am J Respir Crit Care Med 2009.

79. Paredi P, Kharitonov SA, Leak D, Shah PL, Cramer D, Hodson ME, Barnes PJ. Exhaled ethane is elevated in cystic fibrosis and correlates with carbon monoxide levels and airway obstruction. Am J Respir Crit Care Med 2000: 161(4 Pt 1): 1247-1251.

80. Lagrange-Puget M, Durieu I, Ecochard R, Abbas-Chorfa F, Drai J, Steghens JP, Pacheco Y, Vital-Durand D, Bellon G. Longitudinal study of oxidative status in 312 cystic fibrosis patients in stable state and during bronchial exacerbation. Pediatr Pulmonol 2004: 38(1): 43-49.

81. Barker M, Hengst M, Schmid J, Buers HJ, Mittermaier B, Klemp D, Koppmann R. Volatile organic compounds in the exhaled breath of young patients with cystic fibrosis. Eur Respir J 2006: 27(5): 929-936.

82. Horvath I, Hunt J, Barnes PJ, Alving K, Antczak A, Baraldi E, Becher G, van Beurden WJ, Corradi M, Dekhuijzen R, Dweik RA, Dwyer T, Effros R, Erzurum S, Gaston B, Gessner C, Greening A, Ho LP, Hohlfeld J, Jobsis Q, Laskowski D, Loukides S, Marlin D, Montuschi P, Olin AC, Redington AE, Reinhold P, van Rensen EL, Rubinstein I, Silkoff P, Toren K, Vass G, Vogelberg C, Wirtz H. Exhaled breath condensate: methodological recommendations and unresolved questions. Eur Respir J 2005: 26(3): 523-548.

83. Effros RM, Dunning MB, 3rd, Biller J, Shaker R. The promise and perils of exhaled breath condensates. Am J Physiol Lung Cell Mol Physiol 2004: 287(6): L1073-1080. 

SECTION 1

METHODOLOGY
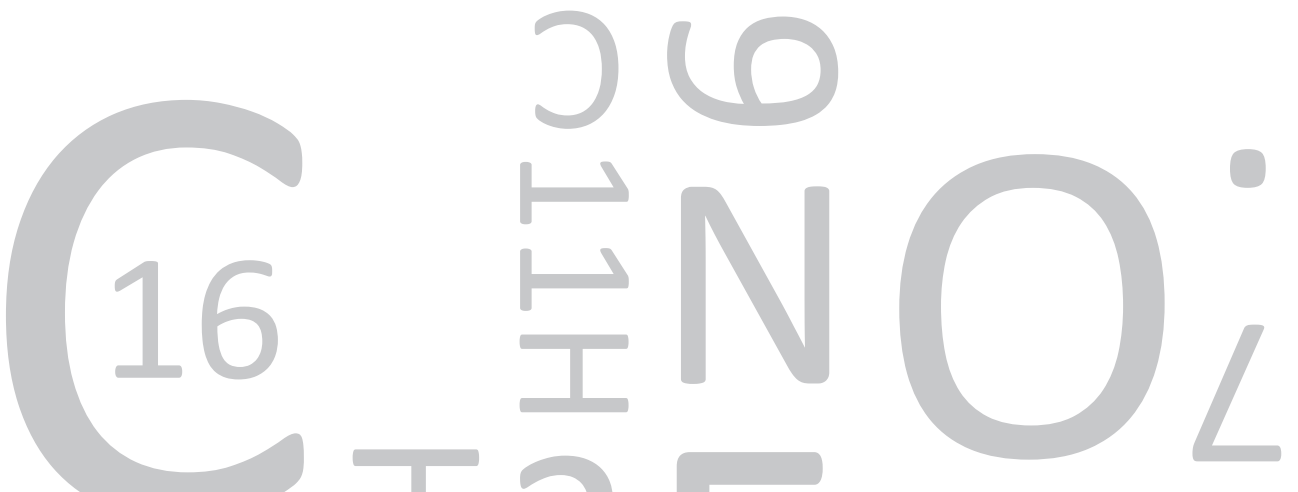



\section{CHAPTER 2}

Feasibility of exhaled nitric oxide measurements at various flow rates in children with asthma

CMHHT Robroeks, D van Vliet, JJE Hendriks, E Dompeling, Q Jöbsis

Pediatric Allergy and Immunology 2010;21(1):e222-e228 


\section{Abstract}

\section{Background}

Measurement of bronchial and alveolar exhaled nitric oxide (NO) levels, could be of clinical importance for the treatment of asthma. To discriminate between alveolar and bronchial NO, measurements need to be assessed at various flow rates.

Aim: To study the feasibility, linearity and long-term repeatability of NO measurements at four different exhalation flow rates, in children with asthma.

\section{Methods}

21 Children with moderate persistent asthma, aged 6 to 12 years, were included in the study. NO was measured according to the ATS/ERS guidelines, using the NIOX analyzer with flow restrictors of $30,50,100$ and $200 \mathrm{~mL} / \mathrm{sec}$. Duration of the measurements ranged from 6-10 seconds, depending on the flow rate. Three and six months after the first NO measurement, the tests were repeated.

\section{Results}

Feasibility of NO measurements at these four flow rates increased from $67 \%$ to $91 \%$ and $95 \%$ at the first, second and third visit, respectively. A significant learning effect was present. Age and lung function indices did not influence success or failure of the tests. At the first measurements occasions, no problems occurred during the NO analysis at a $100 \mathrm{~mL} / \mathrm{sec}$ flow rate. There was a $75-90 \%$ success rate when performing the test using flow rates of $30 \mathrm{~mL} / \mathrm{sec}, 50 \mathrm{~mL} / \mathrm{sec}$, and $200 \mathrm{~mL} / \mathrm{sec}$. However, repeating the tests resulted in a $100 \%$ success rate. Measurements were not successful if: 1) children ran out of air, 2) NO concentration exceeded 200ppb, 3) the measured NO flow was unstable, or, 4) the NO plateau was not formed.

\section{Conclusion}

This study showed good feasibility and linearity of NO measurements in asthmatic children of 6 years and over at flow rates between $50 \mathrm{ml} / \mathrm{sec}$ to $200 \mathrm{ml} / \mathrm{sec}$. A significant learning effect was present. The long-term reproducibility of alveolar and bronchial NO values during 6 months was mode-rate. 


\section{Introduction}

The past years, it has become clear that peripheral airway inflammation, plays an important role in the pathophysiology of asthma [1-3]. Currently, asthma management is based on evaluation of symptoms, physical examination and lung function parameters.

In addition, various studies demonstrated the potential benefit of fractional exhaled nitric oxide (FeNO) in addition to conventional parameters [4-6]. Nitric oxide (NO) is synthesized throughout the respiratory tract by nitric oxide synthases. NO in the airways has important antimicrobial, immunologic and pro-inflammatory properties, and influences, regulation of vascular tone, response to vascular injury and hemostasis [7]. A positive correlation was shown between FeNO and eosinophilic airway inflammation in asthma [8, 9]. FeNO showed predictive value for an asthma relaps after discontinuation of inhaled corticosteroids in symptom-free children [4]. Furthermore, when asthma management was based on FeNO values, patients experienced less exacerbations, and less bronchial hyperresponsiveness was measured [10]. Provided that FeNO manoeuvres are performed correctly, FeNO levels reflect airway inflammation of the lower respiratory tract. Distal airway inflammation may be particularly important in asthma.

Measuring alveolar NO levels $\left(\mathrm{C}_{\mathrm{alv}}\right)$ and bronchial NO flux $\left(\mathrm{J}_{\mathrm{NO}}\right)$, may increase insight in the extent of the inflammation processes in different parts of the lung [11-15]. According to ERS/ATS guidelines, FeNO levels are assessed at an exhalation flow rate of $50 \mathrm{~mL} / \mathrm{sec}$ [16]. These measurements are feasible in children from the age of 4 years and over. $\mathrm{C}_{\mathrm{alv}}$ and $\mathrm{J}_{\mathrm{NO}}$ are calculated from NO flux (úNO) values at various flow rates $[11,17,18]$. There are some studies in children studying bronchial and alveolar NO. However, the feasibility of NO at various flow rates in larger groups in less certain. Moreover, it is important to know the repeatability of NO at these flow rates, and of (the derived) bronchial and alveolar NO levels in children. Therefore, the aim of this study was 1) To study the feasibility and repeatability of NO measurements at four different flow rates, in children with asthma aged 6 years and over, at three occasions three months apart. 2) To study the linearity of the NO flux at exhalation flow rates of 30,50, 100 and $200 \mathrm{~mL} / \mathrm{sec}$. 3) To assess the bronchial NO flux and the alveolar NO concentration in children with asthma.

\section{Methods}

\section{Subjects and Materials}

21 Children with moderate persistent asthma were recruited from our outpatient clinic. Asthma was defined as a chronic inflammatory disorder with wheezing, coughing, chest tightness, or shortness of breath, characterized by a variable, reversible airway obstruction in response to specific or non-specific stimuli. Moderate persistent asthma was defined according to GINA criteria: daytime symptoms more than 2 times per day, or nighttime sleep disruption at least twice a week [19]. Well controlled asthma was defined as the presence of daytime symptoms or the need for reliever medication less than twice a week.

Participating children / parents gave written informed consent. This study was approved by the Ethics Committee of the university hospital Maastricht. During the study height, 
weight, atopy, medication and dosage of inhaled corticosteroids of the patients were assessed. In addition, lung function parameters and NO at different flow rates were measured at the start of the study, and after 3 and 6 months.

\section{NO measurement}

FeNO was measured using the chemiluminescence analyser (NIOX; Aerocrine, Stockholm, Sweden), according to the ATS/ERS guidelines [16]. Children were asked to perform the manoeuvres at four exhalation flow rates, 30, 50, 100 and $200 \mathrm{~mL} / \mathrm{sec}$, guided by an animation. Measurements at 30 and $50 \mathrm{~mL} / \mathrm{sec}$ were established at 10 seconds, whereas the duration of the other flow rates was 6 seconds. Manoeuvres were accepted if a stable NO plateau was reached during the last 3 seconds of each manoeuvre. A test was approved when two FeNO values were within a $10 \%$ range of each other. The mean of these two correctly performed measurements reflect the FeNO at that measured flow rate. Tests with the different exhaled flow rates, were performed in random order. Feasibility of NO measurement at a certain flow rate was defined as the correct performance of the test according to ATS/ERS criteria, like described above. Six attempts were made before the procedure failed.

\section{Alveolar and bronchial NO}

úNO measured at exhalation flows of $30,50,100$ and $200 \mathrm{~mL} / \mathrm{sec}$ at each measurement occasion, were fitted in a linear regression model. The slope of this regression line represents the alveolar NO concentration $\left(\mathrm{C}_{\mathrm{alv}}, \mathrm{ppb}\right)$, and the intercept the bronchial NO flux $\left(\mathrm{J}_{\mathrm{NO}}, \mathrm{pL} / \mathrm{sec}\right.$ ) (Figure 1) [14]. Linearity was considered when $\mathrm{R}$ of the regression models was at least $0.9\left(R^{2}>0.81\right)$. Alveolar and bronchial NO values were only determined when the regression model met these criteria of linearity.

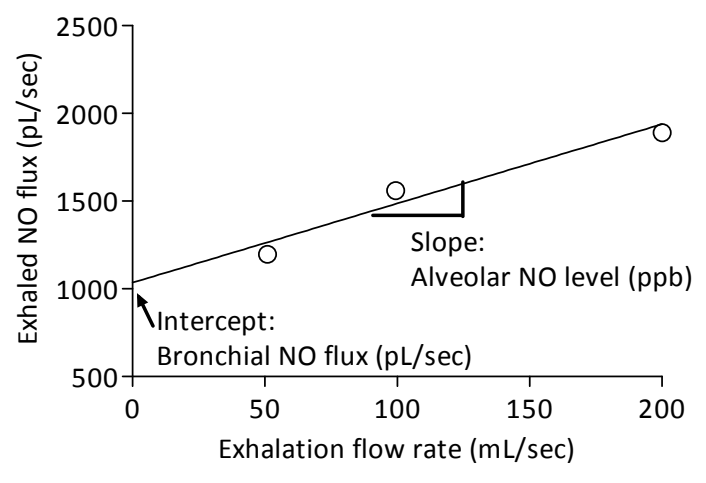




\section{Lung function parameters}

Lung function was measured using the Flowscreen (Viasys ${ }^{\circledR}$, Hoechberg, Germany). Dynamic spirometry was performed according to ERS guidelines. Measured lung function parameters were: forced expiratory volume in one second $\left(\mathrm{FEV}_{1}\right)$, forced vital capacity (FVC) and maximum expiratory flow at $50 \%$ of FCV $\left(\mathrm{MEF}_{50}\right)$, expressed as a percentage of the predicted value. The highest value of three correctly performed manoeuvres was used for analysis.

\section{Statistic analyses}

Data were analysed using SPSS version 11.5. Differences in unpaired data were tested using the t-test for normally distributed data, and the Mann-Whitney test for not normally distributed parameters. Differences in paired data were tested with the paired t-test or Friedman test for normally and not normally distributed parameters respectively. A statistical test was considered significant when the $p$-value $<0.05$. Linear regression models were used to assess $C_{a l v}$ and $J_{\text {NO }}$. Bland-Altman plots of $J_{\text {no, }}, C_{a l v}$ and FeNO were made in order to assess the repeatability over the 6-month period and coefficients of variation (CV) were calculated.

\section{Results}

21 Children with moderate persistent asthma, aged 6-12 years, were included from our outpatient clinic. All patients were treated with inhaled corticosteroids, with a daily dosage of (mean \pm SE) $682 \pm 99 \mu$ g of budesonide or equivalent. In addition, $48 \%$ of the patients used long acting $\beta_{2}$-agonists. The majority of the children were atopic. Patient characteristics are shown in Table 1.

Table 1: Patient characteristics of the population at inclusion in the study.

\begin{tabular}{ll}
\hline & Mean \pm SE \\
\hline Gender (male / female) & $14 / 7$ \\
Age at inclusion (yrs) & $9.5 \pm 0.4$ \\
Weight (kg) & $34.2 \pm 2.3$ \\
Height (cm) & $135.7 \pm 2.5$ \\
Atopy (\%) & 76 \\
Well controlled asthma (\%) & 52 \\
ICS * ( $\mathrm{g}$ ) & $682 \pm 99$ \\
Total IgE (kU/L) & $638 \pm 211$ \\
$\mathrm{FEV}_{1}(\%$ predicted) & $107.6 \pm 3.4$ \\
$\mathrm{FEV}_{1} / \mathrm{VC}$ (\%) & $85.1 \pm 1.6$ \\
$\mathrm{FVC}(\%$ predicted) & $102.8 \pm 2.9$ \\
$\mathrm{MEF}_{50}$ (\% predicted) & $92.7 \pm 7.5$ \\
\hline
\end{tabular}

Abbreviations: SE, standard error; ICS, inhaled corticosteroid; FEV1, forced expiratory volume in 1 second; FVC, forced vital capacity; MEF50, maximum expiratory flow at $50 \%$ of FCV; * ICS budesonide or equivalent. 


\section{Feasibility of NO measurements}

During the first, second and third measurement, the feasibility of the children, who completed the manoeuvres correctly at all assessed flow rates, was 14 (67\%), 19 (91\%) and 20 (95\%), respectively, $p<0.001$. A significant learning effect was present at an exhalation flow rate of $50 \mathrm{~mL} / \mathrm{sec}\left(\mathrm{Chi}^{2}\right.$-test, $\left.\mathrm{p}<0.05\right)$ (Figure 2 ).

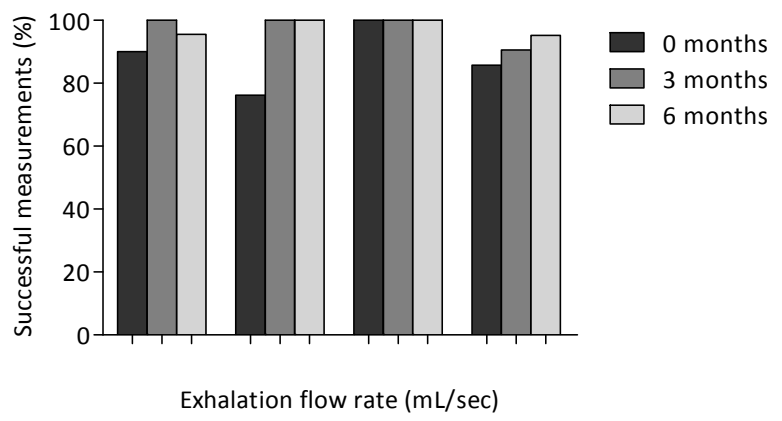

Figure 2: Percentage of successful measurements at the start of the study and after 3 and 6 months at exhalation flow rates of 30,50 , 100 and $200 \mathrm{~mL} / \mathrm{sec}$.

A significant learning effect was present with exhalation flow rate $50 \mathrm{~mL} / \mathrm{sec}\left(\mathrm{Chi}^{2}\right.$-test, $\left.\mathrm{p}<0.05\right)$. Measurements were not successful if: 1) children ran out of air, 2) NO concentration exceeded 200ppb, 3) the measured NO flow was unstable and 4) the NO plateau was not formed.

Only seven children did not perform measurements correctly at exhalation flow rates of $30 \mathrm{~mL} / \mathrm{sec}, 50 \mathrm{~mL} / \mathrm{sec}$ and $200 \mathrm{~mL} / \mathrm{sec}$. All 6 failures at $200 \mathrm{~mL} / \mathrm{sec}$ were due to running out of air and all but one failure at 30 and $50 \mathrm{~mL} / \mathrm{sec}$ were due to no plateau being formed (Table 2).

Table 2: The flow rates at three different measurement occasions in the study during which the patients experienced difficulties with the procedures.

\begin{tabular}{llll}
\hline Patient & $\begin{array}{l}\text { Measurement I } \\
(\mathrm{t}=0 \text { months) }\end{array}$ & $\begin{array}{l}\text { Measurement II } \\
(\mathrm{t}=3 \text { months })\end{array}$ & $\begin{array}{l}\text { Measurement III } \\
\text { ( } \mathrm{t}=6 \text { months) }\end{array}$ \\
\hline 1 & $30 * * *, 50 * * *, 200^{*}$ & & \\
2 & $30 * * *, 50 * * *$ & & \\
3 & $50 * * *$ & $200^{*}$ & $30 *, 200^{*}$ \\
4 & $30 * *, 200^{*}$ & $200^{*}$ & \\
5 & $200^{*}$ & & \\
6 & $50^{* * *}$ & & \\
7 & $30 * * *, 50 * * *$ & & \\
\hline
\end{tabular}

Reasons for failure of NO measurement: * Ran out of air; ${ }^{* *}$ NO flow was unstable (to much fluctuation); ${ }^{* * *}$ NO plateau was not formed (constantly increasing slope).

Age, asthma control, and lung function did not differ between successfully and not successfully performed measurements (unpaired t-test, $p>0.05$ ) for any of the used flow restrictors, Table 3 . None of the children experienced difficulties performing the test at $100 \mathrm{~mL} / \mathrm{sec}$, at any of the measurement occasions. At $200 \mathrm{~mL} / \mathrm{sec}$ flow rate, 3 children ran out of air performing the measurement at some of the measurement occasions. 
Table 3: Characteristics of the patient which completed the tests correctly at all flow rates versus patients who did not fulfil the tests properly.

\begin{tabular}{lll}
\hline & Tests completed & Tests not completed \\
\hline Number of patients & 14 & 7 \\
Well controlled asthma (\%) & 57 & 57 \\
FEV $_{1}$ (\% predicted) & $110.1 \pm 4.2$ & $102.9 \pm 5.6$ \\
FEV 1 VC (\%) & $85.7 \pm 1.5$ & $83.6 \pm 3.9$ \\
FVC (\% predicted) & $104.8 \pm 4.1$ & $99.1 \pm 2.8$ \\
MEF $_{50}$ (\% predicted) & $94.8 \pm 9.6$ & $87.7 \pm 12.7$ \\
\hline
\end{tabular}

Abbreviations: $\mathrm{FEV}_{1}$, forced expiratory volume in 1 second; $\mathrm{FVC}$, forced vital capacity; $\mathrm{MEF}_{50}$, maximum expiratory flow at $50 \%$ of FCV.

\section{Linearity}

Of all successful NO measurements with flow rates ranging from $50-200 \mathrm{~mL} / \mathrm{sec}$, a linear relationship of $R^{2} \geq 0.81$ was found in 13 (61.9\%), 15 (71.4\%) and 15 (71.4\%) patients, at 0 , 3 and 6 months, respectively. At flow rates ranging from $30-200 \mathrm{~mL} / \mathrm{sec}$, linearity was present in 9 (42.9\%), 16 (76.2\%) and 13 (61.9\%) patients, at the first, second and third measurement, respectively. Figure 3 shows linearity between exhalation flow rate and NO output only when flow rates exceed $50 \mathrm{~mL} / \mathrm{sec}$.

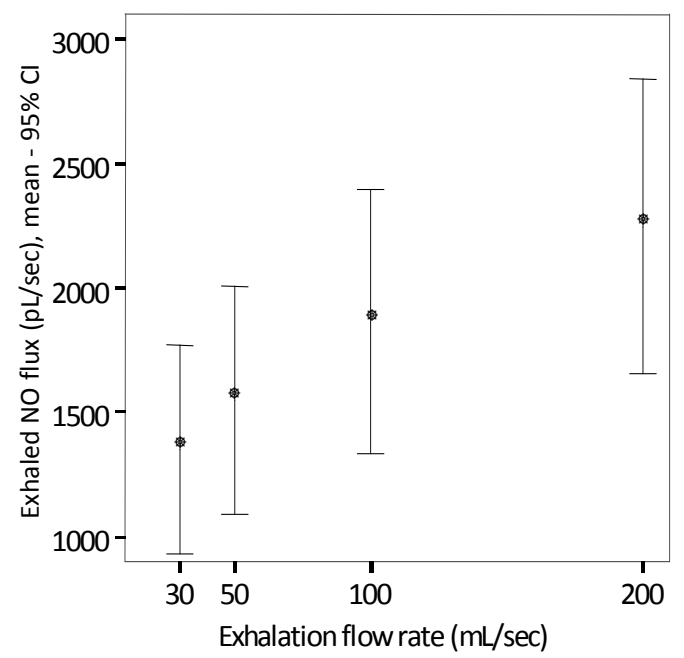

Figure 3: Relationship between exhalation flow rates and the exhaled NO output.

This figures shows linearity between exhalation flow rate and NO flux only when flow rates exceed $50 \mathrm{~mL} / \mathrm{sec}$.

\section{Bronchial and alveolar NO}

Inclusion of $30 \mathrm{~mL} / \mathrm{sec}$ in the regression model influenced, in particular, $\mathrm{C}_{\text {alv }}$ levels: the percentage difference in $\mathrm{C}_{\mathrm{alv}}$ and $\mathrm{J}_{\mathrm{NO}}$ flux between values with and without inclusion of the $30 \mathrm{~mL} / \mathrm{sec}$ flow restrictor were (mean $\pm \mathrm{SE}$ ) $-8.9 \pm 3.5 \%$ and $-0.7 \pm 4.3 \%$ respectively. $\mathrm{C}_{\mathrm{alv}}$ concentrations using the flow restrictors $50-200 \mathrm{~mL} / \mathrm{sec}$ were (median(range) $3.9(2.8-5.2) \mathrm{ppb}$, $5.1(3.2-7.1) \mathrm{ppb}$ and $4.7(3.4-5.3) \mathrm{ppb}$, at the 3 measurement occasions, respectively. $\mathrm{J}_{\mathrm{NO}}$ 
flux levels were 855.0(476.7-2955.0)pL/sec, 855.8(447.5-1947.5)pL/sec and 926.2(560.0$1700.0) \mathrm{pL} / \mathrm{sec}$, at the 3 measurements, respectively. FeNO values did not differ between 0,3 and 6 months (Friedman tests, $\mathrm{p} \geq 0.61$ ). Bland-Altman plots of FeNO, $\mathrm{J}_{\mathrm{NO}}$ and $\mathrm{C}_{\mathrm{alv}}$ over a 6 month interval present the variability in measurements (Figure 4A-C).

Mean $\pm \mathrm{SE}$ of $\mathrm{CV}(\%)$ of FeNO, $\mathrm{J}_{\mathrm{NO}}$ and $\mathrm{C}_{\text {alv }}$ over a 6 month interval were $38 \pm 7 \%, 40 \pm 8 \%$, $45 \pm 8 \%$, respectively.

\section{Lung function parameters}

Patients successfully performed spirometry at all occasions. Levels of lung function parameters at inclusion of the study are shown in Table 1 . No significant difference was found between lung function indices of the first, second and third measurement $(p>0.05)$.

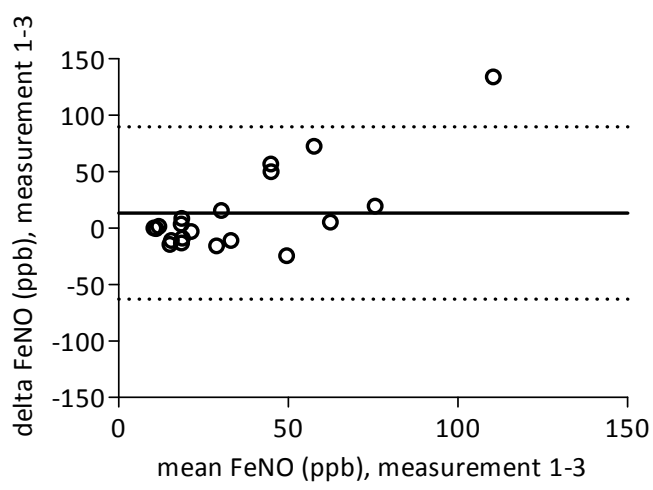

Figure 4a: Bland-Altman plot of FeNO showing the agreement between measurements over a 6 month interval. The $95 \%$ confidence interval of delta FeNO ranged from $-63.0 p p b$ to $+89.8 p p b$.

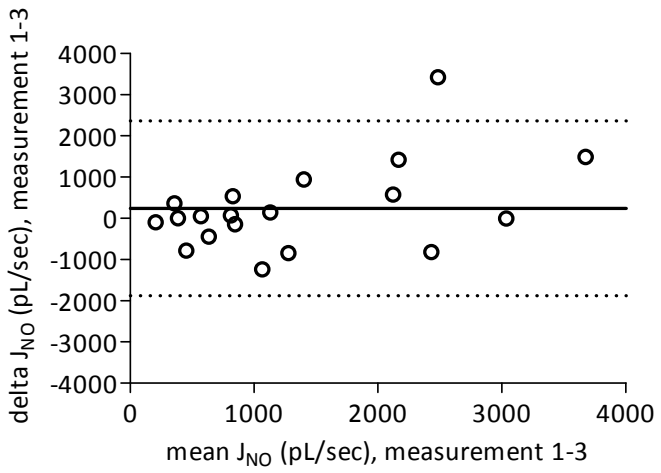

Figure 4b: Bland-Altman plot of $\mathrm{J}_{\mathrm{NO}}$ showing the agreement between measurements over a 6 month interval. The $95 \%$ confidence interval of delta $\mathrm{J}_{\text {NO }}$ ranged from $1875.2 \mathrm{pL} / \mathrm{sec}$ to $+2365.8 \mathrm{pL} / \mathrm{sec}$. 


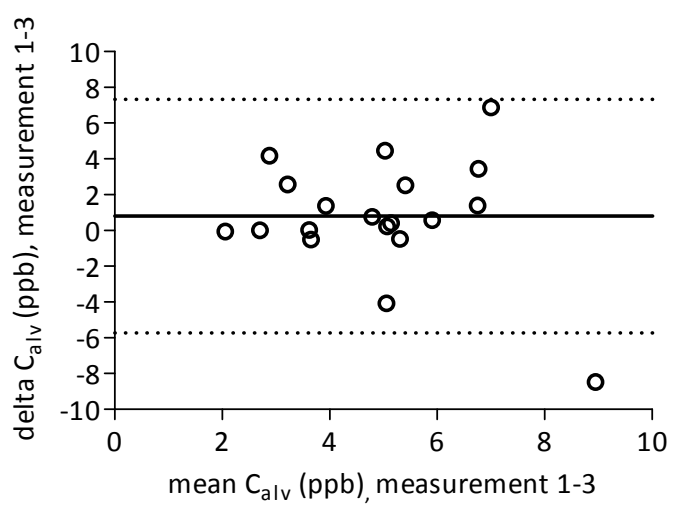

Figure 4c: Bland-Altman plot of Calv showing the agreement between measurements over a 6 month interval. The $95 \%$ confidence interval of delta Calv ranged from $-5.7 p p b$ to 7.3ppb.

\section{Discussion}

This study showed good feasibility of NO measurements at different flows in asthmatic children from the age of 6 years. A significant learning effect was present, feasibility increased from $67 \%$ to $95 \%$ within six months. Failure to perform NO tests, was not related to age or degree of airway obstruction. Reasons of failure of $C_{\text {alv }} / J_{\text {NO }}$ assessment were the inability of children to perform the manoeuvres correctly, or, parameters did not fit in a linear model. Linear regression models of úNO and exhaled flow rates, ranging from 50$200 \mathrm{~mL} / \mathrm{sec}$, met the criteria for linearity more often compared to the models based on flow restrictors ranging from $30-200 \mathrm{~mL} / \mathrm{sec}$. Inclusion of the flow restrictor of $30 \mathrm{~mL} / \mathrm{sec}$, influenced mainly the slope of the linear regression model $\left(C_{a l v}\right.$ levels). The intercepts of the curves, corresponding with the $J_{\text {NO }}$ values, were hardly altered. Mean alveolar NO levels ranged from 3.9 to $5.2 \mathrm{ppb}$, and, median bronchial NO flux ranged from 855 to 926 $\mathrm{pL} / \mathrm{sec}$ in childhood asthma.

Some other studies assessed the feasibility of NO measurements at different flow rates in children [20-23]. In a recent study in children with cystic fibrosis and healthy controls with a mean age of 13 to 14 years, Suri et al. found good feasibility and linearity at flows of 50 tot $260 \mathrm{~mL} / \mathrm{sec}$ when subjects with more severe airway obstruction $\left(\mathrm{FEV}_{1}<40 \%\right.$ of predicted value) were excluded [20]. Mahut and co-workers studied exhaled NO concentrations in 15 asthmatic children, 30 asymptomatic subjects, and 15 healthy children with a mean age of 12.3 years. Although all subjects were able to perform at least one stable prolonged expiratory flow manoeuvre, FeNO at flow rates above $150 \mathrm{~mL} / \mathrm{sec}$ and below $40 \mathrm{~mL} / \mathrm{sec}$ could be obtained in only 20 and 25 of the 60 subjects respectively [21]. This is lower than the feasibility in our study. Van Amsterdam et al. found good feasibility of a single offline NO measurement at a flow rate of $350 \mathrm{~mL} / \mathrm{sec}$ in children aged $4.5-5$ years but not at a flow rate of $8.3 \mathrm{~mL} / \mathrm{sec}$ [22]. As the rate of $8.3 \mathrm{~mL} / \mathrm{sec}$ is far below the lowest flow of $30 \mathrm{~mL} / \mathrm{sec}$ in our study, it is difficult to compare their offline results with our study. Paraskakis and co-workers, reported on feasibility and linearity of NO measurements in children, using exhalation flow restrictors ranging from $50-260 \mathrm{~mL} / \mathrm{sec}$ [23]. He described a feasibility of $85 \%$. In 20 of 112 children, the result did not fit the linear model so that $J_{\mathrm{NO}}$ 
and $\mathrm{C}_{\mathrm{alv}}$ could finally be assessed in 92 subjects (70\%). This is in consistence with our results (44 of 63 measurements (70\%) lead to a reliable result). In addition to the study of by Paraskakis et al, we showed a significant learning effect, increasing the feasibility of NO measurements at various exhalation flow rates to $95 \%$. Thus, the main limiting factor to determine alveolar and bronchial NO is the linearity of the regression model. In our patient group, defining linearity as a correlation coefficient of at least 0.9 , about $30 \%$ of the children had insufficient linear regression lines so that estimation of bronchial or alveolar NO was not possible in this group. This emphasizes the importance of criteria of linearity, which should be defined and standardized.

FeNO levels can be measured from the age of 4 years. This study was performed in children from the age of 6 years old. It is likely that feasibility of NO measurements at multiple flows in younger children is lower than observed in this study. In addition, the studied asthmatic population did not experience an exacerbation, which may have a positive effect on feasibility too. The results of these study may be different in children with more severe asthma and lower levels of asthma control.

Of the 7 children with unsuccessful manoeuvres during the first measurement, only 2 children experienced difficulties at the other measurement occasions. As mentioned before, this may reflect a learning effect, since no relationship between age / lung function indices and feasibility was found. Linearity of the regression models was influenced by the inclusion of the úNO levels measured at a $30 \mathrm{~mL} / \mathrm{sec}$ flow rate. This underlines the nonlinear shape of the curve below $50 \mathrm{~mL} / \mathrm{sec}$ as described by Silkoff et al. and George et al. $[24,25]$. In a study with 10 normal subjects and 25 asthmatic patients, Silkoff and coworkers found a nonlinear relationship between NO output and expiratory flow below $20 \mathrm{ml} / \mathrm{s}$ [24]. Therefore, we suggest to measure úNO levels at minimally three flow rates to test linearity, and, from the flow rate of $50 \mathrm{~mL} / \mathrm{sec}$ and over.

The repeatability of FeNO, $\mathrm{J}_{\mathrm{NO}}$ and $\mathrm{C}_{\mathrm{alv}}$ during a 6-month interval demonstrated considerable variability with CVs ranging from $38-45 \%$. In the study of Paraskakis, the short-term repeatability of $J_{\text {NO }}$ and $C_{\text {alv }}$ was investigated: intraclass correlation coefficients ranged from 0.999 for $\mathrm{J}_{\mathrm{NO}}$ to 0.809 for $\mathrm{C}_{\mathrm{alv}}$. The repeatability in our study was probably higher because not only short-term measurement variation was included but also long-term changes in airway inflammation and disease control.

The most important limiting factor to determine alveolar and bronchial NO, is linearity of the regression model. The additional value of $\mathrm{C}_{\mathrm{alv}} / \mathrm{J}_{\mathrm{NO}}$ compared to FeNO levels for clinical practice needs to be elucidated. In addition, relations between $\mathrm{C}_{\text {alv }} / \mathrm{J}_{\mathrm{NO}}$ with symptoms, bronchial hyperresponsiveness, and airway resistance need to be studied.

This study showed good feasibility of NO measurements in asthmatic children of 6 years and over. A significant learning effect was present. Alveolar NO levels and bronchial NO flux could be assessed in asthmatic children with good feasibility and linearity at flow rates of $50 \mathrm{~mL} / \mathrm{sec}$ to $200 \mathrm{~mL} / \mathrm{sec}$. The long-term reproducibility of alveolar and bronchial NO values during 6 months was moderate.

\section{References}

1. Kraft M. The distal airways: are they important in asthma? Eur Respir J 1999: 14: 1403-1417. 
2. Hamid Q, Song Y, Kotsimbos TC, Minshall E, Bai TR, et al. Inflammation of small airways in asthma. J Allergy Clin Immunol 1997: 100: 44-51.

3. Bjermer L. History and future perspectives of treating asthma as a systemic and small airways disease. Respir Med 2001: 95: 703-719.

4. Pijnenburg MW, Hofhuis W, Hop WC, De Jongste JC. Exhaled nitric oxide predicts asthma relapse in children with clinical asthma remission. Thorax 2005: 60: 215-218.

5. Jones SL, Kittelson J, Cowan JO, Flannery EM, Hancox RJ, et al. The predictive value of exhaled nitric oxide measurements in assessing changes in asthma control. Am J Respir Crit Care Med 2001: 164: 738-743.

6. Berkman N, Avital A, Breuer R, Bardach E, Springer C, et al. Exhaled nitric oxide in the diagnosis of asthma: comparison with bronchial provocation tests. Thorax 2005: 60: 383-388.

7. Taylor DR, Pijnenburg MW, Smith AD, De Jongste JC. Exhaled nitric oxide measurements: clinical application and interpretation. Thorax 2006: 61: 817-827.

8. Warke TJ, Fitch PS, Brown V, Taylor R, Lyons JD, et al. Exhaled nitric oxide correlates with airway eosinophils in childhood asthma. Thorax 2002: 57: 383-387.

9. Piacentini GL, Bodini A, Costella S, Vicentini L, Mazzi P, et al. Exhaled nitric oxide and sputum eosinophil markers of inflammation in asthmatic children. Eur Respir J 1999: 13: 1386-1390.

10 Pijnenburg MW, Bakker EM, Hop WC, De Jongste JC. Titrating steroids on exhaled nitric oxide in children with asthma: a randomized controlled trial. Am J Respir Crit Care Med 2005: 172: 831-836.

11. Lehtimaki L, Turjanmaa V, Kankaanranta H, Saarelainen S, Hahtola P, et al. Increased bronchial nitric oxide production in patients with asthma measured with a novel method of different exhalation flow rates. Ann Med 2000: 32: 417-423.

12. Gelb AF, Taylor CF, Nussbaum E, Gutierrez C, Schein A, et al. Alveolar and airway sites of nitric oxide inflammation in treated asthma. Am J Respir Crit Care Med 2004: 170: 737-741.

13. Latzin P, Beck J, Griese M. Exhaled nitric oxide in healthy children: variability and a lack of correlation with atopy. Pediatr Allergy Immunol 2002: 13: 37-46.

14. Lehtimaki L, Kankaanranta H, Saarelainen S, Hahtola P, Jarvenpaa R, et al. Extended exhaled NO measurement differentiates between alveolar and bronchial inflammation. Am J Respir Crit Care Med 2001: 163: 1557-1561.

15. Pietropaoli AP, Perillo IB, Torres A, Perkins PT, Frasier LM, et al. Simultaneous measurement of nitric oxide production by conducting and alveolar airways of humans. J Appl Physiol 1999: 87: 1532-1542.

16. ATS/ERS Recommendations for Standardized Procedures for the Online and Offline Measurement of Exhaled Lower Respiratory Nitric Oxide and Nasal Nitric Oxide, 2005. Am J Respir Crit Care Med 2005: 171: 912-930.

17. Lehtimaki L, Kankaanranta H, Saarelainen S, Turjanmaa V, Moilanen E. Inhaled fluticasone decreases bronchial but not alveolar nitric oxide output in asthma. Eur Respir J 2001: 18: 635-639.

18. Tsoukias NM, George SC. A two-compartment model of pulmonary nitric oxide exchange dynamics. J Appl Physiol 1998: 85: 653-666.

19. Rabe KF, Adachi M, Lai CK, Soriano JB, Vermeire PA, et al. Worldwide severity and control of asthma in children and adults: the global asthma insights and reality surveys. J Allergy Clin Immunol 2004: 114: 40-47.

20. Suri R, Paraskakis E, Bush A. Alveolar, but not bronchial nitric oxide production is elevated in cystic fibrosis. Ped Pulmonol 2007;42:1215-1221.

21. Mahut B, Delacourt C, Zerah-Lancner, Blic Jacques de, Harf Alain, Delclaux C. Increase in alveolar nitric oxide in the presence of symptoms in childhood asthma. Chest 2004;125:1012-18.

22. Amsterdam JGC van, Zanen P, Somer S, Loveren $H$ van, Opperhuizen A, Steerenberg PA. Flow dependency and off-line measurement of exhaled NO in children. Ped Allergy Immunol 2003;14:266-71.

23. Paraskakis E, Brindicci C, Fleming L, Krol R, Kharitonov SA, et al. Measurement of bronchial and alveolar nitric oxide production in normal children and children with asthma. Am J Respir Crit Care Med 2006: 174: 260267.

24. Silkoff PE, Sylvester JT, Zamel N, Permutt S. Airway nitric oxide diffusion in asthma. Am J Respir Crit Care Med 2000;161:1218-1228.

25. George SC, Hogman M, Permutt S, Silkoff PE. Modeling pulmonary nitric oxide exchange. J Appl Physiol 2004: 96: 831-839. 



\section{CHAPTER 3}

\section{Biomarker reproducibility in exhaled breath condensate collected with different condensers}

PPR Rosias, CMHHT Robroeks, A Kester, GJM den Hartog, WK Wodzig, GT Rijkers, LI Zimmermann, CP van Schayck, Q Jöbsis, E Dompeling European Respiratory Journal 2008;31(5):934-942 


\section{Abstract}

\section{Background}

Optimal collection and analysis of exhaled breath condensate (EBC) are prerequisites for standardisation and reproducibility of assessments. The present study aimed to assess reproducibility of EBC volume, hydrogen peroxide $\left(\mathrm{H}_{2} \mathrm{O}_{2}\right)$, 8-isoprostane and cytokine measurements using different condensers, including a newly developed glass condenser.

\section{Methods}

At four points in time, 30 healthy subjects performed sequential EBC collections randomly using the following four condensers: glass, silicone, EcoScreen (Erich Jaeger $\mathrm{GmbH}$, Hoechberg, Germany) and an optimised glass condenser. In small EBC samples, $\mathrm{H}_{2} \mathrm{O}_{2}$ was measured by spectrophotometer, 8-isoprostane by enzyme immunoassay, and cytokines by multiplexed XMAP technology (Luminex Corporation, Austin, TX, USA).

\section{Results}

The optimised glass condenser yielded significantly more EBC volume (median 2,025 $\mu \mathrm{L}$, interquartile range 1,600-2,525). The reproducibility of EBC volume, yielded by the new glass condenser, was comparable with EcoScreen (19-20 coefficients of variation (CV)\%), but was significantly better compared with silicone and glass (29-37 CV\%). The new condenser was associated with significantly more detections of $\mathrm{H}_{2} \mathrm{O}_{2}$, 8-isoprostane, interleukin-2, -4, -5 and -13 , and tumour necrosis factor-alpha. Isoprostane concentrations were significantly higher using the new condenser, whereas $\mathrm{H}_{2} \mathrm{O}_{2}$ and cytokine concentrations were not. Reproducibility of biomarkers was equally variable for all condenser types.

\section{Conclusion}

In conclusion, significantly more exhaled breath condensate volume and biomarker detections were found using the optimised glass condenser, including higher 8-isoprostane levels. However, biomarker reproducibility in exhaled breath condensate in healthy adults was not influenced by the type of condenser. 


\section{Introduction}

The collection of exhaled breath condensate $(E B C)$ is a non-invasive, safe technique to obtain direct samples from the lower respiratory tract, without disturbing an ongoing inflammation [1-3]. Analysis of EBC reveals the presence of inflammatory markers such as eicosanoids, hydrogen peroxide, and cytokines [1-3]. Although the American Thoracic Society and European Respiratory Society Task Force on EBC published general methodological recommendations on the collection and analysis of EBC, there are still some unresolved methodological pitfalls, as illustrated by the use of various nonstandardised collection systems [1-3].

Optimal condensate collection, and optimal biomarker detection and measurement in EBC are reciprocal prerequisites for any standardisation. However, current condensation systems are suboptimal, with relatively short-measured, open ended designs and loss of noncondensed exhaled breath, as reflected by variable EBC volumes and biomarker reproducibility [1]. Logically, modification may improve condensation, using guided breath flows, enlarged condensation surfaces, and optimised condensate recovery. Moreover, current designs have different inner coatings, featuring different adhesive interactions with exhaled markers [4]. Recently, loss of biomarker within the sampling system was demonstrated, in vitro and in vivo, for 8-isoprostane and albumin, at the expense of nonglass condenser systems, and in favour of glass and silicone condensers [4]. To assess clinical relevance, a study on the reproducibility of these biomarker measurements in vivo, using different condenser coatings including glass, is needed [4]. Furthermore, conventional biomarker assays are not always suitable for use in even large sample volumes of condensate, a bio fluid highly 'diluted' by water vapour [5], whereas new analytical techniques are rapidly emerging and may offer new perspectives [6-7]. Recently, multiplexed cytometric bead array was used in children to simultaneously measure different cytokines in only $50 \mu \mathrm{L}$ condensate samples, however, the detection level did not reach $50 \%$ [6]. Liquid bead-based multiplexing XMAP technology (Luminex Corporation, Austin, USA), based on flow cytometry and (faster) liquid microspheres reaction kinetics, is less laborious, highly sensitive and specific, allows simultaneous measurements in small sample sizes, and improves interarray reproducibility [7]. Therefore, we hypothesised that optimised condensate collection with minimal adhesive properties, improves the reproducibility of different measurements in EBC.

The aim of the present study is to assess the effect of four different condensers (glass, silicone, EcoScreen , and a new, optimised glass condenser) on the reproducibility of breath condensate volume, and the detection, concentration and reproducibility of inflammatory biomarkers, including hydrogen peroxide, 8-isoprostane, interleukin (IL)-2, IL4, IL-5, IL-6, IL-8, IL-10, IL-13, and tumor necrosis factor-alpha (TNF- $\alpha$ ), in EBC in healthy nonasthmatic adults. 


\section{Methods}

\section{Study subjects}

A total of 30 eligible healthy nonasthmatic adult volunteers were recruited (Table 1) among medical students and staff at the University Hospital Maastricht (Maastricht, the Netherlands), based on the criterion that each volunteer was able to breath tidally into a mouthpiece for $\geq 15 \mathrm{~min}$. Exclusion criteria were as follows: history of asthma, upper or lower airway infection, and use of antibiotics, corticosteroids, cromoglycate, nedocromil, theophylline or leukotriene antagonist. Nonasthmatic healthy adults were chosen to eliminate possible confounding factors attributable to heterogeneous disease expression and/or variability of disease control.

Table 1: Subject characteristics of healthy nonasthmatic adult volunteers $\#$.

\begin{tabular}{ll}
\hline Male/Female & $19: 11$ \\
Age $(\mathrm{yrs})$ & $23(22-36)$ \\
Weight $(\mathrm{kg})$ & $75(62-87)$ \\
Height $(\mathrm{cm})$ & $180(171-185)$ \\
Nonsmoking & 28 \\
History of eczema and/or hay fever & 6
\end{tabular}

Data are presented as $n$ or median (interquartile range). ${ }^{\#}: n=30$.

\section{Study design}

To assess within-day, between-day and between-week reproducibility, each volunteer was asked to perform the following four tests. Test 1: on the first day in the morning; test 2: on the first day in the afternoon; test 3: the second day at the same time as test 1 ; and test 4 : 1 week after test 1 at the same time. In turn, each test consisted of four sequential EBC collections, using random different types of condenser.

\section{Exhaled breath condensate collection}

EBC was collected using one of the following condensers: the commercial Teflon-like EcoScreen, a condenser with exchangeable inner cylinder of silicone or glass, as described previously [4], and a new, optimised glass condenser that was developed in close collaboration with the Department of Instrument Development Engineering \& Evaluation (IDEE) of the Maastricht University (patent number EP 07102586), as extensively described in figure 1. Briefly, the inclined condensation surface is enlarged (using a length of $90 \mathrm{~cm}$ ), condensate recovery is optimised (using a downwards moveable plunger), and breath flows are turbinately directed towards the condenser wall (by the plunger's multiple breath channels). To perform one EBC collection, each subject was asked to exhale tidally, while using a nose clip, through a mouthpiece and two-way nonrebreathing valve connected with the condenser, during a fixed period of 15 minutes. 


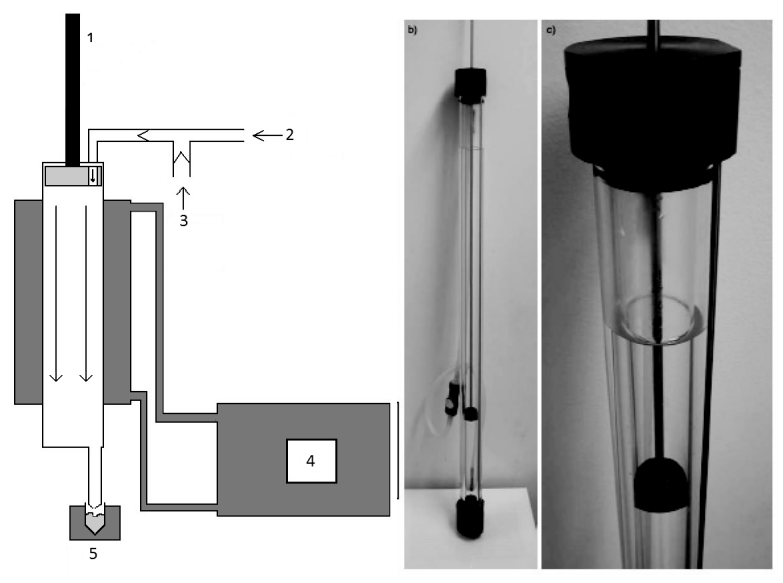

Figure 1 a: A diagrammatic description of the new, optimised glass condenser system. b: The glass condenser tube with plunger in situ. c: detail of the glass condenser tube with the downwards moveable plunger in situ. The plunger has three tangential and axial breath flow channels (to turbinately direct the tidally exhaled breath flows towards the cooled inner condenser wall).

1: inclined glass condenser with a tube length of $90 \mathrm{~cm}$ (to enlarge the condensation surface) and downwards moveable plunger (to optimise condensate recovery from the inner condenser wall), with tangential and axial breath flow channels (to turbinately direct the tidally exhaled breath flows towards the cooled inner condenser wall). 2: swan-neck tubing (serving as gravitational saliva trap) and two-way nonrebreathing valve, connected to a mouthpiece. 3: entrance of inspired room air. 4: cooling unit consisting of a counter-current circulating ice-water pump ( 1 ). 5: removable, cooled glass sample vial (to collect exhaled breath condensate).

\section{Condensate sample processing}

Immediately after collection, condensate samples were snap-frozen at $-78^{\circ} \mathrm{C}$ using dry ice and stored at $-80^{\circ} \mathrm{C}$. Analysis was performed within three months from sampling time.

Hydrogen peroxide was measured in $50 \mu \mathrm{LEBC}$, in duplicate by spectrophotometer (Perkin Elmer UV-VIS Lambda 10 Spectrometer, Shelton/Norwalk, Connecticut, USA) with a lower detection limit of $0.05 \mu \mathrm{M}$, as described previously [8].

Isoprostane was measured in $100 \mu \mathrm{L}$ EBC, by specific enzyme immunoassay (Cayman Chemical , Ann Arbor, Michigan, USA), that was modified to reach a lower limit of detection of $1.0 \mathrm{pg} / \mathrm{mL}$. The standard curve of this assay ranged $250-1.95 \mathrm{pg} / \mathrm{mL}$. It was possible to report 8 -isoprostane values as low as $1.0 \mathrm{pg} / \mathrm{mL}$, as a logit/log transformation was used. Isoprostane recovery experiments were performed, and coefficients of variation (CV) of the absorption signals were assessed. When spiking for the lower 8-isoprostane values of 3.9 and $7.8 \mathrm{pg} / \mathrm{mL}$, an isoprostane recovery of $92 \%$ (CV of concentration 16\%) and $95 \%$ (CV of concentration 15\%) were found, respectively. A CV (of absorption signals) $<15 \%$ was considered highly acceptable. Hence, the corresponding CV of concentrations may be higher. Therefore, the CVs found in the 8-isoprostane recovery experiments using these low values were considered good. Standard curves, patient samples and quality control samples of $2.5 \mathrm{pg} / \mathrm{mL}$ and $10 \mathrm{pg} / \mathrm{mL}$ were assayed in triplicate. Intra-assay variation of standard curves, patient samples and quality control samples had to be $<15 \%$, otherwise all samples measured in that assay were excluded and reanalysed. Finally, in all accepted samples, 8-isoprostane concentrations were determined from mean absorption signal intensities. 
Cytokines were measured in $50 \mu \mathrm{L} E B C$, using the liquid bead-based multiplexing $\mathrm{XMAP}^{\circ}$ technology (Luminex Corporation, Austin, USA). Multiplex immunoassay was performed as described previously [7,9-10]. The corresponding lower limits of cytokine and chemokine detection were (in pg/mL): IL-2 (1.0), IL-4 (1.2), IL-5 (1.2), IL-6 (0.4), IL-8 (1.1), IL-10 (1.2), IL-13 (1.0), and TNF- $\alpha$ (1.3). Measurement and data analysis were performed using the Bioplex 100 system and Bioplex Manager software version 3.0 (Bio-Rad Laboratories, Hercules, CA). All multiplex immunoassays were performed in 96 well format $1.2 \mu \mathrm{m}$ filter bottom plates (Millipore, Amsterdam, The Netherlands) and a 12 point standard curve in duplicate was included on every plate. In order to minimise interassay variation, positive and negative control samples were included. As far as possible, EBC samples from one donor series of experiments were run on one plate. Three EBC samples were spiked with either 100 or $10 \mathrm{pg} / \mathrm{mL}$ cytokines (IL-2, $-4,-5,-6,-8,-10$ and -13 , and TNF- $\alpha$ ), and were measured in quadruplicate. The mean CV of these cytokine measurements was $12.2 \%$. The mean recovery of $100 \mathrm{pg} / \mathrm{mL}$ spiked cytokine was $103 \%$ (range $71-129 \%$ ). At $10 \mathrm{pg} / \mathrm{mL}$, the mean recovery was somewhat lower at $89 \%$ (ranging from $64 \%$ in IL-13 to $111 \%$ in IL-4). It was concluded that there was a slight matrix effect of EBC, but this did not result in an overestimation of cytokine measurements.

\section{Statistics}

Not normally distributed data were expressed as median and interquartile range (IQR). Normally distributed data were expressed as mean and standard error (SE). To estimate variance within a single method of measurement (coating), CV used.

$$
\mathrm{CV}=(\mathrm{SD} / \mathrm{mean}) \times 100 \%[1]
$$

The CV was calculated as the mean of individual CVs, calculated over the two or four relevant measurements. Within-subject CVs were used as data distribution was normal. When the distribution of individual CVs was not normal, the nonparametric Friedman test was used to see if these CVs were different between coatings. ANOVA was used to test for differences among normally distributed repeated measures. The Chi-squared test was used to statistically evaluate the differences between proportions for four groups in a data set. Condensate samples with a biomarker concentration below the lower detection limit, i.e. in strictu sensu negative detections, were not considered as missing values because they actually informed that marker concentrations were below the lower detection limit. Therefore, these negative detections were given an arbitrary value between zero and the lower detection limit: $0.025 \mu \mathrm{M}$ for hydrogen peroxide, $0.1 \mathrm{pg} / \mathrm{mL}$ for 8 -isoprostane, and $0.1 \mathrm{pg} / \mathrm{mL}$ for cytokines. Samples were defined missing if the EBC volume, yielded after 15 minutes collection time, was zero, or insufficient to analyse.

\section{Power calculation}

Ideally, power calculations should be based on the expected changes in biomarker concentrations and on their variability. However, these changes in biomarker concentrations and their variability were unknown. In fact, the objective of this study was to answer this question. Therefore, the uncertainty of the estimated variability was taken as a basis for power calculations. Data from 30 volunteers were thus available for each of the estimated 
variances (within-day, between-day and between-week). Using standard results for the variance of a Chi-squared random variable, the present authors inferred that, with the 30 subjects, the relative confidence limits (relative to the observed value of the variance) would be 0.634 and 1.807 . For the standard deviation this implies relative limits of 0.80 and 1.34 [11].

\section{Ethics}

All parents gave written informed consent. The study was approved by the Medical Ethics Committee of the University Hospital of Maastricht.

\section{Results}

\section{$E B C$ volume}

In five out of 480 manoeuvres performed, the present authors were unable to collect condensate due to erroneously connected tubing. EBC collection by the optimised glass condenser yielded significantly more median condensate volume compared with the other condensers ( $p=0.001$, Friedman-test; Table 2). Within-day, between-day, between-week and overall reproducibility of EBC volume (expressed as CV\%) were comparable in the new condenser and EcoScreen ( $p=0.715$, Chi-square), but was significantly better compared to silicone and glass ( $p<0.028$, Chi-square test).

Table 2: Volume of exhaled breath condensate, and reproducibility (expressed as coefficients of variation) in four different condenser systems.

\begin{tabular}{llllll}
\hline EBC & Silicone & Glass & New & EcoScreen & p-value $^{\text {" }}$ \\
\hline Failed ${ }^{\#}$ total $n$ & $4 / 120$ & $0 / 120$ & $1 / 120$ & $0 / 120$ & - \\
Volume of EBC $(\mu \mathrm{L})^{*}$ & 525 & 712 & 2025 & 1237 & $\mathbf{0 . 0 0 1}$ \\
& $(300-700)$ & $(500-912)$ & $(1600-2525)$ & $(950-1800)$ & $\mathbf{0 . 0 0 2}$ \\
CV within-day \% & $20(10-37)$ & $21(12-31)$ & $11(5-20)$ & $9(4-25)$ & $\mathbf{0 . 0 2 7}$ \\
CV between-day \% & $28(11-47)$ & $30(11-37)$ & $11(4-30)$ & $13(5-28)$ & $\mathbf{0 . 0 0 3}$ \\
CV between-week \% & $35(19-55)$ & $26(31-41)$ & $15(6-28)$ & $15(6-27)$ & $\mathbf{0 . 0 0 1}$ \\
CV overall \% & $37(27-49)$ & $29(22-38)$ & $20(10-27)$ & $19(11-38)$ & \\
\hline
\end{tabular}

Data are presented as median (interquartile range), unless otherwise indicated. EcoScreen is manufactured by Erich Jaeger $\mathrm{GmbH}$ (Hoechberg, Germany). New: optimised glass condenser; ": after 15 min; ": Friedman test.

\section{$\mathrm{H}_{2} \mathrm{O}_{2}$ measurements in $\mathrm{EBC}$}

Overall, $29 \%$ of $\mathrm{H}_{2} \mathrm{O}_{2}$ measurements were missing (Table 3). Significantly more positive $\mathrm{H}_{2} \mathrm{O}_{2}$ detections were found using the optimised glass condenser, compared with silicone and glass ( $p<0.050$, Chi-squared test). Median $\mathrm{H}_{2} \mathrm{O}_{2}$ concentrations and reproducibility (expressed as CV\%) did not significantly differ between the four condensers ( $p=0.286$ and $\mathrm{p}>0.080$, respectively, Friedman test). 
Table 3: Hydrogen peroxide measurements in exhaled breath condensate: detection, concentration, and reproducibility (expressed as coefficients of variation (CV)) in four different condenser systems.

\begin{tabular}{llllll}
\hline $\mathrm{H}_{2} \mathrm{O}_{2}$ & Silicone & Glass & New & EcoScreen & p-value \\
\hline $\begin{array}{l}\text { Missing/total } n \\
\text { Ratio of positive to }\end{array}$ & $59 / 120$ & $49 / 120$ & $10 / 120$ & $23 / 120$ & - \\
negative detections $(n / n)$ & $52 / 9$ & $62 / 9$ & $77 / 33$ & $75 / 22$ & $\mathbf{0 . 0 0 3}^{\#}$ \\
$\mathrm{H}_{2} \mathrm{O}_{2}$ in EBC $(\mu \mathrm{M})$ & & & & \\
$\mathrm{CV}$ within-day \% & $2.6(1.4-4.1)$ & $2.1(1.0-3.3)$ & $1.8(1.0-2.6)$ & $2.2(0.3-2.8)$ & $0.286^{\pi}$ \\
$\mathrm{CV}$ between-day \% & $17(6-31)$ & $12(6-34)$ & $46(15-86)$ & $13(1-22)$ & $0.271^{\pi}$ \\
$\mathrm{CV}$ between-week \% & $22(7-40)$ & $30(4-46)$ & $30(1-119)$ & $16(8-60)$ & $0.821^{\pi}$ \\
$\mathrm{CV}$ overall \% & $18(3-49)$ & $26(5-47)$ & $32(13-57)$ & $12(5-37)$ & $0.256^{\pi}$ \\
\hline
\end{tabular}

Data are presented as median (interquartile range), unless otherwise indicated. EcoScreen is manufactured

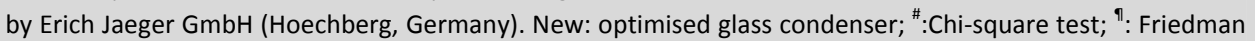
test.

\section{8-Isoprostane measurements in EBC}

Overall, $13 \%$ of 8 -isoprostane measurements were missing (table 4 ). Significantly more positive 8-isoprostane detections were found using the optimised glass condenser, compared with silicone and glass ( $p<0.023$, Chi-squared test). The median concentration of 8 isoprostane was significantly higher using the new condenser compared with the other three condensers ( $p=0.001$, Friedman test). Statistically, 8-isoprostane reproducibility (expressed as CV\%) did not significantly differ between the four condensers $(p>0.151$, Friedman test).

Table 4: 8-Isoprostane (IP) measurements in exhaled breath condensate (EBC) presented as detection, concentration, and reproducibility (expressed as coefficients of variation (CV)) in four different condenser systems.

\begin{tabular}{|c|c|c|c|c|c|}
\hline $8-I P$ & Silicone & Glass & New & EcoScreen & $p$-value \\
\hline Missing/total $n$ & $27 / 120$ & $16 / 120$ & $9 / 120$ & $10 / 120$ & - \\
\hline $\begin{array}{l}\text { Ratio of positive to } \\
\text { negative detections }(n / n)\end{array}$ & $89 / 4$ & $98 / 6$ & $110 / 1$ & $109 / 1$ & $0.001^{\#}$ \\
\hline 8-isoprostane $(\mathrm{pg} / \mathrm{mL})$ & $2.0(1.3-3.5)$ & $2.9(1.4-3.7)$ & $3.6(2.2-4.9)$ & $2.5(1.6-3.4)$ & $0.001^{9}$ \\
\hline CV within-day $\%$ & $6(1-30)$ & $20(6-61)$ & $23(6-41)$ & $24(8-37)$ & $0.297^{\uparrow}$ \\
\hline CV between-day \% & $31(7-72)$ & $26(7-64)$ & $23(10-42)$ & $15(7-39)$ & $0.559^{\natural}$ \\
\hline CV between-week \% & $22(9-51)$ & $22(2-59)$ & 15 (8-39) & $25(8-36)$ & $0.954^{9}$ \\
\hline CV overall \% & $31(11-68)$ & $48(14-69)$ & $29(18-40)$ & $26(18-44)$ & $0.151^{9}$ \\
\hline
\end{tabular}

Data are presented as median (interquartile range), unless otherwise indicated. EcoScreen is manufactured by Erich Jaeger $\mathrm{GmbH}$ (Hoechberg, Germany). New: optimised glass condenser; ":Chi-square test; ": Friedman test.

\section{Cytokine measurements in $E B C$}

Overall, $20 \%$ of cytokine measurements were missing (Table 5). 
Table 5: Number of positive to negative detections of cytokines in exhaled breath condensate, collected with four different condenser systems.

\begin{tabular}{llllll}
\hline \multicolumn{7}{l}{ Type of condenser } & & & \\
\hline Cytokine & Silicone & Glass & New & EcoScreen & p-value $^{\#}$ \\
\hline $\begin{array}{l}\text { Missings/total n positive } \\
\text { to negative detections }\end{array}$ & $49 / 120$ & $37 / 120$ & $1 / 120$ & $10 / 120$ & - \\
IL-2 & $50 / 21$ & $60 / 23$ & $\mathbf{8 2 / 3 7}$ & $68 / 42$ & $\mathbf{0 . 0 0 1}$ \\
IL-4 & $69 / 2$ & $81 / 2$ & $\mathbf{1 1 3 / 6}$ & $108 / 2$ & $\mathbf{0 . 0 0 1}$ \\
IL-5 & $58 / 13$ & $65 / 18$ & $\mathbf{8 7 / 3 2}$ & $70 / 40$ & $\mathbf{0 . 0 0 1}$ \\
IL-6 & $41 / 30$ & $45 / 38$ & $48 / 71$ & $43 / 67$ & 0.881 \\
IL-8 & $44 / 27$ & $45 / 38$ & $44 / 75$ & $44 / 66$ & 0.999 \\
IL-10 & $54 / 17$ & $57 / 26$ & $63 / 56$ & $61 / 49$ & 0.654 \\
IL-13 & $53 / 18$ & $58 / 25$ & $\mathbf{8 8 / 3 1}$ & $71 / 39$ & $\mathbf{0 . 0 0 1}$ \\
TNF- $\alpha$ & $52 / 19$ & $57 / 26$ & $\mathbf{7 2 / 4 7}$ & $65 / 45$ & $\mathbf{0 . 0 5 0}$ \\
\hline
\end{tabular}

Data are presented as median (interquartile range), unless otherwise indicated. EcoScreen is manufactured by Erich Jaeger GmbH (Hoechberg, Germany). IL; interleukin; TNF; tumor necrosis factor. New: optimised

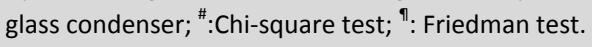

The levels of cytokine detection using XMAP technology were as follows: IL-2 (68\%), IL-4 (97\%), IL-5 (73\%), IL-6 (46\%), IL-8 (46\%), IL-10 (61\%), IL-13 (70\%) and TNF- $\alpha$ (64\%). The optimised glass condenser had significantly more positive detections of IL-2, -4, -5, -13 and TNF- $\alpha$, compared with silicone and glass ( $p<0.050$, Chi-squared test), and more IL-5 and 13 detections, compared with EcoScreen ( $p<0.021$, Chi-squared test). Cytokine concentrations were not significantly different between the four condenser types ( $p>0.113$, Friedman test or ANOVA, respectively; Table 6).

Table 6: Concentration $(\mathrm{pg} / \mathrm{mL})$ of cytokine measurements in exhaled breath condensate $(E B C)$, collected with four different condenser systems.

\begin{tabular}{llllll}
\hline \multicolumn{7}{l}{ Type of condenser } & & \\
\hline Cytokine & Silicone & Glass & New & EcoScreen & p-value \\
\hline Missings/total $n$ & $49 / 120$ & $37 / 120$ & $1 / 120$ & $10 / 120$ & - \\
IL-2 $^{\#}$ & $1.9(5.0)$ & $1.6(4.4)$ & $1.2(3.5)$ & $1.6(4.0)$ & $0.494^{+}$ \\
IL-4" & $5.8(0.3)$ & $5.1(0.3)$ & $4.7(0.3)$ & $5.4(0.3)$ & $0.129^{\S}$ \\
IL-5 & $4.4(13.8)$ & $3.0(12.1)$ & $3.2(11.4)$ & $3.1(12.1)$ & $0.544^{+}$ \\
IL-6 & $0.9(4.3)$ & $0.9(4.5)$ & $0.7(3.8)$ & $0.6(4.4)$ & $0.113^{+}$ \\
IL-8 & $2.0(4.6)$ & $2.1(4.0)$ & $2.7(4.8)$ & $2.1(3.9)$ & $0.341^{+}$ \\
IL-10" & $1.5(0.3)$ & $1.6(0.9)$ & $1.4(0.8)$ & $1.3(0.7)$ & $0.872^{\S}$ \\
IL-13" & $6.6(1.1)$ & $6.6(1.1)$ & $6.3(0.7)$ & $5.9(0.8)$ & $0.946^{\S}$ \\
TNF- $\alpha^{\text {q }}$ & $2.1(0.4)$ & $2.0(0.4)$ & $1.8(0.3)$ & $1.7(0.3)$ & $0.876^{\S}$ \\
\hline
\end{tabular}

Unless otherwise indicated, data are presented as: ${ }^{*}$ median (interquartile range); ${ }^{\text {}}$ : mean ( $95 \%$ confidence interval). EcoScreen is manufactured by Erich Jaeger GmbH (Hoechberg, Germany). IL; interleukin; TNF; tumor necrosis factor. New: optimised glass condenser; ${ }^{+}$: Friedman test; ${ }^{\wp}$ : ANOVA.

Reproducibility (expressed as CV\%) of cytokine measurements did not significantly differ, either overall (Table 7) or within-day, between-day and between-week (data not shown). The best range of overall CV\% was found for the measurement of IL-6 (11-14\%), IL-8 (2- 
15\%), IL-10 (11-30\%) and TNF- $\alpha$ (8-22\%). When CVs were evaluated using the positive values only (without negative and/or arbitrary values), again no differences between condensers were found (data not shown).

Table 7: Overall reproducibility (expressed as coefficients of variation) of cytokine measurements in exhaled breath condensate, collected with four different condenser systems.

\begin{tabular}{llllll}
\hline \multicolumn{7}{l}{ Type of condenser } \\
\hline Cytokine & Silicone & Glass & New & EcoScreen & p-value \\
\hline $\begin{array}{l}\text { Missings/total n } \\
\text { Overall reproducibility \% }\end{array}$ & $49 / 120$ & $37 / 120$ & $1 / 120$ & $10 / 120$ & - \\
IL-2 & & & & \\
IL-4 & $80(12)$ & $75(11)$ & $84(10)$ & $70(11)$ & $0.802^{+}$ \\
IL-5 & $33(7)$ & $32(6)$ & $36(5)$ & $26(4)$ & $0.494^{+}$ \\
IL-6" & $74(13)$ & $71(12)$ & $73(11)$ & $81(13)$ & $0.941^{+}$ \\
IL-8" & $12(77)$ & $12(68)$ & $14(78)$ & $11(69)$ & $0.690^{f}$ \\
IL-10" & $15(28)$ & $12(21)$ & $2(18)$ & $9(25)$ & $0.766^{f}$ \\
IL-13 & $30(48)$ & $17(48)$ & $16(39)$ & $11(53)$ & $0.719^{f}$ \\
TNF- & $51(11)$ & $68(12)$ & $73(10)$ & $63(10)$ & $0.574^{+}$ \\
\hline
\end{tabular}

Unless otherwise indicated, data are presented as: \# mean (95\% confidence); १: median (confidence interval). New: optimised glass condenser; IL; interleukin; TNF; tumor necrosis factor. +; ANOVA; f: Friedman test.

\section{Discussion}

The present authors have demonstrated that EBC volume, and the detection of biomarkers were significantly influenced by the condenser system, in favour of the new glass condenser, whereas biomarker reproducibility was not influenced by the type of condenser. The proposed optimised glass condenser yielded significantly more condensate volume compared to the silicone, glass and EcoScreen condensers. Reproducibility of EBC volume was comparable for the new condenser and EcoScreen , and was significantly better compared to the other two condenser types.

In EBC collected with this new condenser, significantly more positive $\mathrm{H}_{2} \mathrm{O}_{2}$, 8-isoprostane, IL-2, IL-4, IL-5, IL-13 and TNF- $\alpha$ detections were found, supporting improved sampling of EBC. Moreover, 8-isoprostane concentrations were significantly increased in EBC yielded by the new condenser, compared to the other three condensers, which is in accordance with former findings [4]. Conversely, reproducibility of $\mathrm{H}_{2} \mathrm{O}_{2}, 8$-isoprostane, and cytokine measurements in EBC did not significantly differ between the four condensers, suggesting no significant influence of the type of condenser coating on reproducibility. In literature and to date, no formal study addressing this issue has been published, although reproducibility using other analytical techniques has been reported [12-14], and/or could not be calculated due to small number of subjects [15-17].

Variations in biomarker measurements in EBC may be attributed to variations in the dilution and/or quality of condensate that is influenced by different collection techniques and procedures, sample processing and storage conditions, and/or sensitivity of the analytical techniques used [17]. The levels of the highly volatile hydrogen peroxide in EBC may be susceptible to different cooling temperatures during collection [18], circadian rhythm [19], flow dependency [20], different methods of measurement with widely varying values 
(even in healthy subjects) close to lower detection limits [3,17,21-22], and high chemical reactivity with salivary and exhaled compounds by which (some) $\mathrm{H}_{2} \mathrm{O}_{2}$ is consumed over time during collection and storage $[3,17,22]$. Isoprostanes are relatively stable endproducts of in vivo arachidonate peroxidation, and are measured in EBC by immunoassay, that may be influenced by cross reactivity with closely related substances [17]. Other confounding factors may be age, diets rich in antioxidants, and smoking habits [17].

The use of CV is not always the ideal way to express variability: when mean values are low, $\mathrm{CV}$ values can be abnormally high. Therefore, results in the present study were also expressed as standard deviation values and intraclass correlation coefficients. When using both of these alternative expression methods, results were comparable: no difference in biomarker variability between condensers was found (data not shown). Furthermore, biomarker levels were assessed in condensate originating from healthy adults and, thus, may have been more pronounced in a steroid-naïve population with documented chronic respiratory inflammation and comparable levels of disease control. Hence, increased mean concentrations and lowered CVs in diseased subjects could be expected. Conversely, with these very low concentrations of cytokines and 8-isoprostane, an influence of analytical variability cannot be ruled out.

Cytokines were simultaneously measured by xMAP technology in small $50 \mu \mathrm{L}$ EBC samples. The overall level of detection was $46-97 \%$, that was much better compared to cytometric bead array in small samples in children $(<50 \%)$, and in large 1000-2000 $\mu \mathrm{L}$ lyophilisated samples in adults (3-100\%) [6,23-26]. When compared with conventional (solid-phase) immunoassays, multiplexed immunoassays detect bioactive and inactive molecules, have a growing analytical range, are rapid (hours instead of days), have good precision (CV 10-15\%), are not interfered by drugs, and have simple protocols [7].

Currently, different nonstandardised techniques to collect EBC are in use [1-3]. All systems are based on the cooling of exhaled breath, whereas their design may vary from immersed plastic tubing, over glass distilling columns, to commercial systems, such as the lamellar Teflon-like EcoScreen condenser (Erich Jaeger $\mathrm{GmbH}$, Hoechberg, Germany), the handheld disposable polypropylene RTube ${ }^{\mathrm{TM}}$ (Respiratory Inc., Charlottesville, Virginia, USA), and the thermostatically-controlled polyethylene Turbo-Deccs (ItalChill, Parma, Italy) [1$3,27]$. These designs implicate relatively short-measured and open-ended systems that tolerate the needless loss of noncondensed exhaled breath, whether initially, or after a prolonged collection time. Moreover, also biomarkers may be lost within these collection systems, as recently demonstrated for 8-isoprostane and albumin measurements, in vitro and in vivo, in nonglass condensers (including EcoScreen ) [4]. This superiority of glass coatings may be mainly related to the behaviour of water as bipolar vehicle [4]. Three other studies report the influence of sampling systems on biomarkers in EBC [28-30]. Tufvesson et al proposed to coat EcoScreen collection surfaces with bovine serum albumin and Tween-20, to measure cytokines and eicosanoids, respectively [28]. However, they also reported possible false-positive (eicosanoid) results, and the need of sample concentration, by vacuum-centrifugation, prior to analysis [28]. Soyer et al [29] found significantly higher cysteinyl leukotrienes and eotaxin using EcoScreen, compared to $\mathrm{RTube}^{\mathrm{TM}}$, due to susceptibility of precooled RTube ${ }^{\mathrm{TM}}$ sleeves to (increased) ambient temperatures during collection, and due to different materials that could affect sample recovery. Prieto et al [30] compared RTube ${ }^{\mathrm{TM}}$ and EcoScreen , and reported that EBC $\mathrm{pH}$ values are dependent on the collection device used. Furthermore, the EcoScreen has been asso- 
ciated with deposition of frozen condensate on its lamellar walls. For all the above mentioned reasons, we developed an optimised glass condenser system. The new glass condenser had an improved condensation process and condensate recovery, using an inclined and enlarged condensation surface, with a condensate sweeping plunger, having tangentially and axially guiding breath flow channels.

In the present study, these improvements resulted in significantly increased EBC volumes, and increased biomarker detections with the new glass condenser, compared to silicone, glass and EcoScreen ${ }^{\circ}$. This suggests both an improved condensation process, and an increased opportunity to perform a broad spectrum of analyses. Moreover, the optimised glass and EcoScreen condenser were both significantly associated with less variation in the generated EBC volume, compared to the other condensers. Hereby, reducing a possible confounding influence of the variable quantity of EBC collected over a given time, and even within individuals.

Optimisation of EBC collection, using the modified new glass condenser with statistically equivalent CVs compared to the commercial EcoScreen , and optimisation of EBC analysis, using rapid, multiplexed measurement of cytokines in small EBC sample sizes, may all together open a window of opportunities, even in strained collection procedures, such as in young or dyspnoeic subjects (with less sustained efforts to cope with sampling procedures), by allowing the search for, and identification of, particular profiles of different exhaled markers, involved in the regulation of chronic respiratory inflammation, for diagnostic and monitoring purposes.

In conclusion, the optimised glass condenser yielded significantly more EBC volume, with good reproducibility. Furthermore, significantly more positive detections of hydrogen peroxide, 8-isoprostane, IL-2, IL-4, IL-5, IL-13 and TNF- $\alpha$ were found in EBC collected with the new condenser, hereby offering an increased capacity to analyse for complex biomarker profiles. Moreover, concentrations of 8-isoprostane were significantly increased using the optimised glass condenser, compared to the other three condensers. However, reproducibility of biomarker measurements in EBC was not influenced by the type of condenser.

\section{Acknowledgements}

The authors wish to thank J. Suykerbuyk (Dept of Paediatrics, University Hospital Maastricht, Maastricht, the Netherlands) for her technical support in the collection of exhaled breath condensate; M. Meers (Dept of Clinical Chemistry, University Hospital Maastricht) for her technical support in the measurement of 8-isoprostane; N. van Uden (Dept of Immunology, University Medical Centre Wilhelmina Children's Hospital, Utrecht, the Netherlands) for her technical support in the measurement of cytokines; and, last but not least, the volunteers for their participation.

\section{References}

1. Rosias PPR, Dompeling E, Hendriks JJE, Heijnens JWCM, Donckerwolcke RAMG, Jöbsis Q. Exhaled breath condensate in children: pearls and pitfalls. Pediatr Allergy Immunol 2004; 15: 4-19.

2. Jöbsis $Q$, Rosias PPR. Analysis of exhaled breath condensate in children. In: New perspectives in monitoring lung inflammation. Montuschi P, editor. Taylor \& Francis Publishers, London, 2004. (ISBN 0415324653) 
3. Horváth I, Hunt J, Barnes PJ, on behalf of the ATS / ERS Task Force, Exhaled breath condensate: methodological recommendations and unresolved questions. Eur Respir J 2005; 26: 523-548.

4. Rosias PP, Robroeks CM, Niemarkt HJ, Kester AD, Vernooy JH, Suykerbuyk J, Teunissen J, Heynens J, Hendriks $\mathrm{HJ}$, Jöbsis Q, Dompeling E. Breath condenser coatings affect measurement of biomarkers in exhaled breath condensate. Eur Respir J 2006; 28: 1036-1041.

5. Huszar E, Szabo Z, Jakab A, Barta I, Herjavecz I, Horvath I. Comparative measurement of thromboxane A2 metabolites in exhaled breath condensate by different immunoassays. Inflamm Res 2005; 54: 350-355.

6. Robroeks CMHHT, Jöbsis Q, Damoiseaux JGMC, Heijmans PHM, Rosias PPR, Hendriks HJE, Dompeling E. Cytokines in exhaled breath condensate of children with asthma and cystic fibrosis. Ann Allergy Asthma Immunol 2006; 96: 349-355.

7. de Jager W, Rijkers GT. Solid-phase and bead-based cytokine immunoassay: a comparison. Methods 2006; 38: 294-303.

8. Dekhuijzen PN, Aben KK, Dekker I, Aarts LP, Wielders PN, van Herwaarden CL, Bast A. Increased exhalation of hydrogen peroxide in patients with stable and unstable chronic obstructive pulmonary disease. Am J Resp Crit Care Med 1996; 154: 813-816.

9. de Jager W, Prakken BJ, Bijlsma JW, Kuis W, Rijkers GT. Improved multiplex immunoassay performance in human plasma and synovial fluid following removal of interfering heterophilic antibodies. J Immunol Methods 2005; 300: 124-135.

10. de Jager W, te Velthuis H, Prakken BJ, Kuis W, Rijkers GT. Simultaneous detection of 15 human cytokines in a single sample of stimulated peripheral blood mononuclear cells. Clin Diagn Lab Immunol 2003; 10: 133-139.

11. Rosner B. Fundamentals of Biostatistics. $6^{\text {th }}$ Edn. Dixbury Press, Belmont, CA, 2006; p.200.

12. Brooks WM, Lash $\mathrm{H}$, Kettle AJ, Epton MJ. Optimising hydrogen peroxide measurement in exhaled breath condensate. Redox Rep 2006; 11: 78-84.

13. Gerritsen WB, Zanen P, Bauwens AA, van den Bosch JM, Haas FJ. Validation of a new method to measure hydrogen peroxide in exhaled breath condensate. Respir Med 2005; 99: 1132-1137.

14. Gonzalez-Reche LM, Musiol AK, Muller-Lux A, Kraus T, Goen T. Method optimization and validation for the simultaneous determination of arachidonic acid metabolites in exhaled breath condensate by liquid chromatography-electrospray ionization tandem mass spectrometry. J Occup Med Toxicol 2006, doi:10.1186/17456673-1-5.

15. Van Hoydonck PG, Wuyts WA, Vanaudenaerde BM, Schouten EG, Dupont L, Temme EH. Quantitative analysis of 8-isoprostane and hydrogen peroxide in exhaled breath condensate. Eur Respir J 2004; 23: 189-192.

16. Kharitonov SA. Exhaled markers of inflammatory lung diseases: ready for routine monitoring? Swiss Med Wkly 2004; 134: 175-192.

17. Rahman I, Biswas SK. Non-invasive biomarkers of oxidative stress: reproducibility and methodological issues. Redox Rep 2004; 9: 125-143.

18. Goldoni M, Caglieri A, Andreoli R, Poli D, Manini P, Vettori MV, Corradi M, Mutti A. Influence of condensation temperature on selected exhaled breath parameters. BMC Pulmonary Medicine 2005, doi: 10.1186/14712466-5-10.

19. Nowak D, Kalucka S, Bialasiewicz $\mathrm{P}$, Krol M. Exhalation of $\mathrm{H}_{2} \mathrm{O}_{2}$ and thiobarbituric acid reactive substances (TBARs) by healthy subjects. Free Radic Biol Med 2001; 30: 178-186.

20. Schleiss MB, Holz O, Behnke M, Richter K, Magnussen H, Jorres RA. The concentration of hydrogen peroxide in exhaled air depends on expiratory flow rate. Eur Respir J 2000; 16: 1115-1118.

21. Rahman I. Reproducibility of oxidative stress biomarkers in breath condensate: are they reliable? Eur Respir J 2004; 23: 183-184.

22. American Thoracic Society. Workshop Proceedings: Exhaled nitric oxide and nitric oxide oxidative metabolism in exhaled breath condensate. Proc Am Thorac Soc 2006; 3: 131-145.

23. Sack U, Scheibe R, Wötzel M, Hammerschmidt S, Kuhn H, Emmrich F, Hoheisel G, Wirtz H, Gessner C. Multiplex analysis of cytokines in exhaled breath condensate. Cytometry A 2006; 69: 169-172.

24. Gessner C, Scheibe R, Wötzel M, Hammerschmidt S, Kuhn H, Engelmann L, Hoheisel G, Gillissen A, Sack U, Wirtz $\mathrm{H}$. Exhaled breath condensate cytokine patterns in chronic obstructive pulmonary disease. Respir Med 2005; 99: 1229-1240. 
25. Matsunaga K, Yanagisawa S, Ichikawa T, Ueshima K, Akamatsu K, Hirano T, Nakanishi M, Yamagata T, Minakata $\mathrm{Y}$, Ichinose $\mathrm{M}$. Airway cytokine expression measured by means of protein array in exhaled breath condensate: correlation with physiologic properties in asthmatic patients. J Allergy Clin Immunol 2006; 118: 8490.

26. Schumann C, Triantafilou K, Krueger S, Hombach V, Triantafilou M, Becher G, Lepper PM. Detection of erythropoietin in exhaled breath condensate of nonhypoxic subjects using a multiplex bead array. Mediators Inflamm 2006, doi:10.1155/MI/2006/18061.

27. Caglieri A, Goldoni M, Acampa O, Andreoli R, Vettori MV, Corradi M, Apostoli P, Mutti A. The effect of inhaled chromium on different exhaled breath condensate biomarkers among chrome-plating workers. Environ Health Perspect 2006; 114: 542-546.

28. Tufvesson E, Bjermer L. Methodological improvements for measuring eicosanoids and cytokines in exhaled breath condensate. Respir Med 2006; 100: 34-38.

29. Soyer OU, Dizdar EA, Keskin O, Lilly C, Kalayci O. Comparison of two methods for exhaled breath condensate collection. Allergy 2006; 61: 1016-1018.

30. Prieto L, Ferrer A, Palop J, Domenech J, Llusar R, Rojas R. Differences in exhaled breath condensate $\mathrm{pH}$ measurements between samples obtained with two commercial devices. Respir Med 2007, doi: 10.1016/j.rmed.2007.02.023. 


\section{CHAPTER 4}

\section{Feasibility of new method to collect exhaled breath condensate in preschool children}

PPR Rosias, CMHHT Robroeks, KDG van de Kant, GT Rijkers, LI Zimmermann, $\mathrm{CP}$ van Schayck, J Heynens, Q Jöbsis, E Dompeling Pediatric Allergy and Immunology 2010;21(1):e235-e244 


\section{Abstract}

\section{Background}

Exhaled breath condensate $(E B C)$ is a promising non-invasive method to assess respiratory inflammation in adults and children with lung disease. Especially in pre-school children, condensate collection is hampered by long sampling times because of open-ended collection systems. We aimed to assess the feasibility of condensate collection in pre-school children using a closed glass condenser with breath recirculation system, which also collects the residual non-condensed exhaled breath, and subsequently recirculates it back into the condenser.

\section{Methods}

Condensate was collected before and after breath recirculation in 70 non-sedated preschool children with and without recurrent wheeze. Cytokines (IL-4, IL-5, IL-6, IL-8, IL-10, IL-12p70, IL-13, TNF- $\alpha$ ) were measured in $50 \mu \mathrm{L}$ samples using ultrasensitive multiplexed liquid bead array.

\section{Results}

The success rate of condensate collection increased from $64 \%$ (without recirculation) to $83 \%$ (after breath recirculation), and mean condensate volume from 214 to $465 \mu \mathrm{L}$ respectively. Detection of cytokines was successful in $95-100 \%$ of samples. Cytokine concentrations before and after breath recirculation were not different ( $p>0.232)$. In asthmatic children, only TNF- $\alpha$ concentrations were significantly decreased, compared to nonasthmatics.

\section{Conclusion}

In pre-school children, the collection of EBC is feasible using a new closed glass condenser with breath recirculation system. This new method may help to assess-non-invasivelycytokine profiles in asthmatic and non-asthmatic pre-school children. 


\section{Introduction}

Pre-school wheezing is reported in no less than $30 \%$ of children, and may indicate a trivial cold, as well as an ongoing chronic lung inflammation, such as asthma [1-3]. Atopic asthma is associated with a cytokine pattern compatible with predominant activation of the Thelper (Th)-2 mediated immune response [4]. However, in the developing pre-school child, accurate assessment of different wheezing phenotypes is currently difficult [1-3]. Moreover, proper diagnosis is hampered by the current lack of a reliable routine technique to assess different types of respiratory inflammation in pre-school children. Ideally, such a technique would be non-invasive, rapid and easy to perform, without any risks, successful at any age, standardized, sensitive, specific and highly reproducible. The collection of exhaled breath condensate $(E B C)$ is a non-invasive technique to obtain direct samples from the lower respiratory tract, without disturbing an ongoing inflammation $[5,6]$. In children, analysis of EBC reveals the presence of various types of inflammatory biomarkers $[7,8]$, including cytokines $[5,6,9-11]$.

Although general methodological recommendations on EBC collection are published, there are still unresolved questions, resulting in low feasibility of EBC collection in (nonsedated) pre-school children [6,12-14]. Main issues are: (i) the variable efficacy of different condenser coatings, (ii) the variable efficacy of open-ended collection systems and (iii) the use of suboptimal analytical techniques. At first, different condenser coatings may each have different adhesive interactions with a specific type of biomarker that is to be measured in the collected condensate. Therefore, the efficacy of different breath condenser coatings, such as glass, polypropylene and Teflon ${ }^{\mathrm{TM}}$ (DuPont Inc., New Jersey, USA), was studied $[15,16]$. The use of a newly developed and optimized glass condenser was found to be more efficient, in terms of the volume of EBC, and the detection of cytokines and 8 -isoprostane $[15,16]$. Secondly, all EBC collection systems have an open-ended design. Inevitably, such a design is associated with a variable non-standardized loss of noncondensed exhaled breath. In turn, this loss may contribute to unnecessarily prolonged EBC collection times (from a toddler's point of view), as well as to (intra- and inter-subject) variability of EBC dilution, and thus may influence subsequent analysis $[17,18]$. Thirdly, feasibility of EBC is also hampered by the use of suboptimal immunoassays with questionable reproducibility, that often require relatively too large sample volumes that often cannot be collected in pre-school children $[18,19]$.

Taken together, this has led us to make the following methodological adjustments for the purpose of this study in pre-school children: (i) the introduction of a closed optimized glass condenser design with breath recirculation system, which collects all non-condensed residual exhaled breath, and subsequently recirculates it back into the condenser (to optimize condensation and minimize collection time) and (ii) the simultaneous measurement of cytokines in the resulting EBC by means of XMAP technology (Luminex Corporation, Austin, TX, USA), an ultrasensitive multiplexed liquid bead array that requires only one small $(50 \mu \mathrm{L})$ sample volume. We hypothesized that the combination of these methodological adjustments enhances the feasibility of EBC collection and analysis in pre-school children. Therefore, the aim of the present study was to assess the feasibility of EBC collection using a modified closed glass condenser in non-sedated pre-school children with and without wheezing. 


\section{Methods}

\section{Study subjects}

Eligible preschool children were recruited at the paediatric outpatient department of the Maasland Hospital of Sittard and the University Hospital of Maastricht. Healthy preschool children were recruited based on a non-respiratory indication for referral to the paediatric outpatient department; the main indications were routine follow-up of growth and development. According to parent-completed questionnaires, medical documents, and information of the responsible paediatric pulmonologist, children were allocated to one of the following study groups:

1. The doctor's diagnosed atopic asthma group characterized by: (i) recurrent wheezing (three or more episodes of wheeze within the preceding 12 months or less), (ii) not 'exclusively' triggered by viral infections, (iii) with a documented history of a favourable corticosteroid effect (clinical improvement, assessed and documented by the child's physician, after at least 3 months of inhaled corticosteroid therapy, or after a short course of oral prednisone therapy during a wheezy episode), (iv) positive family history of atopy in the first degree (parent or sibling with asthma, hay fever, food allergy or eczema) and (v) atopy (positive Phadiatop or radioallergo-sorbent test class $\geq 2$, and/or elevated blood level of total immunoglobulin-E). Current corticosteroid maintenance therapy was not an obligatory criterion.

2. The viral-triggered recurrent wheeze group characterized by: (i) recurrent wheezing, (ii) 'exclusively' triggered by viral infections, (iii) without a documented history of steroid therapy, or with documented history of no favourable corticosteroid effect and (iv) without corticosteroid maintenance therapy in the preceding 3 months. Lack of atopy was not an obligatory criterion.

3. The healthy control group characterized by: (i) the absence of recurrent wheezing and other respiratory symptoms (including dyspnoea, recurrent cough and signs of upper airway infection), as excluded by means of standardized ISAAC questionnaires and (ii) without a history of corticosteroid maintenance therapy [20]. Lack of atopy was not an obligatory criterion.

4. The unclassifiable recurrent wheeze group consisted of infants and toddlers with recurrent wheezing, but not eligible for the asthmatic or viral wheeze group. They participated only in the feasibility study.

Overall exclusion criteria were cardiac disease, anatomic or congenital defects of the airways or lungs, syndromes, systemic disease, and/or mental retardation. Presumed inability to use a face mask did not preclude participation into this study. Subject characteristics are shown in Table 1. 
Table 1: Characteristics of 70 preschool children, allocated to one of the four study groups, based on parentcompleted questionnaires and available medical documents.

\begin{tabular}{|c|c|c|c|c|}
\hline Study group & Atopic asthma & $\begin{array}{l}\text { Viral } \\
\text { wheezing }\end{array}$ & Healthy control & $\begin{array}{l}\text { Unclassifiable } \\
\text { wheezing }\end{array}$ \\
\hline Numbert & $23(33 \%)$ & $22(31 \%)$ & $20(29 \%)$ & $5(7 \%)$ \\
\hline Age (months) $\ddagger$ & $31(21-40)^{*}$ & $28(21-37)^{*}$ & $13(8-26)^{*}$ & $25(22-37)$ \\
\hline Male/female & $13 / 10$ & $17 / 5$ & $12 / 8$ & $2 / 3$ \\
\hline Weight $(\mathrm{kg}) \ddagger$ & $14(12-15)^{* *}$ & $13(12-14)^{* *}$ & $11(8-13)^{* *}$ & $12(11-14)$ \\
\hline Z-score weight $¥$ & $0[(-1.0)-0.4]$ & $0[(-1.5)-1.0]$ & $0[(-0.6)-0.5]$ & $0[(-0.5)-0.5]$ \\
\hline Height $(\mathrm{cm}) \ddagger$ & 95 (84-98) & $94(81-98)$ & 85 (70-93) & $92(80-95)$ \\
\hline Z-score height $¥$ & $0.0[(-0.5)-1.0]$ & $-0.3[(-1.0)-1.1]$ & $0.5[(-1.0)-1.1]$ & $-1.0[(-2.0)-0.0]$ \\
\hline \multicolumn{5}{|l|}{ Elevated serum IgE, or } \\
\hline positive RAST $\geq$ class $2 \dagger$ & $23(100 \%)$ & $4(18 \%)$ & $0(0 \%)$ & $2(40 \%)$ \\
\hline Current use of steroids ${ }^{\dagger}$ & $15(65 \%)$ & - & - & $5(100 \%)$ \\
\hline Inhaled steroid dose $\ddagger$ & $400(300-400)$ & - & - & $400(200-400)$ \\
\hline
\end{tabular}

IgE, immunoglobulin-E; RAST, radioallergo-sorbent test; Steroids, inhaled corticosteroids (and one subject in the atopic asthma group also ed a short course of oral prednisone); ${ }^{*} \mathrm{p}=0.012$ (multiple comparisons, Hochberg correction); ${ }^{* *} \mathrm{p}=0.046$ (multiple comparisons, Hochberg correction); † Absolute number (\%); $¥$ Median (IQR).

\section{Exhaled breath condensate collection}

This cross-sectional study comprised one attempt, between wheezing episodes, to collect EBC in a pre-school child. Precedingly, parents received thorough instructions on the collection procedure by one of the coaching childhood asthma nurses and were asked to bring along the child's favourite cartoon, cd-song, booklet and/or puppet.

EBC was collected using a modified closed glass condenser system that was developed in close collaboration with the department of Instrument Development Engineering \& Evaluation (IDEE) of the Maastricht University (patent number EP-07102586). The EBC collection system consisted of an inclined and closed glass condenser with a tube length of $90 \mathrm{~cm}$ (to enlarge the condensation surface to $1046 \mathrm{~cm}^{2}$ ), a downwards moveable plunger (to optimize condensate recovery from the inner wall of the condenser), with tangential and axial breath flow channels (to turbinately direct the tidal breath flows towards the cooled inner condenser wall), and an unique breath recirculation system, heated at $37^{\circ} \mathrm{C}$ (Figure 1). The breath recirculation system consisted of heated tubing (to guide the noncondensed residual exhaled breath, and to avoid condensation outside the condenser tube), a humidity sensor, a heated inert single use Tedlar ${ }^{\mathrm{TM}}$ (SKC Ltd., Dorset, UK) gas sample bag (to collect all non-condensed residual exhaled breath) and a small ventilator system to recirculate the non-condensed residual exhaled breath back into the cooled condenser system (to allow secondary condensation of exhaled breath) (Figure 1). 


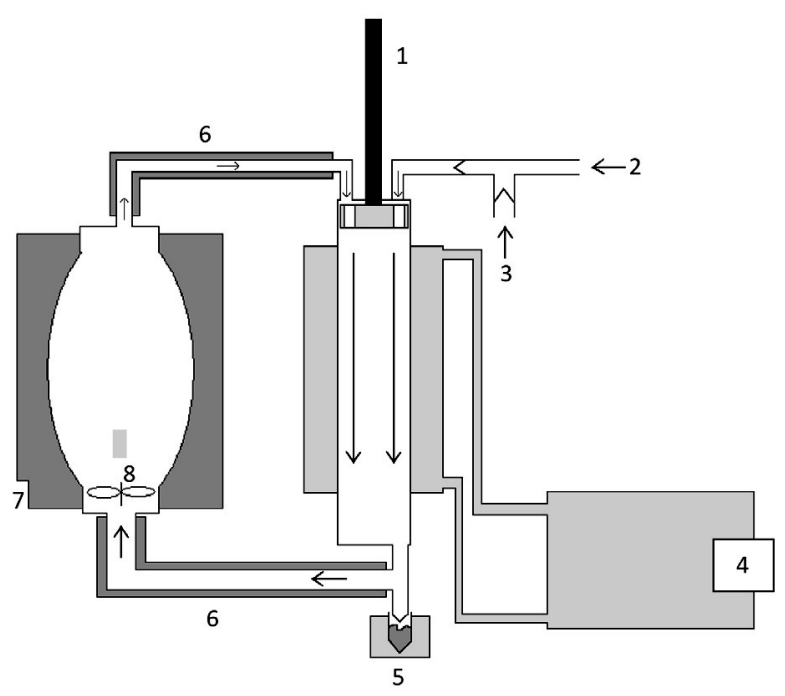

Figure 1: Schematic representation of the new closed glass condenser system, that enabled condensate collection in pre-school children before and after breath recirculation.

1. Inclined and closed glass condenser, and downwards moveable plunger with tangential and axial breath flow channels. 2. Swan-neck tubing, two-way nonrebreathing valve, and face mask with separated nose and mouth cavity. 3. Entrance of inspired room air. 4. Cooling unit consisting of a counter-current circulating icewater pump. 5. Removable glass sample vial, to collect exhaled breath condensate. 6 . Heated (at $37^{\circ} \mathrm{C}$ ) tubing, to guide the residual noncondensed exhaled breath. 7. Humidity sensor and heated (at $37^{\circ} \mathrm{C}$ ) inert Ted$\operatorname{lar}^{\mathrm{TM}}$ gas sample bag. 8. Small ventilator system to recirculate the residual noncondensed exhaled breath back into the condenser system.

The connecting tubing was made of Hytrel thermoplastic polyester elastomer (Du Pont Plastics, Mechelen, Belgium). The total expiratory resistance of the condenser system with breath recirculation was $2.7 \mathrm{cmH}_{2} \mathrm{O} / \mathrm{l} / \mathrm{s}$ at a flow of $50 \mathrm{l} / \mathrm{min}$, and $1.9 \mathrm{cmH}_{2} \mathrm{O} / \mathrm{l} / \mathrm{s}$ at a flow of $30 \mathrm{l} / \mathrm{min}$. The glass condenser was cooled using a counter-current circulating ice-water pump. The cooling temperature was preset at $0^{\circ} \mathrm{C}$ (Figure 1). The child exhaled tidally, as long as possible, through a face mask which separated air from mouth and nasal cavity, and with a two-way non-rebreathing valve (8900 paediatric sizes oro-nasal masks; Hans Rudolph Inc., Kansas City, MO, USA), connected to the glass condenser system by means of a swan-neck tubing (with a length of $38 \mathrm{~cm}$; and serving as gravitational saliva trap). The Hans Rudolph face mask with separated mouth and nasal cavity (which occludes the nasal air flow) was used to sample exhaled breath only from the child's oral cavity [6]. It simultaneously served as an alternative noseclip to limit the loss of exhaled air through the nose, and to prevent accidental inhalation-exhalation through the nose during sample collection [6]. During the condensation process, the formerly lost and non-condensed exhaled breath was separately being collected in the gradually inflating inert Tedlar ${ }^{\mathrm{TM}}$ gas sample bag. When the child refused to proceed and/or started to cry, the procedure was stopped and EBC was collected by pushing the plunger downwards. Subsequently, the plunger was pulled up again, and the separately collected exhaled breath was recirculated from the Tedlar ${ }^{\mathrm{TM}}$ gas sample bag back into the condenser system, to perform a secondary breath condensation. The non-condensed exhaled breath was passed again and again through the condenser until the degree of humidity was $\leq 22 \%$. 
Before and after each EBC collection procedure, the respiratory status was checked by using a clinical asthma score (skin colour changes, breath sounds including coughing and wheeze, breathing rate, retractions and level of alertness). To improve the toddler's coping with distress from the unfamiliar procedure, they were distracted while breathing tidally through a facemask. We primarily combined strict parental cooperation, with an age-appropriate cartoon, that was already started prior to entrance. In case of imminent failure to cope, a more active strategy was used on parent's advice, including children's stories, puppetry and/or children's songs.

EBC collection was defined successful when at least $60 \mu \mathrm{L}$ of condensate was produced, comprising the minimal required volume of $50 \mu \mathrm{L}$ to perform one multiplexed cytokine analysis, and an additional $10 \mu \mathrm{L}$ to compensate for loss because of sample handling.

After each procedure, the Hans Rudolph face mask and two-way non-rebreathing valve were cleaned with Cidex OPA Solution (Advanced Sterilization Products, Johnson \& Johnson Medical BV, Amersfoort, the Netherlands). The non-disposable tubing (Tefa Portanje, Woerden, the Netherlands) and the glass condenser were disconnected, rinsed repeatedly with bi-distilled water and subsequently dried at room air. The non-disposable parts of the heated recirculation system were disconnected, rinsed repeatedly with bi-distilled water and alcohol and subsequently dried at room air.

\section{Condensate sample processing}

Immediately after collection, condensate samples were snap-frozen at $-78^{\circ} \mathrm{C}$ using dry ice and stored at $-80^{\circ} \mathrm{C}$. Analysis was performed within 3 months from sampling time. Cytokines in EBC were measured in duplicate using a liquid bead array multiplexing system or $\mathrm{XMAP}^{\circ}$ technology (Luminex Corporation). Multiplex immunoassay was performed as described previously, to measure the following cytokines (with corresponding lower limits of detection): interleukin (IL)-4 (1.2 pg/mL), IL-5 (1.2 pg/mL), IL-6 $(2.4 \mathrm{pg} / \mathrm{mL})$, chemokine IL-8 $(2.9 \mathrm{pg} / \mathrm{mL}), \mathrm{IL}-10(2.3 \mathrm{pg} / \mathrm{mL}), \mathrm{IL}-12 \mathrm{p} 70(1.2 \mathrm{pg} / \mathrm{mL}), \mathrm{IL}-13(1.0 \mathrm{pg} / \mathrm{mL})$ and tumour necrosis factor-alpha (TNF- $\alpha, 1.3 \mathrm{pg} / \mathrm{mL}$ ) [21-23]. Measurement and data analysis were performed using the Bioplex 100 system and Bioplex Manager software version 3.0 (BioRad Laboratories, Hercules, CA, USA). All multiplex immunoassays were performed in 96 well format $1.2 \mu \mathrm{m}$ filter bottom plates (Millipore, Amsterdam, the Netherlands) and a 12point standard curve in duplicate was included on every plate. To minimize interassay variation, positive and negative control samples were included. The intrasubject variability of cytokines in EBC was assessed in seven children, aged 2-3 yr, with recurrent wheeze, as follows: at $\mathrm{t}=0$ and $30 \mathrm{~min}, 100 \mu \mathrm{L}$ of condensate was collected using the closed glass condenser system. The short-term intrasubject variability was expressed as the coefficient of variation (s.d./mean $\times 100 \%$ ).

\section{Statistics}

Statistical calculations were performed using spss 11.5 (SPSS Inc., Chicago, IL, USA). Normally distributed data were expressed as mean and standard deviation The Student's ttest was used to test for differences among dependent, paired or repeated samples. Pearson's correlation coefficients were used to assess the degree of association between two 
normally distributed variables. anova was used to test for differences among normally distributed variables for more than two study groups.

Not normally distributed data were expressed as median with interquartile ranges (IQR). The Wilcoxon-signed rank test was used to test for differences among not normally distributed paired data, whereas the Mann-Whitney U-test was used for unpaired data. Spearman's rank correlation coefficients were used to assess the degree of association between two not normally distributed variables. The Kruskal-Wallis test was used to test for differences among non-normal variables for more than two study groups. The Hochberg correction for multiple comparisons was applied. The chi-squared test was used to evaluate the distribution of categorical variables (number of occurrences) in four study groups.

\section{Power Calculation}

Assuming a success rate of $80 \%$, the standard error of feasibility is $5.1 \%$ using $n=60$ subjects. This was considered sufficiently accurate for the purpose of this study.

\section{Ethics}

The study was approved by the Medical Ethics Committee of the University Hospital of Maastricht and the Maasland Hospital Sittard. All parents gave written informed consent.

\section{Results}

\section{Feasibility of EBC collection}

A total number of 70 preschool children participated. Feasibility of the new EBC collection method in preschool children is shown in Table 2. Before breath recirculation, the mean duration of $E B C$ collection was 11 minutes $(S D=6)$, the mean $E B C$ volume was $206 \mu \mathrm{L}$ $(S D=198)$, and the success rate was $72 \%$ in these preschool children. After breath recirculation, the mean $\mathrm{EBC}$ volume was $347 \mu \mathrm{L}(\mathrm{SD}=339)$. In total, the mean collected $\mathrm{EBC}$ volume was $466 \mu \mathrm{L}(S D=400)$. The success rate of $E B C$ collection with breath recirculation was $83 \%$ in these preschool children, but differed significantly between the four study groups ( $p<0.001$, Chi-square test).

In the healthy group, the mean duration of EBC collection was $7 \mathrm{~min}$, and mean EBC volume before breath recirculation was $113 \mu \mathrm{L}$, whereas both were significantly decreased compared to the asthmatic and viral wheeze group $(p<0.031$, anova). A younger age significantly correlated with less duration of EBC collection $(R=0.481, p<0.001$, Spearman's correlation coefficient). A duration of EBC collection time of 10 min or more, was associated with successful EBC collection before breath recirculation. In due course of the collection procedure, the cooling temperature slightly rised from $0.00^{\circ} \mathrm{C}$ to a median of $2.15^{\circ} \mathrm{C}(\mathrm{IQR}=0.90-4.85)$ at the end of EBC collection.

Four children refused to start because of sudden anxiety, associated with insufficient parental stimulation and/or recent ear-nose-throat surgery (adenoidectomy, tonsillectomy and/or myringotomy with insertion of tympanostomy tube). Distraction during EBC collection was achieved by combined parental cooperation (86\%) and watching age-appropriate 
cartoons (76\%), listening to children's stories (36\%), using puppetry (15\%) and/or listening to children's songs (12\%). The Hans Rudolph face mask was applied in 60 children, whereas five preferred the babyhaler (GlaxoSmithKline BV, Zeist, the Netherlands) and one the nebuchamber (AstraZeneca BV, Zoetermeer, the Netherlands) face mask. Two collection procedures were not successful because of leakage of exhaled breath from defective Tedlar ${ }^{\mathrm{TM}}$ gas sample bags. Neither adverse effects on respiration, nor other sideeffects were seen during the collection procedures.

\section{Cytokine measurements before and after breath recirculation}

Paired EBC samples were taken from the condensate volume collected before breath recirculation, and from the condensate volume collected after the breath recirculation manoeuvre, separately. Table 3 shows the detection and concentration of IL-4, IL-5, IL-6, IL-8, IL-10, IL-12p70, IL-13, and TNF-alpha in EBC collected before, as well as after breath recirculation, in 43 preschool children. The detection of cytokines in EBC was successful in $95-100 \%$ of the collected $50 \mu \mathrm{L}$ condensate samples. There was no statistical difference between cytokine concentrations before and after breath recirculation ( $p>0.117$, Wilcoxon signed rank test).

The coefficients of variation of the short-term intrasubject variability were $13.9 \%$ for IL-4, $14.3 \%$ for IL-5, 20.0\% for IL-6, 33.3\% for IL-8, 14.3\% for IL-10, 14.3\% for IL-12p70, 8.7\% for IL-13 and $3.7 \%$ for TNF- $\alpha$.

Table 2: Feasibility of exhaled breath condensate (EBC) collection, before and after breath recirculation in 70 pre-school children.

\begin{tabular}{|c|c|c|c|c|c|}
\hline Study group & Atopic asthma & $\begin{array}{l}\text { Viral } \\
\text { wheezing }\end{array}$ & $\begin{array}{l}\text { Healthy } \\
\text { control }\end{array}$ & $\begin{array}{l}\text { Unclass } \\
\text { wheezing }\end{array}$ & $\mathrm{p}$-value \\
\hline Number* & $23(33 \%)$ & $22(31 \%)$ & $20(29 \%)$ & $5(7 \%)$ & - \\
\hline Age (months) ${ }^{\dagger}$ & $31(21-40)$ & $28(21-37)$ & $13(8-26)$ & $25(22-37)$ & 0.012 \\
\hline \multicolumn{6}{|c|}{ Before breath recirculation } \\
\hline $\begin{array}{l}\text { Duration of collection } \\
\text { (min) } \ddagger\end{array}$ & $13(6)$ & $13(5)$ & $7(4)$ & $14(7)$ & 0.004 \\
\hline EBC volume $(\mu \mathrm{L}) \ddagger$ & $199(169)$ & $291(223)$ & $113(168)$ & $320(295)$ & 0.031 \\
\hline Success rate* & $15(65 \%)$ & $20(91 \%)$ & $6(30 \%)$ & $4(80 \%)$ & 0.241 \\
\hline \multicolumn{6}{|c|}{ After breath recirculation } \\
\hline EBC volume $(\mu \mathrm{L}) \ddagger$ & $438(408)$ & $298(239)$ & $271(337)$ & $513(301)$ & 0.385 \\
\hline \multicolumn{6}{|c|}{ Total (before and after breath recirculation) } \\
\hline EBC volume $(\mu \mathrm{L}) \ddagger$ & $491(444)$ & $447(354)$ & $383(372)$ & $730(223)$ & 0.351 \\
\hline Success rate* & 17 (74\%) & $21(95 \%)$ & $15(75 \%)$ & $5(100 \%)$ & $<0.001$ \\
\hline
\end{tabular}

IgE, immunoglobulin-E; RAST, radioallergo-sorbent test; Steroids, inhaled corticosteroids (and one subject in the atopic asthma group also ed a short course of oral prednisone); ${ }^{*} p=0.012$ (multiple comparisons, Hochberg correction); ${ }^{* *} p=0.046$ (multiple comparisons, Hochberg correction); † Absolute number (\%); $¥$ Median (IQR). 
Table 3: Detection (\%) and concentration $(\mathrm{pg} / \mathrm{mL}$ ) of cytokines in exhaled breath condensate (EBC) collected before and after breath recirculation (BR) in 45 preschool children.

\begin{tabular}{|c|c|c|c|c|c|c|}
\hline \multirow[b]{2}{*}{ EBC } & \multicolumn{2}{|c|}{ Before breath recirculation } & \multicolumn{2}{|c|}{ After breath recirculation } & \multirow[b]{2}{*}{ p-value* } & \multirow[b]{2}{*}{ LLD } \\
\hline & $\begin{array}{l}\text { Detection } \\
\%\end{array}$ & $\begin{array}{l}\mathrm{pg} / \mathrm{mL} \\
\text { Median (IQR) }\end{array}$ & $\begin{array}{l}\text { Detection } \\
\%\end{array}$ & $\begin{array}{l}\mathrm{pg} / \mathrm{mL} \\
\text { Median (IQR) }\end{array}$ & & \\
\hline IL-4 & 100 & $2.1(1.7-2.4)$ & 100 & $2.2(1.7-2.6)$ & 0.346 & 1.2 \\
\hline IL-5 & 98 & $16.7(16.7-18.3)$ & 97 & $16.7(16.7-18.3)$ & 0.278 & 1.2 \\
\hline IL-6 & 96 & $3.3(2.5-3.4)$ & 95 & $2.9(2.9-3.3)$ & 0.611 & 2.4 \\
\hline IL-8 & 100 & $3.9(3.4-4.4)$ & 100 & $4.4(3.9-5.0)$ & 0.117 & 2.9 \\
\hline IL-10 & 98 & $4.7(4.5-5.2)$ & 100 & $4.7(4.7-5.2)$ & 0.455 & 2.3 \\
\hline IL-12p70 & 100 & $3.4(3.4-3.8)$ & 100 & $3.4(3.4-3.7)$ & 0.301 & 1.2 \\
\hline IL-13 & 100 & $8.5(7.6-9.4)$ & 100 & $8.5(8.0-9.4)$ & 0.603 & 1.0 \\
\hline TNF- $\alpha$ & 96 & $40.9(39.0-44.8)$ & 95 & $40.9(37.1-43.2)$ & 0.746 & 1.3 \\
\hline
\end{tabular}

IL, interleukin; IQR, interquartile range; TNF, tumor necrosis factor. *: Wilcoxon-signed rank; ": LLD, lower limit of detection $(\mathrm{pg} / \mathrm{mL})$.

\section{Cytokines in subgroups with and without wheeze}

For further cytokine analysis between the different study groups, the separately collected condensate samples before as well as after breath recirculation in each subject, were considered as one donor series. For reasons of disproportion, data of the children that did not use the Hans Rudolph face mask (with separated mouth and nasal cavitiy) were excluded. Between the asthmatic $(n=17)$, viral wheeze $(n=21)$ and healthy $(n=10)$ group, the median cytokine concentrations of IL-4, IL-5, IL-6, IL-8, IL-10, IL-12p70 and IL-13 did not differ (data not shown), whereas the median concentration of TNF- $\alpha$ was significantly decreased in the asthmatic group [median $=38.8 \mathrm{pg} / \mathrm{mL}$ (IQR=37.1-41.3) in asthma, $43.1 \mathrm{pg} / \mathrm{mL}$ (IQR=40.2-46.1) in viral wheeze and $40.9 \mathrm{pg} / \mathrm{mL}$ (IQR=39.7-41.9) in healthy controls, respectively] ( $p=0.018$, Kruskal-Wallis). Only a weak correlation existed between age and cytokine concentrations $(-0.120<R<0.392$, Spearman's correlation coefficient). In atopic asthmatic children, inhaled corticosteroids did in general not influence cytokine levels in EBC, with one exception: TNF- $\alpha$ concentrations were significantly lowered in children with inhaled corticosteroid therapy, compared with steroid naïve children (median=37.1 pg/mL vs. $41.7 \mathrm{pg} / \mathrm{mL}$, respectively) ( $p=0.013$, Mann-Whitney U-test). When asthmatic and non-asthmatic (viral wheeze and healthy) children were compared, TNF- $\alpha$ concentrations in the asthmatic group (median $=38.8 \mathrm{pg} / \mathrm{mL}, \mathrm{IQR}=37.1-41.3$ ) were significantly decreased vs. non-asthmathic children (median $=41.7 \mathrm{pg} / \mathrm{mL}, \quad I Q R=40.2-45.4$ ) ( $p=0.013$, Mann-Whitney U-test) (Table 4, Figure 2a). 
Table 4: Cytokine profile in exhaled breath condensate (EBC) in 48 doctor's diagnosed atopic asthmatic, and nonasthmatic preschool children (consisting of the viral-triggered recurrent wheezing group and healthy control group).

\begin{tabular}{lllll}
\hline EBC & $\begin{array}{l}\text { Doctor's diagnosed } \\
\text { atopic asthma }\end{array}$ & $\begin{array}{l}\text { Non-asthmatic pre- } \\
\text { school children }\end{array}$ & p-value (MWU) & $\begin{array}{l}\text { Lower limit of } \\
\text { detection }(\mathrm{pg} / \mathrm{mL})\end{array}$ \\
\hline Number & 17 & 31 & - & - \\
TNF-a* & $38.8(37.1-41.3)$ & $41.7(40.2-45.4)$ & 0.013 & 1.3 \\
IL-5/TNF* & $0.46(0.42-0.52)$ & $0.40(0.38-0.47)$ & 0.018 & - \\
IL-13/TNF* & $0.24(0.20-0.26)$ & $0.20(0.18-0.23)$ & 0.034 & - \\
IL-4* & $2.1(1.9-2.5)$ & $2.0(1.7-2.5)$ & NS & 1.2 \\
IL-5* & $17.5(16.1-19.1)$ & $16.7(15.9-18.3)$ & NS & 1.2 \\
IL-6* & $3.3(2.8-3.6)$ & $3.1(2.7-3.5)$ & NS & 2.4 \\
IL-8* & $4.2(3.9-5.0)$ & $4.2(3.9-4.5)$ & NS & 2.9 \\
IL-10* & $4.7(4.5-5.9)$ & $5.0(4.7-5.2)$ & NS & 2.3 \\
IL-12p70* & $3.6(3.4-4.0)$ & $3.4(3.4-3.7)$ & NS & 1.2 \\
IL-13* & $9.0(8.0-9.9)$ & $8.5(7.6-9.4)$ & NS & 1.0 \\
\hline
\end{tabular}

IL, interleukin; MWU, Mann-Whitney U-test; NS, not significant; TNF, tumour necrosis factor; *Median (IQR).

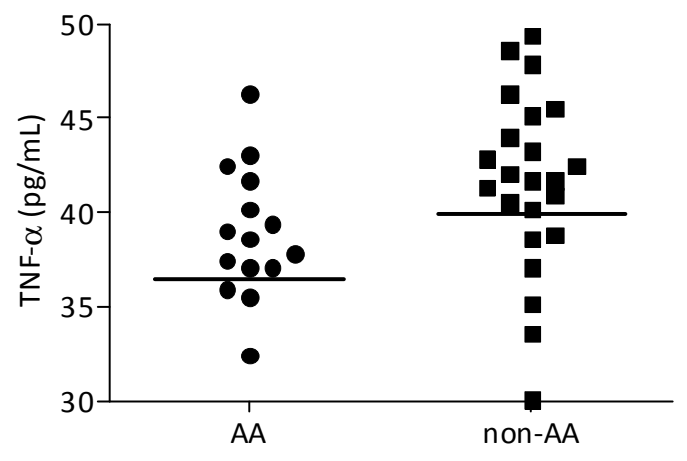

Figure 2a: $\mathrm{T}$ helper 2/T helper 1 cytokine profiles in exhaled breath condensate in doctor's diagnosed atopic asthmatic (AA) and nonasthmatic (non-A) pre-school children.

Condensate TNF- $\alpha$ concentration was significantly decreased in the doctor's diagnosed atopic asthmatic group (median $38.8 \mathrm{pg} / \mathrm{mL}$, IQR 37.1-41.3) compared to nonasthmatic (median $41.7 \mathrm{pg} / \mathrm{mL}$, IQR 40.2- 43.4) preschool children ( $p=0.013, \mathrm{MWU})$.

The ratios of IL-5/TNF- $\alpha$ and IL-13/TNF- $\alpha$ in the asthmatic group (median=0.46, IQR=0.420.52 ; median $=0.24, \mathrm{IQR}=0.20-0.26$, respectively) were significantly higher compared with non-asthmatic children (median $=0.40, I Q R=0.38-0.47$; median $=0.20, I Q R=0.18-0.23$, respectively) ( $p=0.018$ and 0.034 , respectively, Mann-Whitney U-test) (Table 4, Figure $2 b, c)$. 


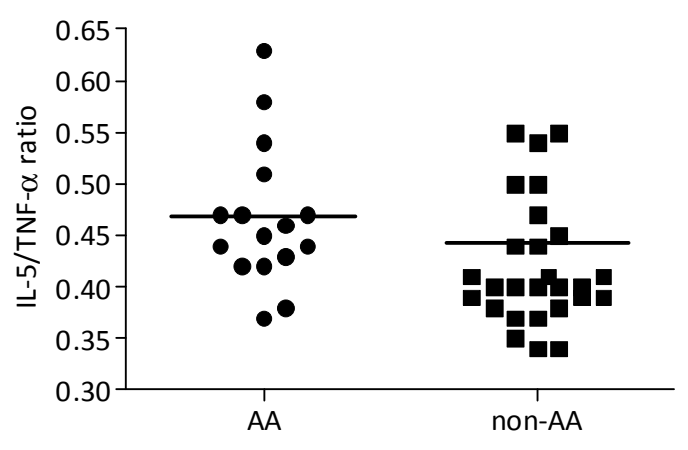

Figure 2b: $T$ helper 2/T helper 1 cytokine profiles in exhaled breath condensate in doctor's diagnosed atopic asthmatic (AA) and nonasthmatic (non-A) pre-school children.

Condensate IL-5/TNF- $\alpha$ ratio was significantly increased in the doctor's diagnosed atopic asthmatic group (median 0.46, IQR 0.42-0.52) compared to nonasthmatic (median 0.40, IQR 0.38-0.47) preschool children $(p=0.018, M W U)$.

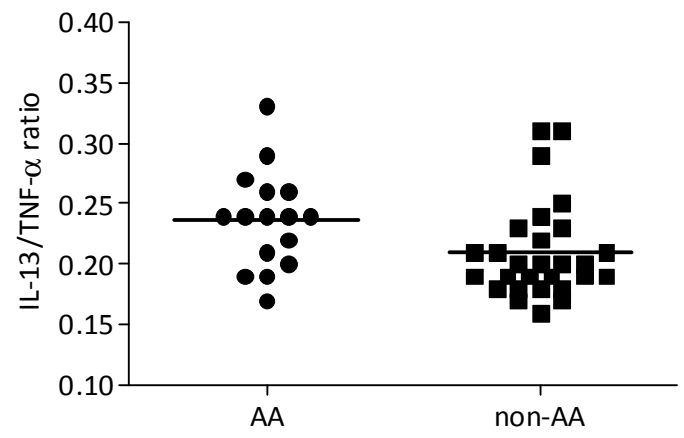

Figure 2c: $T$ helper 2/T helper 1 cytokine profiles in exhaled breath condensate in doctor's diagnosed atopic asthmatic (AA) and nonasthmatic (non-A) pre-school children.

Condensate IL-13/TNF- $\alpha$ ratio was significantly increased in the doctor's diagnosed atopic asthmatic group (median 0.24, IQR 0.20-0.26) compared to nonasthmatic (median 0.20, IQR 0.18-0.23) preschool children $(p=0.034, M W U)$. IL, interleukin; IQR, interquartile range; MWU, Mann Whitney U test; TNF, tumor necrosis factor.

\section{Discussion}

We report an enhanced feasibility of EBC collection in pre-school children with and without wheezing, using a novel closed glass condenser with breath recirculation system, and using multiplexed liquid bead array: (i) the overall success rate increased from $64 \%$ to $83 \%$, and the mean EBC volume increased from 214 to $465 \mu \mathrm{L}$, indicating that breath recirculation indeed improved the feasibility of EBC collection, (ii) the detection of cytokine profiles in $50 \mu \mathrm{L}$ EBC was successful in $95-100 \%$ of the collected samples, (iii) similar cytokine concentrations were found in EBC collected before as well as after breath recirculation and (iv) in the atopic asthmatic group, only TNF- $\alpha$ concentrations in EBC were signifi- 
cantly decreased, compared with pre-school children with viral wheeze and healthy controls.

We simultaneously introduced novel techniques to optimize the condensation process (see Figure 1), and to improve small condensate sample analysis, in non-sedated preschool children with and without recurrent wheezing. Cumulatively, this new closed glass collection method with breath recirculation resulted in an overall success rate of $83 \%$, compared with $64 \%$ when using the 'usual' (without or before breath recirculation) procedure and in a mean total EBC volume of $465 \mu \mathrm{L}$. In the literature on EBC in pre-school children, Moeller et al. [14] proposed a method to collect, both orally and nasally, a median EBC volume of $282 \mu \mathrm{L}$, using two disposable Plastipak syringes (BD Plastipak, Franklin Lakes, NJ, USA), in tidal breathing infants, however, during sedated sleep and without assessment of markers in the collected condensate. Griese et al. presented a nasal polypropylene collection system with air suction pump, to measure hydrogen peroxide and 8isoprostane, and reported $85 \mu \mathrm{L} / \mathrm{min}$ of nasally collected EBC in healthy children aged 4 wk to $18 \mathrm{yr}$, however, with contamination by nasal secretions [24, 25]. The present study reports a correlation between age and duration of EBC collection, which is in accordance with the significantly increased volume of collected EBC with age, reported by both Moeller et al. [14] and Griese et al. [24]. Irrespective of the duration of EBC collection, breath recirculation allowed condensation of the totally exhaled breath volume within the closed glass system, in contrast to maximally $30 \%$ condensation using the open-ended nasal suction system of Griese et al. [24, 25], and in contrast to the open-ended non-standardized EBC collection techniques that are available for use in older children and adults, and, that all have a variable loss of non-condensed exhaled breath, as condensation does not immediately occur, and as condensation does not continue once the surface is saturated [5, $6,26-29]$. Hence, our concept of breath recirculation enabled not only the collection of an increased volume of EBC, but also, in the younger toddlers, with limited cooperation in time (often less than $10 \mathrm{~min}$ ), and often not familiar with the (repeated) use of a face mask, breath recirculation was often crucial to yield a minimal but sufficient volume of condensate. Furthermore, cytokine concentrations in EBC collected before breath recirculation, and after breath recirculation did not differ significantly, demonstrating that condensate cytokine concentrations were not affected by the breath recirculation process and illustrating an efficiency to generate additional and equivalent condensate.

We acknowledge that we cannot exclude a possible influence of the observed small rise in cooling temperature, during the collection procedure. Furthermore, we acknowledge that the use of a face mask without partitioning (mouth-nose) in six out of 66 children, is less ideal, although we did not find a statistical influence on the volume of EBC, or the condensate cytokine concentrations ( $p \geq 0.296$ and 0.188 , respectively, unpaired t-test). In the present study, we did not compare several other collecting devices. The following reasons have led us to decide not to include other devices. At first, pre-school children might have refused to proceed with again another test, which would have biased feasibility. Secondly, we recently demonstrated, in adults, that an open-ended prototype of the glass condenser without breath recirculation yielded significantly more EBC volume (median $=2025 \mu \mathrm{L}$ ), compared with three other condensers (e.g. the EcoScreen (Erich Jaeger $\mathrm{GmbH}$, Hochberg, Germany) yielded $1237 \mu \mathrm{L}$ ), and the glass coating was associated with significantly more detections of IL-2, IL-4, IL-5, IL-13 and TNF- $\alpha$ in EBC $[15,16]$. In preschool children, the feasibility of EBC collection using the most efficient condenser, i.e. the 
open-ended glass prototype, or, in other words, the condenser without breath recirculation was $64 \%$. Hence, we did not find it appropriate to include other, less optimal condensers in these children as this would have resulted in an efficiency even lower than $64 \%$, based on the comparative study in adults [16]. However, to further increase the feasibility, the condenser was equipped with the breath recirculation system, which further increased success rate up to $83 \%$, whereas, cytokine concentrations before and after breath recirculation did not differ significantly, indicating no additional loss of cytokines in the recirculation system.

Multiplexed liquid bead array was used to measure cytokines more accurately in only $50 \mu \mathrm{L}$ sample volumes of EBC. The detection level of cytokines was high, ranging from $95 \%$ to $100 \%$. Multiplexed liquid bead array is based on flow cytometry and microspheres (with faster and more reproducible liquid reaction kinetics, than solid planar assays), and allows multiplexing of up to 100 assays within one sample [21]. Reasons to introduce this technique were: (i) lack of sensitivity of conventional enzyme-linked immunosorbent assays to detect cytokines in 200-500 $\mu \mathrm{L} \mathrm{EBC}$ and (ii) variable level of simultaneous cytokine detection by cytometric bead array ranging from $0 \%$ to $25 \%$ in $50 \mu \mathrm{L} \mathrm{EBC}$ in children and 3$100 \%$ in $1000-2000 \mu \mathrm{L}$ lyophilized EBC in adults [10, 30, 31]. When compared with conventional assays, multiplexed immunoassays are highly sensitive and specific, detect bioactive and inactive molecules, are rapid [hours instead of day(s)], have good (but not yet excellent) precision with coefficients of variation ranging from $10 \%$ to $15 \%$, are not interfered by drugs, have a simple protocol, require small sample volumes and allow simultaneous detection [21].

In pre-school children with different wheezing phenotypes, the central issue consists of finding a non-invasive measure to identify underlying (persisting) airway disease, such as atopic asthma. In contrast to pre-school children with viral wheeze and healthy children, atopic asthma is characterized by a predominantly Th2-mediated chronic eosinophilic airway inflammation $[1,4]$. Statistical analysis only revealed a significantly decreased TNF$\alpha$ in EBC in the atopic asthmatic group, compared with viral wheeze and healthy controls. Steroid-treated atopic asthmatic children had lowered levels of TNF- $\alpha$ in EBC. This is in accordance with studies on bronchial biopsies in atopic asthmatic inflammation showing significantly decreased levels of TNF- $\alpha$ after $8 \mathrm{wk}$ of budesonide treatment [32]. The other EBC cytokine levels measured in the present study were similar in all subject groups, and were close to, but not below the lower limits of detection, in contrast to the few published data in older children and adults $[11,31,33]$. As supported by the findings of Shahid et al. [11], who reported increased levels of IL-4 and decreased IFN- $\gamma$ in EBC in asthmatic children, the ratio's of IL-5/TNF- $\alpha$ and IL-13/TNF- $\alpha$ in EBC in our study were significantly increased in the asthmatic group, compared with non-asthmatics. However, the difference between these fractions of IL-5 and IL-13 to TNF- $\alpha$ was mainly caused by the lowered levels of TNF- $\alpha$ in EBC in asthmatic pre-school children. Obviously, further evaluation of repeated cytokine profile measurements in distinct wheezing phenotypes is warranted. Finally, we speculate that the proposed method of EBC collection may open a new window of opportunities in paediatric research, as it may allow non-invasive inflammatory profiling in non-sedated pre-school children.

In conclusion, we demonstrated the feasibility of EBC collection in non-sedated pre-school children with and without wheezing illness using a novel closed glass condenser with breath recirculation system. Multiplexed liquid bead array enabled more accurate cyto- 
kine measurement in only small EBC sample sizes. The presented method may help to assess-non-invasively-cytokine profiles in asthmatic and non-asthmatic pre-school children.

\section{Acknowledgements}

The authors wish to thank the childhood asthma nurses, Irene Breugelmans, Jacqueline Frenken, and Paula Heggen, for their technical support in the collection of EBC (Dept of Paediatrics, Maasland Hospital, Sittard, the Netherlands); and last but not least the parents and children for their participation.

\section{References}

1. Grigg J, Silverman M. Wheezing disorders in young children: one disease or several phenotypes? In: Frey U, Gerritsen J, eds. Respiratory Diseases in Infants and Children. Eur Respir Mon 2006: 37: 153-69.

2. de Jongste J. To wheeze or not to wheeze. Am J Respir Crit Care Med 2006: 174: 1281-2.

3. Martinez F, Wright A, Taussig L, Holberg C, Halonen M, Morgan W. Asthma and wheezing in the first six years of life. N Eng J Med 1995: 332: 133-8.

4. Robinson D, Hamid Q, Ying S, et al. Predominant TH2-like bronchoalveolar T-lymphocyte population in atopic asthma. N Eng J Med 1992: 326: 298-304.

5. Rosias P, Dompeling E, Hendriks J, Heijnens J, Donckerwolcke R, Jöbsis Q. Exhaled breath condensate in children: pearls and pitfalls. Pediatr Allergy Immunol 2004: 15: 4-19.

6. Horváth I, Hunt J, Barnes P, on behalf of the ATS/ERS Task Force. Exhaled breath condensate: methodological recommendations and unresolved questions. Eur Respir J 2005: 26: 523-48.

7. Linkosalo L, Lehtimäki L, Laitinen J, Kaila M, Holm K, Moilanen E. Increased bronchial NO output in severe atopic eczema in children and adolescents. Pediatr Allergy Immunol 2008: 19: 426-32.

8. Kielbasa B, Moeller A, Sanak M, et al. Eicosanoids in exhaled breath condensates in the assessment of childhood asthma. Pediatr Allergy Immunol 2008: 19: 660-9.

9. Robroeks C, Rosias P, van Vliet D, et al. Biomarkers in exhaled breath condensate indicate presence and severity of cystic fibrosis in children. Pediatr Allergy Immunol 2008: 19: 652-9.

10. Robroeks C, Jöbsis Q, Damoiseaux J, et al. Cytokines in exhaled breath condensate of children with asthma and cystic fibrosis. Ann Allergy Asthma Immunol 2006: 96: 349-55.

11. Shahid S, Kharitonov S, Wilson N, Bush A, Barnes P. Increased interleukin-4 and decreased interferon-gamma in EBC of children with asthma. Am J Respir Crit Care Med 2002: 165: 1290-3.

12. Baraldi E, Ghiro L, Piovan V, Carraro S, Zacchello F, Zanconato S. Safety and success of exhaled breath condensate collection in asthma. Arch Dis Child 2003: 88: 358-60.

13. Cheah F, Darlow B, Winterbourn C. Problems associated with collecting breath condensate for the measurement of exhaled hydrogen peroxide from neonates on respiratory support. Biol Neonate 2003: 84: 33841.

14. Moeller A, Franklin P, Hall G, Horak F, Wildhaber J, Stick S. Measuring exhaled breath condensate in infants. Pediatr Pulmonol 2006: 41: 184-7.

15. Rosias $\mathrm{P}$, Robroeks $\mathrm{C}$, Niemarkt $\mathrm{H}$, et al. Breath condenser coatings affect measurement of biomarkers in exhaled breath condensate. Eur Respir J 2006: 28: 1036-41.

16. Rosias $\mathrm{P}$, Robroeks $\mathrm{C}$, Kester A, et al. Biomarker reproducibility in exhaled breath condensate collected with different condensers. Eur Respir J 2008: 31: 934-42.

17. Rosias P, Robroeks C, Hendriks J, Dompeling E, Jöbsis Q. Exhaled breath condensate: a space odyssey, where no one has gone before... Eur Respir J 2004: 24: 189-90.

18. Rosias $P$, Jöbsis $Q$, van de Kant $K$, et al. Global condensation: a 'climate change' towards better standardisation of exhaled breath condensate measurements. Eur Respir J 2008: 31: 684-5. 
19. Huszár É, Szabó Z, Jakab A, Barta I, Herjavecz I, Horváth I. Comparative measurement of thromboxane A2 metabolites in exhaled breath condensate by different immunoassays. Inflamm Res 2005: 54: 350-5.

20. Asher M, Keil U, Anderson H, et al. International study of asthma and allergies in childhood (ISAAC): rationale and methods. Eur Respir J 1995: 8: 483-91.

21. de Jager W, Rijkers G. Solid-phase and bead-based cytokine immunoassay: a comparison. Methods 2006: 38 : 294-303.

22. de Jager W, Prakken B, Bijlsma J, Kuis W, Rijkers G. Improved multiplex immunoassay performance in human plasma and synovial fluid following removal of interfering heterophilic antibodies. J Immunol Methods 2005: 300: 124-35.

23. de Jager W, te Velthuis H, Prakken B, Kuis W, Rijkers G. Simultaneous detection of 15 human cytokines in a single sample of stimulated peripheral blood mononuclear cells. Clin Diagn Lab Immunol 2003: 10: 133-9.

24. Griese M, Latzin P, Beck J. A noninvasive method to collect nasally exhaled air condensate in humans of all ages. Eur J Clin Invest 2001: 31: 915-20.

25. Latzin P, Beck-Ripp J, Hartl D, Bartenstein A, Noss J, Griese M. 8-Isoprostane in nasally exhaled breath condensate in different pediatric lung diseases. Eur J Med Res 2007: 12: 21-5.

26. Caglieri A, Goldoni M, Acampa O, et al. The effect of inhaled chromium on different exhaled breath condensate biomarkers among chrome-plating workers. Environ Health Perspect 2006: 114: 542-6.

27. Horváth I, Donnelly L, Kiss A, et al. Combined use of exhaled hydrogen peroxide and nitric oxide in monitoring asthma. Am J Respir Crit Care Med 1998: 158: 1042-6.

28. Soyer O, Dizdar E, Keskin O, Lilly C, Kalayci O. Comparison of two methods for exhaled breath condensate collection. Allergy 2006: 61: 1016-8.

29. Tufvesson E, Bjermer L. Methodological improvements for measuring eicosanoids and cytokines in exhaled breath condensate. Respir Med 2006: 100: 34-8.

30. Sack $U$, Scheibe R, Wötzel M, et al. Multiplex analysis of cytokines in exhaled breath condensate. Cytometry A 2006: 69: 169-72.

31. Matsunaga K, Yanagisawa S, Ichikawa T, et al. Airway cytokine expression measured by means of protein array in exhaled breath condensate: correlation with physiologic properties in asthmatic patients. J Allergy Clin Immunol 2006: 118: 84-90.

32. Wilson S, Wallin A, Della-Cioppa G, Sandström T, Holgate S. Effects of budesonide and formoterol on NF-kB, adhesion molecules, and cytokines in asthma. Am J Respir Crit Care Med 2001: 164: 1047-52.

33. Robroeks C, Dompeling E, Rijkers G, Damoiseaux J, Jöbsis Q. Inflammatory markers in exhaled breath condensate, assessed by means of Luminex ${ }^{\circledR}$ technology, can differentiate between children with asthma, cystic fibrosis, and healthy controls. Eur Respir J 2007: 30 (Suppl. 51): 498s-9s. 
SECTION 2

\section{DIAGNOSIS}
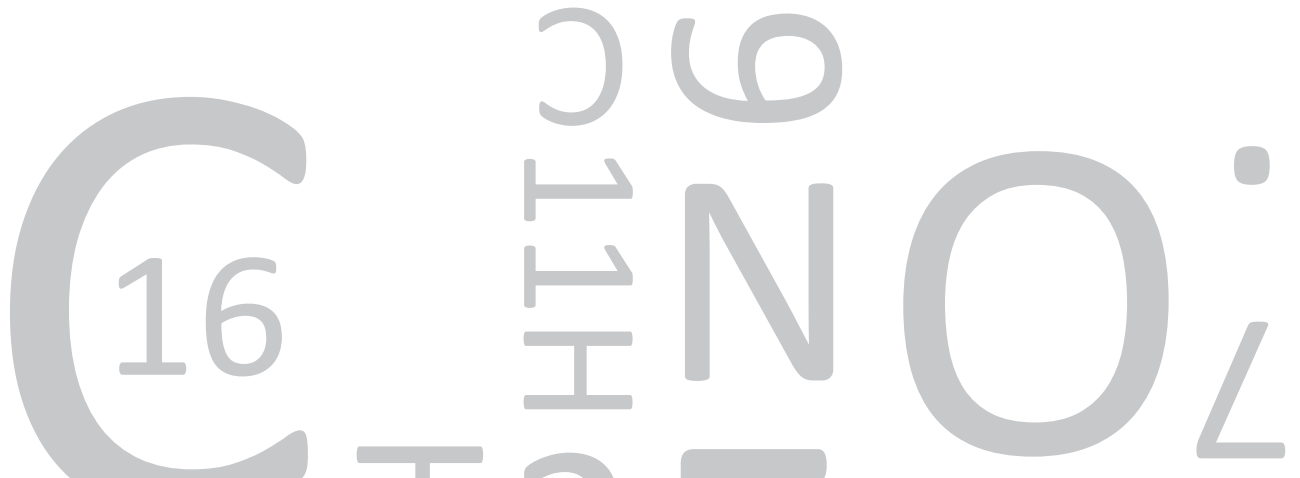



\section{CHAPTER 5}

\section{Cytokines in exhaled breath condensate of children with asthma and cystic fibrosis}

CMHHT Robroeks, Q Jöbsis, JGMC Damoiseaux, PHM Heijmans, PPR Rosias, JJE Hendriks, E Dompeling Annals of Allergy Asthma and Immunology 2006;96(2):349-355 


\section{Abstract}

\section{Background}

Inflammatory mediators in exhaled breath condensate (EBC) indicate ongoing inflammation in the lungs and might differentiate between asthma and cystic fibrosis (CF).

Objective: 1) To assess the presence, concentration, and short-term variability of Th-1 and Th-2 mediated cytokines (INF- $\gamma$, TNF- $\alpha$, IL-10, IL-5, IL-4, IL-2) in EBC of children with asthma, or CF and healthy controls. 2) To analyse the discriminating ability of inflammatory markers in $\mathrm{EBC}$ between children with asthma or $\mathrm{CF}$, and healthy children.

\section{Methods}

Expired air was conducted through a double jacketed glass tube cooled by circulating icewater. In 33 asthmatic children $(9.7 \pm 0.5 \mathrm{yrs}), 12$ children with CF $(13.3 \pm 1.0 \mathrm{yrs})$ and 35 healthy children (10.0 $\pm 0.5 \mathrm{yrs})$, EBC was collected during tidal breathing. Cytokines were measured with flow cytometry.

\section{Results}

IL-2, IL-4, INF- $\gamma$ and IL-10 were detected in respectively $16 \%, 16 \%, 11 \%$ and $9 \%$ of all samples in asthma and CF. IL-5 and TNF- $\alpha$ were not detected in children with CF. Cytokine concentrations did not differ significantly between children with asthma or CF. In the healthy population, INF- $\gamma$, TNF- $\alpha$ and IL-10 were detected in $9 \%, 14 \%$ and $3 \%$ respectively. IL-2, IL-4 and IL-5 were not detected in healthy children.

\section{Conclusions}

Cytokines, like INF- $\gamma$, TNF- $\alpha$, IL-10, IL-5, IL-4 and IL-2 can be detected in EBC of children with asthma and CF. However, the concentrations found are close to the detection limits of the used assay. These findings emphasize the importance to develop more sensitive techniques for analysis of EBC and to standardise the EBC collection method. Several suggestions for future research are discussed. 


\section{Introduction}

Chronic lung diseases, like asthma and cystic fibrosis (CF), are an important source of morbidity and mortality in children [1, 2]. Airway inflammation and oxidative stress are central features in the pathophysiology of asthma and CF [3-6]. Methods available to obtain information about the presence of inflammation in the airways, like induced sputum or bronchoscopy with broncho alveolar lavage (BAL) and biopsies [7], are too invasive to apply routinely or repeatedly. Monitoring of disease activity is currently based on clinical features and lung function tests [7]. If chronic airway inflammation may be demonstrated in a direct and specific way, children may be monitored more closely and by consequence, treatment may be adjusted more appropriately to inflammation activity [8]. A noninvasive method to measure the nature and severity of airway inflammation is to assess inflammatory mediators in exhaled breath condensate (EBC) or in expired air [8-11]. One of the most developed and standardised inflammatory mediator is exhaled nitric oxide (eNO) measurements [12]. eNO is elevated in asthma, and is related to clinical severity, and steroid dosage. Recently, it was shown that eNO was a helpful monitor instrument improving asthma outcome [13].

Cytokine responses are important controlling, and thereby signalling, elements of inflammatory and immune reactions that occur in the course of a disease [14]. Allergic asthma is considered a T-helper (Th)-2 mediated disease characterised by the production of IL-4, IL-5 and IL-6. In allergic asthma, eosinophils become one of the major effector cells when inflammation of the lungs is chronic. IL-5 is the primary cytokine responsible for the selective differentiation of the eosinophils. In contrast, the nature of inflammation in CF is dominated by neutrophils $[15,16]$. The altered cytokine production in patients with CF includes decreased IL-10 and increased TNF- $\alpha$, IL-6 and IL-8 [15]. In CF patients the Th-1 response, characterised by the production of IL-2, INF- $\gamma$ and TNF- $\alpha$, predominates as has been confirmed in patients with a chronic Pseudomonas Aeruginosa lung infection [14].

Despite rapidly increasing data on inflammatory markers in EBC, until now, only two research groups reported on the detection of the cytokines, IL-4 [17, 18], TNF- $\alpha$ [17] and INF- $\gamma$ [18], in EBC of children with asthma, and one group reported on IL-8 in EBC of children with CF [19]. If cytokines can be detected in EBC, one might expect that they are of importance for monitoring airway inflammation and differentiating between distinct diseases.

In an earlier study, we were not able to demonstrate the presence of IL-6, IL-8, TNF- $\alpha$ or sTNF-R75 in EBC of asthmatic children, possibly due to limitations of the ELISA assay with a lower detection limit of $20 \mathrm{pg} / \mathrm{mL}$ [20]. These cytokines do not reflect a possible Th-1/Th-2 imbalance.

Therefore the aim of this study was to assess: 1$)$ The presence, concentration, and shortterm variability of Th- 1 and Th- 2 mediated cytokines INF- $\gamma$, TNF- $\alpha$, IL-10, IL-5, IL-4 and IL-2 in EBC of children with asthma or CF, and healthy controls. 2) The discriminating ability of these inflammatory markers between children with asthma or CF, and healthy children. 


\section{Methods}

\section{Subjects}

Children, aged 5 to 17 years, known with asthma or CF were recruited at the department of Paediatric Pulmonology, University Hospital Maastricht. Asthma was defined as, relevant symptoms combined with presence of reversibility on a bronchodilator (increase FEV F $_{1}$ of $>8 \%$ of predicted value) or bronchial hyperresponsiveness $\left(\mathrm{PC}_{20} \leq 8 \mathrm{mg} / \mathrm{ml}\right.$ histamine). CF was defined as a combination of clinical features and an abnormal sweat test (Chloride $>60 \mathrm{mmol} / \mathrm{L}$ ). The use of antibiotics was allowed in the population with CF. Healthy children, aged 5 to 18 years, were recruited from our outpatient clinic. The main consultation reason was enuresis nocturna and constipation. The ISAAC questionnaire was used to exclude children with any (history of) airway or allergy complains. Exclusion criteria for all populations were: 1) Patients with diseases that might intervene with the result of the study (e.g. upper airway infection, cor vitium, anatomic anomalies of the airways and other chronic inflammatory diseases like M. Crohn and rheumatoid arthritis). 2) Patients with mental retardation. 3) Patients who cannot perform the EBC procedure properly. 4) Active smokers.

Of all subjects, sex, age, height, weight, duration of disease, therapy and co-morbidity were documented. Patients with and without an exacerbation were included. Informed consent to participate was obtained from the parents of all children participating in this study. This study was approved by the Maastricht Ethics Committee of the Maastricht University.

\section{Lung function tests}

Bronchodilator medication was stopped prior to lung function testing: short-acting bronchodilators at least 8 hours before and long-acting bronchodilators at least 36 hours before the test. Dynamic spirometry was performed by means of a pneumotachograph (Masterlab Jaeger, Würzberg, Germany), with measurement of forced expiratory volume in 1 second $\left(\mathrm{FEV}_{1}\right)$ and forced vital capacity (FVC), according to the standards of the European Respiratory Society [21]. The highest values of FEV ${ }_{1}$ and FVC of three forced expiratory manoeuvres were used for data analysis. The bronchodilating response was assessed 15 minutes after administration of a short-acting bronchodilator and expressed as the increase in $\mathrm{FEV}_{1}$ compared to the predicted value of $\mathrm{FEV}_{1}$. Reversibility was defined as an increase in $\mathrm{FEV}_{1}$ of $>8 \%$. This response was not analysed in healthy children. Lung volumes (RV, ITVG and TLC) were assessed by body plethysmography (Jaeger, Würzberg, Germany).

\section{Exhaled Breath Condensate Collection}

EBC was collected in children during tidal breathing while wearing a nose-clip as described previously [20]. A two-way non-rebreathing valve (Hans Rudolph Inc, series 1420, Kansas City, USA), served also as a saliva trap. The expired air was conducted through a $35 \mathrm{~cm}$ double jacketed glass tube cooled by circulating ice-water. The collected condensate was 
directly frozen in small plastic tubes by submerging the tubes in isopentane and dry ice $\left(-80^{\circ} \mathrm{C}\right)$ and stored at the same temperature during a maximum of 4 weeks.

\section{Cytokine Analysis}

Cytokines were assayed with flow cytometry (CBA, BD Bioscience , San Diego, USA) [22]. EBC samples in a volume of $50 \mu \mathrm{L}$ were incubated with beads of the human Th-1/Th-2 cytokine CBA kit (INF- $\gamma$, TNF- $\alpha$, IL-10, IL-5, IL-4, IL-2) in combination with phycoerythrinconjugated detection antibodies. Recombinant cytokine standards were used in different concentrations $(10-10.000 \mathrm{pg} / \mathrm{mL})$ to prepare the respective standard curves. Next, data acquisition was preformed on a FACS-Calibur (BD-science) and analysed by the BD CBA Analysis Software. The detection limit for each cytokine was determined by the fluorescence intensity (FL2 MFI) of a negative control $(0 \mathrm{pg} / \mathrm{mL})$ measured in our hospital, plus one standard deviation given by BD Bioscience. These standard deviations were calculated after measuring the fluorescence of 20 replicates of a negative control. The corresponding concentration of the FL2 MFI signals were calculated using the formula of the standard curves created by us.

To determine intra-assay reproducibility, standard solutions with concentrations of 2.5 $\mathrm{pg} / \mathrm{mL}, 5 \mathrm{pg} / \mathrm{mL}$ and $10 \mathrm{pg} / \mathrm{mL}$, were analysed three times within the same assay. The coefficients of variation (\% CV, standard deviation divided by the mean multiplied by $100 \%$ ) at $2.5 \mathrm{pg} / \mathrm{mL}, 5 \mathrm{pg} / \mathrm{mL}$ and $10 \mathrm{pg} / \mathrm{mL}$ were calculated. In addition, short-term intrasubject reproducibility was assessed in 30 subjects (10 asthma, $10 \mathrm{CF}$ and 10 healthy children), by analysing variation between two different EBC samples with a 30 minute interval.

\section{Statistical Analysis}

All data were expressed as mean \pm SEM. Patient characteristics (sometimes after logarithm transformation) were normally distributed. Cytokine concentrations were not normally distributed. Two-sided independent-Samples T-Tests and Mann-Whitney tests were used for analysis. P-values less than 0.05 were considered statistically significant.

\section{Results}

\section{Clinical Characteristics}

Eighty children of 5 years and over, were included in this study: 33 asthmatic children, 12 children with CF, and 35 healthy controls (Table 1). Children with CF were older compared to the other populations, and taller compared to asthmatic children $(p<0.05)$. The healthy and asthma population were comparable according to the $\mathrm{FEV}_{1}$ and the FEV 1 FVC. In asthma, as expected, a larger reversibility on a bronchodilator $(p<0.01)$ and more atopy was present than in children with CF $(p<0.01)$. In the CF group, three patients were admitted having an exacerbation. Half of the children with CF were colonised with Pseudomonas Aeruginosa and one child was known with Allergic Broncho Pulmonary Aspergillosis (ABPA). 
Table 1: Clinical characteristics of the study population.

\begin{tabular}{|c|c|c|c|c|c|}
\hline & Asthma & & Cystic Fibrosis & & Healthy \\
\hline Number & 33 & & 12 & & 35 \\
\hline Age (yrs) & $9.7 \pm 0.5$ & + & $13.3 \pm 1.0$ & $\#$ & $10.0 \pm 0.5$ \\
\hline Length $(\mathrm{cm})$ & $134.5 \pm 2.7$ & + & $151.4 \pm 5.9$ & & $141.5 \pm 3.0$ \\
\hline Weight (kg) & $33.0 \pm 2.1$ & & $39.3 \pm 3.8$ & & $34.7 \pm 1.9$ \\
\hline Sex (male / female) & $22 / 11$ & & $7 / 5$ & & 19 / 16 \\
\hline $\operatorname{lgE}(\mathrm{kU} / \mathrm{l})$ & $600 \pm 219$ & $\dagger$ & $126 \pm 67$ & & - \\
\hline Exacerbation of disease & $8(24.2 \%)$ & & $3(25.0 \%)$ & & - \\
\hline Phadiatop positive & $25(75.8 \%)$ & $\dagger$ & $1(8.3 \%)$ & & - \\
\hline Active eczema & $4(12.1 \%)$ & & - & & - \\
\hline Allergic rhinitis & $6(18.2 \%)$ & & - & & - \\
\hline Inhaled corticosteroid use & $22(66.7 \%)$ & $\dagger$ & $3(25.0 \%)$ & & - \\
\hline Corticosteroid dosage $(\mu \mathrm{g})$ & $536 \pm 89$ & + & $100 \pm 0$ & & - \\
\hline Antibiotic use & - & & $7(58.3 \%)$ & & - \\
\hline Reversibility $^{* *}$ & $21 \%$ & + & $0 \%$ & & - \\
\hline $\mathrm{FEV}_{1} \%$ predicted $^{* * *}$ & $101.5 \pm 2.9$ & $\dagger$ & $54.9 \pm 6.6$ & $\#$ & $101.3 \pm 1.5$ \\
\hline $\mathrm{FEV}_{1} / \mathrm{VC}^{* * *}$ & $83.0 \pm 3.0$ & $\dagger$ & $69.8 \pm 3.8$ & $\#$ & $87.6 \pm 1.1$ \\
\hline TLC $\%_{\text {predicted }}{ }^{* *}$ & - & & $95.2 \pm 4.8$ & & - \\
\hline RV $\%$ predicted ${ }^{* * *}$ & - & & $182.4 \pm 18.8$ & & - \\
\hline ITVG $\%$ predicted $^{* * *}$ & - & & $114.7 \pm 7.3$ & & - \\
\hline \multicolumn{6}{|c|}{$\begin{array}{l}+\mathrm{p}<0.05 \text { asthma vs. CF; \# } \mathrm{p}<0.05 \mathrm{CF} \text { vs. healthy; Mean values } \pm \mathrm{SEM} \text { are represented unless otherwise } \\
\text { listed; }{ }^{* *} \text { Reversibility is defined as the presence of a change in } \mathrm{FEV}_{1} \text { of }>8 \% \text { after a bronchodilator expressed } \\
\text { as a percentage of the predicted value; }{ }^{* * *} \mathrm{FEV}_{1} \text {, forced expiration volume exhaled in one second; VC, vital } \\
\text { capacity; TLC, total lung capacity; RV, residual volume; ITGV, intra thoracic gas volume; } \% \text { predicted, percent- } \\
\text { age of predicted value. }\end{array}$} \\
\hline
\end{tabular}

\section{Exhaled Breath Condensate Collection}

The duration of EBC collection was not significantly different between the populations. The mean EBC collection time in the asthma and CF population, was $19 \pm 0.4$ and $20 \pm 2$ minutes respectively. The mean volume of collected EBC was $1150 \pm 100$ and $1515 \pm 180$ $\mu \mathrm{l}$, respectively. In the healthy population, the collection time and the volume condensate collected was $29 \pm 0.4$ minutes and $2900 \pm 219 \mu \mathrm{l}$. In the CF and healthy population, more EBC was produced per minute than in asthmatic children $(p<0.05)$. There were no adverse effects.

\section{Exhaled cytokines}

In both asthma and CF, INF- $\gamma$, IL-10, IL-4 and IL-2 were detected (Figure, Table 2). The observed concentrations were close to the lower detection limits of the assay. In EBC of children with asthma, all 6 measured cytokines were detected. However, INF- $\gamma$, IL-4 and IL2 were detected in $12-15 \%$ of the children, whereas TNF- $\alpha$, IL-10 and IL-5 were found in only $3-6 \%$. There was no relationship between the detection of cytokines or the cytokine levels and the use of inhaled corticosteroids. In the CF population, INF- $\gamma$, IL-10, IL-4 and IL2 were found in $8-25 \%$ of the samples. TNF- $\alpha$ and IL-5 were not detected. Cytokine concentrations did not differ significantly between children with asthma and CF. In the healthy population, INF- $\gamma$, TNF- $\alpha$ and IL-10 were detected in $9 \%, 14 \%$ and $3 \%$ respectively. 
There was no detection of IL-5, IL-4 or IL-2 in any of these EBC samples (Table 3). Since the healthy controls were measured in a separate analysis, detection limitis slightly differ from those of the diseased children.

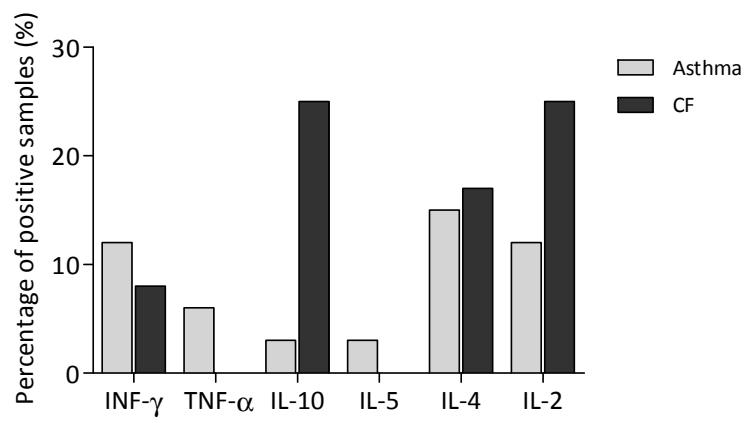

Figure: Percentage of samples in which cytokines were detected in the patient populations.

The percentage of samples in which cytokines were detected of the asthma $(n=33)$ and CF $(n=12)$ population are shown; $n=$ number of patients. TNF- $\alpha$ and IL- 5 were detected in EBC of only children with asthma.

Table 2: Concentrations of cytokines $(\mathrm{pg} / \mathrm{mL})$ detected in $\mathrm{EBC}$ of children with asthma or CF and percentage of samples with positive detection of cytokines.

\begin{tabular}{llllllll}
\hline & \multicolumn{3}{c}{ Total patient group } & \multicolumn{2}{l}{$\begin{array}{l}\text { Asthma } \\
\text { ( } \mathrm{n}=45)\end{array}$} & \multicolumn{2}{l}{ CF } \\
& LLD & N\% & Mean \pm SE (range) & N\% & Mean \pm SE (range) & N\% & Mean \pm SE (range) \\
\hline INF- $\gamma$ & 5.2 & 11 & $6.1 \pm 0.4(5.2-7.2)$ & 12 & $5.8 \pm 0.4(5.2-6.7)$ & 8 & 7.2 \\
TNF- $\alpha$ & 1.3 & 4 & $1.3 \pm 0.0(1.3-1.3)$ & 6 & $1.3 \pm 0.0(1.3-1.3)$ & 0 & \\
IL-10 & 2.0 & 9 & $3.1 \pm 0.8(2.0-5.6)$ & 3 & 5.6 & 25 & $2.3 \pm 0.1(2.0-2.5)$ \\
IL-5 & 1.3 & 2 & 1.3 & 3 & 1.3 & 0 & \\
IL-4 & 2.5 & 16 & $2.8 \pm 0.1(2.7-3.1)$ & 15 & $2.8 \pm 0.1(2.7-3.1)$ & 17 & $2.7 \pm 0.0(2.7-2.7)$ \\
IL-2 & 1.4 & 16 & $2.6 \pm 1.1(1.4-9.1)$ & 12 & $3.5 \pm 1.9(1.4-9.1)$ & 25 & $1.4 \pm 0.0(1.4-1.4)$ \\
\hline
\end{tabular}

LLD, lower limit of detection; SE, standard error; N\%, the percentage of samples in which cytokines were detected.

Table 3: Concentrations of cytokines $(\mathrm{pg} / \mathrm{mL})$ detected in EBC of healthy controls and percentage of samples with positive detection of cytokines.

\begin{tabular}{llll}
\hline & & Healthy population $(\mathrm{n}=35)$ & \\
& LLD & N\% & Mean \pm SE (range) \\
\hline INF- $\gamma$ & 2.7 & $9 \%$ & $2.9 \pm 0.1(2.7-3.0)$ \\
TNF- $\alpha$ & 1.1 & $14 \%$ & $1.6 \pm 0.1(1.3-2.0)$ \\
IL-10 & 1.6 & $3 \%$ & 1.8 \\
IL-5 & 1.1 & $0 \%$ & \\
IL-4 & 2.5 & $0 \%$ & \\
IL-2 & 2.5 & $0 \%$ & \\
\hline
\end{tabular}

LLD, lower limit of detection; SE, standard error; N\%, the percentage of samples in which cytokines were detected. 


\section{Short-term intra-subject reproducibility}

The short-term intra-subject reproducibility of cytokines of all children ranged from $10 \%$ to $32 \%$ (Table 4a). Most reproducible data were obtained with measurements of IL-10 and IL-5 (\% CV 13\% and 10\% respectively). The largest variation was found in INF- $\gamma$ data (range $\%$ CV 22-43\%).

\section{Intra assay reproducibility}

The mean (range) \% CV of samples containing cytokines concentrations $2.5 \mathrm{pg} / \mathrm{mL}$ and 5 $\mathrm{pg} / \mathrm{mL}$, were comparable (31 (22-48)\% vs. 35 (19-46)\% respectively) (Table $4 \mathrm{~b})$. The intra assay reproducibility of samples containing cytokines concentrations of $10 \mathrm{pg} / \mathrm{mL}$, was significantly better (\% CV $20(16-27) \%, p<0.05)$. The intra-assay reproducibility profile of cytokines was in accordance with the profile of in vivo intra-subject reproducibility.

Table 4a: Short-term intra-subject reproducibility (\% CV *).

\begin{tabular}{lllll}
\hline & Healthy & Asthma & CF & Total \\
\hline INF- $\gamma$ & 23 & 43 & 22 & 32 \\
TNF- $\alpha$ & 29 & 21 & 33 & 28 \\
IL-10 & 13 & 16 & 10 & 13 \\
IL-5 & 11 & 6 & 10 \\
IL-4 & 27 & 14 & 21 \\
IL-2 & 26 & 13 & 20 \\
\hline$*$ \%CV: standard deviation divided by the mean value multiplied by $100 \%$. &
\end{tabular}

Table 4b: Intra-assay reproducibility at cytokine concentrations of $2.5 \mathrm{pg} / \mathrm{mL}, 5.0 \mathrm{pg} / \mathrm{mL}$ and $10.0 \mathrm{pg} / \mathrm{mL}$.

\begin{tabular}{lllllll}
\hline & $2.5 \mathrm{pg} / \mathrm{mL}$ & \multicolumn{2}{c}{$5.0 \mathrm{pg} / \mathrm{mL}$} & \multicolumn{3}{l}{$10.0 \mathrm{pg} / \mathrm{mL}$} \\
\hline & $\mathrm{pg} / \mathrm{mL}$ & $\% \mathrm{CV} *$ & $\mathrm{pg} / \mathrm{mL}$ & $\% \mathrm{CV}{ }^{*}$ & $\mathrm{pg} / \mathrm{mL}$ & $\% \mathrm{CV}^{*}$ \\
\hline INF- $\gamma$ & 3.6 & 48 & 5.6 & 46 & 8.8 & 23 \\
TNF- $\alpha$ & 3.6 & 31 & 5.5 & 44 & 8.3 & 27 \\
IL-10 & 3.7 & 32 & 5.4 & 30 & 9.6 & 13 \\
IL-5 & 3.8 & 22 & 5.7 & 31 & 9.0 & 19 \\
IL-4 & 3.9 & 33 & 5.1 & 38 & 7.0 & 20 \\
IL-2 & 2.6 & 22 & 4.6 & 19 & 8.3 & 16 \\
\hline Mean & 3.5 & 31 & 5.3 & 35 & 8.5 & 20 \\
\hline \multirow{2}{*}{$\%$ CV: standard deviation divided by the mean value multiplied by $100 \%}$. &
\end{tabular}

\section{Discussion}

The main finding of this study was the detection of cytokines, INF- $\gamma$, TNF- $\alpha$, IL-10, IL-5, IL-4 and IL-2 in EBC originating from not only children with asthma, but also children with CF, with exception of TNF- $\alpha$ and IL-5. However, cytokine concentrations were low and detection was only possible in a minority of children. No differences in cytokine concentrations were found between asthma and CF. To our knowledge, this is the first EBC study demonstrating IL-4 and INF- $\gamma$ in EBC in children with CF, and IL-2 and IL-5 in EBC of asthmatic 
children. It was shown that EBC collection is a safe and feasible method in children with asthma and CF aged 5.5 years and over, even during an exacerbation. All patients were able to complete the EBC collection procedure and no adverse affects were reported.

One would expect different cytokine profiles in EBC originating from children with asthma and CF, i.e. an increased Th-2 and Th-1 response, respectively. However, these differences were not found, probably due to the limited number of positive cytokine detections. In addition, it was not possible to correlate these findings with patient characteristics or to detect relationships with severity of disease, the use of inhaled corticosteroids or the coexistence of atopy (data not shown).

Data on the variability of cytokines demonstrated a quite acceptable reproducibility of IL-5 and IL-10 but a much larger variation of TNF- $\alpha$ and INF- $\gamma$. No other studies on the variability of inflammatory biomarkers in EBC exist in children. Limited data exist in adults. Reproducibility of inflammatory markers in EBC, has been studied in rather small number of subjects [23-27]. The mean \% CV of eNO described were $9.5 \pm 4.7 \%$ [23], which is close to the reproducibility of IL-5 in our study. It is likely that increased standardisation, and the use of a more optimal coating and condenser system will increase the detection and reproducibility of cytokines in EBC.

EBC data in children are scant [18-20]. Overall, the lack of cytokine detection in EBC of children with asthma or CF, may be due to the following aspects: 1) The inner coating of condenser systems influenced the detection of 8-isoprostane and albumin in EBC. The use of a silicone or a glass coating resulted in a significantly better detection of albumin and 8isoprostane in EBC then teflon, aluminium, and polypropylene coatings [28, 29]. Based on these results, we used a glass condenser coating in this study. However, it is yet unclear which coating is optimal for measuring cytokines. 2) Cytokine concentrations in EBC were close to the detection limits of the assay used. In addition, no emphasis should be given to absolute quantitative values of cytokines in this study. 3) A study population with well controlled disease, while cytokines may only be detectable during an exacerbation, and / or in case of uncontrolled disease.

Based on the results of our current study, the following aspects should be taken into account when measuring cytokines in EBC: 1 ) More sensitive analytical methods are needed to show differences in cytokine concentrations. 2) Techniques to concentrate EBC volume may increase cytokine concentrations and thereby measurements may be more reliably. We concentrated several samples tenfold by means of centrifugation through a $30 \mathrm{kDa}$ molecular filter, but this procedure did not really effect cytokine concentrations measured (data not shown), possibly because of the adhesion of cytokines to the filter device. Other concentration methods, like freeze-drying combined with centrifugation may be beneficial. 3) To prevent degradation of cytokines before analysis, samples may be protected by adding a protease inhibitor or bovine serum albumin [24].

This study stresses the importance of further improvement and standardisation of the EBC technique, as recently reviewed [8]. There are indications that the currently used condensers are not very effective in the sampling of $\operatorname{EBC}[28,29]$. A task force of the European Respiratory Society (ERS) and American Thoracic Society (ATS) is working on the standardisation of the EBC technique. Such recommendations on standardisation of another inflammatory marker -NO in exhaled air- already exist, and had been of great value in the development and application of this marker in clinical studies [12]. As a consequence, some important recent studies demonstrated that eNO is a valuable tool for adjusting 
maintenance treatment in asthma [13], and is a predictor of asthma relapse or asthma exacerbations. Such studies are lacking on EBC. However, eNO has limited value for a diagnosis of asthma, and probably is not very useful in other chronic lung diseases like CF or COPD. After further standardisation, the non-invasive EBC technique may be a valuable additional tool of assessing airway inflammation in various chronic lung diseases.

Altogether, cytokines are potentially important inflammatory markers for both the diagnosis of inflammatory airway diseases and the monitoring of chronic airway inflammation as a result of the primary etiology of asthma and $C F[8,9,30,31]$. Although we were able to demonstrate the presence of INF- $\gamma$, TNF- $\alpha$, IL-10, IL-5, IL-4 and IL-2 in EBC of children with asthma and CF, this study emphasizes the importance of optimising the EBC collection methodology and the development of more sensitive assays or techniques with increased sensitivity for T-cell cytokines.

\section{References}

1. Lenney, W. The burden of pediatric asthma. Pediatr Pulmonol Suppl 1997;15:13-16.

2. Measuring childhood asthma prevalence before and after the 1997 redesign of the National Health Interview Survey-United States. MMWR Morb Mortal Wkly Rep 2000;49(40):908-911.

3. Tattersfield, AE, Knox, AJ, Britton, JR, Hall, IP. Asthma. Lancet 2002;360(9342):1313-1322.

4. Shwachman, H, Kulczycki, LL. Long-term study of one hundred five patients with cystic fibrosis; studies made over a five- to fourteen-year period. AMA J Dis Child 1958;96(1):6-15.

5. Ratjen, F, Doring, G. Cystic fibrosis. Lancet 2003;361(9358):681-689.

6. Cotran RS, KV, Robbins SL. Pathologic basis of disease. 1994.

7. Kharitonov, SA, Barnes, PJ. Exhaled markers of pulmonary disease. Am J Respir Crit Care Med 2001;163(7):1693-1722.

8. Rosias, PP, Dompeling, E, Hendriks, HJ, et al. Exhaled breath condensate in children: Pearls and pitfalls. Pediatr Allergy Immunol 2004;15(1):4-19.

9. Gaston, B. Breath condensate analysis: perhaps worth studying, after all. Am J Respir Crit Care Med 2003;167(3):292-293.

10. Effros, RM, Hoagland, KW, Bosbous, M, et al. Dilution of respiratory solutes in exhaled condensates. Am J Respir Crit Care Med 2002;165(5):663-669.

11. Jobsis, $Q$, Raatgeep, HC, Schellekens, SL, et al. Hydrogen peroxide in exhaled air of healthy children: reference values. Eur Respir J 1998;12(2):483-485.

12. ATS/ERS Recommendations for Standardized Procedures for the Online and Offline Measurement of Exhaled Lower Respiratory Nitric Oxide and Nasal Nitric Oxide, 2005. Am J Respir Crit Care Med 2005;171(8):912-930.

13. Smith, AD, Cowan, JO, Brassett, KP, et al. Use of exhaled nitric oxide measurements to guide treatment in chronic asthma. N Engl J Med 2005;352(21):2163-2173.

14. Moser, C, Jensen, PO, Kobayashi, O, et al. Improved outcome of chronic Pseudomonas aeruginosa lung infection is associated with induction of a Th1-dominated cytokine response. Clin Exp Immunol 2002;127(2):206-213.

15. Epelman, S, Bruno, TF, Neely, GG, et al. Pseudomonas aeruginosa exoenzyme $S$ induces transcriptional expression of proinflammatory cytokines and chemokines. Infect Immun 2000;68(8):4811-4814.

16. Gibson, RL, Burns, JL, Ramsey, BW. Pathophysiology and management of pulmonary infections in cystic fibrosis. Am J Respir Crit Care Med 2003;168(8):918-951.

17. Leung, TF, Wong, GW, Ko, FW, et al. Analysis of growth factors and inflammatory cytokines in exhaled breath condensate from asthmatic children. Int Arch Allergy Immunol 2005;137(1):66-72.

18. Shahid, SK, Kharitonov, SA, Wilson, NM, et al. Increased interleukin-4 and decreased interferon-gamma in exhaled breath condensate of children with asthma. Am J Respir Crit Care Med 2002;165(9):1290-1293. 
19. Cunningham, S, McColm, JR, Ho, LP, et al. Measurement of inflammatory markers in the breath condensate of children with cystic fibrosis. Eur Respir J 2000;15(5):955-957.

20. Rosias, PP, Dompeling, E, Dentener, MA, et al. Childhood asthma: Exhaled markers of airway inflammation, asthma control score, and lung function tests. Pediatr Pulmonol 2004;38(2):107-114.

21. Tammeling, GJ, Quanter, PH, Party., obotRW. Standardized lung function testing. Eur Respir J 1993;6:5-40.

22. Morgan, E, Varro, R, Sepulveda, H, et al. Cytometric bead array: a multiplexed assay platform with applications in various areas of biology. Clin Immunol 2004;110(3):252-266.

23. Kharitonov, SA. Exhaled markers of inflammatory lung diseases: ready for routine monitoring? Swiss Med Wkly 2004;134(13-14):175-192.

24. Tufvesson, E, Bjermer, L. Methodological improvements for measuring eicosanoids and cytokines in exhaled breath condensate. Respir Med 2005.

25. Rahman, I. Reproducibility of oxidative stress biomarkers in breath condensate: are they reliable? Eur Respir J 2004;23(2):183-184.

26. Montuschi, P, Kharitonov, SA, Ciabattoni, G, Barnes, PJ. Exhaled leukotrienes and prostaglandins in COPD. Thorax 2003;58(7):585-588.

27. Carpagnano, GE, Barnes, PJ, Francis, J, et al. Breath condensate pH in children with cystic fibrosis and asthma: a new noninvasive marker of airway inflammation? Chest 2004;125(6):2005-2010.

28. Rosias, PPR, Robroeks, CMHHT, Niemarkt, HJ, et al. The coating of condenser systems influences the detection of 8-isoprostane in exhaled breath. Eur Respir J 2004:468s.

29. Rosias, PP, Vernooij J.J.H., Dentener M.A. The inner coating of condenser systems influences the detection of human albumin in exhaled breath condensate. Eur Respir J 2003;22(S45):280s.

30. Montuschi, P. Indirect monitoring of lung inflammation. Nat Rev Drug Discov 2002;1(3):238-242.

31. Hunt, J. Exhaled breath condensate: an evolving tool for noninvasive evaluation of lung disease. J Allergy Clin Immunol 2002;110(1):28-34. 



\section{CHAPTER 6}

\section{Increased cytokines, chemokines and soluble adhesion molecules in exhaled breath condensate of asthmatic children}

CMHHT Robroeks, GT Rijkers, Q Jöbsis, JJE Hendriks, JGMC Damoiseaux, LI Zimmermann, CP van Schayck, E Dompeling Clinical and Experimental Allergy 2010;40(1):77-84 


\section{Abstract}

\section{Background}

Airway inflammation in asthma is characterised by production of cytokines, chemokines and soluble adhesion molecules. The assessment of these inflammatory biomarkers in exhaled breath condensate $(E B C)$ is hampered by low detection rates. However, the use of a glass condenser system combined with a sensitive analytical technique may increase the possibility to assess these biomarkers in EBC in a reliable way. Objectives: To assess detection rates of cytokines (IL1 $\alpha,-1 \beta,-2,-4,-5,-6,-10,-12 p 70,-13,-18$, IFN $\gamma$, TNF $\alpha$ ), chemokines (MIP1 $\alpha$ (CCL3), MIF, eotaxin (CCL11), RANTES (CCL5), IP10 (CXCL10), IL8 (CXCL8), MCP1), and soluble adhesion molecules (SICAM, SVCAM) in EBC of children with asthma and healthy control children;To study the differences in the biomarker concentration between children with asthma and controls.

\section{Methods}

60 Children were included: 31 asthmatics (71\% atopic), and 29 controls. EBC was collected using a glass condenser system. The inflammatory markers were analysed using multiplex immunoassay technology.

\section{Results}

Detection percentages of cytokines, chemokines and adhesion molecules ranged from 94$100 \%$, except for eotaxin (CCL11) and RANTES (CCL5) (detection rates of $10 \%$ and $45 \%$ in healthy controls respectively). Intra-subject variability of biomarkers in EBC in the group as a whole ranged from $5.2 \%$ to $35.0 \%$. In asthmatics, the levels of cytokines [IL2,-4,-5,-6,-13, IFN $\gamma$ ], chemokines [MIP1 $\alpha$ (CCL3), MIF, RANTES (CCL5), IP10 (CXCL10), IL8 (CXCL8), MCP1], and adhesion molecules [sICAM, SVCAM] were significantly increased in comparison with controls $(p<0.05)$.

\section{Conclusion}

If collected with a glass condenser and analysed by multiplex immunoassay technology, cytokines, chemokines and soluble adhesion molecules can be reliably demonstrated in EBC of children. Most of these inflammatory markers were elevated in EBC of asthmatics compared to controls. 


\section{Introduction}

Cytokines, chemokines and soluble adhesion molecules are soluble proteins with structural similarities and overlapping functions. These markers are secreted during inflammatory responses and are part of a complex regulatory network within the immune system [1]. Cytokines regulate the production of other inflammatory mediators, whereas secreted chemokines function as regulatory molecules that attract (inflammatory) leukocytes to the site of inflammation [1-3]. Soluble intercellular adhesion molecule-1 (sICAM-1) and soluble vascular cell adhesion molecule-1 (sVCAM-1), are expressed by endothelial cells and are upregulated by pro-inflammatory cytokines like tumor necrosis factor- $\alpha$ (TNF $\alpha$ ), IL-1, IL-6 and IL-12 [4-6].

Non-invasive measurement of inflammatory markers may be useful for identification of chronic airway inflammation in asthma. It may provide insight in severity and control of the chronic inflammatory processes [7-13]. Therefore, there is increasing interest in analysing multiple inflammatory markers in exhaled breath condensate (EBC). However, the utility of these inflammatory markers has been hampered by low detection rates and low concentrations in EBC $[10,14]$. We recently demonstrated both in vitro and in vivo that the use of a glass condenser system increased the possibility to detect proteins and 8isoprostane in EBC [14]. Moreover, Th1 and Th2 cytokines in EBC as assessed by flow cytometry were of help in indicating the presence, severity and control of childhood asthma [15]. However, detection rates of cytokines with the flow cytometric technique were still suboptimal: it ranged in children with asthma from $3 \%$ for IL-5 to $31 \%$ for IFN- $\gamma$. A semiquantitative protein array for airway cytokine-chemokine-growth factor production was used in a recent study with ten nonsmoking healthy subjects and 16 adult asthmatic patients [16]. The expressions of most cytokines and chemokines were significantly upregulated in asthmatic airways compared with healthy contols [16]. However, the semiquantitative nature of the technique was a clear draw-back. A very interesting and probably useful technique for analysis of EBC is the multiplex immunoassay technique in which inflammatory markers can be measured simultaneously in a low volume sample with an increased sensitivity and reliability [17]. These features make the multiplex immunoassay possibly very suitable for analysis of EBC. In a study with 30 healthy adults, the use of the multiplex immuno-assay technology in combination with an optimised glass condenser resulted in an average detection rate of 8 different cytokines in EBC of 62\% [18]. The detection rates of IL-2, $-4,-5,-13$ and TNF- $\alpha$ were best with the optimised glass condenser. We studied the detection rates and biomarker concentrations in EBC of cytokines, chemokines and adhesion molecules in both children with asthma and healthy controls. The aim of this study was to assess sensitivity and reliability of cytokine and chemokine measurements in EBC with the sensitive multiplex immune-assay technology in conjunction with a new optimised condenser, and to assess differences between asthmatic children and healthy controls. 


\section{Material and Methods}

\section{Study subjects}

\section{Asthma}

Children with physician-diagnosed asthma of 6 years and over, known at the department of Paediatric Pulmonology of the Maastricht University Medical Centre for at least 6 months were recruited. Asthma was defined as a chronic disorder with wheezing, coughing, chest tightness, or shortness of breath, characterised by a variable but reversible airway obstruction [19]. Reversibility on a beta-2 agonist (increase in $\mathrm{FEV}_{1}$ of at least $9 \%$ of predicted value) was demonstrated in each patient at one or more occasions. Severity of asthma was assessed according to GINA guidelines [19]. Presence of atopy was not an obligatory criterion. A child was considered atopic when the total IgE level exceeded 20 $\mathrm{kU} / \mathrm{l}$ and / or the Phadiatop was positive and / or the Radio Allergo Sorbent Test (RAST) was positive for at least 2 allergens classified as $\geq$ class 2 . The patient characteristics are given in Table 1.

Table 1: Clinical characteristics of the study population.

\begin{tabular}{lll}
\hline Number of patients & Asthma (N=31) & Controls (N=29) \\
\hline Age (yrs) & $11.4 \pm 0.5$ & $10.3 \pm 0.5$ \\
Height (cm) & $150.0 \pm 3.0$ & $145.4 \pm 3.0$ \\
SD height-age (cm) & $0.092 \pm 0.23$ & $0.40 \pm 0.23$ \\
Weight (kg) & $43.0 \pm 2.5$ & $37.9 \pm 2.1$ \\
SD weight-age (kg) & $0.86 \pm 0.40$ & $0.88 \pm 0.32$ \\
Gender (male / female) & $27 / 4$ & $16 / 13$ \\
FEV $(\%$ predicted) & $98.3 \pm 3.4$ & $100.4 \pm 1.8$ \\
FEV 1 (VC (\%) & $83.7 \pm 2.1$ & $88.9 \pm 1.2$ \\
FVC (\%) & $95.4 \pm 2.4$ & $92.6 \pm 1.8$ \\
Reversibility FEV ${ }_{1}(\%) \times$ & 16 & $\mathrm{NA}$ \\
Poor asthma control (\%)* & 49 & $\mathrm{NA}$ \\
Atopy (\%) \# & 71 & 0 \\
ICS (\%) & 87 & 0 \\
\hline
\end{tabular}

Definition of abbreviations: FEV1, forced expiratory volume in 1 second; VC, vital capacity; NA, not applicable; ICS, use of inhaled corticosteroids; Data are given as mean $\pm \mathrm{SE}$, unless indicated otherwise. Differences between population characteristics were statistically tested by means of one way ANOVA (continuous variables). Dichotomous variables (gender and atopy) were tested with $\mathrm{Chi}^{2}$ tests. No significant differences were found between the two groups; $\times$ Reversibility was defined as the increase in $\mathrm{FEV}_{1} \%$ predicted after use of a bronchodilator of $9 \%$ or more. Percentage of children with reversibility at entry into the study are shown; * Classification based on GINA guidelines [34]; \# A child was considered atopic when the total IgE level exceeded $20 \mathrm{kU} / \mathrm{l}$ and / or the Phadiatop was positive and / or the Radio Allergo Sorbent Test (RAST) was positive for at least 2 allergens classified as $\geq$ class 2 .

\section{Controls}

The control population consisted of children without a history or presence of respiratory problems or allergy, objectively checked with the 'International study of asthma and allergies in childhood (ISAAC)' questionnaire [20]. The children were selected from a primary school. The controls did not use medication before or during the study. 


\section{Exclusion criteria}

Exclusion criteria for all study populations were: 1) Presence of a disease that might interfere with the results of this study (airway infection, heart disease, anatomic abnormalities of the airways or other chronic inflammatory diseases such as Crohn's disease and rheumatoid arthritis), 2) Mental retardation, and, 3) Active smoking.

\section{Ethics}

The medical ethics committee of the University Maastricht approved this study. Written informed consent was obtained from the parents and the children. The international trial number was NTC00404976.

\section{Exhaled Breath Condensate}

The EBC collection device consisted of an optimised glass condenser like described previously [18]. Children breathed tidally, while wearing a nose-clip, through a mouthpiece connected to a two-way non-rebreathing valve. The two-way valve and tubing to the condenser served as a saliva trap. The new, optimised glass condenser system consisted of: 1) an inclined glass condenser with a tube length of $90 \mathrm{~cm}$ (to enlarge the condensation surface) and downwards moveable plunger (to optimise condensate recovery from the inner condenser wall), with tangential and axial breath flow channels (to turbinately direct the tidally exhaled breath flows towards the cooled inner condenser wall); 2) swan-neck tubing (serving as gravitational saliva trap) and two-way nonrebreathing valve, connected to a mouthpiece; 3) entrance of inspired room air; 4) cooling unit consisting of a countercurrent circulating ice-water pump; 5) removable, cooled glass sample vial (to collect exhaled breath condensate). After collection (during 10 minutes), EBC was rapidly frozen at $80^{\circ} \mathrm{C}$ using dry ice and was stored at $-80^{\circ} \mathrm{C}$ until analysis $[15,18]$.

\section{Lung function tests}

Subjects were asked to stop bronchodilator medication prior to lung function testing. Short-acting bronchodilators were stopped at least 8 hours, and long-acting bronchodilators at least 36 hours before the test. Dynamic spirometry was performed by means of the Flowscreen (Viasys ${ }^{\circ}$, Hoechberg, Germany). Forced expiratory volume in $1 \mathrm{sec}\left(\mathrm{FEV}_{1}\right)$, vital capacity (VC) and $\mathrm{FEV}_{1} / \mathrm{VC}$ were measured. The highest $\mathrm{FEV}_{1}$ and $\mathrm{VC}$ of three technically correct manoeuvres was used for analysis. Fifteen minutes after inhalation of $400 \mu \mathrm{g}$ salbutamol, dynamic spirometry was repeated in a similar way. Reversibility was expressed as a percentage of $\mathrm{FEV}_{1}$ predicted value.

\section{Multiplex immunoassay technology}

Inflammatory markers were assayed with Luminex 100 analyser (Luminex Corporation, Austin, TX, USA). Colour coded beads with a distinguishable fluorophore were added to the EBC samples [17]. Specific capture antibodies were linked to the beads of a unique fluorescent region, enabling capture and detection of specific molecules in EBC samples. The second antibody was labelled with another fluorescent probe. Within the Luminex analyzer, lasers excite the internal dyes, identifying each bead. A $635 \mathrm{nM}$ laser classified 
the beads, whereas a $523 \mathrm{nM}$ laser detected the fluorescence due to binding of the secondary antibody. All EBC samples were analysed simultaneously in order to minimize inter-assay variation $[1,21]$. The lower limits of detection of the inflammatory markers are given in Table 2. Measurement and data analysis were performed using the Bioplex 100 system and Bioplex Manager software version 3.0 (Bio-Rad Laboratories, Hercules, CA, USA). All multiplex immunoassays were performed in a 96-well format, 1.2- $\mu \mathrm{m}$ filter bottom plates (Millipore, Amsterdam, the Netherlands) and a 12-point standard curve in duplicate was included on every plate. Positive and negative control samples were included.

\section{Intra-subject variability}

Intra-subject variability was assessed in 38 subjects (18 asthma, and 20 control children) by analysing variation between two different EBC samples with a 30 minute interval of collection. The coefficients of variation (SD/mean value $\times 100 \%$ ) were calculated. A CV of $<15 \%$ was considered as acceptable variability.

\section{Statistic analysis}

All data were expressed as mean \pm standard error of the mean (normal distribution) or as median \pm range (not normal distribution). To test differences of parameters between study populations, one-way ANOVA or Kruskal-Wallis tests were used for parametric and non parametric data, respectively. When a test was significant and data distribution was normal, Students t-tests were used for further statistical analysis. Otherwise, Mann Whitney $U$ tests were applied. To correct for multiple tests, the Hochberg correction was performed for Student t-tests and the Bonferoni correction for Mann Whitney $U$ tests. Statistical tests were considered significant with $p$-values $<0.05$.

\section{Power analysis}

Based on earlier data on cytokines in EBC, the expected SD of cytokines like IL-4 and TNF- $\alpha$ in $\mathrm{EBC}$ was $0.35 \mathrm{pg} / \mathrm{mL}$. A total number of 52 subjects are necessary to detect a difference in cytokine concentration of $0.2 \mathrm{pg} / \mathrm{mL}$ between asthmatics and controls, with an alpha of 0.05 and a power of $90 \%$.

\section{Results}

\section{Population characteristics}

60 Children were recruited for this study: 31 with asthma and 29 healthy controls (Table 1). $17 \%$ of the asthmatic children had intermittent disease, $23 \%$ mild persistent, $33 \%$ moderate persistent, and $27 \%$ severe persistent asthma. 


\section{Detections of cytokines, chemokines and soluble adhesion molecules}

The detection percentage in both asthma and controls was high and ranged from 91$100 \%$, except for eotaxin (CCL11) (35\% and 10\% respectively) and RANTES (CCL5) ( $87 \%$ and $45 \%$ respectively). (Table $2 \mathrm{~A}-\mathrm{B})$.

Table 2a: Cytokines in exhaled breath condensate of children with asthma and healthy controls.

\begin{tabular}{|c|c|c|c|c|c|c|}
\hline Marker & LDL & $\mathrm{N} \%$ & Asthma $(\mathrm{N}=31)$ & $\mathrm{N} \%$ & Controls $(\mathrm{N}=29)$ & P-value \\
\hline IL1 $\alpha$ & 2.2 & 100 & $71.4 \pm 2.9$ & 100 & $75.9 \pm 2.4$ & 0.24 \\
\hline IL1 $\beta$ & 2.4 & 97 & $3.9(3.6-4.6)$ & 100 & $3.6(3.2-4.0)$ & 0.02 \\
\hline IL2 & 5.0 & 97 & $68.6 \pm 3.1$ & 100 & $55.4 \pm 1.7$ & 0.001 \\
\hline IL4 & 2.2 & 97 & $16.7 \pm 0.9$ & 100 & $13.8 \pm 0.6$ & 0.01 \\
\hline IL5 & 4.2 & 97 & $79.6(73.7-105.0)$ & 100 & $70.2(64.0-80.9)$ & 0.004 \\
\hline IL6 & 2.7 & 100 & $24.6 \pm 1.2$ & 100 & $21.0 \pm 0.6$ & 0.01 \\
\hline IL10 & 2.0 & 97 & $27.8(24.0-33.4)$ & 100 & $25.8(23.2-29.0)$ & 0.10 \\
\hline IL12p70 & 7.5 & 97 & $1525.8 \pm 67.8$ & 100 & $1400.8 \pm 111.3$ & 0.34 \\
\hline IL13 & 1.9 & 97 & $80.8(75.6-93.3)$ & 100 & 75.7 (67.1-84.4) & 0.02 \\
\hline IL18 & 4.8 & 97 & $88.8 \pm 4.3$ & 100 & $79.6 \pm 3.6$ & 0.11 \\
\hline $\mathrm{IFN} \gamma$ & 12.7 & 97 & $60.0 \pm 4.0$ & 100 & $48.7 \pm 2.0$ & 0.02 \\
\hline TNF $\alpha$ & 1.8 & 97 & $26.8(24.6-32.3)$ & 100 & $25.3(21.6-27.3)$ & 0.02 \\
\hline
\end{tabular}

Data with a normal distribution are expressed as mean $\pm \mathrm{SE}$, otherwise data are expressed as median (range). Unit: $\mathrm{pg} / \mathrm{mL}$. For normally distributed data, one way ANOVA with Hochberg correction for multiple testing was used. For not normally distributed data, Kruskal Wallis tests were applied with Bonferoni correction for multiple testing. Abbreviations: LDL, lower detection limit; N\%, Detection frequency: data are given as percentage of samples above detection limits.

Table $2 \mathbf{b}$ : Chemokines and adhesion molecules in exhaled breath condensate of children with asthma and healthy controls.

\begin{tabular}{|c|c|c|c|c|c|c|c|}
\hline Marker & Marker & LDL & $\mathrm{N} \%$ & Asthma $(\mathrm{N}=31)$ & $\mathrm{N} \%$ & Controls $(\mathrm{N}=29)$ & P-value \\
\hline $\mathrm{CCL2}$ & MCP1 & 2.2 & 97 & $58.5(54.7-64.8)$ & 100 & $52.9(50.4-59.1)$ & 0.008 \\
\hline CCL3 & MIP1 $\alpha$ & 14.8 & 97 & $269.4 \pm 6.5$ & 97 & $241.7 \pm 6.4$ & 0.004 \\
\hline CCL5 & RANTES & 3.9 & 87 & $9.8 \pm 0.7$ & 45 & $6.8 \pm 0.7$ & 0.009 \\
\hline CCL11 & Eotaxin & 5.0 & 35 & $6.3 \pm 0.5$ & 10 & $5.4 \pm 0.4$ & 0.42 \\
\hline CXCL8 & IL8 & 2.1 & 100 & 70.1 (66.7-74.3) & 100 & 65.5 (62.9-67.5) & $<0.001$ \\
\hline \multirow[t]{4}{*}{ CXCL10 } & IP10 & 3038.1 & 94 & $19464.1 \pm 583.8$ & 97 & $17783.3 \pm 412.9$ & 0.02 \\
\hline & MIF & 158.7 & 94 & $993.7 \pm 44.2$ & 97 & $749.3 \pm 32.7$ & $<0.001$ \\
\hline & SICAM & 59.7 & 97 & $763.0 \pm 16.3$ & 97 & $683.5 \pm 14.3$ & 0.001 \\
\hline & sVCAM & 400.0 & 97 & $1998.1 \pm 63.1$ & 91 & $1753.9 \pm 65.6$ & 0.01 \\
\hline
\end{tabular}

Data with a normal distribution are expressed as mean $\pm \mathrm{SE}$, otherwise data are expressed as median (range). Unit: $\mathrm{pg} / \mathrm{mL}$. For normally distributed data, one way ANOVA with Hochberg correction for multiple testing was used. For not normally distributed data, Kruskal Wallis tests were applied with Bonferoni correction for multiple testing. Abbreviations: LDL, lower detection limit; N\%, Detection frequency: data are given as percentage of samples above detection limits.

\section{Intra-subject variability}

The short-term intra-subject variability of cytokines of the group as a whole ranged from $5.2 \%$ to $35.0 \%$ (Table 3 ). Inflammatory markers (IM) with a CV <15\% were: IL-1apha, IL-1 $\beta$, 
IL-5, IL-6, IL-8, IL-18, MCP1, sICAM, MIP1 $\alpha$ (CCL3), IP-10, and eotaxin (CCL11). The CVs of the IM were generally higher in the group of healthy subjects compared to children with asthma (Table 3).

\section{Differences among populations}

\section{Asthma versus controls}

The concentrations of the markers in both study populations are shown in Table $2 a-b$ and in Figures 1a-c and 2a-c. This study showed that both Th1 cytokines [IL2, IFN $\gamma$ ] and Th2 cytokines [IL4, IL5, IL13] were significantly elevated in asthmatic patients compared to controls. Th2/Th1 ratios (IL4, IL5 and IL13 divided by IL2, IFN $\gamma$ and TNF $\alpha$, respectively) were comparable between children with asthma and controls (data not shown). Levels of pro-inflammatory cytokines [IL1 $\alpha$, IL12p70, IL18] were similar in asthmatics compared to controls. However, IL6 was elevated in asthma. Finally, significantly higher levels of chemokines [MIP1 $\alpha$ (CCL3), MIF, RANTES (CCL5), IP10 (CXCL10), IL8 (CXCL8), MCP1] and soluble adhesion molecules [sICAM, SVCAM] were detected in asthmatics compared to controls. In addition, no significant correlations were found between EBC markers and asthma control (Spearman correlation, $\mathrm{p}>0.20$ ).

Table 3: Intra-subject variability of cytokines, chemokines, and soluble adhesion molecules in EBC of children with asthma $(n=18)$, controls $(n=21)$ and the total group $(n=39)$.

\begin{tabular}{|c|c|c|c|c|}
\hline Marker & $\begin{array}{l}\text { Total group } \\
(\mathrm{N}=39)\end{array}$ & $\begin{array}{l}\text { Asthma } \\
(\mathrm{N}=18)\end{array}$ & $\begin{array}{l}\text { Controls } \\
(\mathrm{N}=21)\end{array}$ & $p$-value \\
\hline IL1 $\alpha$ & $8.8 \pm 1.2$ & $7.9 \pm 1.7$ & $9.7 \pm 1.6$ & 0.47 \\
\hline IL1 $\beta$ & $15.0 \pm 2.5$ & $9.9 \pm 1.8$ & $30.5 \pm 4.4$ & 0.004 \\
\hline IL2 & $18.4 \pm 2.1$ & $9.6 \pm 1.8$ & $26.4 \pm 2.6$ & 0.0001 \\
\hline IL4 & $17.2 \pm 2.7$ & $11.2 \pm 3.5$ & $22.7 \pm 3.8$ & 0.032 \\
\hline IL5 & $11.6 \pm 1.8$ & $9.8 \pm 1.9$ & $13.2 \pm 3.0$ & 0.35 \\
\hline IL6 & $12.4 \pm 1.4$ & $9.1 \pm 1.2$ & $15.5 \pm 2.3$ & 0.020 \\
\hline IL-8 (CXCL8) & $5.2 \pm 0.6$ & $4.7 \pm 0.8$ & $5.6 \pm 0.9$ & 0.45 \\
\hline IL10 & $15.4 \pm 2.0$ & $14.8 \pm 2.6$ & $15.8 \pm 3.2$ & 0.80 \\
\hline IL12p70 & $29.3 \pm 4.4$ & $26.5 \pm 5.2$ & $31.8 \pm 7.1$ & 0.55 \\
\hline IL13 & $22.8 \pm 2.6$ & $9.4 \pm 1.8$ & $35.0 \pm 2.5$ & 0.0001 \\
\hline IL18 & $12.3 \pm 1.3$ & $13.0 \pm 1.9$ & $11.6 \pm 1.8$ & 0.60 \\
\hline $\mathrm{IFN} \gamma$ & $32.1 \pm 3.4$ & $14.6 \pm 2.5$ & $47.8 \pm 3.1$ & 0.0001 \\
\hline TNF $\alpha$ & $19.2 \pm 2.0$ & $13.2 \pm 2.5$ & $24.6 \pm 2.6$ & 0.003 \\
\hline MIF & $24.0 \pm 3.2$ & $14.8 \pm 3.4$ & $31.9 \pm 4.5$ & 0.005 \\
\hline MCP1 (CCL2) & $9.7 \pm 1.0$ & $8.9 \pm 1.1$ & $10.4 \pm 1.8$ & 0.47 \\
\hline sICAM & $9.6 \pm 1.1$ & $6.2 \pm 1.3$ & $12.9 \pm 1.4$ & 0.001 \\
\hline SVCAM & $17.0 \pm 1.9$ & $9.2 \pm 2.3$ & $24.3 \pm 1.9$ & 0.0001 \\
\hline MIP1 $\alpha(C C L 3)$ & $8.7 \pm 1.2$ & $5.8 \pm 1.4$ & $11.5 \pm 1.7$ & 0.012 \\
\hline RANTES (CCL5) & $35.0 \pm 6.9$ & $19.4 \pm 6.3$ & $48.9 \pm 9.9$ & 0.025 \\
\hline IP-10 (CXCL10) & $10.7 \pm 1.2$ & $6.8 \pm 1.1$ & $14.2 \pm 1.6$ & 0.001 \\
\hline Eotaxin (CCL11) & $14.3 \pm 11.8$ & $14.3 \pm 11.8$ & - & - \\
\hline
\end{tabular}

Data with a normal distribution are expressed as mean $\pm \mathrm{SE}$, otherwise data are expressed as median (range); Coefficients of variation (SD/mean value $\times 100 \%$ ) are shown. Differences in CVs between asthmatics and controls are tested with the unpaired Student t-test. For most cytokines and chemokines, the CV's in healthy controls were higher than in asthmatics. 


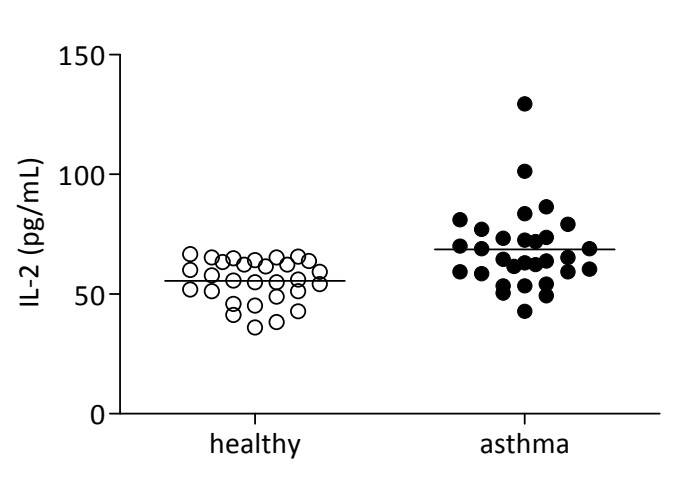

Figure 1a: Interleukin-2.

Figure 1b: Interleukin-4.

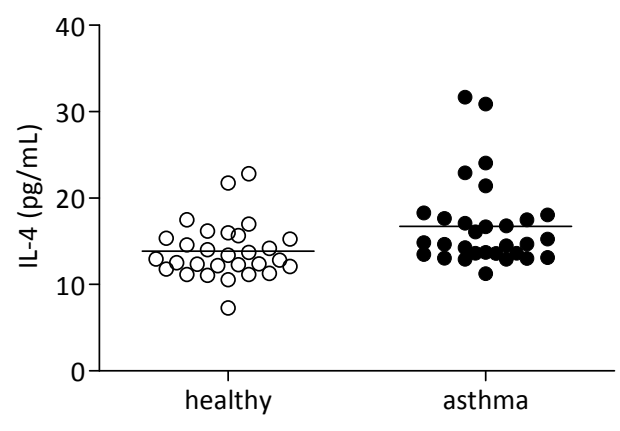

Figure 1c: Interferon-gamma.

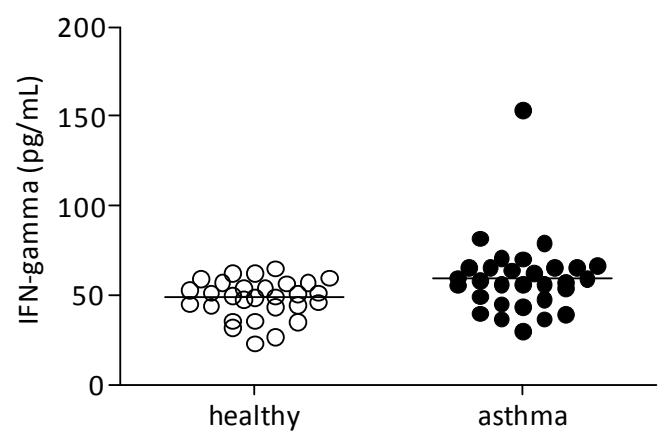

Cytokines in exhaled breath condensate of children with asthma and healthy controls. 


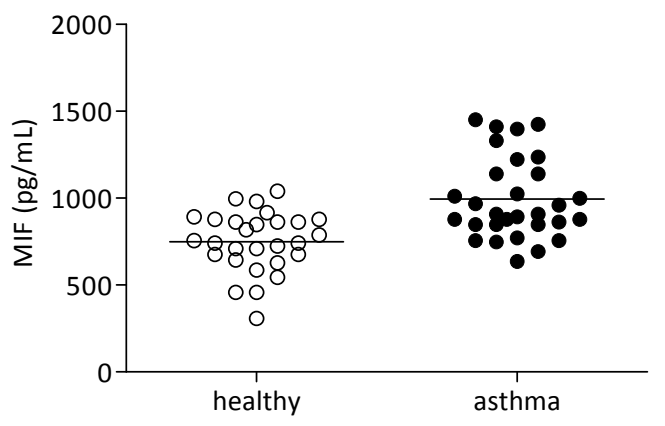

Figure 2a: Macrophage inhibiting factor.

Figure 2b: Macrophage inflamma-

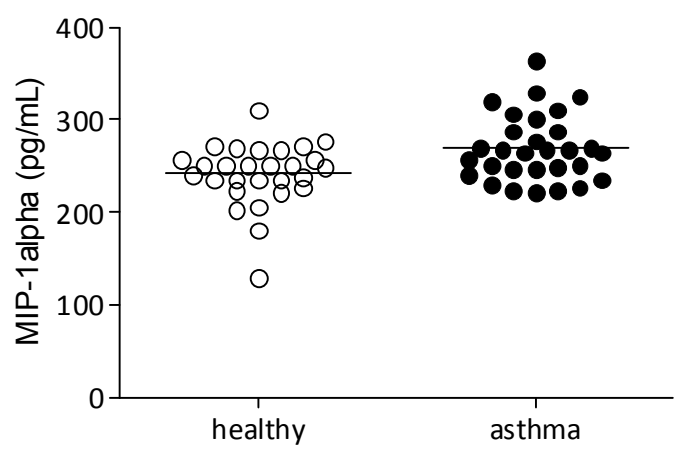
tory protein (MIP)-1alpha (CCL3).

Figure 2c: Soluble intercellular

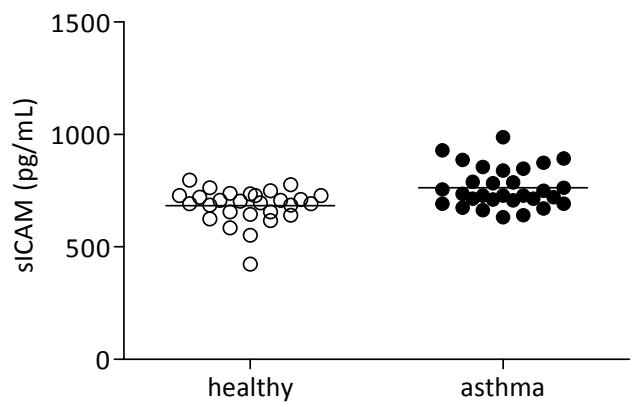
adhesion molecule (sICAM).

Chemokines and soluble adhesion molecules in exhaled breath condensate of children with asthma and healthy controls.

\section{Discussion}

The most important observation of this study is the high detection rates of cytokines $[\mathrm{IL} 1 \alpha,-1 \beta,-2,-4,-5,-6,-10,-12 \mathrm{p} 70,-13,-18, \mathrm{IFN} \gamma, \mathrm{TNF} \alpha]$, chemokines [MIP1 $\alpha$ (CCL3), MIF, eotaxin (CCL11), RANTES (CCL5), IP10 (CXCL10), IL8 (CXCL8), MCP1], and soluble adhesion 
molecules [sICAM, sVCAM] in EBC by using the combination of the optimised glass condenser and the multiplex immunoassay technology. The detection rates were close to $100 \%$ for all IM, except for eotaxin (CCL11) and RANTES (CCL5). In accordance with the pathophysiology of asthma, most inflammatory markers were increased in asthmatic children compared to controls. In addition, intra-subject variability was acceptable for most IM with CVs below $15 \%$.

The successful assessments of inflammatory markers in EBC in our study may be the consequence of two factors : 1 ) the use of a sensitive analysis technique (multiplex immunoassay), and 2) the collection of EBC with a glass condenser. In the literature, few research groups described successful detections of cytokines, chemokines and soluble adhesion molecules in EBC, mainly in asthmatic populations [7, 8, 13], and in COPD patients [10]. Inflammatory markers have been measured using ELISA techniques [7, 8, 13], or with a CBA multiplex bead immunoassay [10]. We succeeded in assessing Th1/Th2 cytokines (TNF $\alpha$, INF $\gamma, \mathrm{IL} 2,-4,-5,-10)$ in EBC of asthma and CF children and controls by using a flow cytometry technique, with detection percentages from 3-25\% [11]. The flow cytometry multiplex immunoassay technology used in the present study has shown to be more sensitive compared to the previously used CBA assay. We emphasize that the overlap between groups was considerable and statistical differences do not necessarily translate into clinical usefulness.

The most frequently used condenser system to collect EBC is the Ecoscreen (Viasys Hoechberg, Germany) [7, 10, 13], or the Rtube (Respiratory Research, Charlottesville, USA) [8]. This is important as we recently demonstrated that the coating of condensers interferes with the detection and concentration of protein mediators and markers of lipid peroxidation in EBC [14]. A glass coating resulted in more detections and higher concentrations of albumine and 8-isoprostane both in vitro and in vivo. In another study in 30 healthy adults we described significantly more detections of IL-2, IL-4, IL-5, IL-13, TNF- $\alpha$, hydrogen peroxide and 8-isoprostane in EBC collected with a glass condenser compared to other condensers [18]. The intra-subject variability of IM in EBC in the present study was acceptable ( CV <15\%) for most biomarkers. However, in healthy subjects, the CVs of most IM were higher than in asthmatic children, which probably has been caused by the lower mean values in healthy children. The CVs for TNF- $\alpha$, IL-6, IL-8, and IL-10 in EBC in healthy children of this study resembled those of a study in healthy adults [18].

\section{Asthma}

\section{Cytokines and chemokines}

Reported cytokines in EBC of asthmatic patients were IL2 [11], IL4 [8, 9, 11, 22], IL5 [23], IL6 [9, 24, 25], IL10 [11], IL13 [7], TNF $\alpha$ [8, 9, 11, 24, 25], and IFN $\gamma[11,22]$. Matsunaga et al. measured 40 cytokines and chemokines in EBC using a semi-quantitative protein array in adult asthmatic patients [16]. An upregulation of IL4, IL5, IL6, TNF $\alpha$, and IFN $\gamma$ has been reported in the above mentioned studies. This is in accordance with the results of our study in which we found increased levels of IL2, IL4, IL5, IL6, and IFN $\gamma$ in childhood asthma, and resembles the findings of others in broncho-alveolar lavage (BAL) fluid [26]. To our knowledge, this was the first study assessing and demonstrating IL18, MIP1 $\alpha$ (CCL3), MIF, RANTES (CCL5), IP10 (CXCL10), IL8 (CXCL8) and MCP1 in EBC samples of asthmatic chil- 
dren. Airway smooth muscle cells secrete a range of chemokines, including MCP, IL8 (CXCL8) and RANTES (CCL5), and are able to recruit and retain mast cells [27]. Increased levels of MCP, IL8 (CXCL8), RANTES (CCL5) and MIP1 $\alpha$ (CCL3) have been observed in BAL samples of patients with allergic asthma [27], and now also have been shown in increased amounts in EBC in our study. TARC, MDC and eotaxin (CCL11) has been measured in EBC of asthmatics children $[8,30]$. Leung et al reported a $100 \%$ detection of these markers in EBC samples of asthmatic patients [28]. In adults, Matsunaga et al. found upregulation of the chemokines IL8 (CXCL8), IP10 (CXCL10), MIP1 $\alpha$ (CCL3), and RANTES (CCL5) in asthmatic patients compared to controls [16]. These results are in concordance with the data in children of the present study.

\section{Soluble adhesion molecules}

Significantly higher levels of sICAM and SVCAM in EBC were found in children with asthma compared to controls. Bronchial eosinophils and $\mathrm{T}_{\mathrm{H}} 2$-cell dominance in asthma are essential in the development and maintenance of the disease $[6,29,30]$. Adherence of these cells to the vascular endothelium is mediated by the interaction with adhesion molecules. In allergic airway inflammation, ICAM-1 on epithelium and endothelial cells, have shown to be upregulated.

\section{Saliva contamination}

The two-way valve and the swan-neck tubing to the condenser served as a gravitational saliva trap. Moreover, children were instructed to immediately rinse their mouth in case of any saliva and the investigators performing the measurements checked this continuously. In an earlier study of one of the authors (dr. Q. Jöbsis), a comparable technique of EBC collection was used. He looked for presence of salivary amylase in EBC of healthy school-aged children but did not found this enzyme in any of the EBC samples [31]. Moreover, we compared the presence of free radicals in EBC with the existence of radicals in saliva of CF patients. In saliva we found no radicals whereas oxygen and carbon centered radicals were found in EBC of CF patients [32]. In the study of Dahlen et al, salivary contamination precautions were less than in our study: no two-way valve, no swann-neck tubing, and no mouth rinsing were applied [33]. With this way of collection, amylase was detected in part of the EBC samples and higher levels of LTB4 were found in these samples. So, saliva only was a source of LTB4 in these samples were amylase was present. On basis of these data and our preventive measures against saliva contamination, we are confident that saliva contamination did not play a role in our study and therefore was not a source of cytokine production in our children.

The results of this study showed that measurements of cytokines, chemokines and adhesion molecules in EBC of children was possible with an on average high success rate and low overall intra-subject variability. This was made possible by using a borosilicate glass condenser system in combination with the sensitive multiplex immunoassay technology. In asthma, cytokines, [IL2,-4,-5,-6,-13, IFN $\gamma$ ], chemokines [MIP1 $\alpha$ (CCL3), MIF, RANTES (CCL5), IP10 (CXCL10), IL8 (CXCL8), MCP1], and adhesion molecules [sICAM, sVCAM] in EBC were upregulated. Therefore, EBC is potentially a useful and reliable non-invasive technique in children with asthma. 


\section{References}

1 de Jager, W, Prakken, BJ, Bijlsma, JW, Kuis, W, Rijkers, GT. Improved multiplex immunoassay performance in human plasma and synovial fluid following removal of interfering heterophilic antibodies. J Immunol Methods 2005;300:124-35.

2 Levine, SJ. Bronchial epithelial cell-cytokine interactions in airway inflammation. J Investig Med 1995;43:2419.

3 Pang, L, Nie, M, Corbett, L, Sutcliffe, A, Knox, AJ. Mast cell beta-tryptase selectively cleaves eotaxin and RANTES and abrogates their eosinophil chemotactic activities. J Immunol 2006;176:3788-95.

4 Kips, JC, Tavernier, JH, Joos, GF, Peleman, RA, Pauwels, RA. The potential role of tumour necrosis factor alpha in asthma. Clin Exp Allergy 1993;23:247-50.

5 Tosi, MF, Stark, JM, Smith, CW, Hamedani, A, Gruenert, DC, Infeld, MD. Induction of ICAM-1 expression on human airway epithelial cells by inflammatory cytokines: effects on neutrophil-epithelial cell adhesion. Am J Respir Cell Mol Biol 1992; 7:214-21.

6 Wong, CK, Ho, CY, Ko, FW, Chan, CH, Ho, AS, Hui, DS, Lam, CW. Proinflammatory cytokines (IL-17, IL-6, IL-18 and IL-12) and Th cytokines (IFN-gamma, IL-4, IL-10 and IL-13) in patients with allergic asthma. Clin Exp Immunol 2001;125:177-83.

7 Tufvesson, E, Bjermer, L. Methodological improvements for measuring eicosanoids and cytokines in exhaled breath condensate. Respir Med 2006;100:34-8.

8 Leung, TF, Wong, GW, Ko, FW, Li, CY, Yung, E, Lam, CW, Fok, TF. Analysis of growth factors and inflammatory cytokines in exhaled breath condensate from asthmatic children. Int Arch Allergy Immunol 2005;137:66-72.

9 Carpagnano, GE, Foschino Barbaro, MP, Resta, O, Gramiccioni, E, Valerio, NV, Bracciale, P, Valerio, G. Exhaled markers in the monitoring of airways inflammation and its response to steroid's treatment in mild persistent asthma. Eur J Pharmacol 2005;519:175-81.

10 Gessner, C, Scheibe, R, Wotzel, M, Hammerschmidt, S, Kuhn, H, Engelmann, L, Hoheisel, G, Gillissen, A, Sack, $\mathrm{U}$, Wirtz, $\mathrm{H}$. Exhaled breath condensate cytokine patterns in chronic obstructive pulmonary disease. Respir Med 2005;99:1229-40.

11 Robroeks, CMHHT, Jöbsis, Q, Damoiseaux, JGMC, Heijmans, PHM, Rosias, PPR, Hendriks, JJE, Dompeling, E. Cytokines in exhaled breath condensate of children with asthma and cystic fibrosis. Ann Allergy Asthma Immunol 2006;96:349-55.

12 Liu, J, Thomas, PS. Exhaled breath condensate as a method of sampling airway nitric oxide and other markers of inflammation. Med Sci Monit 2005;11:MT53-62.

13 Ko, FW, Lau, CY, Leung, TF, Wong, GW, Lam, CW, Lai, CK, Hui, DS. Exhaled breath condensate levels of eotaxin and macrophage-derived chemokine in stable adult asthma patients. Clin Exp Allergy 2006;36:44-51.

14 Rosias, PP, Robroeks, CM, Niemarkt, HJ, Kester, AD, Vernooy, JH, Suykerbuyk, J, Teunissen, J, Heynens, J, Hendriks, HJ, Jobsis, Q, Dompeling, E. Breath condenser coatings affect measurement of biomarkers in exhaled breath condensate. Eur Respir J 2006;28:1036-41.

15 Robroeks, CM, van de Kant, KD, Jobsis, Q, Hendriks, HJ, van Gent, R, Wouters, EF, Damoiseaux, JG, Bast, A, Wodzig, WK, Dompeling, E. Exhaled nitric oxide and biomarkers in exhaled breath condensate indicate the presence, severity and control of childhood asthma. Clin Exp Allergy 2007;37:1303-11.

16 Matsunaga, K, Yanagisawa, S, Ichikawa, T, Ueshima, K, Akamatsu, K, Hirano, T, Nakanishi, M, Yamagata, T, Minakata, $Y$, Ichinose, M. Airway cytokine expression measured by means of protein array in exhaled breath condensate: correlation with physiologic properties in asthmatic patients. J Allergy Clin Immunol 2006;118:84-90.

17 de Jager, W, Rijkers, GT. Solid-phase and bead-based cytokine immunoassay: a comparison. Methods 2006;38:294-303.

18 Rosias, PP, Robroeks, CM, Kester, A, den Hartog, GJ, Wodzig, WK, Rijkers, GT, Zimmermann, LJ, van Schayck, $\mathrm{CP}$, Jobsis, $\mathrm{Q}$, Dompeling, E. Biomarker reproducibility in exhaled breath condensate collected with different condensers. Eur Respir J 2008;31:934-42.

19 Global Initiative for asthma (GINA). Pocket guide for asthma management and prevention in children. National Institute of Health, National Heart, Lung, and Blood Institute 2006. 
20 Asher, MI, Keil, U, Anderson, HR, Beasley, R, Crane, J, Martinez, F, Mitchell, EA, Pearce, N, Sibbald, B, Stewart, AW, et al. International Study of Asthma and Allergies in Childhood (ISAAC): rationale and methods. Eur Respir J 1995;8:483-91.

21 de Jager, W, te Velthuis, H, Prakken, BJ, Kuis, W, Rijkers, GT. Simultaneous detection of 15 human cytokines in a single sample of stimulated peripheral blood mononuclear cells. Clin Diagn Lab Immunol 2003;10:133-9.

22 Shahid, SK, Kharitonov, SA, Wilson, NM, Bush, A, Barnes, PJ. Increased interleukin-4 and decreased interferon-gamma in exhaled breath condensate of children with asthma. Am J Respir Crit Care Med 2002;165:1290-3.

23 Druilhe, A, Pretolani, M. [Eosinophilia and interleukin-5: role in pulmonary atopic processes]. Med Trop (Mars) 1998;58:437-43.

24 Carpagnano, GE, Foschino Barbaro, MP, Cagnazzo, M, Di Gioia, G, Giliberti, T, Di Matteo, C, Resta, O. Use of exhaled breath condensate in the study of airway inflammation after hypertonic saline solution challenge. Chest 2005;128:3159-66.

25 Rosias, PP, Dompeling, E, Dentener, MA, Pennings, HJ, Hendriks, HJ, Van lersel, MP, Jobsis, Q. Childhood asthma: Exhaled markers of airway inflammation, asthma control score, and lung function tests. Pediatr Pulmonol 2004;38:107-14.

26 Stankiewicz, W, Dabrowski, MP, Chcialowski, A, Plusa, T. Cellular and cytokine immunoregulation in patients with chronic obstructive pulmonary disease and bronchial asthma. Mediators Inflamm 2002;11:307-12.

27 Alam, R, York, J, Boyars, M, Stafford, S, Grant, JA, Lee, J, Forsythe, P, Sim, T, Ida, N. Increased MCP-1, RANTES, and MIP-1alpha in bronchoalveolar lavage fluid of allergic asthmatic patients. Am J Respir Crit Care Med 1996;153:1398-404.

28 Leung, TF, Wong, GW, Ko, FW, Lam, CW, Fok, TF. Increased macrophage-derived chemokine in exhaled breath condensate and plasma from children with asthma. Clin Exp Allergy 2004;34:786-91.

29 Bisset, LR, Schmid-Grendelmeier, P. Chemokines and their receptors in the pathogenesis of allergic asthma: progress and perspective. Curr Opin Pulm Med 2005;11:35-42.

30 Schmitz, N, Kurrer, M, Kopf, M. The IL-1 receptor 1 is critical for Th2 cell type airway immune responses in a mild but not in a more severe asthma model. Eur J Immunol 2003;33:991-1000.

31 Jöbsis Q, Raatgeep HC, Schellekens SL, Hop WC, Hermans PW, de Jongste JC. Hydrogen peroxide in exhaled air of healthy children: reference values. Eur Respir J 1998;12:483-5.

32 Rosias PP, Den Hartog GJ, Robroeks CM, Bast A, Donckerwolcke RA, Heynens JW, Suykerbuyk J, Hendriks HJ, Jöbsis $Q$, Dompeling E. Free radicals in exhaled breath condensate in cystic fibrosis and healthy subjects. Free radical research 2006;40:901-9.

33 Gaber F, Acevedo F, Delin I, Sundblad BM, Palmberg L, Larsson K, Kumlin M, Dahlén SE. Saliva is one likely source of leukotriene B4 in exhaled breath condensate. Eur Respir J 2006;28(6):1229-35.

34 Hammer SC, Robroeks CM, van Rij C, Heynens J, Droog R, Jöbsis Q, Hendriks HJ, Dompeling E. Actual asthma control in a paediatric outpatient clinic population: do patients perceive their actual level of control? Pediatr Allergy Immunol 2008;19(7):626-33. 


\section{CHAPTER 7.1}

\section{Exhaled nitric oxide and biomarkers in exhaled breath condensate indicate presence, severity and control of childhood asthma}

CMHHT Robroeks, KDG van de Kant, Q Jöbsis, JJE Hendriks, R van Gent, EFM Wouters, JGMC Damoiseaux, A Bast, WKWH Wodzig, E Dompeling Clinical and Experimental Allergy 2007;37(9):1303-1311 


\section{Abstract}

\section{Background}

Exhaled nitric oxide and inflammatory biomarkers in exhaled breath condensate may be useful to diagnose and monitor childhood asthma. Their ability to indicate an asthma diagnosis, and to assess asthma severity and control, is largely unknown.

The aim was to study 1 ) the ability of exhaled nitric oxide and inflammatory markers in exhaled breath condensate (nitrite, nitrate, hydrogen peroxide, 8-isoprostane, interferon$\gamma$, tumor necrosis factor- $\alpha$, interleukin- $2,-4,-5,-10$, and acidity) to discriminate between childhood asthma and controls. 2) the ability of these biomarkers to indicate asthma severity and control.

\section{Methods}

114 Children were included: 64 asthmatics (10.7 \pm 3.0 years, $67.2 \%$ atopic) and 50 controls $(10.0 \pm 0.4$ years). Condensate was collected using a glass condenser.

\section{Results}

Exhaled nitric oxide, interferon- $\gamma$ and interleukin-4 in exhaled breath condensate, differed significantly between asthma and controls. Multivariate backward logistic regression models demonstrated that interleukin-4 (odds ratio 7.9, 95\% confidence interval 1.2-51.0), was the only significant indicator of an asthma diagnosis. Asthma control was best assessed by exhaled nitric oxide, 8-isoprostane, interferon- $\nu$ and interleukin-4 (sensitivity $82 \%$, specificity $80 \%, p<0.05$ ), whereas, exhaled nitric oxide, 8 -isoprostane, nitrate and nitrite in condensate were best indicators of asthma severity (sensitivity $89 \%$, specificity $72 \%, p<0.05)$.

\section{Conclusion}

Different markers in condensate have additional value to exhaled nitric oxide, and are needed in non-invasive inflammometry. They could be useful to diagnose asthma, and, to indicate asthma control and severity in childhood. 


\section{Introduction}

Asthma is one of the most common occurring chronic diseases in children world-wide. Despite the availability of effective therapies, asthma control is suboptimal in many patients [1, 2]. International criteria for the diagnosis and management of asthma, are currently based on clinical features and lung function tests $[3,4]$. However, symptoms may not accurately reflect the nature and extent of the underlying airway inflammation [5], and lung function tests also show only marginal correlation with markers of airway inflammation [6-9]. A promising tool in asthma is the non-invasive measurement of airway inflammation by analysis of inflammatory markers (IM) in exhaled air, and exhaled breath condensate (EBC) [10-14].

Fractional exhaled nitric oxide (FeNO) is the best studied non-invasive IM. FeNO levels are elevated in children with asthma [15-17], and show a dose-dependent reduction by inhaled corticosteroids [18]. Therefore, FeNO may be useful for tapering ICS therapy [19, 20]. However, FeNO measurements have limitations in asthma; FeNO levels are not disease specific, and increased concentrations do not discriminate between children with atopic symptoms and asthmatics [21,22]. Besides, even small doses of ICS attenuate the increase of FeNO. Overall, the use of FeNO in clinical practice is still controversial [23]. Another non-invasive relatively new technique is assessment of IM in exhaled breath condensate (EBC). Various markers of airway inflammation and oxidative stress, like cytokines, eicosanoids, nitrogen oxides and hydrogen peroxide, are increased in EBC of patients with asthma $[11,14,24-26]$. However, the value of these IM for a diagnosis of asthma is unclear, and the relationships with asthma severity and asthma control are poorly studied. Most studies on EBC in asthmatic children concern relatively small patients groups with one or two IM. So far, no studies compared IM for their ability to indicate control or severity of asthma. Therefore, the aim of this study was to assess: 1 ) the ability of FeNO and IM in EBC (nitrite, nitrate, $\mathrm{H}_{2} \mathrm{O}_{2}$, 8-isoprostane, interferon- $\gamma$, tumor necrosis factor- $\alpha$, interleukin $-2,-4,-5,-10$, and condensate acidity), to discriminate between asthma and controls, and, 2) the ability of these markers to assess asthma severity and asthma control.

\section{Methods}

\section{Study design and subjects}

Asthmatic and control children, aged 5 to 16 years, were included in this cross-sectional study. Children with doctor-diagnosed asthma, known at the department of Paediatric Pulmonology, University Hospital Maastricht, were recruited. Asthma was defined as a chronic inflammatory disorder with intermittent symptoms of cough, dyspnoea, wheezing and chest pain. Severity and control of asthma were assessed according to Global Initiative for Asthma (GINA) guidelines [3]. Poor asthma control was considered if one or more of the following characteristic asthma symptoms were present in the previous week: $\geq 3$ times cough, $\geq 4$ times dyspnoea, $\geq 3$ times wheezing, $\geq 3$ times chest pain, $\geq 2$ times awakening caused by asthma symptoms, $\geq 2$ times use of rescue medication, and/or, $\geq 2$ times limitations in daily activities [3]. The use of anti-inflammatory medication was per- 
mitted (corticosteroids, leukotrien antagonists), during this study. A child was considered atopic when the total IgE level exceeded $20 \mathrm{kU} / \mathrm{L}$ and/or the Phadiatop was positive and/or the Radio Allergo Sorbent Test (RAST) was positive. The RAST test was considered positive when at least 2 allergens were classified as $\geq$ class 2 . Controls, without a history or presence of respiratory problems or allergy, were recruited from the outpatient clinic. The reasons of consultation were constipation and enuresis nocturna. Symptomatic atopy was excluded in the control population, by using the ISAAC questionnaire. However, IgE levels were not assessed in the control group. Age, sex, height, weight, duration of disease, therapy and co-morbidity of all subjects were documented. Exclusion criteria for both groups were: 1) Presence of a disease that might interfere with the results of this study (e.g. recent upper airway infection, heart disease, anatomic abnormalities of the airways and other chronic inflammatory diseases such as Crohns disease and rheumatoid arthritis), 2) Mental retardation, 3) Inability to perform the EBC procedure properly, 4) Active smoking, 5) The use of one of the following medication: Papaverin, Sodium nitroprusside, ACE inhibitors, Oxymetazoline, L-arginine, or NOS inhibitors [4]. Written informed consent was obtained from the parents and children. The medical ethics committee of the University Hospital Maastricht approved this study.

\section{Measurements}

Fractional exhaled nitric oxide (FeNO) levels were measured prior to exhaled breath condensate $(\mathrm{EBC})$ collection and assessment of lung function:

\section{1) NO in exhaled air}

FeNO was measured using a NIOX analyser (Aerocrine, Solna, Sweden) according to the criteria of the American Thoracic Society and European Respiratory Society (ATS/ERS) [27]. After complete exhalation and inhalation, children exhaled at a constant rate of $50 \mathrm{~mL} / \mathrm{sec}$ during 10 seconds. The flow rate was controlled by a dynamic flow restrictor in the apparatus. A balloon animation assisted the children to maintain flow rate during the total length of exhalation. Following this procedure, a NO plateau was reached during at least 3 seconds [9]. The average NO level during the NO-plateau for the last 3 seconds was considered to be FeNO. The mean of three FeNO levels, within a $10 \%$ range of each other, was used for analysis.

\section{2) Exhaled Breath Condensate}

Exhaled breath condensate (EBC) was collected by means of a cooled (1으) $50 \mathrm{~cm}$ double-jacketed condenser, as described previously $[14,24]$. Children, wearing a nose-clip, were breathing tidally through a mouthpiece connected to a two-way non-rebreathing valve. The two-way valve and tubing served as a trap to minimise the possibility of salivary contamination. After collection, acidity of EBC was immediately measured in nondeaerated samples (Radiometer, type PHM201, Zoetermeer, the Netherlands)[9], and, $\mathrm{EBC}$ was rapidly frozen at $-80^{\circ} \mathrm{C}$ using dry ice, and was stored at $-80 \circ \mathrm{C}$ until analysis. In this study, EBC samples were not deaerated, as in an earlier study the clinical usefulness of deaerated and not deaerated samples, was comparable [9].

Cytokines (IFN- - , TNF- $\alpha$, IL-2, IL-4, IL-5, IL-10) were assayed with flow cytometry (CBA, BD Bioscience, San Diego, USA), as described previously [24]. 8-Isoprostane concentrations 
were measured with a specific enzyme immunoassay (EIA) kit (Cayman Chemical Company, Ann Arbor, MI), which was modified in order to lower the detection limit from 5.0 $\mathrm{pg} / \mathrm{mL}$ to $0.5 \mathrm{pg} / \mathrm{mL}$ [13]. Standard curves, patient samples and quality control (QC) samples of $2.5 \mathrm{pg} / \mathrm{mL}$ and $10.0 \mathrm{pg} / \mathrm{mL}$ were measured in triplicate. The coefficients of variation (\% CV) of the absorption signal were assessed. Intra-assay variation of standard curves and QC samples should not exceed $15 \%$, otherwise all samples measured in that assay were excluded. Patient samples with a higher CV than $15 \%$ were excluded and were re-analysed once. In case of acceptance, mean absorption signal intensities were converted to 8isoprostane concentrations.

Nitrate and nitrite concentrations were assessed by means of fluorometry (Cayman Chemical Company, Ann Arbor, MI). The concentration of $\mathrm{H}_{2} \mathrm{O}_{2}$ in $\mathrm{EBC}$ was measured as described by Dekhuizen et al [28]. Briefly, $720 \mu \mathrm{L}$ of EBC was mixed with $220 \mu \mathrm{L} 0.4 \mathrm{M}$ citrate buffer, $50 \mu \mathrm{L}$ of HRP solution $(50 \mathrm{U} / \mathrm{ml})$ and $10 \mu \mathrm{L}$ tetramethylbenzidine (TMB, 20 $\mathrm{mM}$ ) and incubated for $20 \mathrm{~min}$ at room temperature. Then, the sample was mixed with 20 $\mu \mathrm{L}$ sulphuric acid (2 M). The TMB oxidation product as a measure of the amount of $\mathrm{H}_{2} \mathrm{O}_{2}$ was determined spectrometrically using a Perkin Elmer UV-VIS Spectrometer Lambda 10 (Norwalk, CT, USA) at $450 \mathrm{~nm}$. Detection limits of all assays are shown in Table II.

\section{3) Lung function tests}

Subjects were asked to stop bronchodilator medication prior to lung function testing. Short-acting bronchodilators were stopped at least 8 hours, and long-acting bronchodilators were discontinued at least 36 hour before the test. Dynamic spirometry was performed by means of the Flowscreen (Erich Jaeger, Wuerzburg, Germany). Forced expiratory volume in $1 \mathrm{sec}\left(\mathrm{FEV}_{1}\right)$, vital capacity (VC) and $\mathrm{FEV}_{1} / \mathrm{VC}$ were measured. The highest $\mathrm{FEV}_{1}$ and $\mathrm{VC}$, of three technically correct manoeuvres, was used for analysis. Airway reversibility was defined as an increase of $\mathrm{FEV}_{1}$ of $\geq 9 \%$ of the predicted value 30 minutes after administration of $400 \mu \mathrm{g}$ salbutamol [29]. Obstruction of the airways was considered when $\mathrm{FEV}_{1}$ values were lower than $80 \%$ of predicted value.

\section{Statistic analysis}

All data were expressed as mean \pm standard error of the mean. When data distribution was normal, Students t-tests were used for statistical analysis. Otherwise, the Mann Whitney $U$ test was applied. A two tailed test was considered statistically significant with $p-$ values $<0.05$. Logistic regression models were used to assess the relationships between IM with an asthma diagnosis, asthma severity and asthma control. At first, the influence of separate IM was tested in univariate logistic regression analyses. Thereafter, the different IM were combined in multivariate logistic regression models with asthma diagnosis, asthma severity and asthma control as dependent binary variables. Differences between populations tested in the regression models, were introduced in the model as covariates, but were removed if $p$-values exceeded 0.05 , in order to keep the degrees of freedom as high as possible. The stepwise backward method was applied to reduce the number of independent variables and to assess which IM were most predictive: only IM with a pvalue $<0.05$ were kept in the final prediction models. FeNO was categorized in levels below and above 20/30ppb, abbreviated as $\mathrm{FeNO}_{20} / \mathrm{FeNO}_{30}$, respectively. A sample with a negative detection was not considered as a missing value, because it actually informed us 
that the measurement of an IM was below the lower detection limit. Therefore, the negative detection was given an arbitrary value between the lower detection limit and zero.

\section{Power analysis}

Two power calculations were performed: 1) In a pilot of $n=15$ patients expected differences and standard deviations, between IM in EBC of asthmatics compared to control children, of 8-isoprostane, nitrite, IFN- $\gamma$ and IL-4 were: 13.7 (21.5) pg/mL, 1.8 (0.9) $\mu \mathrm{mol}$, 2.8 (1.9) pg/mL and $3.3(1.1) \mathrm{pg} / \mathrm{mL}$, as recently reviewed [14]. Therefore, the total number of subjects necessary $(2 \times N)$ to detect this differences will not exceed 78 when alpha is 0.05 and beta is 0.20 . 2) In a logistic regression analysis, the standard error of the sensitivity and the specificity of an IM will be less than $5 \%$ given a population of 50 children with asthma and 50 controls, and assuming a sensitivity of $70 \%$. Based on these power calculations, we decided to include at least 50 children with asthma and 50 controls.

\section{Results}

\section{Population characteristics}

A total number of 114 children were included (Table 1): 64 asthmatic children $(10.7 \pm 3.0$ $\mathrm{yrs})$, and 50 control children $(10.0 \pm 0.4 \mathrm{yrs})$. Asthmatic patients of all severity categories were represented (range $23-30 \%$ ). In the asthmatic group, more boys were present compared to the control population. As expected, $\mathrm{FEV}_{1} / \mathrm{VC}$ values were significantly lower in children with asthma compared to controls $(p<0.05)$. Poor asthma control was present in $50 \%$ of the children, and $67 \%$ was atopic. The majority of the asthmatic children $(86 \%)$ used inhaled corticosteroids, the mean steroid dose was $576 \mu \mathrm{g} /$ day of budesonide or equivalent. Two children were treated with oral corticosteroids. After administration of $400 \mu \mathrm{g}$ Salbutamol, $\mathrm{FEV}_{1} \%$ of predicted increased with (mean $\pm \mathrm{SE}$ ) $6.3 \pm 0.8 \%$.

\section{The collection of $E B C$}

The EBC collection time and the collected volume were similar in both populations: $29 \pm$ 0.4 minutes in asthma, and $28 \pm 0.4$ minutes in controls. The mean volume of collected EBC in asthmatics of $3005 \pm 159 \mu \mathrm{L}$ was not larger than that of $3119 \pm 157 \mu \mathrm{L}$ in controls. No adverse effects were reported during EBC collection.

\section{Inflammatory markers in exhaled breath condensate}

Table 2 shows the IM detected in EBC of children with asthma and controls. Nitrite, nitrate, $\mathrm{H}_{2} \mathrm{O}_{2}$ and 8-isoprostane were measured in all EBC samples. IL-2 was only detected in one, and IL-5 in two patients. The number of positive detections of IL-4 was higher in children with asthma compared to controls ( $22 \%$ and $2 \%$, respectively, $\mathrm{p}<0.05)$. The positive detections of IL-10, TNF- $\alpha$ and IFN- $\gamma$, varied from $6 \%$ in controls to $31 \%$ in asthma. IFN- $\gamma$ levels were higher in asthmatic children compared to controls $(3.2 \pm 0.2 \mathrm{pg} / \mathrm{mL}$ vs $2.6 \pm 0.2$ $\mathrm{pg} / \mathrm{mL}$ respectively, $\mathrm{p}<0.05)$. Although the total number of positive IL-10 detections was low, the concentrations of IL-10 in EBC were significantly lower in the children with mod- 
erate to severe persistent asthma (number of positive detections $n=5$ ), compared to children with intermittent to mild persistent asthma $(n=4)(1.2 \pm 0.0 \mathrm{pg} / \mathrm{mL}$ vs. $1.6 \pm 0.1 \mathrm{pg} / \mathrm{mL}$ respectively, $p<0.05)$. Furthermore, in children with uncontrolled asthma, IL-10 concentrations were significant lower than IL-10 levels in patients with good asthma control (1.2 \pm $0.0 \mathrm{pg} / \mathrm{mL}$ vs. $1.5 \pm 0.1 \mathrm{pg} / \mathrm{mL}$ respectively, $\mathrm{p}<0.05)$.

Table 1: Clinical characteristics of the study population.

\begin{tabular}{lll}
\hline & Asthma $(\mathrm{n}=64)$ & Controls $(\mathrm{n}=50)$ \\
\hline Age $(\mathrm{yrs})$ & $10.7 \pm 0.4$ & $10.0 \pm 0.4$ \\
Height $(\mathrm{cm})$ & $144 \pm 2$ & $142 \pm 2$ \\
Weight $(\mathrm{kg})$ & $38 \pm 2$ & $36 \pm 2$ \\
Gender (male / female) & $47 / 17$ & $27 / 23$ \\
FEV ${ }_{1}(\%$ predicted) & $96.0 \pm 2.3$ & $101.8 \pm 1.5$ \\
FEV ${ }_{1} /$ VC (\%) & $84.1 \pm 1.3$ & $88.2 \pm 1.0$ \\
Reversibility $(\%) \S$ & 20 & NA \\
Poor asthma control $~$ & $32(50 \%)$ & NA \\
Atopy & $43(67 \%)$ & NA \\
Asthma severity: & & NA \\
- intermittent & $15(23 \%)$ & NA \\
- mild persistent & $12(19 \%)$ & NA \\
- moderate persistent & $17(27 \%)$ & NA \\
- severe persisitent & $19(30 \%)$ & NA \\
ICS use & $55(86 \%)$ & NA \\
ICS daily dosage ( $\mu g)$ & $576 \pm 49$ & NA \\
Antihistamine use & $9(14 \%)$ & + \\
\hline
\end{tabular}

Definition of abbreviations: $\mathrm{FEV}_{1}$, forced expiratory volume in 1 second; VC, vital capacity; NA, not applicable. Data are given as mean \pm SEM except were indicated otherwise; ${ }^{\dagger} p<0.05$, asthma vs. controls; $\S$ Reversibility is defined as the increase in $\mathrm{FEV}_{1} \%$ predicted of $9 \%$ or more after use of a bronchodilator. Percentage of children with reversibility are shown. Severity and control of asthma was classified according to Global Initiative for Asthma (GINA).

\section{Indication of an asthma diagnosis}

When IM were considered separately, FeNO (continuous or categorized), IFN- $\gamma$ and IL-4 were significant indicators of an asthma diagnosis with odds ratios ranging from 1.03 for FeNO to 5.21 for IL-4 in EBC (Table 3). However, in the multivariate backward logistic regression model, only IL-4 was a significant indicator of an asthma diagnosis, with an odds ratio of 7.9 (95\% confidence interval 1.2-51.0) in the multivariate model (Figure 1a). Gender and $\mathrm{FEV}_{1} / \mathrm{VC} \%$ of predicted, were included as covariates. This did not influence the results significantly. Figure 2 shows that $90 \%$ of the children with IL-4 concentrations of $1.4 \mathrm{pg} / \mathrm{mL}$ or higher, were asthmatics. 
Table 2: Concentrations and number of positive detections of non-invasive inflammatory markers in EBC of asthmatics and controls.

\begin{tabular}{|c|c|c|c|c|c|c|c|}
\hline \multirow[b]{2}{*}{ IM } & \multirow[b]{2}{*}{ Unit } & \multirow[b]{2}{*}{ LLD } & \multicolumn{2}{|c|}{ Asthma $(n=64)$} & & \multicolumn{2}{|c|}{ Controls $(n=50)$} \\
\hline & & & $\mathrm{N} \%$ & $\begin{array}{l}\text { mean } \pm \mathrm{SE} \\
\text { median(range) }\end{array}$ & & N\% & $\begin{array}{l}\text { mean } \pm \mathrm{SE} \\
\text { median(range) }\end{array}$ \\
\hline FeNO & $\mathrm{ppb}$ & & 98 & $14.1(8.2-27.3)$ & $*$ & 100 & $15.4 \pm 1.37$ \\
\hline $\mathrm{pH}$ & & & 100 & $6.3 \pm 0.032$ & & 100 & $6.3 \pm 0.033$ \\
\hline Nitrite & $\mu \mathrm{M}$ & 0.05 & 100 & $1.2 \pm 0.10$ & & 100 & $1.4 \pm 0.14$ \\
\hline Nitrate & $\mu \mathrm{M}$ & 0.05 & 100 & $15.5 \pm 1.08$ & & 100 & $16.5 \pm 1.38$ \\
\hline $\mathrm{H}_{2} \mathrm{O}_{2}$ & $\mu \mathrm{M}$ & 0.1 & 100 & $2.0 \pm 0.22$ & & 100 & $2.2 \pm 0.31$ \\
\hline 8-isoprostane & $\mathrm{pg} / \mathrm{mL}$ & 0.5 & 100 & $4.2(1.7-8.7)$ & & 100 & $4.7 \pm 0.64$ \\
\hline IFN- $\nu$ & $\mathrm{pg} / \mathrm{mL}$ & 1.5 & 31 & $3.2 \pm 0.23$ & $*$ & 20 & $2.6 \pm 0.14$ \\
\hline TNF- $\alpha$ & $\mathrm{pg} / \mathrm{mL}$ & 1.2 & 27 & $1.5 \pm 0.048$ & & 15 & $1.6 \pm 0.094$ \\
\hline IL-10 & $\mathrm{pg} / \mathrm{mL}$ & 1.1 & 14 & $1.4 \pm 0.087$ & & 6 & $1.4 \pm 0.22$ \\
\hline IL-5 & $\mathrm{pg} / \mathrm{mL}$ & 1.1 & 3 & 1.1 & & 0 & \\
\hline IL-4 & $\mathrm{pg} / \mathrm{mL}$ & 1.4 & 22 & $2.4 \pm 0.19$ & $\begin{array}{l}* \\
*\end{array}$ & 2 & 2.0 \\
\hline IL-2 & $\mathrm{pg} / \mathrm{mL}$ & 1.2 & 2 & 1.2 & & 0 & \\
\hline
\end{tabular}

Definition of abbreviations: LLD, lower limit of detection; N\%, Detection frequency, data are given as percentage of samples above detection limit; SE, standard error; ${ }^{*} \mathrm{p}<0.05$ concentrations of inflammatory markers asthma versus controls; $* *$ p $<0.05$ detections of inflammatory markers asthma versus controls.

Table 3: Logistic regression models of separate inflammatory markers to indicate an asthma diagnosis.

\begin{tabular}{llll}
\hline & $p(-2 \mathrm{LL})$ & $\beta \pm \mathrm{SE}$ & $\mathrm{OR}(95 \% \mathrm{Cl})$ \\
\hline $\mathrm{FeNO}_{30}$ & 0.03 & $1.20 \pm 0.59$ & $3.32(1.05-10.5)$ \\
$\mathrm{FeNO}_{20}$ & 0.07 & $-0.82 \pm 0.46$ & $2.26(0.92-5.55)$ \\
$\mathrm{FeNO}$ & 0.04 & $0.03 \pm 0.02$ & $1.03(1.00-1.06)$ \\
$\mathrm{pH}$ & 0.60 & $-0.18 \pm 0.88$ & $0.83(0.15-4.69)$ \\
8 -Isoprostane & 0.35 & $0.03 \pm 0.04$ & $1.04(0.96-1.12)$ \\
$\mathrm{H}_{2} \mathrm{O}_{2}$ & 0.77 & $0.04 \pm 0.14$ & $1.04(0.75-1.37)$ \\
Nitrate & 0.86 & $0.004 \pm 0.02$ & $1.00(0.96-1.05)$ \\
Nitrite & 0.61 & $-0.12 \pm 0.24$ & $0.88(0.55-1.42)$ \\
$\mathrm{IFN}-\gamma$ & 0.10 & $0.31 \pm 0.20$ & $1.37(0.93-2.03)$ \\
$\mathrm{IL}-4$ & 0.001 & $1.65 \pm 0.77$ & $5.21(1.15-23.6)$ \\
\hline
\end{tabular}

$0=$ Control; $1=$ Asthma; $\mathrm{p}(-2 \mathrm{LL})$, significance of change in -2 log likelihood; S.E. standard error; ${ }^{*}$ All models were corrected for gender and $\mathrm{FEV}_{1} / \mathrm{VC} \%$ of predicted value; FeNO was categorized in levels below and above 20/30ppb, abbreviated as $\mathrm{FeNO}_{20} / \mathrm{FeNO}_{30}$, respectively. 

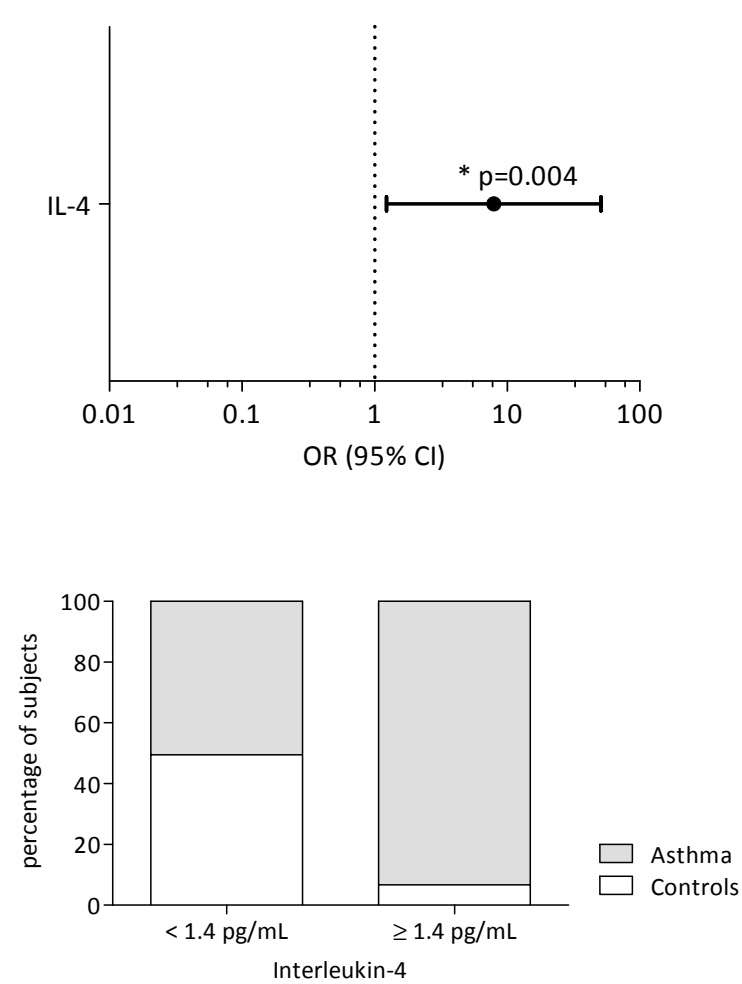

Figure 1a: Odds ratios of the significant inflammatory marker in a multivariate backward logistic model of an asthma diagnosis.
Figure 2: Percentage of asthmatic children with IL-4 concentrations in EBC below or above $1.4 \mathrm{pg} / \mathrm{mL}$.

This figure shows that $90 \%$ of the EBC samples with an IL-4 concentrations exceeding $1.4 \mathrm{pg} / \mathrm{mL}$, originated from asthmatic children.

\section{Indication of asthma control}

Analysis of IM separately, showed that FeNO (continuous or categorized) was a significant indicator of asthma control (Table 4). In the multivariate backward logistic regression model, besides FeNO, 8-isoprostane, IFN- $\gamma$ and IL-4 were significant indicators of asthma control (sensitivity $82 \%$, specificity $80 \%$, area under the curve (AUC) 0.761 ), Figure $1 \mathrm{~b}$. The corresponding receiver operating characteristic (ROC) curve is shown in Figure 3a. Children with poor asthma control used higher inhaled corticosteroid doses than children with good asthma control. Therefore, inhaled corticosteroid daily dose was included as a covariate in the regression models. This increased the influence of FeNO on asthma control. 
Table 4: Logistic regression models of separate inflammatory markers to indicate asthma control.

\begin{tabular}{llll}
\hline & $p(-2 \mathrm{LL})$ & $\beta \pm \mathrm{SE}$ & $\mathrm{OR}(95 \% \mathrm{Cl})$ \\
\hline $\mathrm{FeNO}_{30}$ & 0.03 & $1.58 \pm 0.73$ & $4.85(1.16-20.26)$ \\
$\mathrm{FeNO}_{20}$ & 0.06 & $1.15 \pm 0.62$ & $3.17(0.94-10.68)$ \\
$\mathrm{FeNO}$ & 0.05 & $0.03 \pm 0.01$ & $1.03(1.00-1.05)$ \\
$\mathrm{pH}$ & 0.78 & $-0.33 \pm 1.16$ & $0.72(0.07-7.01)$ \\
$8-$ Isoprostane & 0.13 & $0.06 \pm 0.04$ & $1.06(0.98-1.15)$ \\
$\mathrm{H}_{2} \mathrm{O}_{2}$ & 0.11 & $-0.42 \pm 0.27$ & $0.66(0.38-1.13)$ \\
Nitrate & 0.50 & $0.001 \pm 0.001$ & $0.76(0.35-1.67)$ \\
Nitrite & 0.73 & $-0.01 \pm 0.36$ & $0.99(0.92-1.06)$ \\
IFN- $\gamma$ & 0.18 & $-0.30 \pm 0.23$ & $0.74(0.47-1.16)$ \\
$\mathrm{IL}-4$ & 0.16 & $-0.49 \pm 0.37$ & $0.61(0.29-1.27)$ \\
\hline
\end{tabular}

0=Good asthma control; 1=Poor asthma control; Asthma control was based on GINA criteria; $p$ (-2 LL), significance of change in -2 log likelihood; S.E. standard error; All models were corrected for daily dose of ICS; FeNO was categorized in levels below and above 20/30ppb, abbreviated as $\mathrm{FeNO}_{20} / \mathrm{FeNO}_{30}$, respectively.
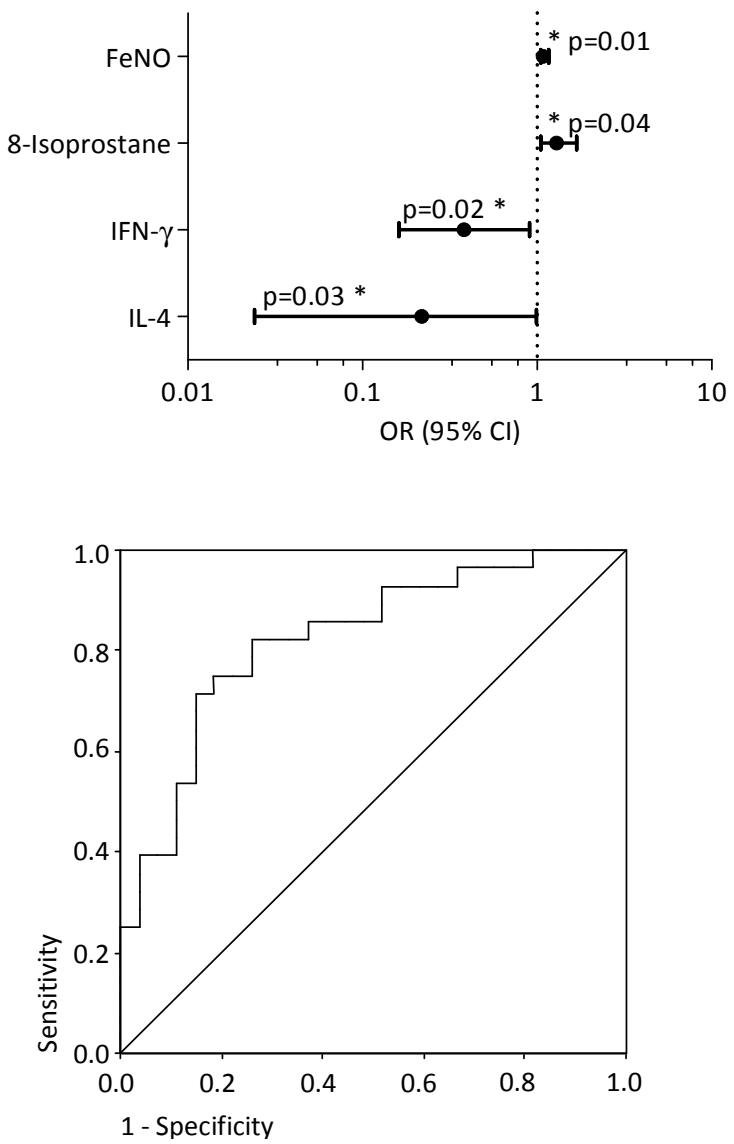

Figure 1b: Odds ratios of significant inflammatory markers in a multivariate logistic model to indicate asthma control.
Figure 3a: Receiver operator characteristic curve of the multivariate backward logistic regression model, of FeNO, 8-isoprotane, INF- $\gamma$ and IL4 , to indicate asthma control.

0=good control; 1=poor control; Asthma control was based on GINA criteria; Sensitivity 82\%; Specificity $80 \%$, AUC 0.761, $\mathrm{p}<0.001$. 


\section{Indication of asthma severity}

As expected, mean $\mathrm{FEV}_{1} \%$ of predicted value was higher in patients with intermittent to mild persistent asthma, compared to children with moderate to severe persistent asthma status. For this reason, $\mathrm{FEV}_{1} \%$ of predicted values was introduced as a covariate in the models, but had no influence on the indication of asthma severity by IM. Table 5 shows that FeNO and IFN- $\gamma$ were significant indicators of asthma severity, when IM were analyzed separately. In the multivariate backward logistic regression model, FeNO, 8isoprostane, nitrate and nitrate were all significant indicators of asthma severity, with a sensitivity of $89 \%$ and a specificity of $72 \%$ (Figure 1c). The corresponding ROC curve had an AUC of 0.830 (Figure $3 b$ ).

Table 5: Logistic regression models of separate inflammatory markers to indicate asthma severity.

\begin{tabular}{llll}
\hline & $\mathrm{p}(-2 \mathrm{LL})$ & $\beta \pm \mathrm{SE}$ & $\mathrm{OR}(95 \% \mathrm{Cl})$ \\
\hline FeNO30 & 0.02 & $1.71 \pm 0.79$ & $5.52(1.17-26.1)$ \\
FeNO20 & 0.07 & $1.08 \pm 0.62$ & $2.95(0.87-9.93)$ \\
FeNO & 0.005 & $0.06 \pm 0.03$ & $1.06(1.01-1.12)$ \\
$\mathrm{pH}$ & 0.91 & $0.14 \pm 1.26$ & $1.15(0.91-13.5)$ \\
8 -Isoprostane & 0.62 & $0.02 \pm 0.04$ & $1.02(0.94-1.11)$ \\
$\mathrm{H}_{2} \mathrm{O}_{2}$ & 0.92 & $0.02 \pm 0.22$ & $1.02(0.66-1.59)$ \\
Nitrate & 0.25 & $-0.07 \pm 0.02$ & $0.94(0.90-0.98)$ \\
Nitrite & 0.23 & $-0.46 \pm 0.40$ & $0.63(0.29-1.36)$ \\
IFN- $\gamma$ & 0.02 & $-0.55 \pm 0.24$ & $0.58(0.36-0.94)$ \\
IL-4 & 0.30 & $-0.36 \pm 0.35$ & $0.70(0.35-1.39)$ \\
\hline
\end{tabular}

$0=$ Intermittent and mild persistent asthma; 1=Moderate and severe persistent asthma; Asthma severity was based on GINA criteria; $\mathrm{p}(-2 \mathrm{LL})$, significance of change in -2 log likelihood; S.E. standard error; Corrected for $\mathrm{FEV}_{1} \%$ of predicted value; FeNO was categorized in levels below and above 20/30ppb, abbreviated as $\mathrm{FeNO}_{20} / \mathrm{FeNO}_{30}$, respectively.

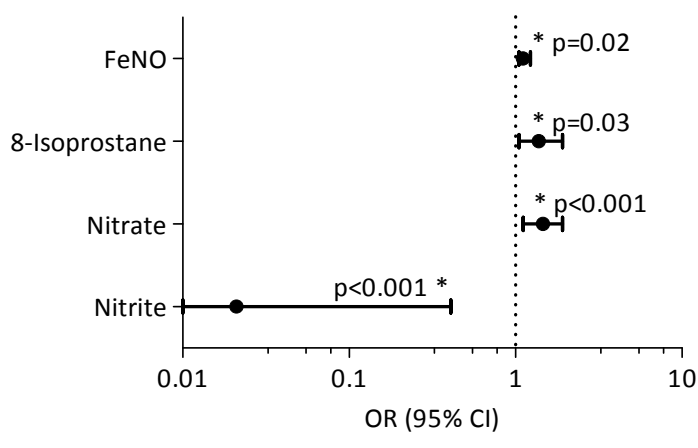

Figure 1c: Odds ratios of significant inflammatory markers in a multivariate logistic model to indicate asthma severity. 


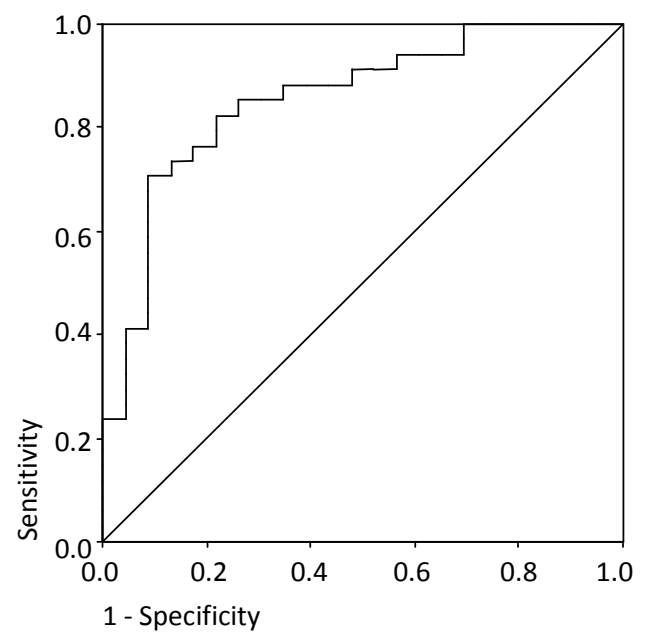

Figure 3b: Receiver operator characteristic curve corresponding to the multivariate backward logistic regression model, of FeNO, 8isoprostane, nitrate and nitrite, to indicate asthma severity.

$0=$ Intermittent and mild persistent asthma; 1=Moderate and severe persistent asthma; Asthma severity was based on GINA criteria; Corrected for $\mathrm{FEV}_{1} \%$ predicted; Sensitivity 89\%; Specificity 72\%, AUC 0.830, p<0.001.

\section{Discussion}

$\mathrm{IM}$ in EBC and FeNO are able to assess an asthma diagnosis, and can indicate asthma severity and control. In this study, FeNO, IFN- $\gamma$ and IL-4 could differentiate between children with asthma and controls. In a multivariate model with all measured IM considered together, IL-4 was the most important indicator of an asthma diagnosis in children above the age of five years. In a significant model of asthma control, FeNO, 8-isoprostane, IFN- $\gamma$ and IL-4 were the most contributing IM, whereas, FeNO, 8-isoprostane, nitrate and nitrite were the best indicators of asthma severity.

Various IM reflect different aspects of airway inflammation in asthma [8, 9, 30]. We compared the ability of several important IM to indicate an asthma diagnosis, and to assess asthma severity and control. This indicates that markers in EBC have additional value to FeNO in the prediction of an asthma diagnosis, severity and control. The asthma population in this study, varied substantially in asthma severity and asthma control, including intermittent as well as severe persistent disease, good controlled and poorly controlled cases. Because anti-inflammatory treatment is important in the management of more severe asthma, the use of corticosteroids and leukotrien antagonists was allowed in this study. By consequence, a large part of this population was treated with inhaled corticosteroids. $14 \%$ Of asthmatic children were treated with $\beta_{2}$-agonists only. Therefore, differences in IM in EBC between populations in this study, are findings between asthma children treated according to international guidelines, and controls. Correction for the use of ICS and for ICS dosage did not influence multivariate logistic models significantly.

The elevated IFN- $\gamma$ concentrations detected in EBC originating from asthmatic children, were in accordance to more abundant IFN- $\gamma$ producing $T$ cells detected in bronchoalveolar lavage (BAL) fluid in asthmatic children compared to controls [31]. Since IFN- $\gamma$ has pro- 
inflammatory effects to stimulate epithelial cells to release inflammatory cytokines, an increase of IFN- $\gamma$ in asthma could indicate inflammation [30]. By contrast, Shahid et al. reported decreased concentrations of IFN- $\gamma$ in EBC of 12 steroid-naïve and 14 steroidtreated children with asthma compared to controls [11]. They also found an elevation of IL-4 concentrations in EBC in children with steroid-naïve asthma. In addition, Leung et al. reported increased IL-4 concentrations in EBC in asthmatic children compared to controls, and IL-4 concentrations were higher in patients with persistent asthma receiving highdose ICS, than those on low-dose ICS [25].

Asthma is characterized by an increase of T helper type 2 (Th2) and a decrease of T helper type 1 (Th1) cells [30,32]. This imbalance may explain the more positive detections of IL-4, a Th2 cytokine, in samples originating from the asthmatic population compared to controls. Furthermore, IL-4 was the most contributing IM in the logistic model to indicate an asthma diagnosis. However, IL-4 was only detected in $22 \%$ of the patients, which limits the utility of this marker as a diagnostic tool until the detection method will become more sensitive. The disturbance of the Th1 and Th2 cell balance, could be caused by regulatory T-cells. IL-10 and IFN- $\gamma$ are important mediators in the development of inducible regulatory T-cells [33]. Suppression of inducible regulatory T-cells is mediated by cytokines, in particular IL-10. Only few data are available on IL-10, TNF- $\alpha$, IL-5 and IL-2 in EBC [9, 24, 34, 35]. Decreased IL-10 levels in more severe and poorly controlled children with asthma in this study, have not been demonstrated before. IL-10 is an important anti-inflammatory cytokine known to be down regulated in asthma. Matsumoto et al. and Takanashi et al. showed decreased levels and positive detections of IL-10 in sputum and serum of adults with asthma compared to control subjects [36, 37].

Higher FeNO levels in children with atopic asthma were demonstrated compared to controls. In contrast, we found no difference in FeNO levels in non-atopic children with asthma compared to controls, which is in accordance to findings of other research groups $[17,22,38]$. This strengthens the theory that FeNO is a better indicator of atopic asthma rather than of non-atopic asthma [17]. Besides, FeNO shows a negative correlation with the inhaled corticosteroid dosage, suggesting that FeNO can be used to titrate steroid treatment in asthma $[19,20]$. Nitrate and nitrite are stable end products of NO metabolism. Concentrations of these IM may reflect NO production, and therefore, may be clinically useful in the management of asthma [39]. Both FeNO and nitrite were important IM in the logistic model to indicate asthma severity. In addition, nitrite and nitrate contributed significantly to the multivariate models indicating asthma severity, with a low and high odds ratio, respectively. An explanation may be, that in more severe asthma, acidity in the airways increases which promotes conversion of nitrite in nitrate. Indeed, more severe asthma in this study was associated with lower nitrite and higher nitrate levels.

Hydrogen peroxide and 8-isoprostane are markers of oxidative stress. Hydrogen peroxide was not a significant indicator in any of the multivariate models, whereas 8-isoprostane was related to both asthma severity and control. This may stress the importance of free radical catalysed peroxidation of arachidonic acid in the pathofysiology of childhood asthma.

Methodological limitations of EBC collection and processing should be taken into account [14]. One of the main limitations is that detection limits of most assays available are often close to the concentrations of many markers in EBC. This may explain why IL-4 was detected more often but without a significant difference in concentration in the asthmatic 
group compared to control children. Moreover, this may also explain the difficulties in the detection of the other cytokines, like IL-2 and IL-5. On the other hand, EBC concentrations of a specific marker below the detection limit are useful as it informs us that its concentration is below the lower detection border. For example, in this study, although IL-4 had a concentration above the detection border in only $22 \%$ of asthma children, it had a high odds ratio of 7.9 for an asthma diagnosis. As the majority of asthmatic children in this study used inhaled corticosteroids, the results can not be easily generalised to children with mild intermittent disease. Collection and analysis of EBC is a non-invasive technique, which may play an important role in diagnosing asthma, especially in young children, and, in monitoring of asthma severity and control throughout childhood. The feasibility of EBC collection in preschool children is yet unclear. Longitudinal studies can further elucidate and confirm the role of different non-invasive markers in monitoring asthma.

In conclusion, non-invasive IM in exhaled breath (condensate) are able to indicate an asthma diagnosis, and to assess asthma control and severity. IL-4 and IFN- $p$ levels in EBC are elevated in a significant part of the children with asthma compared to control children. Although, FeNO levels were related to an asthma diagnosis, asthma severity, asthma control, presence of atopy and steroid dosage, FeNO was not the only indicator in the multivariate models: 1) an asthma diagnosis was indicated by IL-4 in EBC; 2 ) asthma control was best indicated by 8-isoprostane, IFN- - , IL-4 and FeNO; 3) 8-Isoprostane, nitrate, nitrite and FeNO were most significant predictors of asthma severity. The diagnosis and monitoring of childhood asthma may implicate the use of different non-invasive markers of airway inflammation, each representing different aspects of asthma pathophysiology. A longitudinal study will provide further evidence for the value of these different non-invasive IM in exhaled breath (condensate) in the management of asthma.

\section{References}

1. Rabe KF, Adachi M, Lai CK, Soriano JB, Vermeire PA, Weiss KB, Weiss ST. Worldwide severity and control of asthma in children and adults: the global asthma insights and reality surveys. J Allergy Clin Immunol 2004;114(1):40-7.

2. Wolfenden LL, Diette GB, Krishnan JA, Skinner EA, Steinwachs DM, Wu AW. Lower physician estimate of underlying asthma severity leads to undertreatment. Arch Intern Med 2003;163(2):231-6.

3. Global Initiative for asthma (GINA). Pocket guide for asthma management and prevention in children. National Institute of Health, National Heart, Lung, and Blood Institute 2002.

4. Kharitonov SA, Barnes PJ. Exhaled markers of pulmonary disease. Am J Respir Crit Care Med 2001;163(7):1693-722.

5. Kharitonov SA. Exhaled markers of inflammatory lung diseases: ready for routine monitoring? Swiss Med Wkly 2004;134(13-14):175-92.

6. Silvestri M, Sabatini F, Sale R, Defilippi AC, Fregonese L, Battistini E, Biraghi MG, Rossi GA. Correlations between exhaled nitric oxide levels, blood eosinophilia, and airway obstruction reversibility in childhood asthma are detectable only in atopic individuals. Pediatr Pulmonol 2003;35(5):358-63.

7. Sutherland ER, Martin RJ, Bowler RP, Zhang Y, Rex MD, Kraft M. Physiologic correlates of distal lung inflammation in asthma. J Allergy Clin Immunol 2004;113(6):1046-50.

8. Leung TF, Wong GW, Ko FW, Lam CW, Fok TF. Clinical and atopic parameters and airway inflammatory markers in childhood asthma: a factor analysis. Thorax 2005;60(10):822-6. 
9. Rosias PP, Dompeling E, Dentener MA, Pennings HJ, Hendriks HJ, Van lersel MP, Jobsis Q. Childhood asthma: Exhaled markers of airway inflammation, asthma control score, and lung function tests. Pediatr Pulmonol 2004;38(2):107-14.

10. Montuschi P, Barnes PJ. Analysis of exhaled breath condensate for monitoring airway inflammation. Trends Pharmacol Sci 2002;23(5):232-7.

11. Shahid SK, Kharitonov SA, Wilson NM, Bush A, Barnes PJ. Increased interleukin-4 and decreased interferongamma in exhaled breath condensate of children with asthma. Am J Respir Crit Care Med 2002;165(9):12903.

12. Carpagnano GE, Foschino Barbaro MP, Resta O, Gramiccioni E, Valerio NV, Bracciale P, Valerio G. Exhaled markers in the monitoring of airways inflammation and its response to steroid's treatment in mild persistent asthma. Eur J Pharmacol 2005.

13. Montuschi P, Corradi M, Ciabattoni G, Nightingale J, Kharitonov SA, Barnes PJ. Increased 8-isoprostane, a marker of oxidative stress, in exhaled condensate of asthma patients. Am J Respir Crit Care Med 1999;160(1):216-20.

14. Rosias PP, Dompeling E, Hendriks HJ, Heijnens JW, Donckerwolcke RA, Jobsis Q. Exhaled breath condensate in children: Pearls and pitfalls. Pediatr Allergy Immunol 2004;15(1):4-19.

15. Kharitonov SA, Barnes PJ. Clinical aspects of exhaled nitric oxide. Eur Respir J 2000;16(4):781-92.

16. Nelson BV, Sears S, Woods J, Ling CY, Hunt J, Clapper LM, Gaston B. Expired nitric oxide as a marker for childhood asthma. J Pediatr 1997;130(3):423-7.

17. Frank TL, Adisesh A, Pickering AC, Morrison JF, Wright T, Francis H, Fletcher A, Frank PI, Hannaford P. Relationship between exhaled nitric oxide and childhood asthma. Am J Respir Crit Care Med 1998;158(4):1032-6.

18. Kharitonov SA, Donnelly LE, Montuschi P, Corradi M, Collins JV, Barnes PJ. Dose-dependent onset and cessation of action of inhaled budesonide on exhaled nitric oxide and symptoms in mild asthma. Thorax 2002;57(10):889-96.

19. Pijnenburg MW, Bakker EM, Hop WC, De Jongste JC. Titrating steroids on exhaled nitric oxide in children with asthma: a randomized controlled trial. Am J Respir Crit Care Med 2005;172(7):831-6.

20. Smith AD, Cowan JO, Brassett KP, Herbison GP, Taylor DR. Use of exhaled nitric oxide measurements to guide treatment in chronic asthma. N Engl J Med 2005;352(21):2163-73.

21. Prasad A, Langford B, Stradling JR, Ho LP. Exhaled nitric oxide as a screening tool for asthma in school children. Respir Med 2006;100(1):167-73.

22. Gratziou C, Lignos M, Dassiou M, Roussos C. Influence of atopy on exhaled nitric oxide in patients with stable asthma and rhinitis. Eur Respir J 1999;14(4):897-901.

23. Godfrey S. Paediatr Respir Rev 2006;7(suppl1):S96-7.

24. Robroeks CMHHT, Jöbsis Q, Damoiseaux JGMC, Heijmans PHM, Rosias PPR, Hendriks JJE, Dompeling E. Cytokines in exhaled breath condensate of children with asthma and cystic fibrosis. Ann Allergy Asthma Immunol 2006;96(2):349-55.

25. Leung TF, Wong GW, Ko FW, Li CY, Yung E, Lam CW, Fok TF. Analysis of growth factors and inflammatory cytokines in exhaled breath condensate from asthmatic children. Int Arch Allergy Immunol 2005;137(1):6672.

26. Baraldi E, Ghiro L, Piovan V, Carraro S, Ciabattoni G, Barnes PJ, Montuschi P. Increased exhaled 8-isoprostane in childhood asthma. Chest 2003;124(1):25-31.

27. ATS/ERS Recommendations for Standardized Procedures for the Online and Offline Measurement of Exhaled Lower Respiratory Nitric Oxide and Nasal Nitric Oxide, 2005. Am J Respir Crit Care Med 2005;171(8):912-30.

28. Dekhuijzen PN, Aben KK, Dekker I, Aarts LP, Wielders PL, van Herwaarden CL, Bast A. Increased exhalation of hydrogen peroxide in patients with stable and unstable chronic obstructive pulmonary disease. Am J Respir Crit Care Med 1996;154(3 Pt 1):813-6.

29. Dundas I, Chan EY, Bridge PD, McKenzie SA. Diagnostic accuracy of bronchodilator responsiveness in wheezy children. Thorax 2005;60(1):13-6.

30. Chung KF, Barnes PJ. Cytokines in asthma. Thorax 1999;54(9):825-57.

31. Brown V, Warke TJ, Shields MD, Ennis M. T cell cytokine profiles in childhood asthma. Thorax 2003;58(4):311-6. 
32. Koning H, Neijens HJ, Baert MR, Oranje AP, Savelkoul HF. T cell subsets and cytokines in allergic and nonallergic children. I. Analysis of IL-4, IFN-gamma and IL-13 mRNA expression and protein production. Cytokine 1997;9(6):416-26.

33. Damoiseaux J. Regulatory T cells: back to the future. Neth J Med 2006;64(1):4-9.

34. Gessner C, Scheibe R, Wotzel M, Hammerschmidt S, Kuhn H, Engelmann L, Hoheisel G, Gillissen A, Sack U, Wirtz $\mathrm{H}$. Exhaled breath condensate cytokine patterns in chronic obstructive pulmonary disease. Respir Med 2005;99(10):1229-40.

35. Garey KW, Neuhauser MM, Robbins RA, Danziger LH, Rubinstein I. Markers of inflammation in exhaled breath condensate of young healthy smokers. Chest 2004;125(1):22-6.

36. Matsumoto K, Inoue H, Fukuyama S, Tsuda M, Ikegami T, Kibe A, Yoshiura Y, Komori M, Hamasaki N, Aizawa $\mathrm{H}$, Nakanishi Y. Decrease of interleukin-10-producing T cells in the peripheral blood of severe unstable atopic asthmatics. Int Arch Allergy Immunol 2004;134(4):295-302.

37. Takanashi S, Hasegawa Y, Kanehira Y, Yamamoto K, Fujimoto K, Satoh K, Okamura K. Interleukin-10 level in sputum is reduced in bronchial asthma, COPD and in smokers. Eur Respir J 1999;14(2):309-14.

38. Ludviksdottir D, Janson C, Hogman M, Hedenstrom H, Bjornsson E, Boman G. Exhaled nitric oxide and its relationship to airway responsiveness and atopy in asthma. BHR-Study Group. Respir Med 1999;93(8):552-6.

39. Ganas K, Loukides S, Papatheodorou G, Panagou P, Kalogeropoulos N. Total nitrite/nitrate in expired breath condensate of patients with asthma. Respir Med 2001;95(8):649-54. 


\section{CHAPTER 7.2}

\section{Biomarkers in exhaled breath condensate indicate presence and severity of cystic fibrosis in children}

CMHHT Robroeks, PPR Rosias, D van Vliet, Q Jöbsis, JBL Yntema, HJL Brackel, JGMC Damoiseaux, GJM den Hartog, WKWH Wodzig, E Dompeling Pediatric Allergy and Immunology 2008;19(7):652-659 


\section{Abstract}

\section{Background}

Chronic airway inflammation is present in cystic fibrosis. Non-invasive inflammometry may be useful in disease management. The aim of the present cross-sectional study was to investigate: 1 ) the ability of fractional exhaled nitric oxide and inflammatory markers (exhaled breath condensate acidity, nitrite, nitrate, hydrogen peroxide, 8-isoprostane, Th1/Th2 cytokines) to indicate (exacerbations of) cystic fibrosis. 2) The ability of these non-invasive IM to indicate CF disease severity.

\section{Methods}

In 98 children (48 cystic fibrosis / 50 controls), condensate was collected using a glass condenser. Exhaled nitric oxide was measured using the NIOX ${ }^{\circ}$ In all children, exhaled nitric oxide measurements were assessed. In condensate samples, levels of nitrate, nitrite, 8-isoprostane, hydrogen peroxide and acidity were analysed.

\section{Results}

Interferon- $\gamma$ and nitrite concentrations were significantly higher, whereas exhaled nitric oxide levels were significantly lower in cystic fibrosis compared to controls $(3.3 \pm 0.3 \mathrm{pg} / \mathrm{mL}$, $2.2 \pm 0.2 \mu \mathrm{M}, 10.0 \pm 1.2 \mathrm{ppb}$ versus $2.6 \pm 0.2 \mathrm{pg} / \mathrm{mL}, 1.4 \pm 0.1 \mu \mathrm{M}, 15.4 \pm 1.4 \mathrm{ppb}$, respectively). Using multivariate logistic regression models, a diagnosis of cystic fibrosis was best indicated by 8 -isoprostane, nitrite and interferon- $\gamma$ (sensitivity $78 \%$, specificity $83 \%$; area under receiver operating characteristic curve $0.906, p<0.001$ ). An exacerbation of cystic fibrosis was best indicated by 8 -isoprostane and nitrite (sensitivity $40 \%$, specificity $97 \%$, area under receiver operating characteristic curve $0.838, p=0.009$ ). Most indicative biomarkers of cystic fibrosis severity were exhaled nitric oxide, and condensate acidity (sensitivity $96 \%$, specificity 67\%; area under receiver operating characteristic curve 0.751 , $\mathrm{p}=0.008)$.

\section{Conclusions}

In this cross-sectional study, inflammatory markers in exhaled breath condensate could indicate (exacerbations of) cystic fibrosis, and severity of the disease in children. Longitudinal data are necessary to further confirm the role of these markers for the management of this chronic disease. 


\section{Introduction}

Cystic fibrosis (CF) is an autosomal recessive disorder, caused by genetic mutations in CF transmembrane conductance regulator (CFTR) protein. Defective CFTR causes impaired or absent transport of chloride through cell-membranes, with an impaired mucociliary clearance, and viscous mucus in the airways. Moreover, defective mucociliary action results in inability of the airways to clear bacteria, e.g. Pseudomonas aeruginosa [1, 2]. Chronic airway infection, inflammation and oxidative stress are the characteristics of CF disease, which are mainly due to neutrophilic immune responses $[2,3]$. Pulmonary disease is the leading cause of morbidity and mortality in CF patients [4].

Currently, monitoring of pulmonary disease in CF is based on clinical features and pulmonary function tests. However, these tests may not reflect the onset, origin or severity of airway inflammation [5]. Assessment of inflammatory markers (IM) in exhaled breath condensate (EBC) may be a promising tool in patients with chronic lung diseases [5-7]. In $\mathrm{CF}$, non-invasive inflammatory markers may be helpful in the early detection of CF lung disease in infants, the prediction and follow-up of an exacerbation, and the evaluation of treatment effects.

Several research groups detected an increase of IM in EBC of CF patients, compared to controls [8]. However, study populations were small, and data on non-invasive markers in CF are limited and inconsistent. In addition, the relationships between IM and the severity or exacerbations of CF disease, is still unknown. Jöbsis et al. showed high hydrogen peroxide $\left(\mathrm{H}_{2} \mathrm{O}_{2}\right)$ concentrations during $\mathrm{CF}$ exacerbations [9], but it is unclear if $\mathrm{H}_{2} \mathrm{O}_{2}$ is a good indicator of CF exacerbations. Therefore, we assessed several important IM in EBC in almost 100 children with CF and controls, comparing the ability of these markers to indicate (exacerbations of) CF disease, and CF severity. Because of oxidative stress and airway inflammation, $\mathrm{H}_{2} \mathrm{O}_{2}$, 8-isoprostane, nitrate/nitrite are upregulated in the airways of $\mathrm{CF}$ patients, and acidity is increased [10]. In contrast, fractional exhaled nitric oxide (FeNO) levels have shown to be lower in CF. Recently, presence of IL-10, IL-4, TNF- $\alpha$, IFN- $\gamma$ was demonstrated in EBC of CF patients [11].

The aim of the present cross-sectional study was to investigate: 1) The ability of FeNO and IM in EBC ( $\mathrm{pH}$, nitrate, nitrite, $\mathrm{H}_{2} \mathrm{O}_{2}$, Th1/Th2 cytokines), to indicate (exacerbations of) cystic fibrosis. 2) The ability of these non-invasive IM to indicate CF disease severity.

\section{Methods}

\section{Study subjects}

48 Children with CF, and 50 control children without a history of lung disease or atopy were included. Informed consent was obtained from all subjects. The study was approved by the Medical Ethics Committee of the University Hospital of Maastricht.

\section{CF population}

Children known with CF were recruited from the outpatient clinics. CF disease was defined as a combination of typical clinical features (e.g. persistent pulmonary problems, meconium ileus, failure to thrive, steatorrhoe) and an abnormal sweat test (Chloride $>60$ 
$\mathrm{mM}$ ). An exacerbation of CF was diagnosed by the paediatric pulmonologist based on a significant increase in CF related symptoms, and/or a decrease of at least $10 \%$ in $\mathrm{FEV}_{1}$ or FVC compared to previous measurements [12]. Height and weight were expressed as Zscores (age and gender specific standard deviation scores). CF severity was assessed using the Shwachman-Kulczycki score.

\section{Control population}

Control children without lung disease were recruited from the outpatient clinic of the University Hospital Maastricht. The reasons of consultation were constipation and enuresis nocturna. All children completed the 'International Study of Asthma and Allergies in Childhood (ISAAC) questionnaire to exclude children with a (history of) airway or allergy complaints or asthmatic disease [13].

\section{Exclusion criteria}

Exclusion criteria were: 1) Diseases that may interfere with the results of the study (e.g. upper airway infection, heart disease, anatomic abnormalities of the airways and other chronic inflammatory diseases, such as Crohns disease and rheumatoid arthritis), 2) Mental retardation, 3) Inability to perform the EBC collection procedure, 4) Active smoking and, 5) Use of the following medication: papaverin, sodium nitroprusside, angiotensinconverting enzyme (ACE) inhibitors, oxymetazoline, L-arginine, or nitric oxide synthase (NOS) inhibitors.

\section{Design and Measurements}

The design of this study was cross-sectional. At first, FeNO was measured. Subsequently, EBC was collected, and lung function was measured by means of dynamic spirometry. All tests were performed within one hour.

\section{NO in exhaled air}

FeNO was measured using a NIOX chemiluminescence analyser (Aerocrine, Solna, Sweden). Measurements were assessed according to the recommendations of the American Thoracic Society/European Respiratory Society for children (ATS/ERS) [7]. The mean NO value of the plateau reached during the last 3 seconds of exhalation, was used to obtain the FeNO value of that measurement [7]. The mean FeNO of three measurements was used for analysis.

In 14 CF patients, FeNO was measured using the offline method, because there was no NIOX $^{\oplus}$ in these clinics. The first 4 seconds of exhaled air were discarded and during the next 4 seconds, exhaled air was collected in a inert balloon. FeNO levels were analysed on the NIOX ${ }^{\circledR}$ chemiluminescence analyser within 8 hours [14]. The exhalation flow rate during these measurements was $50 \mathrm{~mL} / \mathrm{sec}$.

\section{Exhaled breath condensate}

EBC was collected using a home-made $50 \mathrm{~cm}$ double-wall borosilicate glass condenser, continuously cooled by circulating ice water $\left(1^{\circ} \mathrm{O}\right)$, as described previously $[6,11,15]$. Subjects were asked to breath tidally for 30 minutes through a mouthpiece, while wearing a nose-clip. The mouthpiece was connected to a two-way non-rebreathing valve (Hans 
Rudolph Inc, series 1420 , Kansas City,USA), which also served as a saliva trap. In addition, children were allowed to swallow saliva during EBC collection. The acidity of the EBC was measured immediately after collection (Radiometer, type PHM201, Radiometer Nederland BV, Zoetermeer, the Netherlands). Samples were not deaerated [15]. Subsequently, the $\mathrm{EBC}$ samples were frozen and stored at $-80^{\circ} \mathrm{C}$ in $1 \mathrm{~mL}$ Eppendorf tubes.

In EBC, nitrate and nitrite concentrations were assessed using a fluorimetric assay (Cayman Chemical, Ann Arbor, USA) [16]. To measure 8-isoprostane, an EIA-kit was used (Cayman Chemical, Ann Arbor, USA), which was modified in order to lower the detection limit from $5.0 \mathrm{pg} / \mathrm{mL}\left(80 \% \mathrm{~B} / \mathrm{B}_{0}\right)$ to $0.5 \mathrm{pg} / \mathrm{mL}$. Standard curves, patient samples and quality control (QC) samples of $2.5 \mathrm{pg} / \mathrm{mL}$ and $10 \mathrm{pg} / \mathrm{mL}$, were assayed in triplicate. The coefficients of variation (\%CV) of the absorption signal were assessed. Intra-assay variation of standard curves, patient samples and QC samples should be less than $15 \%$ otherwise all samples measured in that assay were excluded and re-analysed. Finally, in all accepted samples, 8-isoprostane concentrations were determined from mean absorption signal intensities.

$\mathrm{H}_{2} \mathrm{O}_{2}$ measurements were based on a method previously described by Dekhuijzen et al [17]. Briefly, EBC was mixed with $0.4 \mathrm{M}$ citrate buffer, HRP solution $(50 \mathrm{U} / \mathrm{mL}$ ) and tetramethylbenzidine (TMB, $20 \mathrm{mM}$ ) and incubated for $20 \mathrm{~min}$ at room temperature. Then, the sample was mixed with sulphuric acid (2 M). The TMB oxidation product as a measure of the amount of $\mathrm{H}_{2} \mathrm{O}_{2}$ was determined spectrometrically at $450 \mathrm{~nm}$, using a Perkin Elmer UV-VIS Spectrometer Lambda 10 (Norwalk, CT, USA).

Cytokines (TNF- $\alpha$, IFN- $\gamma$, IL-2, IL-4, IL-5, IL-10) were assessed with flow cytometry (CBA, BD Bioscience , San Diego, USA), as described previously [11].

\section{Lung function tests}

Bronchodilator medication was stopped prior to lung function testing: short-acting bronchodilators for at least 8 hours, and long-acting bronchodilators at least for 36 hours. Dynamic spirometry was performed by means of the Flowscreen (Jaeger , Wuerzburg, Germany). The lung function parameters were assessed according to ERS standards. The highest values of one of three correct performed manoeuvres were used for analysis. Recorded parameters were: forced expiratory volume in one second $\left(\mathrm{FEV}_{1}\right)$, and the forced vital capacity (FVC). In addition, in the CF population, residual volume (RV), total lung capacity (TLC) and intrathoracic gas volume (ITVG), were assessed by body plethysmography (Jaeger, Würzberg, Germany). The reversibility on a bronchodilator was determined 15 minutes after inhalation of $400 \mu \mathrm{g}$ salbutamol. Reversibility was defined as an increase in measured $\mathrm{FEV}_{1}$ of at least $9 \%$ of predicted value.

\section{Statistical analysis}

Statistical calculations were performed using SPSS 11.5 (SPSS Inc., Chicago, USA). Data were expressed as mean \pm standard error of the mean (SEM), or as median (interquartile range [IQR]) values for normal and not normal distributed continuous variables, respectively. Comparison between groups was performed using the chi-square for categorical variables, the Student's t-tests for normal distributed parameters, and, Mann Whitney Utests were used when the distribution of the parameters were considered not normal. Pvalues $<0.05$ were considered statistically significant. 
At first, the influence of separate inflammatory markers was tested in univariate logistic regression analyses. Thereafter, the different IM were combined in multivariate logistic regression models with CF disease, CF exacerbation and the CF severity as dependent binary variables. Age, height, weight, $\mathrm{FEV}_{1} \%$ of predicted value, and the $\mathrm{FEV}_{1} / \mathrm{VC} \%$ were introduced in the model as covariates, but were removed if $p$-values exceeded 0.10 , in order to keep the degrees of freedom as high as possible. The stepwise backward method was applied to reduce the number of independent variables and to assess which IM were most predictive: only IM with a p-value $<0.05$ were kept in the final models. Based on these models, receiver operating characteristic (ROC) curves were obtained. EBC samples with negative detections were not considered as missings, because they actually inform us that the measurements of IM were below the lower detection limit. Therefore, the negative detections were given an arbitrary value between the lower detection limit and zero.

\section{Power analysis}

The standard error of the sensitivity and the specificity of an inflammatory marker will be less than $5 \%$, given a population of 50 children with CF and 50 controls, and an assumed sensitivity of $70 \%$. This was considered sufficiently accurate for the purpose of this study.

\section{Results}

\section{Population characteristics}

Two of the 48 CF patients were older than 18 ( 21 and 25 years). Children with CF $(n=48)$ were older, smaller, weighed less and had more airway obstruction compared to controls $(n=50)$ (Table $1 ; p<0.05) .13 \%$ of the CF patients had an exacerbation. 24 Patients were treated with prophylactic antibiotics. All of these patients were received antibiotics orally (co-trimoxazol 17\%, azitromycin 27\%, augmentin 4\%, and, floxapen $2 \%$ ). In addition, 11 patients received inhaled antibiotics (8 (17\% tobramycin, and 6 (13\%) colistin). Therapeutic antibiotic treatment was given to the six patients experiencing an exacerbation (oral: azitromycin 2(4\%), ciprofloxacin 2(4\%), augmentin 1(2\%), and, intra-venous: tobramycin $3(6 \%)$, ceftazidime $1(2 \%)$, tazocin $1(2 \%)$.

\section{Collection of EBC and FeNO measurements}

The mean EBC volume collected was $3.1 \pm 0.2 \mathrm{~mL}$ and $3.0 \pm 0.2 \mathrm{~mL}$ in the CF population and control population, respectively. No side effects were reported during or following FeNO measurements and EBC collection.

\section{Detection of inflammatory markers}

Detections of IM in EBC are shown in Table 2. Nitrite, nitrate, $\mathrm{H}_{2} \mathrm{O}_{2}$ and 8-isoprostane were measured in all EBC samples. Cytokines were not detected in some EBC samples. IFN- $\gamma$ and IL-4 in EBC were detected significantly more often in samples of CF patients ( $42 \%$ and $13 \%$ respectively), compared to samples originating from controls ( $20 \%$ and $2 \%$ respectively; Chi-square test, $\mathrm{p}<0.05)$. 


\section{Concentration of inflammatory markers}

Mean concentrations of IFN- $\gamma$ and nitrite were significantly higher in the CF group (3.3 \pm $0.3 \mathrm{pg} / \mathrm{mL}$ and $2.2 \pm 0.2 \mu \mathrm{M}$ respectively) compared to controls $(2.6 \pm 0.2 \mathrm{pg} / \mathrm{mL}$ and $1.4 \pm$ $0.1 \mu \mathrm{M}$ respectively; Student's t-test, $\mathrm{p}<0.05$ ) (Table 2 ). In contrast, FeNO was significantly lower in children with CF $(10.0 \pm 1.2 \mathrm{ppb})$ compared to control children (15.4 $\pm 1.4 \mathrm{ppb}$; $\mathrm{p}<0.05)$. No influence was present of inhaled corticosteroids, use of antibiotics, or presence of atopy.

Table 1: Clinical characteristics of the study population *

\begin{tabular}{|c|c|c|c|}
\hline & Cystic fibrosis $\mathrm{N}=48$ & & Controls $\mathrm{N}=50$ \\
\hline Age (yrs) & $13.0 \pm 0.6$ & + & $10.0 \pm 0.4$ \\
\hline Height (cm) & $148 \pm 3$ & & $142 \pm 2$ \\
\hline Z-score height \# & $-1.0 \pm 0.2$ & + & $0.1 \pm 0.2$ \\
\hline Z-score weight || & $-1.0 \pm 0.1$ & $\dagger$ & $0.5 \pm 0.2$ \\
\hline Sex (male / female) & $27 / 21$ & & $27 / 23$ \\
\hline $\mathrm{FEV}_{1} \%$ predicted & $76 \pm 4$ & + & $102 \pm 2$ \\
\hline $\mathrm{FEV}_{1} / \mathrm{VC}(\%)$ & $77 \pm 2$ & + & $88 \pm 1$ \\
\hline TLC $\%$ predicted & $104 \pm 2$ & & NA \\
\hline RV \% predicted & $174 \pm 10$ & & NA \\
\hline ITVG \% predicted & $123 \pm 4$ & & NA \\
\hline Reversibility $\S$ & $23 \%$ & & NA \\
\hline CF exacerbation & $6(13 \%)$ & & NA \\
\hline Atopy ** & $17(36 \%)$ & & NA \\
\hline \multicolumn{4}{|l|}{ Positive sputum cultures: } \\
\hline - Pseudomonas Aeruginosa & $23(58 \%)$ & & NA \\
\hline - Staphylococcus Aureus & $29(60 \%)$ & & NA \\
\hline - Haemophilus Influenzae & $14(29 \%)$ & & NA \\
\hline - Haemophilus Parainfluenzae & $3(6 \%)$ & & NA \\
\hline - Aspergillus Fumigatus & $17(35 \%)$ & & NA \\
\hline - Candida Albicans & $6(13 \%)$ & & NA \\
\hline - Hemolytic Streptococcus group A or B & $8(17 \%)$ & & NA \\
\hline \multicolumn{4}{|l|}{ Therapy: } \\
\hline - DNase & $25(52 \%)$ & & NA \\
\hline - Antacids & $24(50 \%)$ & & NA \\
\hline - Corticosteroid & $13(27 \%)$ & & NA \\
\hline - Therapeutic antibiotics & $7(15 \%)$ & & NA \\
\hline - Prophylactic antibiotics & $24(50 \%)$ & & NA \\
\hline
\end{tabular}

Definition of abbreviations: $\mathrm{FEV}_{1}$, forced expiratory volume in 1 second; FVC, forced vital capacity; ITGV, intrathoracic gas volume; RV, residual volume; TLC, total lung capacity; NA, Not applicable; * Data are shown as mean \pm SEM, except indicated otherwise; \# Z-score height: Standard deviation of height corrected for age and sex according to the Dutch standard values; || Z-score weight: Standard deviation of weight corrected for

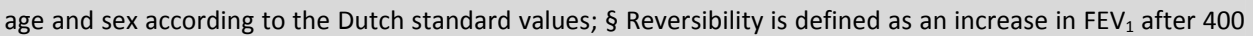
$\mu \mathrm{g}$ salbutamol of of $9 \%$ of predicted or more; + Significantly different from controls $(\mathrm{p}<0.05) .{ }^{* * A}$ child was considered atopic when the total IgE level exceeded $20 \mathrm{kU} / \mathrm{I}$ and / or the Phadiatop was positive and / or the Radio Allergo Sorbent Test (RAST) was positive. 
Table 2: Concentrations and number of positive detection of inflammatory markers in EBC.

\begin{tabular}{|c|c|c|c|c|c|c|c|}
\hline \multirow[b]{2}{*}{ IM } & \multirow[b]{2}{*}{ Unit } & \multirow[b]{2}{*}{$\begin{array}{l}\text { Detection } \\
\text { limit }\end{array}$} & \multicolumn{2}{|c|}{ Cystic fibrosis $(n=48)$} & & \multicolumn{2}{|c|}{ Control $(n=50)$} \\
\hline & & & $\mathrm{N} \%$ & Mean \pm SEM & & $\mathrm{N} \%$ & Mean \pm SEM \\
\hline FeNO & ppb & & 90 & $10.0 \pm 1.2$ & $\mp$ & 100 & $15.4 \pm 1.4$ \\
\hline $\mathrm{pH}$ & & & 100 & $6.3 \pm 0.1$ & & 100 & $6.3 \pm 0.0$ \\
\hline Nitrite & $\mu \mathrm{M}$ & 0.05 & 100 & $2.2 \pm 0.2$ & $\ddagger$ & 100 & $1.4 \pm 0.1$ \\
\hline Nitrate & $\mu \mathrm{M}$ & 0.05 & 100 & $14.4 \pm 2.0$ & & 100 & $16.5 \pm 1.4$ \\
\hline $\mathrm{H}_{2} \mathrm{O}_{2}$ & $\mu \mathrm{M}$ & 0.1 & 100 & $2.4 \pm 0.2$ & & 100 & $2.2 \pm 0.3$ \\
\hline 8-isoprostane & $\mathrm{pg} / \mathrm{mL}$ & 0.5 & 100 & $4.3 \pm 0.5$ & & 100 & $4.7 \pm 0.6$ \\
\hline IFN- $-\gamma$ & $\mathrm{pg} / \mathrm{mL}$ & 1.5 & 42 & $3.3 \pm 0.3$ & $\dagger \ddagger$ & 20 & $2.6 \pm 0.2$ \\
\hline TNF- $\alpha$ & $\mathrm{pg} / \mathrm{mL}$ & 1.2 & 15 & $1.6 \pm 0.1$ & & 15 & $1.6 \pm 0.1$ \\
\hline IL-2 & $\mathrm{pg} / \mathrm{mL}$ & 1.2 & 2 & 1.2 & & 0 & ND \\
\hline IL-4 & $\mathrm{pg} / \mathrm{mL}$ & 1.4 & 13 & $1.8 \pm 0.1$ & $\dagger$ & 2 & 2.0 \\
\hline IL-5 & $\mathrm{pg} / \mathrm{mL}$ & 1.1 & 0 & ND & & 0 & ND \\
\hline IL-10 & $\mathrm{pg} / \mathrm{mL}$ & 1.1 & 10 & $1.3 \pm 0.1$ & & 6 & $1.4 \pm 0.2$ \\
\hline
\end{tabular}

N\% Number of positive detection: data are given as percentage of samples above detection limit; ND, Not detectable; $+p<0.05$ positive detections of cystic fibrosis vs. controls; $\ddagger p<0.05$ concentrations of inflammatory markers in cystic fibrosis vs. controls.

\section{CF disease}

Multivariate logistic regression models showed that EBC 8-isoprostane, nitrite, and IFN- $\gamma$, and, $\mathrm{FEV}_{1} \%$ of predicted value, were all significant indicators for CF disease (Table 3 ). This model had a sensitivity of $78 \%$, a specificity of $92 \%$, and a area under the receiver operating characteristic (AUC) curve of 0.906 (Figure 1a).

Table 3: Multivariate logistic regression model to indicate CF disease.

\begin{tabular}{llll}
\hline & $p(-2 L L)$ & $\beta \pm S E$ & $O R(C l 95 \%)$ \\
\hline 8-Isoprostane & 0.02 & $-0.08 \pm 0.76$ & $0.92(0.88-0.98)$ \\
Nitrite & 0.03 & $0.75 \pm 0.37$ & $2.11(1.35-3.25)$ \\
IFN- $\gamma$ & 0.02 & $0.74 \pm 0.35$ & $2.10(1.17-2.54)$ \\
FEV ${ }_{1} \%$ predicted & $<0.01$ & $-0.07 \pm 0.02$ & $0.93(0.89-0.97)$ \\
\hline
\end{tabular}

$0=$ Controls; $1=$ Cystic fibrosis; Abbreviation: $\mathrm{p}(-2 \mathrm{LL})$, significance of change in -2 log likelihood; SE, standard error; $\mathrm{OR}$, odds ratio; $\mathrm{Cl}$, confidence interval. 


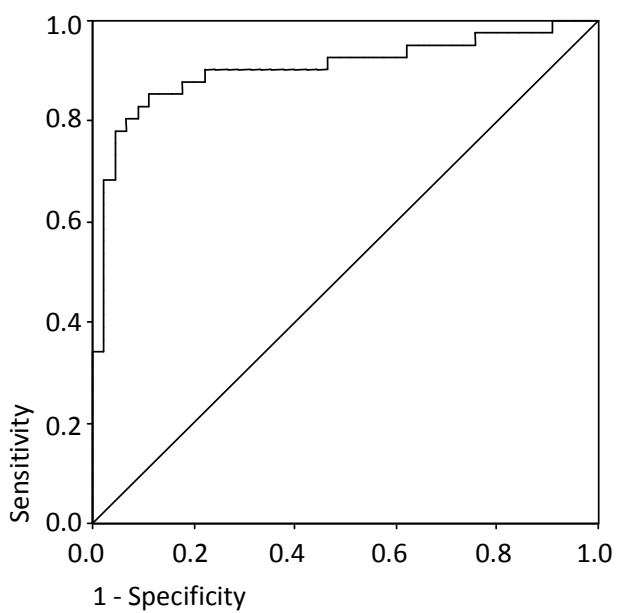

Figure 1a: Receiver operating characteristic curve of the multivariate backward logistic regression model of 8 -isoprostane, nitrite and IFN- $\gamma$, to indicate CF disease.

$0=$ Controls, $1=$ Cystic fibrosis; The area under the curve is 0.906 . The maximum sensitivity is $78 \%$ with a specificity of $83 \%$.

\section{CF exacerbation}

Six (13\%) of the CF population had an exacerbation, based on an increase in symptoms, and/or decrease in lung function parameters. In the sputum of these patients, the following micro-organisms were cultured: Pseudomonas Aeruginosa (100\%), Staphylococcus Aureus (67\%), Haemophilus Influenzae (33\%), Aspergillus Fumigatus (83\%), Candida Albicans (17\%), Hemolytic Streptococcus group A or B (17\%). In a multivariate analysis, there were 8-isoprostane and nitrite indicated a CF exacerbation (Table 4). However, sensitivity was only $40 \%$, with a corresponding specificity of $97 \%$ ( $A \cup C=0.838, p=0.009$ ), (Figure $1 b$ ). Correction for age, gender, presence of atopy, lung function parameters, or use of antibiotics, did not influence the results.

Table 4: Multivariate logistic regression model to indicate a CF exacerbation.

\begin{tabular}{llll}
\hline & $\mathrm{p}(-2 \mathrm{LL})$ & $\beta \pm \mathrm{SE}$ & $\mathrm{OR}(\mathrm{Cl} 95 \%)$ \\
\hline 8-Isoprostane & 0.03 & $-0.42 \pm 0.21$ & $0.70(0.44-0.99)$ \\
Nitrite & 0.01 & $-1.34 \pm 0.65$ & $0.26(0.07-0.93)$ \\
\hline
\end{tabular}

$0=$ no exacerbation; $1=$ =xacerbation; The definition of poor CF lung disease control was based on presence or severity of symptoms, decrease in lung function parameters; Abbreviations: $p(-2 L L)$, significance of change in -2 log likelihood; SE, standard error; OR, odds ratio; $\mathrm{Cl}$, confidence interval. 


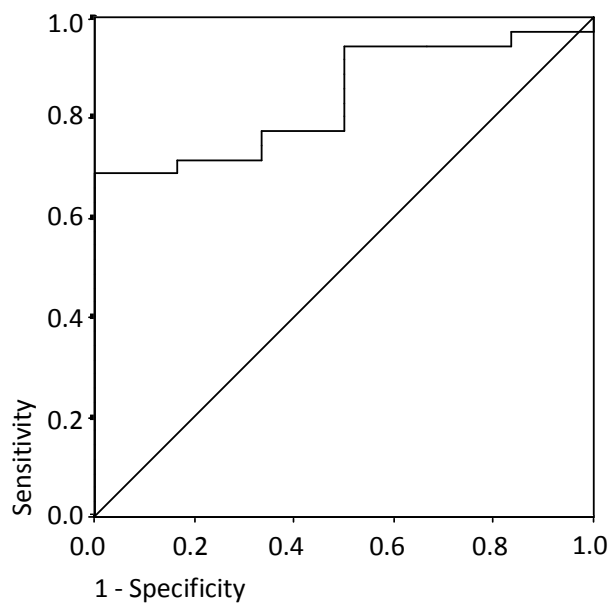

Figure 1b: Receiver operating characteristic curve of the multivariate backward logistic regression model of 8-isoprostane and nitrite, to indicate CF exacerbation.

$0=$ Not excellent CF status, $1=$ Excellent CF status; The area under the curve is 0.838 . The maximum sensitivity is $40 \%$ with a specificity of $97 \%$.

\section{CF severity}

In this part of the analysis, the CF severity classes were reduced to two groups, according to the Shwachman-Kulczycki score: group $1(n=15)$ consisted of patients with no excellent severity status, whereas group $2(n=33)$ consisted of children with an excellent status. In the multivariate logistic regression model, FeNO, EBC acidity and $\mathrm{FEV}_{1} / \mathrm{VC} \%$ were significant markers to indicate CF severity (Table 5 ) with a sensitivity of $96 \%$ and a specificity of $67 \%$ (AUC=0.751, p=0.008), (Figure 1c).

Table 5: Multivariate logistic regression model to indicate CF severity.

\begin{tabular}{llll}
\hline & $p(-2 L L)$ & $\beta \pm S E$ & OR (Cl 95\%) \\
\hline FeNO & 0.01 & $-0.19 \pm 0.09$ & $0.83(0.70-0.98)$ \\
pH & 0.01 & $-6.04 \pm 2.66$ & $0.02(0.00-0.44)$ \\
FEV1/VC \% & $<0.01$ & $0.19 \pm 0.08$ & $1.21(1.03-1.41)$ \\
\hline
\end{tabular}

$0=$ Excellent CF status; 1=Not excellent CF status; CF severity was classified using the Shwachman-Kulczycki score; Abbreviations: p(-2LL), significance of change in -2 log likelihood; SE, standard error; OR, odds ratio; $\mathrm{Cl}$, confidence interval. 


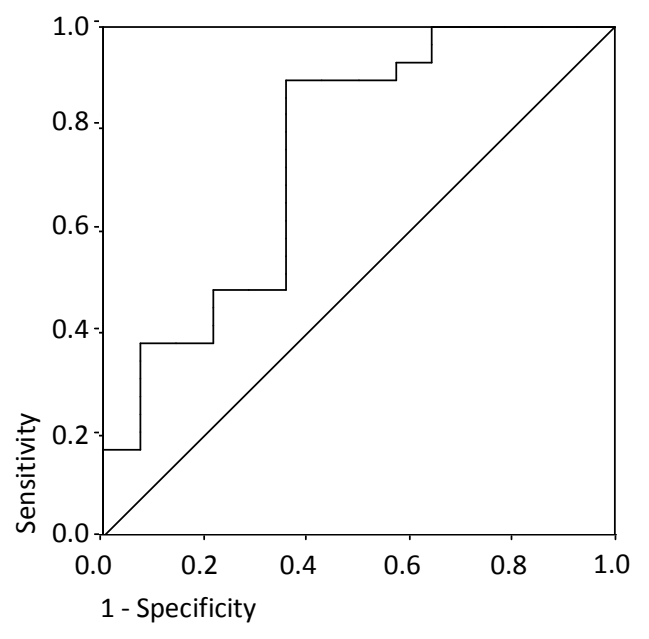

Figure 1c: Receiver operating characteristic curve of the multivariate backward logistic regression model of FeNO, and EBC acidity, to indicate CF severity.

$0=$ Not excellent CF status, 1=Excellent CF status; CF severity was classified using the Shwachman-Kulczycki score. The area under the curve is 0.751 . The maximum sensitivity is $96 \%$ with a specificity of $67 \%$.

\section{Discussion}

This study shows that different non-invasive inflammatory markers in exhaled air (condensate) are able to reflect different aspects of CF disease with considerably good sensitivity and specificity: 1) 8-isoprostane, nitrite and IFN- $\gamma$ concentrations, were able to indicate CF disease. 2) 8-isoprostane and nitrite indicated CF exacerbations, and, 3) CF severity was assessed by FeNO and EBC pH. Therefore, this study indicates that non-invasive inflammatory markers can be helpful in the management of the disease. Longitudinal studies have to confirm that these markers -for instance- are able to indicate the early onset of CF lung disease in young CF children, can detect exacerbations in an early stage, are helpful in the follow up of exacerbations, and can evaluate treatment effects.

The advantages of this study are: 1 ) a relatively large study population of about 100 children (48 CF); 2) the simultaneous assessment of several non-invasive inflammatory biomarkers which allows a comparison between them; 3 ) the possibility to assess relationships with CF severity and CF exacerbation, which has not been attempted before.

To our knowledge, this is the first study, which demonstrates that profiles of IM in EBC and FeNO, can indicate a CF diagnosis, and, are able to assess exacerbation and severity of CF lung disease. The limitation of this study is that no CF patients were actually categorised in the severe category. Therefore, results cannot be generalised to these types of patients.

FeNO levels in the CF population were reduced compared to the control population. This is in accordance to available data in literature [9, 18-26].

Furthermore, we found significantly higher concentrations of nitrite in EBC originating from children with $\mathrm{CF}$, compared to controls. These results are similar to the results of 
Formanek et al. and Cunningham et al., as well as Ho, Ojoo and Tate et al. in young adults with CF $[18,19,27-29]$. Lower FeNO levels and higher nitrite concentrations in the CF population can be explained by several hypotheses $[18,30]$. At first, the viscous mucus in CF airways may impair diffusion of gaseous NO into the airway lumen [19]. This can result in conversion of NO in nitrite, which is consistent with the higher levels of nitrite in CF than in controls. Secondly, decreased expression of inducible NO synthase (NOS) II in epithelial cells may result in low FeNO levels in children with CF [26, 31-34].

More positive detections of IFN- $\gamma$ and IL-4 were found in EBC samples originating from children with $\mathrm{CF}$, and, IFN- $\gamma$ concentrations were higher in this group compared to controls. This is in accordance to the study of Wojnarowski et al., which showed transcription of IFN- $\gamma$ and IL-4 in bronchial biopsies from children with CF, whereas normal controls did not express IFN- $\gamma$ and IL-4 [35]. Immunoregulatory effects of IFN- $\gamma$ on various cells, are extensive and diverse [36, 37]. IFN- $\gamma$ and IL-4 upregulate class II molecules on macrophages and dendritic cells. Thereby, the antigen presenting capacity of epithelial and endothelial cells, among others, is being induced [37]. Additionally, defensive mechanisms to antigens in CF are impaired. By consequence, additional mechanisms need to be addressed to resolve ongoing inflammatory processes and infections [38].

IL-5 was not detected in any sample of the populations, which resembles the findings of Armstrong et al. They could not detect IL-5 in BAL fluid [39]. Also cytokine concentrations were relatively low and close to the detection limits of the assays. Previously, we reported, that IFN- $\gamma$, IL-2, IL-4, IL-10 were detected in $8-25 \%$ of the samples originating from children with CF. TNF- $\alpha$ and IL-5 were not detected in this study as well [11].

In addition, the EBC pH and concentrations of 8-isoprostane and $\mathrm{H}_{2} \mathrm{O}_{2}$ did not differ between the $\mathrm{CF}$ and control populations. This result of $\mathrm{H}_{2} \mathrm{O}_{2}$ is in accordance to the findings of Jöbsis et al. and Ho et al [9, 40]. However, various research groups demonstrated lower EBC $\mathrm{pH}$ levels and higher 8-isoprostane concentrations in children with CF [41-43]. This may be explained by the mild to moderate CF severity in this study, as 8-isoprostane was a significant predictor of CF disease and CF exacerbation in the multivariate models, whereas EBC pH contributed significantly IM to the indication model of CF severity.

We acknowledge limitations in the use of non-invasive measurements of inflammatory biomarkers. At first, sensitive detection techniques are necessary for the measurements of inflammatory biomarkers in EBC, as the concentration of markers is often low. We were not able to detect all cytokines in all subjects. The lack to detect cytokines probably does not mean cytokines are not present. There is an overall agreement that cytokines are involved in the pathophysiology of CF. A sample with negative detection was not considered as a missing value, because it actually informed us that the measurement of an IM was below the lower detection limit. Secondly the value of IM to predict CF severity and CF exacerbation should be confirmed in a longitudinal study design.

This study showed that several non-invasive IM were able to indicate a (exacerbation of) $\mathrm{CF}$, and can assess CF severity with good sensitivity and specificity. These findings emphasise the importance of evaluating several IM reflecting different aspects of the disease, in stead of evaluating one individual IM which may not be able to reflect an evolving chronic inflammatory process appropriately. This study showed that FeNO, and EBC IFN- $\gamma$, nitrite, 8-isoprostane, and acidity were most important inflammatory markers to distinguish between CF and non-CF children, and to indicate the severity of exacerbations in CF children. Currently, management and treatment is mainly based on symptoms and deterioration of 
lung function parameters, which may not reflect ongoing inflammatory processes. In the future, IM in EBC may be a valuable additional tool to monitor inflammatory activity in patients with CF. This may result in earlier detection of a pulmonary CF exacerbation and earlier treatment, which may improve quality of live in children with CF.

\section{References}

1 Ratjen F, Doring G. Cystic fibrosis. Lancet 2003;361:681-689.

2 Kumar AA, N Fausto. In, editors. Pathologic basis of disease. Pennsylvania: 2005.

3 Brown RK, Wyatt H, Price JF, Kelly FJ. Pulmonary dysfunction in cystic fibrosis is associated with oxidative stress. Eur Respir J 1996;9:334-339.

4 FitzSimmons SC. The changing epidemiology of cystic fibrosis. Curr Probl Pediatr 1994;24:171-179.

5 Kharitonov SA, Barnes PJ. Exhaled markers of pulmonary disease. Am J Respir Crit Care Med 2001;163:16931722.

6 Rosias PP, Dompeling E, Hendriks HJ, Heijnens JW, Donckerwolcke RA, Jobsis Q. Exhaled breath condensate in children: Pearls and pitfalls. Pediatr Allergy Immunol 2004;15:4-19.

7 ATS/ERS Recommendations for Standardized Procedures for the Online and Offline Measurement of Exhaled Lower Respiratory Nitric Oxide and Nasal Nitric Oxide, 2005. Am J Respir Crit Care Med 2005;171:912-930.

8 Carpagnano GE, Barnes PJ, Geddes DM, Hodson ME, Kharitonov SA. Increased leukotriene B4 and interleukin-6 in exhaled breath condensate in cystic fibrosis. Am J Respir Crit Care Med 2003;167:1109-1112.

9 Jöbsis Q, Raatgeep HC, Schellekens SL, Kroesbergen A, Hop WC, de Jongste JC. Hydrogen peroxide and nitric oxide in exhaled air of children with cystic fibrosis during antibiotic treatment. Eur Respir J 2000;16:95-100.

10 Rosias PP, Den Hartog, G.J.M., Robroeks, C.M.H.H.T., Bast, A., Donckerwolcke, R.A.M.G., Heynens, J.W.C.M., Suykerbuyk, J., Hendriks, H.J.E., Jöbsis, Q., Dompeling, E. Free radicals in exhaled breath condensate in cystic fibrosis and healthy subjects. Free Radic Res 2006, in press

11 Robroeks CMHHT, Jöbsis Q, Damoiseaux JGMC, et al. Cytokines in exhaled breath condensate of children with asthma and cystic fibrosis. Ann Allergy Asthma Immunol 2006;96:349-355.

12 Rosenfeld M, Emerson J, Williams-Warren J, et al. Defining a pulmonary exacerbation in cystic fibrosis. J Pediatr 2001;139:359-365.

13 Montuschi P. Indirect monitoring of lung inflammation. Nat Rev Drug Discov 2002;1:238-242.

14 Pijnenburg MW, Lissenberg ET, Hofhuis W, et al. Exhaled nitric oxide measurements with dynamic flow restriction in children aged 4-8 yrs. Eur Respir J 2002;20:919-924.

15 Rosias PP, Dompeling E, Dentener MA, et al. Childhood asthma: Exhaled markers of airway inflammation, asthma control score, and lung function tests. Pediatr Pulmonol 2004;38:107-114.

16 Misko TP, Schilling RJ, Salvemini D, Moore WM, Currie MG. A fluorometric assay for the measurement of nitrite in biological samples. Anal Biochem 1993;214:11-16.

17 Dekhuijzen PN, Aben KK, Dekker I, et al. Increased exhalation of hydrogen peroxide in patients with stable and unstable chronic obstructive pulmonary disease. Am J Respir Crit Care Med 1996;154:813-816.

18 Ojoo JC, Mulrennan SA, Kastelik JA, Morice AH, Redington AE. Exhaled breath condensate pH and exhaled nitric oxide in allergic asthma and in cystic fibrosis. Thorax 2005;60:22-26.

19 Ho LP, Innes JA, Greening AP. Nitrite levels in breath condensate of patients with cystic fibrosis is elevated in contrast to exhaled nitric oxide. Thorax 1998;53:680-684.

20 Ho LP, Innes JA, Greening AP. Exhaled nitric oxide is not elevated in the inflammatory airways diseases of cystic fibrosis and bronchiectasis. Eur Respir J 1998;12:1290-1294.

21 Dotsch J, Demirakca S, Terbrack HG, Huls G, Rascher W, Kuhl PG. Airway nitric oxide in asthmatic children and patients with cystic fibrosis. Eur Respir J 1996;9:2537-2540.

22 Grasemann H, Michler E, Wallot M, Ratjen F. Decreased concentration of exhaled nitric oxide (NO) in patients with cystic fibrosis. Pediatr Pulmonol 1997;24:173-177.

23 Lundberg JO, Nordvall SL, Weitzberg E, Kollberg H, Alving K. Exhaled nitric oxide in paediatric asthma and cystic fibrosis. Arch Dis Child 1996;75:323-326. 
24 Thomas SR, Kharitonov SA, Scott SF, Hodson ME, Barnes PJ. Nasal and exhaled nitric oxide is reduced in adult patients with cystic fibrosis and does not correlate with cystic fibrosis genotype. Chest 2000;117:1085-1089.

25 Elphick HE, Demoncheaux EA, Ritson S, Higenbottam TW, Everard ML. Exhaled nitric oxide is reduced in infants with cystic fibrosis. Thorax 2001;56:151-152.

26 Franklin PJ, Hall GL, Moeller A, Horak F, Jr., Brennan S, Stick SM. Exhaled nitric oxide is not reduced in infants with cystic fibrosis. Eur Respir J 2006;27:350-353.

27 Cunningham S, McColm JR, Ho LP, Greening AP, Marshall TG. Measurement of inflammatory markers in the breath condensate of children with cystic fibrosis. Eur Respir J 2000;15:955-957.

28 Formanek W, Inci D, Lauener RP, Wildhaber JH, Frey U, Hall GL. Elevated nitrite in breath condensates of children with respiratory disease. Eur Respir J 2002;19:487-491.

29 Tate S, MacGregor G, Davis M, Innes JA, Greening AP. Airways in cystic fibrosis are acidified: detection by exhaled breath condensate. Thorax 2002;57:926-929.

30 de Winter-de Groot KM, van der Ent CK. Nitric oxide in cystic fibrosis. J Cyst Fibros 2005;4 Suppl 2:25-29.

31 Grasemann H, Ratjen F. Cystic fibrosis lung disease: the role of nitric oxide. Pediatr Pulmonol 1999;28:442448.

32 Meng QH, Springall DR, Bishop AE, et al. Lack of inducible nitric oxide synthase in bronchial epithelium: a possible mechanism of susceptibility to infection in cystic fibrosis. J Pathol 1998;184:323-331.

33 Kelley TJ, Drumm ML. Inducible nitric oxide synthase expression is reduced in cystic fibrosis murine and human airway epithelial cells. J Clin Invest 1998;102:1200-1207.

34 Moeller A, Horak F, Jr., Lane C, et al. Inducible NO synthase expression is low in airway epithelium from young children with cystic fibrosis. Thorax 2006;61:514-520.

35 Wojnarowski C, Frischer T, Hofbauer E, et al. Cytokine expression in bronchial biopsies of cystic fibrosis patients with and without acute exacerbation. Eur Respir J 1999;14:1136-1144.

36 Ijzermans JN, Marquet RL. Interferon-gamma: a review. Immunobiology 1989;179:456-473.

37 Chung KF, Barnes PJ. Cytokines in asthma. Thorax 1999;54:825-857.

38 Watt AP, Courtney J, Moore J, Ennis M, Elborn JS. Neutrophil cell death, activation and bacterial infection in cystic fibrosis. Thorax 2005;60:659-664.

39 Armstrong DS, Hook SM, Jamsen KM, et al. Lower airway inflammation in infants with cystic fibrosis detected by newborn screening. Pediatr Pulmonol 2005;40:500-510.

40 Ho LP, Faccenda J, Innes JA, Greening AP. Expired hydrogen peroxide in breath condensate of cystic fibrosis patients. Eur Respir J 1999;13:103-106.

41 Carpagnano GE, Barnes PJ, Francis J, Wilson N, Bush A, Kharitonov SA. Breath condensate pH in children with cystic fibrosis and asthma: a new noninvasive marker of airway inflammation? Chest 2004;125:2005-2010.

42 Bodini A, D'Orazio C, Peroni D, et al. Biomarkers of neutrophilic inflammation in exhaled air of cystic fibrosis children with bacterial airway infections. Pediatr Pulmonol 2005;40:494-499.

43 Montuschi P, Kharitonov SA, Ciabattoni G, et al. Exhaled 8-isoprostane as a new non-invasive biomarker of oxidative stress in cystic fibrosis. Thorax 2000;55:205-209. 


\section{CHAPTER 8}

\section{Structural lung changes, lung function, and non- invasive inflammatory markers in cystic fibrosis}

CMHHT Robroeks, MH Roozeboom, PA de Jong, HAWM Tiddens, Q Jöbsis, JJE Hendriks, JBL Yntema, HL Brackel, R van Gent, S Robben, E Dompeling Pediatric Allergy and Immunology, in press 


\section{Abstract}

\section{Background}

Cystic fibrosis lung disease is characterised by chronic airway inflammation and recurrent infections, resulting in (ir)reversible structural lung changes and a progressive decline in lung function. The objective of this study was to investigate the relationship between noninvasive inflammatory markers (IM) in exhaled breath condensate (EBC), lung function indices and structural lung changes, visualized by high resolution computed tomography (HRCT) scans in cystic fibrosis.

\section{Methods}

In 34 cystic fibrosis patients, lung function indices (FEV $1, F V C, R V$, and TLC) and noninvasive inflammatory markers (exhaled nitric oxide, and condensate acidity, nitrate, nitrite, 8-isoprostane, hydrogen peroxide, interferon-gamma) were assessed. HRCT scans were scored in a standardized and validated way, a composite score and component scores were calculated.

\section{Results}

In general, the correlations between non-invasive IM and structural lung changes, and between IM and lung function were low (correlation coefficients < 0.40). Patients with positive sputum Pseudomonas cultures had higher EBC nitrite levels and higher parenchymal HRCT subscores than patients with Pseudomonas-negative cultures $(p<0.05)$. Multiple linear regression models demonstrated that FVC was significantly predicted by hydrogen peroxide in EBC, and the scores of bronchiectasis and mosaic perfusion (Pearson correlation coefficient $R=0.78, p<0.001)$. TLC was significantly predicted by 8 isoprostane, nitrate, hydrogen peroxide in EBC, and the mucous plugging subscore $(R=$ $0.92, p<0.01)$.

\section{Conclusion}

Static and dynamic lung function indices in this CF group were predicted by the combination of non-invasive IM in exhaled breath condensate and structural lung changes on HRCT imaging. Future longitudinal studies should reveal whether non-invasive monitoring of airway inflammation in CF adds to better follow-up of patients. 


\section{Introduction}

Cystic fibrosis is an autosomal recessive disorder, caused by genetic mutations in the cystic fibrosis transmembrane conductance regulator protein [1,2]. This results in impaired or absent transport of chloride through cell membranes, with impaired mucociliary clearance, viscous mucus in the airways, and an inability to effectively clear bacteria like Staphylococcus aureus and Pseudomonas aeroginosa [1,2]. Therefore, chronic airway infection, airway inflammation, and oxidative stress are the features of CF disease with mainly neutrophilic immune responses [3]. In the course of time, structural lung changes will occur with diminishment of lung function.

Airway inflammation in patients with CF is already present early in life [1], and plays a major role in CF lung disease. Previous studies showed higher levels in sputum and broncho-alveolar lavage of IFN- $\gamma$ [2], IL-2 [2], IL-4 [2], IL-5 [3], nitrate [4], and nitrite [4, 5] in CF patients compared to controls. An increasing amount of studies emphasize the potential of non-invasive IM to reflect ongoing inflammation and infection [6-11]. Such a new, noninvasive technique is exhaled breath condensate (EBC). Recently, higher EBC levels of 8isoprostane [12], hydrogen peroxide [14], nitrite [6, 13, 14], IFN-gamma [16], and EBC acidity [17], but lower fractional exhaled nitric oxide (FeNO) levels were shown in CF compared to healthy subjects $[15,16]$. However, the relationship between these non-invasive $\mathrm{IM}$ and structural lung abnormalities in CF is unclear.

Structural lung changes in CF can be assessed by HRCT imaging of the lungs, and include bronchiectasis, mucus plugging, peribronchial thickening, parenchymal abnormalities and air trapping [17-21]. HRCT enables visualisation of (small) areas with structural lung changes, even in an early stage $[17,22,23]$. These structural abnormalities may be observed by HRCT imaging, even in the presence of normal lung function parameters and in the absence of pulmonary symptoms [17, 19-27]. Imaging and pathology studies demonstrated that CF lung disease first develops in small peripheral airways, and, that early changes can be restricted to only local areas [28].

Lung function indices show the degree of restriction and obstruction of the overall airway status $[29,30]$, and thereby reflect the consequences of past and presently ongoing inflammatory processes. Lung function is related to morbidity and mortality in CF.

The relationship between lung function on the one hand, structural lung changes as visualized by HRCT scans, and non-invasive IM on the other is yet unclear in CF patients [31]). We hypothesized that structural lung changes on HRCT imaging (as a result of long term damage to the lungs by chronic airway infection and inflammation) together with ongoing airway inflammation (as assessed by IM in EBC) are important determinants of lung function (dynamic as well as static indices) in CF. Data on non-invasive IM in EBC, HRCT imaging, and static and dynamic lung function indices were analyzed in 34 CF patients.

\section{Material and Methods}

\section{Study subjects}

34 patients with CF (19 male), capable of performing lung function tests and EBC manoeuvres, were selected from the outpatient clinic of the CF centers of the Maastricht and 
Nijmegen University Medical Center. The age (mean \pm SD) of the CF population was $12.6 \pm$ 4.4 years. Symptoms, medication use, and the results of physical examination were documented. Standard deviations of weight and height were calculated based on Dutch caucasian population corrected for age and gender [32]. Informed consent was obtained from parents and children, from the age of 12 years, participating in this study. When children were younger than 12 , informed consent was obtained from parents only. The study was approved by the Medical Ethics Committee of the University Hospital Maastricht.

\section{Exclusion criteria}

CF disease was defined as a combination of characteristic clinical features (e.g. persistent pulmonary problems, meconium ileus, failure to thrive, steatorhoe) and an abnormal sweat test (Chloride $>60 \mathrm{mM}$ ) [33]. Exclusion criteria were: 1) Diseases that may interfere with the results of the study (e.g. upper airway infection, congenital heart disease, anatomic abnormalities of the airways and other chronic inflammatory diseases, such as Crohn's disease and rheumatoid arthritis), 2) Mental retardation, 3) Inability to perform the EBC collection procedure, 4) Active smoking and, 5) Use of the following medication: sodium nitroprusside, angiotensin-converting enzyme (ACE) inhibitors, oxymetazoline, Larginine, or nitric oxide synthase (NOS) inhibitors.

\section{HRCT scanning}

HRCT scans were performed in supine position from lung apex to base. In each hospital a sequential scanning technique was used with $1 \mathrm{~mm}$ thick CT slices at intervals ranging from 6-10 mm. Scans were obtained at full inspiration in all subjects and in expiration in a subset. Exposure time ranged from 0.75 tot 1.1 seconds. Beam current ranged from 90200 milli Amperes depending on the patients' age. The peak of beam potential ranged from 120-140 kilo Voltage. All scans were reconstructed using a high-resolution algorithm and displayed at lung settings (window width -400 to -500 hounsfield units and window level 1400-1600 hounsfield units. Different CT scanners were used: a 2-slice spiral CT scanner (Elscint MX Twin, Philips Medical Systems presently, Haifa, Israel), a 16 detector row CT scanner (Siemens sensation 16, Siemens Medical Systems, Erlangen, Germany), a 64 detector row CT scanner (Siemens Somatom 64, Siemens Medical Systems, Erlangen, Germany), and a 4-slice spiral CT scanner (Toshiba Aquillion, Toshiba Medical Systems, Tokyo, Japan).

\section{HRCT scoring}

All inspiratory HRCT-scans were scored using a scoring system described by Brody et al [18] by two blinded and independent observers [27, 34]. The scoring system evaluates the five lobes. The lingual of the left lung was scored as a sixth lobe. The items scored were presence and severity of bronchiectasis, mucous plugging, peribronchial thickening, parenchymal abnormalities and air trapping. Because expiration scans were not available in all patients, mosaic perfusion was scored instead of air trapping in all patients and expiratory images were not used. Component scores and composite scores were calculated as previously reported and expressed on a scale 0-100 [17]. The higher the score, the more a specific item is found in the lungs. 


\section{Clinical scores}

To determine the general clinical condition of the patient we determined the ShwachmanKulczycki score for each subject.

\section{Nitric oxide in exhaled breath}

FeNO was measured using a NIOX chemiluminescence analyser (Aerocrine, Solna, Sweden). Measurements were assessed according to the recommendations of the American Thoracic Society/European Respiratory Society for children (ATS/ERS) [35, 36].

\section{Exhaled breath condensate}

EBC was collected using a glass condenser system [11,12]. Samples were deep frozen ($80^{\circ} \mathrm{C}$ ) immediately after collection. The acidity of the EBC was measured immediately after collection (Radiometer , type PHM201, Radiometer Nederland BV, Zoetermeer, the Netherlands). Samples were not deaerated. In EBC, nitrate and nitrite concentrations were assessed using a fluorimetric assay (Cayman Chemical, Ann Arbor, USA) [36]. To measure 8-isoprostane, an EIA-kit was used (Cayman Chemical, Ann Arbor, USA), which was modified in order to lower the detection limit from $5.0 \mathrm{pg} / \mathrm{mL}\left(80 \% \mathrm{~B} / \mathrm{B}_{0}\right)$ to $0.5 \mathrm{pg} / \mathrm{mL}$, as described previously [8]. $\mathrm{H}_{2} \mathrm{O}_{2}$ measurements were based on a method previously described [37]. Briefly, EBC was mixed with $0.4 \mathrm{M}$ citrate buffer, HRP solution (50 U/mL) and tetramethylbenzidine (TMB, $20 \mathrm{mM})$ and incubated for $20 \mathrm{~min}$ at room temperature. Then, the sample was mixed with sulphuric acid $(2 \mathrm{M})$. The TMB oxidation product as a measure of the amount of $\mathrm{H}_{2} \mathrm{O}_{2}$ was determined spectrometrically at $450 \mathrm{~nm}$, using a Perkin Elmer UV-VIS Spectrometer Lambda 10 (Norwalk, CT, USA). Cytokine IFN- $\gamma$ level was assessed with flow cytometry (CBA, BD Bioscience ${ }^{\bullet}$, San Diego, USA), as described previously [8].

\section{Lung function tests}

Bronchodilator medication was stopped prior to lung function testing: short-acting bronchodilators for at least 8 hours, and long-acting bronchodilators at least for 36 hours. Dynamic and static spirometry were performed by means of the Flowscreen (Viasys ${ }^{\circ}$, Hoechberg, Germany), and by body plethysmography (Viasys ', Hoechberg, Germany). The lung function parameters were assessed according to ERS standards [38]. The highest values of three correctly performed manoeuvres were used for analysis. Recorded parameters were: forced expiratory volume in one second $\left(\mathrm{FEV}_{1}\right)$, forced vital capacity (FVC), $\mathrm{FEV}_{1} / \mathrm{VC}$ and the intrathoracic gas volume (ITGV). The reversibility on a bronchodilator was determined 15 minutes after inhalation of $400 \mu \mathrm{g}$ salbutamol. Reversibility was defined as an increase in measured $\mathrm{FEV}_{1}$ of at least $9 \%$ of predicted value [39]. The static indices TLC and RV were determined.

\section{Statistical analysis}

Intra-observer variability for HRCT scoring (composite score and component scores) was tested with the intra-class correlation coefficient. An intra-class correlation coefficients 
greater than 0.8 represented good agreement between observers. The relationship between HRCT scores (composite and component), lung function indices and non-invasive inflammatory markers were tested using Pearson correlation coefficients. Spearman's rank test was used when distribution of parameters were considered not normal. The mean HRCT scores of both observers were used to calculate Pearson correlation coefficients. For IFN- $\gamma$ samples with concentrations below the detection limit, arbitrary values between 0 and the detection limit were ascribed. Stepwise, backward multiple regression linear analysis was used to analyse the predicting ability of inflammatory markers and HRCT subscores to predict lung function indices (dependent variable). Described tests were considered statistically significant, when $p<0.05$.

\section{Power analysis}

25 Subjects with CF are necessary to assess clinically relevant correlation coefficients of $\geq$ 0.6 between for instance IM and HRCT changes with an alpha of 0.05 , and a power of $90 \%$. Thirty-four subjects were selected for this study.

\section{Results}

\section{Population characteristics}

Of the 34 CF patients, 19 patients (56\%) had a weight one SD below the mean, and a height one SD below the mean (Table 1). Six (18\%) and seven (21\%) of the patients had a weight and height below -2 SD, respectively. Lung function indices showed a mild to moderate impairment with a mixed restrictive and obstructive component. Expiratory volumes and flow rates were decreased, while residual volumes were increased. DNase and corticosteroids (inhaled or oral) were used frequently (62\% and $29 \%$ respectively). Antibiotics were used prophylactically in $41 \%$ (inhaled $18 \%$, oral $3 \%$ ), and therapeutically in $12 \%$ (inhaled $6 \%$, oral $6 \%$, intravenous $9 \%$ ).

\section{FeNO and non-invasive IM in EBC}

Nitrate, nitrite, $\mathrm{H}_{2} \mathrm{O}_{2}$ and 8-isoprostane were detected in all EBC samples. IFN- $\gamma$ was detected in $38 \%$ of the EBC samples. The mean \pm SE of the IM were: FeNO $9.0 \pm 1.5 \mathrm{ppb} ; \mathrm{pH}$ $6.3 \pm 0.1$; Nitrite $2.0 \pm 1.1 \mu \mathrm{M}$; Nitrate $17.7 \pm 2.6 \mu \mathrm{M} ; \mathrm{H}_{2} \mathrm{O}_{2} 2.4 \pm 0.3 \mu \mathrm{M}$; 8-isoprostane 4.2 $\pm 0.5 \mathrm{pg} / \mathrm{mL}$ and IFN- $\gamma$ (range) $0.7-3.5 \mathrm{pg} / \mathrm{mL}$.

\section{Between observer agreement of HRCT scoring}

The intra-class correlation coefficient was 0.98 (95\% confidence interval 0.97 to 0.99 , $\mathrm{p}<0.01)$, for the composite score. The intra-class correlation coefficients for the subscores (with a 95\% confidence interval) were: bronchiectasis $0.91(0.81-0.95, p<0.01)$; airway wall thickening 0.92 (0.85-0.96, $\mathrm{p}<0.01)$; mucous plugging $0.93(0.85-0.96, p<0.01)$; parenchymal abnormalities 0.84 (0.68-0.92, $\mathrm{p}<0.01)$; mosaic perfusion 0.98 (0.95-0.99, $\mathrm{p}<0.01)$. 
Table 1: Clinical characteristics of the CF study population ( $n=34)$.

\begin{tabular}{|c|c|}
\hline Characteristics & Mean \pm SE \\
\hline Age (yrs) & $12.6 \pm 0.7$ \\
\hline Height (cm) & $146 \pm 3.3$ \\
\hline SD height * & $-1.1 \pm 0.2$ \\
\hline Weight (kg) & $36 \pm 2.2$ \\
\hline SD weight * & $-1.2 \pm 0.2$ \\
\hline Gender (male / female) & $19 / 15$ \\
\hline Positive sputum bacterial cultures ( $\mathrm{nr}-\%$ ) & $28 / 34(82)$ \\
\hline Positive sputum Pseudomonas cultures ( $\mathrm{nr}-\%)$ & $14 / 34(41)$ \\
\hline Total SK score, median (range) & $65(55-70)$ \\
\hline \multicolumn{2}{|l|}{ Dynamic lung function indices: } \\
\hline - $\mathrm{FEV}_{1}(\%$ pred) & $72 \pm 4.4$ \\
\hline - FVC (\% pred) & $75 \pm 3.6$ \\
\hline$-\mathrm{FEV}_{1} / \mathrm{VC}(\%)$ & $76 \pm 2.2$ \\
\hline - Reversibility \# (\%) & 15 \\
\hline \multicolumn{2}{|l|}{ Statistic lung function indices: } \\
\hline - TLC (\% pred) & $103 \pm 2.2$ \\
\hline - RV (\% pred) & $181 \pm 12.4$ \\
\hline - ITGV (\% pred) & $120 \pm 4.6$ \\
\hline \multicolumn{2}{|c|}{ 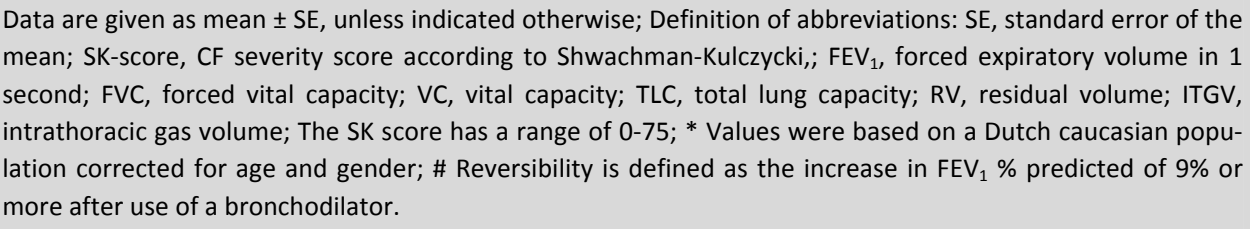 } \\
\hline
\end{tabular}

\section{HRCT scores}

The mean \pm SE of the separate HRCT scores were: bronchiectasis $15 \pm 2.4 \%$; airway wall thickening $19 \pm 2.0 \%$; mucous plugging $19 \pm 2.0 \%$; parenchymal abnormalities $7 \pm 1.1 \%$; mosaic perfusion $24 \pm 4.3 \%$ and total score $17 \pm 1.9 \%$, indicating moderate structural lung changes. The mean \pm SE time span between HRCT imaging and determination of IM and LF was $-2.0 \pm 1.9$ months.

\section{Relationships between IM and HRCT scores}

Overall, the association between IM and HRCT scores was not very strong with correlation coefficients $<0.4$. The only significant correlation was found between the mucous plugging subscore and $\mathrm{H}_{2} \mathrm{O}_{2}(\mathrm{R}=-0.39, \mathrm{p}=0.03)$, Figure 1 . The correlation between $\mathrm{H}_{2} \mathrm{O}_{2}$ and airway wall thickening showed a trend towards significance $(R=-0.32, p=0.07)$. 


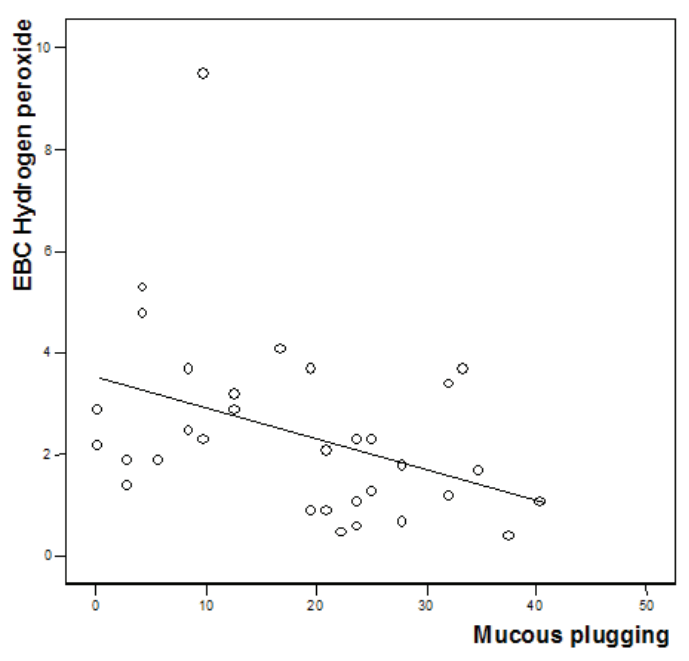

Figure 1: The correlation between hydrogen peroxide in $\mathrm{EBC}$ and the mucous plugging HRCT subscore in 34 CF patients $(R=0.39, p=0.03)$.

Relationships between sputum cultures, non-invasive IM and HRCT scores

Patients with positive sputum Pseudomonas aeruginosa cultures had higher EBC nitrite levels and parenchymal HRCT subscores (mean \pm SE; $2.37 \pm 0.27 \mu \mathrm{M}$ and $10.3 \pm 1.7 \%$ ) compared to CF patients with negative Pseudomonas aeruginosa cultures $(1.55 \pm 0.25 \mu \mathrm{M}$ and $4.23 \pm 1.37 \%, p=0.04$ and $p=0.01$ respectively). In patients with positive sputum cultures of any pathologic micro-organism, higher scores were given to the bronchiectasis $\mathrm{HRCT}$ subscore (mean $\pm \mathrm{SE} ; 17.5 \pm 2.7, \mathrm{p}<0.01$ ), implicating more extensively distributed and more severe bronchiectasis on HRCT scans than patients with no positive sputum cultures $(4.7 \pm 2.7 \%, p=0.04)$.

\section{Relationships between IM and lung function indices}

There were significant correlations between FVC and $\mathrm{H}_{2} \mathrm{O}_{2}(\mathrm{R}=0.39, \mathrm{p}=0.03)$, and between TLC and nitrate $(R=-0.64, p<0.001)$ (Figure 2 and 3 , respectively). 


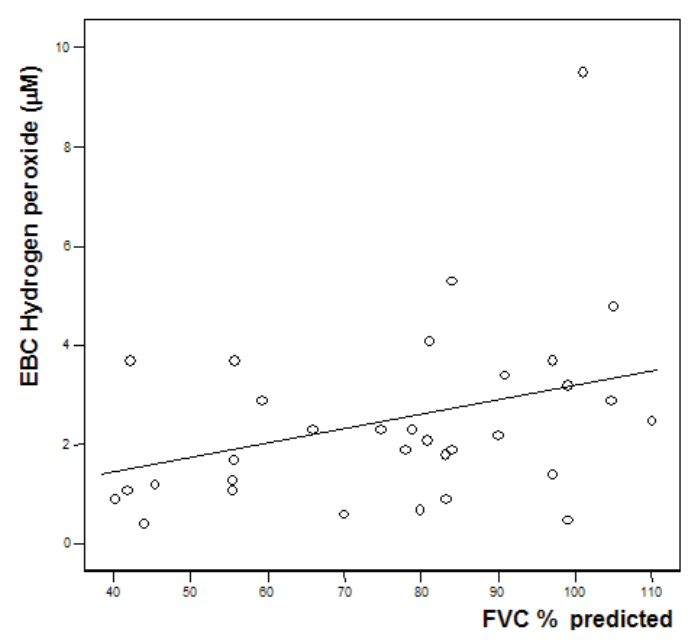

Figure 2: The correlation between hydrogen peroxide in $\mathrm{EBC}$ and the forced vital capacity in $34 \mathrm{CF}$ patients $(R=0.39, p=0.03)$.

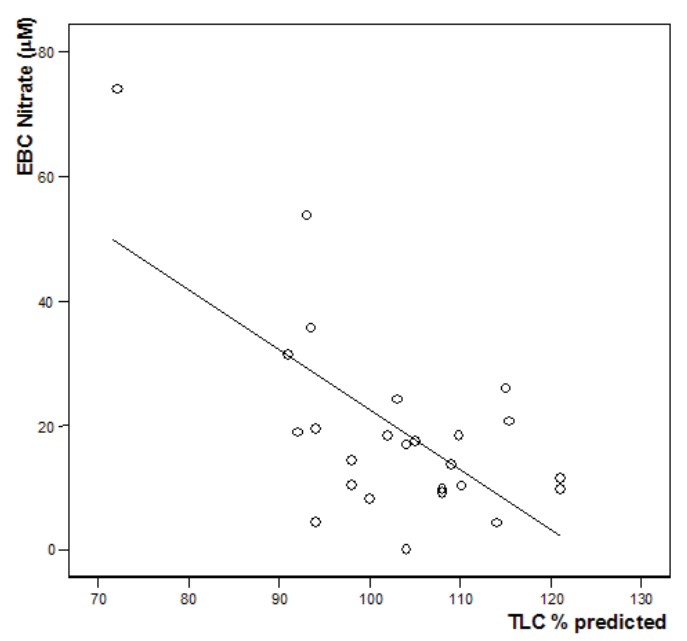

Figure 3: The correlation between nitrate in $\mathrm{EBC}$ and total lung capacity in 34 CF patients $(R=0.64, p<0.001)$.

Relationships between HRCT scores, lung function indices and SK score

Lung function indices ( $\mathrm{FEV}_{1}, \mathrm{FVC}$ and RV) strongly correlated with HRCT scores (Table 2). In general, a stronger correlation was found between the total HRCT score and lung function parameters, than with the HRCT composite and component scores. There were no correlations between TLC \% predicted value and any HRCT composite and component scores. 
Table 2: Relationships between HRCT scores, general clinical (SK) score and lung function indices.

\begin{tabular}{llllllll}
\hline & & $\mathrm{B}$ & $\mathrm{AWT}$ & $\mathrm{Mu}$ & $\mathrm{P}$ & $\mathrm{Mo}$ & Total \\
\hline FEV 1 (\% pred) & $\mathrm{R}$ & -0.64 & -0.62 & -0.54 & -0.48 & -0.64 & -0.77 \\
& $\mathrm{p}$-value & 0.00 & 0.00 & 0.00 & 0.00 & 0.00 & 0.00 \\
FEV $_{1}$ /VC & $\mathrm{R}$ & -0.26 & -0.44 & -0.41 & -0.16 & -0.46 & -0.43 \\
& $\mathrm{p}$-value & 0.13 & 0.01 & 0.02 & 0.37 & 0.01 & 0.01 \\
FVC (\% pred) & $\mathrm{R}$ & -0.59 & -0.55 & -0.47 & -0.56 & -0.62 & -0.73 \\
& $\mathrm{p}$-value & 0.00 & 0.00 & 0.01 & 0.00 & 0.00 & 0.00 \\
TLC (\% pred) & $\mathrm{R}$ & 0.10 & 0.22 & 0.37 & -0.29 & -0.16 & 0.04 \\
& $\mathrm{p}$-value & 0.64 & 0.28 & 0.07 & 0.17 & 0.46 & 0.84 \\
RV (\% pred) & $\mathrm{R}$ & 0.69 & 0.63 & 0.64 & 0.37 & 0.56 & 0.73 \\
& $\mathrm{p}$-value & 0.00 & 0.00 & 0.00 & 0.07 & 0.00 & 0.00 \\
ITGV (\% pred) & $\mathrm{R}$ & 0.61 & 0.56 & 0.52 & 0.26 & 0.39 & 0.59 \\
& $\mathrm{p}$-value & 0.00 & 0.00 & 0.01 & 0.21 & 0.06 & 0.00 \\
SK score & $\mathrm{R}$ & -0.44 & -0.25 & -0.40 & -0.37 & -0.47 & -0.50 \\
& $\mathrm{p}$-value & 0.01 & 0.16 & 0.02 & 0.03 & 0.01 & 0.00 \\
\hline
\end{tabular}

Data were analysed using Pearson correlation tests. Definition of abbreviations: B, bronchiectasis; AWT, airway wall thickening; Mu, mucous plugging; $\mathrm{P}$, Parenchymal abnormalities; Mo, Mosaic perfusion; Total, accumulative score; SK-score, CF severity score according to Shwachman-Kulczycki; FEV $_{1}$, forced expiratory volume in 1 second; VC, vital capacity; FVC, forced vital capacity; TLC, total lung capacity; RV, Residual volume; ITGV, intrathoracic gas volume; $R$, correlation coefficient.

\section{Results of multivariate backward linear regression models}

Multivariate backward linear regression analyses with TLC \% of predicted as dependent variable, and, measured non-invasive IM and HRCT subscores (bronchiectasis, peribronchial thickening, mucous plugging and mosaic perfusion) as independent variables showed that TLC \% of predicted value was significantly predicted by 8-isoprostane, nitrate, $\mathrm{H}_{2} \mathrm{O}_{2}$ and mucous plugging subscore with a high level of explained variance $(R=0.92, p<0.01)$ (Table 3a). In addition, another significant prediction model was possible for the FVC (as dependent variable), which was significantly predicted by $\mathrm{H}_{2} \mathrm{O}_{2}$, and the scores of bronchiectasis and mosaic perfusion ( $R=0.78, p<0.001$ ) (Table $3 b)$.

Table 3a: Backward multiple linear regression model of IM in EBC and the HRCT scores on total lung capacity (dependent variable). TLC was significantly predicted by EBC 8-isoprostane, nitrate, hydrogen peroxide, and the mucous plugging subscore $(R=0.92, p<0.01)$.

\begin{tabular}{lll}
\hline & $\beta \pm \mathrm{SE}$ & $\mathrm{P}$ \\
\hline Constant & $104.10 \pm 4.02$ & \\
8-IP & $-1.92 \pm 4.02$ & 0.006 \\
Nitrate & $-0.52 \pm 0.09$ & 0.000 \\
$\mathrm{H}_{2} \mathrm{O}_{2}$ & $2.38 \pm 0.77$ & 0.010 \\
Mucous plugging & $0.56 \pm 0.15$ & 0.003 \\
\hline
\end{tabular}

Definition of abbreviations: 8-IP, 8-isoprostane; $\mathrm{H}_{2} \mathrm{O}_{2}$, hydrogen peroxide; $\mathrm{SE}$, standard error. 
Table 3b: Backward multiple linear regression model of IM in EBC and HRCT scores on forced vital capacity (dependent variable). The FVC was significantly predicted by EBC hydrogen peroxide and by the bronchiectasis and mosaic perfusion subscores $(R=0.78, p<0.001)$.

\begin{tabular}{lll}
\hline & $\beta \pm \mathrm{SE}$ & $p$ \\
\hline Constant & $83.00 \pm 7.40$ & \\
$\mathrm{H}_{2} \mathrm{O}_{2}$ & $3.75 \pm 1.69$ & 0.037 \\
Bronchiectasis & $-0.62 \pm 0.25$ & 0.019 \\
Mosaic perfusion & $-0.39 \pm 0.13$ & 0.005 \\
\hline \multicolumn{2}{l}{ Definition of abbreviations: $\mathrm{H}_{2} \mathrm{O}_{2}$, hydrogen peroxide; SE, standard error. }
\end{tabular}

\section{Discussion}

This study supports the hypothesis that lung function in CF (both static and dynamic indices) is determined by the combination of structural lung changes on HRCT imaging and airway inflammation, as assessed by non-invasive biomarkers in EBC: the FVC was indicated to a large degree by mosaic perfusion, bronchiectasis and hydrogen peroxide levels, whereas total lung capacity was strongly predicted by mucous plugging, 8-isoprostane, nitrate, and hydrogen peroxide. In general, the correlation between HRCT changes and non-invasive IM in EBC was low: only hydrogen peroxide correlated significantly with mucous plugging. In patients with positive Pseudomonas Aeruginosa sputum cultures, significant higher EBC nitrite levels and more parenchymal abnormalities were found compared to patients with Pseudomonas negative cultures. In general, high correlations were demonstrated between lung function indices and structural lung changes on HRCT imaging.

To our knowledge, this is one of the first studies investigating the relationship between non-invasive IM, structural lung changes, assessed by HRCT scans, and lung function in CF patients. One study by Dakin and co-workers, investigated the relationship between IM in induced sputum and HRCT images in 34 CF patients (mean age 12.3 years; mean FEV $1 \%$ of predicted 67\%) [40]. The time span between assessments of lung function, HRCT scan, and a sputum sample was maximally one month. They found no correlation between induced sputum IL-8 concentrations and the Bhalla HRCT score [40], which is consistent with the low correlations between EBC markers and HRCT in our study. Hilliard et all studied the relationship between structural lung changes as assessed by endobronchial biopsies, and airway inflammation (IM in bronchoalveolar lavage) in a recent cross-sectional study of 43 children with CF (aged 0.3-16.8 years) and 40 control children [37]. The reticular basement membrane thickness was greater in the CF group than in the controls, and correlated positively with levels of transforming growth factor-beta 1, although not with other inflammatory markers or pulmonary function. This is consistent with the generally low correlation between IM in EBC and structural lung changes in our study. In addition, our study examined the relationship between lung function parameters and structural lung changes. $\mathrm{FEV}_{1}, \mathrm{FEV}_{1} / \mathrm{FVC}$, FVC and RV strongly correlated with HRCT scores. In general, lung function parameters correlated better with the total HRCT score, than with the HRCT subscores, which confirms that lung function parameters indeed reflect the overall status of lung changes. This is in accordance to other data in the literature [17, 19, 27]. 
How can we explain the results of this study?

In general, the low correlations between non-invasive IM and structural lung changes indicate that structural lung changes are the consequence of a long-term process of infection and inflammation, and are not adequately described by one discrete assessment of IM. Another explanation is that the relationship between airway inflammation and structural lung changes is modified by an individual (genetic) susceptibility to develop damage. There are several genetic CF components that may influence this susceptibility [41, 42]. Recently, the importance of two modifier genes (mannose-binding lectin 2 and transforming growth factor beta 1) for the levels of lung function in CF was demonstrated in a cohort of 1019 Canadian CF patients [41]. CF patients with mannose-binding lectin 2 deficiency had the more rapid decline in lung function, most significantly in those carrying the high-producing transforming growth factor beta 1 genotype. This last finding points to an important gene-gene interaction. We found that lung function in our group of CF patients was very well predicted by the combination of non-invasive IM in EBC and of structural lung changes on HRCT imaging.

The strengths of our study were: 1 ) we included a relative large study population of $34 \mathrm{CF}$ patients; 2) two observers scored the HRCT scans with an excellent intra-class correlation coefficient; 3 ) the simultaneous assessment of several relevant non-invasive IM in CF, with detection rates of $38-100 \%$.

A limitation of this study may be the time interval between the HRCT imaging and the assessment of lung function and EBC, which on average was 2 months. It is unlikely that the relationships between IM and CT findings was significantly influenced by this time interval as correlation between lung function and HRCT imaging appeared to be very high. This study included patients with mild to moderate CF. In more severe CF, results might be different.

Some correlations in this study were different from expected: decreasing $\mathrm{H}_{2} \mathrm{O}_{2}$ levels were significantly related to a decrease in FVC \% of predicted, whereas lower nitrate values were related to an increase in TLC \% of predicted. This may be the consequence of mucous entrapment of IM. A comparable mechanism had been suggested as one of the explanations for the diminished FeNO values in CF $[43,44]$. The significant correlation between the extent of mucous plugs and $\mathrm{EBC} \mathrm{H}_{2} \mathrm{O}_{2}$ levels in this study demonstrates that this hypothesis of mucus entrapment of IM is probably correct.

\section{How should we monitor CF patients?}

Our cross-sectional study design is not suitable to answer this question. However, the impression from this study is that chronic airway inflammation is an important determinant of structural lung changes and also of lung function. It is possible that monitoring of airway inflammation will add to better follow-up of CF patients. One of the tools for monitoring of airway inflammation in CF may be non-invasive assessment of IM in EBC. However, longitudinal data are necessary to investigate this. HRCT imaging is an interesting technique as it is able to assess early lung changes, but there is concern about the longterm harmful effects of particularly repeated scans and radiation exposures [44]. In summary, this study demonstrated that non-invasive IM in EBC in combination with structural lung changes on HRCT imaging significantly predicted static and dynamic lung function indices in this group of CF patients. Future longitudinal studies should reveal whether noninvasive monitoring of airway inflammation in CF adds to better follow-up of patients. 


\section{References}

1 Khan, TZ, Wagener, JS, Bost, T, Martinez, J, Accurso, FJ, Riches, DW. Early pulmonary inflammation in infants with cystic fibrosis. Am J Respir Crit Care Med. 1995: 151: 1075-82.

2 Hartl, D, Griese, M, Kappler, M, et al. Pulmonary $T(H) 2$ response in Pseudomonas aeruginosa-infected patients with cystic fibrosis. J Allergy Clin Immunol. 2006: 117: 204-11.

3 Koller, DY, Nething, I, Otto, J, Urbanek, R, Eichler, I. Cytokine concentrations in sputum from patients with cystic fibrosis and their relation to eosinophil activity. Am J Respir Crit Care Med. 1997: 155: 1050-4.

4 Linnane, SJ, Keatings, VM, Costello, CM, et al. Total sputum nitrate plus nitrite is raised during acute pulmonary infection in cystic fibrosis. Am J Respir Crit Care Med. 1998: 158: 207-12.

5 Cetin, I, Ozcelik, U, Gocmen, A, Kiper, N, Dogru, D, Yalcin, E. BALF nitrite as an indicator of inflammation in children with cystic fibrosis. Respiration. 2004: 71: 625-9.

6 Carpagnano, GE, Barnes, PJ, Geddes, DM, Hodson, ME, Kharitonov, SA. Increased leukotriene B4 and interleukin-6 in exhaled breath condensate in cystic fibrosis. Am J Respir Crit Care Med. 2003: 167: 1109-12.

7 Kharitonov, SA, Barnes, PJ. Exhaled biomarkers. Chest. 2006: 130: 1541-6.

8 Robroeks, CMHHT, Jöbsis, Q, Damoiseaux, JGMC, et al. Cytokines in exhaled breath condensate of children with asthma and cystic fibrosis. Ann Allergy Asthma Immunol. 2006: 96: 349-55.

9 Carpagnano, GE, Barnes, PJ, Francis, J, Wilson, N, Bush, A, Kharitonov, SA. Breath condensate pH in children with cystic fibrosis and asthma: a new noninvasive marker of airway inflammation? Chest. 2004: 125: 200510.

10 Rahman, I, Kelly, F. Biomarkers in breath condensate: a promising new non-invasive technique in free radical research. Free Radic Res. 2003: 37: 1253-66.

11 Rosias, P, Den Hartog, G, Robroeks, C, et al. Free radicals in exhaled breath condensate in cystic fibrosis and healthy subjects. Free Radic Res. 2006: 40: 901-9.

12 Montuschi, P, Kharitonov, SA, Ciabattoni, G, et al. Exhaled 8-isoprostane as a new non-invasive biomarker of oxidative stress in cystic fibrosis. Thorax. 2000: 55: 205-9.

13 Formanek, W, Inci, D, Lauener, RP, Wildhaber, JH, Frey, U, Hall, GL. Elevated nitrite in breath condensates of children with respiratory disease. Eur Respir J. 2002: 19: 487-91.

$14 \mathrm{Ho}, \mathrm{LP}$, Innes, JA, Greening, AP. Nitrite levels in breath condensate of patients with cystic fibrosis is elevated in contrast to exhaled nitric oxide. Thorax. 1998: 53: 680-4.

15 Grasemann, H, Michler, E, Wallot, M, Ratjen, F. Decreased concentration of exhaled nitric oxide (NO) in patients with cystic fibrosis. Pediatr Pulmonol. 1997: 24: 173-7.

16 Thomas, SR, Kharitonov, SA, Scott, SF, Hodson, ME, Barnes, PJ. Nasal and exhaled nitric oxide is reduced in adult patients with cystic fibrosis and does not correlate with cystic fibrosis genotype. Chest. 2000: 117: 1085-9.

17 Brody, AS, Klein, JS, Molina, PL, Quan, J, Bean, JA, Wilmott, RW. High-resolution computed tomography in young patients with cystic fibrosis: distribution of abnormalities and correlation with pulmonary function tests. J Pediatr. 2004: 145: 32-8.

18 Brody, AS, Molina, PL, Klein, JS, Rothman, BS, Ramagopal, M, Swartz, DR. High-resolution computed tomography of the chest in children with cystic fibrosis: support for use as an outcome surrogate. Pediatr Radiol. 1999: 29: 731-5.

19 Helbich, TH, Heinz-Peer, G, Eichler, I, et al. Cystic fibrosis: CT assessment of lung involvement in children and adults. Radiology. 1999: 213: 537-44.

20 de Jong, PA, Nakano, $\mathrm{Y}$, Lequin, $\mathrm{MH}$, et al. Progressive damage on high resolution computed tomography despite stable lung function in cystic fibrosis. Eur Respir J. 2004: 23: 93-7.

21 Maffessanti, M, Candusso, M, Brizzi, F, Piovesana, F. Cystic fibrosis in children: HRCT findings and distribution of disease. J Thorac Imaging. 1996: 11: 27-38.

22 Bhalla, M, Turcios, N, Aponte, V, et al. Cystic fibrosis: scoring system with thin-section CT. Radiology. 1991: 179: 783-8.

23 Tiddens, HA, de Jong, PA. Update on the application of chest computed tomography scanning to cystic fibrosis. Curr Opin Pulm Med. 2006: 12: 433-9.

24 Tiddens, HA. Detecting early structural lung damage in cystic fibrosis. Pediatr Pulmonol. 2002: 34: 228-31. 
25 Judge, EP, Dodd, JD, Masterson, JB, Gallagher, CG. Pulmonary Abnormalities on High-Resolution CT Demonstrate More Rapid Decline Than FEV1 in Adults With Cystic Fibrosis. Chest. 2006: 130: 1424-32.

26 Robinson, TE. High-resolution CT scanning: potential outcome measure. Curr Opin Pulm Med. 2004: 10: 53741.

27 de Jong, PA, Ottink, MD, Robben, SG, et al. Pulmonary disease assessment in cystic fibrosis: comparison of CT scoring systems and value of bronchial and arterial dimension measurements. Radiology. 2004: 231: 4349.

28 Davis, SD, Fordham, LA, Brody, AS, et al. Computed tomography reflects lower airway inflammation and tracks changes in early cystic fibrosis. Am J Respir Crit Care Med. 2007: 175: 943-50.

29 Gibson, RL, Burns, JL, Ramsey, BW. Pathophysiology and management of pulmonary infections in cystic fibrosis. Am J Respir Crit Care Med. 2003: 168: 918-51.

30 Ratjen, F, Doring, G. Cystic fibrosis. Lancet. 2003: 361: 681-9.

31 Guran, T, Ersu, R, Karadag, B, et al. Association between inflammatory markers in induced sputum and clinical characteristics in children with non-cystic fibrosis bronchiectasis. Pediatr Pulmonol. 2007: 42: 362-9.

32 Gerver, W, de Bruin, R. Paediatric Morphometrics. A Reference Manual. 2nd extended edition ed. Maastricht, the Netherlands: University 2001.

33 Rosenstein, BJ, Cutting, GR. The diagnosis of cystic fibrosis: a consensus statement. Cystic Fibrosis Foundation Consensus Panel. J Pediatr. 1998: 132: 589-95.

34 Brody, AS, Kosorok, MR, Li, Z, et al. Reproducibility of a scoring system for computed tomography scanning in cystic fibrosis. J Thorac Imaging. 2006: 21: 14-21.

35 ATS/ERS Recommendations for Standardized Procedures for the Online and Offline Measurement of Exhaled Lower Respiratory Nitric Oxide and Nasal Nitric Oxide, 2005. Am J Respir Crit Care Med. 2005: 171: 912-30.

36 Misko, TP, Schilling, RJ, Salvemini, D, Moore, WM, Currie, MG. A fluorometric assay for the measurement of nitrite in biological samples. Anal Biochem. 1993: 214: 11-6.

37 Hilliard TN, Regamey N, Shute JK, Nicholson AG, Alton EW, Bush A, Davies JC. Airway remodelling in children with cystic fibrosis. Thorax 2007:62:1074-1080.

38 Miller, MR, Hankinson, J, Brusasco, V, et al. Standardisation of spirometry. Eur Respir J. 2005: 26: 319-38.

39 Dales, RE, Spitzer, WO, Tousignant, P, Schechter, M, Suissa, S. Clinical interpretation of airway response to a bronchodilator. Epidemiologic considerations. Am Rev Respir Dis. 1988: 138: 317-20.

40 Dakin, CJ, Pereira, JK, Henry, RL, Wang, H, Morton, JR. Relationship between sputum inflammatory markers, lung function, and lung pathology on high-resolution computed tomography in children with cystic fibrosis. Pediatr Pulmonol. 2002: 33: 475-82.

41 Zielenski, J, Tsui, LC. Cystic fibrosis: genotypic and phenotypic variations. Annu Rev Genet. 1995: 29: 777-807.

42 Ferrari, M, Cremonesi, L. Genotype-phenotype correlation in cystic fibrosis patients. Ann Biol Clin (Paris). 1996: 54: 235-41.

43 Balfour-Lynn, IM, Laverty, A, Dinwiddie, R. Reduced upper airway nitric oxide in cystic fibrosis. Arch Dis Child. 1996: 75: 319-22.

44 Gaston, B, Ratjen, F, Vaughan, JW, et al. Nitrogen redox balance in the cystic fibrosis airway: effects of antipseudomonal therapy. Am J Respir Crit Care Med. 2002: 165: 387-90. 


\section{CHAPTER 9.1}

\section{Volatile organic compounds in exhaled breath as a diagnostic tool for asthma in children}

JW Dallinga, CMHHT Robroeks, JJBN van Berkel, EJ Moonen, RWL Godschalk, Q Jöbsis, E Dompeling, EFM Wouters, FJ van Schooten

Clinical and Experimental Allergy 2010;40(1):68-76 


\section{Abstract}

\section{Background}

The correct diagnosis of asthma in young children is often hard to achieve, resulting in under-treatment of asthmatic children and over-treatment in transient wheezers.

The objectives were to develop a new diagnostic tool which better discriminates between asthma and transient wheezing and that leads to more accurate diagnosis and hence less under-treatment and over-treatment. A first stage in the development of such a tool is the ability to discriminate between asthmatic children and healthy controls. The integrative analysis of large numbers of volatile organic compounds in exhaled breath has the promise to discriminate between various inflammatory conditions of the respiratory tract.

\section{Methods}

From asthmatic children $(n=63)$ and healthy controls $(n=57)$ breath samples were obtained and analysed for volatile organic compounds (VOC) by gas chromatography-mass spectrometry. A total of 945 determined compounds were subjected to discriminant analysis to find those that could discriminate diseased from healthy children. A set of samples from both asthmatic and healthy children was selected to build a model that subsequently was used to predict the asthma or healthy status of a test group. This way the predictive value of the model could be tested.

\section{Results}

The discriminant analyses demonstrated that asthma and healthy groups are distinct from one another. A number of 8 components discriminated between asthmatic and healthy children with a $92 \%$ correct classification, achieving a sensitivity of $89 \%$ and a specificity of 95\%.

\section{Conclusion}

The results show that a limited number of VOC in exhaled air can well be used to distinguish children with asthma from healthy children. 


\section{Introduction}

Asthma is a frequently occurring chronic inflammatory disease in children world-wide. Although effective therapies are available, asthma control is still not optimal in many patients [1,2]. Until now, the criteria for the diagnosis and management of asthma are based on clinical observations and lung function tests $[3,4]$. However, symptoms may not always accurately represent the nature and extent of the underlying airway condition [5] and lung function tests are not definite in relation to airway inflammation [6-9]. Young children showing symptoms like coughing, wheezing and dyspnoea, may be suffering from various diseases of the respiratory tract, and it is therefore not always easy to reach the proper diagnosis [10-14]. New diagnostic approaches with the potential to make an early diagnosis of asthma possible, may not only greatly decrease under-diagnosis and undertreatment in true asthmatics, but also prevent over-treatment in transient wheezers.

In recent years non-invasive techniques that may be useful for the assessment of airway inflammation have been found in the analysis of exhaled breath. For instance, the collection and analysis of non-volatile compounds in exhaled breath condensate [10] and of volatile compounds such as nitric oxide (NO) [15-18] and carbon monoxide (CO) [19-21] in breath have been used to assess respiratory diseases. Fractional exhaled nitric oxide (FeNO) can be considered as a non-invasive marker of inflammation because its levels are elevated in children with asthma [22-24]. However, FeNO mainly represents eosinophilic inflammation, which may be present in different clinical conditions and therefore its discriminating power is limited $[25,26]$. Also, ethane and pentane which are detectable volatile organic compounds (VOC) in exhaled air have been related to oxidative stress as a result of inflammatory conditions [21, 27-28]. In all these approaches, single or small numbers of components are analysed and attempted to be used as diagnostic tools.

Already in the early 70s Pauling et al. [29] demonstrated the presence of a large variety of VOC in exhaled breath. In 1985 Gordon et al. [30] showed that within this large number of VOC profiles could be found that differentiate between subjects with lung cancer and healthy controls, indicating the use of such profiles as possible biomarkers of disease. More recently, Phillips and co-workers used discriminant analysis to select and measure 22 VOC from exhaled breath to classify subjects with and without lung cancer [31]. In a recent follow up study [32] they demonstrated that a VOC profile consisting of 9 components is capable to classify groups with and without lung cancer with a sensitivity of $85.1 \%$ and a specificity of $80.5 \%$.

VOC can be determined by collection of exhaled breath and quantitative analysis by thermal desorption-gas chromatography time-of-flight mass spectrometry (GC-TOF-MS). Recently we developed a highly accurate methodology to comprehensively analyze the entire range of $\mathrm{VOC}$ in exhaled air to search for unique profiles that can be used to identify certain patient groups [33]. The present study was set up to test the hypothesis that a variable way of sampling and a comprehensive chemical analysis technique in combination with adequate data processing and data analysis will provide a robust and easy to use tool for identifying asthma patients and healthy children based on their exhaled breath. We describe that the use of the totally non-invasive and easy-to-perform sampling method of exhaled air combined with the accurate measurement of a variety of VOC provides information concerning processes in the respiratory tract. Using discriminant analysis, we were able to select a limited number of compounds that discriminates asthmatic children from 
healthy controls and predicts to which group children belong, thus confirming the hypothesis. In a consecutive prospective study we will investigate the potency of this method to distinguish between healthy wheezers and asthmatic children.

\section{Methods}

\section{Study population}

120 children in the age of 5-16 years were included in the study. Of these children 63 were diagnosed with asthma and 57 were healthy controls. The asthmatic children were recruited from the outpatient clinic of the department of paediatric pulmonology of the University Hospital Maastricht. The asthma diagnosis was made by an experienced children's lung specialist on clinical grounds, following the international GINA-guidelines [3]. Appropriate therapy was prescribed by the patients own physician. All 63 patients used an inhaled bronchodilator on demand and 55 of them used an inhaled corticosteroid daily. 67 percent of this population had allergy as documented by radio allergosorbent test (RAST) class 2 or higher for at least one common airborne allergen. The 57 control subjects were recruited from the outpatient paediatric clinic of the University Hospital Maastricht. The ISAAC questionnaire was used to exclude a history of respiratory problems in these children [34]. In the control group enuresis and constipation were the main reasons for consultation.

Patients with diseases that might interfere with the results of this study, mentally retarded children and active smokers were excluded from the study. The two populations were comparable in age, height and weight. Characteristics of patients and controls are presented in table 1. Informed consent was obtained from all children and their legal representatives. The study was approved by the Ethics Committee of Maastricht University, clinical trial registration number NCT 00413140.

\section{Sampling}

The subjects were asked to exhale their breath in a resistance free plastic bag (Tedlar bag, SKC Itd., Dorset, UK). Three to four exhalations were sufficient to fill the 5 litre bag. No special provisions for the manner of exhalation, nor special directions concerning the diet of the subjects were given. The samples of both, patients and controls were collected in the same room, to prevent the occurrence of background bias. Therefore, and because of the data anaysis methods used (i.e. discriminant analysis) no background samples were collected. Within one hour of collection, the bag was emptied under pressure over a stainless steel two-bed sorption tube, filled with carbograph 1TD/Carbopack X (Markes International, Llantrisant, Wales, UK). Before and after loading the tubes were air-tight capped. The tubes were stored at room temperature until analysis.

\section{Analysis}

Our developed GC-TOF-MS methodology to accurately measure VOC and subsequent data handling was recently published and therefore the approach is summarized here shortly 
Table 1: Characteristics of the study population.

\begin{tabular}{|c|c|c|}
\hline & Asthma $(n=63)$ & Controls $(n=57)$ \\
\hline Age (mean \pm SD) & $10.8 \pm 3.1$ & $9.9 \pm 2.8$ \\
\hline Height & $144 \pm 17$ & $141 \pm 17$ \\
\hline Weight & $38.2 \pm 14.9$ & $34.8 \pm 10.9$ \\
\hline Gender male/female & 47 / 16 & $29 / 28+$ \\
\hline $\mathrm{FEV}_{1}(\%$ predicted $)$ & $96.2 \pm 18.2$ & $101.8 \pm 10.6$ \\
\hline $\mathrm{FEV}_{1} / \mathrm{VC}(\%)$ & $84.0 \pm 10.3$ & $88.2 \pm 7.0$ \\
\hline Height & $144 \pm 17$ & $141 \pm 17$ \\
\hline Weight & $38.2 \pm 14.9$ & $34.8 \pm 10.9$ \\
\hline Gender male/female & $47 / 16$ & $29 / 28+$ \\
\hline $\mathrm{FEV}_{1}(\%$ predicted) & $96.2 \pm 18.2$ & $101.8 \pm 10.6$ \\
\hline $\mathrm{FEV}_{1} / \mathrm{VC}(\%)$ & $84.0 \pm 10.3$ & $88.2 \pm 7.0$ \\
\hline Weight & $38.2 \pm 14.9$ & $34.8 \pm 10.9$ \\
\hline Gender male/female & $47 / 16$ & $29 / 28+$ \\
\hline Atopy & 42 (67\%) & \\
\hline \multicolumn{3}{|l|}{ Asthma severity * } \\
\hline - Intermittent & $15(24 \%)$ & \\
\hline - mild persistent & 12 (19\%) & \\
\hline - moderate persistent & $17(27 \%)$ & \\
\hline - severe persistent & $19(30 \%)$ & \\
\hline allergic rhinitis & $11(17 \%)$ & \\
\hline ICS use & 55 (87\%) & \\
\hline ICS daily dosis $(\mu \mathrm{g})$ & $576 \pm 357$ & \\
\hline
\end{tabular}

[33]. Analysis of the samples started with the release of the volatile compounds trapped on the sorption tubes by thermal desorption, using the Markes International Ultra-Unity automated thermal desorption equipment (Markes International, Llantrisant, Wales, UK). The gaseous mixture of released compounds was then split; $90 \%$ of the sample was recollected on a second identical sample tube and stored for an optional second analysis. $10 \%$ of the sample was loaded onto a cold $(5 \stackrel{\circ}{\circ})$ sorption trap, from which it was injected into a gas chromatograph (Trace GC, Thermo Fischer Scientific, Austin, Texas, USA) and analysed by time-of-flight mass spectrometry (Tempus Plus, Thermo Fischer Scientific, Austin, Texas, USA). For the GC-MS measurements the following conditions were used: column: Restek RTX-5ms, $30 \mathrm{~m} \times 0.25 \mathrm{~mm}$ ID, coated with $1.0 \mu \mathrm{m}$ HP-5 phase; helium was used as the carrier gas at a flow rate of $1.5 \mathrm{~mL} / \mathrm{min}$. The temperature of the gas chromatograph

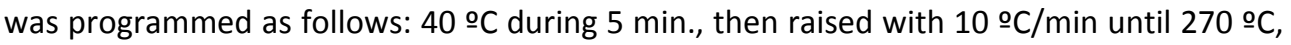
which temperature was maintained for $5 \mathrm{~min}$. The mass spectrometer was set at a scan range of 35-350 AMU and scanned 5 times per second. The complete analytical procedure, including sampling, storage and instrumental analysis was tested for reproducibility. The results of these tests support the methods used and were published elsewhere [33]. 


\section{Data analysis}

The GC-MS chromatograms of the breath samples of the 120 subjects involved in this research were recorded and retention times were normalized by calculating retention indices, relative to toluene and lining up using easily recognizable component peaks, to correct for chromatographic drifting. Parts of the chromatograms that occurred at a retention index $<0.15$ and at a retention index $>2.8$ were removed from the chromatograms, because of unreliable data from these parts, due to noisy mass spectra at the beginning of the chromatograms and column bleeding at the end of each run. The remaining data were carefully transformed to Excel files, containing almost 6000 different chromatographic peaks, determined by retention time and mass spectrum and combined with a relative intensity. Intensities below the detection limit were set at $0 \%$. The detailed descriptions of the data handling procedures can be found elsewhere [33]. We applied no background subtraction, nor any corrections for the diet and the way of exhaling in the sample bag of patients and controls. We realise that this way some noise on the results may be generated, but also that strict rules with regard to exhalation, diet and background subtraction would lead to less robust and less easy sampling, and probably increase of misclassifications. For, background corrections are not only hampered by the complex interdependence of excretion and uptake of VOC, it also increases the measuring fault. Furthermore, data anaysis by discriminant analysis provides the tool to eliminate any random occurring variation, for instance due to diet (there is no reason to expect a difference in diet for patients and controls), breathing (when the subjects are entitled to exhale at their own choice there is no reason to expect differences between the two groups as well) and background (the samples of patients and controls were taken divided over the sampling period and in the same room). It is our goal to select only those compounds that contain information regarding diseased versus healthy status and are independent from other endogenous (gender, age, weight, genetic background) or exogenous factors (ambient air, diet, medication). Before data analysis was executed, all peaks that occurred in less than $8 \%$ of the samples (meaning less than ten times in this study) were removed [33,35]. This resulted in a final data matrix, containing 945 peaks and 120 subjects. This matrix was normalized by adjusting the sum of all peak intensities in each subject at $100 \%$. This way the influence of the infrequently appearing peaks could be diminished. The resulting data were analysed by a stepwise discriminant analysis, using SPSS (SPSS13.0 for windows, SPSS Inc. Chicago, Illinois, USA). The discriminant analyses were performed using a twentyfold cross-over approach. In this method all but 5 percent of the chromatograms (in this study 5 percent equals 6 samples) are used to construct the discriminant function, which is subsequently used to predict to which group the ones left out belong. This is repeated 20 times, until all samples have once been classified. The discriminant functions obtained in this way are based on many (60-80) components and are optimal in terms of differentiation between both groups, but are not necessarily the best predictors for unknown samples, because of obvious overfitting. Therefore, the number of variables is gradually diminished, until a reasonable small number of components remain with sufficient prediction power. The reduction in components is accomplished by starting with the original large set of components from the first discriminant analyses, and then repeating the analysis with the least informative (the last) component of the results left out. 


\section{Results}

The GC-MS analyses resulted in 120 chromatograms, representing the 63 asthmatic patients and the 57 controls. For the discriminant analysis, two groups were defined: group 0 consisted of the samples of the 57 controls, group 1 of the samples of the 63 asthma patients. This discriminant analysis resulted in a discriminant function comprising 72 components that classified all samples correctly, i.e. $100 \%$ sensitivity and $100 \%$ specificity. In the performed discriminant analysis all peaks that enhance the descriptive model were added, but for using the model as a reliably predictive tool the number of components is far too large, and leads to overfitting. Therefore, we gradually diminished the number of components to build the model, and registered the sensitivity and selectivity of the analyses as function of the number of components involved. In this way by using 22 components still a $100 \%$ correct classification was obtained. A sensitivity of $95 \%$ at a specificity of $98 \%$ could be achieved with 14 components, with a number of 8 components still a $92 \%$ correct classification was achieved, with a sensitivity of $89 \%$ and a specificity of $95 \%$, whereas 6 components were sufficient to reach a sensitivity and a specificity of both $84 \%$.

Within this data set validation was performed using 20-fold cross validation: randomly 6 subjects (5\%) were selected of the 120 children involved in this investigation. Subsequently the remaining 114 chromatograms were used to build a model set and a discriminant analysis was performed. Next, the 6 removed chromatograms were added as a test set and their classification into healthy or diseased was determined to test the model based on 114 subjects. This analysis was repeated to a total of 20 times, so that all 120 chromatograms were once part of a test set. Ultimately this approach leads to 20 (slightly) different models predicting the respective unknowns. A $100 \%$ sensitivity and specificity was reached when the number of compounds in the discriminant analysis was 25 or higher. Reducing the number of compounds involved in the analysis to 8 compounds still resulted in $89 \%$ sensitivity at $95 \%$ specificity. The relation between number of components used and the obtained selectivity and specificity using model sets of 114 chromatograms and test sets of 6 chromatograms has been visualized in Figure 1a. Based on GC retention time and mass spectrometric information, we were able to identify the most important compounds that discriminate the asthma patients from the healthy controls. These components are presented in Table 2.

The mean relative intensities of the first ten components that were used to generate the discriminant function that discriminates between asthmatic and healthy children are presented in Figure 2.

In a separate data analysis experiment, we compared the chromatograms of the 42 atopic asthma patients with those of the 57 controls. Discriminant analysis using the 20 -fold cross validation method showed $99 \%$ correct classification down to 25 components used. Using 7 components still gave a $90 \%$ sensitivity and a $89 \%$ specificity. The relationship between the number of components used in the analyses and the sensitivity and specificity is presented in Figure $1 \mathrm{~b}$.

Finally, the chromatograms of the 42 atopic asthma patients and the 21 non-atopic asthma patients were compared using discriminant analyses. Our method, excluding $5 \%$ of the chromatograms from building the discriminant function and introducing these as unknowns, repeated 20 times, resulted in $100 \%$ sensitivity and specificity using 15 components. When 7 components were used to determine the discriminant function the speci- 
ficity and selectivity were $95 \%$ and $84 \%$, respectively, for the cross validation experiment. The relations between number of components involved in the analyses and the sensitivity and specificity are presented in Figure $1 \mathrm{c}$.

Table 2: Overview of the most important components used to discriminate asthma patients from healthy controls.

\begin{tabular}{ll}
\hline number* & Component \\
\hline 1 & (branched) hydrocarbon $\left(\mathrm{C}_{13} \mathrm{H}_{28}\right)$ \\
2 & carbondisulfide $\left(\mathrm{CS}_{2}\right)$ \\
3 & 1-penten-2-on \\
4 & butanoic acid \\
5 & 3 -(1-methylethyl)-benzene \\
6 & (branched) hydrocarbon $\left(\mathrm{C}_{13} \mathrm{H}_{28}\right)$ \\
7 & unsaturated hydrocarbon $\left(\mathrm{C}_{15} \mathrm{H}_{26}\right)$ \\
8 & benzoic acid \\
9 & p-xylene \\
10 & (branched) hydrocarbon $\left(\mathrm{C}_{11} \mathrm{H}_{24}\right)$ \\
\hline$*$ Numbers represent the order in which the components were included in the discriminant analysis.
\end{tabular}




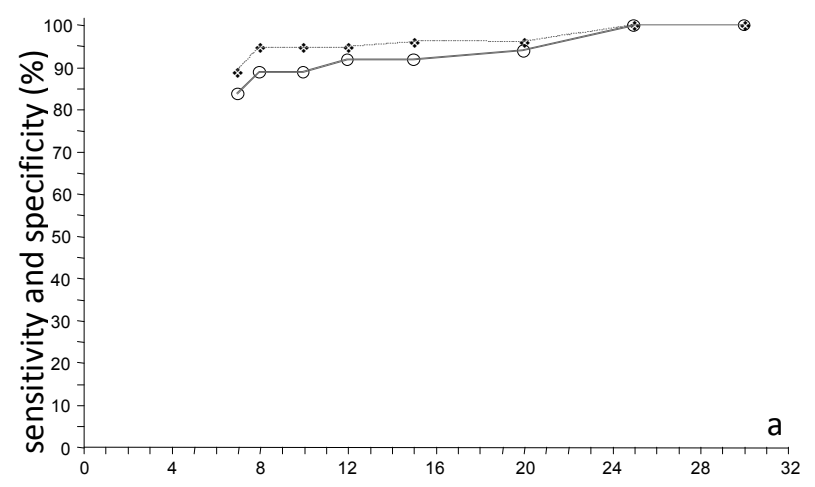

Figure 1: Course of sensitivity and specificity as function of the number of variables involved in the discriminant analyses.
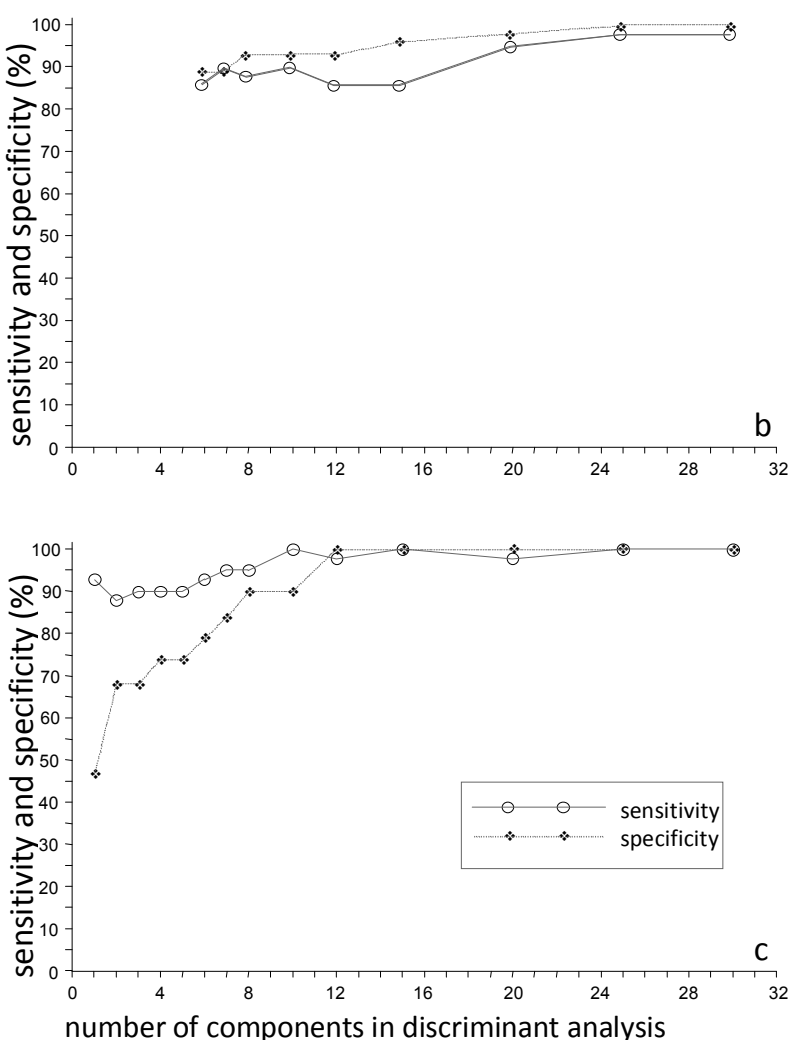

a. analysis of asthma vs healthy controls, using 114 samples for the model and 6 for the test set, 20 times repeated; b. analysis of atopic asthma vs healthy controls, using 95 samples for the model and 5 for the test set, 20 times repeated; c. analysis of atopic vs non-atopic asthma, using 60 samples for the model and 3 for the test set, 20 times repeated. 


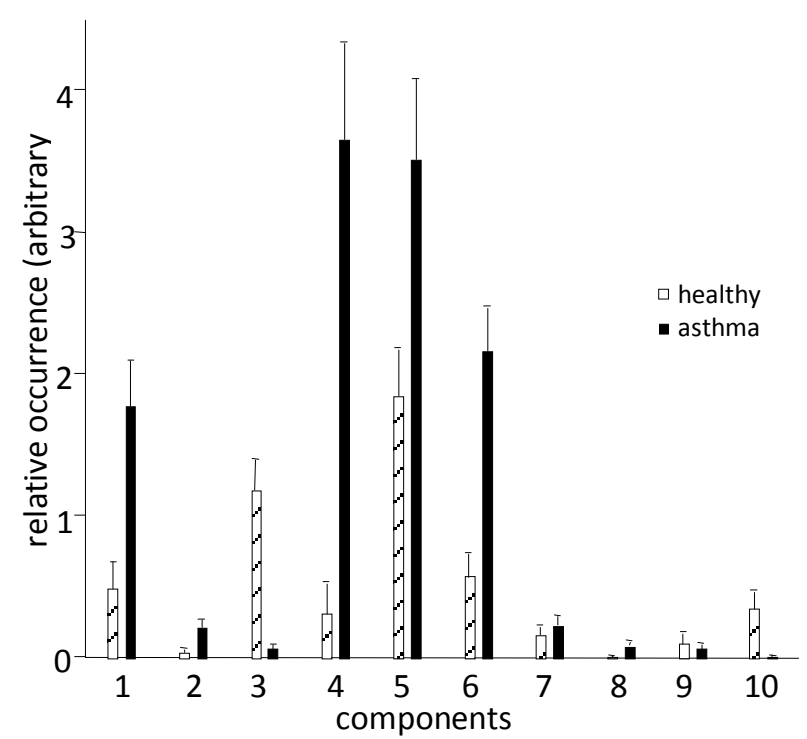

Figure 2: Mean relative intensity (+ standard error) of the ten components that are used to generate the discriminant function that discriminates children with asthma from healthy controls. The number represents the order in which the components were included in the discriminant analysis (see also Table 2).

\section{Discussion}

Inflammation plays a critical role in many physiological changes of the body including inflammatory lung diseases like asthma. Inflammation is accompanied by oxidative stress and subsequently lipid peroxidation and during this process polyunsaturated fatty acids are converted into VOC that are secreted via the lungs. Hundreds of different VOC are present in human breath, and their relative concentrations may alter in the presence of the disease. Recently we developed an approach to comprehensively analyze the entire range of VOC in exhaled air to search for unique profiles that can be used to identify certain patient groups or exposed populations [33]. In the present study we demonstrate the suitability of our highly accurate methodology tested on groups of asthmatic and nonasthmatic children. The results showed that discriminant analysis of exhaled breath chromatograms of 63 asthmatic children and 57 controls using 22 variables led to correct classification and cross validation of all subjects. Of course, it can not be excluded that this number of components used for the classification might partly be based on statistical artefacts. However, reducing the number of components used to describe the discriminant function to 6 components clearly showed that the discriminating information present in the chromatograms was mainly based on real information with regard to the absence or presence of the disease, rather than on coincidence. This became even more evident, when the discriminant analyses was performed on $95 \%$ of the chromatograms (training set), leaving the remaining $5 \%$ (test set) as unknowns to be classified using the training model. In this case 8 components were sufficient to reach $89 \%$ sensitivity at $95 \%$ specificity. As shown in Figure 2, the majority of the discriminating compounds (no. 1, 2, 4, 5, 6, 7, 8) are enhanced but also some compounds (no. 3, 9, 10) are diminished in the exhaledbreath of asthmatic children. Athough a decreased amount in the patients seems unexpected, the reason may be found in the complex equilibrium of formation and removal of VOC in the body. The hypothesis is that the inflammation driven oxidative stress responsi- 
ble for oxidizing polyunsaturated fatty acids abundantly present in cellular membranes, may lead to a gamma of breakdown products excreted as VOC. So it is obvious that the relative composition of $\mathrm{VOC}$ in exhaled breath can change as a result of the disease, and this change can be either an increase or a decrease of certain components. Indeed we observed a considerable number of components that are significantly higher in patients compared to controls but these compounds are not implemented into the optimized discriminating model due to their limited discriminatory power. On the other hand a decline in certain VOCs may occur since especially the longer chain hydrocarbons are further oxidized into smaller compounds due to enhanced oxidative stress and consequently their amounts are decreased in exhaled air of patients. It appeared that the absence of a number of these long chained VOCs is crucial for the diagnostic ability. Apart from the oxidative stress hypothesis explaining changing VOC composition in patients, an alternative reason may be that asthma affected lungs are remodeled resulting in altered gas exchanges over the blood lung barrier.

When we will be able to increase the levels of sensitivity and specificity by increasing the number of samples in the data set, this method may appear valuable for diagnosis of asthma in children below 6 years of age, being an age group in which a definitive diagnosis based on doctors' examination is still difficult. Since under-diagnosis and under-treatment in true asthmatics and over-treatment in transient wheezers are medically and in terms of public health undesirable, an earlier diagnosis would be valuable. The new diagnostic technique described in this study has the potency to make an early diagnosis of asthma possible and therefore a prospective study, aimed to find a relationship between the composition of exhaled air of children at young age, and definite diagnosis of the same children at an age of about 6 years, is worthwhile to be performed. Furthermore, the experiments in which the exhaled breath samples of atopic asthma patients are compared with those of healthy controls showed the potency of this method to discriminate atopic asthma patients from healthy persons. The results of the analyses in which breath samples of atopic and non-atopic asthma patients were compared, seem to indicate that not only healthy from diseased can be separated this way, but that it is also possible to differentiate one form of disease from another. At this stage we did not identify the components involved in the discrimination between atopic asthma patients and healthy controls and non-atopic patients. Resolving the chemical identity of all relevant components will be enormously time consuming: some of the components can readily be identified, but for many there are various alternatives thinkable. To determine the identities, registration of GC retention times of (all) possible candidate compounds is necessary. The relevance of this identification largely depends on the probability to be able to link the components' structure to their respective biological origin.

We are aware of a possible pit-fall in the discrimination between the asthma patients and the healthy controls: because most patients are treated with inhalation steroids and the controls self-evidently not, the discrimination might (partly) be based on remains as for instance metabolites of the medication. However, the medication used was rather diverse and their chemical structures and metabolites were not reflected in the discriminating components. Furthermore, 8 of the 63 asthmatic subjects included were not on treatment and still well classified. Next to that, the fact that the electronic nose does not discriminate treated asthma from non-treated asthma seems to indicate that treatment does not interfere with the discrimination [36]. At the other hand, the medication may quite as well 
have reduced the differences between the breath samples of patients and healthy controls, by suppressing the illness and the accompanying inflammation and thus inflammation products. Therefore, it can be speculated that comparing children with and without asthma, but all (still) without medication, may demonstrate even more difference in exhaled breath profiles than we found in this study. By sampling breath from all children at age 3-4 with respiratory complaints, and comparing the VOC contents of these samples after a reliable diagnosis has been set (typically at age 6) we hope to be able to predict with sufficient sensitivity and specificity the outcome (i.e. asthma positive or negative) of young children with respiratory complaints. The results of the present study may provide a necessary, but of course not yet sufficient step towards this goal.

\section{References}

1. Rabe KF, Adachi M, Lai CK, Soriano JB, Vermeire PA, Weiss KB, Weiss ST. Worldwide severity and control of asthma in children and adults: the global asthma insights and reality surveys. J Allergy Clin Immunol 2004;114:40-47.

2. Wolfenden LL, Diette GB, Krishnan JA, Skinner EA, Steinwachs DM, Wu AW. Lower physician estimate of underlying asthma severity leads to undertreatment. Arch Intern Med 2003;163:231-236.

3. Global Initiative for asthma (GINA). Pocket guide for asthma management and prevention in children. National Institute of Health, National Heart, Lung, and Blood Institute 2002.

4. Kharitonov SA, Barnes PJ. Exhaled markers of pulmonary disease. Am J Respir Crit Care Med 2001;163:16931722.

5. Kharitonov SA. Exhaled markers of inflammatory lung diseases: ready for routine monitoring? Swiss Med Wkly 2004;134:175-192.

6. Silvestri M, Sabatini F, Sale R, Defilippi AC, Fregonese L, Battistini E, Biraghi MG, Rossi GA. Correlations between exhaled nitric oxide levels, blood eosinophilia, and airway obstruction reversibility in childhood asthma are detectable only in atopic individuals. Pediatr Pulmonol 2003;35:358-363.

7. Sutherland ER, Martin RJ, Bowler RP, Zhang Y, Rex MD, Kraft M. Physiologic correlates of distal lung inflammation in asthma. J Allergy Clin Immunol 2004;113:1046-1050.

8. Leung TF, Wong GW, Ko FW, Lam CW, Fok TF. Clinical and atopic parameters and airway inflammatory markers in childhood asthma: a factor analysis. Thorax 2005;60:822-826.

9. Rosias PP, Dompeling E, Dentener MA, Pennings HJ, Hendriks HJ, Van lersel MP, Jobsis Q. Childhood asthma: Exhaled markers of airway inflammation, asthma control score, and lung function tests. Pediatr Pulmonol 2004;38:107-114.

10. Rosias PPR, Dompeling E, Hendriks HJE, Heijnens JWCM, Donckerwolcke RAMG, Jöbsis Q. Exhaled breath condensate in children: pearls and pitfalls. Pediatr Allergy Immunol 2004;15:4-19.

11. Chung KF. Chronic inflammation in asthma. Eur Respir Rev 1998;8:999-1006.

12. Helms PJ. Issues in pediatric asthma. Pediatr Pulmonol 2001;S21:49-56.

13. Hunt J. Exhaled breath condensate: an evolving tool for noninvasive evaluation of lung disease. J Allergy Clin Immunol 2002;110:28-34.

14. Kharitonov SA, Barnes PJ. Biomarkers of some pulmonary diseases in exhaled breath. Biomarkers 2002;7:132.

15. Ashutosh K. Nitric oxide and asthma: a review. Curr Opin Pulm Med 2000;6:21-5.

16. Baraldi E, Pasquale F, Bonetto G, Carraro S, Zanconat S. Exhaled gas analysis and airway inflammation. Pediatr Pulmonol 2004;Suppl.26:16-19.

17. Cicutto LC, Downey GP. Biological markers in diagnosing, monitoring, and treating asthma: a focus on noninvasive measurements. AACN Clin Issues 2004;15:97-111.

18. Malmberg LP, Pelkonen AS, Haahtela T, Turpeinen M. Exhaled nitric oxide rather than lung function distinguishes preschool children with probable asthma. Thorax 2003;58:494-499. 
19. Paredi P, Shah PL, Montuschi P, Sullivan P, Hodson ME, Kharitonov SA, Barnes PJ. Increased carbon monoxide in exhaled air of patients with cystic fibrosis. Thorax 1999;54:917-920.

20. Antuni JD, Kharitonov SA, Hughes D, Hodson ME, Barnes PJ. Increase in exhaled carbon monoxide during exacerbations of cystic fibrosis. Thorax 2000;55:138-142.

21. Paredi P, Kharitonov SA, Barnes PJ. Analysis of expired air for oxidation products. Am J Respir Crit Care Med 2002;166:S31-S37.

22. Kharitonov SA, Barnes PJ. Clinical aspects of exhaled nitric oxide. Eur Respir J 2000;16:781-792.

23. Nelson BV, Sears S, Woods J, Ling CY, Hunt J, Clapper LM, Gaston B. Expired nitric oxide as a marker for childhood asthma. J Pediatr 1997;130:423-427.

24. Frank TL, Adisesh A, Pickering AC, Morrison JF, Wright T, Francis H, Fletcher A, Frank PI, Hannaford P. Relationship between exhaled nitric oxide and childhood asthma. Am J Respir Crit Care Med 1998; 158:10321036.

25. Prasad A, Langford B, Stradling JR, Ho LP. Exhaled nitric oxide as a screening tool for asthma in school children. Respir Med 2006;100:167-173.

26. Gratziou C, Lignos M, Dassiou M, Roussos C. Influence of atopy on exhaled nitric oxide in patients with stable asthma and rhinitis. Eur Respir J 1999;14:897-901.

27. Olopade CO, Zakkar M, Swedler WI, Rubenstein I. Exhaled pentane levels in acute asthma. Chest 1997;111:862-865.

28. Paredi P, Kharitonov SA, leak D, Shah PL, Cramer D, Hodson ME, Barnes PJ. Exhaled ethane is elevated in cystic fibrosis and correlates with carbon monoxide levels and airway obstruction. Am J Respir Crit Care Med 2000;161:1247-1251.

29 Pauling L, Robinson AB, Teranishi R, Cary P. Quantitative analysis of urine vapor and breath by gas-liquid partition chromatography. Proc Natl Acad Sci USA 1971;68:2374-6.

30. Gordon SM, Szidon JP, Krotoszynski BK, Gibbons RD, O'Neill HJ. Volatile organic compounds in exhaled air from patients with lung cancer. Clin Chem 1985;31:1278-82.

31. Phillips M, Gleeson K, Hughes JM, Greenberg J, Cataneo RN, Baker L, McVay WP. Volatile organic compounds in breath as markers of lung cancer: a cross-sectional study. Lancet 1999;353:1930-3.

32. Phillips M, Altorki N, Austin JH, Cameron RB, Cataneo RN, Greenberg J, Kloss R, Maxfield RA, Munawar MI, Pass HI, Rashid A, Rom WN, Schmitt P. Prediction of lung cancer using volatile biomarkers in breath. Cancer Biomark 2007;3:95-109.

33. Van Berkel JJBN, Dallinga JW, Möller GW, Godschalk RWL, Moonen EJ, Wouters EFM, Van Schooten FJ. Development of accurate classification method based on the analysis of volatile organic compounds from human exhaled air. J Chrom B 2008;861:101-7.

34. Variations in the prevalence of respiratory symptoms, self-reported asthma attacks and use of asthma medication in the European Community Respiratory Health Survey (ECRHS). Eur Respir J 1996; 9:687-95.

35. Sanchez JM, Sacks, RD. 2006. Development of a multibed sorption trap, comprehensive two-dimensional gas chromatography, and time-of-flight mass spectrometry system for the analysis of volatile organic compounds in human breath. Anal Chem 2006;78:3046-54.

36. Dragonieri S, Schot R, Mertens BJA, Le Cessie S, Gauw SA, Spanevello A, Resta O, Willard NP, Vink TJ, Rabe $\mathrm{KF}$, Bel EH, Sterk PJ. An electronic nose in the discrimination of patients with asthma and controls. J Allergy Clin Immunol 2007;120;856-62. 



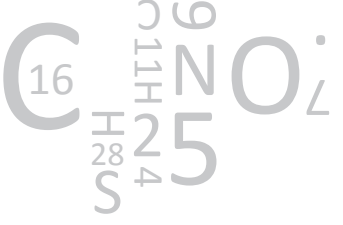

\section{CHAPTER 9.2}

\section{Metabolomics of volatile organic compounds in cystic fibrosis patients and controls}

CMHHT Robroeks, JJBN van Berkel, JW Dallinga, Q Jöbsis, LJI Zimmermann, JJE Hendriks, EFM Wouters, CPM van der Grinten, KDG van de Kant, FJ van Schooten, E Dompeling

Pediatric Research, published ahead of print 


\section{Abstract}

\section{Background}

In cystic fibrosis (CF), airway inflammation causes an increased production of reactive oxygen species, responsible for degradation of cell membranes. During this process, volatile organic compounds (VOCs) are formed. Measurement of VOCs in exhaled breath of CF patients, may be useful for the assessment of airway inflammation. This study investigates whether 'metabolomics' of VOCs could discriminate between CF and controls, and between CF patients with and without Pseudomonas colonization.

\section{Methods}

105 Children (48 CF, 57 controls) were included. After collection, samples were transferred onto tubes containing active carbon in order to adsorb and stabilize VOCs. Samples were analyzed by gas chromatography-time of flight-mass spectrometry to assess VOC profiles.

\section{Results}

1099 VOCs had a prevalence of at least 7\%. By using 22 VOCs, a $100 \%$ correct identification of CF patients and controls was possible. With 10 VOCs, $92 \%$ of the subjects were correctly classified. The reproducibility of VOC measurements with a one hour interval was very good (match factor $0.90 \pm 0.038$.).

\section{Conclusion}

'Metabolomics' of VOCs in exhaled breath was possible in a reproducible way. This new technique was not only able to discriminate between CF patients and healthy controls, but also between CF patients with or without Pseudomonas colonization. 


\section{Introduction}

Airway inflammation plays a central role in the pathophysiology of various chronic lung diseases, such as cystic fibrosis (CF), asthma, and chronic obstructive pulmonary disease (COPD). Diagnosing CF is possible at any age, but it is far less simple to diagnose early pulmonary disease in CF patients, particularly in young children. It is known there is a poor correlation between airway inflammatory processes and respiratory symptoms (1). Airway infection and inflammation can be present before clinical symptoms are evident. Thus monitoring of airway inflammation/oxidative stress in cystic fibrosis may be useful in clinical practice.

There has been an increasing interest in non-invasive assessment of airway inflammation and oxidative stress in chronic lung disease. The collection of broncho-alveolar lavage fluid or lung biopsies is invasive, and therefore cannot be applied very easily in children. Noninvasive techniques include measurement of non-volatile inflammatory markers in exhaled breath condensate, and measurement of volatile inflammatory markers in exhaled breath. Fractional exhaled nitric oxide (FeNO), carbon monoxide (CO), ethane and pentane are the most studied volatile markers $(2,3)$, of which FeNO is most standardized (4). In contrast to assessments of pre-selected inflammatory markers, it is possible to assess profiles of volatile organic compounds (VOCs) in exhaled air.

An increased production of reactive oxygen species (ROS) is caused by the influx of leukocytes, which continuously produce ROS, leading to an imbalance between oxidants and antioxidants (oxidative stress) (5-8). ROS can degrade cell membranes by lipid peroxidation. During this process, VOCs are formed as a result of the degradation of polyunsaturated fatty acids. Endogenous VOCs can be divided in hydrocarbons, oxygen or sulphur containing compounds, and nitrogen containing substances (9).

Analysis of VOCs in exhaled air is possible by using a gas chromatograph - time of flight mass spectrometer (GC-TOF-MS). This technique is highly sensitive and capable of detecting a wide range of VOCs. A predictive model employing nine VOCs was sufficiently sensitive and specific to be considered as a screening tool for lung cancer (10).

The aim of this study was to investigate whether metabolomics of VOCs in exhaled breath was able to discriminate between young subjects with CF and healthy controls, and between subgroups of CF patients (with or without Pseudomonas colonization). It is well known that Pseudomonas colonization of the lung is associated with a less favorable prognosis of CF. The reproducibility of the method, as well as the nature and background of the most discriminating compounds were studied.

\section{Methods and materials}

\section{Subjects}

105 Subjects aged 5-25 years were included in this cross-sectional study: 48 subjects with a CF diagnosis, and 57 control persons. The CF population was recruited from the outpatient clinic of the University Hospital Maastricht, the Netherlands. CF was defined as a combination of typical clinical features, and an abnormal sweat test (Chloride $>60$ $\mathrm{mmol} / \mathrm{L}$ ). Children with CF and/or their parents completed the Shwachman-Kulczycki 
questionnaire, enabling classification of the CF disease status (11). The control group consisted of children without any (history of) respiratory problems, as confirmed by the ISAAC questionnaire (12). 36 (63\%) Of control children were recruited from primary schools, whereas $21(37 \%)$ subjects were included at the outpatient clinic where enuresis nocturna and constipation were the initial reasons for consultation. At the moment of this study, these children were all stable with no signs of infection or any somatic disturbance. We checked the homogeneity of the control group by comparing children recruited from primary schools and outpatient clinic and found no significant differences in VOC patterns between these children (One-way ANOVA tests, p-values>0.108). Exclusion criteria for both CF and control children were: 1) Patients with mental retardation; 2) Active smokers; 3) (Congenital) heart disease; 4) Technical inability to perform the measurements; 5) Patients with an acute respiratory infection.

Informed consent to participate was obtained from the parents of all children participating in this study. The Ethics Committee of the Maastricht University approved this study. The clinical trial registration number is NCT 00413140.

\section{Exhaled breath analysis}

\section{Sampling}

Subjects were asked to exhale into a resistance free polycarbonate (plastic) bag (Tedlar bag, SKC Itd., Dorset, UK). Severe physical exercise before the test was not permitted. At least three exhalations were necessary to fill the 5-liter bag. Within one hour after collection of the sample, the bag was emptied over a stainless steel two-bed sorption tube, filled with carbograph 1TD/Carbopack X (Markes International, Llantrisant, Wales, UK). Before and after loading, the tubes were airtight capped. The desorption tubes were stored at room temperature until analysis.

\section{Analysis}

Analysis of the samples started with releasing the volatile compounds trapped on the sorption tubes by thermal desorption, using the Markes International Ultra-Unity automated thermal desorption equipment (Markes International, Llantrisant, Wales, UK). The gaseous mixture of released compounds was then split; $90 \%$ of the sample was recollected on a second sorption tube and stored for a possible second analysis, $10 \%$ of the sample was loaded onto a cold sorption trap (5 으, Markes U-T2GPH: general purpose hydrophibic trap, designed for sampling VOC C4-C32), from which it was injected into a gas chromatograph (Trace GC, ThermoFischer Scientific, Austin, Texas, USA) and analyzed by time-of-flight mass spectrometry (Tempus Plus, ThermoFischer Scientific, Austin, Texas, USA). For the GC-MS measurements the following conditions were used: column: Restek RTX-5ms, 30m x $0.25 \mathrm{~mm}$ ID; helium was used as the carrier gas at a flow rate of 1.5 $\mathrm{mL} / \mathrm{min}$. Transferline temperature was 250 ㅇ․ The temperature of the gas chromatograph was programmed as follows: $40 \stackrel{\circ}{\circ}$ during $5 \mathrm{~min}$, then raised with $10 \mathrm{o} / \mathrm{min}$ until a final maximum temperature of $270 \stackrel{\circ}{\circ}$. In the final step this temperature was maintained for 5 $\mathrm{min}$. The mass spectrometer was set as follows: Ionizing energy $70 \mathrm{eV}$, source temperature $200 \stackrel{\circ}{ } \mathrm{C}$ at a scan of $5 \mathrm{~Hz}$. 


\section{Data analysis}

The GC-MS chromatograms of the breath samples of the 105 subjects involved in this research were recorded and corrected for retention time differences, using retention indices, and lining up of easily recognizable component peaks. Corrected retention times were based on 6-10 common components with high intensities (e.g. acetone, toluene, phenol and benzene). The parts of the chromatograms that occurred at a retention index $<0.15$ and at a retention index $>2.8$ were removed from the chromatograms, because of unreliable data from these parts, due to noisy mass spectra at the beginning of the chromatograms and column bleeding at the end of the run. The resulting data files were carefully combined. This was done by means of combination of identical compounds from different samples. Compounds were combined based on similarity of mass spectra and retention times. This resulted in a final database file containing almost 6000 different chromatographic peaks. The total surface below the breathogram was set at $100 \%$. The intensity of a specific VOC corresponds with the surface below that peak. The resulting database was used for statistical analysis.

\section{Data mining}

To determine which compounds were of interest with regard to the classification of CF patients and controls, we used stepwise discriminant analysis, using SPSS (SPSS Inc. Chicago, Illinois, USA). An attribute (VOC) was only included in the discriminant analysis if its prevalence was $7 \%$ or more within the study population. Discriminant analysis was assessed with the grouping variables 'diagnosis' ( $C F n=48$, controls $n=57$ ), and 'positive Pseudomonas aeruginosa cultures' (yes $n=23$, no $n=17$ ). To exclude bias by different $C F$ treatments (use of an antibiotic, a corticosteroid or DNase), VOC patterns of patients with or without this treatment were compared. The homogeneity of the control group was assessed by comparing VOC patterns between children selected at the primary school versus the subjects recruited at the outpatient clinic. The quantification of the similarity between the VOC profiles was done by means of calculation of a 'distance measure' (dot product rule) or match factor, as been described in detail by Stein et al (13). This distance measure is based on the similarity of the entire raw chromatogram. A value of ' 1 ' denotes identical samples, the lower the value the lesser the degree of similarity. Values of 0.8 and over can be characterized as very good agreement (13).

\section{Lung function tests}

Lung function tests were performed after exhaled breath sampling. Bronchodilator medication was stopped prior to lung function testing: short-acting bronchodilators at least 8 hours before, and long-acting bronchodilators at least 36 hours before the test. Dynamic spirometry was performed by means of a pneumotachograph (Flowscreen', Viasys, Hoechberg, Germany), with measurement of forced expiratory volume in 1 second (FEV $)_{1}$ and forced vital capacity (FVC), according to the standards of the European Respiratory Society (14). The highest values of $\mathrm{FEV}_{1}$ and FVC of three forced expiratory manoeuvres were used for data analysis. In CF patients, the bronchodilating response was assessed 15 minutes after administration of a short-acting bronchodilator (salbutamol 400 microgram) and expressed as the increase in $\mathrm{FEV}_{1}$ compared to the predicted value of $\mathrm{FEV}_{1}$. In addi- 
tion, in the CF population, static lung volumes, residual volume (RV), intra-thoracic gas volume (ITGV) and total lung capacity (TLC), were assessed by body plethysmography (Viasys, Hoechberg, Germany).

\section{Statistical Analysis}

All data are expressed as mean $\pm \mathrm{SE}$. A normal distribution was present for all patient characteristics. Therefore, two-sided independent-sample T-tests were used for the analysis of the subject characteristics. P-values of $<0.05$ were considered statistically significant. To analyze the VOC profiles, 10 times cross-validation with multiple discriminant analysis was used, to minimize type III errors (15). Of 10 subsamples, 9 subsamples are used as a training set and the remaining single subsample was used to test the model (validation set). The cross-validation process was repeated 10 times, with each of the 10 subsamples used once as the validation data. The 10 results were averaged to produce a single estimation. Discriminant analysis was applied in order to: 1) investigate differences among groups, 2) determine the most parsimonious way to distinguish among groups, 3) discard variables which are of little interest related to group distinctions, 4) classify cases into groups, and 5) test theory by observing whether cases are classified as predicted.

\section{Reproducibility and influence of breathing pattern}

The influence of the breathing pattern (unforced breathing pattern versus hyperventilation) on VOC profiles, and the variation in VOCs across the day and between days was analyzed in 15 healthy control children aged 5-15 yrs (mean \pm SD, 15.5 \pm 3 yrs). Sampling of VOCs was applied during an unforced breathing pattern (conform the entire study population), during hyperventilation, after one hour, and after one day.

\section{Power analysis}

We assume a minimal sensitivity of at least $70 \%$ for a potentially valuable inflammatory marker. The standard error of the sensitivity and the specificity of an inflammatory marker will be less than $5 \%$ when 100 children ( 50 children with CF and 50 controls) are recruited for this study.

\section{Results}

\section{Population characteristics}

Subjects with CF were characterized by a light to moderate airway obstruction, airtrapping, and a mild restrictive impairment in comparison with controls (Table 1 and 2). In the CF group, $49 \%$ of subjects had positive cultures with Pseudomonas aeruginosa in the past 2 years, and 8 persons (17\%) showed a history of allergic bronchopulmonary aspergillosis (ABPA). The mean \pm SE time between sputum collection and breath sample collection was $50 \pm 62$ days. Patients were mainly treated with antibiotics, antacids, DNase, and corticosteroids (Table 2). 
Table 1: Clinical characteristics of the study populations.

\begin{tabular}{|c|c|c|c|c|}
\hline & & Cystic fibrosis & & Control \\
\hline & & $N=48$ & & $N=57$ \\
\hline Age & yrs & $13.0 \pm 0.6$ & * & $9.9 \pm 0.4$ \\
\hline Height & $\mathrm{cm}$ & $148.3 \pm 2.9$ & & $141.5 \pm 2.3$ \\
\hline Weight & $\mathrm{kg}$ & $38.6 \pm 2.1$ & & $34.8 \pm 1.5$ \\
\hline Sex & $M / F$ & $27 / 21$ & & $29 / 28$ \\
\hline $\mathrm{FEV}_{1} \%$ predicted & & $75.5 \pm 3.8$ & $*$ & $101.4 \pm 1.5$ \\
\hline $\mathrm{FEV}_{1} / \mathrm{VC} \%$ & & $76.7 \pm 1.8$ & * & $88.1 \pm 0.9$ \\
\hline
\end{tabular}

Abbreviations: $\mathrm{M}$, male; $\mathrm{F}$, female; $\mathrm{FEV}_{1}$, forced expiratory volume in 1 second; $\mathrm{VC}$, vital capacity; Data are given as mean \pm standard error $(\mathrm{SE}) ;{ }^{*} \mathrm{P}<.05$, cystic fibrosis vs control.

Table 2: Additional characteristics of the cystic fibrosis population $(n=48)$.

\begin{tabular}{lc}
\hline Atopy & $17(36 \%)$ \\
- Total IgE (KU/L) & $625 \pm 263$ \\
- Active eczema & $1(2 \%)$ \\
- Allergic rhinitis & $2(4 \%)$ \\
& \\
Treatment & \\
- Oral steroid & $4(8 \%)$ \\
- Inhaled steroid & $7(15 \%)$ \\
- DNase & $25(52 \%)$ \\
- Antacid & $24(50 \%)$ \\
- Antibiotic & $30(63 \%)$ \\
& \\
Micro-organism colonization & $40(83 \%)$ \\
- Pseudomonas Aeruginosa & $23(48 \%)$ \\
- Staphylococcus Aureus & $29(60 \%)$ \\
- Haemophilus Influenzae & $14(29 \%)$ \\
- Haemophilus Parainfluenzae & $3(6 \%)$ \\
- Aspergillus Fumigatus & $17(35 \%)$ \\
- Candida Albicans & $6(13 \%)$ \\
Lung function indices & \\
- Reversibility § & \\
- TLC \% predicted & $19 \%$ \\
- RV \% predicted & $95.2 \pm 4.8$ \\
- ITGV \% predicted & $182.4 \pm 18.8$ \\
\hline Ahhreyition & $114.7 \pm 7.3$
\end{tabular}

Abbreviations: ITGV, intra-thoracic gas volume; RV, residual volume; TLC, total lung capacity; Data are given as mean $\pm \mathrm{SE}$ except were indicated otherwise; *A child was considered allergic when the total IgE level exceeded $20 \mathrm{kU} / \mathrm{l}$ and / or the Phadiatop was positive and / or the Radio Allergo Sorbent Test (RAST) had two or more allergens $\geq$ class 2 ; $\S$ Reversibility is defined as the presence of an increase in FEV1 of $9 \%$ of predicted value or more after inhalation of $400 \mu \mathrm{g}$ salbutamol [23].

\section{Exhaled breath collection}

All subjects completed the manoeuvre correctly. No adverse effects were reported during the exhaled breath collection. 


\section{VOC profiles}

About 6000 different VOCs were identified in the chromatograms of the entire study population (Figure 1). After selection of attributes with a prevalence of at least 7\%, 1099 VOCs were included in the analysis (16).

\section{Variation across the day and between days}

Distance measure calculation of all complementary files within one hour, resulted in a mean $\pm S D$ match factor of $0.90 \pm 0.038$. Similarity of VOC profiles after one day was $0.85 \pm 0.096$. These data indicate a low within-subject variation in VOC patterns after one hour, and after a day.

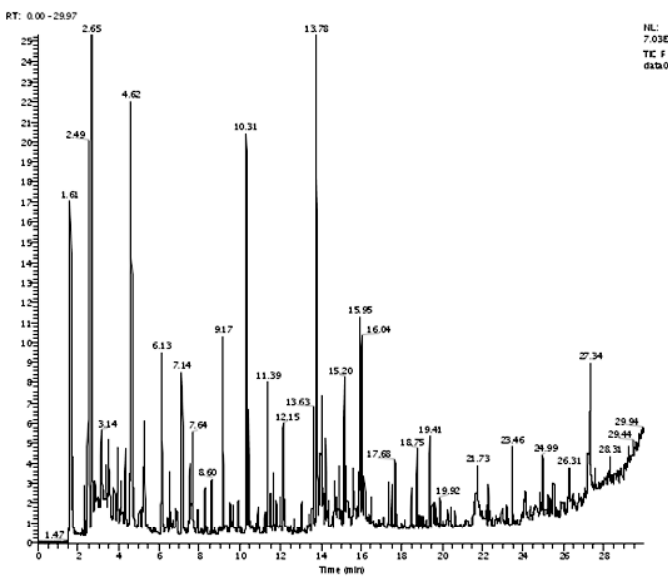
Figure 1: An acquired chroma-
togram of volatile organic com-
pounds.

The $x$-axis represents the gas chromatograph retention time (minutes). The $y$-axis shows the relative abundance of various compound signals. The cumulative area under all peaks used for classification analyses, is by definition $100 \%$.

\section{Influence of breathing patterns}

Samples were assessed with an unforced breathing pattern and during hyperventilation. Characteristics are shown in Table 3. The match factor (mean \pm SD) of these breathing patterns was $0.95 \pm 0.043$ which indicates minimal influence of breathing pattern on VOC profiles.

Table 3: Characteristics of breathing patterns $\left\langle * I^{*}\right\rangle$.

\begin{tabular}{lllll}
\hline Attribute & Units & Standard & Hyperventilation & p-value \\
\hline Exhalation pressure & mbar & $22.3 \pm 11.3$ & $64.2 \pm 15.5$ & $<0.001$ \\
Sampling duration & Seconds & $15: 45 \pm 1: 28$ & $15: 35 \pm 1: 16$ & 0.11 \\
Number of exhalations & & $3.5 \pm 1.6$ & $3.9 \pm 1.3$ & 0.27 \\
Environmental temperature & ${ }^{\circ} \mathrm{C}$ & $23.5 \pm 0.4$ & $23.7 \pm 0.6$ & 0.30 \\
Environmental humidity & $\%$ & $51.7 \pm 9.5$ & $50.5 \pm 8.1$ & 0.27 \\
\hline Data are given as mean \pm SD. & & & &
\end{tabular}


Discriminant analysis: diagnosis of CF

It was possible to assess distinctive VOC profiles, which discriminated between CF children and controls. Based on 10 times cross validation, a 100\% correct classification of CF patients and controls was found using 22 attributes or more (Figure 2 and 3 ). The sensitivity, specificity, and the percentage of correct classification increased with increasing attributes in the analysis.

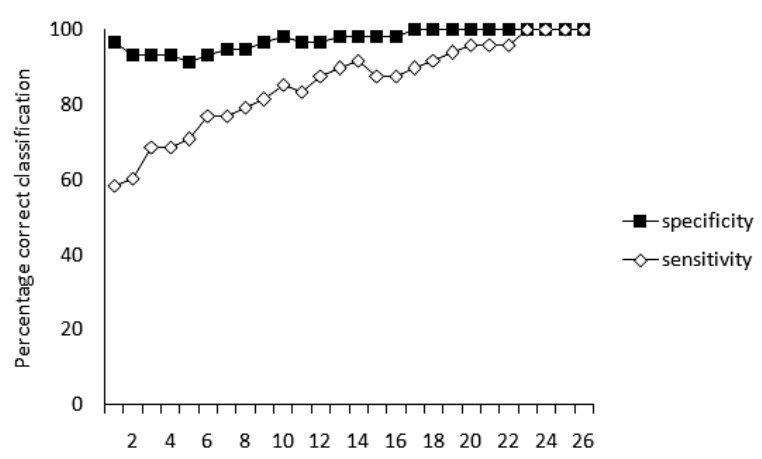

Figure 2: The percentage of correct classification of cystic fibrosis with increasing numbers of attributes, using discriminant analysis.

The discriminant analysis was performed using 10 times cross-validation. An attribute was only included in the analysis if its prevalence was $7 \%$ or more within the study population. This figure shows a $100 \%$ correct classification of cystic fibrosis patients and controls with use of 22 attributes or more. The sensitivity of the models assessed with 1-26 attributes, ranged from $58 \%-100 \%$. The specificity of these models, ranged from 91-100\%.

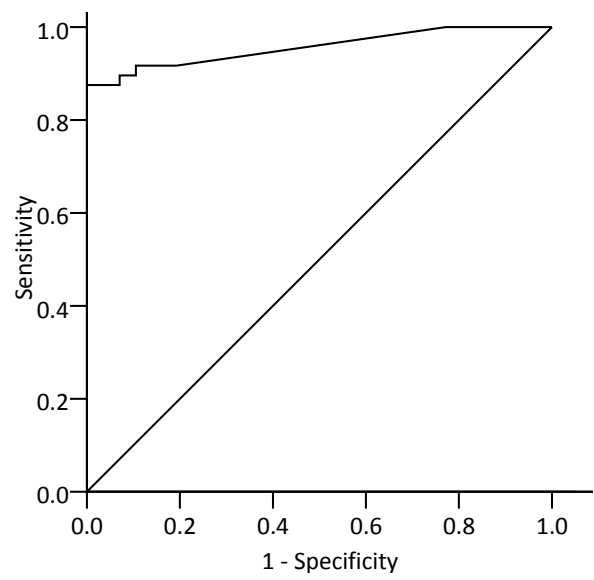

Figure 3: Receiver operating characteristic (ROC) curve of the ten most prominent attributes discriminating between CF and healthy controls.

Area under the ROC curve is $0.962, p<0.001$.

The specificity of the models with 1-26 attributes ranged from $91 \%-100 \%$ with a corresponding sensitivity of $58-100 \%$. Identification of the 10 most important discriminating VOCs was possible in all cases (Table 4). Six of these VOCs were classified as hydrocarbons with 4 to 16 carbon atoms. Figure 4 shows the mean relative intensity of the 10 most prominent attributes which discriminated children with CF from healthy controls. 
Table 4: Identification of 10 most discriminating attributes between CF and controls.

\begin{tabular}{|c|c|c|c|c|}
\hline & Identified as & Chemical formula & Percentage* & $\begin{array}{l}\text { Compound Inten- } \\
\text { sities } * *\end{array}$ \\
\hline 1 & 3,3-dimethylhex-1-ene & $\mathrm{C}_{8} \mathrm{H}_{16}$ & $11.4 \%$ & $72(41-107)$ \\
\hline 2 & 2-buten-1-ol & $\mathrm{C}_{4} \mathrm{H}_{8} \mathrm{O}$ & $46.7 \%$ & $29(14-71)$ \\
\hline 3 & $\mathrm{~N}$-methyl-2-methylpropylamine & $\mathrm{C}_{5} \mathrm{H}_{13} \mathrm{~N}$ & $34.3 \%$ & $8(6-13)$ \\
\hline 4 & C8H16 hydrocarbon (2-octene, 3-octene) & $\mathrm{C}_{8} \mathrm{H}_{16}$ & $19.0 \%$ & $3(1-4)$ \\
\hline 5 & Tolualdehyde (o-, m-, or p- isomers) & $\mathrm{C}_{8} \mathrm{H}_{8} \mathrm{O}$ & $21.9 \%$ & $35(25-48)$ \\
\hline 6 & C16 poly-unsaturated hydrocarbon & $\mathrm{C}_{16}$ & $17.1 \%$ & $7(17-22)$ \\
\hline 7 & C12 saturated hydrocarbon & $\mathrm{C}_{12} \mathrm{H}_{26}$ & $9.5 \%$ & $11(4-19)$ \\
\hline 8 & C13 saturated hydrocarbon & $\mathrm{C}_{13} \mathrm{H}_{28}$ & $10.5 \%$ & $5(1-9)$ \\
\hline 9 & Benzothiazole & $\mathrm{C}_{7} \mathrm{H}_{5} \mathrm{NS}$ & $9.5 \%$ & $10(4-14)$ \\
\hline 10 & Long chain alkylbenzene & $\mathrm{C}_{8}-\mathrm{C}_{16}$ & $8.6 \%$ & $1(0-6)$ \\
\hline
\end{tabular}

* Percentage of compounds with intensities above the detection limit; ${ }^{* *}$ Compound intensities were presented as percentage times $10^{3}$.

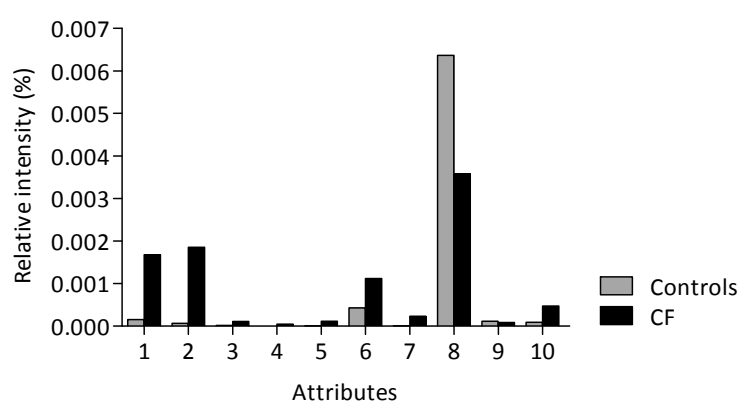

Figure 4: Mean relative intensity of the ten most prominent attributes that are used to generate the discriminant function that discriminates children with CF from healthy controls. The numbers represent the order in which the attributes were included in the disciminant analysis.

All attributes were not significantly different between CF children with of without the use of antibiotics, corticosteroids and DNase use ( $p>0.130$, One way ANOVA). In the control group, there was no significant difference in between subjects recruited from the primary school or the children from the outpatient clinic with initial complaints of constipation or enuresis nocturna.

As acquisition of Pseudomonas aeruginosa in the sputum is associated with increased morbidity and mortality, we were interested in VOC profiles of CF subjects with and without positive cultures of Pseudomonas. Within the CF group, it was possible to identify patients with or without positive Pseudomonas Aeruginosa cultures $100 \%$ correctly by means of 14 VOCs in exhaled breath (Figure 5). 


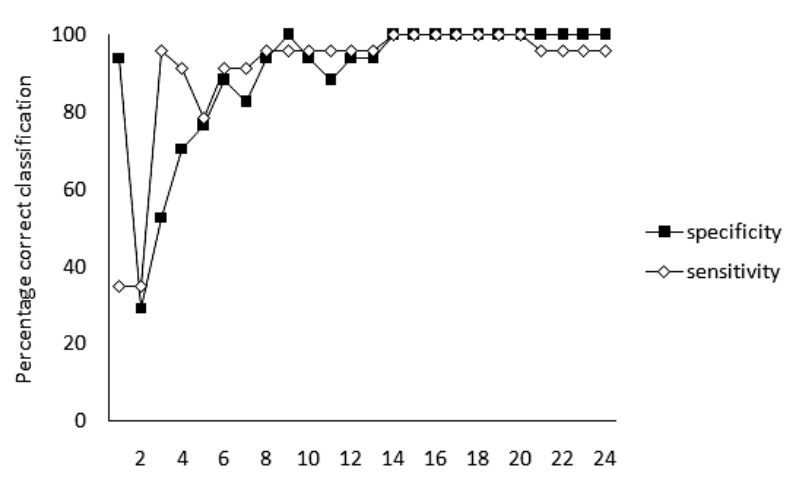

Figure 5: The percentage of correct classification of positive or negative Pseudomonas Aeruginosa cultures in sputum of cystic fibrosis patients with increasing numbers of attributes using discriminant analysis.

The discriminant analysis was performed using 10 times cross-validation. This figure shows a $100 \%$ correct classification of cystic fibrosis patients with and without positive Pseudomonas Aeruginosa cultures in sputum with use of 14 attributes or more. The sensitivity of the models assessed with 1-24 attributes, ranged from $34 \%-100 \%$, the specificity ranged from $29-100 \%$.

\section{Discussion}

This study showed that metabolomics of VOCs in exhaled breath of young subjects with cystic fibrosis and healthy controls was possible in a reproducible way, resulting in a $100 \%$ correct classification rate by using 22 compounds. Moreover, it was possible to discriminate between CF patients with or without positive Pseudomonas aeruginosa cultures, a factor which is clearly associated with CF prognosis. The discrimination between CF and controls was mainly based on $\mathrm{C} 5-\mathrm{C} 16$ hydrocarbons and N-methyl-2-methylpropylamine. This study showed good feasibility of exhaled breath collection in 5 liter Tedlar bags in children of 5 years and over. More than 1000 different VOCs could be measured in exhaled breath of CF patients and controls with a prevalence in the two groups of at least $7 \%$, with good short-term and long-term reproducibility. Breathing patterns did not influence VOC profiles significantly. The match factor is based on a distance measure implementing the dot product rule was applied to establish the degree of similarity between measured chromatograms $(13,17)$.

We acknowledge some limitations in this study: Variance of data is influenced by a large range of patient related and technical factors, therefore there should be some caution in identifying biomarkers. It is of great importance to correct for the selection bias. Ambroise et al. described how selection bias can be assessed and corrected. They recommend 10fold rather than leave-one-out cross-validation to handle overestimation of the results. Therefore, 10-fold cross-validation was applied in our study (17). Although 10 times crossvalidation is a valid method to prevent overestimation of the predictive value of markers, external validation in a control population of patients and controls is necessary in a second stage in order to further validate the results. The control group in this study was signficantly younger that the CF group. This probably did not bias our results as no significant influence of age was found in the discriminant analysis.

As reported previously by van Berkel et al., we did not correct our measurements for chemical background appearing in the samples (18). This is due to the fact that it will not 
be possible to correct for the complex interdependencies between excretion and uptake of VOCs by easily subtracting the inhaled from the exhaled air. Moreover, background noise will be randomly distributed between subjects' samples and would thus neither exert any discriminatory power, nor interfere with the outcome of the analyses. We are aiming with discriminative analysis to select only those compounds that are specific for the disease or condition and should thus principally not depend on background chemicals. In the past few years, there has been increasingly interest in presence of VOCs in exhaled breath of patients with chronic lung diseases. Philips et al. assessed VOCs in exhaled air by means of automated thermal desorption gas chromatography mass spectrometer (ATDGC-MS), and classified patients with and without lung cancer, based on 9-22 VOCs (10, 19). In addition, they studied the potential of VOCs to differentiate between 42 patients with a suspicion of pulmonary tuberculosis and 59 controls, and tuberculosis patients with and without bacterial sputum colonization. These classifications were possible with high sensitivity and specificity (20).

In 2006, Barker et al. studied 12 specific VOCs by means of a customized gas chromatograph in CF patients and controls. They reported a significant lower level of dimethyl sulphide (DMS) in CF compared to controls (21). However, DMS was not identified as an important discriminating component in the present study. Other methods to measure VOCs are sensor systems like the electronic nose, based on chemical vapor arrays responding to VOCs, and colorimetric sensor arrays. These methods were applied to detect lung cancer $(22,23)$. In contrast to VOC assessment with GC-TOF-MS, the electronic nose consists of a nano-composite array of 32 organic polymer sensors. This is a limitation as the significance of specific VOCs for specific lung diseases is not established yet. Firstly, it is important to specify the VOCs important for a specific disease, or a specific question (VOCs important for diagnosis may not be the same ones that appear to be important for disease monitoring). In a second stage, when the relevant VOCs are defined, sensor systems like the 'electronic nose' might be very helpful, as they are rapid, easy to use, and not very expensive.

Currently, diagnosis of pulmonary CF exacerbations and monitoring of disease activity are mainly based on clinical features and lung function tests. These parameters reflect changes in the functional abnormalities in the airways by infective and inflammatory processes, instead of the inflammation itself (24). Therefore, inflammation may be present before clinical parameters change, introducing a time delay between the onset of a pulmonary CF exacerbation and the start of treatment. Persistent inflammation and repeated cycles of infection are present in CF lungs, resulting in progressive lung damage and pulmonary fibrosis (25). Even in stable patients, chronic airway inflammation is present, as reflected by high airway fluid concentrations of pro-inflammatory cytokines (26). Analysis of broncho-alveolar lavage (BAL) fluid has shown a 1.000 fold increase in the number of neutrophils from the lung of patients with CF compared to controls (27).

We hypothesize that VOC profiles may be helpful in the early detection of a CF exacerbation even before symptoms occur and lung function deterioration is present and the decision to stop antibiotic treatment in patients recovering from an exacerbation. However, this should be subject of future longitudinal studies. Based on this study, metabolomics of VOCs in exhaled breath discriminate between CF patients and controls. Dallinga et al and Dragionieri et al showed that VOCs could discriminate between, respectively, children and adults with asthma and healthy controls $(5,6)$. In addition, Fens et al showed good dis- 
crimination between asthmatics and patients with chronic pulmonary obstructive disease by means of the electronic nose (8). Therefore, the hypothesis is that these markers may be of additional value to study on-going processes of airway inflammation and oxidative stress, in addition to lung function and symptoms. As we were interested in the earliest stages of CF lung disease, the CF population of our study had mild to moderately severe pulmonary CF disease. It is not clear whether the results of this study can be generalized to more severe CF. This study showed good feasibility and safety of VOC collection in children. This is an advantage when compared to invasive methods like biopsies, bronchial alveolar lavage (BAL), and induced sputum. The short-term reproducibility of VOCs was excellent. We expect an even higher reproducibility of a typical VOC pattern, because not the absolute values but the values of the spikes relative to each other are important for the reproducibility of a specific pattern. However, this will be subject of future studies. The objective of the present study was to identify VOC profiles of CF disease and control children and to specify which VOCs are important in CF. In our study, products of inhaled medication or their derivates, such as tobramycin or corticosteroids were not recovered in exhaled breath, and therefore did not contribute to the discrimination.

By means of VOC profiles, we were able to differentiate between CF patients with or without positive cultures for Pseudomonas Aeruginosa. This result was consistent, although, other micro-organisms may be concordantly present in the airways of patients, and a certain time period between collection of sputum samples and the sampling of VOCs was present in some subjects.

Future research on VOCs in exhaled breath should not only be focused on clinical questions, but also on methodological issues like the influence of diet and exercise on VOCs. In addition, VOC profiles should be studied in longitudinal studies in order to investigate the additional value of VOC measurements to conventional parameters like symptoms, lung function indices and sputum analyses. Assessment of VOCs in exhaled breath is a new, promising, non-invasive technique, which in addition to conventional parameters can be used to study airway inflammation and oxidative stress in CF patients.

\section{References}

1. Gibson RL, Burns JL, Ramsey BW 2003 Pathophysiology and management of pulmonary infections in cystic fibrosis. Am J Respir Crit Care Med 168:918-951

2. Paredi $P$, Kharitonov SA, Barnes PJ 2000 Elevation of exhaled ethane concentration in asthma. Am J Respir Crit Care Med 162:1450-1454

3. Horvath I, MacNee W, Kelly FJ, Dekhuijzen PN, Phillips M, Doring G, Choi AM, Yamaya M, Bach FH, Willis D, Donnelly LE, Chung KF, Barnes PJ 2001 "Haemoxygenase-1 induction and exhaled markers of oxidative stress in lung diseases", summary of the ERS Research Seminar in Budapest, Hungary, September, 1999. Eur Respir J 18:420-430

4. ATS/ERS Recommendations for Standardized Procedures for the Online and Offline Measurement of Exhaled Lower Respiratory Nitric Oxide and Nasal Nitric Oxide 2005 Am J Respir Crit Care Med 171:912-930

5. Dallinga JW, Robroeks CM, van Berkel JJ, Moonen EJ, Godschalk RW, Jöbsis Q, Dompeling E, Wouters EF, van Schooten FJ 2010 Volatile organic compounds in exhaled breath as a diagnostic tool for asthma in children. Clin Exp Allergy 40:68-76

6. Fens N Zwinderman AH, van der Schee MP, de Nijs SB, Dijkers E, Roldaan AC, Cheung D, Bel EH, Sterk PJ. 2009 Exhaled breath profiling enables discrimination of chronic obstructive pulmonary disease and asthma AJRCCM 180:1076-82 
7. Delfino RJ, Gong H, Linn WS, Hu Y, Pellizzari ED 2003 Respiratory symptoms and peak expiratory flow in children with asthma in relation to volatile organic compounds in exhaled breath and ambient air J Expo Anal Environ Epidemiol 13:348-63

8. Dragonieri S, Schot R, Mertens BJ, Le Cessie S, Gauw SA, Spanevello A, Resta O, Willard NP, Vink TJ, Rabe KF, Bel EH, Sterk PJ 2007 An electronic nose in the discrimination of patients with asthma and controls. J Allergy Clin Immunol. 120:856-62

9. Miekisch W, Schubert JK, Noeldge-Schomburg GF 2004 Diagnostic potential of breath analysis--focus on volatile organic compounds. Clin Chim Acta 347:25-39

10. Phillips M, Cataneo RN, Cummin AR, Gagliardi AJ, Gleeson K, Greenberg J, Maxfield RA, Rom WN 2003 Detection of lung cancer with volatile markers in the breath. Chest 123:2115-2123

11. Shwachman H, Kulczycki LL 1958 Long-term study of one hundred five patients with cystic fibrosis; studies made over a five- to fourteen-year period. JAMA Dis Child 96:6-15

12. Variations in the prevalence of respiratory symptoms, self-reported asthma attacks, and use of asthma medication in the European Community Respiratory Health Survey (ECRHS) 1996 Eur Respir J 9:687-695

13. Stein SE, Scott DR 1994 Optimization and testing of mass-spectral library search algorithms for compound identification. Journal of the American Society of Mass Spectrometry 5:859-866

14. Tammeling GJ, Quanter PH 1993 Standardized lung function testing. Eur Respir J 6:5-40

15. Edwards TL, Torstensen E, Dudek S, Martin ER, Ritchie MD 2010 A cross-validation procedure for general pedigrees and matched odds ratio fitness metric implemented for the multifactor dimensionality reduction pedigree disequilibrium test. Genet Epidemiol 34:194-199

16. Penn DJ, Oberzaucher E, Grammer K, Fischer G, Soini HA, Wiesler D, Novotny MV, Dixon SJ, Xu Y, Brereton RG 2007 Individual and gender fingerprints in human body odour. J R Soc Interface 4:331-340

17. Ambroise C, McLachlan GJ 2002 Selection bias in gene extraction on the basis of microarray gene-expression data PNAS 99:6562-6566

18. van Berkel JJ, Dallinga JW, Moller GM, Godschalk RW, Moonen E, Wouters EF, van Schooten FJ 2008 Development of accurate classification method based on the analysis of volatile organic compounds from human exhaled air. J Chromatogr B Analyt Technol Biomed Life Sci 861:101-107

19. Phillips M, Gleeson K, Hughes JM, Greenberg J, Cataneo RN, Baker L, McVay WP 1999 Volatile organic compounds in breath as markers of lung cancer: a cross-sectional study. Lancet 353:1930-1933

20. Phillips M, Cataneo RN, Condos R, Ring Erickson GA, Greenberg J, La Bombardi V, Munawar MI, Tietje O 2007 Volatile biomarkers of pulmonary tuberculosis in the breath. Tuberculosis (Edinb) 87:44-52

21. Barker M, Hengst M, Schmid J, Buers HJ, Mittermaier B, Klemp D, Koppmann R 2006 Volatile organic compounds in the exhaled breath of young patients with cystic fibrosis. Eur Respir J 27:929-936

22. Machado RF, Laskowski D, Deffenderfer O, Burch T, Zheng S, Mazzone PJ, Mekhail T, Jennings C, Stoller JK, Pyle J, Duncan J, Dweik RA, Erzurum SC 2005 Detection of lung cancer by sensor array analyses of exhaled breath. Am J Respir Crit Care Med 171:1286-1291

23. Mazzone PJ, Hammel J, Dweik R, Na J, Czich C, Laskowski D, Mekhail T 2007 Diagnosis of lung cancer by the analysis of exhaled breath with a colorimetric sensor array. Thorax 62:565-568

24. Kharitonov SA, Barnes PJ 2001 Exhaled markers of pulmonary disease. Am J Respir Crit Care Med 163:16931722

25. McGrath LT, Mallon P, Dowey L, Silke B, McClean E, McDonnell M, Devine A, Copeland S, Elborn S 1999 Oxidative stress during acute respiratory exacerbations in cystic fibrosis. Thorax 54:518-523

26. Grasemann H, loannidis I, Tomkiewicz RP, de Groot H, Rubin BK, Ratjen F 1998 Nitric oxide metabolites in cystic fibrosis lung disease. Arch Dis Child 78:49-53

27. Brown RK, Wyatt H, Price JF, Kelly FJ 1996 Pulmonary dysfunction in cystic fibrosis is associated with oxidative stress. Eur Respir J 9:334-339. 
SECTION 3

\section{MONITORING}

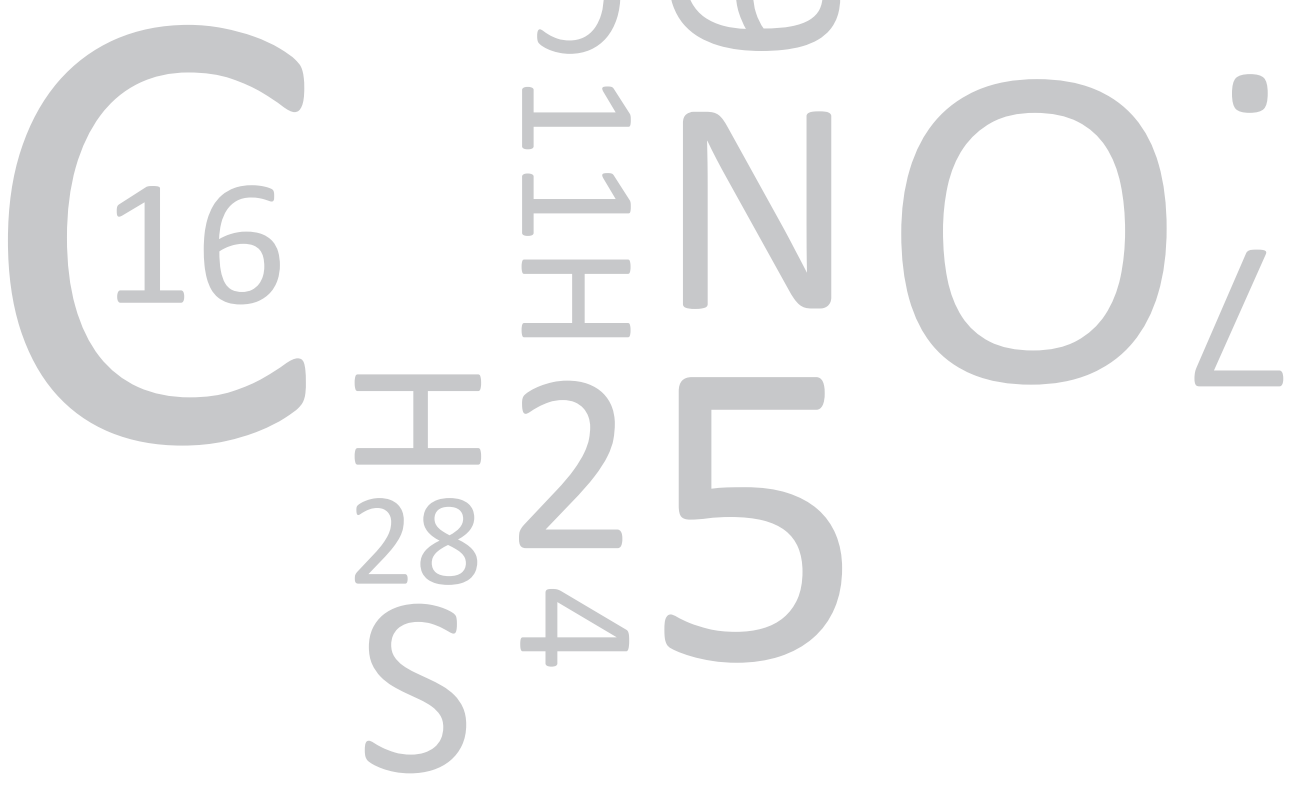





\section{CHAPTER 10}

Comparison of the anti-inflammatory effects of extra-fine HFA-beclomethasone versus fluticasone-DPI on exhaled inflammatory markers in childhood asthma

CMHHT Robroeks, KDG van de Kant, D van Vliet, AD Kester, JJE Hendriks, JGMC Damoiseaux, WKWH Wodzig, GT Rijkers, E Dompeling, Q Jöbsis Annals of Allergy Asthma and Immunology 2008;100(6):601-607 


\section{Abstract}

\section{Background}

Extrafine hydrofluoroalkane (HFA) beclometasone differed from other inhaled corticosteroids by its fine aerosol characteristics. Therefore, extrafine HFA-beclometasone may be particularly useful for treating peripheral airway inflammation in asthma. The aim of this study was to analyse the anti-inflammatory effect of extrafine HFA-beclometasone compared to fluticasone-DPI in asthmatic children by means of bronchial and alveolar nitric oxide (NO), and inflammatory markers in exhaled breath condensate (EBC).

\section{Methods}

In a 6 months cross-over study, 33 children, aged 6-12 years, with moderate persistent asthma, were randomly treated with extrafine HFA-beclometasone (200 $\mu \mathrm{g}$ daily, autohaler) and fluticasone-DPI (200 $\mu \mathrm{g}$ daily, diskus). Primary outcome parameters were: alveolar NO concentration and bronchial NO flux. Secondary outcome parameters were: inflammatory markers in EBC, lung function indices, symptoms, exacerbations, and adverse effects. All parameters were recorded at baseline and after each treatment period.

\section{Results}

Alveolar NO concentration and bronchial NO flux were comparable after treatment with HFA-beclomethasone and fluticasone-DPI (mean \pm SE) $4.7 \pm 0.5 p p b$ vs $4.3 \pm 0.5 p p b, p=0.546$, and $1124.3 \pm 253.6 \mathrm{pL} / \mathrm{sec}$ vs $1029.1 \pm 195.5 \mathrm{pL} / \mathrm{sec}, \mathrm{p}=0.696$, respectively. In addition, inflammatory markers in EBC, lung function indices and symptoms, did not differ between treatments. Patients used less $\beta_{2}$-agonists during the last 2 weeks of HFA-beclomethasone treatment.

\section{Conclusions}

The anti-inflammatory effect of HFA-beclomethasone and fluticasone-DPI, is similar in children with moderate persistent asthma. 


\section{Introduction}

In the past few years, it has become clear that inflammation in especially peripheral airways, plays an important role in the pathophysiology of asthma [1-3]. Inhaled corticosteroid (ICS) therapy reduces airway inflammation and, therefore, plays a central role in current international management guidelines for asthma treatment [4]. In addition, ICS therapy has become the cornerstone of asthma therapy $[4,5]$. Considering that airway inflammation in asthma involves the entire respiratory tract, ICS should reach both central as well as peripheral airways to obtain an optimal effect. Extrafine hydrofluoroalkane (HFA) beclomethasone differs from other ICS because of its extra fine aerosol characteristic [6], with a $1.0 \mu \mathrm{m}$ mass median aerodynamic diameter (MMAD) of HFAbeclomethasone [7] versus less than $6 \mu \mathrm{m}$ of fluticasone via a diskus [8]. The smaller mean particle size results in a greater peripheral airway deposition $[6,9,10]$. Since mean airway diameter in children is smaller compared to adults, this potential advantage of extra-fine HFA-beclomethasone may be especially important in children.

Fractional exhaled nitric oxide (FeNO) is a direct and non-invasive marker of airway inflammation [11-13]. FeNO appears to be a good tool to diagnose asthma, to predict loss of asthma control, and for monitoring the tapering scheme of inhaled corticosteroids [1416]. A correlation is found between FeNO and the single breath nitrogen wash out test. This indicates a relation between FeNO and peripheral airway functions in mild asthma [17]. It is possible to differentiate between alveolar and bronchial FeNO production, by performing NO measurements at various exhalation flow rates (flow-independent method) [18]. Using this method, Lehtimaki et al. reported a reduction of bronchial but not alveolar FeNO output with inhaled fluticasone therapy in adults with asthma [19]. Next to FeNO, measurements of inflammatory markers in exhaled breath condensate (EBC) is an additional non-invasive tool of airway inflammation [20-22]. The collection of $E B C$ is simple, safe and does not enhance ongoing inflammation [23, 24].

The objective of the present study in asthmatic children was to compare the antiinflammatory effects of extrafine HFA-beclomethasone and fluticasone-DPI (dry powder inhaler), on bronchial and alveolar NO levels, inflammatory markers in EBC, and conventional parameters such as lung function indices, local adverse effects and symptoms.

\section{Methods}

\section{Study subjects}

Children with physician-diagnosed moderate persistent asthma, aged 6 to 12 years, were recruited from the outpatient clinic of the Paediatric Pulmonology department, University Hospital Maastricht. Before the start of the study, all patients were being treated with inhaled daily corticosteroids (dose of $\leq 500 \mu \mathrm{g}$ Fluticasone-DPI or equivalent). Exclusion criteria for both groups were: 1 ) serious instability of asthma during the past 3 months, 2) presence of a disease that may intervene with the results of this study, 3) active smoking, 4) mental retardation, 5) inability to perform the measurements properly. 


\section{Study design}

This study had a randomised, prospective, cross-over design during 6 months. Patients were randomized in order to be treated with either: 2 times daily fluticasone-DPI (Flixotide $^{\circledR}$, GlaxoSmithKline Zeist, The Netherlands) $100 \mu \mathrm{g}$ via a diskus ${ }^{\circledR}$, during 3 months, followed by 2 times daily extrafine HFA-beclomethasone (Qvar ${ }^{\circledR}$, IVAX Pharmaceutical Bodegraven, The Netherlands) $100 \mu \mathrm{g}$ via an autohaler ${ }^{\circledR}$ during 3 months, or the same scheme schedule in reverse order. The use of a short-acting $\beta$ agonist was allowed as rescue medication. No dose changes of antihistamines, or long-acting $\beta 2$-agonists, were permitted during the study. When a patient experienced a severe exacerbation requiring oral corticosteroids during the study period, the patient dropped out. This is a real life study in which all patients were treated with inhaled corticosteroids, therefore, a steroid free wash-out period was not ethical to include in the study design. Treatment periods of 3 month exclude period and carry-over effects.

\section{Outcome parameters}

In all children, outcome parameters were measured at baseline $(t=0)$ and after each treatment period ( $\mathrm{t}=3$ months and $\mathrm{t}=6$ months). The primary outcome parameters were: alveolar NO concentration and bronchial NO flux. Secondary outcome parameters were: 1) inflammatory markers in EBC, 2) lung function indices (FEV1, FEV1/ FVC, MEF50, presence and degree of reversibility), 3) symptoms, symptom-free days, exacerbations, 4) adverse effects. Written informed consent was obtained from the parents of all children. The medical ethics committee of the University Hospital Maastricht approved this study.

\section{NO in exhaled air}

FeNO levels were measured, prior to EBC collection and assessment of lung function parameters, using the $\mathrm{NIOX}^{\circledR}$ analyser (Aerocrine, Solna, Sweden). FeNO measurements were performed while children exhaled at a constant flow rate of $50 \mathrm{~mL} / \mathrm{s}$ during 6-10 seconds, according to the criteria of the American Thoracic Society and European Respiratory Society (ATS/ERS) [25]. This procedure has been described previously [26]. To differentiate alveolar NO concentration from bronchial NO flux, NO was assessed at three different flow rates: 50,100 , and $200 \mathrm{~mL} / \mathrm{sec}$. The NO output ( $\mathrm{pL} / \mathrm{sec}, \mathrm{y}$-axis) was plotted against these exhalation flow rates $(\mathrm{mL} / \mathrm{sec}, \mathrm{x}$-axis). The slope of the regression line represents alveolar NO concentration (Calv), the intercept the bronchial NO flux (JNO) [27]. Linear regression models with a $\mathrm{R} 2$ of $\geq 0.81$, were considered reliable, and therefore only those models, Calv and JNO levels were assessed. The ratio between Calv and JNO was assessed to reflect the relative localisation of airway inflammation.

\section{Exhaled Breath Condensate}

EBC was collected using a cooled $(1 \stackrel{\circ}{ } \mathrm{C}) 100 \mathrm{~cm}$ double-jacketed borosilicate glass condenser, while children, wearing a nose-clip, were breathing tidally into a mouthpiece connected to a two-way non-rebreathing valve. The two-way valve and tubing served as a trap to prevent possible salivary contamination. After collection, acidity of EBC was measured immediately (Radiometer, type PHM201, Zoetermeer, the Netherlands), and, EBC was frozen using dry ice and stored thereafter at $-80 \stackrel{\circ}{\circ}$ until analysis of inflammatory 
markers took place. Inflammatory markers, IL-2, IL5 and IL-13, were assayed with Luminex ${ }^{\circledR}$ technology [28]. 8-Isoprostane concentrations were measured with a specific enzyme immunoassay (EIA) kit (Cayman Chemical Company, Ann Arbor, MI), which was modified in order to lower the detection limit from $5.0 \mathrm{pg} / \mathrm{mL}$ to $0.5 \mathrm{pg} / \mathrm{mL}$ [29]. Standard curves, patient samples and quality control (QC) samples of $2.5 \mathrm{pg} / \mathrm{mL}$ and $10.0 \mathrm{pg} / \mathrm{mL}$ were measured in triplicate. The coefficients of variation (\% CV) of the absorption signal were assessed. Intra-assay variation of standard curves and QC samples should not exceed $15 \%$, otherwise all samples measured in that assay were excluded. Patient samples with a higher CV than $15 \%$ were excluded and were re-analysed once. In case of acceptance, mean absorption signal intensities were converted to 8-isoprostane concentrations.

Nitrate and nitrite concentrations were assessed by means of fluorometry (Cayman Chemical Company, Ann Arbor, MI). The concentration of $\mathrm{H} 2 \mathrm{O} 2$ in EBC was measured as described by Dekhuizen et al [30]. Briefly, $720 \mu \mathrm{L}$ of EBC was mixed with $220 \mu \mathrm{L} 0.4 \mathrm{M}$ citrate buffer, $50 \mu \mathrm{L}$ of $\mathrm{HRP}$ solution $(50 \mathrm{U} / \mathrm{ml})$ and $10 \mu \mathrm{L}$ tetramethylbenzidine (TMB, 20 $\mathrm{mM}$ ) and incubated for $20 \mathrm{~min}$ at room temperature. Next, the sample was mixed with 20 $\mu \mathrm{L}$ sulphuric acid ( $2 \mathrm{M}$ ). The TMB oxidation product as a measure of the amount of $\mathrm{H} 2 \mathrm{O} 2$ was determined spectrometrically using a Perkin Elmer UV-VIS Spectrometer Lambda 10 (Norwalk, CT, USA) at $450 \mathrm{~nm}$. Detection limits of all assays are shown in Table 2.

\section{Lung function tests}

Bronchodilator medication was stopped prior to lung function testing. Short-acting bronchodilators were stopped at least 8 hours, and long-acting bronchodilators at least 36 hours, before the test. Dynamic spirometry, (FEV1, FVC, FEV1/VC, MEF50), was performed by means of the Flowscreen (Jaeger, Wuerzburg, Germany). The highest score of a given lung function parameter, of three technically correct manoeuvres, was used for analysis. Fifteen minutes after inhalation of a short-acting bronchodilator, dynamic spirometry was repeated in a similar way. Reversibility was defined as the presence of a change in FEV1 of at least $9 \%$ of predicted value after use of $400 \mu \mathrm{g}$ salbutamol.

\section{Symptoms and adverse effects}

Two weeks prior to the second and third visit, asthma symptoms (dyspnoea, cough, wheeze and awakening caused by asthma), use of rescue medication ( $\beta_{2}$-agonists), and the presence of adverse effects were recorded in a diary. Adverse effects were: hoarseness, oral fungal infection and/or irritation of oral cavity. Moreover, at each visit the degree of asthma severity and control was scored by means of the Asthma Control Questionnaire (ACQ) [31].

\section{Statistic analysis}

All data were expressed as mean \pm standard error of the mean (SE), unless specified otherwise. Parametric tests were performed for normal distributed parameters and nonparametric tests were performed for not normal distributed parameters, such as symptoms and adverse effects [32]. A test was considered significant when $p<0.05$. 


\section{Power analysis}

In a cross-over design, 29 patients were needed, based on a difference in NO of 10ppb between treatment groups, a standard deviation (SD) of $16 \mathrm{ppb}$, and a paired student $\mathrm{t}-$ test with a significant limit of 0.05 and a power of $90 \%$. Assuming a $10 \%$ drop-out rate, 33 children have to be included in this study.

\section{Results}

\section{Study population}

33 Children, aged 6.5 to 12.7 years, were included. After randomisation, patient characteristics (age, lung function, symptoms, adverse effects, dose of inhaled corticosteroids, long acting $\beta_{2}$-agonists (LABA), and number of patients with well controlled asthma) were comparable between the two groups $(p>0.10)$. Patient characteristics and levels of inflammatory markers, at the start of the study, are shown in Table 1.

\section{Drop-outs}

Three patients dropped out because of an exacerbation during the study. These patients dropped-out during the first treatment period of the study, independent of the medication: 2 patients used HFA-beclomethasone, 1 patient was treated with fluticasone-DPI. Before the start of the study, these patients used relatively high daily doses of inhaled corticosteroids: $250 \mu \mathrm{g}, 500 \mu \mathrm{g}$ and $400 \mu \mathrm{g}$ fluticasone or equivalent, respectively.

\section{Period and carry-over effect}

The period and carry-over effect was calculated for the primary outcome parameter FeNO [33]. The carry-over effect, corrected for the period-effect was $3.6 \mathrm{ppb}$, with a SE of $3.4 \mathrm{ppb}$, in favour of fluticasone-DPI. The corresponding $\mathrm{p}$-value was 0.300 (student t-test). The carry-over effect of FeNO was not statistical significant and has no clinical relevance.

\section{NO in exhaled air}

$73 \%$ of the linear regression curves, performed to assess $C_{a l v}$ concentrations and $J_{N O}$ flux, were accepted for final analysis. Mean \pm standard error (SE) of the $\mathrm{R}^{2}$ of the approved models at the 3 measurements occasions, were $0.964 \pm 0.010,0.955 \pm 0.0095$ and $0.945 \pm$ 0.015 , respectively. After a three month treatment period with HFA-beclomethasone and fluticasone-DPI, $C_{\text {alv }}$ (mean $\pm \mathrm{SE}$ ) was $4.7 \pm 0.5 \mathrm{ppb}$ and $4.3 \pm 0.5 \mathrm{ppb}$, respectively (pairedsample $T$ test, $p=0.546)$. $J_{\text {NO }}$ levels were comparable between the two treatments $(1124.3$ $\pm 253.6 \mathrm{pL} / \mathrm{sec}$ during HFA-beclomethasone versus $1029.1 \pm 195.5 \mathrm{pL} / \mathrm{sec}$ during fluticasone-DPI) (paired-sample $T$ test, $\mathrm{p}=0.696)$. The $\mathrm{C}_{\text {alv }} / \mathrm{J}_{\mathrm{NO}}$ ratio (mean $\pm \mathrm{SE}$ ) after treatment with fluticasone-DPI was $0.0072 \pm 0.0015 \mathrm{ppb}^{*} \mathrm{sec} / \mathrm{pL}$ compared to the ratio of $0.0070 \pm$ $0.0021 \mathrm{ppb}^{*} \mathrm{sec} / \mathrm{pL}$ after a 3 months treatment period with HFA-beclomethasone (pairedsample $T$ test, $p=0.906$ ). In addition, FeNO levels (mean $\pm \mathrm{SE}$ ) after treatment with fluti- 
casone-DPI was $24.3 \pm 2.9 \mathrm{ppb}$ compared to $27.4 \pm 3.8 \mathrm{ppb}$ after a 3 months treatment period with HFA-beclomethasone (paired-sample T test, $p=0.414$ ).

Table 1: Patient characteristics at inclusion of the study.

\begin{tabular}{|c|c|c|}
\hline & Unit & Baseline values \\
\hline Age & yrs & $9.3 \pm 0.3$ \\
\hline Gender & $\mathrm{M} / \mathrm{F}$ & $20 / 13$ \\
\hline$S D_{\text {height }}$ & & $-0.5 \pm 0.2$ \\
\hline $\mathrm{SD}_{\text {weight }}$ & & $0.6 \pm 0.3$ \\
\hline Z-score $^{\wedge}$ & & $1.7 \pm 0.4$ \\
\hline $\mathrm{FEV}_{1}$ & $\%$ of predicted & $106.9 \pm 2.6$ \\
\hline FVC & $\%$ of predicted & $100.0 \pm 2.2$ \\
\hline $\mathrm{FEV}_{1} / \mathrm{VC}$ & $\%$ & $87.1 \pm 1.4$ \\
\hline $\mathrm{MEF}_{50}$ & $\%$ of predicted & $96.2 \pm 5.5$ \\
\hline Asthma questionnaire & & $0.8 \pm 0.2$ \\
\hline Well controlled asthma* & & $20(61 \%)$ \\
\hline Dose of fluticasone** & $\mu \mathrm{g}$ & $312.3 \pm 38.0$ \\
\hline Salmeterol & & $14(42 \%)$ \\
\hline $\mathrm{FeNO} \mathrm{N}^{* * *}$ & $\mathrm{ppb}$ & $33.0 \pm 6.4$ \\
\hline Bronchial NO & $\mathrm{pL} / \mathrm{sec}$ & $1093.4 \pm 250.9$ \\
\hline Alveolar NO & $\mathrm{ppb}$ & $4.1 \pm 0.5$ \\
\hline Ratio $C_{\text {alv }} / J_{N O}$ & $\mathrm{ppb}^{*} \mathrm{sec} / \mathrm{pL}$ & $0.011 \pm 0.032$ \\
\hline 8-Isoprostane & $\mathrm{pg} / \mathrm{mL}$ & $4.6 \pm 0.7$ \\
\hline $\mathrm{H}_{2} \mathrm{O}_{2}$ & $\mu \mathrm{M}$ & $1.1 \pm 0.3$ \\
\hline Nitrate & $\mu \mathrm{M}$ & $78.8 \pm 9.2$ \\
\hline Nitrite & $\mu \mathrm{M}$ & $7.4 \pm 1.1$ \\
\hline IL-2 & $\mathrm{pg} / \mathrm{mL}$ & $17.8 \pm 9.2$ \\
\hline IL-5 & $\mathrm{pg} / \mathrm{mL}$ & $3.2 \pm 0.2$ \\
\hline IL-13 & $\mathrm{pg} / \mathrm{mL}$ & $6.9 \pm 0.5$ \\
\hline EBC acidity & & $6.8 \pm 0.1$ \\
\hline
\end{tabular}

Definition of abbreviations: $\mathrm{FEV}_{1}$, forced expiratory volume in 1 second; VC, vital capacity; $\mathrm{MEF}_{50}$, mean expiratory flow at 50\%; ICS, inhaled corticosteroid; ^ Z-score weight to height corrected for age and gender; * Asthma control was based on a daily diary, kept during 2 weeks before the visit at the outpatient clinic; ** Inhaled corticosteroid dosages $(\mu \mathrm{g})$ are fluticasone or equivalent; ${ }^{* * *}$ FeNO measured at a flow rate of $50 \mathrm{~mL} / \mathrm{sec}$.

\section{Exhaled Breath Condensate}

EBC collection was successful in all patients and, at all assessments. In EBC, 8-isoprostane, hydrogen peroxide, nitrate, nitrite, IL-2 and acidity, were detected in all samples. IL-5 and IL-13 were found above the lower detection limit in $95 \%$ and $70 \%$ of the samples, respectively. No significant differences were found between non-invasive inflammatory markers after 3 month treatment periods with HFA-beclomethasone and fluticasone-DPI (Table 2). In addition, there were no significant differences between levels of inflammatory markers at the inclusion of the study, compared to there concentrations after 3 month treatment with HFA-beclomethasone or fluticasone-DPI (paired sample T-tests, p>0.05, Table 1 and 2). 
Table 2: NO and inflammatory markers measured in exhaled breath after 3 month treatment.

\begin{tabular}{|c|c|c|c|c|c|}
\hline & Unit & LDL & HFA-beclomethasone & fluticasone-DPI & $p$-value ${ }^{* *}$ \\
\hline FeNO* & $\mathrm{ppb}$ & & $27.4 \pm 3.8$ & $24.3 \pm 2.9$ & 0.414 \\
\hline Bronchial NO & $\mathrm{pL} / \mathrm{sec}$ & & $1124.3 \pm 253.6_{(n=22)}$ & $1029.1 \pm 195.5_{(\mathrm{n}=22)}$ & 0.696 \\
\hline Alveolar NO & $\mathrm{ppb}$ & & $4.7 \pm 0.5_{(n=21)}$ & $4.3 \pm 0.5_{(n=22)}$ & 0.546 \\
\hline Ratio $C_{\text {alv }} / J_{\text {NO }}$ & $\mathrm{ppb}^{*} \mathrm{sec} / \mathrm{pL}$ & & $0.0072 \pm 0.0015_{(n=19)}$ & $0.0070 \pm 0.0021_{(n=11)}$ & 0.906 \\
\hline 8-Isoprostane & $\mathrm{pg} / \mathrm{mL}$ & 0.5 & $6.7 \pm 1.2$ & $5.1 \pm 0.8$ & 0.288 \\
\hline $\mathrm{H}_{2} \mathrm{O}_{2}$ & $\mu \mathrm{M}$ & 0.1 & $1.2 \pm 0.2$ & $1.5 \pm 0.3$ & 0.331 \\
\hline Nitrate & $\mu \mathrm{M}$ & 0.05 & $68.0 \pm 9.0$ & $61.1 \pm 9.5$ & 0.615 \\
\hline Nitrite & $\mu \mathrm{M}$ & 0.05 & $8.4 \pm 1.9$ & $8.1 \pm 1.2$ & 0.907 \\
\hline IL-2 & $\mathrm{pg} / \mathrm{mL}$ & 2.8 & $16.2 \pm 0.7_{(n=28)}$ & $16.3 \pm 0.6_{(n=29)}$ & 0.907 \\
\hline IL-5 & $\mathrm{pg} / \mathrm{mL}$ & 1.5 & $3.7 \pm 0.4_{(n=26)}$ & $4.4 \pm 0.5_{(n=27)}$ & 0.261 \\
\hline IL-13 & $\mathrm{pg} / \mathrm{mL}$ & 4.4 & $5.4 \pm 0.5_{(n=18)}$ & $7.2 \pm 0.7_{(n=20)}$ & 0.066 \\
\hline EBC acidity & & & $6.1 \pm 0.1$ & $6.3 \pm 0.1$ & 0.092 \\
\hline
\end{tabular}

Data are given as mean $\pm \mathrm{SE}$, except were indicated otherwise. Values are based on 30 observations unless indicated otherwise. Individual ratios of $\mathrm{C}_{\mathrm{alv}} / \mathrm{J}_{\mathrm{NO}}$ can only be calculated when both $\mathrm{C}_{\mathrm{alv}}$ and $\mathrm{J}_{\mathrm{No}}$ values are known; Abbreviations: $\mathrm{H}_{2} \mathrm{O}_{2}$, hydrogen peroxide; IL, interleukin; LDL, lower detection limit; * FeNO measured at a flow rate of $50 \mathrm{~mL} / \mathrm{sec}$; ** paired-sample $\mathrm{T}$ test.

\section{Lung function indices, symptoms and adverse effects}

There was no difference in $\mathrm{FEV}_{1}, \mathrm{FEV}_{1} / \mathrm{VC}, \mathrm{FVC}, \mathrm{MEF}_{50}$ indices between a 3 month treatment period with HFA-beclomethasone and fluticasone-DPI (paired sample t-test, Table $3)$.

Table 3: Lung function parameters after a 3 month treatment period with HFA-beclomethasone and fluticasone-DPI.

\begin{tabular}{lll}
\hline & $\begin{array}{l}\text { HFA-beclomethasone } \\
(\mathrm{N}=30)\end{array}$ & $\begin{array}{l}\text { fluticasone-DPI } \\
(\mathrm{N}=30)\end{array}$ \\
\hline $\mathrm{FEV}_{1}(\%$ of predicted) & Mean $\pm \mathrm{SE}$ & Mean $\pm \mathrm{SE}$ \\
$\mathrm{FEV}_{1} / \mathrm{VC}(\%)$ & $101.7 \pm 2.4$ & $101.5 \pm 3.0$ \\
$\mathrm{FVC}(\%$ of predicted) & $85.0 \pm 1.3$ & $85.6 \pm 1.4$ \\
$\mathrm{MEF}_{50}(\%$ of predicted) & $97.2 \pm 2.2$ & $96.9 \pm 2.6$ \\
\hline
\end{tabular}

Abbreviations: $\mathrm{FEV}_{1}$, forced expiratory volume in 1 second; $\mathrm{VC}$, vital capacity; $\mathrm{MEF}_{50}$, mean expiratory flow at $50 \%$.

The asthma control questionnaire (ACQ) showed no differences in the total score after the 3-month treatment periods. Total ACQ during treatment with HFA-beclomethasone was $0.81 \pm 0.18$ versus $0.94 \pm 0.20$ during fluticasone-DPI (paired t-test, $p=0.471$ ). In addition, the presence and severity of asthma symptoms (dyspnoea, cough, chest pain, wheeze and awakening caused by asthma) did not differ between HFA-beclomethasone and fluticasone-DPI (Table 4). 
Table 4: Symptoms recorded in a daily diary during the last two weeks of the three month treatment period with HFA-beclomethasone and fluticasone-DPI.

\begin{tabular}{lllll}
\hline & & $\begin{array}{l}\text { HFA-beclomethasone } \\
(\mathrm{N}=30)\end{array}$ & $\begin{array}{l}\text { fluticasone-DPI } \\
(\mathrm{N}=30)\end{array}$ & p-value \\
\hline ACQ & Score & $0.81 \pm 0.18$ & $0.94 \pm 0.20$ & $0.471^{*}$ \\
Dyspnoea & Days & $0(0-5)$ & $0(0-7)$ & $0.145^{* *}$ \\
Cough & Days & $4(0-10)$ & $3(0-9)$ & $0.489^{* *}$ \\
Chest pain & Days & $0(0-1)$ & $0(0-1)$ & $0.416^{* *}$ \\
Wheeze & Days & $0(0-5)$ & $0(0-3)$ & $0.311^{* *}$ \\
Awakening & Days & $0(0-5)$ & $0(0-3)$ & $0.648^{* *}$ \\
Symptom-free & Days & 39 & 33 & $0.593^{\#}$ \\
Local adverse effects & $\%$ & 17 & 13 & $0.411^{\#}$ \\
Z-score ${ }^{*}$ & & $1.71 \pm 0.42$ & $1.86 \pm 0.46$ & $0.203^{*}$ \\
Height growth & $\mathrm{cm}$ & $1.07 \pm 0.16$ & $1.41 \pm 0.20$ & $0.299^{*}$ \\
\hline
\end{tabular}

Data are given as mean $\pm \mathrm{SE}$, and, as median (range); ${ }^{*}$ paired T-test; ${ }^{* *}$ Wilcoxon Signed Rank Test; ${ }^{*}$ Chisquare test; $\wedge$ Z-score weight to height to age and gender.

10 Patients in each treatment group were considered to have well controlled asthma. 17\% And $13 \%$ of the children experienced local adverse effects during HFA-beclomethasone and fluticasone-DPI treatment, respectively (paired t-test, $p=0.411$ ).

The number of days with use of a short acting $\beta_{2}$-agonist differed between the studied treatments. Less patients did use $\beta_{2}$-agonists during the HFA-beclomethasone treatment ( $33 \%$ vs $51 \%$, Chi ${ }^{2}$-test, $p<0.001$ ). The frequency of $\beta_{2}$-agonist use did not differ between treatment with extra-fine HFA-belomethasone (median (25\%-75\%), 0 (0-2) times), or fluticasone-DPI treatment (median (25\%-75\%), 1 (0-8) times) (Wilcoxon rank test, $p=0.054$ ).

\section{Discussion}

This study shows equal anti-inflammatory effects of HFA-beclomethasone and fluticasoneDPI as assessed by bronchial and alveolar NO, and inflammatory markers in EBC. Also with respect to lung function and symptoms, no differences were found. This implicates equal effectiveness of treatment with extrafine HFA-beclomethasone 2 times $100 \mu \mathrm{g}$ daily via an autohaler, and, fluticasone-DPI 2 times $100 \mu \mathrm{g}$ daily via a diskus, in controlling moderate childhood asthma [34]. Despite an equal asthma control status, less patients used short acting $\beta_{2}$-agonist during the treatment period with HFA-beclomethasone, compared to the use of rescue medication during treatment with fluticasone-DPI.

Notably, in the present study, a comparable anti-inflammatory effect was established with an equal dose of HFA-beclomethasone and fluticasone-DPI. Based on the greater deposition of HFA-beclomethasone in the airways, a more potent anti-inflammatory effect could have been expected in the peripheral airways, compared to the effect reached with fluticasone-DPI treatment [35]. However, this study showed no additional effect of HFAbeclomethasone in the peripheral airways compared to fluticasone-DPI, as assessed by means of alveolar and bronchial NO or their ratio. Therefore, we found no reasons to recommend HFA-beclomethasone over fluticasone-DPI in children, aged 6-12 years, with moderate asthma in this study. 
Despite fluticasone-DPI is 2.2 more potent on receptor level compared to HFAbeclomethasone, and the use of equal daily dose in both treatments, all measured inflammatory characteristics in this study were similar after 3 months treatment with HFAbeclomethasone and fluticasone-DPI. Probably this is the consequence of the increased peripheral deposition of HFA-beclomethasone. Due to this increased drug deposition in the airways of HFA-beclomethasone, higher plasma steroid levels may be expected [36, 10]. This may increase the rate of systemic adverse effects. Although, plasma levels of the used drugs were not measured in this study, no differences in growth between the 2 groups were observed. Also, local adverse effects were equally present.

Recently, van Schayck et al. and Randell et al. concluded that no clinical relevant adverse effects of HFA-beclomethasone on adrenal function, growth velocity or bone metabolism, were found [37, 38].

To our knowledge, our study was the first on the comparison of HFA-beclomethasone versus fluticasone-DPI in controlling asthma primarily based on exhaled (alveolar and bronchial) NO, and inflammatory markers in EBC in children. In previous studies comparing HFA-beclomethasone with fluticasone-DPI in controlling asthma, lung function indices and symptom scores were used as outcome parameters. Equal effectiveness of extrafine HFA-beclomethasone and fluticasone-DPI with mild-persistent asthma on lung function indices, use of rescue medication, or symptom scores, was also found by Aubier et al. [39]. They performed an open study to demonstrate the equivalence of $800 \mu \mathrm{g}$ HFAbeclomethasone daily and $1000 \mu \mathrm{g}$ HFA-fluticasone daily in 198 adults with moderate to severe asthma.

The present study is a real life study. Before the study, patients were all treated with inhaled corticosteroids. For this reason, it was not ethical to withdraw steroid treatment in order to create a steroid free wash-out period between the treatments. This methodological weakness was resolved by treating the patients during a sufficiently long period of 3 months. This is sufficient for the primary outcome measurement, FeNO and alveolar/bronchial NO. For the inflammatory markers in EBC, it is still unknown whether a carryover effect is present [40]. It is known that NO responds within weeks when starting or stopping inhaled corticosteroids, or with dose changes. Therefore, NO values after 3 months treatment are not influenced by a carry-over effect. In addition, statistical analysis of FeNO based on the results in this study, showed no significant carry-over effect of FeNO. Furthermore, it was not possible to blind the inhaled steroids, because of drug inhalation through different delivery devices. However, drug characteristics and the different inhalation devices may influence patients subjective symptoms or the use of $\beta_{2^{-}}$ agonists, but cannot influence NO or inflammatory markers in EBC. It is important to evaluate the clinical effectiveness of the different inhaled corticosteroids in combination with their inhaler device. And, in this fashion, the use of different inhalation devices reflect the real life situation.

There may be various reasons why the hypothesis, that extra fine HFA-beclomethasone would have better anti-inflammatory properties in peripheral airways than fluticasoneDPI, was not shown in this study: 1) the clinical importance of the extra fine particle characteristic of HFA-beclomethasone, may be increasingly relevant in airways with smaller diameter, like in a population of even smaller children, or asthmatic patients with more airway obstruction. 2) patients with asthma in this study may be controlled with an even lower inhaled corticosteroid dosage. This relative 'over-treatment', could mask a possible 
difference in anti-inflammatory effect between extrafine HFA-beclomethasone and fluticasone-DPI.

In conclusion, this study showed equal anti-inflammatory effects of HFA-beclomethasone 2 times $100 \mu \mathrm{g}$ daily via an autohaler, and, fluticasone-DPI 2 times $100 \mu \mathrm{g}$ daily via a diskus, in children with moderate persistent asthma, assessed by means of exhaled (alveolar and bronchial) NO measurements, inflammatory markers in EBC, lung function indices, asthma symptoms and adverse effects. The presence and extent of airway inflammation can be measured using non-invasive inflammometry. Based on the results of this study, there are no reasons to recommend HFA-beclomethasone over fluticasone-DPI, or vice versa.

\section{Acknowledgement}

We thank Ivax, member of the Teva group, for their financial support.

\section{References}

1 Kraft M. The distal airways: are they important in asthma? Eur Respir J 1999; 14:1403-1417

2 Hamid Q, Song Y, Kotsimbos TC, et al. Inflammation of small airways in asthma. J Allergy Clin Immunol 1997; 100:44-51

3 Bjermer L. History and future perspectives of treating asthma as a systemic and small airways disease. Respir Med 2001; 95:703-719

4 Washington (DC): National Institutes of Health LaBI. Global Initiative for Asthma (GINA). Global strategy for asthma management and prevention, 2006

5 Duiverman EJ, Brackel HJ, Merkus PJ, et al. [Guideline 'Treating asthma in children' for pediatric pulmonologists (2nd revised edition). II. Medical treatment]. Ned Tijdschr Geneeskd 2003; 147:1909-1913

6 Vanden Burgt JA, Busse WW, Martin RJ, et al. Efficacy and safety overview of a new inhaled corticosteroid, QVAR (hydrofluoroalkane-beclomethasone extrafine inhalation aerosol), in asthma. J Allergy Clin Immunol 2000; 106:1209-1226

7 Mitchell JP, Nagel MW, Wiersema KJ, et al. Aerodynamic particle size analysis of aerosols from pressurized metered-dose inhalers: comparison of Andersen 8-stage cascade impactor, next generation pharmaceutical impactor, and model 3321 Aerodynamic Particle Sizer aerosol spectrometer. AAPS PharmSciTech 2003; 4:E54

8 Fuller R. The Diskus: a new multi-dose powder device-efficacy and comparison with Turbuhaler. J Aerosol Med 1995; 8 Suppl 2:S11-17

9 Roller CM, Zhang G, Troedson RG, et al. Spacer inhalation technique and deposition of extrafine aerosol in asthmatic children. Eur Respir J 2007; 29:299-306

10 Leach CL, Davidson PJ, Hasselquist BE, et al. Lung deposition of hydrofluoroalkane-134a beclomethasone is greater than that of chlorofluorocarbon fluticasone and chlorofluorocarbon beclomethasone : a cross-over study in healthy volunteers. Chest 2002; 122:510-516

11 Warke TJ, Fitch PS, Brown V, et al. Exhaled nitric oxide correlates with airway eosinophils in childhood asthma. Thorax 2002; 57:383-387

12 Piacentini GL, Bodini A, Costella S, et al. Exhaled nitric oxide and sputum eosinophil markers of inflammation in asthmatic children. Eur Respir J 1999; 13:1386-1390

13 Kharitonov SA, Barnes PJ. Exhaled markers of pulmonary disease. Am J Respir Crit Care Med 2001; 163:16931722

14 Pijnenburg MW, Hofhuis W, Hop WC, et al. Exhaled nitric oxide predicts asthma relapse in children with clinical asthma remission. Thorax 2005; 60:215-218

15 Jones SL, Kittelson J, Cowan JO, et al. The predictive value of exhaled nitric oxide measurements in assessing changes in asthma control. Am J Respir Crit Care Med 2001; 164:738-743 
16 Berkman N, Avital A, Breuer R, et al. Exhaled nitric oxide in the diagnosis of asthma: comparison with bronchial provocation tests. Thorax 2005; 60:383-388

17 Battaglia S, den Hertog $\mathrm{H}$, Timmers $\mathrm{MC}$, et al. Small airways function and molecular markers in exhaled air in mild asthma. Thorax 2005; 60:639-644

18 Lehtimaki L, Kankaanranta H, Saarelainen S, et al. Extended exhaled NO measurement differentiates between alveolar and bronchial inflammation. Am J Respir Crit Care Med 2001; 163:1557-1561

19 Lehtimaki L, Kankaanranta H, Saarelainen S, et al. Inhaled fluticasone decreases bronchial but not alveolar nitric oxide output in asthma. Eur Respir J 2001; 18:635-639

20 Robroeks CMHHT, Jöbsis Q, Damoiseaux JGMC, et al. Cytokines in exhaled breath condensate of children with asthma and cystic fibrosis. Ann Allergy Asthma Immunol 2006; 96:349-355

21 Kharitonov SA, Barnes PJ. Exhaled markers of inflammation. Curr Opin Allergy Clin Immunol 2001; 1:217-224

22 Montuschi P, Barnes PJ. Analysis of exhaled breath condensate for monitoring airway inflammation. Trends Pharmacol Sci 2002; 23:232-237

23 Rosias PP, Dompeling E, Hendriks HJ, et al. Exhaled breath condensate in children: Pearls and pitfalls. Pediatr Allergy Immunol 2004; 15:4-19

24 Baraldi E, Ghiro L, Piovan V, et al. Safety and success of exhaled breath condensate collection in asthma. Arch Dis Child 2003; 88:358-360

25 ATS/ERS Recommendations for Standardized Procedures for the Online and Offline Measurement of Exhaled Lower Respiratory Nitric Oxide and Nasal Nitric Oxide, 2005. Am J Respir Crit Care Med 2005; 171:912-930

26 Rosias PP, Dompeling E, Dentener MA, et al. Childhood asthma: Exhaled markers of airway inflammation, asthma control score, and lung function tests. Pediatr Pulmonol 2004; 38:107-114

27 Tsoukias NM, George SC. A two-compartment model of pulmonary nitric oxide exchange dynamics. J Appl Physiol 1998; 85:653-666

28 de Jager W, Rijkers GT. Solid-phase and bead-based cytokine immunoassay: a comparison. Methods 2006; 38:294-303

29 Montuschi P, Corradi M, Ciabattoni G, et al. Increased 8-isoprostane, a marker of oxidative stress, in exhaled condensate of asthma patients. Am J Respir Crit Care Med 1999; 160:216-220

30 Dekhuijzen PN, Aben KK, Dekker I, et al. Increased exhalation of hydrogen peroxide in patients with stable and unstable chronic obstructive pulmonary disease. Am J Respir Crit Care Med 1996; 154:813-816

31 Juniper EF, O'Byrne PM, Ferrie PJ, et al. Measuring asthma control. Clinic questionnaire or daily diary? Am J Respir Crit Care Med 2000; 162:1330-1334

32 Schouten HJA. Klinische statistiek. Bohn: Stafleu en van Loghum, 2002

33 Altmann DG. Practical statistics for medical research. First CRC Press Reprint ed. London, 1999

34 van Aalderen WM, Price D, De Baets FM, et al. Beclometasone dipropionate extrafine aerosol versus fluticasone propionate in children with asthma. Respir Med 2007

35 Dekhuijzen PN. Drug targeting in bronchial asthma: inhaled corticosteroids should reach the peripheral airways. Current Drug Therapy 2006; 1:257-261

36 Agertoft L, Laulund LW, Harrison LI, et al. Influence of particle size on lung deposition and pharmacokinetics of beclomethasone dipropionate in children. Pediatr Pulmonol 2003; 35:192-199

37 van Schayck CP, Donnell D. The efficacy and safety of QVAR (hydrofluoroalkane-beclometasone diproprionate extrafine aerosol) in asthma (Part 2): Clinical experience in children. Int J Clin Pract 2004; 58:786-794

38 Randell TL, Donaghue KC, Ambler GR, et al. Safety of the newer inhaled corticosteroids in childhood asthma. Paediatr Drugs 2003; 5:481-504

39 Aubier M, Wettenger R, Gans SJ. Efficacy of HFA-beclomethasone dipropionate extra-fine aerosol (800 microg day(-1)) versus HFA-fluticasone propionate (1000 microg day(-1)) in patients with asthma. Respir Med 2001; 95:212-220

40 van Beurden WJ, Harff GA, Dekhuijzen PN, et al. Effects of inhaled corticosteroids with different lung deposition on exhaled hydrogen peroxide in stable COPD patients. Respiration 2003; 70:242-248 


\section{CHAPTER 11.1}

Non-invasive inflammatory markers in exhaled breath condensate predict exacerbations of childhood asthma; results of a one-year prospective controlled study

CMHHT Robroeks, D van Vliet, Q Jöbsis, R Braekers, GT Rijkers, WKWH Wodzig, A Bast, GJM den Hartog, LJI Zimmermann, E Dompeling Submitted 


\section{Abstract}

\section{Background}

Non-invasive measurements of inflammatory markers in exhaled breath (condensate) reflect airway inflammation and oxidative stress in airways and may be useful in the monitoring of childhood asthma. Aim: To study the ability of non-invasive inflammatory markers to predict exacerbations of childhood asthma; To assess the time course of changes in exhaled inflammatory markers during exacerbations.

\section{Methods}

The design was a prospective one-year longitudinal study. 40 Children with asthma (aged 6-16 yrs) participated. Regular two-monthly visits to the outpatient clinic were required. The primary outcome measure was the occurrence of an exacerbation. Assessment was made of the presence and severity of pulmonary symptoms, use of medication, and measurements of forced expiratory volume in one second using home monitor. The following independent parameters were assessed during outpatient visits: 1) exhaled nitric oxide, 2) inflammatory markers in exhaled breath condensate: acidity, nitrite, hydrogen peroxide, interleukin-1 $\alpha,-5,-13$, interferon- $\gamma, 3)$ lung function indices, 4) asthma control score.

\section{Results}

38 of the 40 children completed the study. Sixteen children developed exacerbations, of which 10 were moderate and 6 severe. Inflammatory markers in exhaled breath condensate were detected in $85-100 \%$ of samples. Univariate Cox regression analysis revealed that condensate acidity, interleukin- 5 and asthma control score were significant predictors of asthma exacerbation $(p<0.05)$. Multivariate Cox regression analysis showed that the time until an exacerbation was significantly predicted by the asthma control score and by the level of interleukin- 5 in exhaled breath condensate. The Kaplan-Meier survival curve of this multivariate model showed that children with the $10 \%$ most optimal values of interleukin-5 and the asthma control score had a more than two times reduced risk of exacerbations in the subsequent year.

\section{Conclusion}

Both exhaled breath condensate interleukin-5 level and asthma control score were significant predictors of asthma exacerbation. These findings open up the possibility of assessing the potential of such parameters to titrate asthma treatment in future studies. 


\section{Introduction}

Since the release of the GINA guidelines, several Asthma Insights and Reality (AIR) surveys worldwide have assessed the severity, control, and management of asthma in children and adults by means of cross-sectional surveys [1,2] Despite variations at country level, all these studies have indicated that the level of asthma control falls short of goals for longterm management set by international guidelines [1-3]. One probable explanation is that monitoring of asthma is currently performed by assessments of symptoms and lung function, but not by measurements of airway inflammation $[4,5]$. Correlation between parameters of airway inflammation on one hand, and symptoms and lung function on the other, is generally low. Moreover, many children with asthma have poor perception of dyspnoea, which results in underreporting of symptoms and even underdiagnosis of asthma in childhood. [3, 6].

Standard conventional techniques to assess airway inflammation in children such as bronchoscopy with bronchoalveolar lavage or bronchial biopsy have to be performed under general anaesthesia, and are therefore far too invasive for routine use. There is a need for specific non-invasive tests. Previous studies have shown that fractional exhaled nitric oxide (FeNO) and inflammatory markers (IM) in exhaled breath condensate (EBC) considered together may be useful in the diagnosis of asthma, and in the identification of asthma stability and severity [7]. Other research groups have been able to discriminate between healthy and diseased children using tests based on FeNO and concentrations of various markers in EBC, such as Th1/Th2 cytokines, nitrate, nitrite, and hydrogen peroxide [8-11]. These cross-sectional findings have shown that $\mathrm{EBC}$ may be a promising tool for diagnostic and monitoring purposes. Longitudinal data, however, are lacking. A prospective longitudinal study over a period of one year was therefore performed in children with asthma, in which regular measurements of biomarkers in EBC were carried out. The aims of this Asthma Inflammation Monitoring (AIM) study were to assess the ability of exhaled inflammatory markers (FeNO, and EBC $\mathrm{pH}$, nitrite, hydrogen peroxide, IL-1 $\alpha$, IL-5, IL-13, IFNv) to predict asthma exacerbation, and, to study the time course of changes in exhaled IM during asthma exacerbations.

\section{Methods}

\section{Patients}

Children aged 6-16 years with medically diagnosed asthma were recruited. They were selected from the outpatient clinic of the Department of Paediatric Pulmonology, Maastricht University Medical Centre, and had been known to have had an asthma diagnosis at our clinic for at least six months. Asthma was defined as a chronic inflammatory disorder with paroxysmal wheezing, breathlessness, chest tightness, or coughing with a variable but reversible airway obstruction and airway hyperresponsiveness [12]. Both allergic and non-allergic asthmatic children were selected. A child was considered atopic when the total IgE level exceeded $20 \mathrm{kU} / \mathrm{L}$, the Phadiatop was positive or the Radio Allergo Sorbent Test (RAST) was positive (at least two RAST classes $\geq$ class 2). Exclusion criteria were: 1 ) diseases that may interfere with the results of the study (e.g. heart disease, anatomic 
abnormalities of the airway, other chronic inflammatory diseases, such as Crohn's disease or rheumatoid arthritis), 2) mental retardation, 3) inability to perform measurements properly, or 4) active smoking. All parents or children gave informed consent. This study was approved by the Medical Ethics Committee of the Maastricht University Medical Centre. The international study number was NCT00404859.

\section{Study design}

The design was a prospective longitudinal study during a one year period. Every two months, routine visits took place at the outpatient clinic. In addition to these fixed visits, patients were asked to visit the outpatient clinic an additional four times during an exacerbation. These extra visits were planned maximally twice during the study. Extra measurements were planned at days one, three, and five of the exacerbation and after stabilisation.

\section{Primary outcome measure: asthma exacerbation}

The occurrence of an asthma exacerbation was the primary outcome measure of this study. A moderate exacerbation was defined as an increase in asthma symptoms (dyspnoea, chough, wheezing) and/or use of short acting $\beta_{2}$-agonists for not more than two days. ${ }^{13}$ In this case, the fall in $\mathrm{FEV}_{1}$ was not below the $80 \%$ personal maximum value. In case of a severe exacerbation, one or more of the following items was present: 1) The $\mathrm{FEV}_{1} \%$ of maximum personal value fell below $80 \%$ of the person maximal value for at least two consecutive days, and/or, 2) need for treatment with oral corticosteroids, and/or, 3) need for hospital admission [13].

To recognise an exacerbation in an early stage, AM1 home monitors (Viasys, Hoechberg, Germany) were used. All patients received a personal home monitor and a corresponding modem (HC1, Viasys, Hoechberg, Germany). Using this monitor, patients could measure $\mathrm{FEV}_{1}$ at home. In addition, use of rescue medication, overall well-being, and the presence and severity of pulmonary symptoms were recorded. The intensity of symptoms was scored on a scale of zero to three. Patients were asked to use the AM1 once daily, at a fixed time of the day. All patients were asked to perform the manoeuvres three times within ten minutes, and the highest $\mathrm{FEV}_{1}[\mathrm{~L}]$ was stored in the memory. Data were sent to a computer at the Maastricht University Medical Centre by means of a modem once per week. In case of deterioration of $\mathrm{FEV}_{1}$ values, and/or an increase in presence and severity of pulmonary symptoms, patients were called into the hospital for additional measurements and a consultation with the paediatric pulmonologist responsible.

\section{Measurements of independent predictors}

At each clinical visit, parameters were assessed in the following order: 1) FeNO, 2) IM in EBC 3) lung function indices, 4) asthma control score, and, 5) asthma severity (criteria used as published previously). ${ }^{7}$ All measurements were performed within one hour. 


\section{NO in exhaled air}

First, FeNO was measured online using a NIOX chemiluminescence analyser (Aerocrine, Solna, Sweden). Measurements were carried out according to the American Thoracic Society/European Respiratory Society (ATS/ERS) criteria for children [14].

\section{Exhaled breath condensate collection and analysis}

EBC was collected by means of an optimised borosilicate glass tube, cooled by countercurrent circulating ice water, as described in previous research [15]. Children breathed tidally for ten minutes, while wearing a nose-clip, through a mouthpiece connected to a two-way non-re breathing valve (Hans Rudolph Inc, series 1420, Kansas City, USA). After collection, $\mathrm{EBC}$ was rapidly frozen at $-80^{\circ} \mathrm{C}$ using dry ice and was stored at $-80^{\circ} \mathrm{C}$ until analysis.

Acidity of EBC was measured immediately after collection (Radiometer, type PHM201, Radiometer Nederland BV, Zoetermeer, NL) without de-aeration. Subsequently, EBC samples were frozen using dry ice and stored at $-80^{\circ} \mathrm{C}$. Levels of nitrite (fluoremetric) and hydrogen peroxide $\left(\mathrm{H}_{2} \mathrm{O}_{2}\right.$, spectrometric) were assessed as described in an earlier study [7]. Concentrations of IL-1 $\alpha$, IL-5, IL-13, and IFN- $\gamma$ in 100 microliters of EBC were determined using the multiplex immunoassay Luminex 100 analyser (Luminex Corporation, Austin, TX, USA) as described earlier [16-18]. Lower limits of detection were included in Table 3. All EBC samples of a specific patient were analysed simultaneously in order to minimise inter-assay variation.

\section{Lung function tests}

Dynamic spirometry was performed by means of the Flowscreen ${ }^{\bullet}$, according to ERS standards (Viasys, Hoechberg, Germany) [19]. Highest values of three correctly performed manoeuvres were used for analysis. Recorded indices were forced expiratory volume in one second $\left(\mathrm{FEV}_{1}\right)$, forced vital capacity $(\mathrm{FVC})$, and maximal expiratory flow at $50 \%$ of FCV $\left(\mathrm{MEF}_{50}\right)$, all expressed as a percentage of the predicted normal value [19]. Reversibility to a beta-2-agonist was determined 15 minutes after inhalation of $400 \mu \mathrm{g}$ salbutamol at zero, six and twelve months of the study. Short-acting bronchodilators were stopped at least eight hours and long-acting bronchodilators at least 36 hours before testing.

\section{Asthma control score}

The asthma control score was assessed two-monthly using a validated questionnaire, as in previous studies $[2,3]$. The questionnaire contained questions about chronic airway symptoms, sleep disturbance, limitation of daily activity, asthmatic attacks, emergency or urgent care visits, and need for short acting beta-2-agonists [3].

\section{Treatment}

During the one-year study period, children were treated by their own paediatric pulmonologist according to GINA guidelines [20]. 


\section{Statistic analysis}

The statistical tests to compare and correlate normally distributed parameters between individuals and populations were Students t- or Wilcoxon's test and the Pearson Rank test, respectively. Non-normally distributed parameters were compared and correlated using the Mann-Whitney U or Friedman tests and Spearman's rank test, respectively. The influence of independent predictors (non-invasive IM, lung function, asthma control) on the time until an exacerbation was analysed by univariate and multivariate Cox regression analyses. Samples with IM levels below the detection limit were given an arbitrary value between 0 and the lower limit of detection. Two-sided P-values $<0.05$ were taken as lower limit of statistical significance.

\section{Power analysis}

In order to find clinically meaningful correlations between independent predictors and exacerbation rate of $0.6, \mathrm{~N}=35$ children are necessary to assess such a relationship with a two-sided alpha of 0.05 and a power of $98 \%$. During the 12 month follow-up period, a drop-out rate of $10 \%$ was assumed. Therefore, 40 children with asthma were included in this study.

\section{Role of the funding source}

AstraZeneca had no role in the study design, in writing the manuscript or in the decision to submit this paper.

\section{Results}

\section{Population characteristics}

All asthma severity classes were present and were almost equally distributed within the population at the start of the study: intermittent $11 / 40$ (27.5\%), mild persistent $8 / 40$ (20\%), moderately persistent $7 / 40(17 \cdot 5 \%)$, and severely persistent $14 / 40$ (35\%). Lung function indices showed no obstructive or restrictive abnormalities (Table 1). The majority of the group was atopic: $29 / 40$ (73\%). With one exception, all patients received maintenance treatment with inhaled corticosteroids.

38 of the 40 children completed the study while two dropped out. Of these two, one patient moved out of the region. Before he had to withdraw, he experienced one exacerbation. The other child lost interest in future participation. She had stable asthma.

\section{Exacerbations}

During the one-year study period, 16 children developed an exacerbation of which 10 were moderate and 6 severe. Only 3 patients developed a second exacerbation. The mean \pm SE time interval between the preceding standard measurement and the onset of exacerbation was $39 \pm 4$ days. 
Table 1: Clinical characteristics of asthmatic children at commencement of study.

\begin{tabular}{|c|c|}
\hline Age (yrs) & $10.7 \pm 0.4$ \\
\hline Weight (kg) & $38.8 \pm 2.0$ \\
\hline Height $(\mathrm{cm})$ & $142.3 \pm 2.4$ \\
\hline Male / Female & $29 / 11$ \\
\hline Asthma control score (minimum-maximum) & $27.6 \pm 2.3(9-64)$ \\
\hline \multicolumn{2}{|l|}{ Lung function indices } \\
\hline - Reversibility (increase in $\mathrm{FEV}_{1} \%$ predicted) & $5.9 \pm 1.1$ \\
\hline - $\mathrm{FEV}_{1} \%$ predicted & $99.6 \pm 2.2$ \\
\hline$-\mathrm{FEV}_{1} / \mathrm{VC} \%$ & $83.9 \pm 1.5$ \\
\hline - FVC \% predicted & $99.2 \pm 2.4$ \\
\hline - $\mathrm{MEF}_{50} \%$ predicted & $82.6 \pm 3.8$ \\
\hline Atopy (yes/no) & $29 / 11$ \\
\hline - Total IgE (kU/L) & $493.3 \pm 120.7$ \\
\hline - Active eczema & $7(18 \%)$ \\
\hline - Allergic rhinitis & $4(10 \%)$ \\
\hline \multicolumn{2}{|l|}{ Treatment } \\
\hline - Dose of inhaled budesonide or equivalent ( $\mu \mathrm{g})$ & $587 \pm 53$ \\
\hline - Long-acting beta-2-agonist & $21(53 \%)$ \\
\hline - Leukotriene receptor antagonist & $8(20 \%)$ \\
\hline
\end{tabular}

Data were given as mean \pm SEM except where indicated otherwise. Abbreviations: $\mathrm{FEV}_{1}$, forced expiratory volume in 1 second; $\mathrm{VC}$, vital capacity; $\mathrm{MEF}_{50} \%$, mean expiratory flow at $50 \%$.

\section{Cox regression analyses}

The results of Cox regression analysis are shown in Table 2. In the univariate analysis, asthma control score, EBC acidity and IL-5 were significantly related to the time until an exacerbation (Table $2 \mathrm{a}, \mathrm{p}<0.05$ ). When analysed using a multivariate model, only EBC IL-5 and asthma control score remained significant (Table $2 \mathrm{~b}$ ).

The Kaplan-Meier survival curve of this multivariate model showed that children with the $10 \%$ most optimal values of IL-5 and asthma control score had a more than two times lower risk of exacerbations after one year (Figure 1).

Table 2a: Univariate Cox regression analysis of the time until an exacerbation.

\begin{tabular}{llll}
\hline Parameter & $\beta \pm \mathrm{SE}$ & $\begin{array}{l}\text { Wald score } \\
\text { Chi-square }(\mathrm{df}=1)\end{array}$ & $\mathrm{p}$-value \\
\hline FEV ${ }_{1} \%$ predicted & $-0.02 \pm 0.03$ & 0.64 & 0.43 \\
Asthma control score & $\mathbf{0 . 0 4} \pm \mathbf{0 . 0 2}$ & $\mathbf{4 . 8 2}$ & $\mathbf{0 . 0 3}$ \\
FeNO $(\mathrm{ppb})$ & $0.01 \pm 0.01$ & 0.27 & 0.60 \\
$\mathrm{EBC} \mathrm{pH}$ & $\mathbf{1 . 1 8} \pm \mathbf{0 . 5 2}$ & $\mathbf{5 . 1 0}$ & $\mathbf{0 . 0 3}$ \\
$\mathrm{Nitrite}(\mu \mathrm{M})$ & $-0.005 \pm 0.03$ & 0.03 & 0.87 \\
$\mathrm{H}_{2} \mathrm{O}_{2}(\mu \mathrm{M})$ & $-0.11 \pm 0.09$ & 1.28 & 0.26 \\
$\mathrm{IL}-1 \alpha$ & $-0.0004 \pm 0.0006$ & 0.45 & 0.50 \\
$\mathrm{IL}-5$ & $\mathbf{0 . 0 0 0 4} \pm \mathbf{0 . 0 0 0 2}$ & $\mathbf{3 . 7 6}$ & $\mathbf{0 . 0 5}$ \\
$\mathrm{IL}-13$ & $0.0001 \pm 0.001$ & 0.01 & 0.92 \\
$\mathrm{IFN}-\gamma$ & $-0.0002 \pm 0.002$ & 0.01 & 0.92 \\
\hline
\end{tabular}


Table 2b: Multivariate Cox regression analysis of the time until an exacerbation.

\begin{tabular}{llll}
\hline Parameter & $\beta \pm$ SE & $\begin{array}{l}\text { Wald score } \\
\text { Chisquare }(\mathrm{df}=1)\end{array}$ & $\mathrm{p}$-value \\
\hline Asthma control score & $0.047 \pm 0.017$ & 7.19 & 0.007 \\
IL-5 & $0.0004 \pm 0.0002$ & 4.44 & 0.035 \\
\hline
\end{tabular}

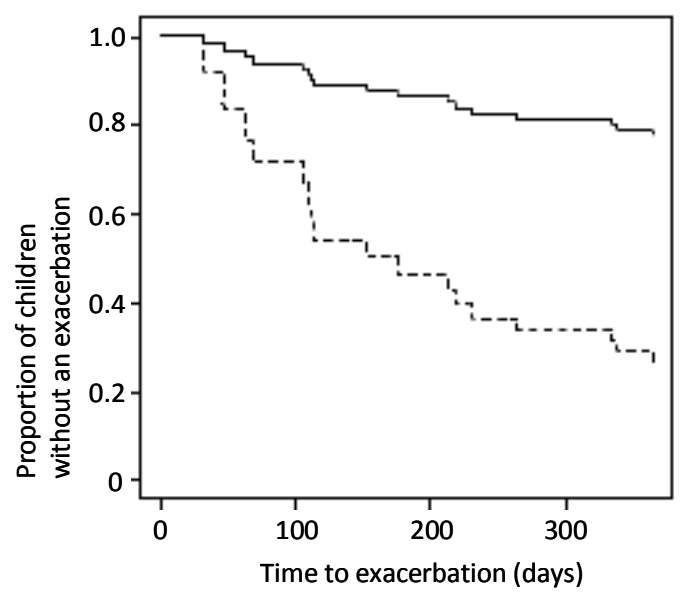

Figure 1: Kaplan-Meier survival curve of the time until an exacerbation in children with the $10 \%$ best values of IL-5 and the asthma control score (dashed line) compared to the $10 \%$ least optimal IL-5 and control score (unbroken line).

\section{Independent predictors before and during exacerbations}

Table 3 shows the independent predictors before and during exacerbations. Significant changes were found in nitrite, IL-13 and IFN- $\gamma$ (Friedman test, $p<0.05$ ). In Figure $2 a-b$, the course of FEV 1 , IL-5 and FeNO are shown, respectively.

Table 3: Course of inflammatory markers until the onset of an asthma exacerbation.

\begin{tabular}{|c|c|c|c|c|c|c|}
\hline Parameter & & LLD & Stable period & $\begin{array}{l}39 \pm 4 \text { days before the } \\
\text { onset of an exacerba- } \\
\text { tion }\end{array}$ & $\begin{array}{l}\text { At the onset of an } \\
\text { exacerbation }\end{array}$ & $\mathrm{p}$-value \\
\hline $\mathrm{FEV}_{1} \%$ pred & $*$ & & $102.8 \pm 4.8$ & $100.0 \pm 4.9$ & $90.9 \pm 5.6$ & 0.14 \\
\hline FeNO (ppb) & $*$ & & $25.5 \pm 5.7$ & $27.7 \pm 5.3$ & $35.1 \pm 8.5$ & 0.70 \\
\hline $\mathrm{EBC} \mathrm{pH}$ & $*$ & & $6.0 \pm 0.2$ & $6.5 \pm 0.1$ & $6.1 \pm 0.1$ & 0.18 \\
\hline Nitrite $(\mu \mathrm{M})$ & $*$ & 0.1 & $2.4 \pm 0.5$ & $5.2 \pm 1.6$ & $3.6 \pm 1.8$ & 0.001 \\
\hline $\mathrm{H}_{2} \mathrm{O}_{2}(\mu \mathrm{M})$ & $* *$ & 0.1 & $1.8(0.3-2.7)$ & $1.7(0.8-2.7)$ & $1.5(0.3-3.2)$ & 0.93 \\
\hline $\mathrm{IL}-1 \alpha(\mathrm{pg} / \mathrm{mL})$ & $* *$ & 2.2 & $50.5(31.9-948.3)$ & $45.1(33.3-321.2)$ & $44.6(29.4-76.2)$ & 0.16 \\
\hline IL-5 (pg/mL) & $* *$ & 4.2 & 244.7(190.2-998.5) & $406.7(206.6-2418.9)$ & $345.1(168.3-514.5)$ & 0.06 \\
\hline $\mathrm{IL}-13(\mathrm{pg} / \mathrm{mL})$ & $*$ & 1.9 & $75.7 \pm 23.0$ & $152.9 \pm 43.8$ & $105.8 \pm 31.5$ & 0.03 \\
\hline IFN- $-\gamma(\mathrm{pg} / \mathrm{mL})$ & $* *$ & 13 & $49.6(31.7-272.0)$ & $44.6(32.3-175.8)$ & $45.4(29.2-66.1)$ & 0.03 \\
\hline
\end{tabular}

Detection frequency (\%) was $100 \%$ expect for IL-5 (85\%). *; Normally distributed date were expressed as mean \pm standard error. ${ }^{* *}$; Non-normally distributed data were expressed as median (inter-quartile range). p-value; Friedman test. LLD: lower limit of detection. 

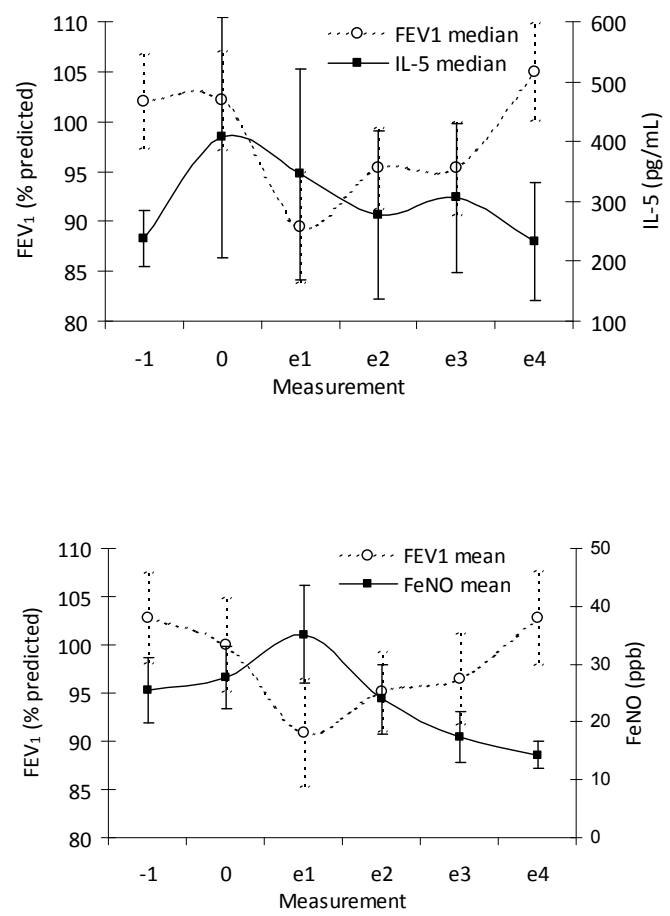

Figure 2a: The course of $\mathrm{FEV}_{1}$ and IL5 before and during an asthma exacerbation.
Figure 2b: The course of $\mathrm{FEV}_{1}$ and FeNO before and during an asthma exacerbation.

Measurements -1 and 0 represent on average 3 and 1 months before the exacerbation; Measurements e1, e2, e3 and e4 represent the measurements at day 1, 3, 5 and at the end of exacerbation.

\section{Discussion}

This study shows that exacerbations of childhood asthma were significantly predicted by regular two-month assessments of EBC IL-5 levels, EBC acidity and asthma control score. In the multivariate analysis, EBC acidity was no longer a significant predictor of the exacerbation rate. The Kaplan-Meier curve showed an increase in exacerbation rate from 0.3 to 0.8 per year in patients with the $10 \%$ least optimal values of IL-5 and asthma control score compared to children with the $10 \%$ most optimal values. Strikingly, lung function and FeNO had no significant influence on the prediction of exa-cerbations.

The strengths of the study are the prospective one-year study period, use of various noninvasive IM as well as lung function and asthma control score as independent predictors, more than $85 \%$ detection frequency of markers in EBC, and use of an optimised glass condenser system [21]. Moreover, the study population was well defined and already known to have had asthma for a for a significant period of time. A relative draw back of the study is the generally good level of asthma control and the occurrence of exacerbations in only a minority of patients (16 out of 40 ). The authors did not incorporate regular measurements of IM in venous blood in this study as this would have increased the drop-out rate consid- 
erably. EBC samples were not de-aerated before measurement of $\mathrm{pH}$ because there is no consensus on this topic $[8,22]$. Moreover, it has been found in an earlier study that non de-aerated EBC samples are of greater clinical value than de-aerated samples in discriminating between asthmatic and control subjects [23]. The two-way valve and tubing served as a saliva trap [24].

IL-5 is an interdigitating homodimeric glycoprotein. It is produced by Th-2 cells and mast cells and induces immunoglobulin synthesis and activation of eosinophils in asthma. The importance of IL-5 in the pathophysiology of asthma has been demonstrated by a recent study showing that a monoclonal antibody against IL-5, Mepolizumab, was able to diminish exacerbations of asthma [25]. To the authors' current knowledge, the study described in this paper is the first research showing that IL-5 in exhaled breath condensate is a significant predictor of asthma exacerbations in children. However, the relationship between IL-5 and exacerbations of asthma has already been demonstrated in a number of other studies. As Norzila and colleagues studied the characteristics of airway inflammation in exacerbations of childhood asthma using sputum cell counts and fluid-phase measurements at presentation to the emergency department and 2 weeks later at recovery. During exacerbation, the total cell count, sputum myeloperoxidase, IL-8 and IL-5 were increased [26]. Kocak and colleagues found an increase in serum IL-5, eosinophilic cationic protein (ECP), and CD4+/CD25+ lymphocytes at day one and five of an exacerbation of childhood asthma compared to control subjects, which normalised after glucocorticoid treatment [27]. Corrigan and co-workers assessed expression of Th- 1 and Th-2 cytokine mRNA and spontaneous secretion of IL-3, IL-5, and GM-CSF by peripheral blood CD4 and CD8 T-lymphocytes from asthmatics before and after oral glucocorticoid therapy, and in healthy controls. In asthmatics, the percentages of CD4 T-lymphocytes expressing IL-5 mRNA correlated with disease severity and the numbers of peripheral blood eosinophils. After oral glucocorticoid therapy, lung function improved and the number of CD4 lymphocytes expressing mRNA encoding IL-5 were reduced [28].

In the univariate analysis, the acidity of EBC was a significant predictor. However, in the multivariate analysis this influence disappeared.

A lower $\mathrm{pH}$ of EBC seems to result from both eosinophilic and neutrophilic airway inflammation and apart from asthma also occurs in other diseases such as cystic fibrosis or COPD [29].

Based on the results of our study, it is possible that EBC IL-5 gives more specific information about airway inflammation in (exacerbations of) asthma than EBC $\mathrm{pH}$.

Potential of FeNO or lung function for the prediction of exacerbations of childhood asthma was not found in this study. Atopic as well as non-atopic subjects were included. It is known that FeNO is particularly a marker of eosinophilic airway inflammation and may not be useful in non-atopic asthmatic children. Pijnenburg and colleagues have determined the value of FeNO to predict asthma relapses in 85 atopic children with clinical asthma remission. Two and four weeks after inhaled corticosteroid withdrawal, more than two times higher FeNO levels were found in those who were about to relapse compared with children who did not relapse, showing that FeNO was a significant predictor of asthma exacerbations in these children [30].

Zacharasiewicz and coworkers performed a comparable study in which weaning of inhaled corticosteroids was studied in 40 asthmatic children. Two-monthly assessments of FeNO and sputum eosinophils were significantly related to failed steroid reduction but lung 
function and nitrate/nitrite in EBC were not [31]. This coincides with the results of the authors' study, in which EBC nitrite and lung function were not related to exacerbations. In contrast, two-monthly assessment of the asthma control score was found to be a significant predictor of the exacerbation rate. This indicates that asthma exacerbations are often preceded by a period with less asthma control, and also demonstrates the relevance of regular assessments of this validated questionnaire, for instance during visits to the outpatient clinic.

More longitudinal studies on the value of non-invasive IM in EBC in asthmatic children are necessary. In the future it is relevant to study whether titration of anti-inflammatory treatment on the basis of IM such as IL-5 in EBC and the asthma control score is of help in the prevention of exacerbations of asthma.

In this one-year longitudinal study, both exhaled breath condensate IL-5 levels and the asthma control score were significant predictors of an exacerbation of childhood asthma. This opens up the possibility of assessing the potential of these parameters to titrate asthma treatment in future studies.

\section{Acknowledgement}

This study was sponsored by AstraZeneca and the prof.dr. Jaap Swieringa Foundation.

\section{References}

1 Gustafsson, PM, Watson, L, Davis, KJ, Rabe, KF. Poor asthma control in children: evidence from epidemiological surveys and implications for clinical practice. Int J Clin Pract 2006;60:321-334.

2 Rabe, KF, Adachi, M, Lai, CK, Soriano, JB, Vermeire, PA, Weiss, KB, Weiss, ST. Worldwide severity and control of asthma in children and adults: the global asthma insights and reality surveys. J Allergy Clin Immunol 2004;114:40-47.

3 Hammer, SC, Robroeks, CM, van Rij, C, Heynens, J, Droog, R, Jobsis, Q, Hendriks, HJ, Dompeling, E. Actual asthma control in a paediatric outpatient clinic population: do patients perceive their actual level of control? Pediatr Allergy Immunol 2008;19:626-633.

4 Kharitonov, SA, Barnes, PJ. Biomarkers of some pulmonary diseases in exhaled breath. Biomarkers 2002;7:132.

5 Kips, JC, Kharitonov, SA, Barnes, PJ. Non invasive assessment of airway inflammation in asthma. Eur Respir Mon 2003;13:164-179.

6 van Gent, R, van Essen-Zandvliet, LE, Rovers, MM, Kimpen, JL, de Meer, G, van der Ent, CK. Poor perception of dyspnoea in children with undiagnosed asthma. Eur Respir J 2007;30:887-891.

7 Robroeks, CM, van de Kant, KD, Jobsis, Q, Hendriks, HJ, van Gent, R, Wouters, EF, Damoiseaux, JG, Bast, A, Wodzig, WK, Dompeling, E. Exhaled nitric oxide and biomarkers in exhaled breath condensate indicate the presence, severity and control of childhood asthma. Clin Exp Allergy 2007;37:1303-1311.

8 Horvath, I, Hunt, J, Barnes, PJ, Alving, K, Antczak, A, Baraldi, E, Becher, G, van Beurden, WJ, Corradi, M, Dekhuijzen, R, Dweik, RA, Dwyer, T, Effros, R, Erzurum, S, Gaston, B, Gessner, C, Greening, A, Ho, LP, Hohlfeld, J, Jobsis, Q, Laskowski, D, Loukides, S, Marlin, D, Montuschi, P, Olin, AC, Redington, AE, Reinhold, P, van Rensen, EL, Rubinstein, I, Silkoff, P, Toren, K, Vass, G, Vogelberg, C, Wirtz, H. Exhaled breath condensate: methodological recommendations and unresolved questions. Eur Respir J 2005;26:523-548.

9 Carpagnano, GE, Barnes, PJ, Francis, J, Wilson, N, Bush, A, Kharitonov, SA. Breath condensate pH in children with cystic fibrosis and asthma: a new noninvasive marker of airway inflammation? Chest 2004;125:20052010. 
10 Kharitonov, SA. Exhaled markers of inflammatory lung diseases: ready for routine monitoring? Swiss Med Wkly 2004;134:175-192.

11 Kharitonov, SA, Barnes, PJ. Exhaled markers of pulmonary disease. Am J Respir Crit Care Med 2001;163:1693-1722.

12 Bateman, ED, Hurd, SS, Barnes, PJ, Bousquet, J, Drazen, JM, FitzGerald, M, Gibson, P, Ohta, K, O'Byrne, P, Pedersen, SE, Pizzichini, E, Sullivan, SD, Wenzel, SE, Zar, HJ. Global strategy for asthma management and prevention: GINA executive summary. Eur Respir J 2008;31:143-178.

13 Demoly, P, Crestani, B, Leroyer, C, Magnan, A, Mounedji, N, Humbert, M. Control and exacerbation of asthma: a survey of more than 3000 French physicians. Allergy 2004;59:920-926.

14 ATS/ERS Recommendations for Standardized Procedures for the Online and Offline Measurement of Exhaled Lower Respiratory Nitric Oxide and Nasal Nitric Oxide, 2005. Am J Respir Crit Care Med 2005;171:912-930.

15 Rosias, PP, Robroeks, CM, Kester, A, den Hartog, GJ, Wodzig, WK, Rijkers, GT, Zimmermann, LJ, van Schayck, $\mathrm{CP}$, Jobsis, $\mathrm{Q}$, Dompeling, E. Biomarker reproducibility in exhaled breath condensate collected with different condensers. Eur Respir J 2008;31:934-942.

16 de Jager, W, Prakken, BJ, Bijlsma, JW, Kuis, W, Rijkers, GT. Improved multiplex immunoassay performance in human plasma and synovial fluid following removal of interfering heterophilic antibodies. J Immunol Methods 2005;300:124-135.

17 de Jager, W, Rijkers, GT. Solid-phase and bead-based cytokine immunoassay: a comparison. Methods 2006;38:294-303.

18 de Jager, W, te Velthuis, H, Prakken, BJ, Kuis, W, Rijkers, GT. Simultaneous detection of 15 human cytokines in a single sample of stimulated peripheral blood mononuclear cells. Clin Diagn Lab Immunol 2003;10:133139.

19 Quanjer, PH, Tammeling, GJ, Cotes, JE, Pedersen, OF, Peslin, R, Yernault, JC. Lung volumes and forced ventilatory flows. Report Working Party Standardization of Lung Function Tests, European Community for Steel and Coal. Official Statement of the European Respiratory Society. Eur Respir J Suppl 1993;16:5-40.

20 Global Initiative for asthma (GINA). Pocket guide for asthma management and prevention in children. National Institute of Health, National Heart, Lung, and Blood Institute 2006.

21 Rosias, PP, Robroeks, CM, van de Kant, KD, Rijkers, GT, Zimmermann, L, van Schayck, CP, Heynens, JW, Jobsis, Q, Dompeling, E. Feasibility of a new method to collect exhaled breath condensate in pre-school children. Pediatr Allergy Immunol 2009.

22 Effros, RM. Exhaled breath condensate pH. Am J Respir Crit Care Med 2006;173:1047-1048.

23 Rosias, PP, Dompeling, E, Hendriks, HJ, Heijnens, JW, Donckerwolcke, RA, Jobsis, Q. Exhaled breath condensate in children: Pearls and pitfalls. Pediatr Allergy Immunol 2004;15:4-19.

24 Rosias, PP, Dompeling, E, Dentener, MA, Pennings, HJ, Hendriks, HJ, Van lersel, MP, Jobsis, Q. Childhood asthma: Exhaled markers of airway inflammation, asthma control score, and lung function tests. Pediatr Pulmonol 2004;38:107-114.

25 Antoniu, SA. Mepolizumab for difficult-to-control asthma with persistent sputum eosinophilia. Expert Opin Investig Drugs 2009;18:869-871.

26 Norzila, MZ, Fakes, K, Henry, RL, Simpson, J, Gibson, PG. Interleukin-8 secretion and neutrophil recruitment accompanies induced sputum eosinophil activation in children with acute asthma. Am J Respir Crit Care Med 2000;161:769-774.

27 Kocak, AK, Bor, O, Yildiz, B, Erdogan, L, Us, T. T-lymphocyte activation and the levels of eosinophilic cationic protein and interleukin-5 in asthmatic children with acute exacerbation and effect of glucocorticoid treatment. Allergy Asthma Proc 2006;27:371-377.

28 Corrigan, CJ, Hamid, Q, North, J, Barkans, J, Moqbel, R, Durham, S, Gemou-Engesaeth, V, Kay, AB. Peripheral blood CD4 but not CD8 t-lymphocytes in patients with exacerbation of asthma transcribe and translate messenger RNA encoding cytokines which prolong eosinophil survival in the context of a Th2-type pattern: effect of glucocorticoid therapy. Am J Respir Cell Mol Biol 1995;12:567-578.

29 Hoffmeyer, F, Raulf-Heimsoth, M, Bruning, T. Exhaled breath condensate and airway inflammation. Curr Opin Allergy Clin Immunol 2009;9:16-22.

30 Pijnenburg, MW, Bakker, EM, Hop, WC, De Jongste, JC. Titrating steroids on exhaled nitric oxide in children with asthma: a randomized controlled trial. Am J Respir Crit Care Med 2005;172:831-836. 
31 Zacharasiewicz, A, Wilson, N, Lex, C, Erin, EM, Li, AM, Hansel, T, Khan, M, Bush, A. Clinical use of noninvasive measurements of airway inflammation in steroid reduction in children. Am J Respir Crit Care Med 2005;171:1077-1082. 



\section{CHAPTER 11.2}

\section{Cystic fibrosis exacerbations are predicted by non-invasive inflammatory markers in exhaled breath condensate: a one-year longitudinal study}

CMHHT Robroeks, Q Jöbsis, GT Rijkers, WKWH Wodzig, A Bast, JJE Hendriks, GJ Wesseling, R Braekers, A Gaddah, LJ Zimmermann, KDG van de Kant, JE Dankert-Roelse, E Dompeling Submitted 


\section{Abstract}

\section{Background}

Morbidity and mortality in cystic fibrosis are mainly caused by pulmonary complications. Non-invasive exhaled inflammatory markers may be suitable for evaluation of oxidative stress and neutrophilic airway inflammation. Aim: 1) To study the ability of non-invasive IM to predict a pulmonary exacerbation of cystic fibrosis; 2) To assess the time course of changes in inflammatory markers during exacerbations.

\section{Methods}

During this prospective longitudinal study, 26 cystic fibrosis patients visited the outpatient clinic every two months during one-year period. In addition to these standard visits, four additional visits were planned during an exacerbation. To monitor an exacerbation, patients were requested three times per week, using a home monitor, to 1 ) assess measurements of forced expiratory volume in one second; 2) record medication use; 3) register the presence and severity of pulmonary symptoms. The primary outcome measure was the occurrence of an exacerbation. Secondary outcome parameters were: 1) fractional exhaled nitric oxide, 2) inflammatory markers in exhaled breath condensate: acidity, 8isoprostane, nitrate, interleukin- $1 \alpha,-5,-6,-8,-10$, tumour necrosis factor- $\alpha$, macrophage inhibiting factor, 3) lung function parameters, 4) cystic fibrosis control (cystic fibrosis clinical score) and severity (Shwachman-Kulczycki score). Anderson-Gill survival analysis for recurrent events was performed for statistical analysis.

\section{Results}

26 Patients, aged 6-30 years, were included. 17 Patients experienced a first exacerbation of which 8 demonstrated a second event during the study period. Univariate Anderson-Gill models showed that macrophage inhibiting factor, tumour necrosis factor-alpha, interleukin-6, -8 and -10 were significant predictors of an exacerbation of cystic fibrosis $(p<0.01)$. Multivariate analysis demonstrated that macrophage inhibiting factor was the strongest predictor. The risk of an exacerbation increased to $100 \%$ in patients with low macrophage inhibiting factor levels $(<130 \mathrm{pg} / \mathrm{mL})(p<0.05)$. Symptoms and lung function indices were not significant predictors.

\section{Conclusions}

This study showed that inflammatory markers in exhaled breath condensate were able to predict a pulmonary exacerbation of cystic fibrosis on average one month before clinical onset. 


\section{Introduction}

In cystic fibrosis (CF) a genetically determined defect in cystic fibrosis transmembrane conductance regulator protein (CFTR) causes impaired chloride transport across epithelial cell membranes. This causes viscous mucus, and diminished mucociliary clearance, and hence decreased airway clearance of bacteria such as Staphylococcus aureus and Pseudomonas aeruginosa. The resulting infection leads to increased oxidative stress, chronic (neutrophilic) airway inflammation, airway obstruction, and structural lung damage [1, 2]. Morbidity and mortality in CF are mainly caused by pulmonary complications [3]. Exacerbations of CF lung disease have an important influence on the well being of patients and are characterised by a deterioration in symptoms, lung function, and quality of life, sometimes requiring hospital admission. Early detection and early treatment of exacerbations leads to improved prognosis. The question is whether early prediction of an exacerbation is possible by means of symptoms and lung function measurements. These reflect functional abnormalities rather than the presence of airway inflammation, and therefore only provide late signs of an exacerbation. This may result in a significant delay between the onset of an exacerbation and the time when treatment will commence. Previous crosssectional studies have shown that non-invasive inflammatory markers (IM), exhaled nitric oxide (FeNO), and markers in exhaled breath condensate (EBC), could predict the presence and severity of CF lung disease [4-12]. Cytokines regulate the production of other inflammatory mediators, whereas secreted chemokines function as regulatory molecules that attract leukocytes to the site of inflammation. Cytokines and chemokines are involved in the pathophysiology of CF and also affect regulation of several chemokines involved in intracellular signalling pathways. Nuclear factor-kappa $B(N K-K B)$ is a particularly important protein active in these mechanisms, resulting in excessive production of NF-KB dependent cytokines and chemokines such as TNF, IL-1, IL-6, and IL-8 [13].

A one-year prospective longitudinal study was performed with the aim of determining the time course of FeNO and non-invasive IM in EBC before, during and after a pulmonary exacerbation of CF and to assess the ability of FeNO and non-invasive IM in EBC to predict a pulmonary exacerbation of CF.

\section{Methods}

\section{CF study population}

CF patients aged 6-30 years were selected from the total patient population under the care of Maastricht CF centre. CF disease was defined as the combination of characteristic clinical features (persistent pulmonary symptoms, failure to thrive, steatorrhoea) and an abnormal sweat test (Chloride $>60 \mathrm{mM}$ ) [14]. The severity of CF was based on the Shwachman-Kulczycki score (SK score) $[15,16]$.

Exclusion criteria were: 1 ) diseases that may interfere with the results of the study (e.g. heart disease, anatomic abnormalities of the airway, other chronic inflammatory diseases, such as Crohn's disease or rheumatoid arthritis), 2) mental retardation, 3) inability to perform measurements properly, and 4) active smoking. Atopy was defined as the presence of 2 RAST classes $\geq 2$. All parents or children gave informed consent. The study was ap- 
proved by the Medical Ethics Committee of University Hospital Maastricht. The international study number was NCT00404859.

\section{Study design}

A prospective controlled study during a one year period was carried out. Every two months, routine clinical visits were made to outpatients, and assessments of clinical parameters were performed by means of questionnaire data (Cystic Fibrosis Clinical Score, CFCS [17] and Shwachman-Kulczycki score (SK-score) [15]), by measurement of lung function indices, and by non-invasive measurement of IM, as described below. In the case of an exacerbation, patients were asked to make four additional visits. These extra visits were planned at day $1,5,10$ and at the end of the exacerbation, Table 1 . A maximum of two events were documented during the study.

Table 1: Study design: codes of measurements points.

Standard
$\mathrm{t}=-3$
The baseline measurement was defined as the sample $0-2$ months (mean 39 days) before the clinical onset of
an asthma exacerbation, denoted by $\mathrm{t}=0$. Measurements before $\mathrm{t}=0$, were coded $\mathrm{t}=-1,-2,-3 \ldots$ etc. The time
interval between these measurements was 2 months. During the exacerbation measurements were denoted
by $\mathrm{t}=\mathrm{e}_{1}, \mathrm{e}_{2}, \mathrm{e}_{3}$ and $\mathrm{e}_{4}$, referring to the additional measurements at day $1,5,10$ and at the end of the event,
respectively. After the exacerbation, standard measurements continued at two month intervals denoted as
$\mathrm{t}=1,2, \ldots$ etc.

\section{Primary outcome measure: CF exacerbation}

The definition of an exacerbation was based on an increase in wheeze, sputum production, chest congestion, decreased exercise tolerance or dyspnoea with exertion, school or work absenteeism, weight loss (more than one kg over the preceding month), crackles or rhonchi on auscultation, and a decrease in $\mathrm{FEV}_{1}$ of more than $20 \%$ of personal maximum value [17]. To monitor the development of an ongoing exacerbation, the CFCS questionnaire of Kanga et al. was applied on each occasion of measurement. This scoring system is based on both subjective and objective observation [18]. Recognition of an exacerbation at an early stage was made possible by means of home monitors (AM1, Viasys, Hoechberg, Germany). With home monitors, the forced expiratory volume in one second $\left(\mathrm{FEV}_{1}\right)$, as well as use of antibiotics, overall well-being, and presence of pulmonary symptoms (cough, sputum, dyspnoea) were recorded. Patients were instructed to use their monitor 3 days a week at a fixed time. All patients were trained to perform the manoeuvres 3 times within 10 minutes and the highest $\mathrm{FEV}_{1}[\mathrm{~L}]$ was stored. Data were sent to a computer at Maastricht University Medical Centre by means of a modem ( $\mathrm{HC1}$, Viasys, Hoechberg, Germany) once per week. In the case of development of an exacerbation, patients were called to the hospital for the additional measurements. All medication use during the study was recorded. 


\section{Measurements of independent predictors}

Every two months, measurements were performed in the following order: 1) clinical evaluation, 2) FeNO, 3) collection of exhaled breath condensate, 4) lung function indices, 5) questionnaires (CFCS and SK-score).

Nitric oxide in exhaled breath

FeNO was measured online using a NIOX ${ }^{\circledR}$ chemiluminescence analyser (Aerocrine, Solna, Sweden). Measurements were carried out according to the American Thoracic Society/European Respiratory Society (ATS/ERS) criteria for children [19].

\section{Exhaled Breath Condensate collection and analysis}

EBC was collected by means of an optimised borosilicate glass tube, cooled by countercurrent circulating ice water (patent EP07102586), as described in previous research [20]. Patients breathed tidally for ten minutes, while wearing a nose-clip, through a mouthpiece connected to a two-way non-rebreathing valve (Hans Rudolph Inc, series 1420, Kansas City, USA). After collection, EBC was rapidly frozen at $-80^{\circ} \mathrm{C}$ using dry ice and was stored at -80 ㄷ $\mathrm{C}$ until analysis.

EBC acidity was measured immediately after collection (Radiometer, type PHM201, Radiometer Nederland BV, Zoetermeer, NL). Levels of nitrate (fluorometric) and 8-isoprostane (enzyme immunoassay) were assessed as described in an earlier study [21]. Cytokines and chemokines in 100 microliters of EBC were assessed using the multiplex immunoassay Luminex 100 analyser (Luminex Corporation, Austin, TX, USA), as described previously [22-24]. All EBC samples of a specific patient were analysed simultaneously in order to minimise inter-assay variation.

\section{Lung function tests}

Dynamic spirometry was performed by means of the Flowscreen ${ }^{\circ}$, according to ERS standards (Viasys, Hoechberg, Germany) [25]. Highest values of three correctly performed manoeuvres were used for analysis. Recorded parameters were: $\mathrm{FEV}_{1}$, forced vital capacity (FVC) and maximum expiratory flow at $50 \%$ of $\mathrm{FCV}\left(\mathrm{MEF}_{50}\right)$, all expressed as a percentage of the predicted normal value [25]. Reversibility was determined at the first, fourth and last standard visit, 15 minutes after inhalation of $400 \mu \mathrm{g}$ salbutamol. Short-acting bronchodilators were stopped at least 8 hours and long-acting bronchodilators were stopped at least 36 hours before testing. Residual volume (RV), total lung capacity (TLC) and intrathoracic gas volume (ITVG), were assessed once during the study period by body plethysmography (Viasys, Hoechberg, Germany).

\section{Treatment}

Patents were treated according to European consensus guidelines [26], which resemble the guidelines of the British and American Thoracic Societies. The main aspects of treatment were: 1) antibiotic treatment in case of an exacerbation, 2) agents to promote airway secretion clearance, such as DNase, and 3) physiotherapy. Changes in therapy were documented. 


\section{Statistical analysis}

The relationship between the non-invasive IM (independent predictors) and an exacerbation (the primary outcome measure), was analysed by survival analysis for recurrent events (Andersen-Gill models). In order to investigate whether the season of year in which a patient entered the study had a significant effect on the time until an exacerbation, seasons of inclusion (winter, spring, summer) were introduced as covariates.

\section{Power analysis}

At least 24 patients are necessary for assessing the relationship between a specific predictor and exacerbations with a power of $90 \%$, an alpha of 0.05 , and a clinically relevant correlation coefficient of $>0.6$. A total of 26 subjects with CF were included.

\section{Results}

\section{Population characteristics}

26 Patients were recruited with a (mean \pm SE) age of $15.8 \pm 2.1$ years (Table 2). Mutation had been identified in the majority (88\%) of patients. In 18 patients, the delta F508/delta F508 mutation was found. Three patients had one delta F508 mutation combined with another mutation, and 2 patients were diagnosed with other CF mutations. Lung function testing showed both a moderate obstructive and restrictive impairment. With one exception, all patients were pancreas insufficient. CF-related diabetes mellitus was present in 4 cases (15\%). All patients entered the study between December 2005 and August 2006.

\section{Exacerbations}

The mean \pm SE time interval between the previous standard measurement and the onset of an exacerbation was $39 \pm 4$ days. During the 12-month observation period, 17 patients experienced one exacerbation and 8 patients developed two. Of the patients with one exacerbation, 2 withdrew from the study after the event. These 2 patients were excluded in the recurrent analysis. No correlation was found between the time until the first and the second exacerbation for patients with 2 events, which suggested that the times until the first and the second exacerbation were independent.

\section{Time course of inflammatory markers before and during and exacerbation}

All IM measured in EBC were detected in at least 92\% of the samples. Median (interquartile range) levels of independent predictors before and during an exacerbation are shown in Table 3. Significant changes in FEV 1 , EBC IL-1 $\alpha$, IL-6, IL-8, and macrophage inhibiting factor (MIF), were measured between these occasions (Friedman test, $p<0.05$ ), Table 3 , Figure 1a-b. Before and during an exacerbation, no significant changes in FeNO were observed. 
Table 2: Characteristics of the 26 CF patients.

\begin{tabular}{|c|c|}
\hline Age (yrs) & $15.8 \pm 2.1$ \\
\hline Weight (kg) & $37.8 \pm 3.1$ \\
\hline Height $(\mathrm{cm})$ & $143.1 \pm 4.1$ \\
\hline Gender (Male / Female) & $13 / 13$ \\
\hline \multicolumn{2}{|l|}{ Lung function indices } \\
\hline - Bronchodilating response in $\mathrm{FEV}_{1}$ ( $\%$ predicted) & $2.9 \pm 1.1$ \\
\hline - $\mathrm{FEV}_{1} \%$ predicted & $68.3 \pm 4.1$ \\
\hline$-\mathrm{FEV}_{1} / \mathrm{VC} \%$ & $77.9 \pm 3.3$ \\
\hline - FVC \% predicted & $72.3 \pm 4.1$ \\
\hline$-\mathrm{MEF}_{50} \%$ predicted & $51.1 \pm 5.9$ \\
\hline - TLC \% predicted & $106.4 \pm 2.6$ \\
\hline - RV \% predicted & $180.1 \pm 13.9$ \\
\hline - ITGV \% predicted & $121.3 \pm 5.2$ \\
\hline Atopy (yes / no) & $12 / 14$ \\
\hline - Total IgE (kU/L) & $299 \pm 117$ \\
\hline \multicolumn{2}{|l|}{ Treatment } \\
\hline - Antibiotics, prophylactic (inhaled or oral) & $7(27 \%)$ \\
\hline - DNase & $18(69 \%)$ \\
\hline - Fat soluble vitamins & $23(89 \%)$ \\
\hline - Antacids & $20(77 \%)$ \\
\hline - Inhaled corticosteroids & $6(23 \%)$ \\
\hline - Long acting $\beta_{2}$-agonist & $5(19 \%)$ \\
\hline Positive sputum cultures (yes / no) & $22 / 4$ \\
\hline - Pseudomonas aeruginosa & $9(43 \%)$ \\
\hline - Staphylococcus aureus & $17(77 \%)$ \\
\hline - Haemophilus influenzae & $6(29 \%)$ \\
\hline - Haemophilus parainfluenzae & $5(24 \%)$ \\
\hline - Aspergillus fumigatus & $6(29 \%)$ \\
\hline - Candida albicans & $4(19 \%)$ \\
\hline
\end{tabular}

Mean values + standard error of the mean are presented unless stated otherwise.

\section{Univariate recurrent event analysis}

Results of the Anderson-Gill models showed that EBC IL1 $\alpha$, IL-5, IL-6, IL-10, TNF- $\alpha$, IL-8, and MIF were very significant predictors of an exacerbation of CF, Table 4.

\section{Multivariate recurrent event analysis}

In the final model of multivariate recurrent event analysis, MIF was the only significant predictor of an exacerbation (Table 5). 
Table 3: Levels of inflammatory markers before and during an exacerbation.

\begin{tabular}{|c|c|c|c|c|c|c|c|}
\hline $\begin{array}{l}\text { Inflammatory } \\
\text { markers }\end{array}$ & $\mathrm{t}_{-1}$ & $t_{0}$ & $t_{e 1}$ & $t_{\mathrm{e} 2}$ & $t_{e 3}$ & $t_{\text {e4 }}$ & p-value* \\
\hline $\mathrm{FEV}_{1} \%$ pred & $65.9 \pm 7.6$ & $66.2 \pm 5.4$ & $59.7 \pm 5.3$ & $61.9 \pm 5.3$ & $69.2 \pm 6.2$ & $69.9 \pm 6.5$ & 0.01 \\
\hline FeNO (ppb) & $12.0 \pm 3.2$ & $8.1 \pm 1.1$ & $10.4 \pm 1.3$ & $6.6 \pm 1.1$ & $7.6 \pm 1.1$ & $9.5 \pm 2.3$ & 0.38 \\
\hline $\mathrm{EBC} \mathrm{pH}$ & $6.2 \pm 0.1$ & $6.2 \pm 0.1$ & $6.3 \pm 0.1$ & $6.3 \pm 0.1$ & $6.2 \pm 0.1$ & $6.2 \pm 0.1$ & 0.65 \\
\hline $\begin{array}{l}\text { 8-isoprostane } \\
(\mathrm{pg} / \mathrm{mL})\end{array}$ & $8.1 \pm 3.4$ & $5.3 \pm 2.0$ & $4.4 \pm 0.9$ & $4.8 \pm 1.2$ & $5.2 \pm 1.5$ & $9.7 \pm 3.5$ & 0.56 \\
\hline Nitrate $(\mu \mathrm{M})$ & $70.7 \pm 7.7$ & $69.5 \pm 12.7$ & $71.8 \pm 8.0$ & $104.5 \pm 11.0$ & $89.4 \pm 9.3$ & $100.5 \pm 15.9$ & 0.20 \\
\hline \multicolumn{8}{|c|}{ Th1 cytokines (pg/mL) } \\
\hline IL-1 $\alpha$ & $481 \pm 164$ & $64 \pm 18$ & $305 \pm 109$ & $554 \pm 190$ & $599 \pm 194$ & $393 \pm 172$ & 0.04 \\
\hline IL-6 & $480 \pm 213$ & $96 \pm 16$ & $245 \pm 66$ & $391 \pm 124$ & $423 \pm 126$ & $293 \pm 102$ & 0.03 \\
\hline TNF- $\alpha$ & $703 \pm 217$ & $212 \pm 44$ & $468 \pm 100$ & $609 \pm 165$ & $685 \pm 174$ & $532 \pm 151$ & 0.10 \\
\hline \multicolumn{8}{|c|}{ Th2 cytokines $(\mathrm{pg} / \mathrm{mL})$} \\
\hline IL-5 & $1285 \pm 367$ & $434 \pm 96$ & $946 \pm 196$ & $879 \pm 245$ & $1145 \pm 231$ & $823 \pm 158$ & 0.26 \\
\hline IL-10 & $62 \pm 30$ & $14 \pm 2$ & $29 \pm 7$ & $48 \pm 16$ & $48 \pm 15$ & $34 \pm 12$ & 0.16 \\
\hline \multicolumn{8}{|c|}{ Chemokines $(\mathrm{pg} / \mathrm{mL})$} \\
\hline IL-8 & $185 \pm 60$ & $69 \pm 7$ & $114 \pm 20$ & $162 \pm 39$ & $177 \pm 37$ & $127 \pm 32$ & 0.03 \\
\hline MIF & $2234 \pm 711$ & $520 \pm 204$ & $1337 \pm 380$ & $2270 \pm 732$ & $1940 \pm 560$ & $1715 \pm 639$ & 0.02 \\
\hline
\end{tabular}

Data are expressed as mean \pm standard error. * Friedman test; $t_{-1}$ : Stable CF : on average $100 \pm 4$ days before diagnosis of exacerbation; $t_{0:}$ on average $39 \pm 4$ days before diagnosis of exacerbation; $t_{\mathrm{e} 1}-t_{\mathrm{e} 4}$ at day $1,5,10$ and at the end of exacerbation, respectively.

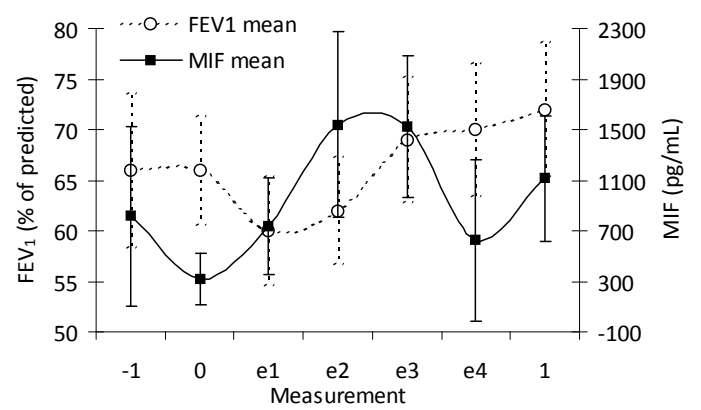

Figure 1a: Time course of $\mathrm{FEV}_{1}$ and MIF (mean \pm SE) levels before, during and after a CF exacerbation.

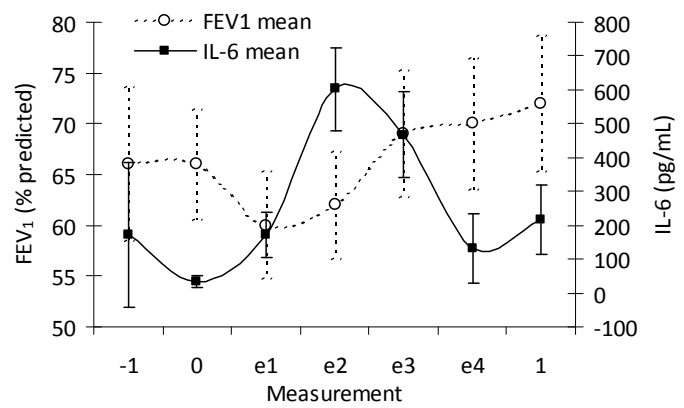

Figure 1b: Time course of $\mathrm{FEV}_{1}$ and IL-6 (mean \pm SE) levels before, during and after a CF exacerbation.

Measurements -1 and 0 represent on average 3 and 1 months before the exacerbation; Measurements e1, e2, e3 and e4 represent the measurements at day 1, 5, 10 and at the end of exacerbation. 
Table 4: Univariate recurrent event analysis.

\begin{tabular}{lll}
\hline Covariate & Estimate \pm SE & p-value \\
\hline FEV $\%$ pred & $-0.0028 \pm 0.0083$ & 0.735 \\
Symptoms & $-0.0806 \pm 0.1956$ & 0.680 \\
FeNO $(\mathrm{ppb})$ & $-0.0717 \pm 0.0509$ & 0.159 \\
EBC $\mathrm{pH}$ & $-0.3746 \pm 0.6334$ & 0.554 \\
8-isoprostane $(\mathrm{pg} / \mathrm{mL})$ & $0.00742 \pm 0.0166$ & 0.655 \\
Nitrate $(\mu \mathrm{M})$ & $-0.0023 \pm 0.0046$ & 0.622 \\
& & \\
Th1 cytokines $(\mathrm{pg} / \mathrm{mL})$ & & 0.006 \\
IL-1 $\alpha$ & $-0.0024 \pm 0.0009$ & 0.005 \\
IL-6 & $-0.0037 \pm 0.0013$ & $<0.001$ \\
TNF- $\alpha$ & $-0.0019 \pm 0.0006$ & \\
Th2 cytokines $(\mathrm{pg} / \mathrm{mL})$ & & 0.014 \\
IL-5 & & 0.007 \\
IL-10 & $-0.0010 \pm 0.0004$ & \\
Chemokines $(\mathrm{pg} / \mathrm{mL})$ & $-0.0297 \pm 0.0109$ & 0.004 \\
IL-8 & & 0.003 \\
MIF & & \\
\hline
\end{tabular}

Table 5: Multivariate recurrent event analysis

\begin{tabular}{lll}
\hline Covariate & Estimate \pm SE & p-value \\
\hline MIF & $-0.0006 \pm 0.0002$ & 0.004 \\
Winter & $-0.53 \pm 0.92$ & 0.563 \\
Spring & $-0.69 \pm 1.01$ & 0.497 \\
\hline Summer was baseline. & &
\end{tabular}

\section{Kaplan-Meier Curve}

A Kaplan-Meier curve of the time until an exacerbation showed that patients with high levels of MIF ( $>5204 \mathrm{pg} / \mathrm{mL}$, upper $10 \%$ of MIF levels), had only a $10 \%$ risk of developing an exacerbation in the following year. However, patients with low MIF levels $(<130 \mathrm{pg} / \mathrm{mL}$, lowest $10 \%$ of assessed MIF levels) had a $100 \%$ risk of an exacerbation, Figure 3. 


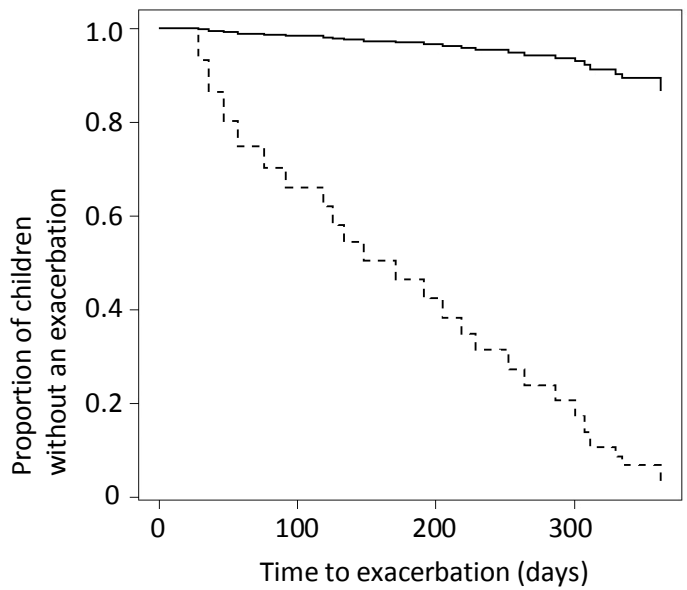

Figure 3: Kaplan Meijer curve of MIF. The continuous line denotes prediction at the $90^{\text {th }}$ percentile of MIF and the dashed line is the prediction at the $10^{\text {th }}$ percentile.

\section{Influences of conductivity}

The reason why MIF is a strong predictor can be seen from the course of MIF levels over time. At the last standard measurement before the onset of an exacerbation ( $t=0)$, MIF levels drop significantly $(p=0.02)$, Figure 1 a. A similar pattern is seen in the significant markers of the univariate analysis. An example of IL-6 is given in Figure 1b. Because of this significant drop before the onset of an exacerbation, the conductivity by means of sodium and potassium levels was assessed.

The method used to assess sodium and potassium in EBC was Flame Emission Spectroscopy (FES). Samples are diluted in a diluent containing known amounts of caesium and aspirated into a propane-air flame. Sodium, potassium and caesium when excited, emit spectra with sharp bright lines at 589, 766 and $852 \mathrm{~nm}$, respectively. Light emitted from the thermally excited ions is directed through separate interference filters to corresponding photodetectors, The caesium $\left(\mathrm{Cs}^{+}\right)$emission signal is used as an internal standard $(1,5$ $\mathrm{mmol} / \mathrm{L})$ against which the sodium $\left(\mathrm{Na}^{+}\right)$and potassium $\left(\mathrm{K}^{+}\right)$signals are compared. The system is calibrated relative to low and high concentrations of each analyte, and the relation of signal to concentration is defined.

\section{Sodium and Potassium levels}

From 12 patients who experienced an event, samples of EBC at $t=-1, t=0$ and $t=e 1$ were analysed. In all 36 samples, sodium and potassium levels were assessed. In order to investigate the influence of conductivity on MIF levels, concentrations of MIF were divided by the sum of the concentrations of sodium and potassium [27]. Before correction for conductivity, MIF levels differed significantly according to time of collection before the onset of an exacerbation (Friedman $\mathrm{p}=0.04$ ). However, corrected MIF levels did not show a significant difference in time (Friedman $p=0.15$ ), Table 6 . This suggests a significant change in conductivity before the onset of a CF exacerbation. 
Table 6: Sodium and potassium levels ( $\mathrm{mmol} / \mathrm{L})$.

\begin{tabular}{lllll}
\hline Measurement & $\mathrm{t}-1$ & $\mathrm{t} 0$ & $\mathrm{te}$ & $\mathrm{p}$-value* \\
\hline Sodium & $1.1(0.5-1.5)$ & $0.9(0.7-1.7)$ & $1.1(0.8-1.2)$ & 0.56 \\
Potassium & $0.08(0.04-0.18)$ & $0.07(0.03-0.15)$ & $0.04(0.02-0.08)$ & 0.70 \\
Sodium+Potassium & $1.2(0.5-1.7)$ & $1.0(0.7-1.9)$ & $1.9(1.7-1.3)$ & 0.50 \\
& & & & \\
MIF & $2096(1394-3826)$ & $207(78-1735)$ & $770(149-3803)$ & 0.04 \\
CMIF* & $1621(720-6561)$ & $426(70-946)$ & $940(104-4502)$ & 0.15 \\
\hline
\end{tabular}

All data were expressed as median (quartiles). *Friedman test. CMIF ${ }^{*}$, MIF levels corrected for sodium and potassium levels.

\section{Discussion}

This study showed that the cytokines (IL-1 $\alpha,-5,-6,-10$, TNF- $\alpha$ ) and chemokines (IL-8 and MIF) in EBC were significant predictors of an exacerbation of CF on average one month before an exacerbation was clinically evident. Al patients with low EBC MIF experienced an exacerbation of CF in the subsequent year. MIF was the only significant predictor in the multivariate model.

MIF inducts pro-inflammatory cytokine secretion and has the ability to induce Toll-like receptor (TLC) 4 on mast cells. Surface molecules of Gram-negative bacteria bind to this TLC receptor, resulting in activation of lipopolysaccharide (LPS)-induced inflammatory cytokine production [28]. Steinkamp et al. have described 23-fold higher plasma levels of MIF in CF patients compared to controls [29]. Plant et al. have reported that patients with less MIF promoter activity had a milder clinical disease phenotype [30].

Based on these mechanisms, an increase in MIF and cytokines in EBC during exacerbations was expected. Indeed, a significant increase was found in IL-1 $\alpha$, IL-6, IL-8, and MIF during exacerbations. However, before the onset of an exacerbation, significant decreases in these markers were measured. The reason for this drop in marker concentrations is unclear. The authors of this study hypothesise that this decrease may be due to: 1) Dilution of EBC: inflammation is characterised by vasodilatation and increased vascular permeability which may result in an amplified effusion of plasma and an increased dilution of EBC; 2 ) Protease activity: it is possible that these enzymes are released more profusely just before the exacerbation with consequently increased breakdown of cytokines and decreased levels of IM; 3) Mucus entrapment: in analogy to low FeNO levels in CF, the increased viscous mucus secretions in CF airways may impair IM to diffuse to the epithelial lining fluid [31]. In order to explore the influence of dilution on samples, the conductivity by means of sodium and potassium levels was assessed in a subset of samples. Samples selected originated from measurement, on average, three months and one month before an exacerbation, and at the onset of the event. Uncorrected MIF levels were significantly different according to time of collection. However, after correction for dilution, no significant changes in this time course was found. This demonstrates that dilution may have had a significant influence on levels of MIF in EBC in CF. Dilution may thus be a valuable marker in the prediction of CF exacerbations. Further testing of this hypothesis is necessary.

To our knowledge, this is the second longitudinal study to analyse the monitoring ability of IM in EBC in paediatric CF lung disease. The strengths of this study are: 1) the longitudinal 
design with a follow-up period of one year; 2 ) the use of a home monitor in order to reliably diagnose an exacerbation of CF at an early stage; 3) regular 2-month assessment at the outpatient clinic; 4) the simultaneous assessment of several non-invasive IM which allows a comparison of the predictive value between them; 5) the need for small amounts of EBC because of the use of the sensitive multiplex immune-assay technology to measure cytokines, chemokines and adhesion molecules in EBC [32], in conjunction with a new optimised condenser [20]. In a one-year prospective longitudinal study Horak et al. researched the monitoring ability of nitrite in EBC in a population of 32 children with CF who suffered from mild lung disease. Samples of nitrite were collected five times during the study. They concluded that nitrite levels in EBC were not helpful for the prediction of a pulmonary exacerbation of CF [33]. The present study revealed similar findings concerning nitrate.

Although the results of the study are promising, the authors acknowledge that further research needs to be carried out before non-invasive measurement of IM can be implemented in daily clinical practice. First, the predictive value of IM in CF exacerbations should be confirmed from a larger population sample using a randomised longitudinal comparative study design in which IM are included as parameters to adjust CF treatment. Second, methodological issues need to be resolved before non-invasive IM can be incorporated in daily practice, as has been stated by the American Thoracic Society / European Respiratory Society (ATS/ERS) Task Force [34]. The general recommendations of the Task Force for oral sample collection were followed in this study [34]. The Task Force states that more research is needed before EBC can be applied in diagnosis or monitoring. Third, the dilution factor was measured retrospectively and thus not assessed in all samples. Fourth, the sample population of patients exhibited a relatively wide age range. Fifth, the range of IM levels was also wide. The combined influence of these five factors, however, seems small when compared with the highly significant differences between IM levels measured before and during exacerbations.

This study responded to some of the important needs for future research: the design was longitudinal and the aim of the study was to investigate which IM in EBC can be used for the monitoring of pulmonary CF disease [34].

In conclusion, the study revealed that MIF, TNF- $\alpha$, IL-6, IL-8 and IL-10 levels in EBC, but neither symptoms, nor lung function indices, nor FeNO were able to predict a pulmonary exacerbation of CF before these events became manifest in lung function tests and clinical symptoms. These results emphasise the potential importance of non-invasive inflammometry in monitoring CF lung disease in order to predict a pulmonary exacerbation of CF. However, in the final model of multivariate recurrent event analysis, MIF was the only significant predictor of a CF exacerbation (uncorrected for dilution).

\section{Acknowledgements}

We would like to thank AstraZeneca and the Dutch Cystic Fibrosis foundation for their financial support. All authors state no conflict of interest.

\section{References}

1 Brown, RK, Wyatt, H, Price, JF, Kelly, FJ. Pulmonary dysfunction in cystic fibrosis is associated with oxidative stress. Eur Respir J 1996;9:334-339. 
2 Ratjen, F, Doring, G. Cystic fibrosis. Lancet 2003;361:681-689.

3 FitzSimmons, SC. The changing epidemiology of cystic fibrosis. Curr Probl Pediatr 1994;24:171-179.

4 Robroeks, CM, Rosias, PP, van Vliet, D, Jobsis, Q, Yntema, JB, Brackel, HJ, Damoiseaux, JG, den Hartog, GM, Wodzig, WK, Dompeling, E. Biomarkers in exhaled breath condensate indicate presence and severity of cystic fibrosis in children. Pediatr Allergy Immunol 2008;19:652-659.

5 Ojoo, JC, Mulrennan, SA, Kastelik, JA, Morice, AH, Redington, AE. Exhaled breath condensate $\mathrm{pH}$ and exhaled nitric oxide in allergic asthma and in cystic fibrosis. Thorax 2005;60:22-26.

6 Moeller, A, Horak, F, Jr., Lane, C, Knight, D, Kicic, A, Brennan, S, Franklin, P, Terpolilli, J, Wildhaber, JH, Stick, $\mathrm{SM}$. Inducible NO synthase expression is low in airway epithelium from young children with cystic fibrosis. Thorax 2006;61:514-520.

7 Narang, I, Ersu, R, Wilson, NM, Bush, A. Nitric oxide in chronic airway inflammation in children: diagnostic use and pathophysiological significance. Thorax 2002;57:586-589.

8 Bodini, A, D'Orazio, C, Peroni, D, Corradi, M, Folesani, G, Baraldi, E, Assael, BM, Boner, A, Piacentini, GL. Biomarkers of neutrophilic inflammation in exhaled air of cystic fibrosis children with bacterial airway infections. Pediatr Pulmonol 2005;40:494-499.

9 Carpagnano, GE, Barnes, PJ, Geddes, DM, Hodson, ME, Kharitonov, SA. Increased leukotriene B4 and interleukin-6 in exhaled breath condensate in cystic fibrosis. Am J Respir Crit Care Med 2003;167:1109-1112.

10 Carpagnano, GE, Barnes, PJ, Francis, J, Wilson, N, Bush, A, Kharitonov, SA. Breath condensate pH in children with cystic fibrosis and asthma: a new noninvasive marker of airway inflammation? Chest 2004;125:20052010.

11 Kharitonov, SA. Exhaled markers of inflammatory lung diseases: ready for routine monitoring? Swiss Med Wkly 2004;134:175-192.

12 Jöbsis, Q, Raatgeep, HC, Schellekens, SL, Kroesbergen, A, Hop, WC, de Jongste, JC. Hydrogen peroxide and nitric oxide in exhaled air of children with cystic fibrosis during antibiotic treatment. Eur Respir J 2000;16:95100.

13 Nichols, D, Chmiel, J, Berger, M. Chronic inflammation in the cystic fibrosis lung: alterations in inter- and intracellular signaling. Clin Rev Allergy Immunol 2008;34:146-162.

14 Rosenstein, BJ, Cutting, GR. The diagnosis of cystic fibrosis: a consensus statement. Cystic Fibrosis Foundation Consensus Panel. J Pediatr 1998;132:589-595.

15 Shwachman, H, Kulczycki, LL. Long-term study of one hundred five patients with cystic fibrosis; studies made over a five- to fourteen-year period. JAMA Dis Child 1958;96:6-15.

16 Oliveira, MC, Reis, FJ, Oliveira, EA, Colosimo, EA, Monteiro, AP, Penna, FJ. Prognostic factors in cystic fibrosis in a single center in Brazil: A survival analysis. Pediatr Pulmonol 2002;34:3-10.

17 Rosenfeld, M, Emerson, J, Williams-Warren, J, Pepe, M, Smith, A, Montgomery, AB, Ramsey, B. Defining a pulmonary exacerbation in cystic fibrosis. J Pediatr 2001;139:359-365.

18 Kanga, J, Kuhn, R, Craigmyle, L, Haverstock, D, Church, D. Cystic fibrosis clinical score: a new scoring system to evaluate acute pulmonary exacerbation. Clin Ther 1999;21:1343-1356.

19 ATS/ERS Recommendations for Standardized Procedures for the Online and Offline Measurement of Exhaled Lower Respiratory Nitric Oxide and Nasal Nitric Oxide, 2005. Am J Respir Crit Care Med 2005;171:912-930.

20 Rosias, PP, Robroeks, CM, van de Kant, KD, Rijkers, GT, Zimmermann, L, van Schayck, CP, Heynens, JW, Jobsis, Q, Dompeling, E. Feasibility of a new method to collect exhaled breath condensate in pre-school children. Pediatr Allergy Immunol 2009.

21 Robroeks, CM, van de Kant, KD, Jobsis, Q, Hendriks, HJ, van Gent, R, Wouters, EF, Damoiseaux, JG, Bast, A, Wodzig, WK, Dompeling, E. Exhaled nitric oxide and biomarkers in exhaled breath condensate indicate the presence, severity and control of childhood asthma. Clin Exp Allergy 2007;37:1303-1311.

22 de Jager, W, Prakken, BJ, Bijlsma, JW, Kuis, W, Rijkers, GT. Improved multiplex immunoassay performance in human plasma and synovial fluid following removal of interfering heterophilic antibodies. J Immunol Methods 2005;300:124-135.

23 de Jager, W, Rijkers, GT. Solid-phase and bead-based cytokine immunoassay: a comparison. Methods 2006;38:294-303. 
24 de Jager, W, te Velthuis, H, Prakken, BJ, Kuis, W, Rijkers, GT. Simultaneous detection of 15 human cytokines in a single sample of stimulated peripheral blood mononuclear cells. Clin Diagn Lab Immunol 2003;10:133139.

25 Quanjer, PH, Tammeling, GJ, Cotes, JE, Fabbri, LM, Matthys, H, Pedersen, OF, Peslin, R, Roca, J, Sterk, PJ, Ulmer, WT, et al. Symbols, abbreviations and units. Working Party Standardization of Lung Function Tests, European Community for Steel and Coal. Eur Respir J Suppl 1993;16:85-100.

26 Kerem, E, Conway, S, Elborn, S, Heijerman, H. Standards of care for patients with cystic fibrosis: a European consensus. J Cyst Fibros 2005;4:7-26.

27 Effros, RM, Biller, J, Foss, B, Hoagland, K, Dunning, MB, Castillo, D, Bosbous, M, Sun, F, Shaker, R. A simple method for estimating respiratory solute dilution in exhaled breath condensates. Am J Respir Crit Care Med 2003;168:1500-1505.

28 Roger, T, David, J, Glauser, MP, Calandra, T. MIF regulates innate immune responses through modulation of Toll-like receptor 4. Nature 2001;414:920-924.

29 Steinkamp, G, Wiedemann, B. Relationship between nutritional status and lung function in cystic fibrosis: cross sectional and longitudinal analyses from the German CF quality assurance (CFQA) project. Thorax 2002;57:596-601.

30 Plant, BJ, Gallagher, CG, Bucala, R, Baugh, JA, Chappell, S, Morgan, L, O'Connor, CM, Morgan, K, Donnelly, SC. Cystic fibrosis, disease severity, and a macrophage migration inhibitory factor polymorphism. Am J Respir Crit Care Med 2005;172:1412-1415.

31 Habib, SS. Exhaled nitric oxide: an emerging marker of inflammation in respiratory diseases. Saudi Med J 2008;29:1697-1702.

32 Robroeks, CMHHT, Rijkers, GT, Jöbsis, Q, Hendriks, JJE, Damoiseaux, JGMC, Zimmermann, LJI, van Schayck, $\mathrm{CP}$, Dompeling, E. Increased cytokines, chemokines and soluble adhesion molecules in exhaled breath condensate of asthmatic children. Clin Exp Allergy 2009;in press.

33 Horak, F, Jr., Moeller, A, Singer, F, Straub, D, Holler, B, Helbich, TH, Schneider, B, Eichler, I, Wildhaber, JH, Hall, GL. Longitudinal monitoring of pediatric cystic fibrosis lung disease using nitrite in exhaled breath condensate. Pediatr Pulmonol 2007;42:1198-1206.

34 Horvath, I, Hunt, J, Barnes, PJ, Alving, K, Antczak, A, Baraldi, E, Becher, G, van Beurden, WJ, Corradi, M, Dekhuijzen, R, Dweik, RA, Dwyer, T, Effros, R, Erzurum, S, Gaston, B, Gessner, C, Greening, A, Ho, LP, Hohlfeld, J, Jobsis, Q, Laskowski, D, Loukides, S, Marlin, D, Montuschi, P, Olin, AC, Redington, AE, Reinhold, P, van Rensen, EL, Rubinstein, I, Silkoff, P, Toren, K, Vass, G, Vogelberg, C, Wirtz, H. Exhaled breath condensate: methodological recommendations and unresolved questions. Eur Respir J 2005;26:523-548. 


\section{CHAPTER 12.1}

\section{Volatile organic compounds in exhaled breath predict exacerbations of childhood asthma in a one year prospective controlled study}

CMHHT Robroeks*, JJBN van Berkel*, Q Jöbsis, FJ van Schooten, EFM Wouters, JW Dallinga, E Dompeling * Shared first authorship Submitted 


\section{Abstract}

\section{Background}

Exacerbations of childhood asthma are characterised by an increase in respiratory symptoms, airway inflammation and deterioration in lung function and quality of life. Currently, monitoring of the disease mostly occurs on the basis of symptoms and lung function, which do not directly reflect airway inflammation. The hypothesis of the present longitudinal study was that prediction of an exacerbation is possible by means of analysis of volatile organic compounds (VOCs) in exhaled breath. The study aims were: 1) to study whether VOCs in exhaled breath are able to predict asthma exacerbations and to assess which combination of VOCs is most predictive; 2 ) to study the chemical background of the most predictive VOCs.

\section{Methods}

A prospective longitudinal study was performed of 40 children with asthma (age[mean \pm $\mathrm{SE}] 10.7 \pm 0.4$ years) during one year. At standard 2-month intervals, exhaled breath samples, lung function and symptoms were determined in a standardised way. Exhaled breath was sampled in 5 litre inert Tedlar bags, stabilised on carbon adsorption tubes and subsequently analysed for VOC profiles using gas chromatography time-of-flight mass spectrometry (GC-TOF-MS).

\section{Results}

16 out of 40 children experienced an exacerbation, of which 3 had a second event during the study. With support vector machine analysis, the most optimal model of baseline measurements versus exacerbation within patients was based on 6 VOCs (correct classification $96 \%$, sensitivity $100 \%$, specificity $93 \%$ ). The model of baseline values of patients with an exacerbation compared to levels of patients without an exacerbation consisted of 7 VOCs (correctly classification $91 \%$, sensitivity $79 \%$, specificity $100 \%$ ).

\section{Conclusion}

This study indicates that a combination of different VOCs in exhaled breath is able to predict exacerbations of childhood asthma on average 39 days before the event is clinically manifest. 


\section{Introduction}

The purpose of asthma treatment is optimal control of the disease [1]. Several crosssectional surveys have demonstrated that actual levels of asthma control fall far below the goals of national and international guidelines [2-4]. Hammer et al. have reported poor control in 55\% of children with asthma in the Netherlands [3]. Poor asthma control and frequent exacerbations may lead to irreversible, pathological and functional airway changes [5]. Children with asthma often have a poor perception of complaints which results in underreporting of symptoms and even underdiagnosis of asthma $[3,6]$. Another explanation for poor asthma control may be that although asthma is characterised by chronic airway inflammation, monitoring is currently not performed by measuring airway inflammation $[7,8]$. Standard assessments of airway inflammation such as bronchoscopy with broncho-alveolar lavage or sputum-induction are far too invasive for repeated use in children.

A non-invasive technique to assess airway inflammation/oxidative stress in the airways is profiling of VOCs in exhaled breath. In patients with asthma, production of reactive oxygen species (ROS) is increased $[9,10]$, which causes lipid peroxidation of cell membranes and subsequent production of VOCs $[11,12]$. Dallinga et al. showed that VOCs in exhaled breath could discriminate between asthmatic children and controls with a high sensitivity and specificity [13]. Dragonieri et al. studied smellprints of VOCs by means of the electronic nose in 10 young adults with mild asthma, 10 older patients with severe asthma and 20 age-matched controls. They found that the electronic nose could discriminate patients with asthma from healthy controls, whereas the distinction between different asthma severities was more difficult [14].

The present longitudinal study investigates the hypothesis that specific VOCs in exhaled breath of children with asthma are able to predict exacerbations at a stage before they are clinically manifest. The specific aims were to investigate: 1 ) whether VOCs in exhaled breath are able to reliably predict asthma exacerbations and to assess which combination of VOCs is most predictive; 2 ) the chemical background of the most predictive VOCs; 3 ) the time course of VOCs during an exacerbation.

\section{Methods}

\section{Study design}

The study design was a prospective longitudinal study during one year. Every two months, routine visits were planned at the outpatient clinic. In addition to these fixed visits, patients were asked to visit the outpatient clinic an additional four times during an exacerbation. These extra visits were planned at most twice during the study. Extra measurements were planned at day 1, 3 and 5 of the exacerbation, and after stabilisation (Table 1). 
Table 1: Study design: abbreviations of measurement points.

\begin{tabular}{|c|c|c|c|c|c|c|c|c|c|}
\hline \multicolumn{4}{|c|}{ Standard } & \multicolumn{2}{|c|}{ Exacerbation } & \multicolumn{4}{|c|}{ Standard } \\
\hline$t=-3$ & -2 & -1 & 0 & e1 & e2 & e3 & e4 & 1 & 2 \\
\hline
\end{tabular}

The baseline measurement was defined as the sample 0-2 months (on average 39 days) before the clinical onset of an asthma exacerbation, denoted by $t=0$. Measurements before $t=0$, were coded $t=-1,-2,-3 \ldots$ etc. The time interval between these measurements was 2 months. During the exacerbation, measurements were denoted by $t=e_{1}, e_{2}, e_{3}$ and $e_{4}$, referring to the additional measurements at day $1,3,5$ and at the end of the event, respectively. After the exacerbation, standard measurements continued ( $t=1,2, \ldots$ etc).

\section{Study subjects}

Children with doctor-diagnosed asthma aged 6-16 years were selected from the outpatient clinic of the department of Paediatric Pulmonology of the Maastricht University Medical Centre. All children were known with a diagnosis of asthma at our clinic for at least 6 months. Asthma was defined as a chronic inflammatory disorder with paroxysmal wheezing, breathlessness, chest tightness, or coughing with variable but reversible airway obstruction and airway hyperresponsiveness [15]. A heterogeneous population of allergic and non-allergic asthmatic children was selected. All children were treated according to GINA guidelines [1]. A child was considered atopic when the total IgE level exceeded 20 $\mathrm{kU} / \mathrm{L}$ and/or the Phadiatop was positive and/or the Radio Allergo Sorbent Test (RAST) was positive (at least two RAST classes $\geq 2$ ). Exclusion criteria were: 1) Diseases which might have interfered with the results of the study (e.g. other chronic inflammatory diseases), 2) Inability to properly perform measurements, or 3) Active smoking.

\section{Ethics}

This study was approved by the Medical Ethics Committee of the Maastricht University Medical Centre. The clinical trial registration number was NCT00404859.

\section{Exhaled breath sample collection}

Subjects were asked to deeply inhale and subsequently exhale into resistance free Tedlar bags (5 litre) as has been previously described [13]. One to 3 exhalations were usually sufficient to inflate the bag. The contents of the bag was transferred under standardised conditions onto stainless steel two-bed sorption tubes containing active carbon (Markes International, Llantrisant, Wales, UK). VOCs were trapped onto the carbon components until analysis of the sample. In order to analyse the sample, VOCs were released from the tubes by thermal desorption at 270 ㅇ (Marks Unity desorption unit, Markes International Limited, Llantrisant, Wales, UK). VOCs were then separated by gas chromatography (GC) (ThermoFisher Scientific., Austin, Texas, USA) and subsequently detected by a time-offlight mass spectrometer (TOF-MS) (Thermo Electron Tempus Plus time-of-flight mass spectrometer, ThermoFisher Scientific, Austin, Texas, USA) as previously reported $[13,16]$. 


\section{Outcome measures}

Primary outcome measure: asthma exacerbation

The occurrence of an asthma exacerbation was the primary outcome measure of this study. A moderate exacerbation was defined as an increase in asthma symptoms (dyspnoea, cough, wheezing) and/or use of short acting $\beta_{2}$-agonists for not more than two days [17]. In this case, the drop in forced expiratory volume in one second (FEV 1 ) was not below the $80 \%$ personal maximum value. In the case of a severe exacerbation, one or more of the following criteria applied: 1 ) The $\mathrm{FEV}_{1} \%$ of maximum personal value fell below $80 \%$ of the subject's maximum value for at least two consecutive days, and/or 2) need for treatment with oral corticosteroids, and/or 3) need for hospital admission [17].

To recognise an exacerbation at an early stage, AM1 home monitors (Viasys ${ }^{\circledR}$, Hoechberg, Germany) and modems ( $\mathrm{HC} 1$, Viasys, Hoechberg, Germany) were used. FEV 1 measurements, use of rescue medication, the overall well-being and the presence and severity of pulmonary symptoms were recorded on a daily base and at a fixed time. The intensity of symptoms was scored on a scale from zero to three. All patients were asked to perform the manoeuvres 3 times within 10 minutes and the highest $\mathrm{FEV}_{1}[\mathrm{~L}]$ was stored. Data were send digitally by telephone modem to a personal computer at the Maastricht University Medical Centre once per week. In the case of deterioration of $\mathrm{FEV}_{1}$ values, and/or an increase in presence and severity of pulmonary symptoms, patients were called to the hospital for additional measurements and a consultation of the responsible Paediatric Pulmonologist. At the outpatient clinic, asthma control scores and lung function tests were assessed and breath samples were collected.

\section{Secondary outcome measure 1: Asthma control score}

The asthma control score was assessed two-monthly by using a validated questionnaire as has been described before $[3,4]$. The questionnaire contained questions about chronic airway symptoms, sleep disturbance, limitation of daily activity, asthmatic attacks, emergency or urgent care visits, and need for short acting beta-2 agonists [3].

\section{Secondary outcome measure 2: Lung function tests}

Short-acting bronchodilators were stopped at least 8 hours and long-acting bronchodilators at least 36 hours before testing. Dynamic spirometry was performed by means of the Flowscreen (Viasys ${ }^{\circledR}$, Hoechberg, Germany) according to ERS standards [18]. Recorded parameters were: $\mathrm{FEV}_{1}$, forced vital capacity (FVC), and maximum expiratory flow at $50 \%$ of FCV $\left(\mathrm{MEF}_{50}\right)$, all expressed as a percentage of the predicted normal value [18]. The reversibility to a $\beta_{2}$-agonist was determined 15 minutes after inhalation of $400 \mu \mathrm{g}$ salbutamol via a spacer (Volumatic) at 0, 6 and 12 months of the study.

\section{Analysis of data}

\section{Data-acquisition \& mining}

Analysis of the data output files from GC-TOF-MS was performed in successive steps as previously described in detail [16]. In summary, the first step was to perform peak detection and baseline corrections on all analysis output files. Normalisation of the calculated 
peak areas was performed using an area scaling factor based on the cumulative area under the detected peaks. Retention times (RT) of all samples were assessed. Finally, the output files were merged by combining corresponding compounds based on degree of similarity of the corresponding mass spectra, by determining the match factor values (MFs) and similarity of RT. The degree of mass spectra similarity was calculated using a match factor based on the similarity index as described by Stein et al. [19].

\section{Analysed settings}

In order to analyse which VOCs are predictive of an asthma exacerbation, analysis was performed in two settings. Both settings were explored since both settings might provide different information with regards to the compounds that contribute to an early detection of exacerbations in asthma patients.

\section{Setting 1}

The first explored setting was an intra-subject comparison between baseline measurements $(t=0)$ and the first measurement of an exacerbation $\left(e_{1}\right)$. The baseline measurement was defined as the sample taken before the start of the exacerbation $\left(\mathrm{e}_{0}\right)$, on average one month prior to measurement $e_{1}$. The measurements during the exacerbation were chronologically denoted by $e_{1}, e_{2}, e_{3}$ and $e_{4}$. The baseline measurement before $t=0$ is denoted by $\mathrm{t}=-1$, the one before that $\mathrm{t}=-2$, as shown in Table 1 .

\section{Setting 2}

The second setting was based on an inter-subject comparison. Baseline samples $(t=0)$ of subjects suffering an exacerbation were compared to baseline measurements of subjects not suffering an exacerbation. Sampling dates of all incorporated samples in this setting were chosen as close to one another as possible.

\section{Component selection}

Subsequent component selection and determination of interesting compounds regarding both settings ( 1 and 2 ) was performed in two ways:

a) Compounds detected in less than $8 \%$ of the samples were deleted from the dataset according to Penn et al. [20].

b) VOCs with significant different intensities between children with and without exacerbations were selected (student T-tests with Bonferroni correction). Samples of measurement $\mathrm{t}=0$ originating from the patients with an exacerbation were compared to samples of the group without an exacerbation (Table 1). These samples matched the dates of the $t=0$ samples.

c) Support vector machine (SVM) classification models were used to analyse the ability of VOCs to predict an asthma exacerbation. The SVM approach was chosen for its ability to construct predictive models with large generalisation power even in the case of large dimensionality of the data when the number of observations available for training is low [21]. SVMs are specifically useful since they seek a globally optimised solution and avoid over-fitting, allowing for a large number of features or compounds. In order to obtain the 
best subset of compounds, the attribute selection option implemented in Weka [22] was used. Compounds were selected using an SVM attribute evaluator. The attribute evaluator evaluated the worth of a subset of compounds by considering the individual predictive ability of each compound along with the redundancy between them. Preferably compounds were selected showing high correlations within the class and low inter-correlation. A subset of the highest ranking compounds was implemented into an SVM classifier trained with John Platt's sequential minimal optimisation algorithm [23]. The SVM classifiers were validated and performance was tested using 10-fold cross-validation in which the entire dataset is split repeatedly into a test set $(90 \%$ of samples) and a validation set $(10 \%$ of samples).

\section{Identification of VOCs}

Identification of VOCs was performed by comparing the mass spectra with data from the NIST library and by interpretation of the mass spectra by a specialised spectrometrist.

\section{Power analysis}

In order to find clinically meaningful correlations between independent predictors and the exacerbation rate of at least $0.6, \mathrm{~N}=35$ children are necessary to assess such a relationship with a two-sided alpha of 0.05 and a power of $98 \%$. During the 12 months follow-up, a drop out of $10 \%$ was assumed. 40 children with asthma were thus included in this study.

\section{Results}

\section{Patient characteristics}

Clinical characteristics at inclusion of the study are shown in Table 2. All asthma severity classes, based on GINA criteria [1], were present at the start of the study: intermittent disease $11 / 40(27.5 \%)$, mild persistent $8 / 40$ (20\%), moderately persistent $7 / 40$ (17.5\%), and severely persistent $14 / 40$ (35\%). The majority of the group was atopic: $29 / 40$ (73\%). All but one patient were receiving maintenance treatment with inhaled corticosteroids. 38 of the 40 children completed the study, while two patients dropped out. One patient moved out of the region. Before he dropped out, he experienced one exacerbation. The data of this exacerbation were included in the analysis. The other child was not motivated to further participate. She had stable asthma.

\section{Exacerbation rate}

During the one-year study period, 16 children developed an exacerbation of which 10 were moderate and 6 were severe. Only 3 patients developed a second exacerbation. The mean $\pm S E$ time interval between the preceding standard measurement and the onset of an exacerbation was $39 \pm 4$ days. 
Table 2: Clinical characteristics of the asthmatic children at inclusion of the study.

\begin{tabular}{|c|c|}
\hline Age (yrs) & $10.7 \pm 0.4$ \\
\hline Weight (kg) & $38.8 \pm 2.0$ \\
\hline Height $(\mathrm{cm})$ & $142.3 \pm 2.4$ \\
\hline Male / Female & $29 / 11$ \\
\hline ACQ score (minimum-maximum) & $27.6 \pm 2.3(9-64)$ \\
\hline \multicolumn{2}{|l|}{ Lung function indices } \\
\hline - Reversibility (increase in $\mathrm{FEV}_{1} \%$ predicted) & $5.9 \pm 1.1$ \\
\hline - $\mathrm{FEV}_{1} \%$ predicted & $99.6 \pm 2.2$ \\
\hline - $\mathrm{FEV}_{1} / \mathrm{VC} \%$ & $83.9 \pm 1.5$ \\
\hline - FVC \% predicted & $99.2 \pm 2.4$ \\
\hline$-\mathrm{MEF}_{50} \%$ predicted & $82.6 \pm 3.8$ \\
\hline Atopy (yes/no) & $29 / 11$ \\
\hline - Total IgE (kU/L) & $493.3 \pm 120.7$ \\
\hline - Active eczema & $7(18 \%)$ \\
\hline - Allergic rhinitis & $4(10 \%)$ \\
\hline \multicolumn{2}{|l|}{ Treatment } \\
\hline - Dose of inhaled budesonide or equivalent ( $\mu \mathrm{g})$ & $587 \pm 53$ \\
\hline - Long-acting $\beta_{2}$-agonist & $21(53 \%)$ \\
\hline - Antihistamines & $9(23 \%)$ \\
\hline - Leukotrien receptor antagonist & $8(20 \%)$ \\
\hline
\end{tabular}

Data are given as mean \pm SEM except where indicated otherwise. Abbreviations: FEV $_{1}$, forced expiratory volume in 1 second; VC, vital capacity; $\mathrm{MEF}_{50} \%$, mean expiratory flow at 50\%; ACQ, Asthma control score.

\section{Volatile organic compounds}

VOCs included in data analysis

In all 39 children with asthma, a total amount of 3434 different VOCs were detected in the exhaled breath samples. On average, 343 VOCs per individual were detected. Each subject delivered 7 to 15 samples depending on the occurrence of an exacerbation.

\section{Significant different VOCS}

In total for both settings, independent student T-tests showed 30 significantly different VOCs after Bonferroni correction $(p<0.05)$. These VOCs were identified as markers associated with exacerbations.

\section{Course of VOCS}

After selection of the significantly different compounds these VOCs were tracked in all other samples $\left(\mathrm{e}_{-2}\right.$ to $\left.\mathrm{e}_{4}\right)$. Their relative intensity was determined in all samples in order to provide more insight in the course of intensities of these compounds prior to and during exacerbations. As an example, the course of the intensity of three VOCs during an exacerbation are shown in Figure. 
p-xylene
(Friedman test, $p=0.005$ )

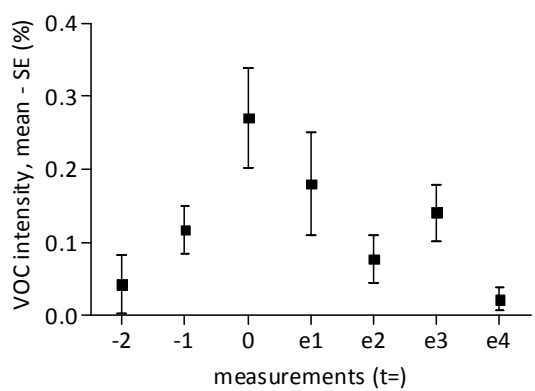

3,6-octadecadiynoic acid, methylester (Friedman test, $\mathrm{p}=0.004$ )

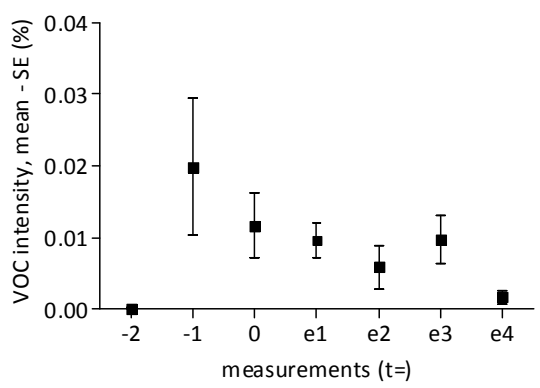

octadecane

(Friedman test, $\mathrm{p}=0.01$ )

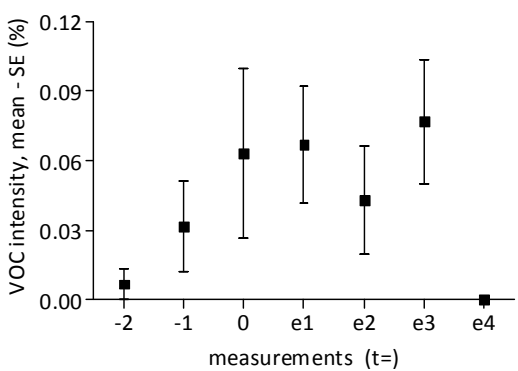

8,11-octadecadiynoic acid, methyl ester (Friedman test, $\mathrm{p}=0.01$ )

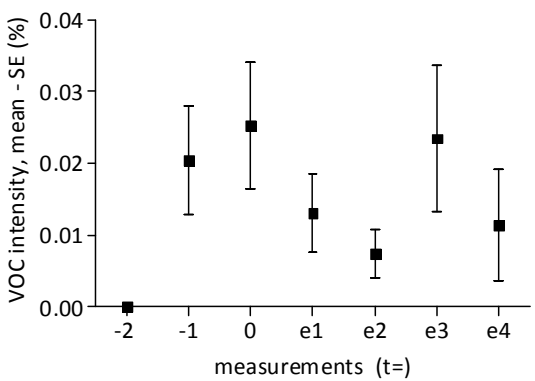

Figure 1: Mean \pm SE intensities (\%) of 4 significant different compounds demonstrating their course before and during an asthma exacerbation.

Ability to predict an asthma exacerbation (Support Vector Machine classifier)

All VOCs that were present in at least $8 \%$ of the samples were included in the SVM analyses in order to assess the ability of VOCs to predict an asthma exacerbation. Results of SVM models are based on VOCs considered together. In setting 1 (baseline versus exacerbation within patients), the most optimal performing SVM was based on 6 VOCs. Exacerbations were correctly classified in $96 \%$ of samples (sensitivity $100 \%$, specificity $93 \%$ ) as shown in table 3a. The optimal performing SVM of setting 2 (baseline resulting in exacerbation versus baseline not resulting in exacerbation, between patients) was based on 7 VOCs. A correct classification rate of $91 \%$ was found with a corresponding sensitivity and specificity of $78 \%$ and $100 \%$, respectively, Table $3 \mathrm{~b}$. The relationship between the number of different VOCs in the SVM model and the corresponding sensitivity and specificity is shown in Table 3a-b. In summary, different VOCs predict the onset of an exacerbation or monitor the course of the exacerbation. 
Table 3a: The relationship between the number of VOCs in the SVM model and the prediction of exacerbations of setting 1.

\begin{tabular}{llll}
\hline Number of VOCs & Sensitivity (\%) & Specificity (\%) & Correct classification (\%) \\
\hline 9 & 100 & 100 & 100 \\
8 & 100 & 100 & 100 \\
7 & 100 & 93 & 96 \\
6 & 100 & 93 & 96 \\
5 & 100 & 79 & 89 \\
4 & 100 & 71 & 86 \\
3 & 100 & 64 & 82 \\
2 & 100 & 50 & 75 \\
1 & 100 & 29 & 64 \\
\hline
\end{tabular}

The sensitivity, specificity and the percentages of correct classification of the SVM models are given. An optimal model contained 6 VOCs and had a sensitivity of $100 \%$ and a specificity of $93 \%$.

Table 3b: The relationship between the number of VOCs in the SVM model and the prediction of exacerbations of setting 2 .

\begin{tabular}{llll}
\hline Number of VOCs & Sensitivity (\%) & Specificity (\%) & Correct classification (\%) \\
\hline 9 & 79 & 95 & 88 \\
8 & 71 & 95 & 85 \\
7 & 79 & 100 & 91 \\
6 & 64 & 100 & 85 \\
5 & 71 & 100 & 88 \\
4 & 64 & 100 & 85 \\
3 & 43 & 100 & 76 \\
2 & 36 & 100 & 73 \\
1 & 21 & 100 & 67 \\
\hline The sensitivity, specificity and the percentages of correct classification of the SVM models are given. An \\
optimal model contained 7 VOCs and had a sensitivity of 79\% and a specificity of 100\%.
\end{tabular}

\section{Identification of VOCs}

it was possible to identify 5 out of 6 VOCs of the optimally discriminating SVM model of setting 1 , and 6 out of 7 VOCs of setting 2 . (Table $4 a-b)$.

Table 4a: Identified compounds as implemented into the most optimal SVM classifiers of setting 1.

\begin{tabular}{ll}
\hline 1 & p-xylene \\
2 & 3-methyl-pentane \\
3 & 2-ethyl-4-methyl-1-pentanol \\
4 & unknown \\
5 & 1-phenyl-1-butene \\
6 & 4,6,9-nonadecatriene \\
\hline
\end{tabular}


Table 4b: Identified compounds as implemented into the most optimal SVM classifiers of setting 2.

\begin{tabular}{ll}
\hline 1 & unknown \\
2 & 2-ethyl-1,3-butadiene \\
3 & cyclohexane \\
4 & 2-octen-1-ol \\
5 & $1.2-$-Methyl-4H-1,3-benzoxathiine \\
6 & benzene \\
7 & $1,4-$ hexadiene \\
\hline
\end{tabular}

\section{Discussion}

This study indicates that VOCs in exhaled breath are able to predict an asthma exacerbation on average 39 days before the event is clinically manifest. Setting 1: Nineteen VOCs were significantly different between measurements before the onset of an exacerbation $(t=0)$ compared to the first measurement of an exacerbation $\left(e_{1}\right)$. Six VOCs combined into a SVM classifier could correctly classify $96 \%$ demonstrating a sensitivity of $100 \%$ and and specificity $93 \%$ respectively. Setting 2: Eleven VOCs showed a significant change in the course of an exacerbation, whereas a combination of 7 VOCs could classify stable disease or pre-clinical exacerbations with a correct classification rate of $91 \%$ and a sensitivity and specificity of $78 \%$ and $100 \%$ respectively. The identified VOCs were mainly classified as hydrocarbons.

To our knowledge this is the first longitudinal study to analyse the ability of VOCs in exhaled breath to predict exacerbations of childhood asthma, and to study the course of VOCs during an exacerbation. In a cross-sectional study of 120 children (57 controls, 63 asthma), we found a good discrimination between asthma and controls based on an optimal combination of eight VOCs in exhaled breath [13]. In adults, Dragonieri et al. have assessed VOC patterns in exhaled breath by means of the electronic nose. They found that asthma could be differentiated from controls but the discrimination between different degrees of asthma severity was not satisfactory [14]. In contrast to GC-TOF-MS used in our study, the electronic nose in the study of Dragonieri et al. was used to assess "smellprints" of different VOCs. These techniques are fundamentally different, since the smellprints are based on pattern recognition instead of assessing single mass spectra. The disadvantage of smell-prints is that it may not measure VOCs which are important for the discrimination. The advantage of the approach used in the present study is that the most predictive VOCs are detected and that the chemical background of the compounds can be elucidated. This information can be used in the future to construct an 'electronic nose' which is specifically useful for a certain disease (e.g. childhood asthma) and a specific clinical question (e.g. the early detection of exacerbations).

Several studies have assessed the potential of FeNO to predict exacerbations of childhood asthma. In a 6-month study by Fritsch et al. of 47 children with mild to moderate asthma, FeNO at a cut-off point of $29 \mathrm{ppb}$ was best predictive for an exacerbation with a sensitivity of $80 \%$ and a specificity of $60 \%$ [24]. In the inhaled corticosteroid withdrawal study of Pijnenburg et al., FeNO values $>49$ ppb 4 weeks after discontinuation had a sensitivity for 
an asthma relapse of $71 \%$ and a specificity of $93 \%$ [25]. In non-atopic patients with asthma, FeNO is usually not elevated [26]. It is questionable whether one inflammatory parameter like FeNO provides sufficiently adequate information about the extensive and complicated inflammatory mechanisms underlying asthma. Regular two-month assessments of FeNO in this study were not predictive of exacerbations (data not shown). The advantage of VOC analysis in exhaled breath is that several inflammatory markers are measured in exhaled breath simultaneously and that the most predictive biomarkers can be selected, as indicated by the high sensitivity and specificity of the combination of six VOCs in our study.

Several elements of our study are relevant to discuss. First, a group of 40 children with asthma was included of which 16 children experienced an exacerbation during the year of follow-up. Although internal validation was performed in our study by 10 -fold crossvalidation and exacerbations could be predicted with good sensitivity and specificity, external validation of our study in larger study populations will be necessary to confirm the optimal combination of VOCs [27].

Second, presence of VOCs may be influenced by intrinsic (gender, age, weight, atopy, lung function) and extrinsic factors (ambient air, diet or medication). Most of these intrinsic factors were well controlled in our study. Moreover, no relationship was found between these intrinsic factors, medication and exacerbations during the study. This almost rules out a possible confounding influence of these factors on the results of this study. We did not correct our data for VOCs in ambient air. Currently, there is no consensus on this methodological issue. Children were all measured in the same room at each session, and therefore, were all influenced by ambient air in the same way. In the predicting models, the presence of VOCs in the chromatogram resulting from ambient air pollution will not appear as a clear predictor of exacerbations. An earlier report by our research group concluded that the procedure followed of sampling, chemical analysis, data handling and accurate data mining [16] was highly reproducible. In addition, chemical structures and metabolites of medication used were not reflected in the significantly predictive VOCs. Third, the origin of the identified VOCs remains in some instances uncertain. We were able to identify the majority of the predicting VOCs.

We hypothesize that discriminating VOCs reflect the degree of airway inflammation and oxidative stress. Saturated hydrocarbons (VOCs) in exhaled breath are formed during lipid peroxidation of fatty acid components of cell membranes. This process is triggered by reactive oxygen species (ROS) [16, 28-33].

In the future, it is relevant to study whether titration of anti-inflammatory treatment on the basis of VOCs is of help in the prevention of exacerbations of asthma. In addition, studies are necessary to clarify the biochemical origin, the pathophysiological function and the optimal way of measuring predictive VOCs.

Breath analysis mirrors biochemical and immunological processes in the human lung. The present study indicates that by means of VOCs analysis in exhaled breath, an asthma exacerbation may be predicted on average 39 days before the event became clinically manifest. Future studies are needed to explore the nature of these VOCs, and to investigate whether better asthma control can be achieved when exhaled breath analysis is included as a parameter in daily clinical practice. 


\section{Acknowledgements}

The authors gratefully acknowledge AstraZeneca, MSD, the "Jaap Swieringa Foundation" and the province of Limburg (Netherlands) for their financial support.

\section{References}

1. Global Initiative for asthma (GINA). Pocket guide for asthma management and prevention in children. National Institute of Health, National Heart, Lung, and Blood Institute 2006.

2. Gustafsson PM, Watson L, Davis KJ, Rabe KF. Poor asthma control in children: evidence from epidemiological surveys and implications for clinical practice. Int J Clin Pract 2006: 60(3): 321-334.

3. Hammer SC, Robroeks CM, van Rij C, Heynens J, Droog R, Jobsis Q, Hendriks HJ, Dompeling E. Actual asthma control in a paediatric outpatient clinic population: do patients perceive their actual level of control? Pediatr Allergy Immunol 2008: 19(7): 626-633.

4. Rabe KF, Adachi M, Lai CK, Soriano JB, Vermeire PA, Weiss KB, Weiss ST. Worldwide severity and control of asthma in children and adults: the global asthma insights and reality surveys. J Allergy Clin Immunol 2004: 114(1): 40-47.

5. O'Connell EJ. Optimizing inhaled corticosteroid therapy in children with chronic asthma. Pediatr Pulmonol 2005: 39(1): 74-83.

6. van Gent R, van Essen-Zandvliet LE, Rovers MM, Kimpen JL, de Meer G, van der Ent CK. Poor perception of dyspnoea in children with undiagnosed asthma. Eur Respir J 2007: 30(5): 887-891.

7. Kharitonov SA, Barnes PJ. Biomarkers of some pulmonary diseases in exhaled breath. Biomarkers 2002: 7(1): 1-32.

8. Kips JC, Kharitonov SA, Barnes PJ. Non invasive assessment of airway inflammation in asthma. Eur Respir Mon 2003: 13: 164-179.

9. Rahman I, Kelly F. Biomarkers in breath condensate: a promising new non-invasive technique in free radical research. Free Radic Res 2003: 37(12): 1253-1266.

10. Kinnula VL. Production and degradation of oxygen metabolites during inflammatory states in the human lung. Curr Drug Targets Inflamm Allergy 2005: 4(4): 465-470.

11. Antczak A, Nowak D, Shariati B, Krol M, Piasecka G, Kurmanowska Z. Increased hydrogen peroxide and thiobarbituric acid-reactive products in expired breath condensate of asthmatic patients. Eur Respir J 1997: 10(6): 1235-1241.

12. Montuschi P, Corradi M, Ciabattoni G, Nightingale J, Kharitonov SA, Barnes PJ. Increased 8-isoprostane, a marker of oxidative stress, in exhaled condensate of asthma patients. Am J Respir Crit Care Med 1999: 160(1): 216-220.

13. Dallinga JW, Robroeks CMHHT, Van Berkel JJ, Moonen E, Godschalk RW, Jöbsis Q, Dompeling E, Wouters EF, Van Schooten FJ. Analysis of volatile organic compounds in exhaled breath as a diagnostic tool for asthma in children. Clinical and Experimental Allergy 2009: in press.

14. Dragonieri S, Schot R, Mertens BJ, Le Cessie S, Gauw SA, Spanevello A, Resta O, Willard NP, Vink TJ, Rabe KF, Bel EH, Sterk PJ. An electronic nose in the discrimination of patients with asthma and controls. J Allergy Clin Immunol 2007: 120(4): 856-862.

15. Washington (DC): National Institutes of Health LaBI. Global Initiative for Asthma (GINA). Global strategy for asthma management and prevention. publication No. 02-3659. 2002.

16. Van Berkel JJ, Dallinga JW, Moller GM, Godschalk RW, Moonen E, Wouters EF, Van Schooten FJ. Development of accurate classification method based on the analysis of volatile organic compounds from human exhaled air. J Chromatogr B Analyt Technol Biomed Life Sci 2008: 861(1): 101-107.

17. Demoly P, Crestani B, Leroyer C, Magnan A, Mounedji N, Humbert M. Control and exacerbation of asthma: a survey of more than 3000 French physicians. Allergy 2004: 59(9): 920-926.

18. Quanjer PH, Tammeling GJ, Cotes JE, Fabbri LM, Matthys H, Pedersen OF, Peslin R, Roca J, Sterk PJ, Ulmer WT, et al. Symbols, abbreviations and units. Working Party Standardization of Lung Function Tests, European Community for Steel and Coal. Eur Respir J Suppl 1993: 16: 85-100. 
19. Stein SE, Scott DR. Optimization and Testing of Mass-Spectral Library Search Algorithms for Compound Identification. Journal of the American Society for Mass Spectrometry 1994: 5(9): 859-866.

20. Penn DJ, Oberzaucher E, Grammer K, Fischer G, Soini HA, Wiesler D, Novotny MV, Dixon SJ, Xu Y, Brereton RG. Individual and gender fingerprints in human body odour. J R Soc Interface 2007: 4(13): 331-340.

21. Guyon I, Weston J, Barnhill S, Vapnik V. Gene selection for cancer classification using support vector machines. Machine Learning 2002: 46(1-3): 389-422.

22. Frank E, Hall M, Trigg L, Holmes G, Witten IH. Data mining in bioinformatics using Weka. Bioinformatics 2004: 20(15): 2479-2481.

23. Platt JC. Fast training of support vector machines using sequential minimal optimization. MIT press, 1998.

24. Fritsch M, Uxa S, Horak F, Jr., Putschoegl B, Dehlink E, Szepfalusi Z, Frischer T. Exhaled nitric oxide in the management of childhood asthma: a prospective 6-months study. Pediatr Pulmonol 2006: 41(9): 855-862.

25. Pijnenburg MW, Hofhuis W, Hop WC, De Jongste JC. Exhaled nitric oxide predicts asthma relapse in children with clinical asthma remission. Thorax 2005: 60(3): 215-218.

26. Silvestri M, Sabatini F, Spallarossa D, Fregonese L, Battistini E, Biraghi MG, Rossi GA. Exhaled nitric oxide levels in non-allergic and allergic mono- or polysensitised children with asthma. Thorax 2001: 56(11): 857862.

27. Steyerberg EW, Bleeker SE, Moll HA, Grobbee DE, Moons KG. Internal and external validation of predictive models: a simulation study of bias and precision in small samples. J Clin Epidemiol 2003: 56(5): 441-447.

28. Sanchez JM, Sacks RD. Development of a multibed sorption trap, comprehensive two-dimensional gas chromatography, and time-of-flight mass spectrometry system for the analysis of volatile organic compounds in human breath. Anal Chem 2006: 78(9): 3046-3054.

29. Ashley DL, Bonin MA, Hamar B, McGeehin M. Using the blood concentration of 2,5-dimethylfuran as a marker for smoking. Int Arch Occup Environ Health 1996: 68(3): 183-187.

30. Corradi M, Pignatti P, Manini P, Andreoli R, Goldoni M, Poppa M, Moscato G, Balbi B, Mutti A. Comparison between exhaled and sputum oxidative stress biomarkers in chronic airway inflammation. Eur Respir J 2004: 24(6): 1011-1017.

31. Lex C, Zacharasiewicz A, Payne DN, Wilson NM, Nicholson AG, Kharitonov SA, Barnes PJ, Bush A. Exhaled breath condensate cysteinyl leukotrienes and airway remodeling in childhood asthma: a pilot study. Respir Res 2006: 7: 63.

32. Paredi P, Kharitonov SA, Leak D, Ward S, Cramer D, Barnes PJ. Exhaled ethane, a marker of lipid peroxidation, is elevated in chronic obstructive pulmonary disease. Am J Respir Crit Care Med 2000: 162(2 Pt 1): 369373.

33. Phillips M, Herrera J, Krishnan S, Zain M, Greenberg J, Cataneo RN. Variation in volatile organic compounds in the breath of normal humans. J Chromatogr B Biomed Sci Appl 1999: 729(1-2): 75-88. 


\section{CHAPTER 12.2}

\section{Volatile organic compounds in exhaled breath predict exacerbations in cystic fibrosis: results in a one-year longitudinal study}

JJBN Van Berkel*, CMHHT Robroeks*, JW Dallinga, Q Jöbsis, EJ Moonen, EFM Wouters, E Dompeling, FJ van Schooten * shared first authorship Submitted 


\section{Abstract}

\section{Background}

Pulmonary exacerbations of CF are an important cause for the experienced disability of patients, respiratory symptoms, and decrease in lung function, which require antibiotic therapy at home or in the hospital. Therefore, prevention of exacerbations in CF is important. Airway infections in CF and changes due to exacerbations are characterized by oxidative stress/airway inflammation and the subsequent changes regarding the profile of volatile organic compounds (VOCs) in exhaled breath. We recently developed a methodological approach to identify profiles of VOCs in exhaled breath that are unique for patient groups. In the present longitudinal study we studied whether changes in VOC profiles can predict pulmonary exacerbations of CF.

\section{Methods}

A one year longitudinal study was performed in 26 CF patients (age [mean \pm SE] 15.8 \pm 2.1 years). At 2-month intervals, exhaled breath samples were obtained and lung function and symptoms were determined in a standardized way. The VOCs were analyzed by GC-TOFMS. Advanced statistical unsupervised learning algorithms were applied to find relevant VOCs to predict the occurrence of exacerbations.

\section{Results}

Seventeen CF patients experienced an exacerbation of which 8 experienced a second event during the study. In total, 2667 different VOCs were analyzed. It appeared that 15 VOCs were differentially present in exhaled breath from patients not having an exacerbation versus patients suffering an exacerbation event within 3-8 weeks after sampling, indicating that these VOCs might predict an exacerbation (between-subject analysis). A support vector machine classifier based on this dataset contained 8 VOCs and classified $92 \%$ of exacerbations (sensitivity $100 \%$,specificity $84 \%$ ). The VOCs with predictive value of a CF exacerbation, were mainly long-chain hydrocarbons.

\section{Conclusions}

This study shows that VOCs in exhaled breath are able to indicate CF exacerbations weeks before these adverse events are clinically manifest. 


\section{Introduction}

Cystic fibrosis is a hereditary disease affecting exocrine glands. It will, among other multisystem failures, manifest itself as a progressive lung disease characterized by recurrent infectious events. These exacerbations lead to acute changes in pulmonary symptoms related to increased airway secretions. Pulmonary symptoms has a great impact on the quality of life and is the main reason of morbidity in cystic fibrosis. In case of an exacerbation intravenous antibiotics and hospital admission are necessary and exacerbations have also been associated with diminished lung function in later life [1]. The yearly exacerbation rate increases with age and more severe pulmonary impairment and is clearly related to survival To date there is no predictive parameters regarding the occurrence of an exacerbation by means of an objective chemical, physiologic or histologic marker. Therefore, it is not possible to anticipate and by means of treatment prevent lung damage on the long term. An early and accurate diagnosis of an exacerbation will facilitate the possibilities to treat these adverse events before they are clinically manifest.

Oxidative stress plays a major role in CF pulmonary exacerbations. Before exacerbations become clinically manifest the increase in oxidative stress might be useful as an indicator of early manifestation of the exacerbations. Recent publications stated that the analysis of exhaled breath might prove useful as a diagnostic tool regarding the detection of oxidative stress and might prove useful in the diagnosis and/or early prediction of exacerbations providing clinicians with the possibility to antibiotically treat these patients in an early stage in order to reduce the severity of the exacerbation or even prevent it [2-6].

Regarding the analysis of exhaled breath several biomarkers for diseases have already been identified. Nitric oxide (NO) levels are generally accepted as a marker of eosinophilic airway inflammation and oxidative stress in the respiratory tract in patients with asthma [7]. The availability of hundreds of different VOCs in exhaled breath was already demonstrated by Pauling et al. in 1971 [8] and many more have been identified since. Philips et al. have recently successful demonstrated the possibility of using VOC profiles in exhaled breath as biomarkers of lung cancer and pulmonary tuberculosis [9-11]. In previous research we identified subsets of VOCs that proved to correctly classify COPD patients from non-diseased controls [12] and children with asthma or CF from healthy controls [4, 13]. Therefore, we are particularly interested in multi-component analysis of VOCs in CF patients suffering an exacerbation in order to increase diagnostic performance using a biomarker profile approach.

These previous studies applied a cross sectional setup whereas the study described in this article demonstrates the longitudinal approach to the analysis of exacerbations in CF patients. This approach may have great potential regarding clinical application for the assessment of exacerbations. Especially since the analysis of breath provides a fast, noninvasive, cost beneficial and easy to perform diagnostic tool.

The aim of this study was to identify VOCs in exhaled breath which are able to 1) predict CF exacerbations reliably and 2) could monitor the course of exacerbations in CF. 


\section{Methods}

\section{Study subjects}

A total of 26 subjects (age [mean $\pm S E$ ] 15.8 \pm 2.1 years) suffering from cystic fibrosis (CF) were recruited at the Maastricht University Medical Centre, The Netherlands. Subject characteristics are presented in Table 1. CF disease was defined as the combination of characteristic clinical features (persistent pulmonary symptoms, meconium ileus, failure to thrive, steatorrhoe) and an abnormal sweat test (Chloride $>60 \mathrm{mM}$ ) [14]. CF severity was based on the Shwachman-Kulczycki score (SK-score) [15].

The following exclusion criteria were applied: diseases that may interfere with the results of the study (e.g. other chronic inflammatory diseases, such as Crohn's disease or rheumatoid arthritis), mental retardation, inability to perform measurements properly or active smoking.

Table 1: Subject characteristics (26 CF patients).

\begin{tabular}{|c|c|}
\hline Age (yrs) & $15.8 \pm 2.1$ \\
\hline Weight (kg) & $37.8 \pm 3.1$ \\
\hline Height $(\mathrm{cm})$ & $143.1 \pm 4.1$ \\
\hline Gender (M/F) & $13 / 13$ \\
\hline \multicolumn{2}{|l|}{ Lung function indices } \\
\hline Bronchodilating response in $\mathrm{FEV}_{1}(\%)$ & 2.9 \\
\hline $\mathrm{FEV}_{1}(\%$ predicted $)$ & $68.3 \pm 4.1$ \\
\hline $\mathrm{FEV}_{1} / \mathrm{VC}(\%)$ & $77.9 \pm 3.3$ \\
\hline FVC (\% predicted) & $72.3 \pm 4.1$ \\
\hline $\mathrm{MEF}_{50}$ (\% predicted) & $51.1 \pm 5.9$ \\
\hline TLC (\% predicted) & $106.4 \pm 2.6$ \\
\hline RV (\% predicted) & $180.1 \pm 13.9$ \\
\hline ITGV (\% predicted) & $121.3 \pm 5.2$ \\
\hline \multicolumn{2}{|l|}{ Treatment } \\
\hline Antibiotics, prophylactic (inhaled or oral) & $7(27 \%)$ \\
\hline DNase & $18(69 \%)$ \\
\hline Fat soluble vitamins & $23(89 \%)$ \\
\hline Antacids & $20(77 \%)$ \\
\hline Inhaled steroids & $6(23 \%)$ \\
\hline Long acting beta-2 agonist & $5(19 \%)$ \\
\hline \multicolumn{2}{|l|}{ Sputum cultures } \\
\hline Positive sputum cultures (yes/no) & $22 / 4$ \\
\hline Pseudomonas Aeruginosa & $9(43 \%)$ \\
\hline Staphylococcus Aureus & $17(77 \%)$ \\
\hline Haemophilius Influenzae & $6(29 \%)$ \\
\hline Haemophilus Parainfluenzae & $5(24 \%)$ \\
\hline Aspergillus Fumigatus & $6(29 \%)$ \\
\hline Candida Albicans & $4(19 \%)$ \\
\hline
\end{tabular}

Mean \pm standard error are presented unless stated otherwise. 


\section{Study Design}

The design was a one-year prospective controlled longitudinal study. At 2-month intervals, routine clinical visits with assessments of clinical parameters, Shwachman-Kulczycki score, measurement of lung function indices and collection of exhaled breath samples were carried out. In case of an exacerbation, patients were asked to come for four extra visits at day $1,5,10$ and at the end of the exacerbation. These additional visits were planned at most twice during the study per patient.

In order to diagnose a CF exacerbation in a standardized manner, the scoring system of Rosenfeld et al. was applied [16]. The definition of an exacerbation was based on respiratory symptoms, school or work absenteeism, weight loss (over one kg over the preceding month), crackles or rhonchi on auscultation, and a decrease in $\mathrm{FEV}_{1}$ of more than $20 \%$ of personal maximum value. Recognition of an exacerbation in an early stage was important in this study. It was made possible by means of home monitors (AM1, Viasys, Hoechberg, Germany). With the home monitor, the forced expiratory volume in one second $\left(\mathrm{FEV}_{1}\right)$, as well as use of antibiotics, overall well-being, and presence of pulmonary symptoms (cough, sputum, dyspnoea) were recorded 3 times a week at fixed times. These data were sent to a computer at the Maastricht University Medical Centre by means of a modem (HC1, Viasys, Hoechberg, Germany) once per week. In case of an exacerbation, patients were called to the hospital for the additional measurements. To monitor the development of an ongoing exacerbation the cystic fibrosis clinical score questionnaire (CFCS) of Kanga et al. was used [17].

All subjects or their parents gave their informed consent and the study protocol was approved by the medical ethics committee of Maastricht University. The international study number was NCT00404859.

\section{Sample collection and analysis}

Subjects were sampled at a centrally ventilated treatment rooms located in the hospital as described previously by van Berkel et al. [5]. Subjects were asked to deeply inhale and subsequently exhale into resistance free Tedlar bags (1L). Usually 2 to 3 exhalations were sufficient to inflate the bag. The contents of the bags were transported under standardized conditions onto stainless steel two-bed sorption tubes, filled with carbograph 1TD/Carbopack X (Markes International, Llantrisant, Wales, UK) that trap VOCs. Desorption tubes were stored at room temperature until analysis. For the analysis these desorption tubes were placed inside a thermal desorption unit (Marks Unity desorption unit, Markes International Limited, Llantrisant, Wales, UK) and subsequently heated to 270 degrees Celsius in order to release all VOCs onto a cold trap. This preconcentration step is necessary to increase sensitivity and to provide the means to release all compounds onto the gas chromatograph (GC) at the same time. VOCs were separated by GC (ThermoFisher Scientific., Austin, Texas, USA). The gas chromatography capillary column RTX-5ms, $30 \mathrm{~m} \mathrm{x}$ $0.25 \mathrm{~mm}$ 5\% diphenyl, 95\% dimethylsiloxane capillary, film thickness 1.0 micron was used for separation. Subsequently detection by a time-of-flight mass spectrometer (TOF-MS) (Thermo Electron Tempus Plus time-of-flight mass spectrometer, ThermoFisher Scientific, Austin, Texas, USA) was applied. The temperature of the gas chromatograph was programmed as follows: 40 degrees Cesius during 5 min., then raised with 10 degrees Cel- 
sius/min until the final temperature of 270 degrees Cesius which was maintained for 5 min. Electron ionization at $70 \mathrm{eV}$ was used with a $5 \mathrm{~Hz}$ scanning rate over a mass range of $\mathrm{m} / \mathrm{z}$ 35-350 amu. An example of a breathogram and the identification of a few peaks is shown in Figure 1.

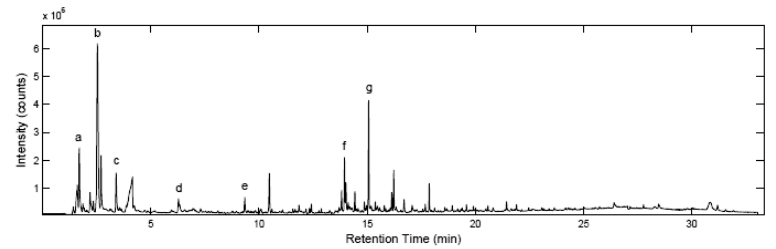

Figure 1: Example of a breathogram from a control subject demonstrating a few identified compounds.

a) acetone; b) 1,3-pentadiene; c) 2-methyl-pentane; d) benzene; e) toluene; f) 6-methyl-5-hepten-2-one; g) phenol.

\section{Data-acquisition}

Analysis of the data output files from the GC-TOF-MS was performed in successive steps as previously described in detail [5] . In summary, the first step was to perform peak detection and baseline corrections on all analysis output files. Normalization of the calculated peak areas was performed using an area scaling factor based on the cumulative area under the detected peaks; since all chromatograms display rather similar profiles this method of normalization proved most robust.

The retention times (RT) of all samples were corrected for chromatographic drifting. Applying the correction for retention times is very effective and easy to perform. The use of an added internal standard is avoided, since already available compounds that demonstrate availability in a large number of samples are used, adding to the straightforwardness and robustness of the presented methodology.

Finally, the output files were merged by combining corresponding compounds based on retention time and on similarity of the corresponding mass spectra. The degree of mass spectra similarity was calculated using a match factor (MF) based on the similarity index as described by Stein et al. [18]. These match factors were only determined for compounds within a selectable RT-window, this value was chosen based on analysis of the maximum RT-range of identical compounds found in several subject files. MF-threshold values were determined based on a variety of complementary compounds manually combined which resulted in a used MF of 0.859 .

\section{Selection of informative compounds}

To gain more insight of compounds regarding prediction of the occurrence of these exacerbations analysis of two settings was performed comparing different phases of the exacerbations.

\section{Setting 1}

The first explored setting (setting 1 ) was the comparison of baseline measurements $(t=0)$ to the first exacerbation measurement $\left(t=e_{1}\right)$ at the start of the exacerbation event. The 
baseline measurement was defined as the standard sample taken on average one month prior to the start of the exacerbation event and denoted by $t=0$. The measurements during the exacerbation were chronologically denoted by $t=e_{1}, e_{2}, e_{3}$ and $e_{4}$. If subjects suffered from a second exacerbation during the time of research these measurements were denoted by $t=e_{5}, e_{6}, e_{7}$ and $e_{8}$. These phases were treated as $t=e_{1}, e_{2}, e_{3}$ and $e_{4}$ respectively. The baseline measurement before $t=0$ is denoted by $t=-1$, the one before that $t=-2$ as shown in Figure 2.

Figure 2: Study timeline demonstrating standard and exacerbation measurements.

\begin{tabular}{|c|c|c|c|c|c|c|c|c|c|}
\hline \multicolumn{4}{|c|}{ Standard } & \multicolumn{2}{|c|}{ Exacerbation } & \multicolumn{4}{|c|}{ Standard } \\
\hline$t=-3$ & -2 & -1 & 0 & e1 & e2 & e3 & e4 & 1 & 2 \\
\hline
\end{tabular}

The baseline measurement was defined as the sample $0-2$ months (on average 39 days) before the clinical onset of an CF exacerbation, denoted by $t=0$. Measurements before $t=0$, were coded $t=-1, t=-2$...etc. The time interval between these measurements was 2 months. During the exacerbation, measurements were denoted by $e_{1}, e_{2}, e_{3}$ and $e_{4}$, referring to the additional measurements at day $1,3,5$ and at the end of the event, respectively. After the exacerbation, standard measurements continued at two months intervals denoted as $t=+1, t=+2, \ldots$ etc.

\section{Setting 2}

The second setting (setting 2) explored dealt with comparing baseline samples $(t=0)$ of subjects suffering an exacerbation to baseline measurements $(t=0)$ of subjects not suffering an exacerbation. Sampling dates of all incorporated samples in this setting were chosen as close to one another as possible.

Both settings were explored since both settings might provide different information with regards to the compounds that contribute to an early detection of exacerbations in $\mathrm{CF}$ patients. Setting 1 analysed the intra-individual differences before and during exacerbations while setting 2 analysed the inter-individual differences prior to exacerbations.

\section{Component Selection}

Subsequent component selection and determination of interesting compounds regarding both settings ( 1 and 2 ) was performed in two ways:

1) Analyzing the compounds and determining the significantly different compounds based on a t-test combined with a Bonferroni correction as the dataset demonstrated to be normally distributed. The significantly different compounds could hold valuable information and effort was made to find those compounds that might hold the potential of a single biomarker for the early detection of exacerbations in CF patients. In addition to that, analysis of all compounds individually might provide future insight into the changes in pathophysiology of exacerbations. We are aware that numerous compounds in breath are still not biologically linked to metabolic or disease pathways, and studying the pathophysiology of these exacerbations in relation to extracted compounds is currently out of scope. 
2) By selecting informative compounds fitted into a support vector machines (SVM) classification model that would preferably be able to (1) correctly classify samples of baseline measurements $(t=0)$ compared to the first exacerbation measurement $\left(t=e_{1}\right)$ at the start of the exacerbation event and (2) baseline samples from subjects suffering from an exacerbation from baseline samples from subjects not suffering from an exacerbation. This might provide information regarding a profile of VOCs combined into an SVM model that holds predictive power with regards to early detection of exacerbations. Previous research has concluded that classification based on a profile of VOCs in breath is far superior compared to classification based on single compounds.

The SVM approach was chosen for its ability to construct predictive models with large generalization power even in the case of large dimensionality of the data when the number of observations available for training is low, which is obviously the case here. SVM are specifically useful since they seek a globally optimized solution and avoid over-fitting, so the large number of features or compounds is allowed [19]. The compounds are selected through a number of variable selection criteria. This selection algorithm will select the optimal subset of compounds able to correctly classify the dataset. A variety of subset selection methods was tested, among which the gain-ratio attribute evaluator. In order to obtain the best subset of compounds the attribute selection option implemented in Weka [20] was used. Compounds were selected using an SVM attribute evaluator. The attribute evaluator we used evaluated the worth of a subset of compounds by considering the individual predictive ability of each compound along with the redundancy between them. Preferably compounds were selected showing high correlations within the class and low inter-correlation. After every run the least efficient compound was removed. A subset of the highest ranking compounds was implemented into an SVM classifier trained with John Platt's sequential minimal optimization algorithm [21]. The SVM classifiers were validated and performance was tested with use of 10 times cross-validation in which the entire dataset is split repeatedly into a test set ( $90 \%$ of samples) and a validation set $(10 \%$ of samples).

\section{Results}

Of 26 included patients, 17 patients experienced one exacerbation and 8 patients had a second exacerbation. Of the patients with one exacerbation, 2 withdrew from the study after the event. These patients were excluded from the analysis.

\section{Significantly different compounds}

The analysis of exhaled breath based on 24 demonstrated that the breath contained a wide variety of VOCs. A total of 2667 different compounds were found and a mean of 332 different VOCs were detected per sample. Each subject delivered 7 to 15 samples depending on the occurrence of exacerbations or not. Table 2 shows the significantly different compounds in both settings. For each setting the significantly different compounds $(p<0.05)$ were determined by a t-test with a Bonferroni correction. Significantly different compounds regarding setting 1 : $t=0$ compared to $t=e_{1}$ (for subjects experiencing an exac- 
erbation) were obtained using a paired t-test. Regarding setting 2: $t=0$ from subjects that would experience an exacerbation within 2-4 weeks compared to baseline samples from subjects not suffering an exacerbation were analyzed with a non-paired t-test. Identification of the compounds was performed by comparing the mass spectra to a library and by interpretation of the mass spectra by an experienced mass spectrometrist.

Table 2: Identified VOCs.

\begin{tabular}{|c|c|}
\hline Compound & p-value \\
\hline \multicolumn{2}{|l|}{ Setting 1} \\
\hline 2-methyl-2-penten-1-ol & 0.047 \\
\hline Unknown & 0.004 \\
\hline 2-methyl-1-hexadecanol & 0.044 \\
\hline 2,3,5,8-tetramethyl-decane & 0.020 \\
\hline Unknown & 0.047 \\
\hline 1,4-dione-2,5-cyclohexadiene & 0.039 \\
\hline 2-methyl-nonane & 0.010 \\
\hline 2,3-dimethyldecane & 0.049 \\
\hline 2-chloro-benzenamine & 0.015 \\
\hline Unknown & 0.008 \\
\hline butyl-cyclopropane & 0.006 \\
\hline 3-buten-2-one & 0.035 \\
\hline Heptane & 0.010 \\
\hline 2,3,4-trimethyl-pentane & 0.049 \\
\hline Unknown & 0.050 \\
\hline \multicolumn{2}{|l|}{ Setting 2} \\
\hline 1-methylthiol-1-propeen & 0.029 \\
\hline 5-tridecene & 0.008 \\
\hline 2-tetradecen-1-ol & 0.042 \\
\hline Unknown & 0.047 \\
\hline 3,5-dimethyl-1-hexene & 0.033 \\
\hline Unknown & 0.022 \\
\hline Unknown & 0.042 \\
\hline 2-pentene & 0.043 \\
\hline 3,6-dimethyl-octane & 0.022 \\
\hline Nonadecane & 0.013 \\
\hline Diphenylmethane & 0.028 \\
\hline Dodecanal & 0.040 \\
\hline Unknown & 0.020 \\
\hline Unknown & 0.038 \\
\hline 3,3-dimethyl-1-hexene & 0.029 \\
\hline 1-propene-1-thiol & 0.024 \\
\hline Unknown & 0.025 \\
\hline 1,5-pentanediol & 0.048 \\
\hline
\end{tabular}

Significantly different compounds regarding both setting 1 and setting 2. P-value is corrected for with Bonferonni correction.

After selection of the significantly different compounds these VOCs were tracked in all other samples ( $\mathrm{t}=-2$ to $\mathrm{t}=\mathrm{e}_{4}$ ) and their relative intensity was determined in all samples in 
order to provide more insight in the course of intensities of these compounds prior to and during exacerbations. Analysis of the availability of the selected VOCs prior to and during the exacerbations ranging from phases $t=-2$ to $t=e_{4}$ demonstrated that some of the significantly different compounds do display a correlation towards the phase of the exacerbation and might hold potential as biomarkers for monitoring of an exacerbation. As an example, the course of 4 of the 7 significant VOCs were shown in Figure 3. One compound (1-amino-2-butanol) was found to be significantly different in both settings.
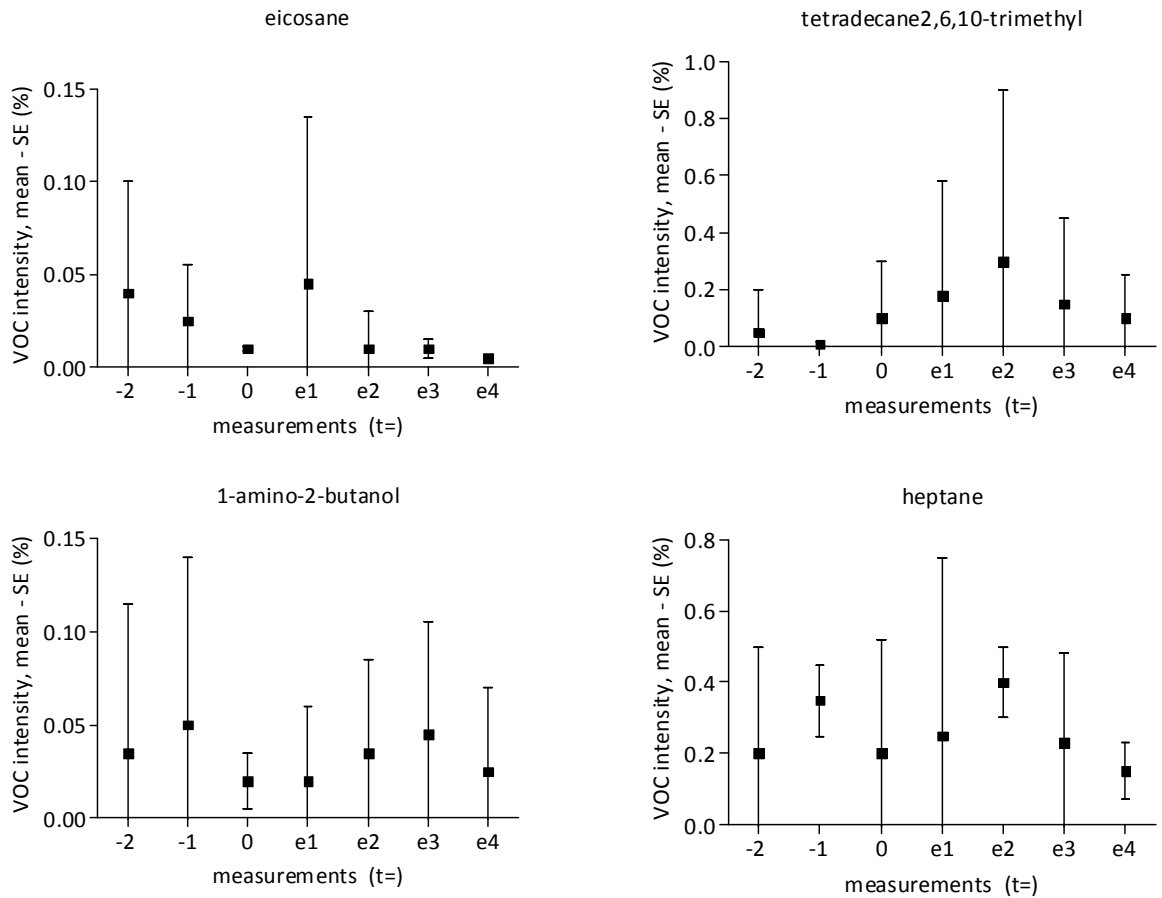

Figure 3: Mean \pm SE intensities (\%) of 4 significant different compounds demonstrating their course before and during a $\mathrm{CF}$ exacerbation.

\section{Support Vector Machine classifier}

The second approach to determine which combined compounds might be useful for the prediction of an exacerbation and classification of the exacerbation stages was performed by means of building an SVM model. Compounds detected in less than $8 \%$ of samples were deleted from the dataset according to Penn et al. [22] in order to avoid inclusion of compounds demonstrating availability in a very low number of samples resulting in a very high discriminatory power of these compounds with regards to samples they were detected in. This will result in overfitting and has to be avoided.

The 912 compounds that were available in at least $8 \%$ of samples were introduced to the SVM analyses. The resulting datasets were selected as described in the previous paragraph (Selection of informative compounds). The identified compounds and their performance when introduced into an SVM model are shown in Tables 3 and 4. It should be 
emphasized that the best performing compounds implemented in an SVM model do not necessarily exhibit high individual sensitivity or specificity since the performance of the SVM model is based on the cumulative information of all implemented compounds. As demonstrated in Table 3 the most optimal performing SVM for setting 1 (baseline versus exacerbation within patients) is based on a subset of 6 VOCs classifying $94 \%$ of samples correctly (sensitivity $94 \%$, specificity $95 \%$ ). Adding more VOCs does, to some degree, increase performance, but, as little VOCs as necessary are to be incorporated in order to minimize or avoid the effect of over-fitting. The most optimal SVM model regarding setting 2 (baseline resulting in exacerbation versus baseline not resulting in exacerbation) is based on 8 VOCs. Exacerbations were correctly classified in $92 \%$ of samples (sensitivity $100 \%$, specificity $84 \%$ ) as shown in Table 3 . In summary, different VOCs predict the onset of an exacerbation or monitor the course of the exacerbation.

Table 3: SVM performance as based on a number of VOCs.

\begin{tabular}{|c|c|c|c|}
\hline Number of VOCs & Specificity & Sensitivity & $\%$ Correct \\
\hline \multicolumn{4}{|l|}{ Setting 1} \\
\hline 9 & 0.75 & 0.89 & 83 \\
\hline 8 & 0.94 & 0.89 & 91 \\
\hline 7 & 0.94 & 0.95 & 94 \\
\hline $6^{*}$ & 0.94 & 0.95 & 94 \\
\hline 5 & 0.81 & 0.89 & 86 \\
\hline 4 & 0.69 & 0.95 & 83 \\
\hline 3 & 0.63 & 1.00 & 83 \\
\hline 2 & 0.56 & 0.89 & 74 \\
\hline 1 & 0.44 & 0.79 & 63 \\
\hline \multicolumn{4}{|l|}{ Setting 2} \\
\hline 11 & 1.00 & 1.00 & 100 \\
\hline 10 & 0.84 & 0.95 & 89 \\
\hline 9 & 0.89 & 0.95 & 92 \\
\hline $8^{*}$ & 0.84 & 1.00 & 92 \\
\hline 7 & 0.79 & 1.00 & 89 \\
\hline 6 & 0.79 & 0.84 & 82 \\
\hline 5 & 0.74 & 0.89 & 82 \\
\hline 4 & 0.63 & 0.89 & 76 \\
\hline 3 & 0.47 & 0.84 & 66 \\
\hline 2 & 0.32 & 0.95 & 63 \\
\hline 1 & 0.26 & 0.84 & 55 \\
\hline
\end{tabular}


Table 4: Identified VOCs as implemented into the most optimal SVM classifiers.

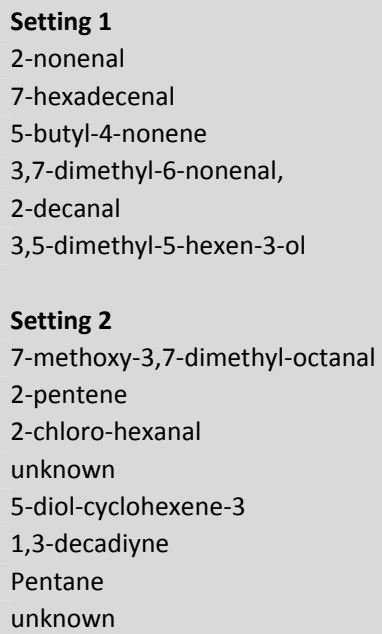

\section{Discussion}

This paper describes our effort to develop and test a methodology based on the analysis of exhaled breath to obtain information regarding the exacerbations patients suffering from CF experience. We could identify 8 different VOCs which (as a combination) were able to predict $92 \%$ of exacerbations (or stable disease) correctly with a corresponding sensitivity of $92 \%$ and a specificity of $84 \%$. Moreover, we found 8 VOCs which could be useful as biomarkers of the follow-up of an exacerbation. Based on the presented data, early recognition of an up-coming exacerbation and consequently early treatment with antibiotics, leading to prevention or suppression of exacerbations in future studies seems feasible [23].

The principle is based on identification of VOCs in exhaled breath, a totally non-invasive, easy to perform, cost effective and accurate methodology. We identified 32 VOCs that demonstrated a significant difference in availability in exhaled breath in the two mentioned settings. Setting 1 : $t=0$ compared to $t=e_{1}$ (for subjects experiencing an exacerbation) using a paired t-test in which 15 compounds proved to be significantly different. Setting 2: $t=0$ from subjects that would retrospectively experience an exacerbation within 2-4 weeks compared to baseline samples from a subject not suffering an exacerbation in which 18 compounds proved to be significantly different. Further analysis of these 33 significantly different compounds revealed that 7 compounds significantly changed in the course of the exacerbation. This demonstration might provide the means for detailing an exacerbation or monitoring the phase of the exacerbation. It also demonstrates that prediction of an exacerbation in all subjects with acceptable sensitivity and specificity could not be accomplished based on a single compound. External validation of these results in a larger study population has to be performed. 
Our study also demonstrates that SVM classifiers are able to predict an exacerbation with acceptable sensitivity and specificity. To classify subjects suffering an exacerbation from subjects not suffering an exacerbation a model based on VOCs in exhaled breath resulted in $94 \%$ correct classification of the samples as validated with 10 times cross validation. Using sophisticated biostatistical tools we extracted 6 VOCs out of nearly 912 exhaled compounds that combined into an accurate SVM model. This model proved to be highly sensitive and specific regarding the prediction of exacerbations intra-individually. The second SVM model was based on baseline measurement comparing patients suffering an exacerbation 2-4 weeks after the baseline measurement versus patients not suffering an exacerbation. This resulted in an SVM model comprised of 8 VOCs able to classify correctly $92 \%$ of exacerbations.

The selected compounds appeared to be mainly long chain hydrocarbons, however, the origin of some of the identified VOCs remains elusive. Bacterial infections might give rise to changes in the profile of exhaled VOCs as metabolic products are degraded or formed as described by Syhre et al. who monitored bacterial headspace changes [24]. Whether it is possible to detect such small changes in profiles of VOCs produced by these bacteria in human breath - that is if these bacterial VOCs are not altered by human metabolic processes - is unknown. Another origin for the discriminating VOCs might be due to changes in the degree of oxidative stress. Generation of reactive oxygen species (ROS) by activated neutrophils cause degradation of polyunsaturated fatty acids (PUFAs) in a process called lipid peroxidation, giving rise to VOCs like ethane and pentane. These hydrocarbons demonstrate a low solubility in blood and if the blood stream passes the lung tissue these hydrocarbons are excreted into exhaled breath. In earlier research we have been able to identify compounds in exhaled breath from smokers possibly related to oxidative stress [5]. These compounds were already mentioned in literature as smoking behavior related and were not exogenous of origin $[5,25,26]$. We also build an SVM model based on a set of 6 compounds able to discriminate between COPD patients and healthy controls [12]. Especially the compound 2-nonenal and its hydroxilized form 4-hydroxy-2-nonenal are described in the literature as reaction products of lipid peroxidation of omega-7 fatty acids and has already in 2003 been linked to inflammatory processes in COPD patients [27]. Also the chlorinated compound 2-chloro-hexanal has been described to be generated by a chlorination process in case of a neutrophilic response [28]. Although different scenarios regarding the origin of VOCs from breath samples are possible, it still remains speculative to draw any conclusions. Further studies are necessary in clinical settings but also in inflammation models to explain the biochemical origin, the physiological meaning and exhalation kinetics of our selected VOCs. Analyses of all individual VOCs might provide future insight into the changes in pathophysiology of exacerbations although we are aware that numerous VOCs in exhaled breath are still not biologically linked to metabolic or disease pathways and studying the pathophysiology of these exacerbations in relation to extracted VOCs is currently out of scope. Nevertheless, without this mechanistic knowledge the compounds may already be of value as a monitoring tool for clinical studies.

In the research field of breath analyses, there is an ongoing discussion whether or not background measurements should be taken into account in order to correct for ambient air influences. In the presented work, no background extraction was applied to keep the methodology as easy and straightforward as possible. Moreover, Miekisch et al. mentioned that it will not be possible to correct for the complex interdependencies between 
excretion and uptake of VOCs by easily subtracting the peak areas obtained from inhaled air and breath [29]. In addition, the VOC selection procedure will only select those compounds that contain information about the disease status and since the subjects have been randomly sampled, all VOCs that are indeed influenced by some confounding factors, like background or time-of-day, will not display information regarding the disease or severity. This random sampling limits the possibility of an exogenous compound showing up only in a certain measurement $\left(\mathrm{t}=-2\right.$ to $\left.\mathrm{t}=\mathrm{e}_{8}\right)$ in all subjects in order to constitute a highly significant effect.

Another point of discussion is the influence of dead space. No correction for dead space was applied since omitting the start volume of breath might add noise to the samples and reduce sensitivity. In our research we did not correct for dead space by means of sampling only the alveolar air. The applied methodology samples a mixture of alveolar air and dead space air, which is about $90 \mathrm{ml}$ in children of $40 \mathrm{~kg}$. Since most of the subjects were able to fully inflate the $5 \mathrm{~L}$ Tedlar bag in 2 to 3 expirations the contribution of dead space to the total volume is not so significant $90 \%$ of the obtained exhaled volume originates from alveolar air and in our setup the dilution with dead space air does not lead to sensitivity issues. In addition we wanted to provide a methodology as easy and straightforward as possible. Results regarding the reproducibility of the methodology are provided in previous research [5].

One of the innovative steps of our approach compared to other studies in this field is that we used the raw mass spectra data to find the matching compounds in all subjects instead of searching for matching compounds based on their chemical identification. This results in a far more precise and accurate dataset since chemical identification remains elusive and numerous misidentifications result in corrupt datasets. Our solution was to introduce the match factor in order to directly match compounds from different samples based on their mass spectra. This increases accuracy since compounds are compared to one another as measured with the same experimental setup thus eliminating the differences between library mass spectra and measured mass spectra arising from use of different setups. This results in a superior database as compared to a database based on identification of compounds. The final identification of the compounds as shown in Table 4 remains difficult and in a few cases questionable, due to GC-resolution limitations.

Our main goal was to develop a robust diagnostic methodology as a whole not influenced by too many confounding factors. In principal the excretion of VOCs can be influenced by many factors of intrinsic nature (gender, age, weight, genetic background) and of exogenous origin (ambient air, diet or medication). It is practically impossible to control for all these potentially confounding factors. Therefore it was our goal to select those compounds that provide information independently from other endogenous or exogenous confounding factors. We recently concluded that our sampling procedure, chemical analysis, data handling and accurate data mining provide a highly reproducible methodology [5]. Now that interesting VOCs have been selected new advances in diagnostic tools like the electronic nose [30] could be specifically tuned to detect the mentioned VOCs in order to provide a fast, non-invasive cost effective and efficient diagnostic device to detect exacerbations in an early phase. When combined with early (antibiotic) treatment, this method may result in the prevention of exacerbations, an improvement in quality of life and lung function and a reduction in healthcare cost which clearly is a topic for future prospec- 
tive studies. In further research the pathophysiological meaning of the detected compounds could be tracked.

Also, further studies are necessary in clinical settings to explain the biochemical origin, the physiological meaning and exhalation kinetics of selected VOCs. However, without this mechanistic knowledge these VOCs may already be used as a diagnostic tool for exacerbations in future clinical CF studies.

\section{Acknowledgements}

The authors gratefully acknowledge the province of Limburg (The Netherlands), the Jaap Swieringa Foundation, the NCFS and AstraZeneca for their financial support.

\section{References}

1. Emerson, J, Rosenfeld, M, McNamara, S, Ramsey, B, Gibson, RL. Pseudomonas aeruginosa and other predictors of mortality and morbidity in young children with cystic fibrosis. Pediatr Pulmonol 2002;34:91-100.

2. Robroeks, CM, Rosias, PP, van Vliet, D, Jobsis, Q, Yntema, JB, Brackel, HJ, Damoiseaux, JG, den Hartog, GM, Wodzig, WK, Dompeling, E. Biomarkers in exhaled breath condensate indicate presence and severity of cystic fibrosis in children. Pediatr Allergy Immunol 2008;19:652-659.

3. Robroeks, CM, van de Kant, KD, Jobsis, Q, Hendriks, HJ, van Gent, R, Wouters, EF, Damoiseaux, JG, Bast, A, Wodzig, WK, Dompeling, E. Exhaled nitric oxide and biomarkers in exhaled breath condensate indicate the presence, severity and control of childhood asthma. Clin Exp Allergy 2007;37:1303-1311.

4. Robroeks, CMHHT, van Berkel, JBN, Dallinga, JW, Jöbsis, Q, Zimmermann, LI, Hendriks, JJE, Wouters, EFM, van der Grinten, CPM, van de Kant, KDG, van Schooten, FJ, Dompeling, E. Metabolomics of volatile organic compounds in cystic fibrosis patients and controls Pediatric Research 2010;in press.

5. Van Berkel, JJ, Dallinga, JW, Moller, GM, Godschalk, RW, Moonen, E, Wouters, EF, Van Schooten, FJ. Development of accurate classification method based on the analysis of volatile organic compounds from human exhaled air. J Chromatogr B Analyt Technol Biomed Life Sci 2008;861:101-107.

6. Matsunaga, K, Ichikawa, T, Yanagisawa, S, Akamatsu, K, Koarai, A, Hirano, T, Sugiura, H, Minakata, Y, Ichinose, M. Clinical application of exhaled breath condensate analysis in asthma: prediction of FEV(1) improvement by steroid therapy. Respiration 2009;78:393-398.

7. Kharitonov, SA, Barnes, PJ. Clinical aspects of exhaled nitric oxide. Eur Respir J 2000;16:781-792.

8. Pauling, L, Robinson, AB, Teranishi, R, Cary, P. Quantitative analysis of urine vapor and breath by gas-liquid partition chromatography. Proc Natl Acad Sci U S A 1971;68:2374-2376.

9. Phillips, M, Altorki, N, Austin, JH, Cameron, RB, Cataneo, RN, Greenberg, J, Kloss, R, Maxfield, RA, Munawar, MI, Pass, HI, Rashid, A, Rom, WN, Schmitt, P. Prediction of lung cancer using volatile biomarkers in breath. Cancer Biomark 2007;3:95-109.

10. Phillips, M, Cataneo, RN, Condos, R, Ring Erickson, GA, Greenberg, J, La Bombardi, V, Munawar, MI, Tietje, O. Volatile biomarkers of pulmonary tuberculosis in the breath. Tuberculosis (Edinb) 2007;87:44-52.

11. Phillips, M, Cataneo, RN, Cummin, AR, Gagliardi, AJ, Gleeson, K, Greenberg, J, Maxfield, RA, Rom, WN. Detection of lung cancer with volatile markers in the breath. Chest 2003;123:2115-2123.

12. Van Berkel, JJ, Dallinga, JW, Moller, GM, Godschalk, RW, Moonen, EJ, Wouters, EF, Van Schooten, FJ. A profile of volatile organic compounds in breath discriminates COPD patients from controls. Respir Med 2009.

13. Dallinga, JW, Robroeks, CM, van Berkel, JJ, Moonen, EJ, Godschalk, RW, Jobsis, Q, Dompeling, E, Wouters, EF, van Schooten, FJ. Volatile organic compounds in exhaled breath as a diagnostic tool for asthma in children. Clin Exp Allergy 2009.

14. Rosenstein, BJ, Cutting, GR. The diagnosis of cystic fibrosis: a consensus statement. Cystic Fibrosis Foundation Consensus Panel. J Pediatr 1998;132:589-595.

15. Shwachman, H, Kulczycki, LL. Long-term study of one hundred five patients with cystic fibrosis; studies made over a five- to fourteen-year period. AMA J Dis Child 1958;96:6-15.

16. Rosenfeld, M, Emerson, J, Williams-Warren, J, Pepe, M, Smith, A, Montgomery, AB, Ramsey, B. Defining a pulmonary exacerbation in cystic fibrosis. J Pediatr 2001;139:359-365.

17. Kanga, J, Kuhn, R, Craigmyle, L, Haverstock, D, Church, D. Cystic fibrosis clinical score: a new scoring system to evaluate acute pulmonary exacerbation. Clin Ther 1999;21:1343-1356. 
18. Stein, SE, Scott, DR. Optimization and Testing of Mass-Spectral Library Search Algorithms for Compound Identification. Journal of the American Society for Mass Spectrometry 1994;5:859-866.

19. Guyon, I, Weston, J, Barnhill, S, Vapnik, V. Gene selection for cancer classification using support vector machines. Machine Learning 2002;46:389-422.

20. Frank, E, Hall, M, Trigg, L, Holmes, G, Witten, IH. Data mining in bioinformatics using Weka. Bioinformatics 2004;20:2479-2481.

21. Platt, JC. Fast training of support vector machines using sequential minimal optimization: MIT press 1998.

22. Penn, DJ, Oberzaucher, E, Grammer, K, Fischer, G, Soini, HA, Wiesler, D, Novotny, MV, Dixon, SJ, Xu, Y, Brereton, RG. Individual and gender fingerprints in human body odour. J R Soc Interface 2007;4:331-340.

23. Johnson, C, Butler, SM, Konstan, MW, Morgan, W, Wohl, ME. Factors influencing outcomes in cystic fibrosis: a center-based analysis. Chest 2003;123:20-27.

24. Syhre, M, Chambers, ST. The scent of Mycobacterium tuberculosis. Tuberculosis (Edinb) 2008;88:317-323.

25. Ashley, DL, Bonin, MA, Hamar, B, McGeehin, M. Using the blood concentration of 2,5-dimethylfuran as a marker for smoking. Int Arch Occup Environ Health 1996;68:183-187.

26. Sanchez, JM, Sacks, RD. Development of a multibed sorption trap, comprehensive two-dimensional gas chromatography, and time-of-flight mass spectrometry system for the analysis of volatile organic compounds in human breath. Anal Chem 2006;78:3046-3054.

27. Rahman, I, Kelly, F. Biomarkers in breath condensate: a promising new non-invasive technique in free radical research. Free Radic Res 2003;37:1253-1266.

28. Thukkani, AK, Hsu, FF, Crowley, JR, Wysolmerski, RB, Albert, CJ, Ford, DA. Reactive chlorinating species produced during neutrophil activation target tissue plasmalogens: production of the chemoattractant, 2chlorohexadecanal. J Biol Chem 2002;277:3842-3849.

29. Miekisch, W, Schubert, JK, Noeldge-Schomburg, GF. Diagnostic potential of breath analysis--focus on volatile organic compounds. Clin Chim Acta 2004;347:25-39.

30. Di Natale, C, Macagnano, A, Martinelli, E, Paolesse, R, D'Arcangelo, G, Roscioni, C, Finazzi-Agro, A, D'Amico, A. Lung cancer identification by the analysis of breath by means of an array of non-selective gas sensors. Biosens Bioelectron 2003;18:1209-1218. 


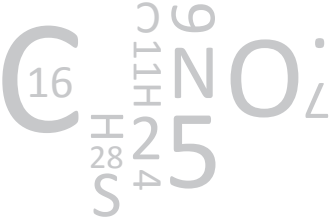

CHAPTER 13

General discussion 


\section{Contents}

13.1 Methodology

13.1.1 Fractional exhaled nitric oxide

13.1.2 Exhaled breath condensate

13.1.3 Volatile organic compounds

13.2 Diagnosis

251

13.2.1 Asthma

13.2.2 Cystic fibrosis

13.3 Monitoring

13.3.1 Asthma

13.3.2 Cystic fibrosis

$13.4 \quad$ Future research

254

13.4.1 Methodology

13.4.2 Diagnosis

13.4.3 Monitoring 
The paradox in daily practice is that although chronic airway inflammation is the hallmark of asthma and cystic fibrosis (CF), methods to evaluate this inflammation are not routinely involved in the diagnosis and management of these diseases. However, respiratory symptoms, physical examination and lung function parameters mainly reflect the consequence but not the origin of this airway inflammation. Only by means of invasive techniques like induced sputum, broncho-alveolar lavage, and lung biopsies, ongoing inflammation can be assessed [1]. However, this is not applicable in children on a regular basis because it has to be performed under general anaesthesia in children and is far too invasive for routine use. In contrast, breath analysis is non-invasive, it is relatively easy to perform and does not interact with ongoing processes in the airways. It is safe in children of all ages and repeated testing is possible [2-5]. Different forms of breath analysis are possible: 1) measurement of fractional exhaled nitric oxide (FeNO) in exhaled breath; 2) analysis of biomarkers in exhaled breath condensate (EBC); 3) assessment of volatile organic compounds (VOCs) in exhaled breath. Therefore, breath analysis may be of help in the diagnosis and monitoring of chronic inflammatory airway diseases in children. In the past decade, the number of scientific reports on non-invasive inflammometry increased exponentially, Figure 1. For both FeNO and EBC, joint task forces of the European Respiratory Society (ERS) and the American Thoracic Society (ATS) were formed. They published recommendations on measurement of FeNO $[6,7]$ and on collection and analysis of biomarkers in EBC [8]. Their aim was to identify methodological issues, to provide recommendations for measurements and to underline areas for further research. The use of VOCs in exhaled breath for the diagnosis and monitoring of chronic inflammatory diseases is an even more innovative tool studied recently [9].

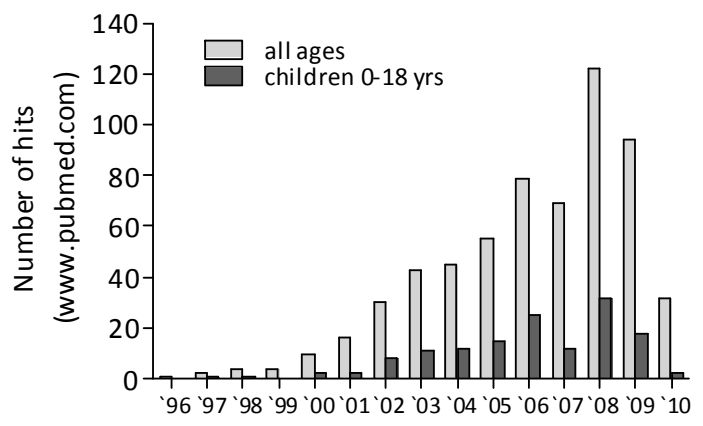

Figure 1: The number of publications with keyword 'exhaled breath condensate. (www.pubmed.com).

*The number of publications in 2009 until november 15th.

This thesis is divided in three sections: 1) methodology, 2) diagnosis and 3) monitoring. Moreover, several techniques of non-invasive inflammometry are considered in this thesis simultaneously (FeNO, biomarkers in EBC, and volatile organic compounds (VOCs) in exhaled breath) in both childhood asthma and CF. 


\subsection{Methodology}

\subsubsection{Fractional exhaled nitric oxide}

In the past, various studies demonstrated the potential benefit of FeNO in addition to conventional parameters [10-12]. It is non-invasive, easy to perform, rapid, reproducible, repeatable, and it may be helpful for asthma management in children [13]. According to ERS/ATS guidelines, FeNO levels are assessed at an exhalation flow rate of $50 \mathrm{~mL} / \mathrm{sec}$ [7]. These measurements are mostly feasible in children of 5 years and over. However, there are still methodological questions of FeNO measurements in children younger than 5 years of age that need to be resolved. In young children, two offline methods are available [14, 15]: 1) Tidal breathing with or without expiratory resistance. Or 2) Single exhalation against a resistance into a reservoir. There is no generally accepted standard method for this age group [16-22].

\section{Feasibility and reproducibility of NO measurements at different flow rates}

Measuring alveolar NO levels and bronchial NO flux gives insight in the extent of the inflammation processes in different parts of the lung [23-27]. Alveolar and bronchial NO are calculated from NO flux values at various flow rates $[23,28,29]$. Linearity between NO flux levels is obligatory in order to assess alveolar and bronchial levels reliably. In children, the feasibility and repeatability of NO at various flow rates, and of (the derived) bronchial and alveolar NO levels in larger groups is uncertain. In chapter 2 we demonstrated a good feasibility of NO measurements at flows ranging from 30 till $200 \mathrm{~mL} / \mathrm{sec}$ in asthmatic children from the age of 6 years. However, a significant learning effect was present. Failure to perform NO tests was not related to age or degree of airway obstruction. Inclusion of the flow restrictor of $30 \mathrm{~mL} / \mathrm{sec}$ influenced mainly the slope of the linear regression model, and therefore had an effect on the alveolar NO levels. Reasons of failure of assessment were the inability of children to perform the manoeuvres correctly, or, that the measured parameters did not fit in a linear model. The long-term reproducibility of alveolar and bronchial NO values during 6 months were moderate.

The ability to measure alveolar and bronchial NO may be helpful for the differentiation between different chronic lung diseases in children. Especially in asthma, where distal airway inflammation is more prominent, alveolar NO may help to discriminate between asthma-like diseases and "true asthma". This hypothesis needs to be studied in future research.

\subsubsection{Exhaled breath condensate}

The collection of EBC is a non-invasive technique to obtain direct samples from the lower respiratory tract which, in contrast to induced sputum, does not disturb the ongoing inflammation $[8,30]$. There is a current consensus defined by the task force of the ATS/ERS: the inner surface of the condenser tube need to be inert, a saliva trap is essential, it is important to prevent contamination with ambient air in the condenser, and two-way valves are necessary to prevent inhalation through the condenser [8]. However, the influence of condenser coatings on biomarker reproducibility, condenser temperature, storage temperature and duration, influences of diet, exercise, and breathing pattern are not fully 
understood yet. In addition, it is not known whether biomarkers in EBC should be standardized for collection time, collected volume or the dilution factor. Because there is a lack of standardization, it is difficult to compare and/or reproduce data of different research groups which hampers the further development of the technique. In children, analysis of EBC reveals the presence of various types of inflammatory biomarkers. Condensation systems are suboptimal, with relatively short-measured, open ended designs and loss of exhaled breath, as reflected by variable EBC volumes and biomarker reproducibility [8]. As a result, EBC collection was hardly possible in children below 4 years of age.

\section{Development of condenser systems}

The ERS / ATS taskforce on EBC published general methodological recommendations on the collection and analysis of EBC. However, there are still some unresolved methodological pitfalls, as illustrated by the use of various non-standardised collection systems [8]. There are few studies investigating and comparing different condenser systems, all performed in adult subjects. Currently, different research groups use different condenser equipments, all based on the same principle of cooling exhaled breath [8]. Noncommercial designs include immersion of a Teflon-lined tubing in an ice-filled bucket, and a double-wall borosilicate glass condenser system (Figure 2a) [31-33]. Although cooling of exhaled breath, conducted through a Teflon-lined tubing, can be readily achieved using wet ice as well as dry ice or liquid nitrogen, complete obstruction of the collection tube has been reported [31]. The commercially available condensers are the EcoScreen (Erich Jaeger $\mathrm{GmbH}$, Hoechberg, Germany) (Figure $2 \mathrm{~b}$ ), the Rtube ${ }^{\mathrm{TM}}$ breath condensate collector (Respiratory Research Inc., Charlottesville, VA, USA) (Figure 2c), and the TURBO-DECCS (Transportable Unit for Research on Biomarkers Obtained from Disposable Exhaled Condensate Collection Systems: ItalChill, Parma, Italy) (Figure 2d). The EcoScreen system conducts exhaled breath through a lamellar condenser with a sample collection vial inserted into a cooling cuff (electric refrigerator) [34]. The condenser maintains a temperature of -10 degrees Celsius during a 10-15 min collection period. An advantage of the system is its pretty high recovery (in terms of EBC volume per minute collection). A drawback may be the Teflon coating (see below) and the freezing of EBC during collection which requires defrosting before the EBC can be put into separate collection vials [30]. Defrosting may interfere with the stability of biomarkers, e.g. proteins/cytokines are disrupted by freezing/defrosting. A second version of the apparatus separates EBC from proximal and more distal airways by means of a valve system (www.viasyshealthcare.com). It is unknown yet whether this separation will increase the possibility to detect differences between healthy and diseased subjects.

The Rtube ${ }^{\mathrm{TM}}$ breath condensate collector is a portable device and consist of a disposable polypropylene tube acting as condenser and collector, which is placed into a pre-cooled aluminium sleeve $[35,36]$. The device uses an exhalation valve that also serves as a syringe-style plunger to pool fluid from the condenser walls, which allows reducing collection time. The latter system is not associated with the problem of complete obstruction. During the collection procedure, the pre-cooled Rtube ${ }^{\mathrm{TM}}$ is highly sensitive to the higher ambient temperatures [37].

The TURBO-DECCS is a relatively new, commercially available device [38, 39]. 'TURBO' is a refrigerating system relying on a thermo electrical module giving rise to the Peltier effect. 
The cold side of the Peltier module is connected to an aluminium support shaped to house the test tube. The collecting temperature is adjustable from $-10{ }^{\circ} \mathrm{C}$ to room temperature or higher, with a tolerance of $\pm 1{ }^{\circ} \mathrm{C}$. 'TURBO' is supplied with 'DECCS', a disposable polyethylene device for collecting EBC that consists of a mouthpiece equipped with a one-way valve and saliva trap, connected to a collecting vial $(50 \mathrm{~mL})$ by means of a tube.

One of the limitations of EBC methodology is the low biomarker concentration [8]. Some years ago, we hypothesized that adhesive properties of different condenser coatings could interfere with measurements of biomarkers in EBC [40]. In both an in vitro study and an in vivo experiment, the influence of various condenser coatings on the detection and concentration of eicosanoids and proteins in EBC was assessed. We found that a condenser with silicone or glass coating was more efficient for measurement of 8-isoprostane and albumin in EBC, than EcoScreen , aluminium, polypropylene or Teflon coating [40].

Only $25-30 \%$ of the total expired volume (tidal volume * weight * breath frequency * collection duration) is condensed by means of open-ended condensers. With this low efficiency, collection time increases considerably, resulting in poor feasibility in young children. Because of these limitations of existing condensing devices, an optimized glass condenser system was developed as described in chapter $4[41,42]$. This condenser was patented in Europe, United States of America and in Canada (patent EP07102586, figure 2e). The instrument consists of an increased condenser surface (tube length $90 \mathrm{~cm}$, surface $1046 \mathrm{~cm}^{2}$ ), a downwards moveable plunger (to optimize condensate recovery from the inner condenser wall), and tangential and axial breath flow channels in the plunger (to directly turbinate the tidal breath flows towards the cooled inner condenser wall), and a removable, cooled inert sample vial (to collect EBC). The use of a plunger increases the recovery of $E B C$ from the condenser wall. In chapter 3, the detection rates and reproducibility of inflammatory markers in EBC collected with the optimized condenser were compared with the EcoScreen , and an older glass and silicone condenser in 30 healthy volunteers at four time points [41]. The optimized glass condenser yielded almost two times more $\mathrm{EBC}$ volume than the EcoScreen ${ }^{\circ}$. Moreover, the new condenser was associated with significantly more detections of $\mathrm{H}_{2} \mathrm{O}_{2}, 8$-isoprostane, and cytokines. The reproducibility of biomarkers was equal for all condenser types.

The increased EBC volume collection enables use of EBC in younger children. To increase the efficiency of the condenser even further, a breath recirculation unit was added to the optimized condenser as used and described in chapter 3 . The non-condensed exhaled breath was collected in an inert single use Tedlar ${ }^{\mathrm{TM}}$ (SKC Ltd., Dorset, UK) gas sample bag, heated at $37{ }^{\circ} \mathrm{C}$. When the child is finished, a small ventilator recirculate the noncondensed residual exhaled breath back into the cooled condenser system (to allow secondary condensation of exhaled breath) (Chapter 4, Figure 1). In chapter 4 we studied the feasibility of this condenser with recirculation system in 70 pre-school children. The success rate of obtaining at least $60 \mu \mathrm{L} E B C$ within 10 minutes collection time, was $64 \%$ (without recirculation) and increased to $83 \%$ (with breath recirculation). This volume is enough to assess cytokines in EBC. The detection of cytokine profiles in $50 \mu \mathrm{LEBC}$ was successful in $95-100 \%$ of the collected samples. Similar cytokine concentrations were found in EBC collected before as well as after breath recirculation.

These results show that recirculation of non-condensed breath increases efficiency of EBC collection. As a consequence of these improvements, EBC collection has become feasible 


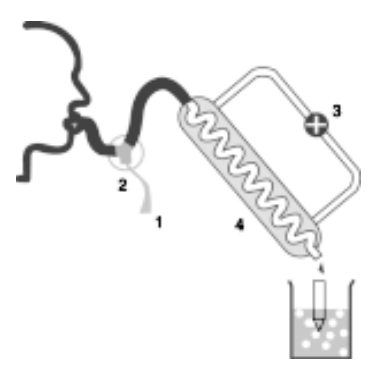

Figure 2a: Older non-commercial designs.

Figure 2b: EcoScreen (first type).
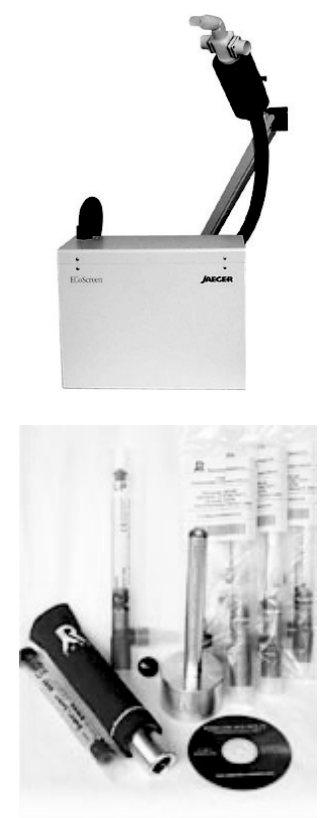

Figure 2c: Rtube ${ }^{\mathrm{TM}}$.

A
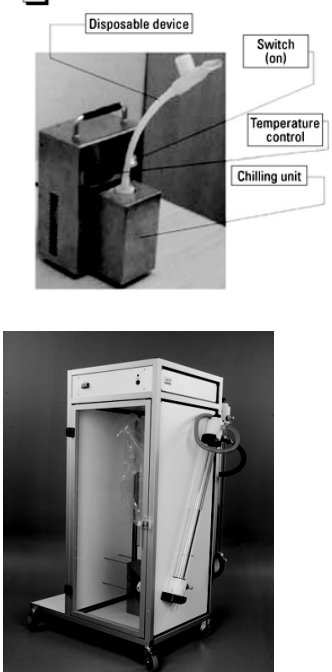

B

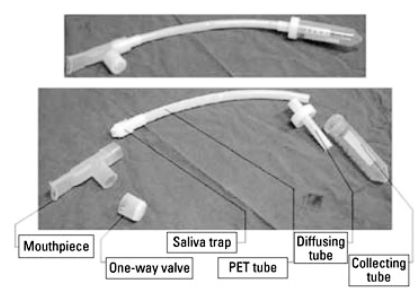

Figure 2d: TURBO-DECCS condenser (A. TURBO-DECCS condenser; B. DECCS system disposable device, $\mathrm{PET}$, polyethylene).

Figure 2e: Optimized glass condenser with breath recirculation. 
in children below 4 years of age. This enlarges the possibilities for future research. It is worthwhile to assess the value of EBC for an early and reliable asthma diagnosis, to study different wheezing phenotypes in young children, and to monitor the development of CF lung disease in relation to lung function and imaging.

\section{Assessments of markers in EBC}

An advantage of EBC analysis, in contrast to FeNO, is that multiple inflammatory markers can be measured simultaneously. On the other hand, simultaneous measurement may be complicated as the optimal collection device and collection method may vary between markers.

\section{Acidity of EBC}

Among all parameters in $\mathrm{EBC}$, acidity $(\mathrm{pH})$ is probably the best studied one $[8,30]$. The degree of airway acidification may serve as a simple and inexpensive method to monitor airway inflammation. The acidity can be immediately tested after collection with a $\mathrm{pH}$ probe. There is a clear tendency towards higher EBC pH values in the hour following collection $[43,44]$. This probably is related to the release of $\mathrm{CO}_{2}$ from $\mathrm{EBC}$. A method to overcome this is to de-aerate samples with a $\mathrm{CO}_{2}$-free gas (argon, nitrogen, or oxygen). The $\mathrm{pH}$ of deaerated samples is not affected by hyperventilation, duration of collection, storage, exclusion of oral ammonia, or airway obstruction following methacholine, with acceptable day-to-day intra-subject coefficients of variation of $4.5 \%$ [45]. So far, it is questionable whether deaeration increases the diagnostic value: some studies found a better diagnostic values whereas others did not $[43,44]$. In our studies, we did not deaerate samples.

\section{Markers of oxidative stress}

The best studied parameters is hydrogen peroxide $\left(\mathrm{H}_{2} \mathrm{O}_{2}\right)$ [46-49]. It is produced after converting superoxide anions to $\mathrm{H}_{2} \mathrm{O}_{2}$ by superoxide dismutase in neutrophils, eosinophils and epithelial cells, and has been demonstrated in EBC by several research groups [8]. As the product is unstable, EBC samples should be immediately frozen at $-80^{\circ} \mathrm{C}$. For analysis of hydrogen peroxide in $\mathrm{EBC}$, fluorimetric and colorimetric techniques, a fast chemiluminescent method, and online measurement with a biosensor with electrochemical detection have been used $[50,51]$. There is concern about the reproducibility of the colorimetric method [8]. More sensitive and reproducible assays are necessary. Stability of samples is at least 40 days [52]. In chapter 3, the intra-subject variability of $\mathrm{H}_{2} \mathrm{O}_{2}$ (determined fluorimetrically) in 30 healthy adult volunteers was, dependent of the type of condenser, $22 \%$ to $29 \%$ [41]. Other markers of oxidative stress in EBC include glutathione and malondialdehyde, which can be measured by mass spectrometry.

\section{Nitrative stress}

Nitrate/nitrite, S-nitrosothiols and 3-nitrotyrosine are nitrogen-reactive species. The reaction between nitric oxide and superoxide anions in the airways results in the formation of peroxynitrite, which can be trapped by thiol-containing biomolecules such as cysteine and glutathione to form S-nitrosothiols, or can be oxidized to nitrate and nitrite $[8,30]$. 3Nitrotyrosine is a stable end-product of peroxynitrite and tyrosine. The nitrite/nitrate balance is determined by colorimetric assay (based on the Griess reaction), by fluorimetric assay (based on the 2,3-diaminoaphtalene reaction), by ionic chromatograph analyzer or 
by chromatography/mass spectrometry [53-58]. Several methods are available for the detection of S-nitrosothiols and nitrotyrosine. Sensitivity of the fluorimetric assay of nitrite/nitrate is $0.1 \mu \mathrm{M}$. Data on intra-subject variability are not available. Nitric oxides are present on every laboratory surface and profound rinsing of the EBC collection system and of other materials is important to prevent contamination [8].

\section{Eicosanoids}

Eicosanoids are potent mediators of inflammation, which are derived from arachidonic acid, and include prostaglandins, thromoboxane, isoprostanes, leukotriens and lipoxins [8, 30]. The best studied biomarkers are 8-isoprostane and (cysteinyl) leucotrienes. 8Isoprostane is mostly measured by an EIA (detection limit of $4 \mathrm{pg} / \mathrm{mL}$ ) or a RIA. Other methods include a reverse-phase high-performance liquid chromatography or by gas chromatography/mass spectrometry [59]. Recently, the detection limit of the EIA was decreased to $1 \mathrm{pg} / \mathrm{mL}$ [58]. With this assay, the intra-subject variability of 8-isoprostane with an optimized glass condenser system in 30 healthy volunteers was $29 \%$ [41]. Leucotrienes can be measured by EIA or by reverse-phase high performance liquid chromatography [60]. $\mathrm{LTB}_{4}$ is formed by hydrolysis of $\mathrm{LTA}_{4}$, a pro-inflammatory mediator and activator of neutrophils. The day-to-day variability of $\mathrm{LTB}_{4}$ was $2 \%$ in a small group of healthy subjects [61]. The cysteinyl leukotrienes (leukotriene $C_{4}, D_{4}$, and $E_{4}$ ) are released from mast cells and eosinophils. No data on variability have been reported.

\section{Cytokines / chemokines / soluble adhesion molecules}

Cytokines are usually quantified by EIA/ELISA kits, flow cytometry, liquid bead-based multiplexing immunoassay technology, and protein array [32, 58, 62-66]. The detection of proteins and cytokines is probably better with a glass or silicone condenser coating [40, 41]. Cytokines are sensitive to frosting-defrosting cycles, which should be avoided [30]. Biomarker concentrations are low and in many studies close to the detection limit of the assay [32]. In the chapters 5, 7.1 and 7.2, we demonstrated Th-1 and Th-2 cytokines in EBC samples of children with asthma, CF and in controls by means of flow cytometry. However, cytokine concentrations were low and detection was only possible in a minority of children. In chapter 6, we determined (variability in) cytokines, chemokines and soluble adhesion molecules using liquid bead-based multiplexing immunoassay (XMAP ) technology in 60 children with asthma and healthy controls [65]. Intra-subject variability of these biomarkers ranged from $5.2 \%$ for CXL-8 (IL-8) to $35.0 \%$ for CCL5 (RANTES), and was acceptable $(C V<15 \%)$ for most cytokines. By means of the XMAP technique, cytokines, chemokines and adhesion molecules could be detected in $94-100 \%$ of the samples. When compared with conventional immunoassays, multiplexed immunoassay detects bioactive and inactive molecules, has a wider analytical range, is rapid (hours instead of days), have a high reproducibility (coefficient of variation 10-15\%), are not interfered by drugs, and have simple protocols. Therefore, in order to assess cytokines, chemokines and adhesion molecules in EBC, XMAP technology is a valuable technique

\section{Proteomics and metabolomics in EBC}

In proteomic and metabolomic, the "-omic" term was used to define methods capable of identifying groups of biomarkers (making a fingerprint) characteristic of a particular disease within the set of proteins and metabolites, respectively $[67,68]$. 
Proteomics may be defined as the large-scale characterization of proteins expressed by the genome. Proteins were identified in EBC of adults [69]. There is an advice to exclude proteins of ambient air from the analysis of the proteome [70]. The value of proteomics has been studied in adult patients with pulmonary emphysema, lung cancer, and in smokers [71, 72].

Only few rapports have been published on metabolomics in EBC. De Laurentiis et al. showed that metabolomics of EBC is feasible by means of nuclear magnetic resonance in adults [73]. Nuclear magnetic resonance-based metabolomic analyses of EBC could discriminate between asthmatic and healthy children, with a $95 \%$ correct classification rate [74].

Proteomics and metabolomics may be a valuable tool for: 1) diagnosis of various lung diseases; 2) characterisation of disease sub-phenotypes; 3 ) monitoring and management of various diseases. It may help to adjust therapy, identify responders and predict toxicity [75].

\subsubsection{Volatile organic compounds}

Besides measurement of FeNO and specific inflammatory markers in EBC, discriminative patterns of volatile organic compounds (VOCs) in exhaled breath can be assessed. These techniques are relatively new. Therefore, there are only few published data available [7681]. VOCs can be measured by mass-spectroscopy, gas chromatography time of flight mass spectroscopy (GC-TOF-MS) and with the electronic nose (eNose). Breath sampling proved to be feasible without discomfort or adverse events in children and adults [80]. Barker et al. published moderate to good intra-subject coefficients of variation of 12 different hydrocarbons in exhaled breath originating from healthy controls and CF patients, ranging from 9 to 34\% [80]. In contrast to mass spectroscopy, the eNose was used to distinguish VOC mixtures by pattern recognition, or "smell-prints". These techniques are fundamentally different, since the smell-prints are based in pattern recognition instead of single mass spectra. Dragonieri et al used this technique in adults with asthma and controls [82]. In chapter 9.1 and 9.2, we demonstrate feasibility of exhaled breath collection and analysis by means of GC-TOF-MS in children with asthma, CF and in healthy controls. In chapter 9.2 variation of VOCs across the day and between days was analyzed. These data indicate a low within-subject variation in VOC patterns after one hour, and after a day. In addition, the influence of breathing patterns was studied. Samples were assessed with an unforced breathing pattern and during hyperventilation. Influences of breathing patterns of VOC profiles were minimal. Van Berkel et al. used the same methodology to measure VOCs in a smoking/non-smoking population [83]. They assessed reproducibility and variability measurements (instrumental, inter- and intra-individual). They showed that inter-individual variability is larger than intra-individual variability. The instrumental variability was the smallest, which confirms the reliability of the methodology used.

Use of raw mass spectra to find the matching compounds in all subjects results in a precise and accurate dataset, since chemical identification remains elusive and numerous misidentifications are made. Sensitivity is high and a wide range of VOCs can be measured with good short-term and long-term reproducibility [83]. The advantage of this method is that there is no pre-selection of markers. Afterwards, VOCs of interest can be identified. With this method it is able to search for unique profiles that can be used to identify cer- 
tain patient groups [33]. Nevertheless, final identification of VOCs remains difficult and in a few cases questionable. VOCs in exhaled breath is promising; it is feasible, non-invasive, and it may hold potential for non-invasive diagnosis and monitoring of inflammatory processes. However, future research needs to resolve methodological issues.

\subsection{Diagnosis}

\subsubsection{Asthma}

Differentiation of wheezing phenotypes in preschool children is challenging. So far, it is not possible to discriminate between children with "true asthma" or children with "transient, viral wheeze". Non-invasive inflammometry may help to discriminate these phenotypes correctly. In older children, an asthma diagnosis is more easily made. Children with viral wheezing are often symptom-free at this age and lung function measurements can be performed. The level of asthma control falls short of the goals for long-term management in international guidelines $[84,85]$. Patients can get used to their symptoms which may lead to underdiagnosis and undertreatment of uncontrolled asthma [86]. The use of noninvasive inflammometry may be of help for a correct diagnosis of the asthma control and severity status. In chapter 6 we demonstrated that a range of cytokines, chemokines, and soluble adhesion molecules could be assessed in EBC samples of asthmatic children and healthy controls in almost all samples with good reliability. In addition, most of these inflammatory markers in samples originating from children with asthma were elevated. In chapter 7.1, analysis of FeNO, EBC 8-isoprostane, nitrate, nitrite, hydrogen peroxide, cytokines and conventional parameters (lung function, symptoms) were considered together. This study showed that the diagnosis of asthma, the severity level and the level of disease control, could all be predicted with good sensitivity and specificity be means of noninvasive inflammometry. Dependent on the outcome variable (diagnosis / severity / level of control), different combinations of markers were predictive. These data show that a combination of markers is more informative than a single marker, and that non-invasive inflammometry may be a valuable additional tool in daily clinical practice. In chapter 9.1, we showed that asthma could be distinguished from healthy children by a limited number of 8 VOCs with high a sensitivity of $89 \%$ and a specificity of $95 \%$. These data imply that in addition to conventional parameters, FeNO, biomarkers in EBC and VOCs in exhaled breath can be of help for various aspects of an asthma diagnosis.

\subsubsection{Cystic fibrosis}

Diagnoses of CF disease can be done in a reliable, non-invasive way by means of a sweat test. However, diagnosing the earliest stages of CF lung disease is more challenging. Airway inflammation in patients with CF is already present early in life $[87,88]$, and plays a major role in CF lung disease. Non-invasive inflammometry may give insight in airway inflammation and infection in CF lungs. The aim of the study of chapter 7.2 was to investigate the ability of FeNO and markers in EBC to indicate CF lung disease, exacerbations of CF lung disease and the severity of CF lung disease. This study demonstrated that a combination of FeNO and markers in EBC can indicate a diagnosis of CF lung disease, and ex- 
acerbations of CF lung disease. Also, severity could be assessed with good sensitivity of $96 \%$ and moderate specificity of $67 \%$. These findings emphasise the importance of evaluating several IM reflecting different aspects of the disease, instead of evaluating one individual marker. Interpretation of non-invasive inflammatory markers is complicated because there are many influencing factors. For instance, both inflammation and infection are present in CF. For FeNO, the viscous mucus in the airways may impair diffusion of gaseous NO into the airway lumen [89]. Also a decreased expression of inducible NO synthase (NOS) II in epithelial cells was found, which result in low FeNO levels in children with CF [90-94]. It is unknown whether these mechanisms also are important for non-volatile markers in EBC. In chapter 8, the relationships between non-invasive markers, structural lung changes, assessed by HRCT scans, and lung function in CF patients were studied. This study supports the hypothesis that lung function in CF (both static and dynamic indices) is determined to a large extent by the combination of structural lung changes on HRCT imaging and non-invasive biomarkers in EBC.

Not only biomarkers in EBC but also metabolomics of VOCs in exhaled breath was helpful in CF. This new technique was able to discriminate between CF patients and healthy controls, and between CF patients with or without Pseudomonas colonization, as described in chapter 9.2. It is possible that analysis of VOCs will be of help by the early diagnosis of Pseudomonas colonisation in the lung which enables proper treatment in an early stage thereby minimizing persistent colonisation and more irreversible lung damage. This is particularly important as sputum cultures can be false negative for Pseudomonas and delay can occur [95].

\subsection{Monitoring}

Currently monitoring of chronic lung disease in children is based on symptoms and lung function. These reflect only the consequences of the airway inflammation. We hypothesised that direct measurement of airway inflammation, increases insight in disease control and severity, effects of treatment can be evaluated, and exacerbations can be diagnosed before this is clinically evident, enabling early treatment.

\subsubsection{Asthma}

\section{Evaluation of treatment effects}

Inhaled corticosteroid therapy is the mainstay of asthma treatment. They should be used in the lowest possible doses. Non-invasive methods may help to taper anti-inflammatory treatment. Extrafine hydrofluoroalkane (HFA) beclometasone differs from other inhaled corticosteroids by its fine aerosol characteristics. Therefore, extrafine HFA-beclometasone may be particularly useful for treating peripheral airway inflammation in childhood asthma. In chapter 10 we compared the anti-inflammatory effect of extrafine HFAbeclometasone with fluticasone-DPI in asthmatic children by means of bronchial and alveolar NO levels, and biomarkers in EBC in a 6 months cross-over study. Although fluticasone is 2.2 more potent on a receptor level than HFA-beclomethasone, and the use of equal daily doses for both treatments, all measured inflammatory and conventional out- 
come measures in this study were similar after 3 months treatment with each drug. Probably this is the consequence of the increased peripheral deposition of HFAbeclomethasone [96]. Biomarkers in EBC and bronchial / alveolar NO can be used to evaluate treatment effects in children with asthma.

\section{Prediction of exacerbations}

In a study with forty asthmatic children, Pijnenburg et al found that FeNO has predictive value for an asthma relapse after discontinuation of inhaled corticosteroids in symptomfree children [10]. Furthermore, in 59 children of a one-year prospective study, asthma management based on FeNO values was accompanied by less bronchial hyperresponsiveness [97]. However, a recent Cochrane analysis reviewed all available studies in adults and children on the efficacy of tailoring asthma interventions by means of FeNO in comparison to clinical symptoms for asthma related outcomes $[98,99]$. They concluded that guiding of treatment on basis of FeNO did not result in a better outcome of asthma. In addition, it is debatable whether a single parameter like FeNO can give a good reflection of the complex inflammatory pathways underlying asthma.

Therefore, in this thesis we analyzed the value of biomarkers in EBC and of VOCs in exhaled breath for the monitoring of asthma. In chapter 11.1, we studied the ability of FeNO, markers in EBC, lung function and symptoms to predict exacerbations of childhood asthma and analysed changes in time of these exhaled markers during exacerbations. The design was a prospective longitudinal study during one year in which 40 children with asthma participated. We were able to predict exacerbations of childhood asthma significantly by regular two-month assessments of EBC IL-5 levels and the asthma control score. However, FeNO or lung function indices were not able to predict exacerbations of childhood asthma. In this study, atopic as well as non-atopic subjects were included which may be an explanation for our results and those of Pijnenburg et al. Zacharasiewicz et al performed a comparable study in which weaning of inhaled corticosteroids was studied in 40 asthmatic children. Two-monthly assessments of FeNO and sputum eosinophils were significantly related to failed steroid reduction but lung function and nitrate/nitrite in EBC were not [100]. This is in line with our results as we also did not find a relationship between EBC nitrate/nitrite and exacerbations.

In the study described above we also analysed the predictive ability of two-monthly assessed breathogram of VOCs for an asthma exacerbation in these 40 children (chapter 12.1). We were able to identify 6 VOCs which -as a combination- could predict exacerbations or stable disease with a correct classification rate of $96 \%$ with a corresponding sensitivity and specificity of $100 \%$ and $93 \%$ respectively. To our knowledge this is the first longitudinal study to analyze the predictive ability of VOCs in exhaled breath to predict exacerbations of childhood asthma, and to study the course of VOCs during an exacerbation. No comparable studies have been finished yet. Our study indicates that by means of VOCs analysis in exhaled breath, an asthma exacerbation could be predicted on average 40 days before the event was clinically manifest. Breath analysis may reflect biochemical and immunological processes of airway inflammation and oxidative stress in the human lung. This opens the possibility to assess the potential of non-invasive inflammometry to titrate asthma treatment in future studies. 


\subsubsection{Cystic fibrosis}

Exaggerated airway inflammation and recurrent infections in CF result in irreversible structural changes of the airways, eventually leading to respiratory failure and death [101, 102]. Currently, the diagnosis of pulmonary exacerbations in CF is based on clinical features and pulmonary function tests. However, these tests may not reflect the onset, origin or severity of airway inflammation [103]. In chapter 11.2 we therefore studied the ability of FeNO, markers in EBC, and lung function symptoms to predict pulmonary exacerbations of CF. Also the time course of changes in inflammatory markers during exacerbations was investigated. In this one year prospective longitudinal study in 26 CF patients, we found that several markers in EBC, but not symptoms, lung function indices or FeNO, were able to predict a pulmonary CF exacerbation before these events became clinically manifest. Several cytokines (IL-1 $\alpha,-5,-6,-10$, TNF- $\alpha$ ) and chemokines (IL-8 and MIF) were significant predictors of a cystic fibrosis exacerbation, of which macrophage inhibiting factor was the strongest predictor. The risk of an exacerbation increased to $100 \%$ in patients with low macrophage inhibiting factor levels in $E B C(<130 \mathrm{pg} / \mathrm{mL})$. These results emphasize the potential importance of non-invasive inflammometry during the monitoring of CF lung disease in order to predict a pulmonary CF exacerbation. In a comparable study, Horak et al. studied the monitoring ability of nitrite in EBC in children with mild CF in a prospective longitudinal study during one year. They concluded that nitrite levels in EBC were not helpful to predict a pulmonary CF exacerbation which is in accordance with our results [104].

In contrast to our study, Horak et al did not assess cytokines or chemokines in EBC. We also investigated the predictive ability of VOCs for the detection of CF exacerbations (chapter 12.2). This was done in the same study as described above in chapter 11.2. We were able to define 8 different VOCs which could predict $92 \%$ of the exacerbations correctly with a corresponding sensitivity of $100 \%$ and a specificity of $84 \%$. The VOCs with predictive value for a CF exacerbation were chemically identified as mainly long chain hydrocarbons. Non-invasive inflammometry is also a promising tool in patients with CF. Earlier detection of a pulmonary CF exacerbation will lead to earlier treatment, which may result in (partial) prevention of exacerbations, better disease control, less hospitalisation, a better lung function, and an improved quality of live.

\subsection{Future research}

When methodological issues are further clarified, analyses of exhaled breath is a promising tool for the diagnosis and monitoring of children with asthma and CF, which should be applied in addition to conventional parameters, like symptoms, physical examination and lung function indices. Future studies are needed to investigate which (combination of) markers of breath analysis are most helpful for answering everyday clinical questions for a specific disease. 


\subsubsection{Methodology}

\section{Exhaled breath condensate}

Some methodological issues need to be resolved. Although the ATS/ERS guideline gives clear recommendations on EBC standardization, further research on EBC methodology and standardization is still needed [8]. Methodological issues for future research are: 1) development of an optimal standardized condenser for all age categories: the development of a highly efficient standard condenser for use in both children and adults is not only necessary to assess the full potential of the technique, but will also improve the standardization within and between centers. As ideal condenser dimensions and coatings may differ from one marker to another, the design must be flexible and adjustable; 2) standardisation for collection time, exhaled volume, or dilution factor. So far, biomarker amounts in EBC are expressed as concentrations. Although this is a practical and simple approach, it may be that standardisation for collection time, volume of exhaled breath, or the dilution factor of EBC may increase the possibility to compare different samples between and within subjects, and may lower the variability; 3 ) development of more sensitive assays in a lower volume of EBC: most analytical methods used in the literature on EBC are not specifically developed for use in EBC. Although considerable progress has been made, for instance in the assessment of cytokines/chemokines, new sensitive analytical methods in lower EBC volumes are needed for several other biomarkers; 4) increasing reproducibility; the low reproducibility is still an issue for several biomarkers and may mainly be the consequence of the intra-assay variability. Therefore, new assays and methods yielding highly reproducible results are necessary; 5) identifying new markers by means of "-omic" techniques; 6) influence of ambient air pollution, cleaning of the condenser equipment and storage conditions: as on-line measurements are largely not available, EBC samples are usually stored before analysis at $-80{ }^{\circ} \mathrm{C}$ until analysis $[8,30]$. For many mediators, the relationship between storage time and mediator stability is unknown. Although the recent guideline does not advocate the use of an inspiration filter, it is possible that ambient air pollutants can disturb the measurements of at least some biomarkers in EBC. It has been shown that higher ambient NO levels reduced hydrogen peroxide concentrations in EBC [47]. More data comparing EBC markers with and without filtering of the inspired air are needed. In addition, very little is known about the influence of different cleaning protocols on biomarker concentration which is an important topic for future studies.

In order to investigate the origin and the processes of EBC formation, in vitro studies may be necessary to increase insight in the mechanism of EBC formation. Also the relationship between the position in the airways (proximal or more distal airways) and the formation of EBC particles is not known yet. This is difficult to investigate. Tracer techniques with radioactive labelling may be useful to unravel this issue. Studies in patients with a tracheostoma can clarify the possible influence of particle formation in the upper airways. Future studies in which the diagnostic value of selective sampling of EBC from more distal airways is investigated will also help to clarify this issue. New condensers like the newly developed EcoScreen in which proximal and distal breath can be separated, are useful on this topic. In vitro studies may also be of help to elucidate other queries, like the influence of exhalation flow and the dilution factor. 


\section{Volatile organic compounds}

In VOCs analysis, future studies are necessary in clinical settings for identification of compounds, to explain the biochemical origin, the physiological meaning and exhalation kinetics of selected markers. In analogy with NO and EBC, the institution of an international ERS/ATS task force on VOCs analysis will help to standardize the measurements within and between centers. Many methodological studies performed on FeNO and biomarkers in EBC should be specifically repeated for VOCs in exhaled breath. Moreover, the development of a standard sampling and collection technique for use in children and adults will help to increase the standardization of measurements. Meanwhile, more clinically orientated studies assessing the potential of the technique for diagnostic and monitoring purposes in different diseases should go on.

\subsubsection{Diagnosis}

\section{Wheezing phenotypes, asthma and cystic fibrosis}

As stated in the introduction of this thesis, there is a major overlap between asthma phenotypes. Currently, these phenotypes are categorized in different ways [105-107]. Noninvasive inflammometry may clarify: 1 ) differences and overlaps between these phenotypes based on the actual ongoing type of airway inflammation; 2) the presence of neutrophilic or eosinophilic inflammation; 3 ) the response to various treatments, like inhaled corticosteroids of leucotriene receptor antagonists.

An early, reliable diagnosis of asthma in preschool children by non-invasive markers in exhaled breath (condensate) will have a major influence on health care. It will prevent underdiagnosis and undertreatment in "true asthmatics". On the other hand, exclusion of asthma at an early age prevents overtreatment in the large group of children with "transient wheezing or viral wheeze". In chapter 4, we demonstrate that the ratio of Th2/Th1 cytokines (e.g. IL-5 to TNF- $\alpha$ ) was higher in children with a high probability of asthma. Longitudinal studies (for instance the ADEM study, Asthma DEtection and Monitoring study) are currently being performed to assess whether these or other non-invasive markers at an early age are related to persistent wheezing and asthma the age of 6 years. [108]. When different phenotypes of asthma can be defined, more specific treatment may be given. In theory, this could result in use of less medication, better asthma control, prevention of anxiety of parents, less hospitalisation, higher quality of life, and less economic costs.

The relationship between non-invasive parameters of airway inflammation with more conventional parameters like lung function, imaging techniques (CT), broncho-alveolar lavage, or biopsies is clearly a topic of interest for future research.

In CF, non-invasive tests of airway inflammation may help in assessing the severity of airway inflammation, and the decision to start or to stop antibiotic treatment. Early detection of exacerbations may help to start treatment early and to prevent at least part of the exacerbations. This prevention of exacerbations in CF can have a major impact on the morbidity and progression of the disease [109].

\section{Other (respiratory) diseases}

Endogenous metabolic processes can be reflected in the exhalation of various biomarkers in exhaled breath (condensate). The simultaneous measurements of hundreds of exhaled 
markers by means of the "-omic" technologies, makes these techniques especially suitable for the definition and monitoring of the time course of evolving, complex metabolic conditions, including, among others, diabetes mellitus, chronic inflammatory bowel diseases (colitis ulcerosa, Crohns disease), and chronic infections. The potential role of non-invasive inflammometry in these diseases is currently under investigation. Besides, non-invasive techniques may have a role for the (rapid) characterisation of respiratory infections in children and adults (viral, bacterial or atypical pathogen) which may help with the institution of specific antibiotic treatment, which will result in less antibiotic resistance and more cost-effective treatment.

\subsubsection{Monitoring}

Longitudinal studies and randomized controlled trials contribute significantly to basic science. It will greatly increase our knowledge about the most important features of the underlying airway inflammation in chronic inflammatory respiratory diseases, differences in inflammatory characteristics, and characteristics of stable and instable disease. In future studies, the predictive value of biomarkers in EBC or VOCs in exhaled breath for disease exacerbations should be confirmed in an enlarged study population. In a randomized longitudinal comparative study design, biomarkers should be used to adjust disease treatment. If proven effective for the prevention of exacerbations in these studies, noninvasive monitoring may be implemented in every day clinical practice.

Technical advances include online measurements of biomarkers or eNose. Depending on the application/clinical questions (e.g. diagnosis of chronic inflammatory disorders, monitoring disease control, identification of pulmonary infections in CF), different chips for EBC or eNoses may be composed. This may enable fast assessments of non-invasive inflammometry. In addition to conventional markers (like symptoms and lung function), this way of fast non-invasive inflammometry may improve health care in both the outpatient clinic, but also in everyday practice of general practitionars. In addition, patients with chronic diseases may perform home measurements. Combined with online feedback, patients may be controlled better. This is very important as exacerbations are a major source of disability, hospitalisation, progression of disease, reduced quality of life, and large economic costs $[109,110]$.

\section{References}

1. Wilson NM, Bridge P, Spanevello A, Silverman M. Induced sputum in children: feasibility, repeatability, and relation of findings to asthma severity. Thorax 2000: 55(9): 768-774.

2. Moeller A, Franklin P, Hall GL, Horak F, Jr., Wildhaber JH, Stick SM. Measuring exhaled breath condensates in infants. Pediatr Pulmonol 2006: 41(2): 184-187.

3. Griese $\mathrm{M}$, Latzin $\mathrm{P}$, Beck J. A noninvasive method to collect nasally exhaled air condensate in humans of all ages. Eur J Clin Invest 2001: 31(10): 915-920.

4. Muller WG, Morini F, Eaton S, Peters M, Jaffe A. Safety and feasibility of exhaled breath condensate collection in ventilated infants and children. Eur Respir J 2006: 28(3): 479-485.

5. Baraldi E, Ghiro L, Piovan V, Carraro S, Zacchello F, Zanconato S. Safety and success of exhaled breath condensate collection in asthma. Arch Dis Child 2003: 88(4): 358-360. 
6. Recommendations for standardized procedures for the on-line and off-line measurement of exhaled lower respiratory nitric oxide and nasal nitric oxide in adults and children-1999. This official statement of the American Thoracic Society was adopted by the ATS Board of Directors, July 1999. Am J Respir Crit Care Med 1999: 160(6): 2104-2117.

7. ATS/ERS Recommendations for Standardized Procedures for the Online and Offline Measurement of Exhaled Lower Respiratory Nitric Oxide and Nasal Nitric Oxide, 2005. Am J Respir Crit Care Med 2005: 171(8): 912930.

8. Horvath I, Hunt J, Barnes PJ, Alving K, Antczak A, Baraldi E, Becher G, van Beurden WJ, Corradi M, Dekhuijzen R, Dweik RA, Dwyer T, Effros R, Erzurum S, Gaston B, Gessner C, Greening A, Ho LP, Hohlfeld J, Jobsis Q, Laskowski D, Loukides S, Marlin D, Montuschi P, Olin AC, Redington AE, Reinhold P, van Rensen EL, Rubinstein I, Silkoff P, Toren K, Vass G, Vogelberg C, Wirtz H. Exhaled breath condensate: methodological recommendations and unresolved questions. Eur Respir J 2005: 26(3): 523-548.

9. Miekisch W, Schubert JK, Noeldge-Schomburg GF. Diagnostic potential of breath analysis-focus on volatile organic compounds. Clin Chim Acta 2004: 347(1-2): 25-39.

10. Pijnenburg MW, Hofhuis W, Hop WC, De Jongste JC. Exhaled nitric oxide predicts asthma relapse in children with clinical asthma remission. Thorax 2005: 60(3): 215-218.

11. Jones SL, Kittelson J, Cowan JO, Flannery EM, Hancox RJ, McLachlan CR, Taylor DR. The predictive value of exhaled nitric oxide measurements in assessing changes in asthma control. Am J Respir Crit Care Med 2001: 164(5): 738-743.

12. Berkman N, Avital A, Breuer R, Bardach E, Springer C, Godfrey S. Exhaled nitric oxide in the diagnosis of asthma: comparison with bronchial provocation tests. Thorax 2005: 60(5): 383-388.

13. Pijnenburg MW, Jobsis $Q$, de Jongste JC. ['Inflammometry' with nitric oxide in exhaled air: a new test for lung diseases]. Ned Tijdschr Geneeskd 2001: 145(20): 946-950.

14. Jobsis Q, Schellekens SL, Kroesbergen A, Hop WC, de Jongste JC. Sampling of exhaled nitric oxide in children: end-expiratory plateau, balloon and tidal breathing methods compared. Eur Respir J 1999: 13(6): 1406-1410.

15. Jobsis Q, Schellekens SL, Kroesbergen A, Hop WC, de Jongste JC. Off-line sampling of exhaled air for nitric oxide measurement in children: methodological aspects. Eur Respir J 2001: 17(5): 898-903.

16. Baraldi E, Dario C, Ongaro R, Scollo M, Azzolin NM, Panza N, Paganini N, Zacchello F. Exhaled nitric oxide concentrations during treatment of wheezing exacerbation in infants and young children. Am J Respir Crit Care Med 1999: 159(4 Pt 1): 1284-1288.

17. Franklin PJ, Turner SW, Hall GL, Moeller A, Stick SM. Exhaled nitric oxide is reduced in infants with rhinorrhea. Pediatr Pulmonol 2005: 39(2): 117-119.

18. Franklin PJ, Turner SW, Mutch RC, Stick SM. Measuring exhaled nitric oxide in infants during tidal breathing: methodological issues. Pediatr Pulmonol 2004: 37(1): 24-30.

19. Gabriele C, van der Wiel EC, Nieuwhof EM, Moll HA, Merkus PJ, de Jongste JC. Methodological aspects of exhaled nitric oxide measurements in infants. Pediatr Allergy Immunol 2007: 18(1): 36-41.

20. Mieskonen ST, Malmberg LP, Kari MA, Pelkonen AS, Turpeinen MT, Hallman NM, Sovijarvi AR. Exhaled nitric oxide at school age in prematurely born infants with neonatal chronic lung disease. Pediatr Pulmonol 2002: 33(5): 347-355.

21. Moeller A, Franklin P, Hall GL, Turner S, Straub D, Wildhaber JH, Stick SM. Inhaled fluticasone dipropionate decreases levels of nitric oxide in recurrenty wheezy infants. Pediatr Pulmonol 2004: 38(3): 250-255.

22. Ratjen F, Kavuk I, Gartig S, Wiesemann HG, Grasemann H. Airway nitric oxide in infants with acute wheezy bronchitis. Pediatr Allergy Immunol 2000: 11(4): 230-235.

23. Lehtimaki L, Turjanmaa V, Kankaanranta H, Saarelainen S, Hahtola P, Moilanen E. Increased bronchial nitric oxide production in patients with asthma measured with a novel method of different exhalation flow rates. Ann Med 2000: 32(6): 417-423.

24. Gelb AF, Taylor CF, Nussbaum E, Gutierrez C, Schein A, Shinar CM, Schein MJ, Epstein JD, Zamel N. Alveolar and airway sites of nitric oxide inflammation in treated asthma. Am J Respir Crit Care Med 2004: 170(7): 737741.

25. Latzin P, Beck J, Griese M. Exhaled nitric oxide in healthy children: variability and a lack of correlation with atopy. Pediatr Allergy Immunol 2002: 13(1): 37-46. 
26. Lehtimaki L, Kankaanranta H, Saarelainen S, Hahtola P, Jarvenpaa R, Koivula T, Turjanmaa V, Moilanen E. Extended exhaled NO measurement differentiates between alveolar and bronchial inflammation. Am J Respir Crit Care Med 2001: 163(7): 1557-1561.

27. Pietropaoli AP, Perillo IB, Torres A, Perkins PT, Frasier LM, Utell MJ, Frampton MW, Hyde RW. Simultaneous measurement of nitric oxide production by conducting and alveolar airways of humans. J Appl Physiol 1999: 87(4): 1532-1542.

28. Lehtimaki L, Kankaanranta H, Saarelainen S, Turjanmaa V, Moilanen E. Inhaled fluticasone decreases bronchial but not alveolar nitric oxide output in asthma. Eur Respir J 2001: 18(4): 635-639.

29. Tsoukias NM, George SC. A two-compartment model of pulmonary nitric oxide exchange dynamics. J Appl Physiol 1998: 85(2): 653-666.

30. Rosias PP, Dompeling E, Hendriks HJ, Heijnens JW, Donckerwolcke RA, Jobsis Q. Exhaled breath condensate in children: pearls and pitfalls. Pediatr Allergy Immunol 2004: 15(1): 4-19.

31. Mutlu GM, Garey KW, Robbins RA, Danziger LH, Rubinstein I. Collection and analysis of exhaled breath condensate in humans. Am J Respir Crit Care Med 2001: 164(5): 731-737.

32. Robroeks CM, Jobsis Q, Damoiseaux JG, Heijmans PH, Rosias PP, Hendriks HJ, Dompeling E. Cytokines in exhaled breath condensate of children with asthma and cystic fibrosis. Ann Allergy Asthma Immunol 2006: 96(2): 349-355.

33. Jobsis Q, Raatgeep HC, Hermans PW, de Jongste JC. Hydrogen peroxide in exhaled air is increased in stable asthmatic children. Eur Respir J 1997: 10(3): 519-521.

34. Montuschi P, Barnes PJ. Analysis of exhaled breath condensate for monitoring airway inflammation. Trends Pharmacol Sci 2002: 23(5): 232-237.

35. Soyer OU, Dizdar EA, Keskin O, Lilly C, Kalayci O. Comparison of two methods for exhaled breath condensate collection. Allergy 2006: 61(8): 1016-1018.

36. Prieto L, Ferrer A, Palop J, Domenech J, Llusar R, Rojas R. Differences in exhaled breath condensate $\mathrm{pH}$ measurements between samples obtained with two commercial devices. Respir Med 2007: 101(8): 17151720.

37. www.rtube.com.

38. Carraro S, Andreola B, Alinovi R, Corradi M, Freo L, Da Dalt L, Baraldi E. Exhaled leukotriene B4 in children with community acquired pneumonia. Pediatr Pulmonol 2008: 43(10): 982-986.

39. Goldoni M, Caglieri A, Andreoli R, Poli D, Manini P, Vettori MV, Corradi M, Mutti A. Influence of condensation temperature on selected exhaled breath parameters. BMC Pulm Med 2005: 5: 10.

40. Rosias PP, Robroeks CM, Niemarkt HJ, Kester AD, Vernooy JH, Suykerbuyk J, Teunissen J, Heynens J, Hendriks $\mathrm{HJ}$, Jobsis Q, Dompeling E. Breath condenser coatings affect measurement of biomarkers in exhaled breath condensate. Eur Respir J 2006: 28(5): 1036-1041.

41. Rosias PP, Robroeks CM, Kester A, den Hartog GJ, Wodzig WK, Rijkers GT, Zimmermann L, van Schayck CP, Jobsis $Q$, Dompeling E. Biomarker reproducibility in exhaled breath condensate collected with different condensers. Eur Respir J 2008: 31(5): 934-942.

42. Rosias PP, Robroeks CM, van de Kant KD, Rijkers GT, Zimmermann L, van Schayck CP, Heynens JW, Jobsis Q, Dompeling E. Feasibility of a new method to collect exhaled breath condensate in pre-school children. Pediatr Allergy Immunol 2009.

43. Rosias PP, Dompeling E, Dentener MA, Pennings HJ, Hendriks HJ, Van lersel MP, Jobsis Q. Childhood asthma: exhaled markers of airway inflammation, asthma control score, and lung function tests. Pediatr Pulmonol 2004: 38(2): 107-114.

44. Hunt J. Exhaled breath condensate pH: reflecting acidification of the airway at all levels. Am J Respir Crit Care Med 2006: 173(4): 366-367.

45. Vaughan J, Ngamtrakulpanit L, Pajewski TN, Turner R, Nguyen TA, Smith A, Urban P, Hom S, Gaston B, Hunt J. Exhaled breath condensate $\mathrm{pH}$ is a robust and reproducible assay of airway acidity. Eur Respir J 2003: 22(6): 889-894.

46. Doniec Z, Nowak D, Tomalak W, Pisiewicz K, Kurzawa R. Passive smoking does not increase hydrogen peroxide (H2O2) levels in exhaled breath condensate in 9-year-old healthy children. Pediatr Pulmonol 2005: 39(1): 41-45. 
47. Latzin P, Griese M. Exhaled hydrogen peroxide, nitrite and nitric oxide in healthy children: decrease of hydrogen peroxide by atmospheric nitric oxide. Eur J Med Res 2002: 7(8): 353-358.

48. Robroeks CM, Rosias PP, van Vliet D, Jobsis Q, Yntema JB, Brackel HJ, Damoiseaux JG, den Hartog GM, Wodzig WK, Dompeling E. Biomarkers in exhaled breath condensate indicate presence and severity of cystic fibrosis in children. Pediatr Allergy Immunol 2008: 19(7): 652-659.

49. Epton MJ, Dawson RD, Brooks WM, Kingham S, Aberkane T, Cavanagh JA, Frampton CM, Hewitt T, Cook JM, McLeod S, McCartin F, Trought K, Brown L. The effect of ambient air pollution on respiratory health of school children: a panel study. Environ Health 2008: 7: 16.

50. Lehmann C RM, Becher G. A new method for rapid measurement of hydrogen peroxide in exhaled breath condensate. Am J Respir Crit Care Med 2002: 165: A53.

51. Zappacosta B, Persichilli S, Mormile F, Minucci A, Russo A, Giardina B, De Sole P. A fast chemiluminescent method for $\mathrm{H}(2) \mathrm{O}(2)$ measurement in exhaled breath condensate. Clin Chim Acta 2001: 310(2): 187-191.

52. van Beurden WJ, Harff GA, Dekhuijzen PN, van den Bosch MJ, Creemers JP, Smeenk FW. An efficient and reproducible method for measuring hydrogen peroxide in exhaled breath condensate. Respir Med 2002: 96(3): 197-203.

53. Balint B, Kharitonov SA, Hanazawa T, Donnelly LE, Shah PL, Hodson ME, Barnes PJ. Increased nitrotyrosine in exhaled breath condensate in cystic fibrosis. Eur Respir J 2001: 17(6): 1201-1207.

54. Ganas K, Loukides S, Papatheodorou G, Panagou P, Kalogeropoulos N. Total nitrite/nitrate in expired breath condensate of patients with asthma. Respir Med 2001: 95(8): 649-654.

55. Formanek W, Inci D, Lauener RP, Wildhaber JH, Frey U, Hall GL. Elevated nitrite in breath condensates of children with respiratory disease. Eur Respir J 2002: 19(3): 487-491.

56. Kharitonov SA, Donnelly LE, Montuschi P, Corradi M, Collins JV, Barnes PJ. Dose-dependent onset and cessation of action of inhaled budesonide on exhaled nitric oxide and symptoms in mild asthma. Thorax 2002: 57(10): 889-896.

57. Vogelberg C, Kahlert A, Wurfel C, Marx K, Bohm A, Range U, Neumeister V, Leupold W. Exhaled breath condensate nitrite-methodological problems of sample collection. Med Sci Monit 2008: 14(8): CR416-422.

58. Robroeks CM, van de Kant KD, Jobsis Q, Hendriks HJ, van Gent R, Wouters EF, Damoiseaux JG, Bast A, Wodzig WK, Dompeling E. Exhaled nitric oxide and biomarkers in exhaled breath condensate indicate the presence, severity and control of childhood asthma. Clin Exp Allergy 2007: 37(9): 1303-1311.

59. Montuschi P. LC/MS/MS analysis of leukotriene B4 and other eicosanoids in exhaled breath condensate for assessing lung inflammation. J Chromatogr B Analyt Technol Biomed Life Sci 2009: 877(13): 1272-1280.

60. Montuschi P, Ragazzoni E, Valente S, Corbo G, Mondino C, Ciappi G, Barnes PJ, Ciabattoni G. Validation of leukotriene B4 measurements in exhaled breath condensate. Inflamm Res 2003: 52(2): 69-73.

61. Csoma Z, Kharitonov SA, Balint B, Bush A, Wilson NM, Barnes PJ. Increased leukotrienes in exhaled breath condensate in childhood asthma. Am J Respir Crit Care Med 2002: 166(10): 1345-1349.

62. Brunetti L, Francavilla R, Tesse R, Fiermonte P, Fiore FP, Lore M, Margiotta M, Armenio L. Exhaled breath condensate cytokines and $\mathrm{pH}$ in pediatric asthma and atopic dermatitis. Allergy Asthma Proc 2008: 29(5): 461-467.

63. Profita M, La Grutta S, Carpagnano E, Riccobono L, Di Giorgi R, Bonanno A, Pace E, Bonsignore G, Bousquet J, Vignola AM, Gjomarkaj M. Noninvasive methods for the detection of upper and lower airway inflammation in atopic children. J Allergy Clin Immunol 2006: 118(5): 1068-1074.

64. Shahid SK, Kharitonov SA, Wilson NM, Bush A, Barnes PJ. Increased interleukin-4 and decreased interferongamma in exhaled breath condensate of children with asthma. Am J Respir Crit Care Med 2002: 165(9): 1290-1293.

65. Robroeks C, Rijkers G, Jobsis Q, Hendriks JE, Damoiseaux JGMC, Zimmermann LI, Schayck CP van, Dompeling E. Increased cytokines, chemokines and soluble adhesion molecules in exhaled breath condensate of asthmatic children. Clin Exp Allergy 2009 (in press).

66. Matsunaga K, Yanagisawa S, Ichikawa T, Ueshima K, Akamatsu K, Hirano T, Nakanishi M, Yamagata T, Minakata $\mathrm{Y}$, Ichinose $\mathrm{M}$. Airway cytokine expression measured by means of protein array in exhaled breath condensate: correlation with physiologic properties in asthmatic patients. J Allergy Clin Immunol 2006: 118(1): 84-90. 
67. Nicholson JK, Wilson ID. Opinion: understanding 'global' systems biology: metabonomics and the continuum of metabolism. Nat Rev Drug Discov 2003: 2(8): 668-676.

68. Sauer U, Heinemann M, Zamboni N. Genetics. Getting closer to the whole picture. Science 2007: 316(5824): 550-551.

69. Griese M, Noss J, von Bredow C. Protein pattern of exhaled breath condensate and saliva. Proteomics 2002: 2(6): 690-696.

70. Kurova VS, Anaev EC, Kononikhin AS, Fedorchenko KY, Popov IA, Kalupov TL, Bratanov DO, Nikolaev EN, Varfolomeev SD. Proteomics of exhaled breath: methodological nuances and pitfalls. Clin Chem Lab Med 2009: 47(6): 706-712.

71. Conrad DH, Goyette J, Thomas PS. Proteomics as a method for early detection of cancer: a review of proteomics, exhaled breath condensate, and lung cancer screening. Journal of general internal medicine 2008: 23 Suppl 1: 78-84.

72. Gianazza E, Allegra L, Bucchioni E, Eberini I, Puglisi L, Blasi F, Terzano C, Wait R, Sirtori CR. Increased keratin content detected by proteomic analysis of exhaled breath condensate from healthy persons who smoke. Am J Med 2004: 117(1): 51-54.

73. de Laurentiis G, Paris D, Melck D, Maniscalco M, Marsico S, Corso G, Motta A, Sofia M. Metabonomic analysis of exhaled breath condensate in adults by nuclear magnetic resonance spectroscopy. Eur Respir J 2008: 32(5): 1175-1183.

74. Carraro S, Rezzi S, Reniero F, Heberger K, Giordano G, Zanconato S, Guillou C, Baraldi E. Metabolomics applied to exhaled breath condensate in childhood asthma. Am J Respir Crit Care Med 2007: 175(10): 986-990.

75. Baraldi E, Carraro S, Giordano G, Reniero F, Perilongo G, Zacchello F. Metabolomics: moving towards personalized medicine. Italian journal of pediatrics 2009: 35(1): 30.

76. Phillips M, Gleeson K, Hughes JM, Greenberg J, Cataneo RN, Baker L, McVay WP. Volatile organic compounds in breath as markers of lung cancer: a cross-sectional study. Lancet 1999: 353(9168): 1930-1933.

77. Phillips M, Cataneo RN, Cummin AR, Gagliardi AJ, Gleeson K, Greenberg J, Maxfield RA, Rom WN. Detection of lung cancer with volatile markers in the breath. Chest 2003: 123(6): 2115-2123.

78. Phillips M, Cataneo RN, Condos R, Ring Erickson GA, Greenberg J, La Bombardi V, Munawar MI, Tietje O. Volatile biomarkers of pulmonary tuberculosis in the breath. Tuberculosis (Edinb) 2007: 87(1): 44-52.

79. Sanchez JM, Sacks RD. Development of a multibed sorption trap, comprehensive two-dimensional gas chromatography, and time-of-flight mass spectrometry system for the analysis of volatile organic compounds in human breath. Anal Chem 2006: 78(9): 3046-3054.

80. Barker M, Hengst M, Schmid J, Buers HJ, Mittermaier B, Klemp D, Koppmann R. Volatile organic compounds in the exhaled breath of young patients with cystic fibrosis. Eur Respir J 2006: 27(5): 929-936.

81. Machado RF, Laskowski D, Deffenderfer O, Burch T, Zheng S, Mazzone PJ, Mekhail T, Jennings C, Stoller JK, Pyle J, Duncan J, Dweik RA, Erzurum SC. Detection of lung cancer by sensor array analyses of exhaled breath. Am J Respir Crit Care Med 2005: 171(11): 1286-1291.

82. Dragonieri S, Schot R, Mertens BJ, Le Cessie S, Gauw SA, Spanevello A, Resta O, Willard NP, Vink TJ, Rabe KF, Bel EH, Sterk PJ. An electronic nose in the discrimination of patients with asthma and controls. J Allergy Clin Immunol 2007: 120(4): 856-862.

83. Van Berkel JJ, Dallinga JW, Moller GM, Godschalk RW, Moonen E, Wouters EF, Van Schooten FJ. Development of accurate classification method based on the analysis of volatile organic compounds from human exhaled air. J Chromatogr B Analyt Technol Biomed Life Sci 2008: 861(1): 101-107.

84. Gustafsson PM, Watson L, Davis KJ, Rabe KF. Poor asthma control in children: evidence from epidemiological surveys and implications for clinical practice. Int J Clin Pract 2006: 60(3): 321-334.

85. Rabe KF, Adachi M, Lai CK, Soriano JB, Vermeire PA, Weiss KB, Weiss ST. Worldwide severity and control of asthma in children and adults: the global asthma insights and reality surveys. J Allergy Clin Immunol 2004: 114(1): 40-47.

86. van Gent R, van Essen-Zandvliet LE, Rovers MM, Kimpen JL, de Meer G, van der Ent CK. Poor perception of dyspnoea in children with undiagnosed asthma. Eur Respir J 2007: 30(5): 887-891.

87. Khan TZ, Wagener JS, Bost T, Martinez J, Accurso FJ, Riches DW. Early pulmonary inflammation in infants with cystic fibrosis. Am J Respir Crit Care Med 1995: 151(4): 1075-1082. 
88. Sly PD, Brennan S, Gangell C, de Klerk N, Murray C, Mott L, Stick SM, Robinson PJ, Robertson CF, Ranganathan SC. Lung disease at diagnosis in infants with cystic fibrosis detected by newborn screening. Am J Respir Crit Care Med 2009: 180(2): 146-152.

89. Ho LP, Innes JA, Greening AP. Nitrite levels in breath condensate of patients with cystic fibrosis is elevated in contrast to exhaled nitric oxide. Thorax 1998: 53(8): 680-684.

90. Grasemann H, Ratjen F. Cystic fibrosis lung disease: the role of nitric oxide. Pediatr Pulmonol 1999: 28(6): 442-448.

91. Meng QH, Springall DR, Bishop AE, Morgan K, Evans TJ, Habib S, Gruenert DC, Gyi KM, Hodson ME, Yacoub $\mathrm{MH}$, Polak JM. Lack of inducible nitric oxide synthase in bronchial epithelium: a possible mechanism of susceptibility to infection in cystic fibrosis. J Pathol 1998: 184(3): 323-331.

92. Kelley TJ, Drumm ML. Inducible nitric oxide synthase expression is reduced in cystic fibrosis murine and human airway epithelial cells. J Clin Invest 1998: 102(6): 1200-1207.

93. Franklin PJ, Hall GL, Moeller A, Horak F, Jr., Brennan S, Stick SM. Exhaled nitric oxide is not reduced in infants with cystic fibrosis. Eur Respir J 2006: 27(2): 350-353.

94. Moeller A, Horak F, Jr., Lane C, Knight D, Kicic A, Brennan S, Franklin P, Terpolilli J, Wildhaber JH, Stick SM. Inducible NO synthase expression is low in airway epithelium from young children with cystic fibrosis. Thorax 2006: 61(6): 514-520.

95. Tramper-Stranders GA, van der Ent CK, Wolfs TF. Detection of Pseudomonas aeruginosa in patients with cystic fibrosis. J Cyst Fibros 2005: 4 Suppl 2: 37-43.

96. Leach CL, Davidson PJ, Boudreau RJ. Improved airway targeting with the CFC-free HFA-beclomethasone metered-dose inhaler compared with CFC-beclomethasone. Eur Respir J 1998: 12(6): 1346-1353.

97. Pijnenburg MW, Bakker EM, Hop WC, De Jongste JC. Titrating steroids on exhaled nitric oxide in children with asthma: a randomized controlled trial. Am J Respir Crit Care Med 2005: 172(7): 831-836.

98. Petsky HL, Cates CJ, Li A, Kynaston JA, Turner C, Chang AB. Tailored interventions based on exhaled nitric oxide versus clinical symptoms for asthma in children and adults. Cochrane Database Syst Rev 2009(4): CD006340.

99. Petsky HL, Cates CJ, Li AM, Kynaston JA, Turner C, Chang AB. Tailored interventions based on exhaled nitric oxide versus clinical symptoms for asthma in children and adults. Cochrane Database Syst Rev 2008(2): CD006340.

100.Zacharasiewicz A, Wilson N, Lex C, Erin EM, Li AM, Hansel T, Khan M, Bush A. Clinical use of noninvasive measurements of airway inflammation in steroid reduction in children. Am J Respir Crit Care Med 2005: 171(10): 1077-1082.

101.Birrer P, McElvaney NG, Rudeberg A, Sommer CW, Liechti-Gallati S, Kraemer R, Hubbard R, Crystal RG. Protease-antiprotease imbalance in the lungs of children with cystic fibrosis. Am J Respir Crit Care Med 1994: 150(1): 207-213.

102. Konstan MW, Hilliard KA, Norvell TM, Berger M. Bronchoalveolar lavage findings in cystic fibrosis patients with stable, clinically mild lung disease suggest ongoing infection and inflammation. Am J Respir Crit Care Med 1994: 150(2): 448-454.

103. Kharitonov SA, Barnes PJ. Exhaled markers of pulmonary disease. Am J Respir Crit Care Med 2001: 163(7): 1693-1722.

104. Horak F, Jr., Moeller A, Singer F, Straub D, Holler B, Helbich TH, Schneider B, Eichler I, Wildhaber JH, Hall GL. Longitudinal monitoring of pediatric cystic fibrosis lung disease using nitrite in exhaled breath condensate. Pediatr Pulmonol 2007: 42(12): 1198-1206.

105.Wenzel SE. Asthma: defining of the persistent adult phenotypes. Lancet 2006: 368(9537): 804-813.

106. Henderson J, Granell R, Sterne J. The search for new asthma phenotypes. Arch Dis Child 2009: 94(5): 333336.

107.Simpson JL, Grissell TV, Douwes J, Scott RJ, Boyle MJ, Gibson PG. Innate immune activation in neutrophilic asthma and bronchiectasis. Thorax 2007: 62(3): 211-218.

108.van de Kant KD, Klaassen EM, Jobsis Q, Nijhuis AJ, van Schayck OC, Dompeling E. Early diagnosis of asthma in young children by using non-invasive biomarkers of airway inflammation and early lung function measurements: study protocol of a case-control study. BMC public health 2009: 9: 210. 
109.Gibson RL, Burns JL, Ramsey BW. Pathophysiology and management of pulmonary infections in cystic fibrosis. Am J Respir Crit Care Med 2003: 168(8): 918-951.

110.Global Initiative for asthma (GINA). Pocket guide for asthma management and prevention in children. National Institute of Health, National Heart, Lung, and Blood Institute 2006. 



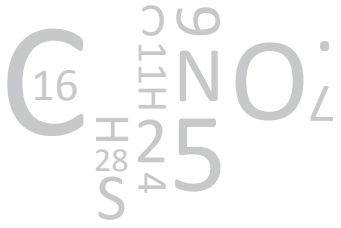

CHAPTER 14

Summary

Samenvatting 


\section{Summary}

In the introduction (chapter 1), we describe problems in childhood asthma and in cystic fibrosis (CF) and the potential additional value of non-invasive inflammometry. There is a major overlap of asthma phenotypes. The relationship between phenotypes, genotypes and responds to treatment is not clear yet. In spite of marked progress in several aspects of asthma management and treatment in the past 30-50 years, worldwide large problems exist with the control of asthma in both adults and children. The consequences of childhood asthma for daily life are huge and comprise respiratory complaints, diminished quality of life, disturbed physical activities, school absence or work absence for the parents, extra visits to a doctor, emergency care visits, and hospital admissions. A CF diagnosis can be made properly by means of a sweat test, however, there is a wide variety of CF phenotypes among individuals with a similar CFTR genotype. Chronic inflammation and infections in CF lung disease results in a progressive decline in lung function and results in irreversible lung damage. Pulmonary CF disease is the major cause of mortality. Available techniques to measure airway inflammation, like broncho-alveolar lavage, induced sputum and lung biopsy, are to invasive to used in children regularly. In both asthma and CF, lung function parameters and presence of symptoms do not reflect ongoing inflammation in a direct manner and treatment cannot be adjusted based on inflammation activity but only on the consequences of the disease. In the past decade, there has been an increasingly interest in non-invasive measurement of airway inflammation. Non-invasive measurements of chronic airway inflammation by means of inflammatory markers in exhaled breath (condensate) is completely non-invasive, has a $100 \%$ success rate in children from the age of 6 years, takes little time, and has the advantage that several parameters can be assessed simultaneously.

By applying non-invasive inflammatory markers in exhaled breath (condensate) in order to monitor children with chronic inflammatory lung diseases like asthma and CF, we hypothesise that there will be an improved level of disease control, a reduced number of exacerbations, less severe exacerbations and, consequently, an increase quality of life in these children.

\section{Section one of this thesis focuses on the methodology of non-invasive inflammometry.}

In chapter 2 we studied the methodology of bronchial and alveolar nitric oxide (NO) measurement assessment in children. These parameters could be of clinical importance for the treatment of asthma, as airway inflammation is more prominent in distal airways of patients with asthma. To discriminate between alveolar and bronchial NO, measurements need to be assessed at various flow rates. The feasibility, linearity and long-term repeatability of NO measurements at four different exhalation flow rates, in 21 children with moderate persistent asthma was studied. Three and six months after the first NO measurement, the tests were repeated. A significant learning effect was present. Feasibil- 
ity of NO measurements at these four flow rates increased from $67 \%$ to $91 \%$ and $95 \%$ at the first, second and third visit, respectively. This study showed good feasibility and linearity of NO measurements in asthmatic children of 6 years and over at flow rates between $50 \mathrm{~mL} / \mathrm{sec}$ to $200 \mathrm{~mL} / \mathrm{sec}$. The long-term reproducibility of alveolar and bronchial NO values during 6 months was moderate.

Chapter $\mathbf{3}$ and $\mathbf{4}$ focuses on the methodology of exhaled breath condensate (EBC). Optimal collection and analysis of EBC are prerequisites for standardisation and reproducibility of assessments. In chapter $\mathbf{3}$ we aimed to assess reproducibility of EBC volume, hydrogen peroxide, 8-isoprostane and cytokine measurements using different condensers. At four points in time, 30 healthy subjects performed sequential EBC collections randomly using the following four condensers: glass, silicone, EcoScreen (Erich Jaeger $\mathrm{GmbH}$, Hoechberg, Germany) and an optimised glass condenser. The optimised glass condenser yielded significantly more EBC volume. The reproducibility of EBC volume, of the new glass condenser was comparable with EcoScreen ", but was significantly better compared with silicone and glass. The new condenser was associated with significantly more detections of hydrogen peroxide, 8-isoprostane, and cytokines. Reproducibility of biomarkers was equally variable for all condenser types. In conclusion, significantly more EBC volume and biomarker detections were found using the optimised glass condenser. However, biomarker reproducibility in EBC in healthy adults was not influenced by the type of condenser. EBC is a promising non-invasive method to assess respiratory inflammation in adults and children with lung disease. Especially in pre-school children, condensate collection is hampered by long sampling times because of open-ended collection systems.

In chapter 4 we aimed to assess the feasibility of condensate collection in pre-school children using a closed glass condenser with breath recirculation system, which also collects the residual non-condensed exhaled breath, and subsequently recirculates it back into the condenser. Condensate was collected before and after breath recirculation in 70 preschool children with and without recurrent wheeze. Cytokines were measured in $50 \mu \mathrm{L}$ samples using ultrasensitive multiplexed liquid bead array. The success rate of condensate collection increased from $64 \%$ (without recirculation) to $83 \%$ (after breath recirculation), and mean condensate volume from 214 to $465 \mu \mathrm{L}$ respectively. Detection of cytokines was successful in $95-100 \%$ of samples. Cytokine concentrations before and after breath recirculation were not different ( $p>0.232$ ). In asthmatic children, only TNF- $\alpha$ concentrations were significantly decreased, compared to non-asthmatics. In pre-school children, the collection of EBC is feasible using a new closed glass condenser with breath recirculation system. This new method may help to assess, non-invasively, cytokine profiles in asthmatic and non-asthmatic pre-school children.

\section{Section two of this thesis focussed on an asthma and CF lung disease diagnosis by means of non-invasive inflammometry.}

Inflammatory markers in EBC indicate ongoing inflammation in the lungs and might differentiate between asthma and CF. The objective of chapter 5 was to assess the presence, concentration, and short-term variability of Th- 1 and Th- 2 mediated cytokines (interferon$\gamma$, tumour necrosis factor- $\alpha$, interleukin-10, $-5,-4,-2$ ) in EBC of children with asthma, or CF 
and healthy controls. In addition, we analysed the discriminating ability of these markers in EBC between children with asthma or CF, and healthy children. In 33 asthmatic children, 12 children with CF and 35 healthy children, EBC was collected with a glass condenser during tidal breathing. Cytokines were measured with flow cytometry. Detections of cytokines in the EBC samples were low. Interleukin-2, -4, interferon- $\gamma$ and interleukin-10 were detected in respectively $16 \%, 16 \%, 11 \%$ and $9 \%$ of all samples in asthma and CF. In the healthy population, interferon- $\gamma$, tumour necrosis factor- $\alpha$ and interleukin-10 were detected in $9 \%, 14 \%$ and $3 \%$ respectively. Interleukin-5 and tumour necrosis factor- $\alpha$ were not detected in children with CF, and interleukin-2, -4 and -5 were not detected in healthy children. Cytokine concentrations did not differ significantly between children with asthma or CF. Based on these results we concluded that cytokines can be detected in EBC of children with asthma and CF, however, concentrations found are close to the detection limits of the used assay. These findings emphasize the importance to develop more sensitive techniques for analysis of EBC and to standardise the EBC collection method.

In chapter 6, we combined the use of a glass condenser system with a sensitive analytical technique (multiplex immunoassay technology) in order to improve assessments of cytokines, chemokines and soluble adhesion molecules in EBC of children with asthma and healthy controls. Measured markers were 1$)$ cytokines: interleukin $1 \alpha,-1 \beta,-2,-4,-5,-6,-10,-$ 12 p70,-13,-18, interferon- $\gamma$, tumour necrosis factor- $\alpha, 2)$ chemokines: MIP1 $\alpha$ (CCL3), MIF, eotaxin (CCL11), RANTES (CCL5), IP10 (CXCL10), IL8 (CXCL8), MCP1, and 3) soluble adhesion molecules (sICAM, sVCAM). Sixty children were included: 31 asthmatics (71\% atopic), and 29 controls. Detection percentages of cytokines, chemokines and adhesion molecules ranged from 94-100\%, except for eotaxin (CCL11) and RANTES (CCL5) (detection rates of $10 \%$ and $45 \%$ in healthy controls respectively). Intra-subject variability of biomarkers in EBC in the group as a whole ranged from $5.2 \%$ to $35.0 \%$. In asthmatics, the levels of cytokines [interleukin-2,-4,-5,-6,-13, interferon- $\gamma$ ], chemokines [MIP1 $\alpha$ (CCL3), MIF, RANTES (CCL5), IP10 (CXCL10), IL8 (CXCL8), MCP1], and adhesion molecules [sICAM, sVCAM] were significantly increased in comparison with controls. This study shows that in EBC, collected with a glass condenser, cytokines, chemokines and adhesion molecules can be detected by means of multiplex immunoassay technology. In addition these data show that these noninvasive markers were elevated in EBC of asthmatics compared to controls.

Fractional exhaled nitric oxide (FeNO) and various solitaire markers in EBC have shown to be significant different between asthma, CF, and controls. However, the ability of these markers to indicate an asthma diagnosis, to assess asthma severity and control, is largely unknown. In chapter $\mathbf{7 . 1}$ we studied the discriminating ability of non-invasive markers considered together between children with asthma and controls, and between levels of asthma control and severity. Assessed inflammatory markers in EBC were nitrite, nitrate, hydrogen peroxide, 8-isoprostane, interferon- $\gamma$, tumour necrosis factor- $\alpha$, interleukin-2,4,-5,-10, and acidity. 114 Children were included: 64 asthmatics and 50 controls. FeNO, and EBC interferon- $\gamma$ and interleukin- 4 differed significantly between asthma and controls. Multivariate backward logistic regression models demonstrated that interleukin-4 was the only significant indicator of an asthma diagnosis. Asthma control was best assessed by exhaled nitric oxide, 8-isoprostane, interferon- $\gamma$ and interleukin- 4 with a sensitivity of $82 \%$ and a specificity of $80 \%$, whereas, FeNO, 8 -isoprostane, nitrate and nitrite in condensate 
were significant indicators of asthma severity (sensitivity $89 \%$, specificity $72 \%$ ). The similar study was performed in $48 \mathrm{CF}$ patients and 50 controls (chapter 7.2). Interferon- $\gamma$ and nitrite concentrations were significantly higher, whereas FeNO levels were significantly lower in CF compared to controls. Using multivariate logistic regression models, a diagnosis of CF was significantly indicated by 8 -isoprostane, nitrite and interferon- $\gamma$ (sensitivity $78 \%$, specificity $83 \%$ ). An exacerbation of CF was significantly indicated by 8 -isoprostane and nitrite (sensitivity $40 \%$, specificity $97 \%$ ). Most indicative biomarkers of CF severity were FeNO, and condensate acidity (sensitivity 96\%, specificity $67 \%$ ). In these crosssectional studies we demonstrated that non-invasive markers could indicate (exacerbations of) asthma and CF, and severity of the disease in children.

Structural lung changes in CF can be assessed by HRCT imaging of the lungs, and include bronchiectasis, mucus plugging, peribronchial thickening, parenchymal abnormalities and air trapping. HRCT enables visualisation of (small) areas with structural lung changes, even in an early stage. These structural abnormalities may be observed by HRCT imaging, even in the presence of normal lung function parameters and in the absence of pulmonary symptoms. The relationship between lung function on the one hand, structural lung changes as visualized by HRCT scans, and non-invasive IM on the other is yet unclear in CF patients. In chapter $\mathbf{8}$ we hypothesized that structural lung changes on HRCT imaging (as a result of long term damage to the lungs by chronic airway infection and inflammation) together with ongoing airway inflammation (as assessed by non-invasive markers) are important determinants of lung function (dynamic as well as static indices) in CF. Data on non-invasive markers (FeNO, and EBC acidity, nitrate, nitrite, 8-isoprostane, hydrogen peroxide, interferon- $\gamma$ ), HRCT imaging, and static and dynamic lung function indices were analyzed in $34 \mathrm{CF}$ patients. HRCT scans were scored in a standardized and validated way, a composite score and component scores were calculated. In general, the correlations between non-invasive markers and structural lung changes, and between IM and lung function were low. Patients with positive sputum Pseudomonas cultures had higher EBC nitrite levels and higher parenchymal HRCT subscores than patients with Pseudomonas-negative cultures. Multiple linear regression models demonstrated that FVC was significantly predicted by hydrogen peroxide in EBC, and the scores of bronchiectasis and mosaic perfusion. Total lung capacity was significantly predicted by 8-isoprostane, nitrate, hydrogen peroxide in $\mathrm{EBC}$, and the mucous plugging subscore. In this study we show that static and dynamic lung function indices in this CF group were predicted by the combination of noninvasive markers and structural lung changes on HRCT imaging.

In exhaled breath, hundreds of volatile organic compounds (VOCs) can be detected. The integrative analysis of large numbers of VOCs in exhaled breath has the promise to discriminate between various inflammatory conditions of the respiratory tract. In chapter $\mathbf{9 . 1}$ and 9.2 we studied the potential of this novel technique to discriminate asthma and CF from healthy controls. In chapter 9.1, breath samples were analysed from 63 children with asthma and 57 healthy controls. Analysis of VOCs was assessed by means of gas chromatography-time of flight-mass spectrometry (GC-TOF-MS). A total of 945 determined compounds were subjected to discriminant analysis to find those that could discriminate diseased from healthy children. A set of samples from both asthmatic and healthy children was selected to build a model that subsequently was used to predict the asthma or 
healthy status of a test group. This way the predictive value of the model could be tested. Discriminant analyses demonstrated that based on 8 VOCs asthmatic and healthy children were correctly classified in $92 \%$, with a sensitivity of $89 \%$ and a specificity of $95 \%$. In chapter 9.2 we investigated whether 'metabolomics' of VOCs could discriminate between CF and controls, and between CF patients with and without Pseudomonas colonization. 105 Children (48 CF, 57 controls) were included. By using 22 VOCs, a 100\% correct identification of CF patients and controls was possible. With 10 VOCs, $92 \%$ of the subjects were correctly classified. The reproducibility of VOC measurements with a one hour interval was very good. Chapter 9.1 and 9.2 show that 'metabolomics' of VOCs in exhaled breath was possible in a reproducible way. This new technique was able to discriminate between children with asthma and controls, and between CF patients and healthy controls. In addition, a good separation could be made between CF patients with or without Pseudomonas colonization.

\section{Section three focussed on non-invasive monitoring airway inflammation in asthma and CF.}

Extrafine hydrofluoroalkane (HFA) beclomethasone differed from other inhaled corticosteroids by its fine aerosol characteristics. Therefore, extrafine HFA-beclomethasone may be particularly useful for treating peripheral airway inflammation in asthma. The aim of chapter $\mathbf{1 0}$ was to analyse the anti-inflammatory effect of extrafine HFA-beclomethasone compared to fluticasone-DPI (which is 2.2 times more potent) in asthmatic children by means of bronchial and alveolar NO, and inflammatory markers in EBC. In a 6 months cross-over study, 33 children, aged 6-12 years, with moderate persistent asthma, were randomly treated with extrafine HFA-beclomethasone (200 $\mu \mathrm{g}$ daily, autohaler) and fluticasone-DPI (200 $\mu \mathrm{g}$ daily, discus). Primary outcome parameters were: alveolar NO concentration and bronchial NO flux. Secondary outcome parameters were: inflammatory markers in EBC, lung function indices, symptoms, exacerbations, and adverse effects. All parameters were recorded at baseline and after each treatment period. Results show that alveolar NO concentration and bronchial NO flux were comparable after treatment with HFA-beclomethasone and fluticasone-DPI. In addition, inflammatory markers in EBC, lung function indices and symptoms, did not differ between treatments. Patients used less $\beta_{2^{-}}$ agonists during the last 2 weeks of HFA-beclomethasone treatment. Based on this study, we concluded that the anti-inflammatory effect of HFA-beclomethasone and fluticasoneDPI, is similar in children with moderate persistent asthma.

Chapter $\mathbf{1 1 . 1}$ and $\mathbf{1 1 . 2}$ investigate the ability of non-invasive inflammatory markers to predict exacerbations of childhood asthma and CF, respectively. In addition, the time course of changes in exhaled inflammatory markers during exacerbations was studied. The design was a prospective one-year longitudinal study. Regular two-monthly visits to the outpatient clinic were required. In addition to these standard 2 monthly visits, four additional visits were planned during an exacerbation. In asthmatics these visits were planned on day 1, 3, 5 and at the end of the exacerbation. However, additional measurements in CF were planned on day 1, 5, 10 and after clinical stabilisation. The primary outcome measure was the occurrence of an exacerbation. Assessment was made of the presence and severity of pulmonary symptoms, use of medication, and measurements of forced 
expiratory volume in one second $\left(\mathrm{FEV}_{1}\right)$ using home monitor. To monitor an exacerbation, patients were requested three times per week, using a home monitor, to 1 ) assess $\mathrm{FEV}_{1}$ measurements; 2) record medication use; 3 ) register the presence and severity of pulmonary symptoms. The following independent parameters were assessed during outpatient visits: 1) FeNO, 2) inflammatory markers in EBC: acidity, nitrite, hydrogen peroxide, interleukin-1 $\alpha,-5,-13$, interferon- $\gamma, 3$ ) lung function indices, 4) control scores. In chapter 11.1, 40 children with asthma (aged 6-16 yrs) participated. 38 of the 40 children completed the study. Sixteen children developed exacerbations, of which 10 were moderate and 6 severe. Inflammatory markers in EBC were detected in $85-100 \%$ of samples. Univariate Cox regression analysis revealed that condensate acidity, interleukin- 5 and asthma control score were significant predictors of asthma exacerbation. Multivariate Cox regression analysis showed that the time until an exacerbation was significantly predicted by the asthma control score and by the level of interleukin-5 in EBC. The Kaplan-Meier survival curve of this multivariate model showed that children with the $10 \%$ most optimal values of interleukin-5 and the asthma control score had a more than two times reduced risk of exacerbations in the subsequent year. In chapter 11.2, 26 CF patients, aged 6-30 years, were included. 17 Patients experienced a first exacerbation of which 8 demonstrated a second event during the study period. In this study the cystic fibrosis control (cystic fibrosis clinical score) and severity (Shwachman-Kulczycki score) was used. Univariate Anderson-Gill models showed that macrophage inhibiting factor (MIF), tumour necrosis factor- $\alpha$, interleukin-6, -8 and -10 were significant predictors of an exacerbation of CF. Multivariate analysis demonstrated that MIF was the strongest predictor. The risk of an exacerbation increased to $100 \%$ in patients with low MIF levels. Symptoms and lung function indices were not significant predictors. These studies showed that by means of inflammatory markers in EBC, an asthma exacerbation and a pulmonary CF exacerbation could be predicted on average one month before clinical onset.

In similar study designs as described in chapter 11.1 and 11.2, we studied whether VOCs are able to predict an asthma and pulmonary CF exacerbation reliably in chapter $\mathbf{1 2 . 1}$ and 12.2, respectively. In addition, the chemical background of the most predicting VOCs was studied. In chapter 12.1, 16 of the 40 included children with asthma experienced an exacerbation, of which 3 had a second event during the study. With support vector machine analysis, the most optimal model of baseline measurements versus exacerbation within patients was based on 6 VOCs (correct classification 96\%, sensitivity 100\%, specificity $93 \%)$. The model of baseline values of patients with an exacerbation compared to levels of patients without an exacerbation consisted of 7 VOCs (correct classification 91\%, sensitivity $79 \%$, specificity $100 \%$ ). All VOCs were identified as hydrocarbons. In chapter $12.2,17$ of the 26 included CF patients experienced an exacerbation of which 8 experienced a second event during the study. The occurrence of 18 VOCs was significantly different in samples of exhaled breath obtained 3-8 weeks prior to exacerbation compared to samples obtained during an exacerbation (intra-subject). A support vector machine classifier based on this dataset contained 6 VOCs and classified $94 \%$ of exacerbations (sensitivity $94 \%$, specificity $95 \%$ ). In addition, 15 VOCs were differentially present in exhaled breath from patients not having an exacerbation versus patients suffering an exacerbation event within 3-8 weeks after sampling, indicating that these VOCs might predict an exacerbation (intersubject). A support vector machine classifier based on this dataset contained 8 VOCs and 
classified $92 \%$ of exacerbations (sensitivity $100 \%$, specificity $84 \%$ ). The VOCs with predictive value of a CF exacerbation, were mainly long chain hydrocarbons. These studies shows that VOCs in exhaled breath are able to indicate CF exacerbations weeks before these adverse events are clinically manifest.

Chapter 13 includes a general discussion including directions for future research. The paradox in daily practice is that although chronic airway inflammation is the hallmark of asthma and CF, methods to evaluate this inflammation are not routinely involved in the diagnosis and management of these diseases. In the past decade, the number of scientific reports on non-invasive inflammometry increased exponentially. Different forms of breath analysis are possible: 1) measurement of FeNO; 2) analysis of biomarkers in EBC; 3) assessment of VOCs. This thesis shows that breath analysis may be of help in the diagnosis and monitoring of chronic inflammatory airway diseases in children. When methodological issues are further clarified, analyses of exhaled breath is a promising tool for the diagnosis and monitoring of children with asthma and CF, which should be applied in addition to conventional parameters, like symptoms, physical examination and lung function indices. Future studies are needed to investigate which (combination of) markers of breath analysis are most helpful for answering everyday clinical questions for a specific disease. 


\section{Samenvatting}

In de introductie (hoofdstuk 1) hebben we de klinische problemen bij astma en cystic fibrosis (CF) op de kinderleeftijd beschreven en de mogelijk aanvullende waarde van het meten van inflammatoire stoffen in de uitademingslucht (niet-invasieve inflammometrie). $\mathrm{Er}$ is een grote overlap tussen de verschillende fenotypen van astma. De relatie tussen fenotype, genotype en reactie op behandeling is nog niet volledig bekend. Wereldwijd blijkt zowel bij kinderen als bij volwassenen met astma ondanks evidence-based behandelrichtlijnen, nog steeds onvoldoende controle van de ziekte te bestaan. De gevolgen van astma op kinderleeftijd in het dagelijks leven zijn groot zijn wat geïllustreerd wordt door, afgenomen kwaliteit van leven, verstoorde lichamelijke activiteiten, schoolverzuim en ziekteverlof van de ouders, extra poliklinische- en extra eerste hulp bezoeken alsmede ziekenhuis opnamen. CF kan goed worden gediagnosticeerd met behulp van een zweettest. Er bestaat echter een grote variëteit van fenotypen bij eenzelfde genotype. De chronische luchtwegontsteking en infecties bij CF resulteren in een progressieve afname van de longfunctie en heeft irreversibele longschade tot gevolg. De chronische longziekte is verantwoordelijk voor de hoge mortaliteit bij CF patiënten.

Bij zowel astma en CF zijn longfunctietesten en ziekte symptomen niet toereikend om een goed luchtwegontsteking weer te geven. De huidige technieken om luchtwegontsteking te meten, zoals de broncho-alveolaire lavage, geïnduceerd sputum en de longbiopsie, zijn dusdanig invasief voor kinderen dat ze niet voor routinematig gebruik geschikt zijn. Zodoende kan er niet gericht behandeld worden op geleide van ontstekingsactiviteit maar slechts op de gevolgen ervan. Het afgelopen decennium is er toenemend belangstelling ontstaan voor het meten van luchtwegontsteking op een niet-invasieve wijze. Luchtwegcondensaat (EBC) wordt verkregen door uitademingslucht af te koelen. Hierin zijn voornamelijk niet-vluchtige stoffen aanwezig. Door uitademingslucht op te vangen kunnen vluchtige stoffen (vluchtige organische componenten, VOCs) gemeten worden. Het meten van chronische luchtwegontsteking door middel van ontstekingsmarkers in uitademingslucht (condensaat), is niet-invasief, heeft een hoge succes percentage bij kinderen vanaf 6 jaar, is snel uit te voeren en heeft het voordeel dat meerdere parameters simultaan verkregen worden.

De hypothese van dit proefschrift is dat het meten van ontstekingsstoffen in uitademingslucht bij kinderen met astma en CF leidt tot een betere diagnostiek en monitoring van deze ziektebeelden wat op termijn zal leiden tot een toename van de kwaliteit van leven bij kinderen met astma en CF.

Het eerste deel van dit proefschrift (sectie 1) richt zich op de methodologie van nietinvasieve inflammometrie.

In hoofdstuk 2 wordt de methodologie van bronchiaal en alveolair stikstofmonoxide (NO) metingen bij kinderen bestudeerd. Deze parameters kunnen van klinische betekenis zijn 
bij de behandeling van astma aangezien luchtwegontsteking zich met name presenteert in de distale luchtwegen. Om onderscheid te maken tussen alveolair en bronchiaal NO is het noodzakelijk om metingen bij verschillenden uitademingssnelheden uit te voeren. De toepasbaarheid, lineariteit en de lange termijn reproduceerbaarheid van NO metingen bij kinderen zijn bij vier verschillende uitademingssnelheden onderzocht. In deze studie zijn 21 kinderen met matig ernstig astma tussen de 6 en 12 jaar oud geïncludeerd. Drie en zes maanden na de eerste NO metingen werden de testen herhaald. Er werd een significant leereffect gezien. De toepasbaarheid van NO metingen met verschillende uitademingssnelheden $(20 \mathrm{~mL} / \mathrm{sec}, 50 \mathrm{~mL} / \mathrm{sec}, 100 \mathrm{~mL} / \mathrm{sec}, 200 \mathrm{~mL} / \mathrm{sec})$ verbeterde van $67 \%$ naar $91 \%$ en $95 \%$ bij respectievelijk het eerste, tweede en derde bezoek. Deze studie laat een goede toepasbaarheid en lineariteit van NO metingen zien bij kinderen met astma vanaf de leeftijd van 6 jaar bij verschillende uitademingssnelheden. De lange termijn reproduceerbaarheid van alveolair en bronchiaal NO gedurende 6 maanden was redelijk.

De hoofdstukken 3 en 4 richten zich op de verbetering van de methodologie van EBC. Een gestandaardiseerde verzamelmethode en analyse methode van EBC zijn essentieel voor een goede reproduceerbaarheid. In hoofdstuk $\mathbf{3}$ was het doel te onderzoeken of toepassing van een geoptimaliseerde glazen condensor gepaard ging met een groter condensaat volume en hogere concentraties van waterstofperoxide, 8-isoprostaan en cytokines in $E B C$, en een betere reproduceerbaarheid van biomarkers in vergelijking met andere condensors. Bij 30 gezonde vrijwilligers is op vier tijdstippen met vier verschillende condensors EBC verzameld in willekeurige volgorde: glas, silicone, EcoScreen ${ }^{\circledR}$ (Erich Jaeger Gm$\mathrm{bH}$, Hoechberg, Duitsland) en met een nieuwe geoptimaliseerde glazen condensor. De resultaten lieten zien dat met de geoptimaliseerde condensor een significant hoger EBC volume werd verzameld. De reproduceerbaarheid van het EBC volume van de nieuwe glazen condensor was vergelijkbaar met die van de EcoScreen ${ }^{\circledR}$ maar was significant beter dan de reproduceerbaarheid van de siliconen en glazen condensors. Daarnaast was met de nieuwe condersor het percentage EBC samples waarin waterstofperoxide, 8isoprostaan en cytokines gedetecteerd werd hoger. De reproduceerbaarheid van verschillende biomarker in condensaat was vergelijkbaar tussen alle gebruikte condensoren. Concluderend, met de nieuwe geoptimaliseerde glazen condensor werden significant hogere volumes EBC verzameld en was er een hoger percentage detecties van biomarkers. Er was geen invloed van de condensor op de reproduceerbaarheid van EBC biomarkers.

$E B C$ is een veelbelovende niet-invasieve methode om luchtwegontsteking bij kinderen en volwassenen met een longziekte te kunnen meten. Echter, met name bij jonge kinderen ( $<4$ jaar), is het moeilijk condensaat te verzamelen in verband met de verzamelduur met open systemen waarbij uitademingslucht kan ontsnappen. In hoofdstuk 4 hebben we gekeken naar de toepasbaarheid van EBC verzameling bij kinderen onder de 4 jaar met een geoptimaliseerde glazen condensor in combinatie met een gesloten recirculatie systeem. Met deze nieuwe toepassing wordt de waterdamp in de uitademingslucht nagenoeg volledig gecondenseerd. Hierdoor wordt sneller een bepaalde hoeveelheid condensaat volume verkregen waardoor de tijd van verzamelen/verzamelduur aanzienlijk bekort kan worden. Condensaat werd verzameld voor en na recirculatie bij 70 jonge kinderen met en zonder luchtwegklachten van piepen. De concentratie van cytokines werden gemeten met behulp van ultragevoelige 'multiplexed liquid bead array' methode. Het succes-percentage van condensaatverzameling nam door de recirculatie significant toe van $64 \%$ (zonder re- 
circulatie) naar $83 \%$ (met recirculatie). Het gemiddelde EBC volume nam door recirculatie toe van $214 \mu \mathrm{L}$ naar $465 \mu \mathrm{L}$. Detectie van cytokines was succesvol in $95-100 \%$ van de EBC samples. De concentraties van cytokines waren niet significant verschillend voor en na recirculatie $(p>0.232)$. Bij kinderen met piepen waren tumor necrosis factor (TNF)- $\alpha$ concentraties significant lager in vergelijking met kinderen zonder klachten. Concluderend is de verzameling van EBC bij jonge kinderen toepasbaar door gebruikt te maken van de aangepaste glazen condensor met een gesloten systeem en recirculatie. Deze nieuwe methode maakt het mogelijk om niet-invasief verkregen cytokine profielen te bepalen bij jonge kinderen ( $<4$ jaar) met en zonder luchtwegklachten.

Het tweede deel van dit proefschrift (sectie 2) richt zich op de diagnostiek van astma en CF met behulp van niet-invasieve inflammometrie.

De hypothese van dit deel van het proefschrift was dat ontstekingsmarkers in EBC de aanwezige luchtweginflammatie weergeven en kunnen differentiëren tussen astma en CF. Het doel van hoofdstuk 5 was om de aanwezigheid, concentratie en de korte termijn reproduceerbaarheid van Th-1 en Th- 2 gemedieerde cytokines (interferon- $\gamma$, tumor necrosis factor- $\alpha$, interleukine-10, $-5,-4,-2$ ) in EBC van kinderen met astma, CF en van gezonde kinderen, te bestuderen. Daarnaast hebben we de discriminerende waarde van deze cytokines bij kinderen met astma, CF en gezonden geanalyseerd. In 33 kinderen met astma, 12 kinderen met $\mathrm{CF}$, en in 35 gezonde kinderen is $\mathrm{EBC}$ verzameld tijdens rustademhaling met een glazen condensor. De cytokines werden gemeten met behulp van flow cytometry. De detectie van cytokines in EBC was erg laag. Interleukine-2, -4 , interferon- $\gamma$ en interleukine10 zijn gedetecteerd in respectievelijk 16\%, 16\%, 11\% en $9 \%$ van de condensaat samples. In de gezonde populatie werden interferon- $\gamma$, tumor necrosis factor- $\alpha$ en interleukine-10 gedetecteerd in respectievelijk $9 \%, 14 \%$ en $3 \%$ van de samples. Interleukine-5 en tumor necrosis factor- $\alpha$ werden niet gevonden bij kinderen met $C F$, en interleukine-2, -4 en -5 werden niet gedetecteerd in de gezonde populatie. Ook was er geen verschil in cytokine concentraties tussen kinderen met astma en CF. Op basis van deze resultaten kunnen we concluderen dat cytokines gedetecteerd kunnen worden in EBC van kinderen met astma en CF. Echter, concentraties zijn dicht bij de detectielimieten van de gebruikte analyse techniek. Deze resultaten bevestigen het belang van meer gevoelige analyse technieken van EBC..

Om de betrouwbaarheid van metingen van cytokines, chemokines en oplosbare adhesie moleculen in EBC bij kinderen met astma en bij gezonde kinderen te optimaliseren hebben we in hoofdstuk 6 een combinatie gebruikt van een glazen condensor systeem en een zeer gevoelige analyse techniek (multiplex immunoassay technologie). Markers die werden bepaald zijn: 1) cytokines: interleukine- $1 \alpha,-1 \beta,-2,-4,-5,-6,-10,-12 p 70,-13,-18$, interferon- $\gamma$, tumor necrosis factor- $\alpha, 2)$ chemokines: MIP1 $\alpha$ (CCL3), MIF, eotaxin (CCL11), RANTES (CCL5), IP10 (CXCL10), IL8 (CXCL8), MCP1, en 3) oplosbare adhesie moleculen (sICAM, sVCAM).

Zestig kinderen werden in de studie geïncludeerd: 31 met astma (71\% atopisch), en 29 gezonde controles. Het percentage positieve detecties van de biomarkers varieerde van 94-100\%, met uitzondering van eotaxin (CCL11) en RANTES (CCL5) (10\% en $45 \%$ bij gezonden respectievelijk). De variatie van biomarkers binnen personen bedroeg $5,2 \%$ tot $35.0 \%$. Bij kinderen met astma, waren de concentraties van cytokines [interleukine-2,-4,-5,-6,-13, interferon- $\gamma$ ], chemokines [MIP1 $\alpha$ (CCL3), MIF, RANTES (CCL5), IP10 (CXCL10), IL8 (CXCL8), 
MCP1], en adhesie moleculen [sICAM, sVCAM] significant hoger in vergelijking met de gezonde populatie. Deze studie laat zien dat in EBC, verzameld met een glazen condensor, cytokines, chemokines en adhesive moleculen gedetecteerd kunnen worden met behulp van multiplex immunoassay technologie. Daarnaast werden ook hogere concentraties van ontstekingsstoffen gevonden bij kinderen met astma ten opzichte van gezonde controles.

Gefractioneerd uitgeademde stikstofmonoxide (FeNO) en verschillende markers in EBC zijn significant verschillend tussen patiënten met astma, CF en gezonde controles. Desondanks, is het onduidelijk welke plaats deze markers hebben bij diagnose stelling van astma en CF en bij de bepaling van de ernst en de mate van controle van deze ziekten. In hoofstuk 7.1 is de waarde van combinaties van niet-invasieve makers geanalyseerd om onderscheid te kunnen maken tussen kinderen met astma en gezonde controles, en tussen patiënten met wisselende mate van ernst en ziekte controle. In EBC werden nitriet, nitraat, waterstofperoxide, 8-isoprostaan, interferon- $\gamma$, tumor necrosis factor- $\alpha$, interleukine-2,-4,-5,-10, en de zuurgraad $(\mathrm{pH})$ gemeten. 114 Kinderen werden geïncludeerd: 64 astma en 50 gezonde controles. FeNO, en interferon- $\gamma$ en interleukine- 4 in condensaat waren significant verschillend tussen astma en controles. Multivariate 'backward' logistische regressie modellen lieten zien dat interleukine-4 de enige significante marker was voor een astma diagnose. De mate van astmacontrole werd voorspeld door FeNO, 8isoprostaan, interferon- $\gamma$ en interleukine-4 (sensitiviteit $82 \%$, specificiteit $80 \%$ ). Voorspellers voor de ernst van astma waren FeNO, 8-isoprostaan, nitraat en nitriet (sensitiviteit $89 \%$, specificiteit $72 \%$ ).

Een vergelijkbare studie werd uitgevoerd bij 48 patiënten met CF en 50 gezonde controles (hoofdstuk 7.2). Concentraties van interferon- $\gamma$ en nitriet in $E B C$ waren significant hoger terwijl FeNO waarden significant verlaagd waren bij kinderen met CF in vergelijking met de gezonden. Multivariaat logistische regressie analyse liet zien dat een CF diagnose significant werd weergegeven door 8-isoprostaan, nitriet en interferon- $\gamma$ in EBC (sensitiviteit $78 \%$, specificiteit $83 \%$ ). Een exacerbatie van CF longziekte werd significant weergegeven door 8-isoprotaan en nitriet (sensitiviteit 40\%, specificiteit 97\%), en ernst van CF longziekte door FeNO en EBC zuurgraad (sensitiviteit 96\%, specificiteit 67\%). In deze crosssectionele studies hebben we aangetoond dat verschillende combinaties van biomarkers astma, CF, en de mate van ziekte controle en ziekte ernst kunnen weergeven.

Structurele longafwijkingen bij CF kunnen goed gezien worden op hoog resolutie computer tomografie (HRCT) scans. Er kan onder andere onderscheid worden gemaakt tussen bronchiectasiën, mucus plugging, peribronchiale verdikking, parenchym afwijkingen en air trapping. Deze afwijkingen zijn in een vroege fase al zichtbaar. Longfunctie parameters kunnen in die fase nog normaal zijn en de patiënt kan nog symptoomvrij zijn. De relatie tussen longfunctie, structurele longafwijkingen en niet-invasieve markers in CF is nog niet bekend. Hoofdstuk 8 had als hypothese dat structurele longafwijkingen (als gevolg van chronisch inflammatie en infecties) en luchtweginflammatie (vastgesteld met nietinvasieve biomarkers) belangrijke voorspellende factoren zijn voor de longfunctie in CF. In 34 patiënten met CF zijn HRCT scans, niet-invasieve markers (FeNO, en EBC-pH, nitraat, nitriet, 8-isoprostaan, waterstofperoxide, interferon- $\gamma$ ), en statistische en dynamische longfunctiewaarden geanalyseerd. HRCT scans zijn op een gestandaardiseerde en gevalideerde wijze bekeken. Over het algemeen waren correlaties tussen niet-invasieve mar- 
kers, structurele longschade en biomarkers laag. Patiënten met Pseudomonas in het sputum hadden hogere nitriet waarden en meer parenchym afwijkingen in vergelijking met $\mathrm{CF}$ patiënten zonder positieve sputumkweek met Pseudomonas. Multipele liniaire regressie modellen lieten zien dat de geforceerde vitale capaciteit (FVC), significant voorspeld werd door waterstofperoxide in EBC, en de mate van bronchiectasiën en mosaïc perfusie op de CT scan. De totale long capaciteit werd significant voorspeld door 8-isoprostaan, nitraat, waterstofperoxide in EBC en mucus pluggen op de CT scan. Concluderend, laat deze studie zien dat longfunctie waarden werden voorspeld door een combinatie van (niet-invasieve) onstekingsmarkers en structurele longafwijkingen op de HRCT scans.

Bij inflammatie bestaat er een dysbalans tussen oxidanten en anti-oxidanten (oxidatieve stress). Dit leidt tot meer lipide peroxidatie. Dit proces kan de membraanfunctie verstoren, membraan gebonden receptoren en enzymen inactiveren, en de permeabiliteit verhogen. Ook kan het reageren met antioxidanten of decomposeren na een reactie met metaal ionen (zoals ijzer en koper). De hierbij vrijkomende (bij)producten kunnen als VOCs in de uitademings lucht worden gedetecteerd.

Het analyseren van deze VOCs is een nieuwe veelbelovende techniek waarmee mogelijk verschillende inflammatoire longziekten kunnen worden gediscrimineerd. In hoofdstukken 9.1 en 9.2 zijn de mogelijkheden van deze techniek geanalyseerd om kinderen met, respectievelijk, astma en CF te onderscheiden van gezonde kinderen. In hoofdstuk 9.1, werden VOC profielen van 63 kinderen met astma en 57 gezonde controles bestudeerd. Analyse van uitgeademde lucht werd verricht met behulp van een gas chromatograaf time of flight- massa spectrometer (GC-TOF-MS). In totaal zijn 945 VOCs gebruikt in een discriminant analyse. Deze analyse liet zien dat op basis van 8 VOCs kinderen met astma en gezonde controles konden worden onderscheiden met een correcte classificatie van 92\%, een sensitiviteit van $89 \%$ en een specificiteit van $95 \%$. In hoofdstuk $\mathbf{9 . 2}$ hebben we onderzocht of 'metabolomics' van VOCs kon discrimineren tussen CF en gezonden, en tussen CF patiënten met en zonder positieve sputumkweek met Pseudomonas. Er werden 105 kinderen (48 CF, 57 controles) geïncludeerd. Met 22 VOCs kon een 100\% correcte classificatie worden gemaakt. Met 10 VOCs, was dit in $92 \%$ van de kinderen het geval. De reproduceerbaarheid van VOCs in uitgeademde lucht na een uur was goed (gemiddelde match factor tussen de profielen van 0.9). Samenvattend laten hoofdstukken 9.1 en 9.2 zien dat 'metabolomics' van VOCs in uitgeademde lucht mogelijk is en een goede reproduceerbaarheid heeft. Deze nieuwe techniek kan kinderen met astma of CF onderscheiden van gezonden. Daarnaast kan met deze techniek ook een scheiding gemaakt worden tussen CF patiënten met en zonder positieve sputum kweek met Pseudomonas.

Deel 3 van dit proefschrift richt zich op het niet-invasief monitoren van luchtweginflammatie bij astma en CF.

Extrafijn hydrofluoroalkaan (HFA) beclomethason verschilt van andere inhalatie corticosteroiden door de kleine aerosol deeltjes welke diep in de perifere luchtwegen kunnen doordringen. Daarom wordt extrafijn HFA-beclomethason met name gebruikt om perifere luchtweginflammatie bij astma te behandelen. Het doel van hoofdstuk 10 was om het anti-inflammatoire effect van extrafijn HFA-beclomethason te vergelijken met fluticasonDPI (wat een minder gunstige deeltjes grootte heeft maar wel 2,2 maal zo potent is) bij kinderen met astma aan de hand van bronchiaal en alveolair NO en inflammatoire mar- 
kers in EBC. In een prospectieve, longitudinale cross-over studie werden 33 kinderen in de leeftijd van 6 tot 12 jaar met matig ernstig astma afwisselend gedurende 3 maanden behandeld met extrafijn HFA-beclomethason (200 $\mu \mathrm{g}$ dagelijks, autohaler) en 3 maanden met fluticason-DPI (200 $\mu \mathrm{g}$ dagelijks, diskus). De primaire uitkomstparameters waren: alveolaire NO concentratie en bronchiale NO-flux. De secundaire uitkomstparameters waren inflammatoire markers in EBC, longfunctiewaarden, symptomen, exacerbaties en bijwerkingen. Alle parameters werden gemeten bij aanvang van de studie en na elke behandelperiode. De resultaten lieten zien dat alveolair en bronchiaal NO vergelijkbaar waren na de verschillende behandelingen. Ook was er geen verschil in inflammatoire markers in EBC, longfunctiewaarden en symptomen. Tijdens de laatste 2 weken van de behandeling met extrafijn HFA-beclomethason gebruikten patiënten minder $\beta 2$-agonisten. Gebaseerd op deze studie concluderen we dat het anti-inflammatoire effect van extrafijn HFAbeclomethason en fluticason-DPI in bovengenoemde doseringen vergelijkbaar is bij kinderen met matig ernstig astma.

In de hoofdstukken $\mathbf{1 1 . 1}$ en $\mathbf{1 1 . 2}$ werd de waarde onderzocht van niet-invasieve inflammometrie om exacerbaties van respectievelijk astma en CF te voorspellen. Ook is er gekeken naar het tijdsverloop van inflammatoire markers tijdens exacerbaties. Tijdens deze prospectieve, longitudinale studies gedurende 1 jaar, waren er twee maandelijkse reguliere controles op de polikliniek. Daarnaast werden er 4 extra bezoeken gepland tijdens een exacerbatie. Bij astma werd dit gepland op dag 1, 3, 5 en aan het eind van de exacerbatie. Bij CF werden deze afspraken gepland op dag 1, 5, 10 en na klinische stabilisatie. De primaire uitkomstparameter was het vóórkomen van een exacerbatie. Met behulp van een thuismonitor, werden driemaal per week de aanwezigheid, de ernst van luchtweg klachten, het medicatiegebruik en een flow-volume curve vastgelegd om een exacerbatie vroegtijdig en betrouwbaar vast te stellen. De volgende onafhankelijke parameters werden gemeten tijdens bezoeken op de polikliniek: FeNO, EBC-pH, nitriet, waterstofperoxide, interleukine-1 $\alpha,-5,-13$, interferon- $\gamma$, longfuncties en vragenlijsten. Hoofdstuk $\mathbf{1 1 . 1}$ beschrijft de resultaten van deze studie bij 40 kinderen met astma (6-16 jaar) In totaal hebben 38 van de 40 kinderen de studie afgerond. Er waren 16 kinderen die een exacerbatie doormaakten. Inflammatoire markers in EBC werden gedetecteerd in $85-100 \%$ van de samples. Univariate Cox regressie analyse liet zien dat EBC pH, interleukine-5 en de astma controle-score significante voorspellers waren voor een astma exacerbatie. Een multivariate Cox regressie analyse toonde aan dat de tijd tot het voorkomen van een exacerbatie significant voorspeld werd door de astma control score en het EBC interleukine-5. De Kaplan-Meier overlevingscurve van dit multivariate model liet zien dat kinderen met de $10 \%$ meest optimale waarden van IL-5 en astma control score, een meer dan twee keer zo laag risico hebben op het ontwikkelen van een exacerbatie in het volgende jaar.

In hoofdstuk 11.2 werden 26 patiënten met CF (6-30 jaar) geïncludeerd. 17 Patiënten ontwikkelden een exacerbatie. Hiervan kregen 8 patiënten tevens een tweede exacerbatie. In deze studie zijn CF controle (cystic fibrosis clinical score) en ernst (ShwachmanKulczycki score) scorelijsten gebruikt. In verband met het frequent voorkomen van herhaalde exacerbaties werd survival-analyse voor recurrent events ( Anderson-Gill models) toegepast. De univariate analyses toonden aan dat macrophage inhibiting factor (MIF), tumor necrosis factor- $\alpha$, interleukine- 6 , -8 en -10 significante voorspellers waren van een CF exacerbatie. Uit de multivariate analyse bleek dat MIF de sterkste voorspeller was. Het 
risico op het krijgen van een exacerbatie liep op tot $100 \%$ bij patiënten met lage MIF concentraties. Symptomen en longfunctie waren geen significante voorspellers. Samenvattend tonen deze studies dat met behulp van niet-invasieve inflammatoire markers in EBC een astma en CF exacerbatie kunnen worden voorspeld, gemiddeld een maand voordat een exacerbatie klinisch manifest werd.

In vergelijkbare studies hebben we in hoofdstukken $\mathbf{1 2 . 1}$ en $\mathbf{1 2 . 2}$ de voorspellende waarde van VOCs in uitademingslucht onderzocht. In deze studies werd ook de chemische achtergrond van de meest voorspellende VOCs geanalyseerd. In hoofdstuk $\mathbf{1 2 . 1}$ hadden 16 patiënten van de 40 geïncludeerde kinderen met astma een exacerbatie. Drie van deze kinderen maakten een tweede exacerbatie door. Op basis van 6 VOCs konden exacerbaties van stabiele ziekten correct worden geclassificeerd in $96 \%$ van de gevallen. Met support vector machine analyse, bestond het meest optimale model, bij de vergelijking tussen metingen tijdens een stabiele fase versus metingen voorafgaande aan een exacerbatie, uit 6 VOCs (correcte classificatie 96\%, sensitiviteit 100\%, specificiteit 93\%). Het model op basis van metingen van patiënten zonder een exacerbatie en metingen voorafgaande aan een exacerbatie, bestond uit VOCs (correcte classificatie 91\%, sensitiviteit 79\%, specificiteit 100\%). Alle VOCs werden geïdentificeerd als koolwaterstoffen. In hoofdstuk $\mathbf{1 2 . 2}$ maakten 17 van de 26 geïncludeerde CF patiënten een exacerbatie door. Hiervan hadden 8 patiënten een 2 e exacerbatie tijdens de studie. Het voorkomen van 18 VOCs waren significant verschillend tussen samples van 3-8 weken voor het ontstaan van de exacerbatie ten opzichte van tijdens de exacerbatie (binnen personen vergelijk). Met behulp van support vector machine analyse was er op basis van 6 VOCs een correcte classificatie van deze binnen patiënt vergelijking mogelijk in $94 \%$ (sensitiviteit 94\%, specificiteit 95\%). Daarnaast waren er 15 VOCs significant verschillend tussen patiënten die geen exacerbatie hebben gehad tijdens de studie en patiënten met een exacerbatie (tussen personen vergelijk). Analyse van deze dataset liet zien dat met 8 VOCs $92 \%$ van de exacerbaties goed kon worden geclassificeerd (sensitiviteit $100 \%$, specificiteit $84 \%$ ). De VOCs met een voorspellende waarde voor een CF exacerbatie waren met name lange keten koolwaterstoffen. Samenvattend laat deze studie zien dat VOCs in uitgeademde lucht een CF exacerbatie enkele weken voordat deze klinisch manifest is voorspeld.

Hoofdstuk 13 bevat een algemene discussie inclusief mogelijkheden voor toekomstig onderzoek. De paradox in de dagelijkse praktijk is, dat hoewel chronische luchtweginflammatie in de pathofysiologie van astma en CF centraal staat, deze ontsteking niet routinematig gemeten wordt. In het laatste decennium is het aantal publicaties betreffende niet-invasieve inflammometrie exponentieel gestegen. Verschillende methoden van uitgeademde lucht analyse zijn mogelijk: 1) metingen van FeNO, 2) analyse van biomarkers in EBC, 3) metingen van VOCs in uitgeademde lucht. Dit proefschrift laat zien dat uitgeademde luchtweganalyse een goede aanvulling kan zijn op conventionele parameters zoals symptomen, lichamelijk onderzoek en longfunctie bij de diagnostiek en monitoring van kinderen met astma en CF. Toekomstige studies zijn nodig om te onderzoeken welke (combinatie van) markers van uitgeademde lucht het meest waardevol zijn in de dagelijkse praktijk bij een specifieke ziekte en vraagstelling (b.v. diagnostiek versus monitoring). 



\section{Dankwoord}

Het dankwoord is wellicht het meest gelezen stuk van een proefschrift. Zonder de hulp, ondersteuning en medewerking van vele mensen was deze promotie niet mogelijk geweest.

Allereerst wil ik de hoofdpersonen van mijn proefschrift bedanken; de kinderen en hun ouders. Zonder jullie hadden we nooit zoveel resultaten kunnen boeken. Zeker in de FLAME studie hebben we veel van jullie gevraagd. Ik vind het super dat jullie medewerking aan al die metingen hebben gegeven! Ik wens jullie het beste en hoop dat het goed met jullie gaat.

Dr. E. Dompeling en dr. Q. Jöbsis, mijn co-promotoren. Beste Edward, beste Rijn, bedankt voor jullie begeleiding. Dankzij jullie tussenkomst is mijn wetenschapsstage uitgegroeid tot een promotie-onderzoek. Vanaf het eerste begin heb ik onze samenwerking als heel prettig, persoonlijk en gezellig ervaren. Jullie hebben me altijd bij alle facetten van het onderzoek volledige vrijheid en ondersteuning gegeven. Dat ging overigens niet ten koste van mijn eigen ideeën waardoor dit proefschrift echt mijn 'kindje' is geworden. Edward, ik waardeer je om je positieve instelling, je doorzettingsvermogen, je open houding alsmede je persoonlijke belangstelling. Ondanks de drukte was er ook altijd tijd voor overleg en een kop koffie. Een goede kinder(long)arts en een goede onderzoeker. Je hebt me laten zien wat je allemaal kunt bereiken in een relatief korte tijd met een aanstekelijk enthousiasme. Dit heeft me vaak geïnspireerd. Rijn, een goede reisleider en altijd in voor een grapje. Jij kunt heel goed prioriteiten stellen en bent voor mij een goed voorbeeld hoe je werk en privé kan combineren. Ook bij jou heb ik me altijd veilig gevoeld om dingen te bespreken. In het onderzoek ben je heel precies en kun je erg kritisch zijn waardoor je me weer aan het denken had gezet. Ik had me geen betere co-promotoren kunnen wensen en hoop dan ook dat de samenwerking na het beëindigen van mijn proefschrift zal doorgaan.

Prof.dr. L.J.I. Zimmermann, mijn promotor en opleider. Beste Luc, bedankt voor je begeleiding en je kritische noten.

Dr. J.J.E. Hendriks, kinderlongarts. Beste Han, van jou heb ik met name veel geleerd over de behandeling en begeleiding van patiënten met CF. Bedankt voor jou hulp met het includeren van de CF patiënten voor de studies, je begeleiding en voor de feedback op de artikelen.

Dr. P.P.R. Rosias, kinderarts en mijn voorganger in EBC. Beste Philippe, we hebben een tijd veel samengewerkt waardoor ik goed op de hoogte was van de ontwikkelingen van jouw projecten. Dat heeft zeker positief bijgedragen in mijn wetenschappelijke ontwikkeling. Bedankt voor de samenwerking. 
Dr. H.J.L. Brackel (Catharina ziekenhuis), drs. J.L. Yntema (Sint Radboud ziekenhuis) en dr. R. van Gent (Máxima Medisch Centrum), kinderlongartsen. Beste Hein, Jan-Bart en René, bedankt voor jullie hulp met het includeren van de laatste CF patiënten die we nodig hadden om de studies af te ronden.

Dr. J.E. Dankert-Roelse, kinderlongarts en opleider kindergeneeskunde in het Atrium Medisch Centrum. Beste Jeannette, Heerlen was mijn startpunt voor de opleiding tot kinderarts. Bij jullie heb ik een fijne en leerzame tijd gehad. Bedankt voor je openheid en het begrip voor mijn situatie. Wellicht kunnen we in de toekomst samenwerken op het gebied van CF.

Drs. K.D.G. van de Kant en drs. D. van Vliet, Kim en Dillys, mijn eerste student-assistenten. Twee vriendinnen van elkaar, beiden goede, enthousiaste, hardwerkende en leergierige studenten. Het idee van de stage was dat jullie mij zouden ontlasten met de metingen, maar ook zelf veel zouden leren van onderzoek opzetten, analyseren en opschrijven. Ik denk dat dit zeker is gelukt. Het is een vruchtbare periode geweest voor ons allen. Beste Kim, ik vind het geweldig dat je nu ook bezig bent met je promotie in de niet-invasieve inflammometrie bij jonge kinderen. Je bent altijd in om iets gezelligs te ondernemen. Het is fijn om met je samen te werken. Beste Dillys ook de samenwerking met jou was heel prettig. $\mathrm{Na}$ je stage heb je me nog een paar maanden geholpen. Je bent een hele harde werker met een heel groot hart. Ik vind het super dat je nu ook geneeskunde hebt afgerond en zou graag je directe collega zijn. Dames, nogmaals bedankt voor jullie werk en veel succes in jullie toekomst. Drs. M. Roozeboom en drs. J. Theunissen, destijds geneeskunde studenten. Marieke en Jasmijn, ook jullie hebben de wetenschapsstage goed volbracht. Bij deze wil ik jullie ook bedanken voor jullie hulp en inzet. Heel veel succes in jullie verdere carrière.

Peggy van den Heuvel, secretaresse. Beste Peggy, al die jaren heb ik op je kunnen rekenen. Je hebt veel werk voor me uit handen gehaald en denkt altijd een stap vooruit. Bedankt voor al je werk.

Sandra, Peggy, Carmen, Gabby, Jolanda en Petra, de poli dames. Allereerst was het heel gezellig bij jullie. Ik kan het erg waarderen dat jullie het geen probleem vonden dat ik een kamer bezet hield met alle spullen voor de metingen van de kinderen. Bedankt voor jullie hartelijkheid.

D. Holslag, CF verpleegkundige. Beste Dorien, jij hebt me een hele andere kant laten zien van de begeleiding van CF patiënten waar je als arts minder mee te maken hebt. Je bent altijd bereid geweest me te helpen met het benaderen van patiënten. De samenwerking was altijd ontspannen en gezellig. Bedankt voor je inzet en behulpzaamheid.

Een dankjewel voor de longfunctie afdeling is zeker op zijn plaats. Bij jullie voelde ik me altijd welkom. Een woord van speciale dank voor Marie-José Hermans en Paul Bongaerts voor hun flexabiliteit en medewerking, en Stefan Vervuren, op wie ik altijd kon rekenen voor technische ondersteuning. 
Meer dan 1000 metingen leveren meer dan 5000 cupjes met luchtwegcondensaat op die, na het vinden van de juiste analyse techniek, het liefst gelijktijdig op een zo kort mogelijke termijn bepaald moesten worden. Deze uitdaging is uitzonderlijk goed gelukt. Dat was alleen mogelijk door de prettige, open, directe samenwerking met de verschillende laboratoria. Graag wil ik de volgende mensen, en de betreffende analisten hier niet bij naam genoemd, bedanken: prof.dr. A. Bast en dr. G.J.M. den Hartog (farmacologie en toxicologie), dr. J.G.M.C. Damoiseaux (klinische en experimentele immunologie, academisch ziekenhuis Maastricht), prof.dr. F.J. van Schooten (gezondheidsrisicoanalyse en toxicologie, GRAT, universiteit Maastricht), dr. W.K.W.H. Wodzig (klinische chemie, academisch ziekenhuis Maastricht), dr. G.T. Rijkers (medische microbiologie en immunologie, Sint Antonius ziekenhuis, Nieuwegein).

Ook wil ik de andere co-auteurs van mijn publicaties dr. S. Robben, prof.dr. H.A.W.M. Tiddens, dr. P.A. de Jong, prof.dr. C.P. van Schayck, prof.dr. E.F.M. Wouters, dr. J.W. Dallinga, J.B.N. van Berkel, dr. A. Kester, prof.dr. R. Braeker, dr A. Gaddah bedanken voor het delen van hun expertise.

Zonder financiële ondersteuning kan geen onderzoek worden gedaan. Hierbij wil ik AstraZeneca B.V., Nederlandse CF stichting, Teva Pharma Nederland B.V., prof.dr. Jaap Swieringa stichting en Merck Sharp \& Dohme B.V. bedanken voor hun financiële steun voor de onderzoeksprojecten.

Dr. B. Panis, drs. J.V. Been en drs. R. Moonen, mijn kamergenoten. Beste Bianca en Jasper, met jullie heb ik het langst samen gezeten op de kamer. We waren een goed team met voldoende afleiding tussen het werken door. Samen koffie drinken, samen squashen, samen mopperen op de ethische commissie, en samen blij zijn met weer een geaccepteerd artikel. Bedankt voor de gezelligheid. Beste Rob, met jou heb ik minder lang op de kamer gezeten. Maar ook met jou heb ik graag de kamer gedeeld. Veel geluk in jullie verdere carrières.

Waar ben je zonder je vrienden: Greteke Heule, Marjolein en Ewout Boogaart-Nijsen, Marieke Bekker, Ingeborg en Jan-Willem van der Velden-Pater, Vera Ruchti, Miriam Pluym, Monique Venema, Lisette Walbeehm-Hol, Mascha Becker, Rosalie van StormboekBurgers, Olga Thönissen, Astrid Cantineau, Pepijn Gielkens, Sjoerd Dijkema, Hendrik Hanssens, Marrit Hilwerda, Remco Stoutenbeek, Marlous Hamersma, Mike Vervoort, Floris Mulkens en Mireille Pot, Sabine Janssen. Lieve vrienden, jullie vriendschappen zijn erg belangrijk voor me. Ik hoop dat we elkaar nog lang blijven zien.

Connie Dees, Svintha Bootsma, Eva Bootsma, Oma Dees, Fabrice Papa, Bé de Boer, mijn schoonfamilie. Lieve allemaal, ondanks dat het soms moeilijk te begrijpen was waarom altijd de laptop mee ging, hebben jullie me wel gesteund in het proces. Ik vind het fijn dat jullie mijn schoonfamilie zijn.

Lieve Anne-Claire, Georgette en Daniel, mijn zussen en broer, Martijn Joon en Merel Slingenberg. We gaan allemaal een eigen pad maar we staan altijd voor elkaar klaar. Ik ben er trots op dat jullie mijn familie zijn. Bedankt dat jullie er zijn! Lieve Georgette, bedankt met 
het helpen ontwerpen van de kaft. Het is precies geworden wat ik wilde. Lieve Daniel, ik vind het super dat je naast me staat als mijn paranimf.

Lieve mama, lieve papa, het is moeilijk in woorden uit te drukken wat jullie voor mij betekenen. Jullie staan altijd voor me klaar en hebben mij altijd alles gegeven wat ik kon wensen. Dat heeft gezorgd voor een hele sterke basis van waaruit ik me heb kunnen ontwikkelen. Lieve mama, wij hebben een nauwe band. Je bent lief, energiek, creatief, veelzijdig, verzorgend, hebt altijd een luisterend oor, je bent een harde werker. Niets is je teveel en je zorgt voor alles en iedereen in de familie. Je staat zeker met Lucas altijd voor ons klaar waarvoor ik je ook via deze weg wil bedanken. Lieve papa, je hebt me geleerd dat niks onmogelijk is. Werk hard en met plezier, volg je droom en geniet van het leven, zijn enkele van je uitspraken. Je hebt een brede interesse en een kritische blik die me meerdere malen aan het denken heeft gezet. We lijken veel op elkaar. Ik ben trots dat jij mijn paranimf bent.

Sander en Lucas, mijn lieve mannen. Lieve Sander, mijn rots in de branding. Woorden die weergeven wat jij voor mij betekent schieten tekort. We hebben de laatste jaren veel samen meegemaakt. Na mijn ziekte beginnen met de opleiding gecombineerd met het afronden van mijn proefschrift, was pittig. Maar met jou aan mijn zijde heb ik het gehaald. Je bent sterk, lief, vrolijk, creatief, nuchter en staat altijd voor me klaar. Je kunt me in elke gemoedstoestand laten lachen en mijn zorgen verlichten. Ik ben heel dankbaar dat je er voor me bent en ben trots je vrouw te zijn. Ik kijk met veel plezier naar het verleden, het heden en naar de toekomst.

Lieve Lucas, tot nu toe heb je mama vaak met de laptop gezien. Dat gaat nu zeker veranderen. Ook jij hebt me met de laatste loodjes geholpen. Als ik aan het werk ben, trek je aan mijn arm en zeg je 'mama, eraf'. Jouw lach, je armen om mijn nek, alle verhalen die je te vertellen hebt, eigenlijk je hele zijn, maakt me heel blij en relativeert mijn zorgen. Ik kijk ernaar uit vaker met jou naar de bloemen te kijken, de eendjes te voeren, lekker naar buiten te gaan, boekjes te lezen en nieuwe avonturen te beleven. 


\section{Curriculum Vitae}

Charlotte MHHT Robroeks is geboren op 24 juni 1978 te Boxtel. In 1997 behaalde zij het VWO diploma aan het Sint Maartenscollege te Maastricht. Daarna volgde zij haar studie geneeskunde aan de Rijksuniversiteit Groningen. In december 2003 ontving Charlotte haar doctoraal- en artsenbul. De co-schappen doorliep zij grotendeels in het Sint Elisabeth Ziekenhuis te Willemstad, Curaçao. Zij volbracht haar wetenschapsstage binnen de afdeling kinderlongziekten in het academisch ziekenhuis Maastricht.

Geïnspireerd door haar begeleiders en kinderlongartsen dr. Edward Dompeling en dr. Rijn Jöbsis, werd zij per januari 2004 arts-onderzoeker en bouwde zij deze stage uit tot een wetenschappelijk onderzoek met als resultaat 17 WI 1 publicaties en 2 nationale publicaties. Hiervan zijn 15 publicaties opgenomen in dit proefschrift. Tijdens de weg die gevolgd werd naar verbetering van analyse- en onderzoeksmethoden van uitademingsluchtcondensaat werd een efficiënte condensor met een gesloten systeem ontwikkeld waarvan Charlotte mede patenthouder is. Met deze nieuwe toepassing kan luchtwegcondensaat op alle leeftijden verzameld worden.

Sinds 15 oktober 2007 is Charlotte begonnen met haar opleiding tot kinderarts in het academisch ziekenhuis Maastricht (opleider prof.dr. Luc JI Zimmermann). De perifere stage volbracht zij in het Atrium Medisch Centrum te Heerlen (opleider dr. Jeannette E Dankert-Roelse).

In juni 2007 trouwde zij met Sander Bootsma. Zij zijn trotse ouders van Lucas. 


\section{List of publications}

Robroeks CMHHT, Dompeling E, Jöbsis Q. Exhaled breath analysis as a non-invasive monitoring instrument of chronic airway inflammation in children with cystic fibrosis. CF Nieuws NCFS 2005;36:38-39.

Robroeks CMHHT, Jöbsis Q, Damoiseaux JGMC, Heijmans PHM, Rosias PPR, Hendriks JJE, Dompeling E. Cytokines in exhaled breath condensate of children with asthma and cystic fibroris. Annals of Allergy, Asthma and Immunology 2006;96:349-355.

Robroeks CMHHT, van de Kant KDG, Jöbsis Q, Hendriks JJE, van Gent R, Wouters EFM, Damoiseaux JGMC, Bast A, Wodzig WKWH, Dompeling E. Inflammatory biomarkers in exhaled breath (condensate) predict asthma control, severity and diagnosis. Clinical and Experimental Allergy 2007;39:1303-1311.

Robroeks CMHHT, van de Kant KDG, van Vliet D, Kester AD, Hendriks JJE, Damoiseaux JGMC, Wodzig WKWH, Rijkers GT, Dompeling E, Jöbsis Q. Comparison of the antiinflammatory effects of extra-fine hydrofluoroalkane-beclomethasone vs fluticasone dry powder inhaler on exhaled inflammatory markers in childhood asthma. Annals of Allergy, Asthma and Immunology 2008;100:601-607.

Robroeks CMHHT, Rijkers GT, Jöbsis Q, Hendriks, JJE, Damoiseaux JGMC, Zimmermann LI, van Schayck CP, Dompeling E. Increased cytokines, chemokines and soluble adhesion molecules in breath condensate of asthmatics. Clinical Experimental Allergy 2010;40(1):77-84.

Robroeks CMHHT, Rosias PPR, van Vliet D, Jöbsis Q, Yntema JL, Brackel HJL, Damoiseaux JGMC, den Hartog GJM, Wodzig WKWH, Dompeling E. Biomarkers in exhaled breath condensate indicate presence and severity of cystic fibrosis in children. Pediatric Allergy and Immunology 2008;19(7):652-659.

Robroeks CMHHT, Roozeboom MH, de Jong PA, Tiddens HAWM, Jöbsis Q, Hendriks JJE, Yntema JL, Brackel HL, van Gent R, Robben S, Dompeling E. Structural lung changes, lung function, and inflammatory markers in cystic fibrosis. Pediatric Allergy and Immunolo, in press.

Robroeks CMHHT, van Vliet D, Dompeling E, Hendriks JJE, Jöbsis Q. Feasibility of exhaled nitric oxide measurements at various flow rates in children with asthma. Pediatric Allergy and Immunology 2010;21(1):e222-e228. 
Robroeks CMHHT, Schrander JJP, Heurn van LWE, Sie GH, Schrander-Stumpel CThRM. Multiple Endocrine Neoplasia (MEN) type 2B: a plea for prophylactic thyroidectomy and lymph node dissection in the first year of life. Journal of Pediatric Surgery, in press.

Robroeks CMHHT, van Berkel JJBN, Dallinga JW, Jöbsis Q, Zimmermann LI, Hendriks JJE, Wouters EFM, van Schooten FJ, Dompeling E. Metabolomics of volatile organic compounds in exhaled breath of subjects with cystic fibrosis and controls. Pediatric Research, in press.

+Robroeks CMHHT, Jöbsis Q, Rijkers GT, Wodzig WK, Bast A, Hendriks JJE, Wesseling GJ, Braekers R, Gaddah A, Zimmermann LI, van de Kant KDG, Dankert-Roelse JE, Dompeling E. Cystic fibrosis exacerbations are predicted by non-invasive markers: a one-year longitudinal study. Submitted.

Robroeks CMHHT, van Vliet D, Jöbsis Q, Braekers R, Rijkers GT, Wodzig WKWH, Bast A, den Hartog GJM, Zimmermann LI, Dompeling E. Non-invasive inflammatory markers in exhaled breath condensate predict exacerbations of childhood asthma; results of a oneyear prospective controlled study. Submitted.

Robroeks CMHHT*, van Berkel JJBN*, Jöbsis Q, van Schooten FJ, Dallinga JW, Dompeling E (* Shared first authorship). Volatile organic compounds in exhaled breath predict exacerbations of childhood asthma in a one year prospective controlled study. Submitted.

van Berkel JJBN*, Robroeks CMHHT*, Dallinga JW, Jöbsis Q, Moonen EJ, Wouters EFM, Dompeling E, van Schooten FJ (* Shared first authorship). Volatile organic compounds in exhaled breath predict exacerbations in cystic fibrosis in a one-year longitudinal study. Submitted.

Rosias PPR, Robroeks CMHHT, Hendriks JJE, Dompeling E, Jöbsis Q. Exhaled breath condensate: a space odessey, where no one has gone before... European Respiratory Journal 2004; 24(1):189-190.

Rosias PPR, Robroeks CMHHT, Niemarkt HJ, Kester AD, Vernooy JA, Suykerbuyk J, Teunissen J, Heynens J, Hendriks JJE, Jöbsis Q, Dompeling E. Breath condenser coatings affect the measurement of biomarkers in exhaled breath condensate. European Respiratory Journal 2006;28:1036-1041.

Jöbsis Q, Robroeks CMHHT, Dompeling E. Non-invasive assessment of airway inflammation by means of exhaled breath condensate analysis in children with respiratory symptoms. CARA visie 2007;2:7-11.

Hammer S, Robroeks CMHHT, van Rij C, Heynens JW, Droog R, Hendriks JJE, Dompeling E. Actual asthma control in a pediatric outpatient clinic population; Relationship with patient-perceived and doctor-assessed control, and prescribed medication. Pediatric Allergy and Immunology 2008;19(7):626-633. 
Rosias PPR, Robroeks CMHHT, Kester AD, den Hartog GJM, Damoiseaux JGMC, Rijkers GT, Zimmermann LI, Schayck CP, Jöbsis Q, Dompeling E. Biomarker reproducibility in exhaled breath condensate collected with different condensers. European Respiratory Journal 2008;31:934-942.

Rosias PPR, Robroeks CMHHT, van de Kant KDG, Rijkers GT, Zimmermann LI, Schayck CP, Jöbsis Q, Dompeling E. Feasibility of a new method to collect exhaled breath condensate in preschool children. Pediatric Allergy and Immunology 2010;21(1):e235-e244.

Dallinga JW, Robroeks CMHHT, van Berkel JJBN, Moonen EJ, Godschalk RWL, Jöbsis Q, Dompeling E, Wouters EFM, van Schooten FJ. Analysis of volatile organic compounds in exhaled breath as a diagnostic tool for asthma in children. Clinical and Experimental Allergy 2010;40:68-76.

Rosias PPR, Den Hartog GJ, Robroeks CMHHT, Bast A, Donckerwolcke RAMG, Heynens JW, Suykerbuyk J, Hendriks JJE, Jobsis Q, Dompeling E. Free radicals in exhaled breath condensate in cystic fibrosis and healthy subjects. Free Radical Research 2006;40:901-909.

Rosias PPR, Jöbsis Q, van Schayck CP, Zimmermann LI, Robroeks CMHHT, van de Kant KDG, Dompeling E. Global condensation: a 'climate change' towards better standardisation of exhaled breath condensate measurements. European Respiratory Journal 2008;31:684-685. 